Prepared in cooperation with the Georgia Department of Natural Resources, Environmental Protection Division

\title{
Water Use in Georgia by County for 2005; and Water-Use Trends, 1980-2005
}

Scientific Investigations Report 2009-5002 
Cover:

The city of Albany has a very complete system of water-works which cost about $\$ 80,000$.

The water is pumped directly from the wells to the water-tower, from which it is dis-

tributed through the city by hydrostatic pressure. This plant furnishes to the city, daily, aboout 25,000 gallons of water, which is used for all purposes.

S.W. McCallie

Assistant Geologist

A Preliminary Report on the Artesian-Well System of Georgia

Bulletin No. 7, 1898 


\section{Water Use in Georgia by County for 2005; and Water-Use Trends, 1980-2005}

By Julia L. Fanning and Victoria P. Trent

Prepared in cooperation with the Georgia Department of Natural Resources Environmental Protection Division

Scientific Investigations Report 2009-5002 


\section{U.S. Department of the Interior DIRK KEMPTHORNE, Secretary}

\section{U.S. Geological Survey \\ Mark D. Myers, Director}

\section{U.S. Geological Survey, Reston, Virginia: 2009}

For product and ordering information:

World Wide Web: http://www.usgs.gov/pubprod

Telephone: 1-888-ASK-USGS

For more information on the USGS--the Federal source for science about the Earth, its natural and living resources, natural hazards, and the environment:

World Wide Web: http://www.usgs.gov

Telephone: 1-888-ASK-USGS

Any use of trade, product, or firm names is for descriptive purposes only and does not imply endorsement by the U.S. Government.

Although this report is in the public domain, permission must be secured from the individual copyright owners to reproduce any copyrighted materials contained within this report.

Suggested citation:

Fanning, J.L. and Trent, V.P., 2009, Water Use in Georgia by County for 2005; and Water-Use Trends, 1980-2005:

U.S. Geological Survey Scientific Investigations Report 2009-5002, 186 p., Web-only publication available at http://pubs.usgs.gov/sir/2009/5002/ 


\section{Contents}

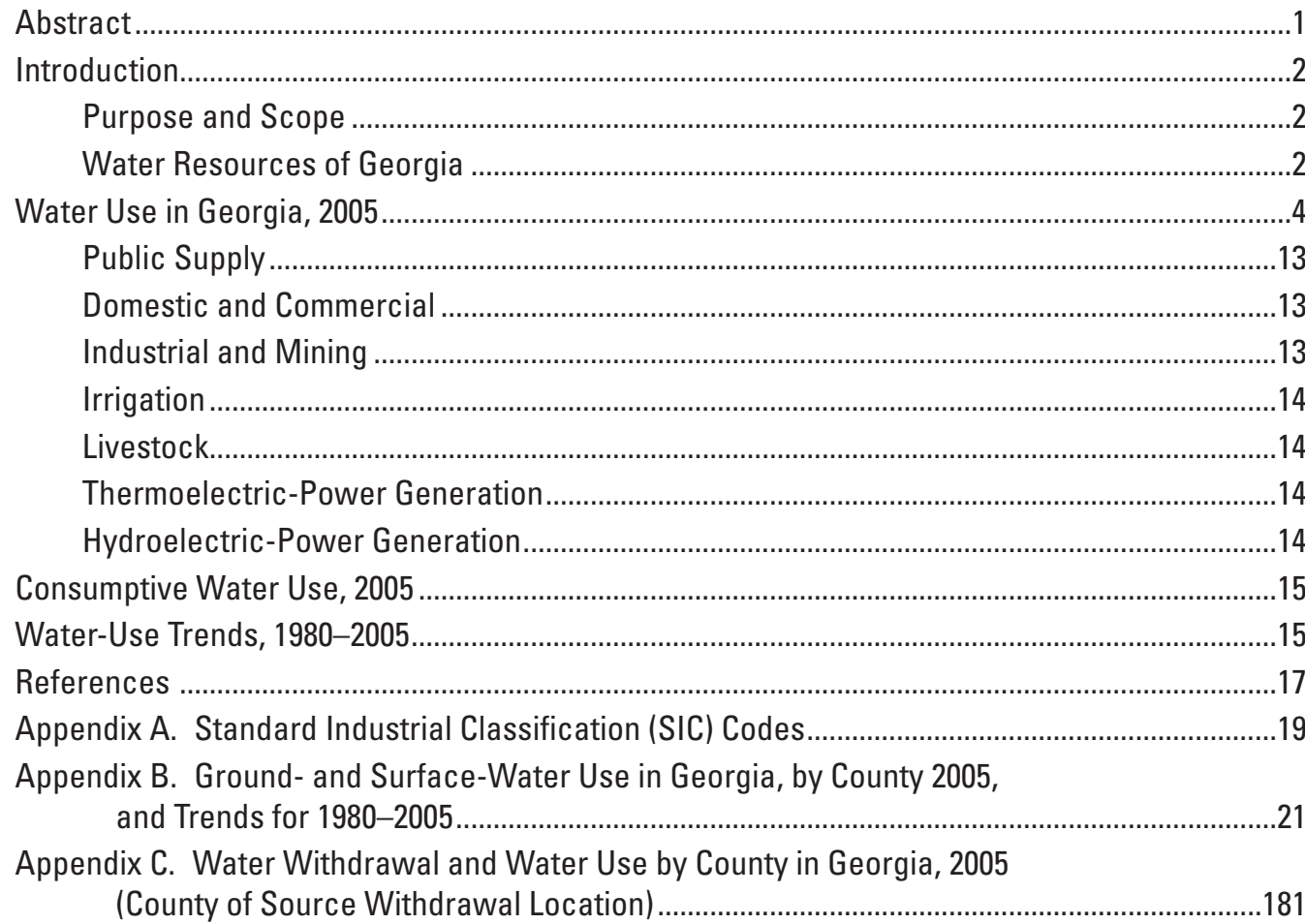

\section{Figures}

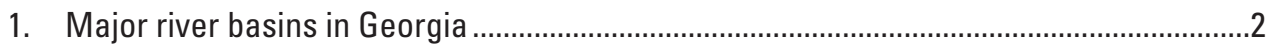

2. Area of use of principal aquifers and physiographic provinces in Georgia .......................

3. Water use by county and source in Georgia, 2005 .......................................................

4. Water withdrawals by category and source in Georgia, 2005 .......................................

5. Percentage of total water use by county and source in Georgia, 2005...........................6

6. Population and water-use trends by source in Georgia, 1980-2005 ................................16

7. Trends in total water use by category in Georgia, 1980-2005 ......................................16

\section{Tables}

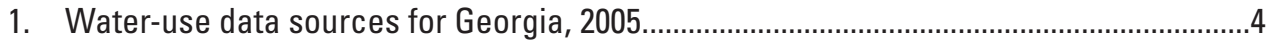

2. Water use by source and category in Georgia, 2005.....................................................

3. Industrial water use by major Standard Industrial Classification code

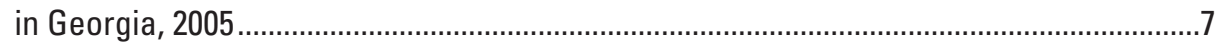

4. Surface-water use by category, by major river basin, in Georgia, 2005 .........................8

5. Ground-water use by category, by principal aquifer, in Georgia, 2005 ...........................8

6. Water use and consumptive use by county in Georgia, 2005 ......................................... 


\section{Conversion Factors}

\begin{tabular}{lcl}
\hline \multicolumn{1}{c}{ Multiply } & By & \multicolumn{1}{c}{ To obtain } \\
\hline acre & Area & \\
acre & 4,047 & square meter $\left(\mathrm{m}^{2}\right)$ \\
square mile $\left(\mathrm{mi}^{2}\right)$ & 0.00156 & square mile $\left(\mathrm{mi}^{2}\right)$ \\
& 2.59 & square kilometer $\left(\mathrm{km}^{2}\right)$ \\
\hline & Volume & \\
\hline acre-foot (acre-ft) & 0.325851 & million gallons $(\mathrm{Mgal})$ \\
gallons per day (gal/d) & 3.785 & liters per day $(\mathrm{L} / \mathrm{d})$ \\
million gallons per day (Mgal/d) & 0.003785 & million cubic meters per day $\left(\mathrm{Mm}^{3} / \mathrm{d}\right)$ \\
\hline & Energy & \\
\hline gigawatthour $(\mathrm{GWh})$ & 1,000 & megawatthour $(\mathrm{mWh})$ \\
gigawatthour $(\mathrm{GWh})$ & $1,000,000$ & kilowatthour $(\mathrm{kWh})$ \\
\hline
\end{tabular}

Horizontal coordinate information is referenced to the North American Datum of 1983 (NAD 83). 


\title{
Water Use in Georgia by County for 2005; and Water-Use Trends, 1980-2005
}

\author{
By Julia L. Fanning and Victoria P. Trent ${ }^{1}$
}

\begin{abstract}
Water use for 2005 for each county in Georgia was estimated using data obtained from various Federal and State agencies and local sources. Total consumptive water use also was estimated for each county in Georgia for 2005. Estimates of offstream water use include the categories of public supply, domestic, commercial, industrial, mining, irrigation, livestock, and thermoelectric power. The only category of instream use estimated was hydroelectric-power generation.

Total offstream water use from ground- and surface-water sources was about 5,471 million gallons per day (Mgal/d) in 2005. Surface water used in the process of thermoelectricpower generation was the largest volume of water withdrawn with withdrawals of $2,717 \mathrm{Mgal} / \mathrm{d}$ in 2005 . Estimated instream water use for hydroelectric-power generation was $54,096 \mathrm{Mgal} / \mathrm{d}$. Withdrawals for irrigation totaled $752 \mathrm{Mgal} / \mathrm{d}$ with 65 percent supplied by ground-water sources. Surface water provided 78 percent of the $1,180 \mathrm{Mgal} / \mathrm{d}$ withdrawn for public supply. Many counties in the northern Piedmont physiographic province of Georgia, an area of dense population, had a large percentage of withdrawals from surface-water sources. In contrast, in the southern Coastal Plain physiographic province part of the State, many counties had more withdrawals from ground-water sources.

As part of the Georgia Water-Use Program, statewide water-use estimates have been compiled every 5 years since 1980. During this period, water use was greatest in 1980 at $6,725 \mathrm{Mgal} / \mathrm{d}$. Water use decreased by 31 percent to 5,353 Mgal/d in 1990 then increased to 6,487 Mgal/d in 2000.
\end{abstract}

By 2005, water withdrawals had decreased to an estimated 5,471 Mgal/d primarily because of a decline in withdrawals for thermoelectric-power generation and a decline in demands as 2005 was a normal year for precipitation compared to 2000, which was in drought. Throughout the period 1980-2005, water withdrawn for thermoelectric-power generation made up the largest volume of offstream water use in Georgia. Total withdrawals for thermoelectric-power generation decreased about 24 percent in 2005 compared to 2000, due to the decommissioning of three power plants in the State. In addition, several plants operated by Georgia Power Company were retooled during this period to increase water conservation. Public-supply use steadily increased from 1980 to 2000, concurrent with increasing population in the State; however, in 2005 , there was a slight decrease in public-supply use. Conversely, industrial water use decreased during the period 1980-2005. Water withdrawals for irrigation during 1980-2005 followed changing hydrologic conditions, increasing during drier years (1980 and 2000) and decreasing during normal or wetter years. Withdrawals for the categories of domestic and commercial use remained about the same during 1980-2005. Livestock and mining use increased in 2005 compared to the 2000 estimates because of changes in estimation techniques.

Consumptive water use was determined for each category of use and compiled for each county. Estimation techniques vary for each water-use category. While consumptive use varied for each county in 2005, from about 1 percent to nearly 100 percent of total withdrawals, consumptive-use estimates for the entire State totaled 1,310 Mgal/d, about 24 percent of total withdrawals. 


\section{Introduction}

Knowledge of the quantity of water withdrawn from available water resources and how that water is used is necessary for resource management. Estimates of how much water is consumed and, therefore, no longer available for immediate use, helps quantify the amount of water available to downstream users. In addition to water withdrawal and consumption, determining water-use trends can provide a basis for estimating future needs. By examining historical patterns and current practices, local and State managers can make more realistic projections of water needs.

To provide water withdrawal and consumptive-use estimates needed for water management, the U.S. Geological Survey (USGS) - in cooperation with the Georgia Department of Natural Resources, Environmental Protection Division (GaEPD)—developed the Georgia Water-Use Program (GWUP) in 1979 (Fanning, 1985). This ongoing program focuses on collecting, compiling, and disseminating wateruse information for the State. These data are stored in a central database known as the Georgia Water-Use Data System (GWUDS) maintained by the USGS.

The report for 2005 is the seventh in a series of reports describing water use by county in Georgia. Previous reports published as part of the GWUP include summaries for 1980 (Pierce and others, 1982); 1985 (Turlington and others, 1987); 1987 (Trent and others, 1990); 1990 (Fanning and others, 1992); 1995 (Fanning, 1997); and 2000 (Fanning, 2003). This report is the first in the series to include county summaries of consumptive water use. The report also summarizes surfacewater use by basin and ground-water use by aquifer.

\section{Purpose and Scope}

This report presents water-use estimates in Georgia for selected water-use categories and estimated consumptive use during 2005. Water-use trends during the period 1980-2005 are also presented. Water-use estimates are reported by county, major river basin, and principal aquifer. Data are presented in tabular form, with maps showing locations of major water users in 2005 and graphs showing 25-year water-use trends.

\section{Water Resources of Georgia}

Water resources in Georgia are abundant and have been developed extensively. Climate, geology, and landforms control the distribution of Georgia's water resources. Georgia is a "headwaters" State, with most of the rivers beginning in northern Georgia and increasing in size downstream. Surface water is the primary source of water in northern Georgia; obtaining ground water from the underlying fractured crystalline rock in this area is more difficult than in the Coastal Plain physiographic province because of the complex geology of the area. Most ground-water withdrawals are in the Coastal Plain physiographic province of Georgia where aquifers are well defined and highly productive.

The major river basins in Georgia are the Savannah, Ogeechee, Altamaha, Satilla and St. Marys, Suwannee, Ochlockonee, Apalachicola, Mobile, and Tennessee (fig. 1). The major ground-water sources include the Floridan aquifer system, Claiborne aquifer, Clayton aquifer, Cretaceous aquifer system, crystalline-rock aquifers, and Paleozoic-rock aquifers (fig. 2).

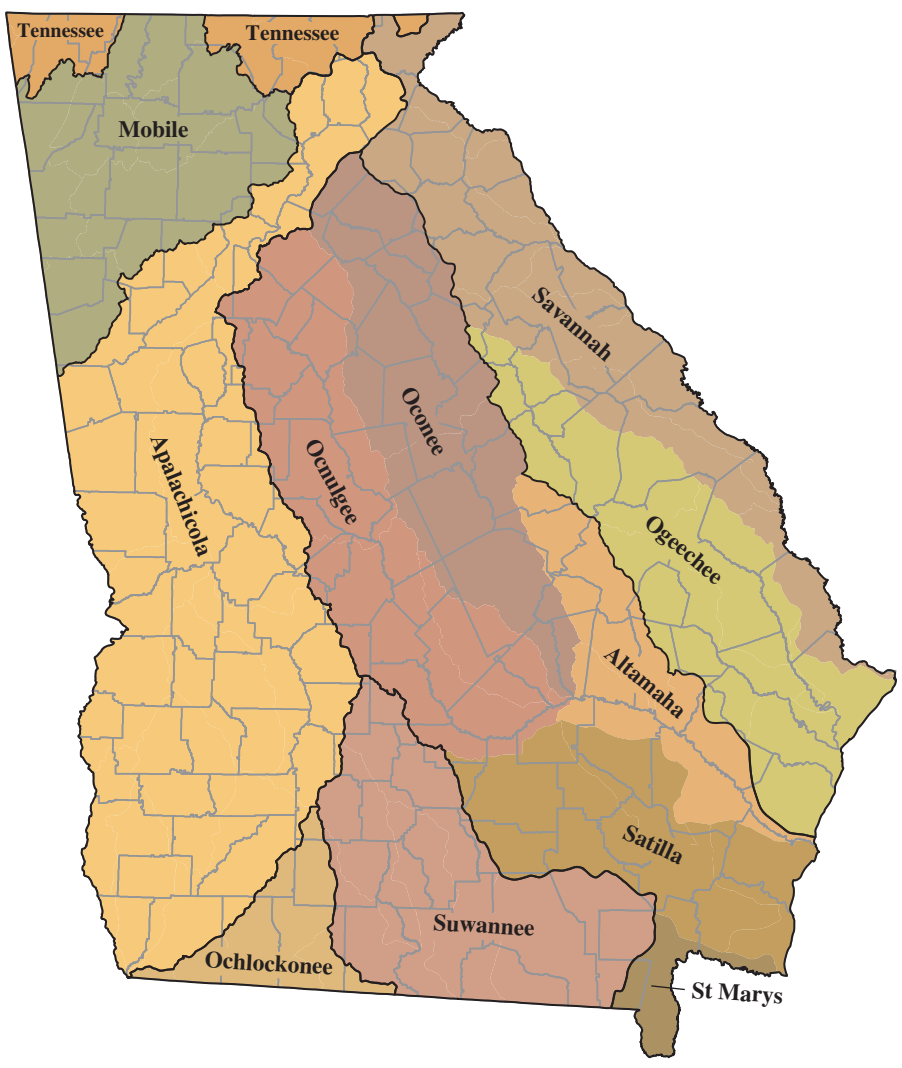

Figure 1. Major river basins in Georgia. 


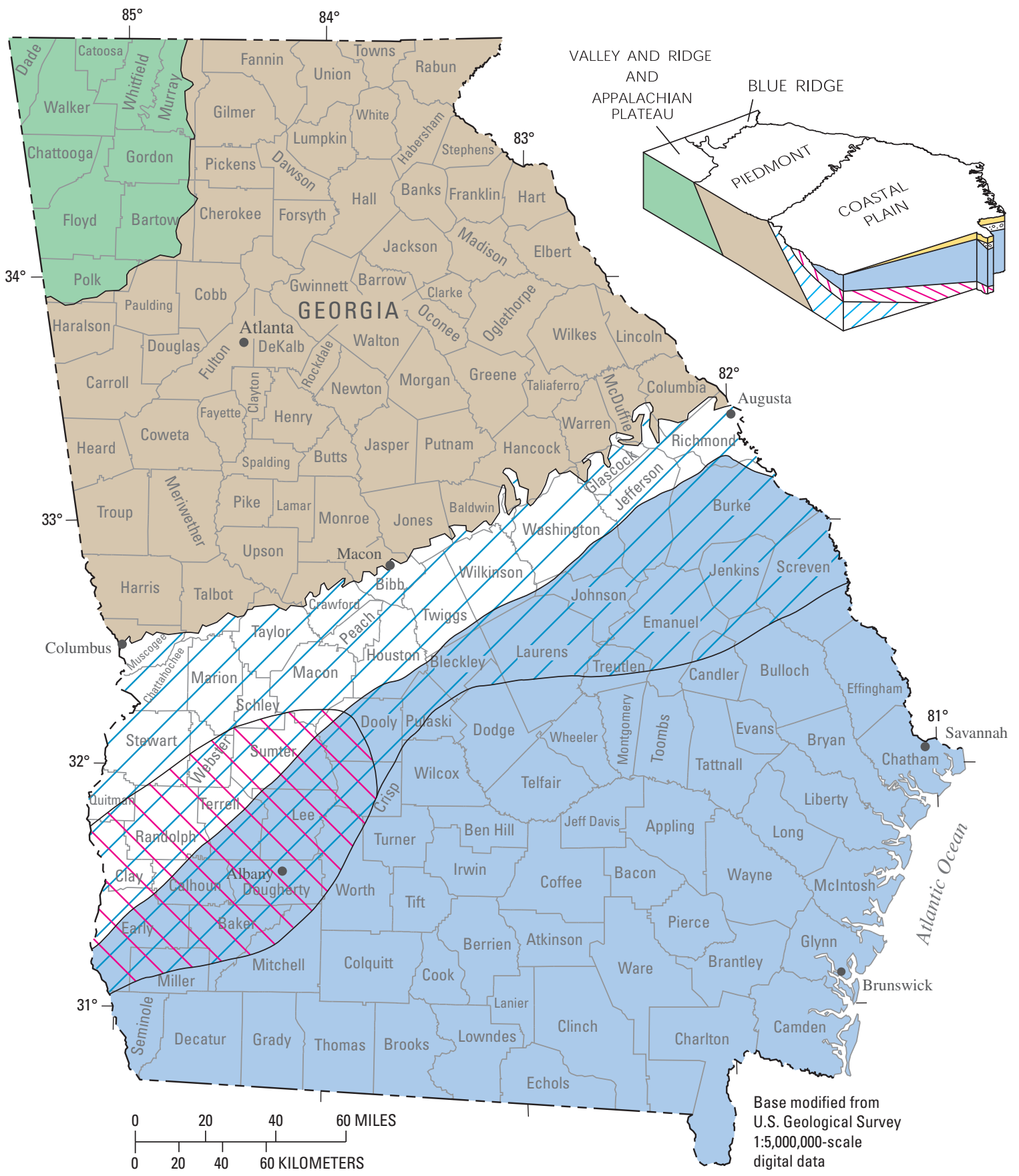

EXPLANATION

Coastal Plain aquifers

Surficial aquifer system—Not a principal aquifer. Shown on block only

Floridan aquifer system

Claiborne and Clayton aquifers

Cretaceous aquifer system

\section{Piedmont and Blue Ridge aquifers}

Crystalline-rock aquifers

\section{Valley and Ridge and Appalachian} Plateau aquifers

Paleozoic-rock aquifers

Figure 2. Area of use of principal aquifers and physiographic provinces in Georgia (modified from U.S. Geological Survey, 2006). 


\section{Water Use in Georgia, 2005}

Water use is subdivided according to offstream and instream use. Offstream use is defined as water withdrawn or diverted from a ground- or surface-water source and transported to the place of use. Offstream water-use categories include public supply, domestic, commercial, industrial, mining, irrigation, livestock, and thermoelectric power. Instream use is that which occurs within a stream channel for such purposes as hydroelectric-power generation, navigation, water-quality improvement, fish propagation, and recreation (Solley and others, 1988).

Georgia law (the Georgia Ground-Water Use Act of 1972 and the Georgia Water Supply Act of 1978) requires any water user who withdraws more than 100,000 gallons per day (gal/d) on a monthly average to obtain a withdrawal permit from the GaEPD. Permit holders generally must report their withdrawals by month. Agricultural permit holders initially were exempt from reporting, and the agricultural water-use reporting system is not yet complete. The GWUP collects the reported information under the withdrawal permit system and the drinkingwater permit system and stores the data in GWUDS (table 1).
An estimated 5,471 Mgal/d were withdrawn for offstream uses throughout Georgia in 2005 (fig. 3). An estimated 4,291 Mgal/d were withdrawn from surface-water sources (78 percent), and of that amount, 63 percent was for thermoelectric-power generation (fig. 4). Estimated ground-water withdrawals (fig. 3 , table 2), which include water obtained from springs, were 1,180 Mgal/d in 2005, mostly for irrigation (nearly 41 percent) and industrial and mining uses (nearly 24 percent). The greatest offstream use $(60 \mathrm{Mgal} / \mathrm{d}$ or greater) occurred in counties where thermoelectric-power plants are located. Exceptions include Cobb, DeKalb, Fulton, and Gwinnett Counties, located in the Metropolitan Atlanta area, where large amounts of water are used for public supply; and Early, Glynn, and Wayne Counties, where large amounts of water are withdrawn for industrial use. The percentage of county water withdrawals by ground- and surface-water sources provides an indication of the relative importance of these resources in each county (fig. 5). The only instream use summarized by the GWUP for this report is for hydroelectricpower generation. In 2005, an estimated 54,096 Mgal/d was used to generate hydroelectric power.

Table 1. Water-use data sources for Georgia, 2005.

[GaEPD, Georgia Environmental Protection Division; WPB, Water Protection Branch; USGS, U.S. Geological Survey]

\begin{tabular}{|c|c|c|}
\hline Water-use category & Data source & Type of data \\
\hline \multirow[t]{2}{*}{ Public supply } & $\begin{array}{l}\text { Permit files, GaEPD, WPB, water withdrawal } \\
\text { permitting }\end{array}$ & Withdrawals by permitted users \\
\hline & $\begin{array}{l}\text { Drinking Water Program, GaEPD, WPB, } \\
\text { water withdrawal permitting }\end{array}$ & $\begin{array}{l}\text { Population served by small public suppliers } \\
\text { (subdivisions and mobile home parks) }\end{array}$ \\
\hline Industrial & $\begin{array}{l}\text { Permit files; GaEPD, WPB, water } \\
\text { withdrawal permitting }\end{array}$ & Withdrawals by permitted users \\
\hline Mining & $\begin{array}{l}\text { J.K. Lovelace, USGS, written commun., } 2006 \\
\text { (estimated from) }\end{array}$ & Withdrawal estimates by county \\
\hline Livestock and aquaculture & National Agricultural Statistics Service data & \\
\hline \multirow[t]{4}{*}{ Irrigation } & $\begin{array}{l}\text { Cooperative Extension Service, } \\
2004 \text { irrigation survey }\end{array}$ & $\begin{array}{l}\text { Withdrawal estimates and irrigated } \\
\text { acreage by county }\end{array}$ \\
\hline & Permit files & \\
\hline & GaEPD, WPB, water withdrawal permitting & Withdrawals by permitted users \\
\hline & University of Georgia, 1999 golf survey & Acreage, water application rates \\
\hline Thermoelectric power & $\begin{array}{l}\text { Permit files; GaEPD WPB, water } \\
\text { withdrawal permitting }\end{array}$ & $\begin{array}{l}\text { Withdrawals by thermoelectric- } \\
\text { power plants generation }\end{array}$ \\
\hline
\end{tabular}



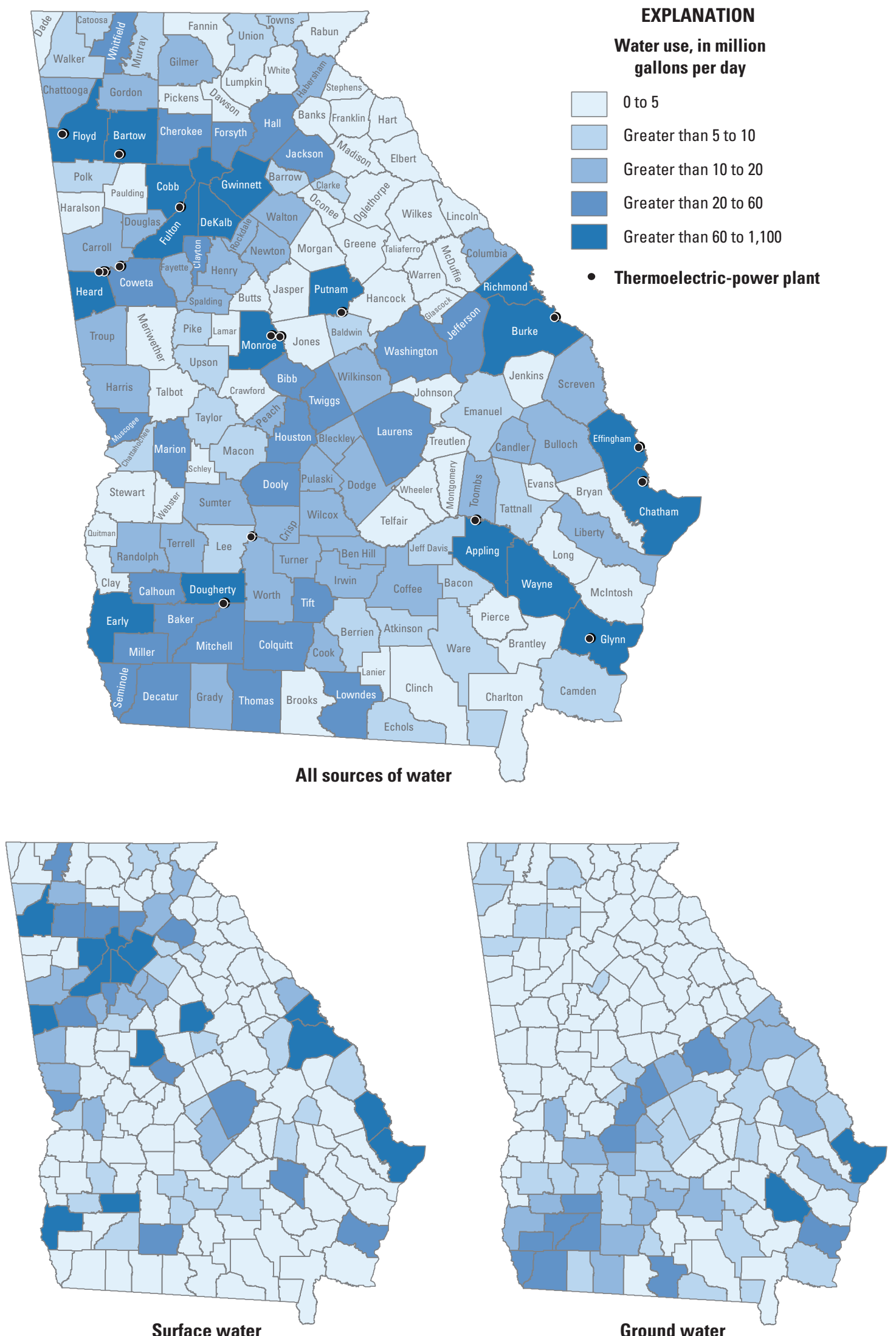

Figure 3. Water use by county and source in Georgia, 2005. 


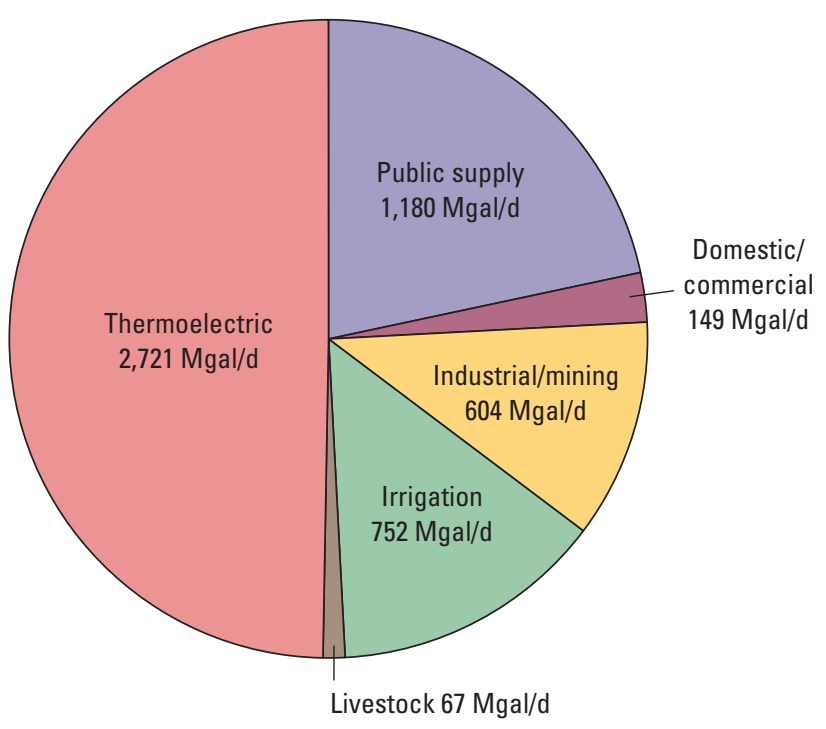

Total water withdrawals-5,471 Mgal/d

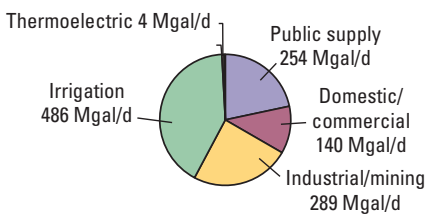

Ground-water withdrawals-1,180 Mgal/d

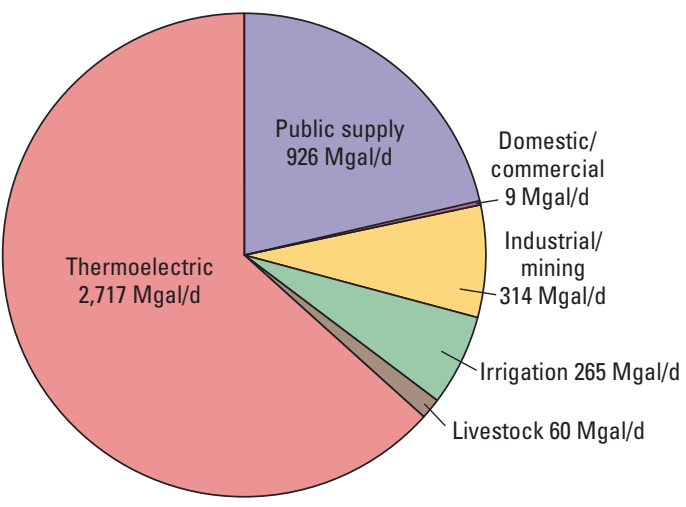

Surface-water withdrawals-4,291 Mgal/d

Figure 4. Water withdrawals by category and source in Georgia, 2005.

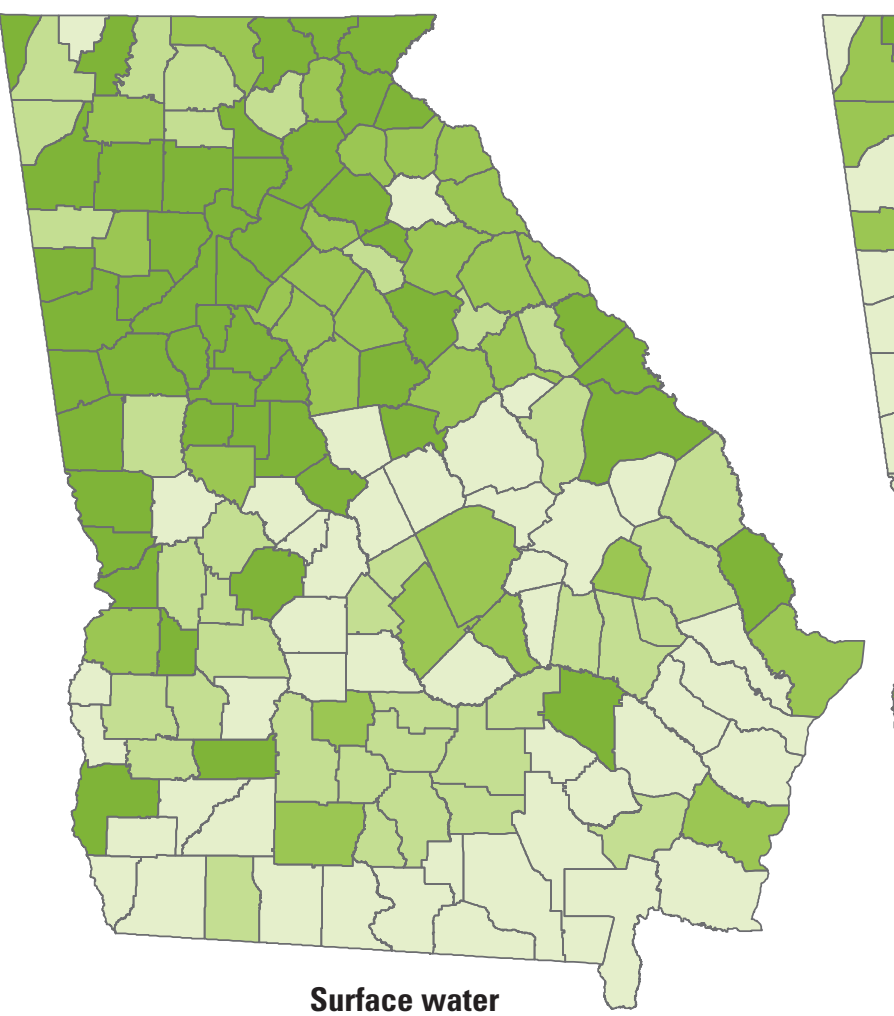

Surface water



EXPLANATION

Percentage of total water use

0 to 25

Greater than 25 to 50

Greater than 50 to 75

Greater than 75 to 100

Figure 5. Percentage of total water use by county and source in Georgia, 2005. 
Table 2. Water use by source and category in Georgia, 2005.

Population: 9,072,576

Population served by public supply: 7,472,550

Acres irrigated: $1,514,620$

Hydroelectric use (million gallons per day): 54,095.63

\begin{tabular}{|c|c|c|c|c|c|c|c|c|c|c|}
\hline & \multicolumn{10}{|c|}{ Withdrawals, in million gallons per day } \\
\hline & $\begin{array}{l}\text { Public } \\
\text { supply }\end{array}$ & Domestic & Commercial & Industrial & Mining & Irrigation & Livestock & $\begin{array}{l}\text { Aqua- } \\
\text { culture }\end{array}$ & $\begin{array}{l}\text { Thermo- } \\
\text { electric }\end{array}$ & Totals \\
\hline Surface water & 925.68 & 0.00 & 9.20 & 314.00 & 0.47 & 265.28 & 26.29 & 33.44 & $2,717.07$ & $4,291.43$ \\
\hline Total & $1,179.85$ & 119.68 & 29.05 & 554.16 & 49.40 & 751.64 & 28.44 & 38.42 & $2,720.83$ & $5,471.47$ \\
\hline
\end{tabular}

In general, surface-water resources provide the largest percentage of water withdrawal for counties in the northern half of the State, whereas ground water provides the greatest percentage for counties in the southern half of the State. There are exceptions. For several northern Georgia counties, more than 50 percent of the total county withdrawal is obtained from ground water, with ground-water withdrawals exceeding 75 percent in the relatively low water-usage counties of Catoosa, Madison, and Murray. Similarly, there are several southern Georgia counties where surface water provides more than 50 percent of the total withdrawal primarily because of thermoelectric-power plants, with percentages exceeding 75 percent in Appling, Burke, Dougherty, Early, Effingham, Macon, Richmond, and Webster Counties.

This report provides summaries of water use during 2005 in a variety of formats. The total statewide water withdrawals for 2005 by ground- and surface-water sources for public supply, domestic and commercial, industrial and mining, irrigation, livestock, aquaculture, and thermoelectric power are shown in table 2. Industrial water use by major Standard Industrial Classification (SIC) code (Executive Office of the President, Office of Management and Budget, 1987; Appendix A) is listed in table 3. Surface-water withdrawal estimates by major river basin are listed in table 4; and ground-water withdrawals by principal aquifer in the State are listed in table 5. Water withdrawal totals by category and consumptive-use totals for each county are listed in table 6 . County summaries of water use are given in Appendix B, including ground- and surfacewater withdrawals by water-use category, major public-supply systems, industrial use by SIC code, trend graphs, and a map of withdrawal locations. Small public-water suppliers (subdivisions, mobile home parks, and small towns) and military bases are not listed individually in Appendix B, but the withdrawal for each is included in the county totals.

In this report, water withdrawals are mostly reported according to the county in which the water is used; however, in some counties, water supply is derived from sources located in an adjacent county. For example, Gwinnett County derives most of its water supply from Lake Sidney Lanier in Hall County. For this reason, Appendix C presents withdrawals according to the location of the water source. Thus, the total withdrawal for Gwinnett County listed in table 6 is $94.2 \mathrm{Mgal} / \mathrm{d}$, whereas the total withdrawal listed in Appendix C is $2.9 \mathrm{Mgal} / \mathrm{d}$. The smaller amount listed in Appendix $\mathrm{C}$ indicates supplies are derived from sources outside of the county. Other counties in which water is obtained from sources in adjacent counties for 2005 include Banks, Bartow, Bibb, Clayton, Cobb, DeKalb, Douglas, Fulton, Henry, Jackson, Jones, Meriwether, Pike, Spalding, and Talbot.

Table 3. Industrial water use by major Standard Industrial Classification code in Georgia, 2005.

[SIC, Standard Industrial Classification]

\begin{tabular}{lcc}
\hline \multirow{2}{*}{ SIC } & \multicolumn{2}{c}{ Withdrawals, in million gallons per day } \\
\cline { 2 - 3 } & Ground water & Surface water \\
\hline 14-Mining & 48.93 & 0.47 \\
20-Food & 9.61 & 1.94 \\
22-Textiles & 8.85 & 6.19 \\
23-Apparel & 0.15 & 2.16 \\
26-Paper & 76.27 & 276.65 \\
28-Chemicals & 80.31 & 18.08 \\
29-Petroleum & 0.37 & 0.09 \\
30-Rubber & 0.57 & 0.01 \\
32-Stone, clay & 60.80 & 7.44 \\
33-Primary metals & 0.03 & 0.25 \\
34-Fabricated metal products & 1.10 & 0.00 \\
36-Electrical machinery & 0.42 & 0.00 \\
\hline
\end{tabular}


Table 4. Surface-water use by category, by major river basin, in Georgia, 2005.

[Figures may not sum to totals because of independent rounding]

\begin{tabular}{|c|c|c|c|c|c|c|c|}
\hline $\begin{array}{l}\text { Major river basin } \\
\text { (fig. 1) }\end{array}$ & \multicolumn{7}{|c|}{ Withdrawals, in million gallons per day } \\
\hline $\begin{array}{l}\text { Altamaha and Satilla } \\
\text { and St. Marys }\end{array}$ & 133.00 & 0.06 & 52.88 & 75.28 & 8.03 & $1,245.53$ & $1,514.78$ \\
\hline Ochlockonee & 0.00 & 0.00 & 0.54 & 16.40 & 0.34 & 0.00 & 17.28 \\
\hline Apalachicola & 525.75 & 6.90 & 121.84 & 75.92 & 16.06 & 580.04 & $1,326.51$ \\
\hline Mobile & 154.78 & 0.30 & 32.49 & 11.31 & 16.18 & 573.92 & 788.98 \\
\hline Tennessee & 13.50 & 0.00 & 2.00 & 2.09 & 9.79 & 0.00 & 27.38 \\
\hline Total & 925.68 & 9.20 & 314.47 & 265.28 & 59.73 & 2,717.07 & $4,291.43$ \\
\hline
\end{tabular}

Table 5. Ground-water use by category, by principal aquifer, in Georgia, 2005.

[Figures may not sum to totals because of independent rounding]

\begin{tabular}{|c|c|c|c|c|c|c|c|}
\hline \multirow[b]{2}{*}{$\begin{array}{l}\text { Aquifer } \\
\text { (fig. 2) }\end{array}$} & \multicolumn{7}{|c|}{ Withdrawals, in million gallons per day } \\
\hline & $\begin{array}{l}\text { Public } \\
\text { supply }\end{array}$ & $\begin{array}{c}\text { Domestic and } \\
\text { commercial }\end{array}$ & $\begin{array}{l}\text { Industrial } \\
\text { and mining }\end{array}$ & Irrigation & Livestock & Thermoelectric & Totals \\
\hline Claiborne aquifer & 18.14 & 6.07 & 2.65 & 59.99 & 1.15 & 0.85 & 88.85 \\
\hline Clayton aquifer & 9.22 & 1.64 & 6.87 & 51.94 & 0.16 & 0.18 & 70.01 \\
\hline Crystalline-rock aquifers & 18.26 & 66.42 & 15.42 & 7.80 & 0.29 & 0.02 & 108.21 \\
\hline Paleozoic-rock aquifers & 25.84 & 6.27 & 15.37 & 2.03 & 0.64 & 0.00 & 50.15 \\
\hline Total & 254.17 & 139.53 & 289.09 & 486.36 & 7.13 & 3.76 & $1,180.04$ \\
\hline
\end{tabular}


Table 6. Water use and consumptive use by county in Georgia, 2005.

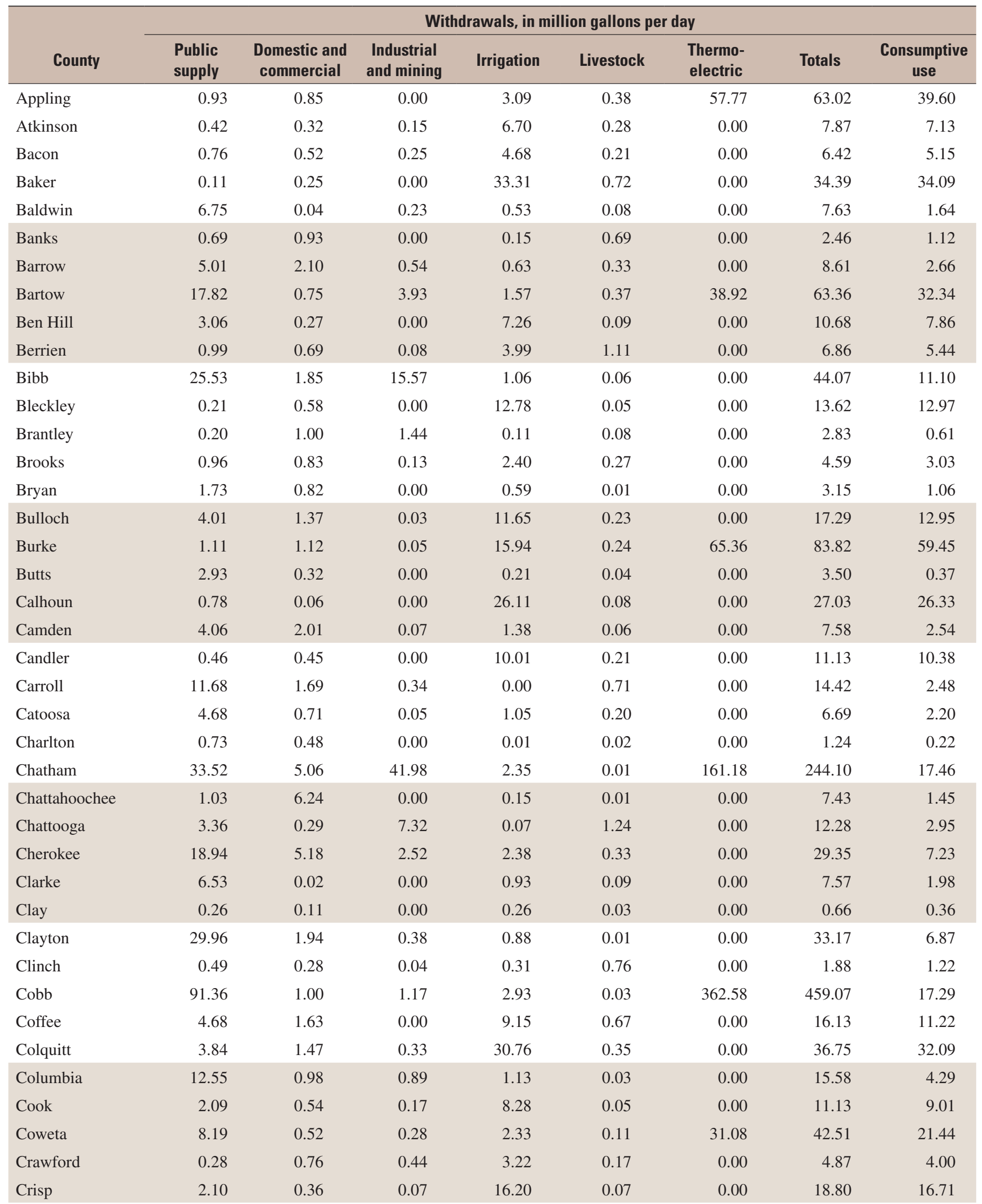


Table 6. Water use and consumptive use by county in Georgia, 2005.-Continued

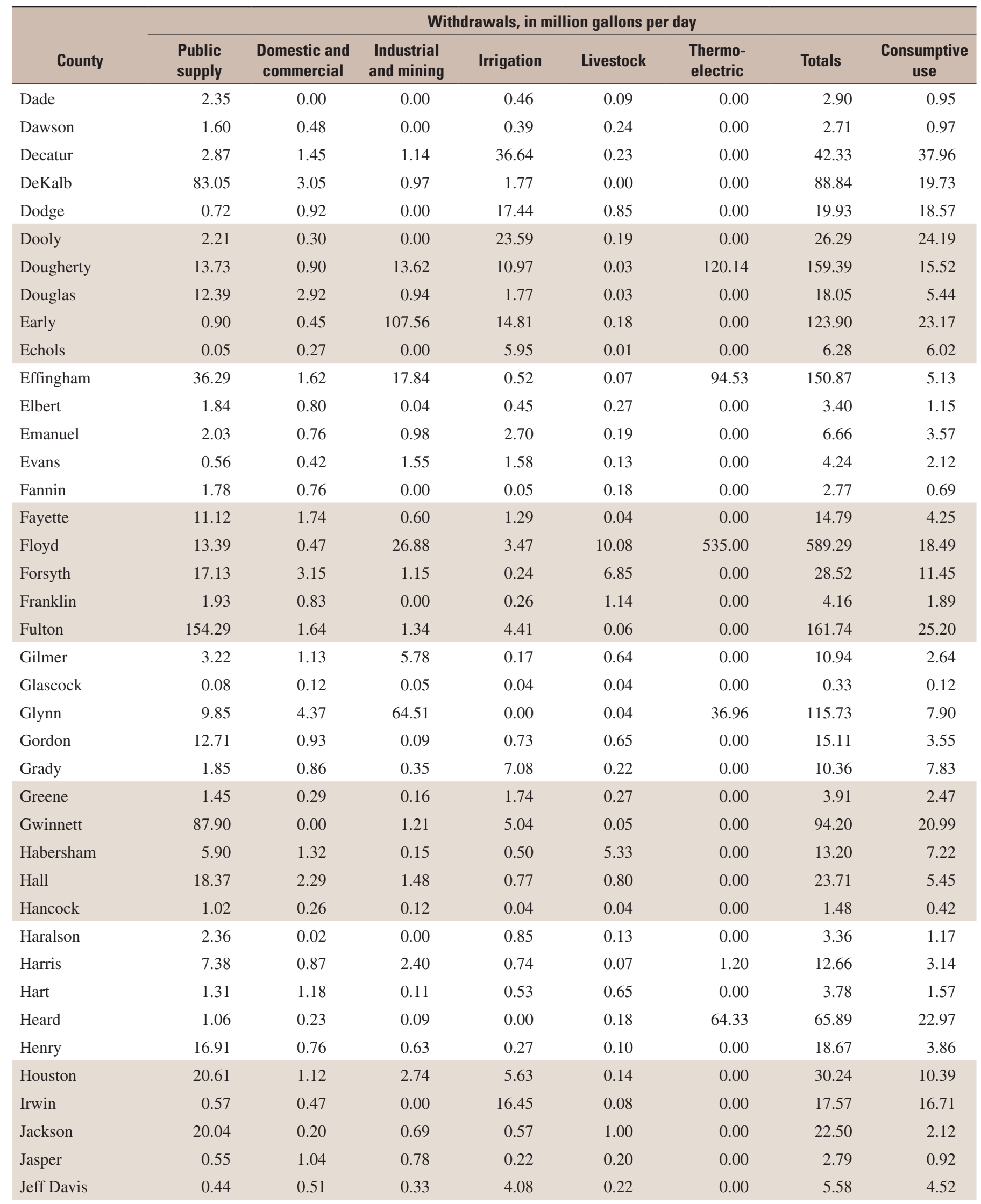


Table 6. Water use and consumptive use by county in Georgia, 2005.—Continued




Table 6. Water use and consumptive use by county in Georgia, 2005.-Continued

\begin{tabular}{|c|c|c|c|c|c|c|c|c|}
\hline \multirow[b]{2}{*}{ County } & \multicolumn{8}{|c|}{ Withdrawals, in million gallons per day } \\
\hline & $\begin{array}{l}\text { Public } \\
\text { supply }\end{array}$ & $\begin{array}{l}\text { Domestic and } \\
\text { commercial }\end{array}$ & $\begin{array}{l}\text { Industrial } \\
\text { and mining }\end{array}$ & Irrigation & Livestock & $\begin{array}{l}\text { Thermo- } \\
\text { electric }\end{array}$ & Totals & $\begin{array}{c}\text { Consumptive } \\
\text { use }\end{array}$ \\
\hline Richmond & 43.13 & 2.44 & 69.28 & 2.99 & 0.03 & 0.00 & 117.87 & 19.24 \\
\hline Rockdale & 12.54 & 4.97 & 0.15 & 0.31 & 0.02 & 0.00 & 17.99 & 3.19 \\
\hline Schley & 0.63 & 0.05 & 0.00 & 0.28 & 0.08 & 0.00 & 1.04 & 0.49 \\
\hline Screven & 1.02 & 0.68 & 1.73 & 10.79 & 0.11 & 0.00 & 14.33 & 11.41 \\
\hline Seminole & 0.63 & 0.39 & 0.00 & 38.30 & 0.12 & 0.00 & 39.44 & 38.62 \\
\hline Spalding & 8.07 & 0.87 & 0.21 & 0.81 & 0.07 & 0.00 & 10.03 & 2.57 \\
\hline Stephens & 3.97 & 0.19 & 0.11 & 0.07 & 0.23 & 0.00 & 4.57 & 1.32 \\
\hline Stewart & 0.22 & 0.04 & 0.00 & 0.78 & 0.06 & 0.00 & 1.10 & 0.88 \\
\hline Sumter & 3.05 & 0.87 & 0.71 & 9.10 & 0.23 & 0.00 & 13.96 & 10.32 \\
\hline Talbot & 0.18 & 0.14 & 0.91 & 0.00 & 0.14 & 0.00 & 1.37 & 1.14 \\
\hline Taliaferro & 0.05 & 0.08 & 0.00 & 0.00 & 0.06 & 0.00 & 0.19 & 0.08 \\
\hline Tattnall & 1.12 & 1.80 & 0.09 & 5.40 & 0.57 & 0.00 & 8.98 & 6.60 \\
\hline Taylor & 0.78 & 0.31 & 2.56 & 1.46 & 0.15 & 0.00 & 5.26 & 2.76 \\
\hline Telfair & 1.27 & 0.31 & 0.09 & 3.02 & 0.06 & 0.00 & 4.75 & 3.37 \\
\hline Terrell & 1.48 & 0.24 & 0.00 & 14.40 & 0.08 & 0.00 & 16.20 & 14.82 \\
\hline Thomas & 5.55 & 0.92 & 3.04 & 10.40 & 0.23 & 0.00 & 20.14 & 14.64 \\
\hline Tift & 4.99 & 1.00 & 0.00 & 19.89 & 0.10 & 0.00 & 25.98 & 20.77 \\
\hline Toombs & 2.67 & 0.68 & 0.04 & 7.53 & 0.16 & 0.00 & 11.08 & 8.34 \\
\hline Towns & 2.11 & 0.05 & 0.05 & 0.33 & 4.04 & 0.00 & 6.58 & 4.68 \\
\hline Treutlen & 0.40 & 0.27 & 0.00 & 0.85 & 0.02 & 0.00 & 1.54 & 0.99 \\
\hline Troup & 10.33 & 0.89 & 0.27 & 1.05 & 0.09 & 0.00 & 12.63 & 2.75 \\
\hline Turner & 0.88 & 0.24 & 0.00 & 14.61 & 0.14 & 0.00 & 15.87 & 14.96 \\
\hline Twiggs & 0.30 & 0.59 & 19.89 & 1.40 & 0.02 & 0.00 & 22.20 & 8.77 \\
\hline Union & 1.91 & 0.52 & 0.10 & 0.15 & 5.40 & 0.00 & 8.08 & 6.03 \\
\hline Upson & 2.86 & 1.16 & 0.23 & 0.50 & 0.28 & 0.00 & 5.03 & 1.40 \\
\hline Walker & 7.61 & 0.02 & 1.23 & 0.71 & 0.35 & 0.00 & 9.92 & 2.53 \\
\hline Walton & 5.20 & 2.61 & 0.00 & 2.68 & 0.26 & 0.00 & 10.75 & 3.93 \\
\hline Ware & 3.44 & 1.27 & 0.00 & 2.01 & 0.13 & 0.00 & 6.85 & 3.02 \\
\hline Warren & 0.39 & 0.20 & 2.17 & 1.74 & 0.10 & 0.00 & 4.60 & 3.19 \\
\hline Washington & 3.24 & 0.70 & 26.06 & 5.59 & 0.14 & 0.00 & 35.73 & 16.63 \\
\hline Wayne & 2.09 & 1.21 & 59.23 & 2.60 & 0.09 & 0.00 & 65.22 & 10.39 \\
\hline Webster & 0.17 & 0.08 & 0.00 & 2.64 & 0.05 & 0.00 & 2.94 & 2.73 \\
\hline Wheeler & 0.31 & 0.34 & 0.00 & 4.17 & 0.03 & 0.00 & 4.85 & 4.31 \\
\hline White & 1.89 & 1.10 & 0.01 & 0.29 & 0.37 & 0.00 & 3.66 & 1.19 \\
\hline Whitfield & 35.38 & 0.07 & 0.22 & 0.51 & 0.71 & 0.00 & 36.89 & 5.89 \\
\hline Wilcox & 0.69 & 0.28 & 0.00 & 11.42 & 0.19 & 0.00 & 12.58 & 11.78 \\
\hline Wilkes & 1.20 & 0.40 & 0.00 & 0.02 & 0.32 & 0.00 & 1.94 & 0.60 \\
\hline Wilkinson & 0.93 & 0.23 & 17.27 & 0.08 & 0.02 & 0.00 & 18.53 & 6.02 \\
\hline Worth & 1.34 & 0.89 & 0.00 & 10.66 & 0.14 & 0.76 & 13.79 & 11.18 \\
\hline Totals & $1,179.85$ & 148.73 & 603.56 & 751.64 & 66.86 & $2,720.83$ & $5,471.47$ & $1,310.21$ \\
\hline
\end{tabular}




\section{Public Supply}

Public-supply water use is water that is withdrawn by public and private water suppliers and delivered for a variety of uses, including domestic, commercial, industrial, and other uses. Small communities and subdivisions are considered to be public-supply systems if at least 25 people are served or if there are a minimum of 15 hookups or water connections (Hutson and others, 2004).

Data for public-supply water use generally were provided by the GaEPD, Watershed Protection Branch. Additional information was obtained from city managers and operators (table 1). In previous years, the GWUP conducted a survey of the largest public water-supply users in the State for this report. In 2005, however, the survey was expanded to include all permitted public-supply water users in the State. Data were obtained on the population served; the number of connections for each system; and the percentage of water delivered to wholesale, residential, commercial, and industrial customers.

Estimated public-supply withdrawal during 2005 was about 1,180 Mgal/d, of which surface water accounted for about 78 percent (926 Mgal/d), and ground water accounted for about 22 percent (254 Mgal/d) (table 2). The largest public suppliers are located in counties in the Metropolitan Atlanta area in northern Georgia, where population is greatest. Thus, surface water is the principal water source. Several counties in the area reported a decline in withdrawals in 2005, collectively totaling more than $20 \mathrm{Mgal} / \mathrm{d}$. The decrease in 2005 has been attributed to conservation measures at public-supply systems, better reporting by public-supply systems as more systems are metered, and a decrease in outdoor water use (2000 was a drought year and outdoor use, primarily lawn watering, was above normal as compared to 2005 when precipitation was normal, and outdoor uses were lower).

\section{Domestic and Commercial}

Domestic water use is water used for normal household purposes, such as drinking, food preparation, bathing, washing clothes and dishes, flushing toilets, washing cars, and watering lawns and gardens. In Georgia, most water for domestic use is provided by public suppliers; however, some households, generally in rural areas, are supplied by individual water systems, primarily wells. These households are termed selfsupplied domestic water users. Because self-supplied domestic water use generally is not metered, the GWUP conducted a survey in 1983 in the Athens, Georgia, area and estimated per capita water use was $75 \mathrm{gal} / \mathrm{d}$. Withdrawals by self-supplied domestic users were estimated by subtracting estimates of the number of persons served by public-supply systems from the total county population obtained from the U.S. Bureau of the Census, 2005, and multiplied by 75 (gal/d).
Commercial users include restaurants, hotels, retail stores, and other businesses, government and military facilities, prisons, schools, hospitals, office parks, and other institutions, and recreational facilities. Golf course irrigation is also included as a commercial use. In Georgia, there are very few commercial users large enough to require a GaEPD permit, so reported commercial water-use data are minimal, and these data were combined with estimates for smaller users.

Domestic and commercial water use was estimated to be $149 \mathrm{Mgal} / \mathrm{d}$ in 2005 (table 2), of which $120 \mathrm{Mgal} / \mathrm{d}$ was for self-supplied domestic use, and $29 \mathrm{Mgal} / \mathrm{d}$ was for commercial use. The estimated self-supplied population was 1,600,026. Statewide, an estimated 18 percent of the population is selfsupplied, and this water is assumed to be from ground water (wells and springs). Sixty-eight percent of commercial water use was from ground-water sources, and 32 percent was from surface-water sources.

\section{Industrial and Mining}

Industrial water use includes fabrication, processing, washing, and cooling for facilities that manufacture products, including steel, chemical and allied products, paper and allied paper products, mining, and petroleum refining. The largest industrial water users in Georgia are pulp and paper mills concentrated along the coast, the textile industries concentrated in northwestern Georgia, chemical manufacturers, and the mining and mineral industries.

Mining water use is water used for the extraction of minerals, including water used for quarrying, milling, and other typical preparations at a mine site. Granite, clay, marble, and other materials are mined extensively in the State, but these mining operations, other than clay, do not require large quantities of water. Mining water-use estimates were provided by the USGS National Water-Use Program from mine production data for 2004 from the USGS Minerals Information Team and the Energy Information Administration and commodity-specific coefficients (J.K. Lovelace, U.S. Geological Survey, written commun., 2007). The total mining withdrawal estimate was allocated between ground and surface water using the ratio by county for mining withdrawals for 2000 (Fanning, 2003).

Industrial and mining water-use estimates totaled $603 \mathrm{Mgal} / \mathrm{d}$ (table 2) during 2005. Self-supplied industries in Georgia used about $554 \mathrm{Mgal} / \mathrm{d}$ of water in 2005, of which 43 percent was from ground water, and 57 percent was from surface water. The industrial water-use estimate was determined from withdrawals reported to the GaEPD for 2005. There was a decrease in industrial water withdrawals between 2000 and 2005, mostly because of the closure of a major pulp and paper company in Camden County, Georgia. During 2005, the estimated mining withdrawal was about $49 \mathrm{Mgal} / \mathrm{d}$. Nearly, 99 percent of the withdrawals for mining were from groundwater sources, and 1 percent were from surface-water sources. 


\section{Irrigation}

Irrigation water use is water used for crops, large nurseries, athletic fields, and golf courses. Estimates of irrigation water use are based on a 2004 irrigation survey conducted by the University of Georgia, Cooperative Extension Service (Kerry Harrison, Cooperative Extension Service, written commun., 2004). County extension agents were asked to provide data on crop acreage, water application rates by crop for each county, and percentages of ground- and surface-water use. Withdrawal data for permitted users were provided by GaEPD. Withdrawals for golf irrigation were estimated using water application rates provided by the University of Georgia (Gil Landy and Clint Waltz, University of Georgia, written commun., 1999). In this report, the 2004 irrigation data were used because 2005 data were not available.

Estimated total irrigation water use in 2004 was about $752 \mathrm{Mgal} / \mathrm{d}$ (65 percent from ground water and 35 percent from surface water; table 2), a decrease of about $338 \mathrm{Mgal} / \mathrm{d}$ from the 2000 estimate. This 30-percent decrease results from decreased irrigation as compared to irrigation during the drought in 2000. Most irrigation occurs in the Coastal Plain physiographic province, with the largest withdrawal in the southwestern part of the State. Irrigation withdrawal accounts for more than 95 percent of total water use in some counties in southwestern Georgia. Irrigation is the largest category of ground-water use in Georgia, accounting for 41 percent of all ground water withdrawn.

\section{Livestock}

Livestock water use is water used for livestock watering, feed lots, dairy operation, and farm operation for poultry, horses, cattle, hogs, and catfish and trout farms. Estimates of water use for livestock were compiled by animal type (cattle, hogs, horses, and several kinds of poultry) and by pond acreage for catfish and trout farming. Livestock water-use estimates were provided by the USGS National Water-Use Program from U.S. Department of Agriculture, National Agricultural Statistics Service livestock population estimates, and animal specific (J.K. Lovelace, U.S. Geological Survey, written commun., 2007). Coefficients were the same as those used for the 2000 estimate of water use in Georgia (Fanning, 2003). The total withdrawal estimate was allocated between ground and surface water, using the county ratio for livestock withdrawals for 2000 (Fanning, 2003).
In this report, the livestock category also includes aquaculture, which is the farming of organisms that live in water, such as fish, shellfish, and algae. In 2005, aquaculture in Georgia included catfish and trout farms. Aquaculture water use was estimated using data for commercial and non-commercial aquaculture operations (J.K. Lovelace, U.S. Geological Survey, written commun., 2007).

Livestock water use in 2005 was estimated to be about $67 \mathrm{Mgal} / \mathrm{d}$ (table 2), with $28 \mathrm{Mgal} / \mathrm{d}$ for livestock animals and $38 \mathrm{Mgal} / \mathrm{d}$ for catfish and trout farms. About 89 percent of all livestock withdrawals were from surface-water sources, and only 11 percent were from ground-water sources.

\section{Thermoelectric-Power Generation}

Thermoelectric-power water use is water used in the generation of thermoelectric power, mainly for cooling purposes. Thermoelectric-power water use includes 13 plants that operate on fossil fuels, such as oil, coal, or natural gas, and 2 nuclear-powered plants. Data were obtained from the GaEPD Water Resources Management Program, which collects data on the amount of water treated for potable use and for boiler makeup. Also, plant operators were contacted directly for additional information on cooling water and other water uses at each power plant. Estimated thermoelectric-power water use during 2005 was about 2,721 Mgal/d (table 2), a decrease of $589 \mathrm{Mgal} / \mathrm{d}$ from 2000. Three thermoelectric-power plants-Arkwright (Bibb County), Atkinson (Cobb County), and Riverside (Chatham County)—have closed since 2000. Plant Yates, a facility in Coweta County, converted from once-through cooling to a closed cooling system with cooling towers in 2004.

\section{Hydroelectric-Power Generation}

Instream water use for hydroelectric-power generation is the largest water-use category for Georgia, with an estimated $54,096 \mathrm{Mgal} / \mathrm{d}$ used in 2005 (table 2). Hydroelectric-power water use is instream water used for the generation of electricity at plants where turbine generators are driven by falling water. Data on the number of gallons used by each plant to generate one megawatt-hour of electricity was obtained from plant operators and multiplied by the monthly power generation to estimate the water used at each plant in 2005. Hydroelectric-power water use for 2005 increased 41 percent over use in 2000, a year of drought. 


\section{Consumptive Water Use, 2005}

The distinction between the amount of water withdrawn for use and the amount of water consumed during use is important for water planning and management. Consumptive water use is the part of water withdrawn that is evaporated, transpired, incorporated into products or crops, consumed by humans or livestock, or otherwise removed from the immediate water environment (Hutson and others, 2004, p. 44). For many withdrawals, data on the amount withdrawn that was returned to the immediate water environment (either a groundor surface-water system) are not available. Water sales and purchases and interbasin transfers make it difficult to relate withdrawals to return flows. For example, an industrial facility might withdraw some water from wells and purchase additional water from a public supplier. Some of the total water used at that facility then might be returned directly to a stream (if it was noncontact cooling water) and some might be treated at a public or private waste treatment plant and the treated wastewater returned to a stream. Because of the difficulties in obtaining and compiling data on the amount of water returned to the environment for each withdrawal, consumptive use was estimated from the withdrawal amounts and consumptive-use coefficients by water-use category. Although estimates derived using coefficients can provide insight into consumptive water use, the results are not as accurate as metered data on withdrawals and return flows.

The consumptive-use coefficients vary for each of the water-use categories. Because public supply delivered water to domestic, commercial, industrial, and thermoelectric-power users, consumptive use is estimated for those use categories and not for public supply. The total water used in the categories is the sum of water withdrawn (self-supplied water) and water delivered from public suppliers. For domestic water use, consumptive use was estimated at 18 percent of the total use (Atlanta Regional Commission, written commun., 2007). The consumptive-use coefficient for commercial use was developed by the GWUP and supported by calculations using withdrawal and discharge data for some commercial users and estimated to be 18 percent.

For industrial and mining use, consumptive-use coefficients were determined by industry type and type of mining activity. For example, the estimated consumptiveuse coefficient for the pulp and paper industry is 7 percent for 2005, whereas for the textile industry the coefficient is 13 percent. Although the coefficient is less, more water actually is consumed by pulp and paper industries than by textile industries in Georgia because of the larger amount of water being withdrawn for production.
In this report, irrigation and livestock water uses are considered to be 100 percent consumed; that is, all of the water withdrawn was evaporated or transpired, incorporated into crops, or consumed by livestock. Irrigation in Georgia uses sprinkler and micro-irrigation methods, which do no have the large nonconsumptive amounts of water as do flood irrigation methods. Consumptive-use coefficients for thermoelectric power ranged from 0 to nearly 70 percent, and is determined by the type of plant cooling (once-through cooling or cooling towers or ponds). Consumptive use is negligible for instream hydroelectric-power generation.

Estimated consumptive use by county for 2005 is listed in table 6. Total consumptive use was estimated to be about $1,310 \mathrm{Mgal} / \mathrm{d}$ or 24 percent of total withdrawals in the State.

\section{Water-Use Trends, 1980-2005}

Since 1980, the GWUP has compiled and published statewide water-use estimates by category at 5-year intervals. National water-use compilations began in 1950. However, during the period 1950-2005, water-use estimates were not always comparable. Category definition and estimation techniques have been the most consistent since 1980 because of efforts of the National Water-Use Information Program (NWUIP). In Georgia, total estimated water use for 1980 was 6,725 Mgal/d (Pierce and others, 1982). By 1990, total estimated water use had declined about 31 percent to $5,353 \mathrm{Mgal} / \mathrm{d}$. Statewide water use increased in 1995 and 2000 to reach $6,487 \mathrm{Mgal} / \mathrm{d}$. In 2005, estimated water use was $5,471 \mathrm{Mgal} / \mathrm{d}$, a decrease of $1,015 \mathrm{Mgal} / \mathrm{d}$ (about 19 percent) since 2000 (fig. 6).

Statewide public-supply water use steadily increased from 1980 to 2000, corresponding to an increase in population (from 4,189,000 to 8,186,450) during the same period; however, water use slightly decreased from 2000 to 2005 (fig. 7). Commercial, domestic, and livestock uses remained about the same for the period 1980-2005. Industrial water use fluctuated during the period 1980-2005, mostly because of improved water efficiency, water recycling, and conservation measures at industrial plants and changes in the number and type of industrial facilities. Major decreases in industrial water use in Glynn and Camden Counties were likely caused by chemical and paper plant closings and more efficient water-use practices at the remaining plants.

Irrigation water use declined from 1980 to 1985 and again to 1990, increased from 1995 to 2000 , and decreased markedly from 2000 to 2005. Estimated irrigation withdrawal was 30 percent lower in 2005 than in 2000 because 


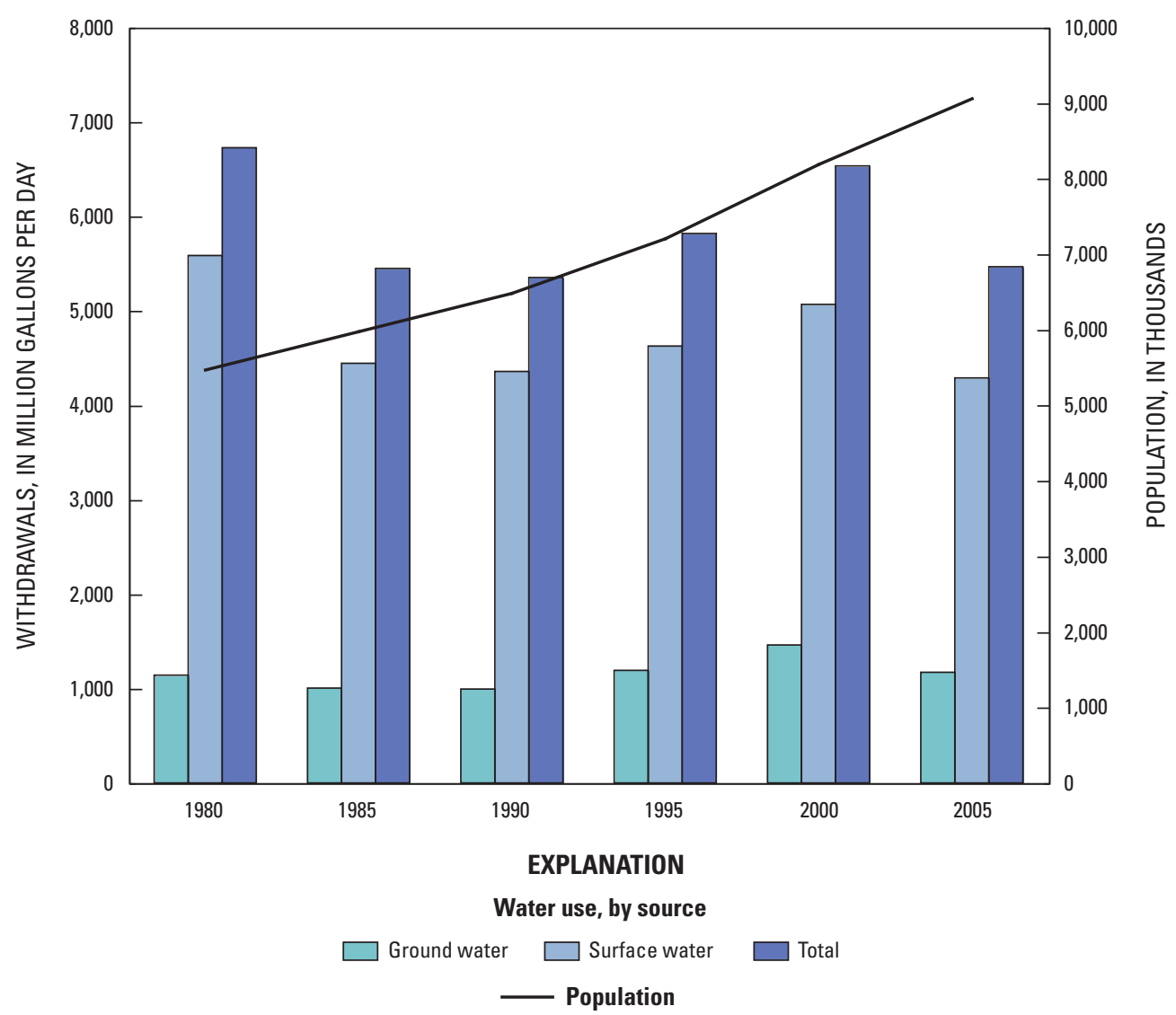

Figure 6. Population and water-use trends by source in Georgia, 1980-2005.

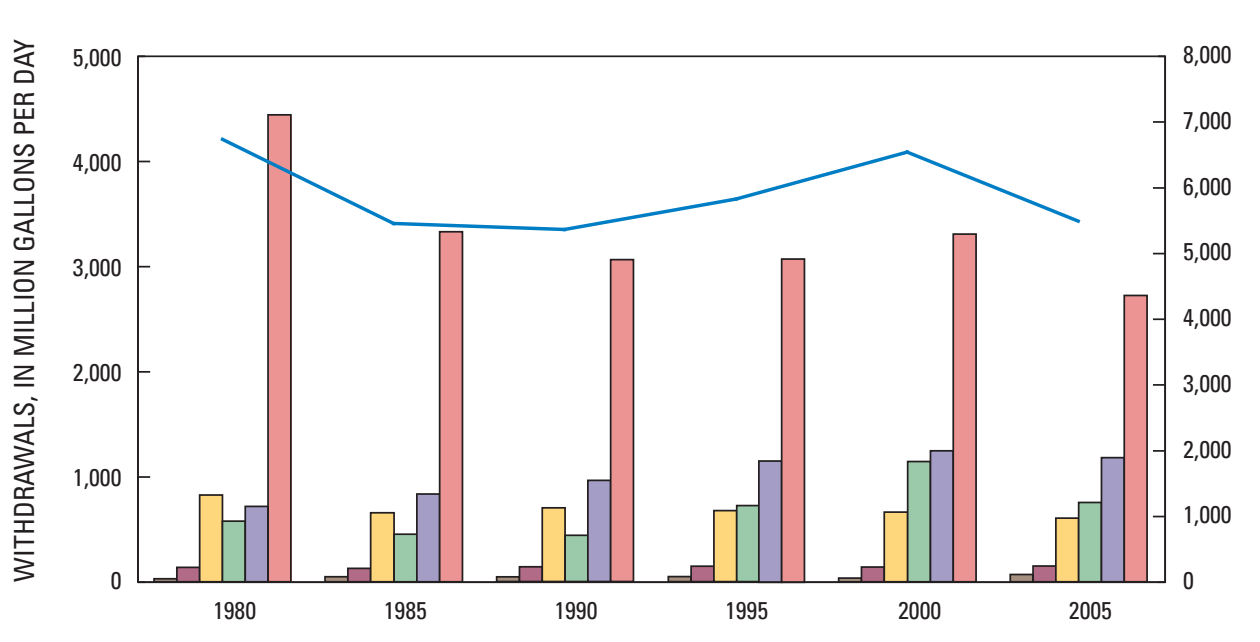

EXPLANATION

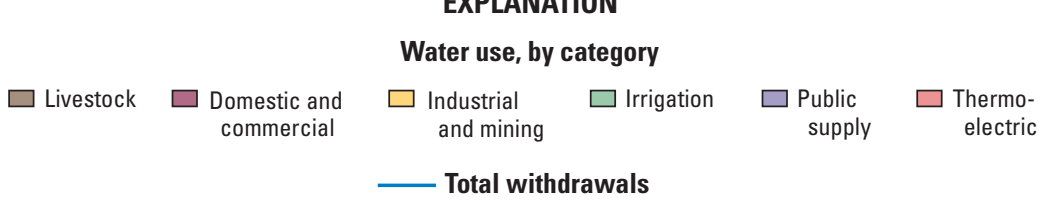

Figure 7. Trends in total water use by category in Georgia, 1980-2005. 
of decreased irrigation demands resulting from the greater amount of rainfall in 2005, which relieved the prolonged drought that began in 1998 and continued through 2002 (Georgia Department of Natural Resources, 2000). The total number of acres irrigated in the State increased by nearly 32 percent between 1995 and 2005.

Thermoelectric-power water continues to be the largest of any offstream category in Georgia. Thermoelectric-power withdrawal peaked in 1980, dropped sharply in 1985, and declined again in 1990 before increasing in 1995 and 2000. This rising trend was reversed in 2005 when thermoelectric power decreased by 24 percent from 2000 because of the decommissioning of three power plants and retrofitting cooling towers at several other plants.

Instream hydroelectric-power generation requires the use of significant amounts of water. From 1980 to 1995 , hydroelectric-power water use was increasing. However, because hydroelectric-power water use is dependent on the availability of surface-water flow, low-flow conditions related to drought in 1995 and 2000 resulted in a 19,000 Mgal/d decrease in usage. In 2005, water used for hydroelectricpower generation rose significantly to $54,096 \mathrm{Mgal} / \mathrm{d}$, an increase of 22,206 Mgal/d (59 percent) from 2000, because of the increased surface-water flow.

The GWUP has been compiling and disseminating water-use information on water users in the State for more than 25 years. While these data have been useful to managers making policy decisions for the water resources in Georgia, continued monitoring of water-use information is necessary. The GWUP is working to develop a site-specific database of reported information to improve data aggregation techniques, provide better quality assurance, and improve trend data. An Internet-based system capable of online interactive updating and reporting of water withdrawal by permitted user would allow for more timely data collection and data accuracy. Additionally, the site-specific database could be improved further to be aligned with other databases and could incorporate metered irrigation data. Also, data on public supply and industrial discharges in the State could be added. Other considerations, such as the reduction in permit limits from $100,000 \mathrm{gal} / \mathrm{d}$ to $10,000 \mathrm{gal} / \mathrm{d}$, which has been adopted in some adjoining States, would improve the available data for smaller industrial and mining users and some small public suppliers.

\section{References}

Executive Office of the President, Office of Management and Budget, 1987, Standard industrial classification manual, 1987: Springfield, Va., National Technical Information Service, Order no. PB 87-100012, 649 p.
Fanning, J.L., 1985, The Georgia water-use program: U.S. Geological Survey Open-File Report 85-481, pamphlet.

Fanning, J.L., 1997, Water use in Georgia by county for 1995: Georgia Geologic Survey Information Circular 101, 95 p.

Fanning, J.L., 2003, Water use in Georgia by county for 2000 and water-use trends for 1980-2000: Georgia Geologic Survey Information Circular 106, 176 p.

Fanning, J.L., Doonan, G.A., and Montgomery, L.T., 1992, Water use in Georgia by county for 1990: Georgia Geologic Survey Information Circular 90, 98 p.

Georgia Department of Natural Resources, Environmental Protection Division, 2000, 1998-2000 Georgia drought report: Georgia Department of Natural Resources, 43 p.

Georgia Department of Natural Resources, Environmental Protection Division, 2008a, Existing rules and corresponding laws, 391-3-2 Groundwater Use, accessed July 24, 2008, at http://www.georgiaepd.org/Documents/rules_exist.html

Georgia Department of Natural Resources, Environmental Protection Division, 2008b, Existing rules and corresponding laws, 391-3-5 Safe Drinking Water, accessed July 24, 2008, at http://www.georgiaepd.org/Documents/ rules_exist.html

Hutson, S.S., Barber, N.L., Kenny, J.F., Linsey, K.S., Lumia, D.S., and Maupin, M.A., 2004, Estimated use of water in the United States in 2000: U.S. Geological Survey Circular 1268, 46 p.

Pierce, R.R., Barber, N.L., and Stiles, H.R., 1982, Water use in Georgia by county for 1980: Georgia Geologic Survey Information Circular 59, $180 \mathrm{p}$.

Solley, W.B., Merk, C.F., and Pierce, R.R., 1988, Estimated use of water in the United States in 1985: U.S. Geological Survey Circular 1004, 82 p.

Trent, V.P., Fanning, J.L., and Doonan, G.A., 1990, Water use in Georgia by county for 1987: Georgia Geologic Survey Information Circular 85, $112 \mathrm{p}$.

Turlington, M.C., Fanning, J.L., and Doonan, G.A., 1987, Water use in Georgia by county for 1985: Georgia Geologic Survey Information Circular 81, 110 p.

U.S. Bureau of the Census, 2005, Estimates and projections of population, 2005: Washington, D.C., U.S. Department of Commerce, series P25, variously paged.

U.S. Geological Survey, 2006, Georgia's ground-water resources and monitoring network, 2006: U.S. Geological Survey Fact Sheet 2006-3077, 2 p. 


\section{Appendix A. Standard Industrial Classification (SIC) Codes}

Industrial water use is given by industry type, classified by Standard Industrial Classification (SIC) Code. A brief description of the codes used in this publication is given below.

14-Mining and quarrying of nonmetallic minerals, except fuels. This major group includes establishments engaged in mining or quarrying, developing mines, or exploring for nonmetallic minerals, except fuels. Also included are certain well and brine operations, and primary preparation plants, such as those engaged in crushing, grinding, washing, or other concentration.

20-Food and kindred products. This major group includes establishments manufacturing or processing foods and beverages for human consumption, and certain related products, such as manufactured ice, chewing gum, vegetable and animal fats and oils, and prepared feeds for animals and fowls.

22-Textile mill products. This major group includes establishments engaged in performing any of the following operations: (1) preparation of fiber and subsequent manufacturing of yarn, thread, braids, twine, and cordage; (2) manufacturing broad woven fabric, narrow woven fabric, knit fabric and carpets and rugs from yarn; (3) dyeing and finishing fiber, yarn, fabric, and knit apparel; (4) coating, waterproofing, or otherwise treating fabric; (5) the integrated manufacture of knit apparel and other finished articles from yarn; and (6) the manufacture of felt goods, lace goods, non-woven fabrics and miscellaneous textiles.

23-Apparel and other finished products made from fabrics and similar materials. This major group, known as the cutting-up and needle trades, includes establishments producing clothing and fabricating products by cutting and sewing purchased woven or knit textile fabrics and related materials such as leather, rubberized fabrics, plastics, and furs.

24-Lumber and wood products, except furniture. This major group includes logging camps engaged in cutting timber and pulpwood; merchant sawmills, lathe mills, shingle mills, cooperage stock mills, planing mills, and pulpwood mills and veneer mills engaged in producing lumber and wood basic materials; and establishments engaged in manufacturing finished articles made entirely or mainly of wood or wood substitutes.

26-Paper and allied products. This major group includes the manufacture of pulps from wood and other cellulose fibers, and from rags; the manufacture of paper and paperboard; and the manufacture of paper and paperboard into converted products such as paper coated off the paper machine, paper bags, paper boxes, and envelopes.

28-Chemicals and allied products. This major group includes establishments producing basic chemicals, and establishments manufacturing products by predominantly chemical processes. Establishments classified in this major group manufacture three general classes of products: (1) basic chemicals such as acids, alkalies, salts, and organic chemicals; (2) chemical products to be used in further manufacture such as synthetic fibers, plastics materials, dry colors, and pigments; and (3) finished chemical products to be used for ultimate consumption such as drugs, cosmetics, and soaps, or to be used as materials or supplies in other industries such as paints, fertilizers, and explosives.

29-Petroleum refining and related industries. This major group includes establishments primarily engaged in petroleum refining, manufacturing paving and roofing materials, and compounding lubricating oils and greases from purchased materials.

30-Rubber and miscellaneous plastics products. This major group includes establishments manufacturing from natural, synthetic, or reclaimed rubber, gutta percha, balata, or gutta siak, rubber products such as tires, rubber footwear, mechanical rubber goods, heels and soles, flooring, and rubber sundries.

32-Stone, clay, glass, and concrete products. This major group includes establishments engaged in manufacturing flat glass and other glass products, cement, structural clay products, pottery, concrete and gypsum products, cut stone, abrasive and asbestos products, etc., from materials taken principally from the earth in the form of stone, clay, and sand.

33-Primary metal industries. This major group includes establishments engaged in the smelting and refining of ferrous and nonferrous metals from ore, pig, or scrap; in the rolling, drawing, and alloying of ferrous and non-ferrous metals; in the manufacture of castingsand other basic products of ferrous and nonferrous metals; and in the manufacture of nails, spikes, and insulated wire and cable.

\section{4-Fabricated metal products, except machinery and transportation equipment. This major group includes establishments engaged in fabricating ferrous and nonferrous metal products such as metal cans, tinware, hand tools, cutlery, general hardware, non-electric heating apparatus, fabricated structural metal products, metal forging, metal stamping, ordnance (except vehicles and guided missiles), and a variety of metal and wire products not elsewhere classified.}

35-Machinery, except electrical. This major group includes establishments engaged in manufacturing machinery and equipment, other than electrical equipment (Major Group 36) and transportation equipment (Major Group 37).

36-Electrical and electronic machinery, equipment, and supplies. This major group includes establishments engaged in manufacturing machinery, apparatus, and supplies for the generation, storage, transmission, transformation, and utilization of electrical energy. 
Water Use in Georgia by County for 2005; and Water-Use Trends, 1980-2005 


\section{Appendix B. Ground- and Surface-Water Use in Georgia, by County 2005, and Trends for 1980-2005}

The following tables list ground- and surface-water withdrawals by water-use category. Also listed is information about:

- major public suppliers,

- major industrial groups,

- county populations,

- population served by public supply, and

- acres irrigated.

Trend graphs and maps showing ground- and surface-water system locations are included. 


\section{APPLING COUNTY}

Population: 17,954

Population served by public supply: 6,660

Acres irrigated: 7,110

Hydroelectric use (Mgal/d): 0.00

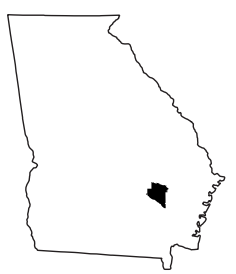

2005 WITHDRAWALS, IN MILLION GALLONS PER DAY

\begin{tabular}{|c|c|c|c|c|c|c|c|}
\hline & $\begin{array}{l}\text { Public } \\
\text { Supply }\end{array}$ & $\begin{array}{l}\text { Domestic \& } \\
\text { Commercial }\end{array}$ & $\begin{array}{c}\text { Industrial \& } \\
\text { Mining }\end{array}$ & Irrigation & Livestock & $\begin{array}{l}\text { Thermo- } \\
\text { electric }\end{array}$ & Totals \\
\hline Ground Water & 0.93 & 0.85 & 0.00 & 2.24 & 0.07 & 0.18 & 4.27 \\
\hline Surface Water & 0.00 & 0.00 & 0.00 & 0.85 & 0.31 & 57.59 & 58.75 \\
\hline TOTALS & 0.93 & 0.85 & 0.00 & 3.09 & 0.38 & 57.77 & 63.02 \\
\hline
\end{tabular}

Withdrawals by Major Public Suppliers (Mgal/d):

Name

City of Baxley

City of Surrency
GW SW

$0.87 \quad 0.00$

$0.04 \quad 0.00$
Withdrawals by Major Industrial Groups (Mgal/d):

SIC

GW

SW

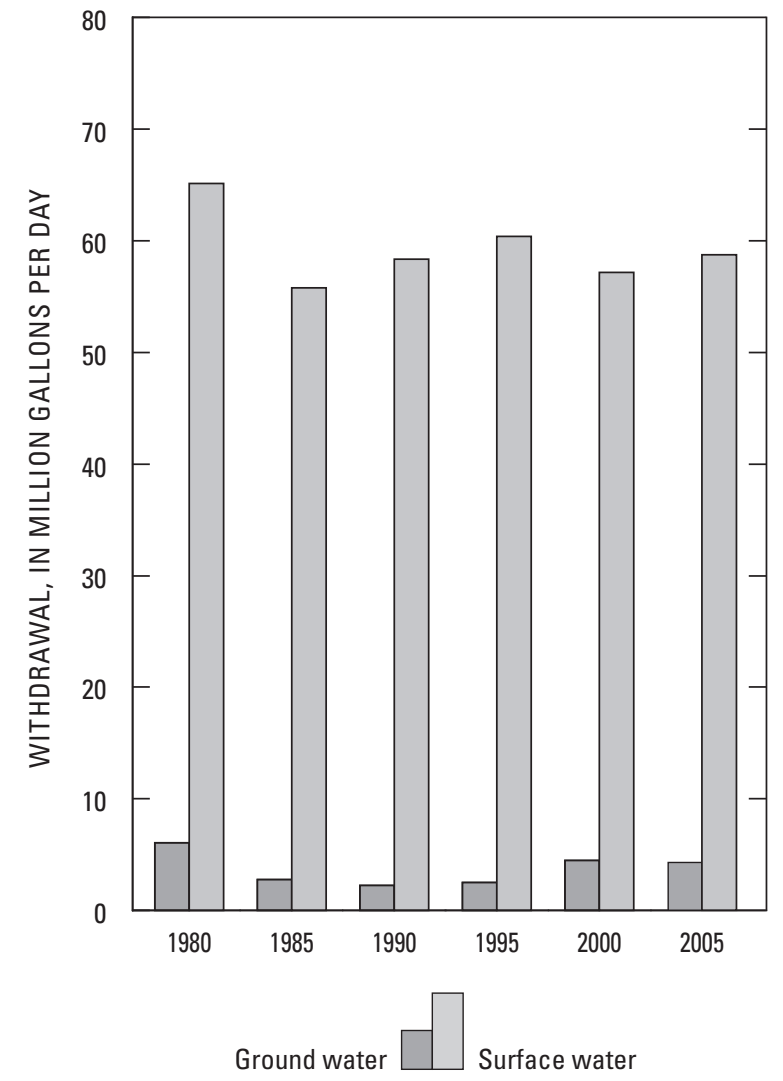

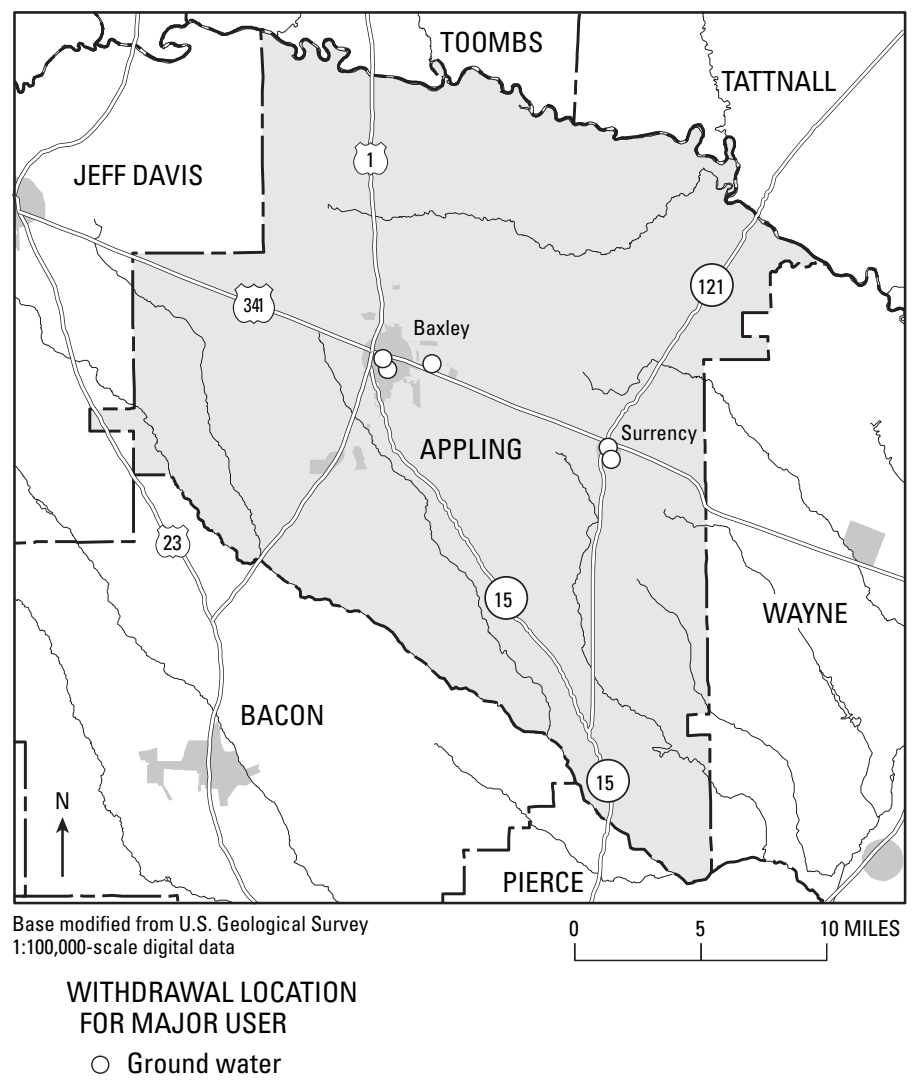




\section{ATKINSON COUNTY}

Population: 8,030

Population served by public supply: 3,750

Acres irrigated: 9,330

Hydroelectric use (Mgal/d): $\quad 0.00$

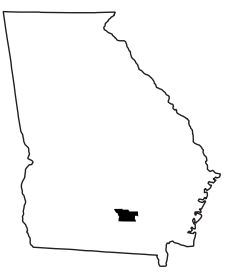

2005 WITHDRAWALS, IN MILLION GALLONS PER DAY

\begin{tabular}{|c|c|c|c|c|c|c|c|}
\hline & $\begin{array}{l}\text { Public } \\
\text { Supply }\end{array}$ & $\begin{array}{l}\text { Domestic \& } \\
\text { Commercial }\end{array}$ & $\begin{array}{c}\text { Industrial \& } \\
\text { Mining }\end{array}$ & Irrigation & Livestock & $\begin{array}{l}\text { Thermo- } \\
\text { electric }\end{array}$ & Totals \\
\hline Ground Water & 0.42 & 0.32 & 0.15 & 3.89 & 0.04 & 0.00 & 4.82 \\
\hline Surface Water & 0.00 & 0.00 & 0.00 & 2.81 & 0.24 & 0.00 & 3.05 \\
\hline TOTALS & 0.42 & 0.32 & 0.15 & 6.70 & 0.28 & 0.00 & 7.87 \\
\hline
\end{tabular}

Withdrawals by Major Public Suppliers (Mgal/d):

Name

City of Pearson

GW

0.22

0.19

SW

0.00

0.00

Town of Willacoochee
Withdrawals by Major Industrial Groups (Mgal/d):

SIC

GW

SW
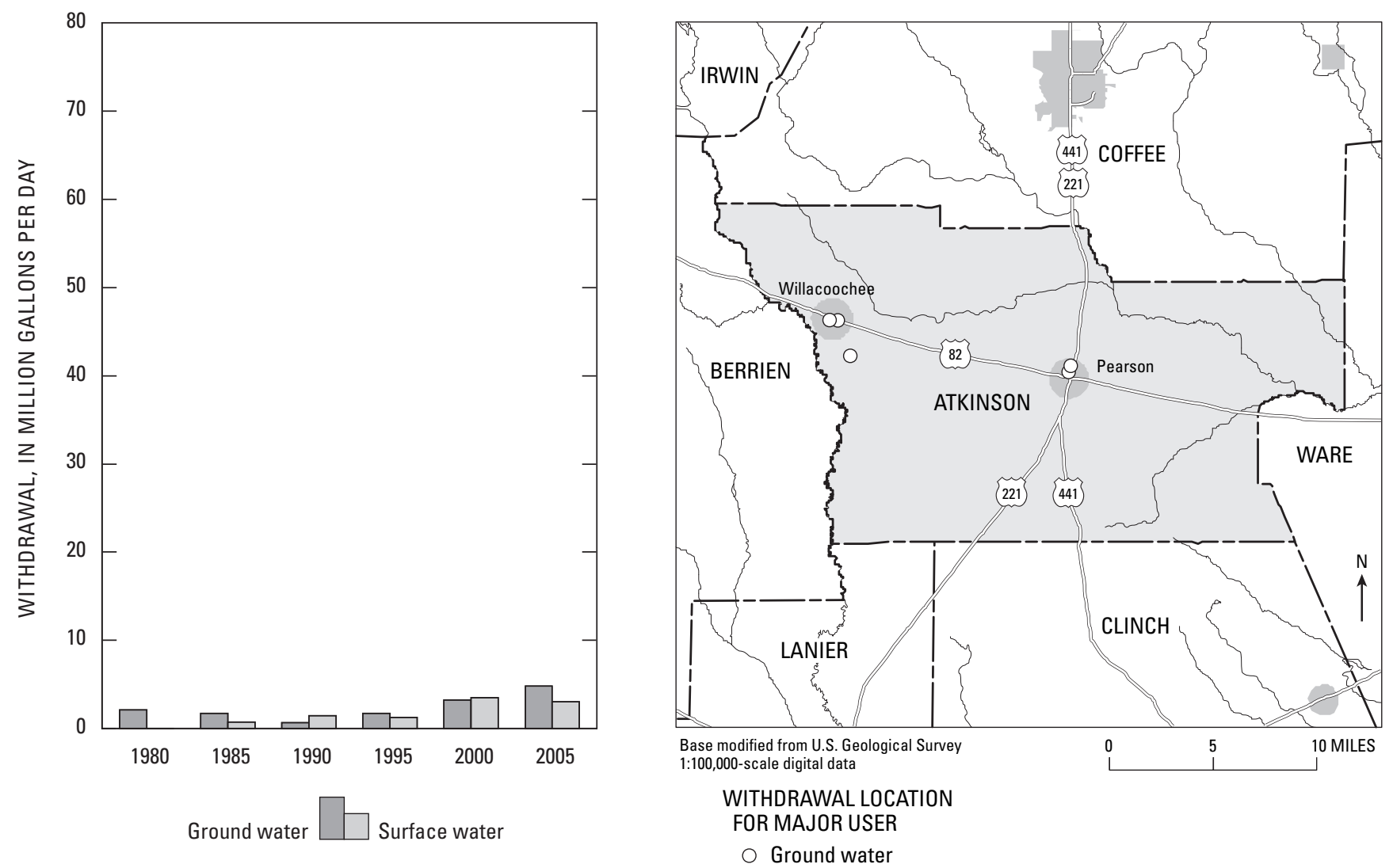


\section{BACON COUNTY}

Population: $\quad 10,379$

Population served by public supply: 3,380

Acres irrigated: 7,990

Hydroelectric use (Mgal/d): $\quad 0.00$

2005 WITHDRAWALS, IN MILLION GALLONS PER DAY

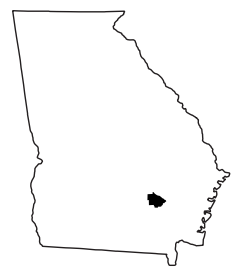

\begin{tabular}{|c|c|c|c|c|c|c|c|}
\hline & $\begin{array}{l}\text { Public } \\
\text { Supply }\end{array}$ & $\begin{array}{l}\text { Domestic \& } \\
\text { Commercial }\end{array}$ & $\begin{array}{c}\text { Industrial \& } \\
\text { Mining }\end{array}$ & Irrigation & Livestock & $\begin{array}{l}\text { Thermo- } \\
\text { electric }\end{array}$ & Totals \\
\hline Ground Water & 0.76 & 0.52 & 0.25 & 3.68 & 0.02 & 0.00 & 5.23 \\
\hline Surface Water & 0.00 & 0.00 & 0.00 & 1.00 & 0.19 & 0.00 & 1.19 \\
\hline TOTALS & 0.76 & 0.52 & 0.25 & 4.68 & 0.21 & 0.00 & 6.42 \\
\hline
\end{tabular}

Withdrawals by Major Public Suppliers (Mgal/d):

Name GW SW

Withdrawals by Major Industrial Groups (Mgal/d):

City of Alma

$0.75 \quad 0.00$

SIC

GW

SW

22 - Textiles

0.24

0.00
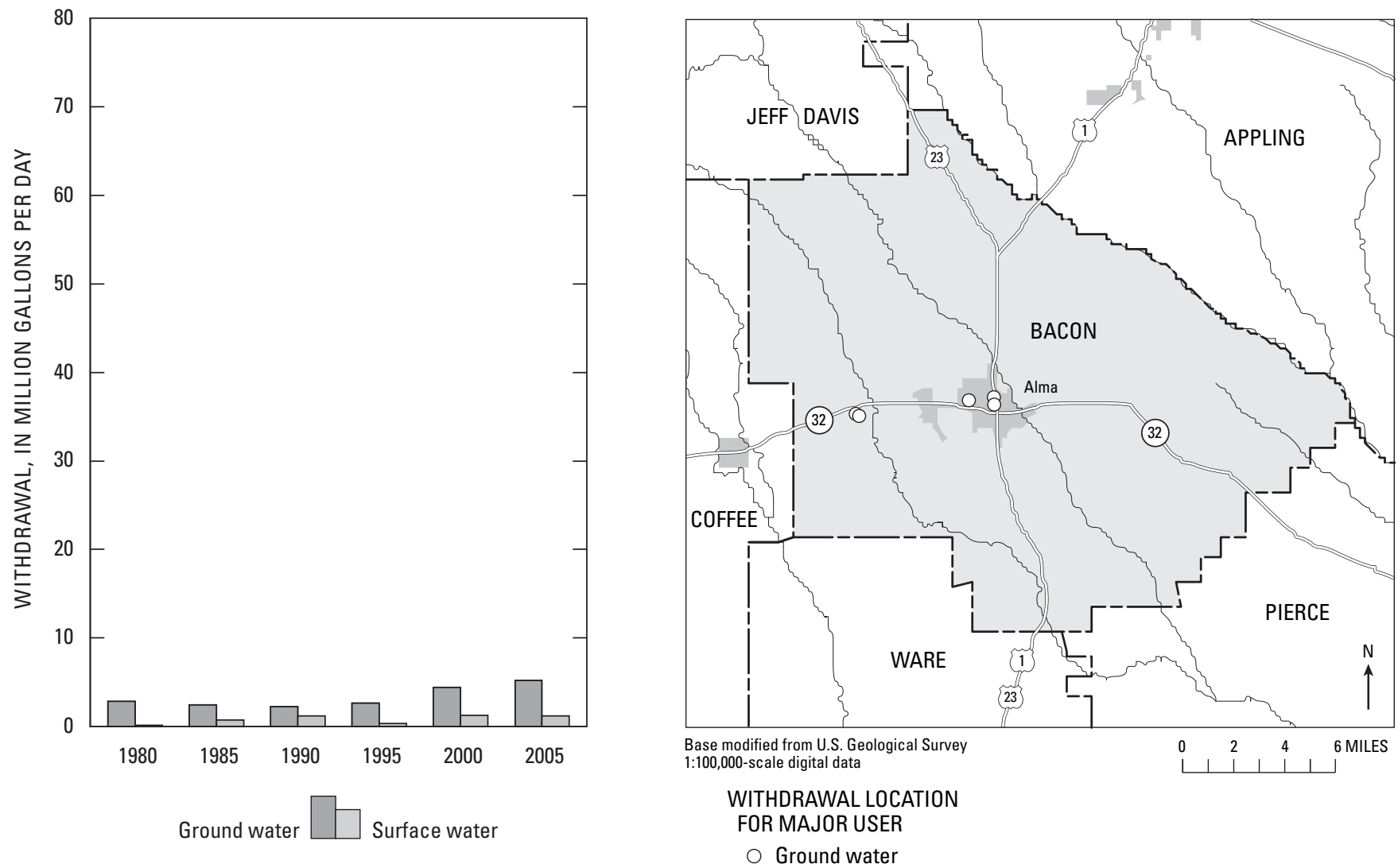


\section{BAKER COUNTY}

Population: 4,154

Population served by public supply: 850

Acres irrigated: 49,580

Hydroelectric use (Mgal/d): $\quad 0.00$

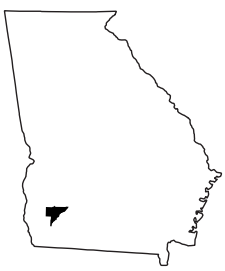

2005 WITHDRAWALS, IN MILLION GALLONS PER DAY

\begin{tabular}{|c|c|c|c|c|c|c|c|}
\hline & $\begin{array}{l}\text { Public } \\
\text { Supply }\end{array}$ & $\begin{array}{l}\text { Domestic \& } \\
\text { Commercial }\end{array}$ & $\begin{array}{c}\text { Industrial \& } \\
\text { Mining }\end{array}$ & Irrigation & Livestock & $\begin{array}{l}\text { Thermo- } \\
\text { electric }\end{array}$ & Totals \\
\hline Ground Water & 0.11 & 0.25 & 0.00 & 30.75 & 0.00 & 0.00 & 31.11 \\
\hline Surface Water & 0.00 & 0.00 & 0.00 & 2.56 & 0.72 & 0.00 & 3.28 \\
\hline TOTALS & 0.11 & 0.25 & 0.00 & 33.31 & 0.72 & 0.00 & 34.39 \\
\hline
\end{tabular}

Withdrawals by Major Public Suppliers (Mgal/d):

Name

GW

0.11

SW

0.00
Withdrawals by Major Industrial Groups (Mgal/d):

SIC

GW

SW

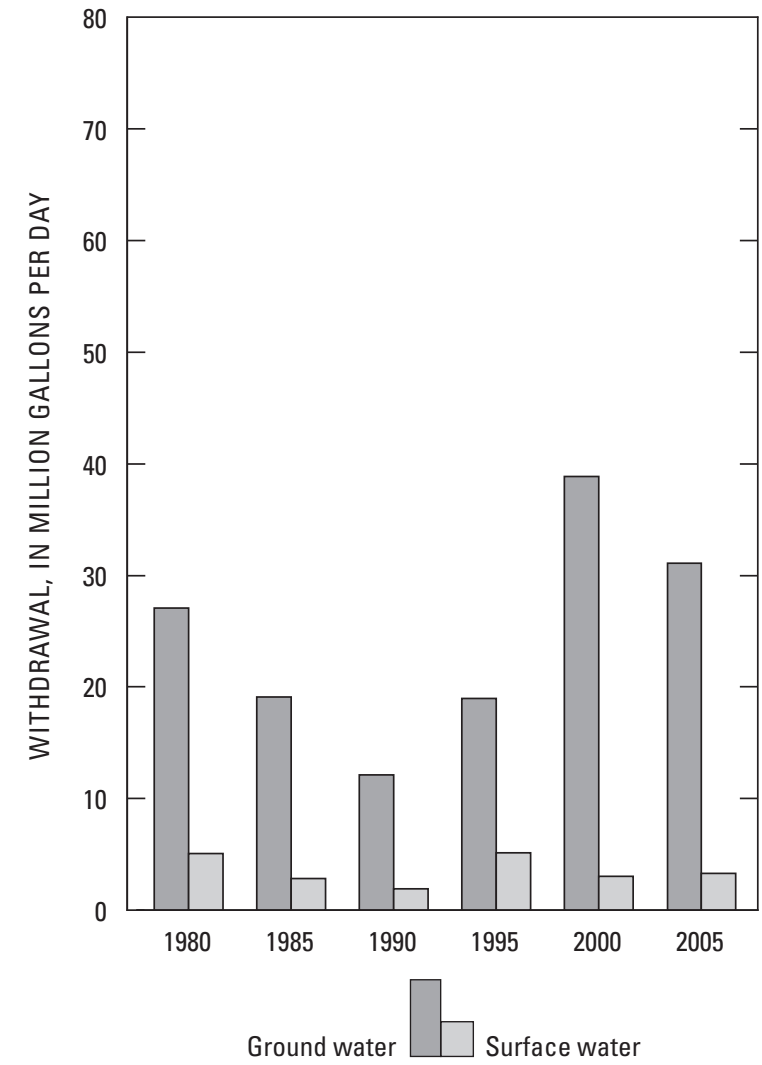

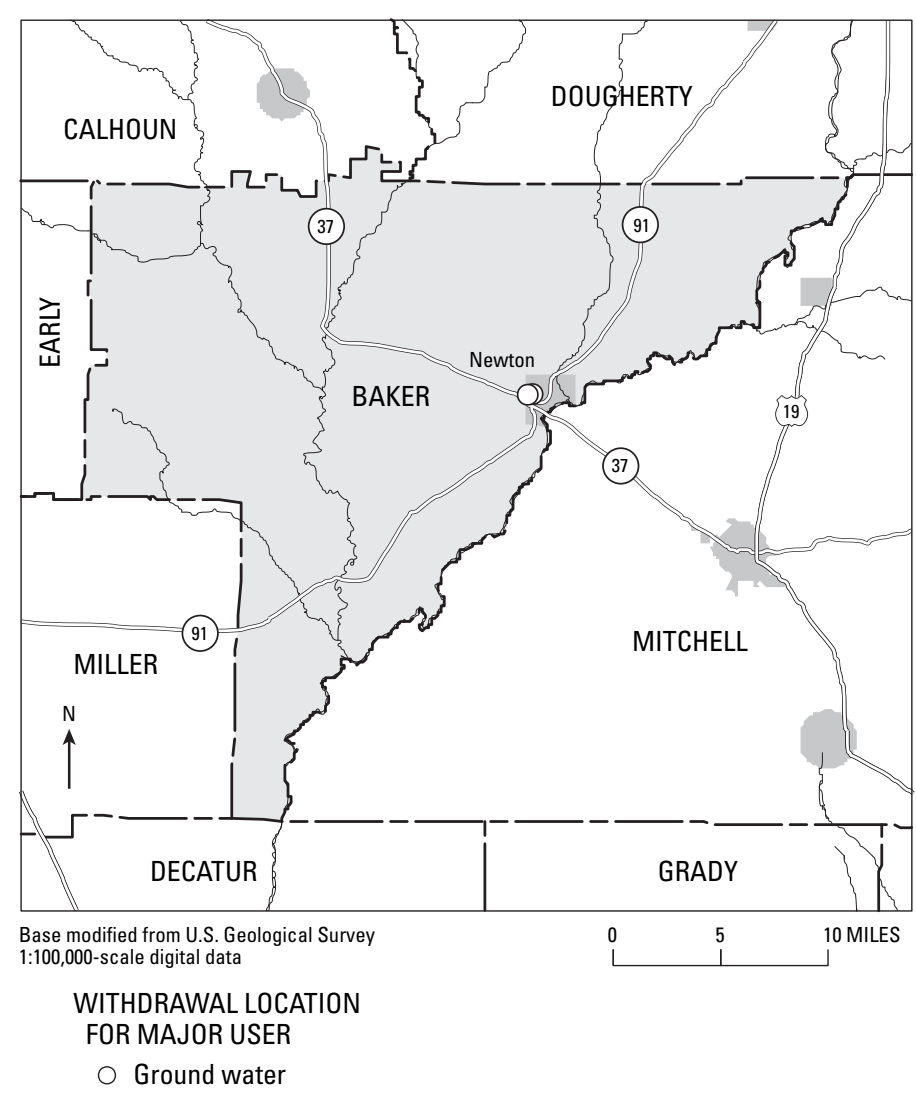




\section{BALDWIN COUNTY}

Population: 45,230

Population served by public supply: 44,750

Acres irrigated: 400

Hydroelectric use (Mgal/d): 1,692.16

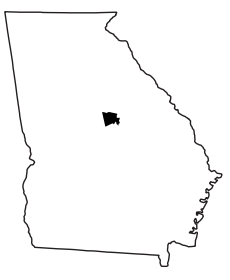

2005 WITHDRAWALS, IN MILLION GALLONS PER DAY

\begin{tabular}{|c|c|c|c|c|c|c|c|}
\hline & $\begin{array}{l}\text { Public } \\
\text { Supply }\end{array}$ & $\begin{array}{l}\text { Domestic \& } \\
\text { Commercial }\end{array}$ & $\begin{array}{c}\text { Industrial \& } \\
\text { Mining }\end{array}$ & Irrigation & Livestock & $\begin{array}{l}\text { Thermo- } \\
\text { electric }\end{array}$ & Totals \\
\hline Ground Water & 0.00 & 0.04 & 0.23 & 0.24 & 0.00 & 0.00 & 0.51 \\
\hline Surface Water & 6.75 & 0.00 & 0.00 & 0.29 & 0.08 & 0.00 & 7.12 \\
\hline TOTALS & 6.75 & 0.04 & 0.23 & 0.53 & 0.08 & 0.00 & 7.63 \\
\hline
\end{tabular}

Withdrawals by Major Public Suppliers (Mgal/d):

Name

City of Milledgeville
SW 6.75
Withdrawals by Major Industrial Groups (Mgal/d):

SIC

None
SW

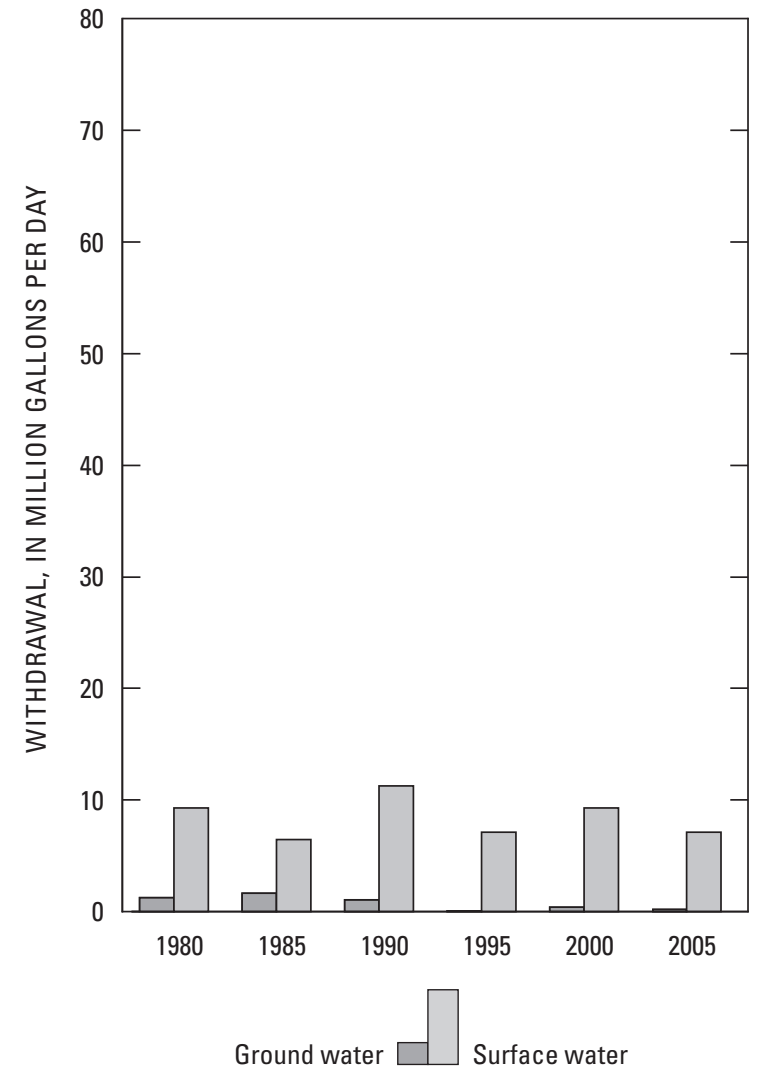

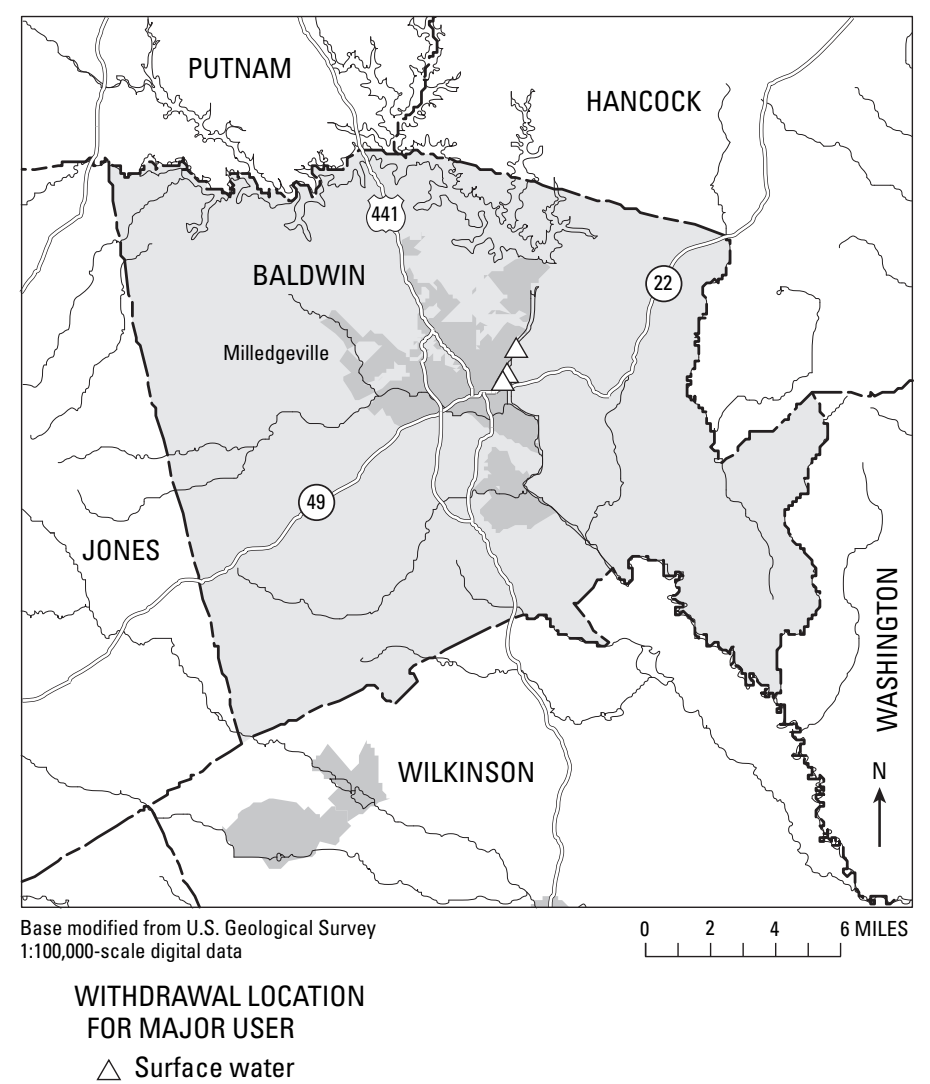




\section{BANKS COUNTY}

Population: 16,055

Population served by public supply: $\quad 6,850$

Acres irrigated: 100

Hydroelectric use (Mgal/d): $\quad 0.00$

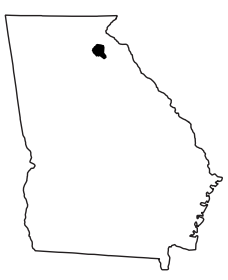

2005 WITHDRAWALS, IN MILLION GALLONS PER DAY

\begin{tabular}{|c|c|c|c|c|c|c|c|}
\hline & $\begin{array}{l}\text { Public } \\
\text { Supply }\end{array}$ & $\begin{array}{l}\text { Domestic \& } \\
\text { Commercial }\end{array}$ & $\begin{array}{c}\text { Industrial \& } \\
\text { Mining }\end{array}$ & Irrigation & Livestock & $\begin{array}{l}\text { Thermo- } \\
\text { electric }\end{array}$ & Totals \\
\hline Ground Water & 0.07 & 0.69 & 0.00 & 0.00 & 0.00 & 0.00 & 0.76 \\
\hline Surface Water & 0.62 & 0.24 & 0.00 & 0.15 & 0.69 & 0.00 & 1.70 \\
\hline TOTALS & 0.69 & 0.93 & 0.00 & 0.15 & 0.69 & 0.00 & 2.46 \\
\hline
\end{tabular}

Withdrawals by Major Public Suppliers (Mgal/d):

Name

Banks County Board

of Commissioners

Town of Homer

GW

0.00

SW

0.07

0.62

0.00
Withdrawals by Major Industrial Groups (Mgal/d):

SIC

None
GW

SW
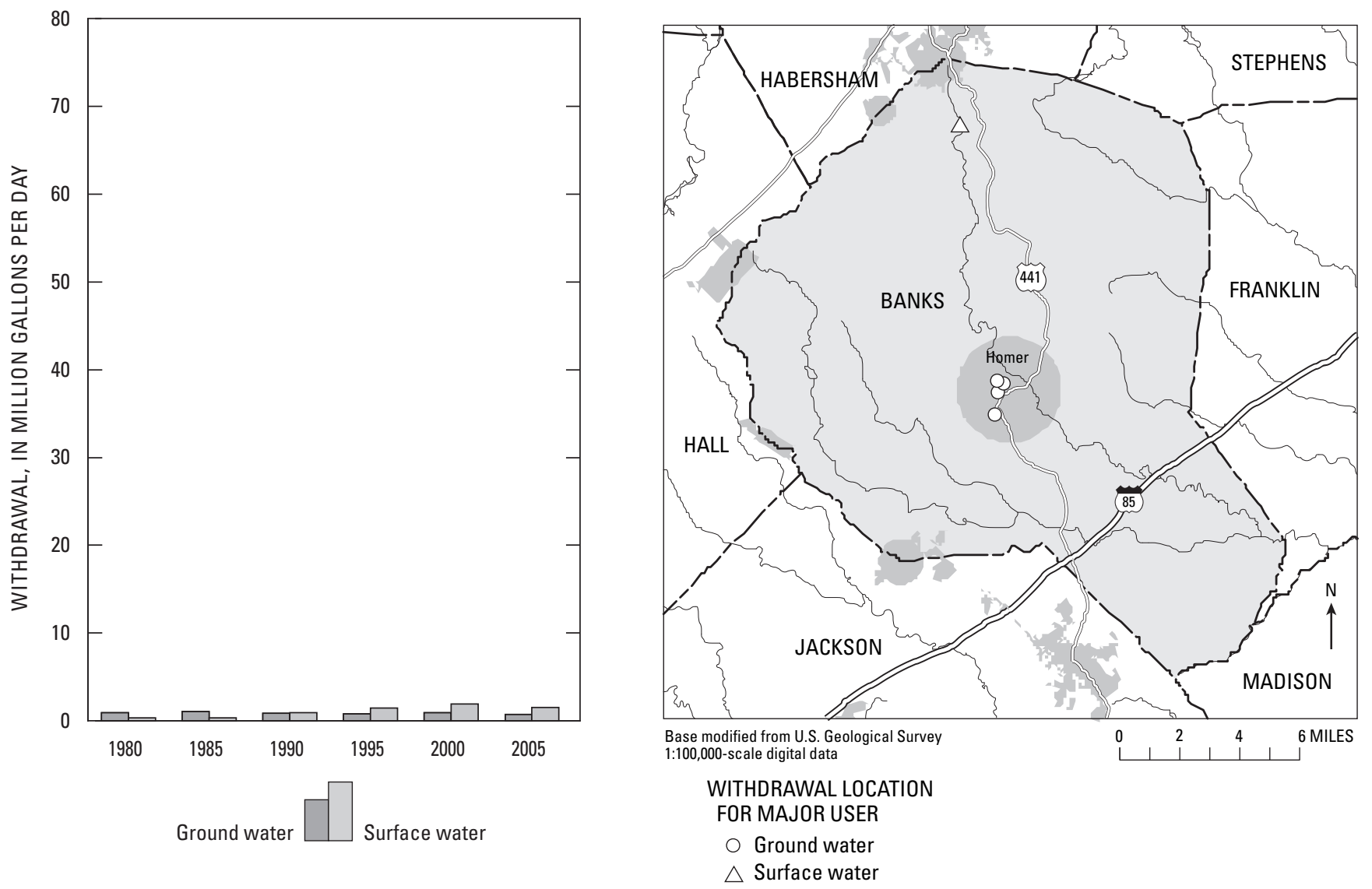


\section{BARROW COUNTY}

Population: 59,954

Population served by public supply: $\quad 31,890$

Acres irrigated: 670

Hydroelectric use (Mgal/d): $\quad 0.00$

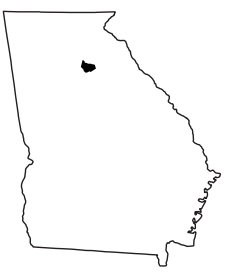

2005 WITHDRAWALS, IN MILLION GALLONS PER DAY

\begin{tabular}{|c|c|c|c|c|c|c|c|}
\hline & $\begin{array}{l}\text { Public } \\
\text { Supply }\end{array}$ & $\begin{array}{l}\text { Domestic \& } \\
\text { Commercial }\end{array}$ & $\begin{array}{c}\text { Industrial \& } \\
\text { Mining }\end{array}$ & Irrigation & Livestock & $\begin{array}{l}\text { Thermo- } \\
\text { electric }\end{array}$ & Totals \\
\hline Ground Water & 0.57 & 2.10 & 0.54 & 0.29 & 0.00 & 0.00 & 3.50 \\
\hline Surface Water & 4.44 & 0.00 & 0.00 & 0.34 & 0.33 & 0.00 & 5.11 \\
\hline TOTALS & 5.01 & 2.10 & 0.54 & 0.63 & 0.33 & 0.00 & 8.61 \\
\hline
\end{tabular}

Withdrawals by Major Public Suppliers (Mgal/d):

\section{Name}

Town of Auburn

City of Statham

City of Winder

$\begin{array}{cc}\text { GW } & \text { SW } \\ 0.52 & 0.00 \\ 0.00 & 0.19 \\ 0.00 & 4.24\end{array}$

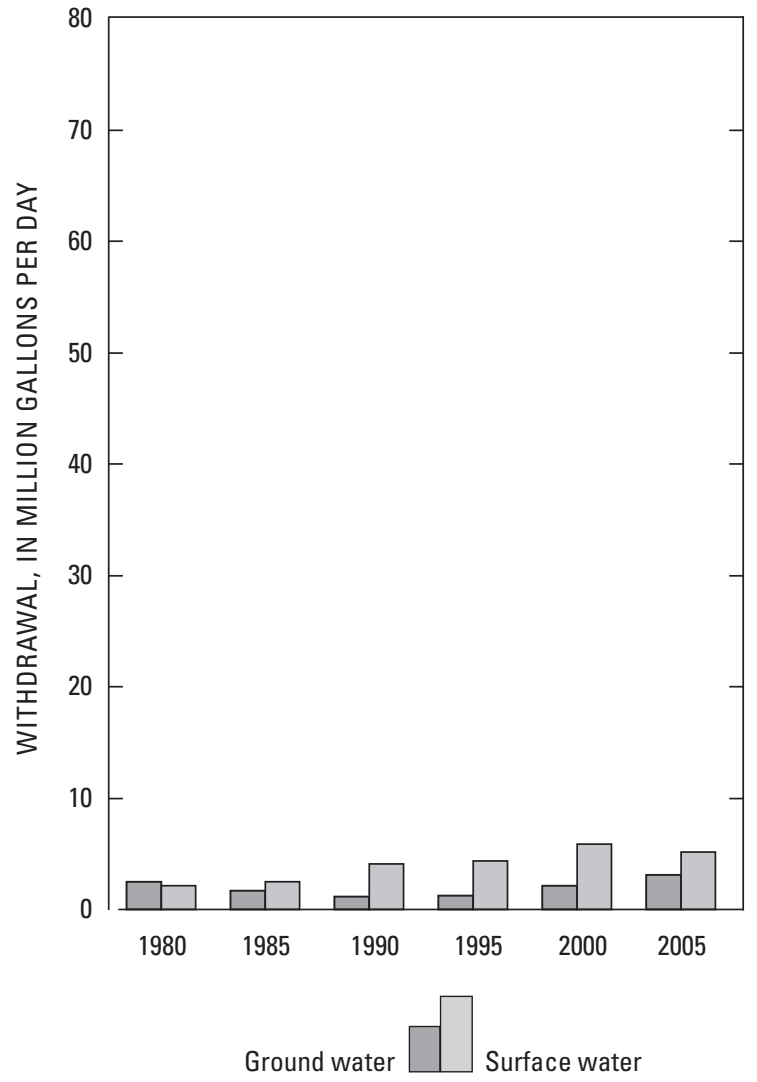

Withdrawals by Major Industrial Groups (Mgal/d):

$\begin{array}{lcc}\text { SIC } & \text { GW } & \text { SW } \\ 20 \text { - Food } & 0.27 & 0.00\end{array}$

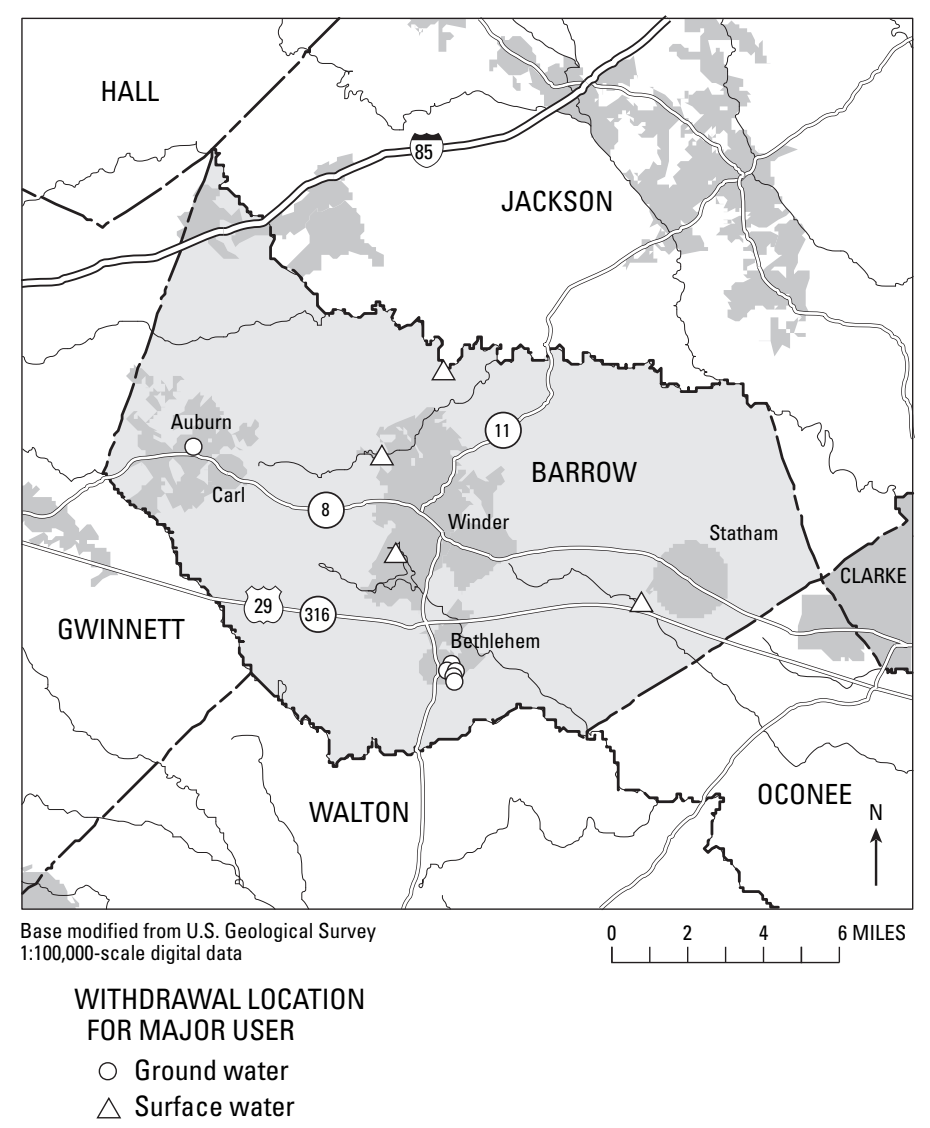




\section{BARTOW COUNTY}

Population: 89,229

Population served by public supply: 79,290

Acres irrigated: 1,560

Hydroelectric use (Mgal/d): $\quad 1,201.30$

2005 WITHDRAWALS, IN MILLION GALLONS PER DAY

\begin{tabular}{|c|c|c|c|c|c|c|c|}
\hline & $\begin{array}{l}\text { Public } \\
\text { Supply }\end{array}$ & $\begin{array}{l}\text { Domestic \& } \\
\text { Commercial }\end{array}$ & $\begin{array}{c}\text { Industrial \& } \\
\text { Mining }\end{array}$ & Irrigation & Livestock & $\begin{array}{l}\text { Thermo- } \\
\text { electric }\end{array}$ & Totals \\
\hline Ground Water & 4.31 & 0.75 & 2.10 & 0.00 & 0.02 & 0.00 & 7.18 \\
\hline Surface Water & 13.51 & 0.00 & 1.83 & 1.57 & 0.35 & 38.92 & 56.18 \\
\hline TOTALS & 17.82 & 0.75 & 3.93 & 1.57 & 0.37 & 38.92 & 63.36 \\
\hline
\end{tabular}

Withdrawals by Major Public Suppliers (Mgal/d):

Name

GW SW

City of Adairsville

$2.68 \quad 0.00$

Bartow County Water System

0.54

0.00

City of Cartersville

0.00

0.95

0.10
13.26

0.00

0.00
Withdrawals by Major Industrial Groups (Mgal/d):

$\begin{array}{lcc}\text { SIC } & \text { GW } & \text { SW } \\ 28 \text { - Chemicals } & 1.24 & 1.67 \\ 33 \text { - Primary metals } & 0.00 & 0.16\end{array}$

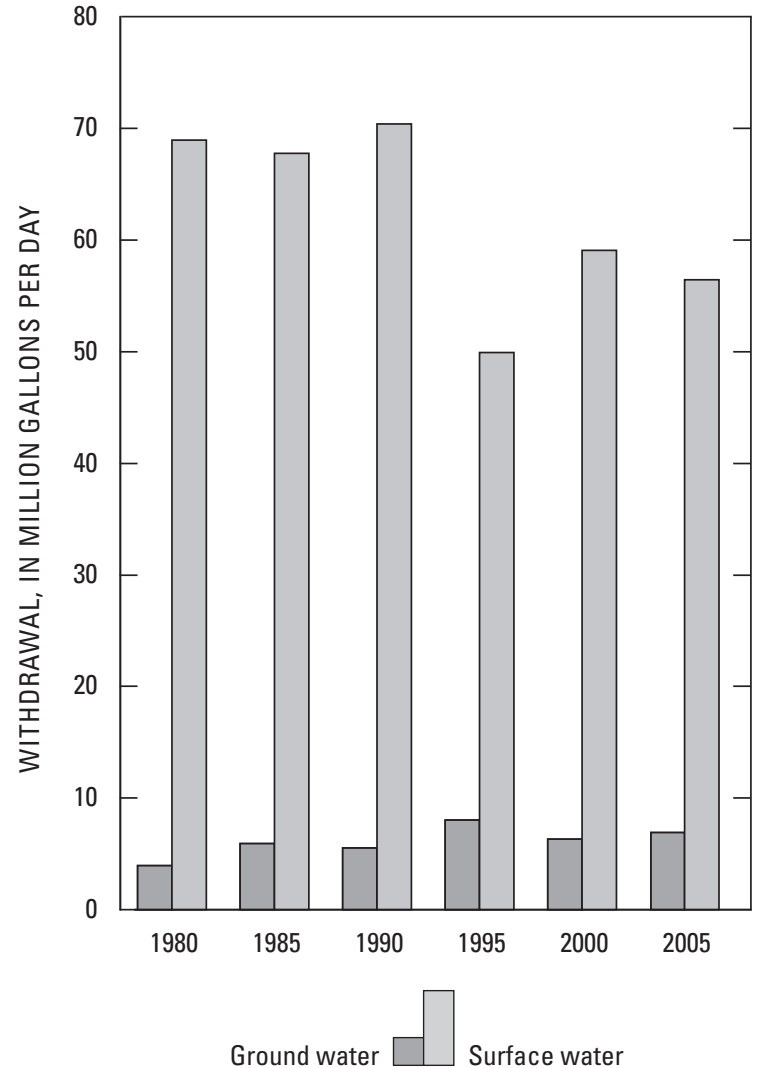

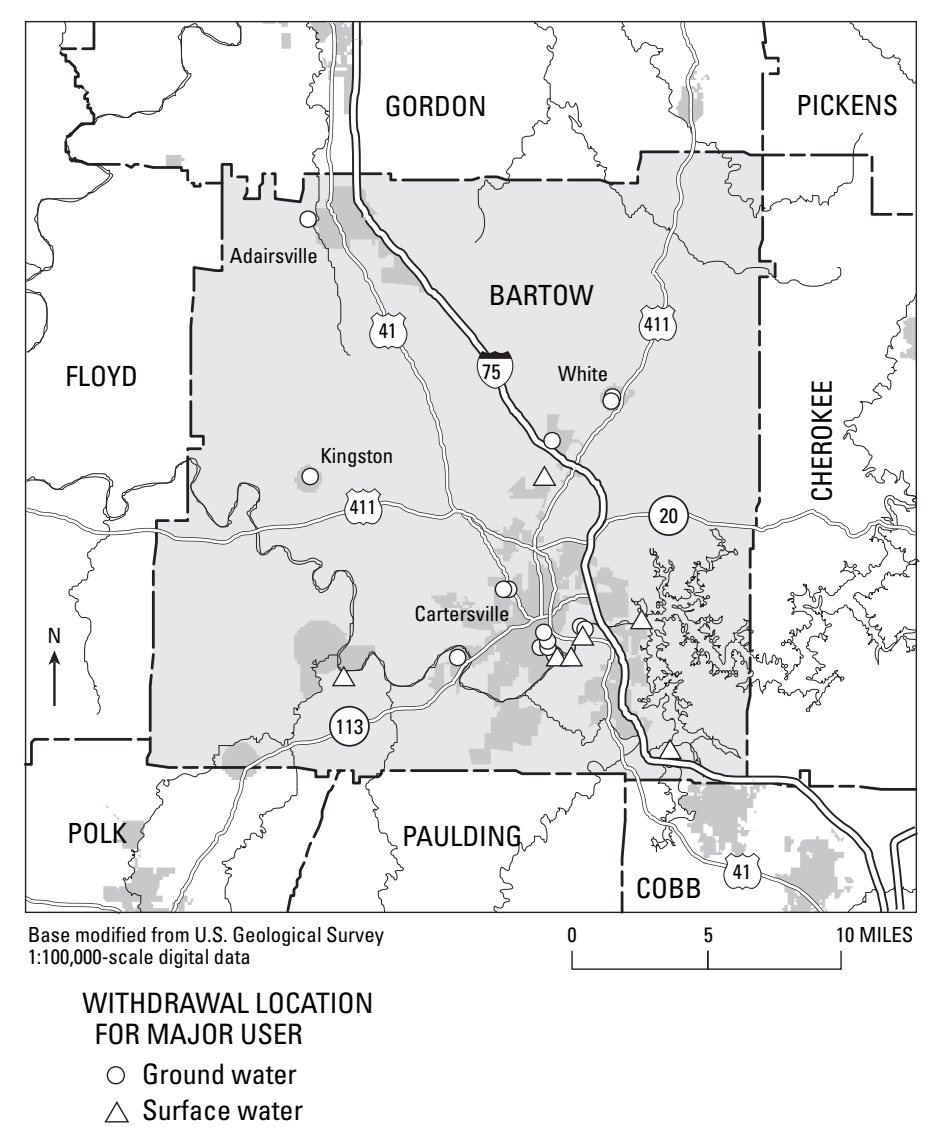




\section{BEN HILL COUNTY}

Population: 17,316

Population served by public supply: 13,670

Acres irrigated: 16,300

Hydroelectric use (Mgal/d): $\quad 0.00$

2005 WITHDRAWALS, IN MILLION GALLONS PER DAY

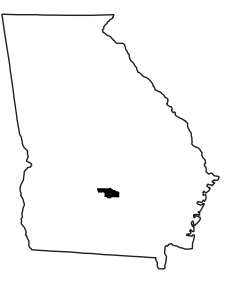

\begin{tabular}{|c|c|c|c|c|c|c|c|}
\hline & $\begin{array}{l}\text { Public } \\
\text { Supply }\end{array}$ & $\begin{array}{l}\text { Domestic \& } \\
\text { Commercial }\end{array}$ & $\begin{array}{c}\text { Industrial \& } \\
\text { Mining }\end{array}$ & Irrigation & Livestock & $\begin{array}{l}\text { Thermo- } \\
\text { electric }\end{array}$ & Totals \\
\hline Ground Water & 3.06 & 0.27 & 0.00 & 4.13 & 0.02 & 0.00 & 7.48 \\
\hline Surface Water & 0.00 & 0.00 & 0.00 & 3.13 & 0.07 & 0.00 & 3.20 \\
\hline TOTALS & 3.06 & 0.27 & 0.00 & 7.26 & 0.09 & 0.00 & 10.68 \\
\hline
\end{tabular}

Withdrawals by Major Public Suppliers (Mgal/d):

Name GW SW

Fitzgerald Water/Light $\quad 3.06 \quad 0.00$

Withdrawals by Major Industrial Groups (Mgal/d):

SIC

GW

SW

None
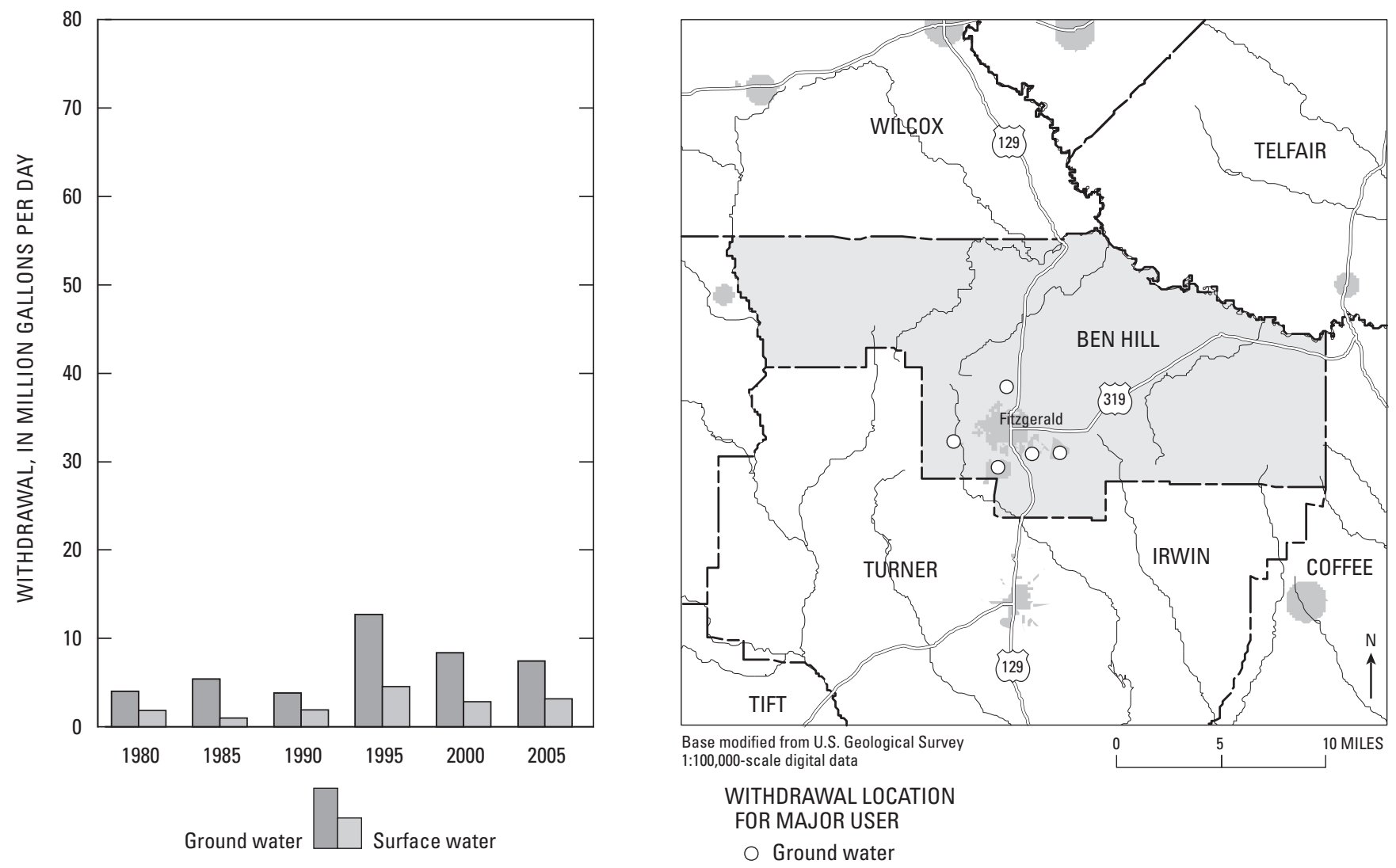


\section{BERRIEN COUNTY}

Population: 16,708

Population served by public supply: 7,540

Acres irrigated: 36,310

Hydroelectric use (Mgal/d): 0.00

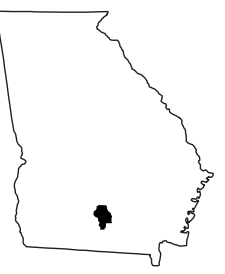

2005 WITHDRAWALS, IN MILLION GALLONS PER DAY

\begin{tabular}{|c|c|c|c|c|c|c|c|}
\hline & $\begin{array}{l}\text { Public } \\
\text { Supply }\end{array}$ & $\begin{array}{l}\text { Domestic \& } \\
\text { Commercial }\end{array}$ & $\begin{array}{c}\text { Industrial \& } \\
\text { Mining }\end{array}$ & Irrigation & Livestock & $\begin{array}{l}\text { Thermo- } \\
\text { electric }\end{array}$ & Totals \\
\hline Ground Water & 0.99 & 0.69 & 0.08 & 2.07 & 0.97 & 0.00 & 4.80 \\
\hline Surface Water & 0.00 & 0.00 & 0.00 & 1.92 & 0.14 & 0.00 & 2.06 \\
\hline TOTALS & 0.99 & 0.69 & 0.08 & 3.99 & 1.11 & 0.00 & 6.86 \\
\hline
\end{tabular}

Withdrawals by Major Public Suppliers (Mgal/d):

Name

Town of Alapaha

Town of Enigma

City of Nashville

City of Ray City
GW SW

$0.06 \quad 0.00$

$0.08 \quad 0.00$

$0.78 \quad 0.00$

$0.06 \quad 0.00$
Withdrawals by Major Industrial Groups (Mgal/d):

SIC

GW

SW

22 - Textiles

0.04

0.00

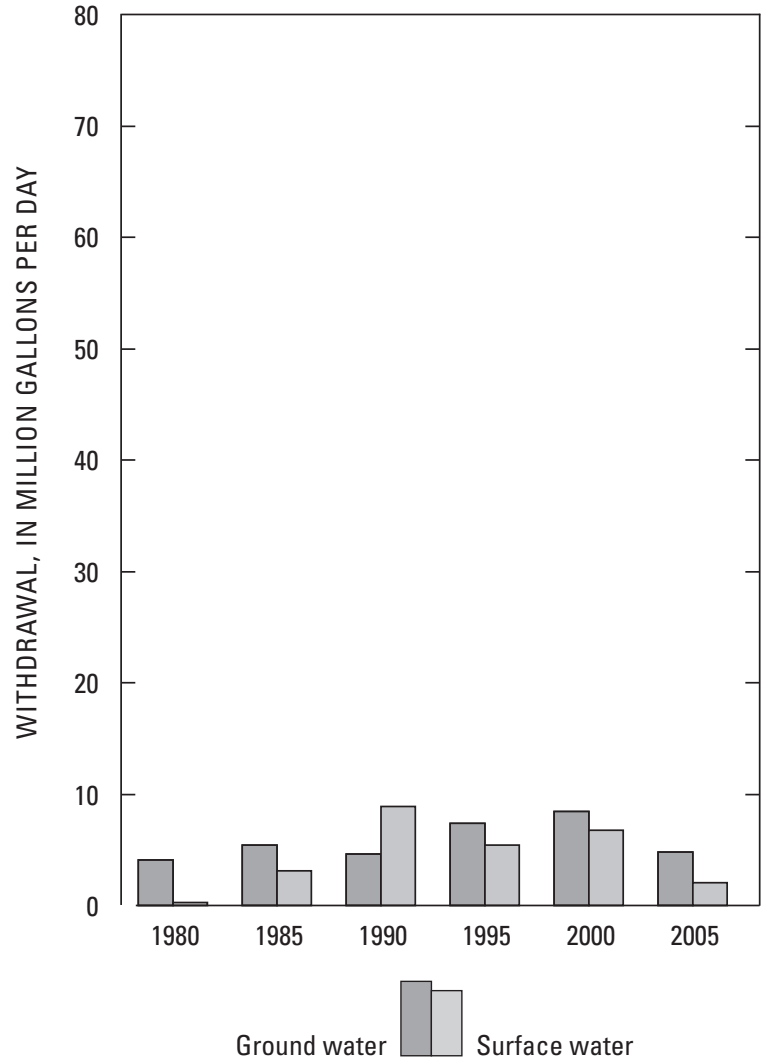

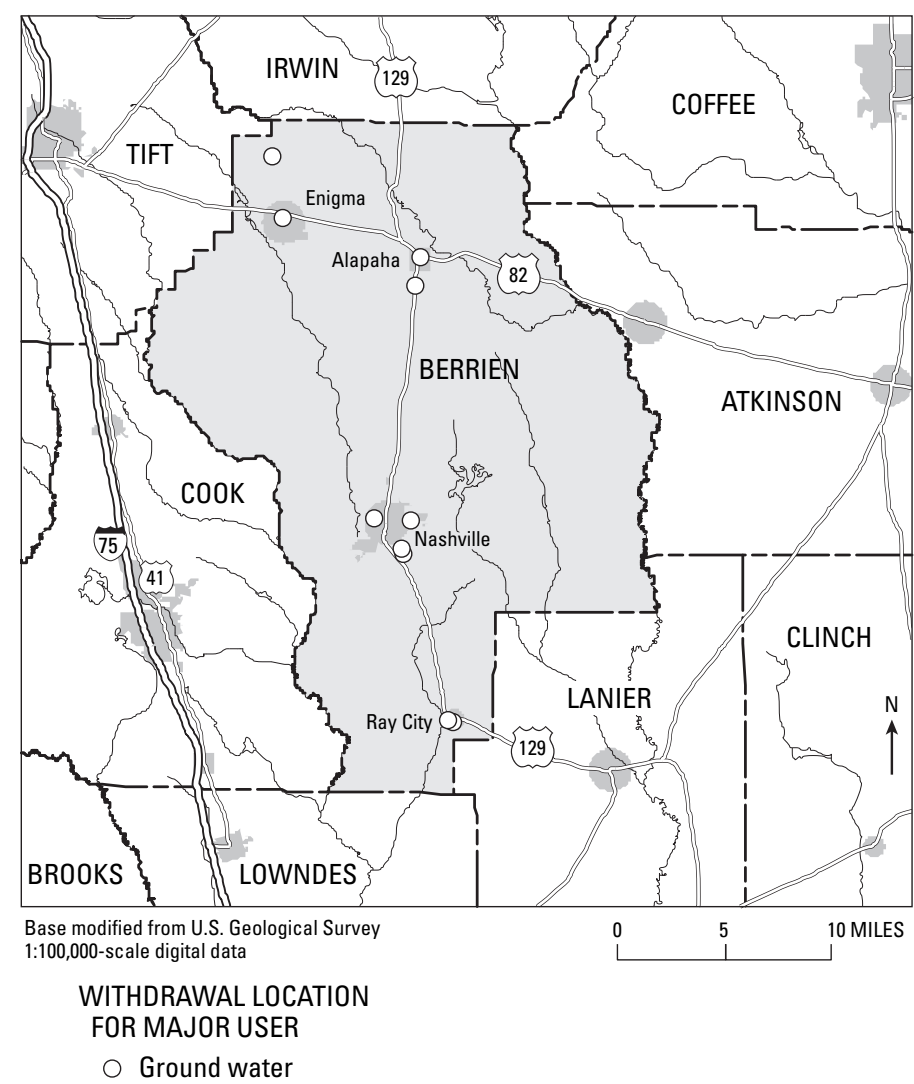




\section{BIBB COUNTY}

Population: 154,918

Population served by public supply: 130,280

Acres irrigated: 790

Hydroelectric use (Mgal/d): $\quad 0.00$

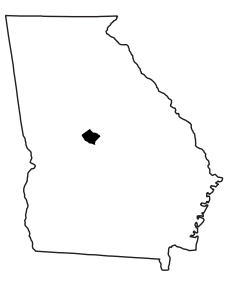

2005 WITHDRAWALS, IN MILLION GALLONS PER DAY

\begin{tabular}{|c|c|c|c|c|c|c|c|}
\hline & $\begin{array}{l}\text { Public } \\
\text { Supply }\end{array}$ & $\begin{array}{l}\text { Domestic \& } \\
\text { Commercial }\end{array}$ & $\begin{array}{c}\text { Industrial \& } \\
\text { Mining }\end{array}$ & Irrigation & Livestock & $\begin{array}{l}\text { Thermo- } \\
\text { electric }\end{array}$ & Totals \\
\hline Ground Water & 0.14 & 1.85 & 3.12 & 1.06 & 0.01 & 0.00 & 6.18 \\
\hline Surface Water & $25.39^{\mathrm{a}}$ & 0.00 & 12.45 & 0.00 & 0.05 & 0.00 & 37.89 \\
\hline TOTALS & 25.53 & 1.85 & 15.57 & 1.06 & 0.06 & 0.00 & 44.07 \\
\hline
\end{tabular}

Withdrawals by Major Public Suppliers (Mgal/d):

\section{Name}

Macon Water Authority

GW SW

$0.00 \quad 25.39^{\mathrm{a}}$

Withdrawals by Major Industrial Groups (Mgal/d):

$\begin{array}{lcc}\text { SIC } & \text { GW } & \text { SW } \\ 26 \text { - Paper } & 0.64 & 12.43 \\ 32 \text { - Stone, clay } & 1.17 & 0\end{array}$

${ }^{\mathrm{a}}$ Water used in Bibb County but withdrawn from Jones County.
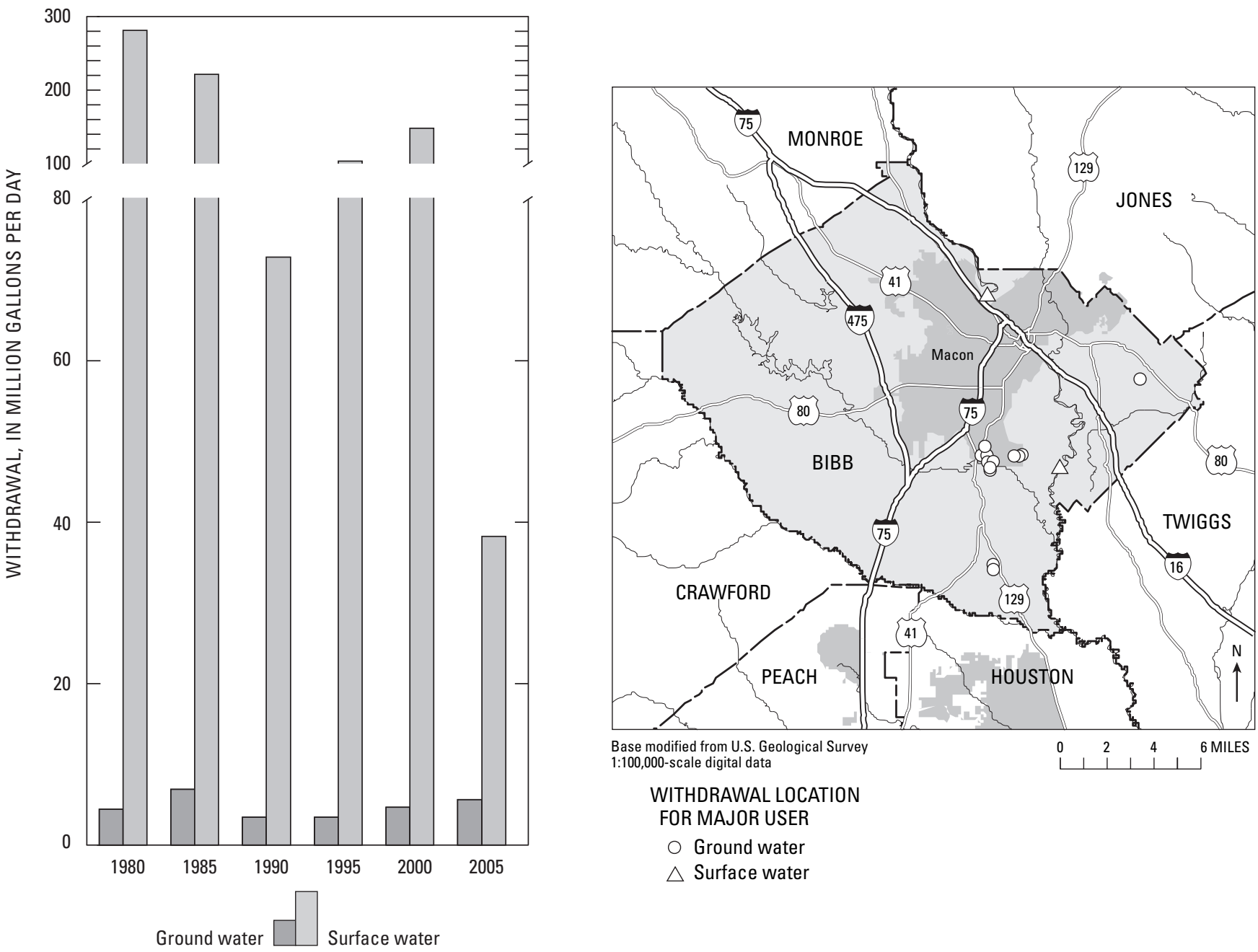


\section{BLECKLEY COUNTY}

Population: 12,141

Population served by public supply: $\quad 5,380$

Acres irrigated: 12,850

Hydroelectric use (Mgal/d): $\quad 0.00$

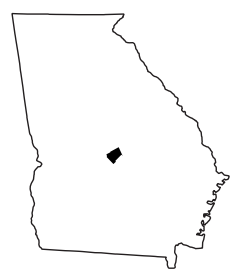

2005 WITHDRAWALS, IN MILLION GALLONS PER DAY

\begin{tabular}{|c|c|c|c|c|c|c|c|}
\hline & $\begin{array}{l}\text { Public } \\
\text { Supply }\end{array}$ & $\begin{array}{l}\text { Domestic \& } \\
\text { Commercial }\end{array}$ & $\begin{array}{c}\text { Industrial \& } \\
\text { Mining }\end{array}$ & Irrigation & Livestock & $\begin{array}{l}\text { Thermo- } \\
\text { electric }\end{array}$ & Totals \\
\hline Ground Water & 0.21 & 0.58 & 0.00 & 7.04 & 0.01 & 0.00 & 7.84 \\
\hline Surface Water & 0.00 & 0.00 & 0.00 & 5.74 & 0.04 & 0.00 & 5.78 \\
\hline TOTALS & 0.21 & 0.58 & 0.00 & 12.78 & 0.05 & 0.00 & 13.62 \\
\hline
\end{tabular}

Withdrawals by Major Public Suppliers (Mgal/d):

Name

GW SW

City of Cochran

0.21

0.00
Withdrawals by Major Industrial Groups (Mgal/d):

SIC

GW

SW
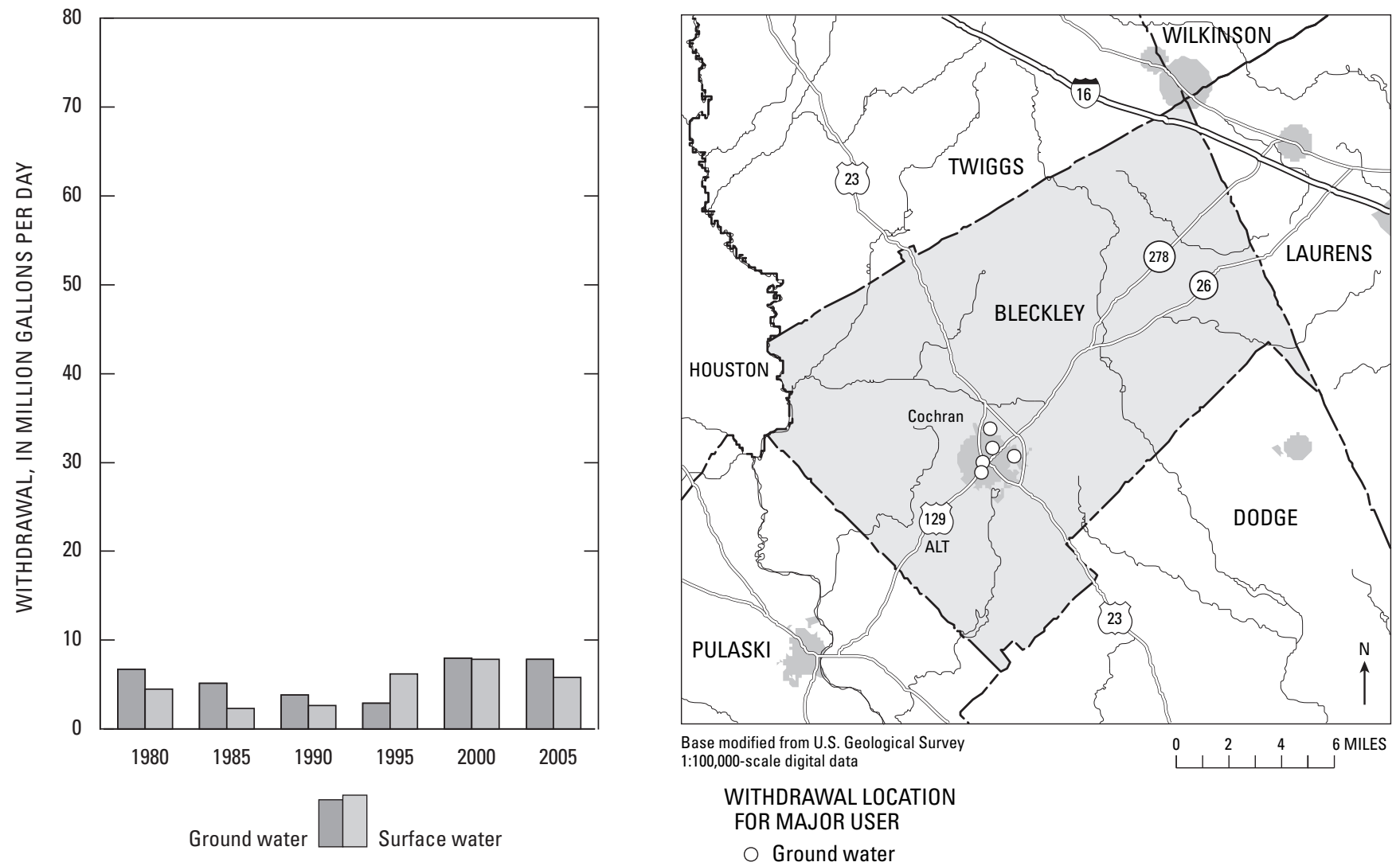


\section{BRANTLEY COUNTY}

Population: 15,491

Population served by public supply: $\quad 2,160$

Acres irrigated: 300

Hydroelectric use (Mgal/d): $\quad 0.00$

2005 WITHDRAWALS, IN MILLION GALLONS PER DAY

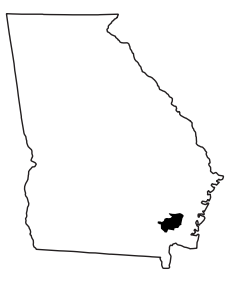

\begin{tabular}{lccccccc}
\hline & $\begin{array}{c}\text { Public } \\
\text { Supply }\end{array}$ & $\begin{array}{c}\text { Domestic \& } \\
\text { Commercial }\end{array}$ & $\begin{array}{c}\text { Industrial \& } \\
\text { Mining }\end{array}$ & Irrigation & Livestock & $\begin{array}{c}\text { Thermo- } \\
\text { electric }\end{array}$ & Totals \\
\cline { 1 - 4 } Ground Water & 0.20 & 1.00 & 0.42 & 0.05 & 0.01 & 0.00 & 1.68 \\
Surface Water & 0.00 & 0.00 & 1.02 & 0.06 & 0.07 & 0.00 & 1.15 \\
TOTALS & 0.20 & 1.00 & 1.44 & 0.11 & 0.08 & 0.00 & 2.83 \\
\hline
\end{tabular}

Withdrawals by Major Public Suppliers (Mgal/d):

$\begin{array}{lcc}\text { Name } & \text { GW } & \text { SW } \\ \text { City of Hoboken } & 0.04 & 0.00 \\ \text { City of Nahunta } & 0.12 & 0.00\end{array}$

Withdrawals by Major Industrial Groups (Mgal/d):

SIC

GW

SW

City of Nahunta
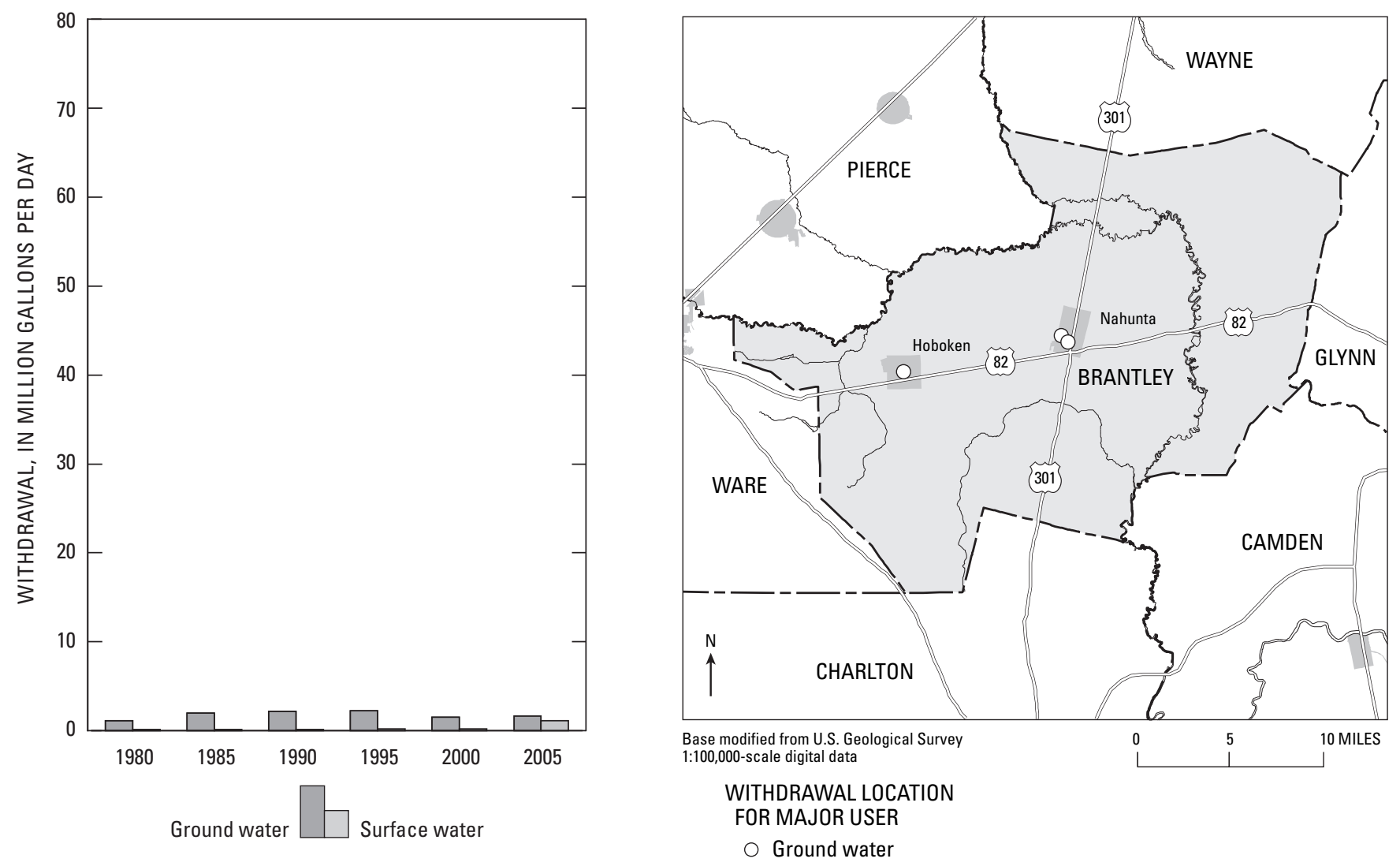


\section{BROOKS COUNTY}

Population: 16,327

Population served by public supply: $\quad 6,990$

Acres irrigated: 8,330

Hydroelectric use (Mgal/d): 0.00

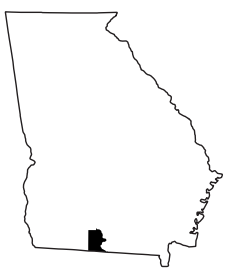

2005 WITHDRAWALS, IN MILLION GALLONS PER DAY

\begin{tabular}{|c|c|c|c|c|c|c|c|}
\hline & $\begin{array}{l}\text { Public } \\
\text { Supply }\end{array}$ & $\begin{array}{l}\text { Domestic \& } \\
\text { Commercial }\end{array}$ & $\begin{array}{c}\text { Industrial \& } \\
\text { Mining }\end{array}$ & Irrigation & Livestock & $\begin{array}{l}\text { Thermo- } \\
\text { electric }\end{array}$ & Totals \\
\hline Ground Water & 0.96 & 0.83 & 0.13 & 2.29 & 0.05 & 0.00 & 4.26 \\
\hline Surface Water & 0.00 & 0.00 & 0.00 & 0.11 & 0.22 & 0.00 & 0.33 \\
\hline TOTALS & 0.96 & 0.83 & 0.13 & 2.40 & 0.27 & 0.00 & 4.59 \\
\hline
\end{tabular}

Withdrawals by Major Public Suppliers (Mgal/d):

\section{Name}

Town of Barwick

City of Morven

City of Quitman
GW SW

$0.03 \quad 0.00$

$0.05 \quad 0.00$

$0.78 \quad 0.00$
Withdrawals by Major Industrial Groups (Mgal/d):

SIC

GW

SW
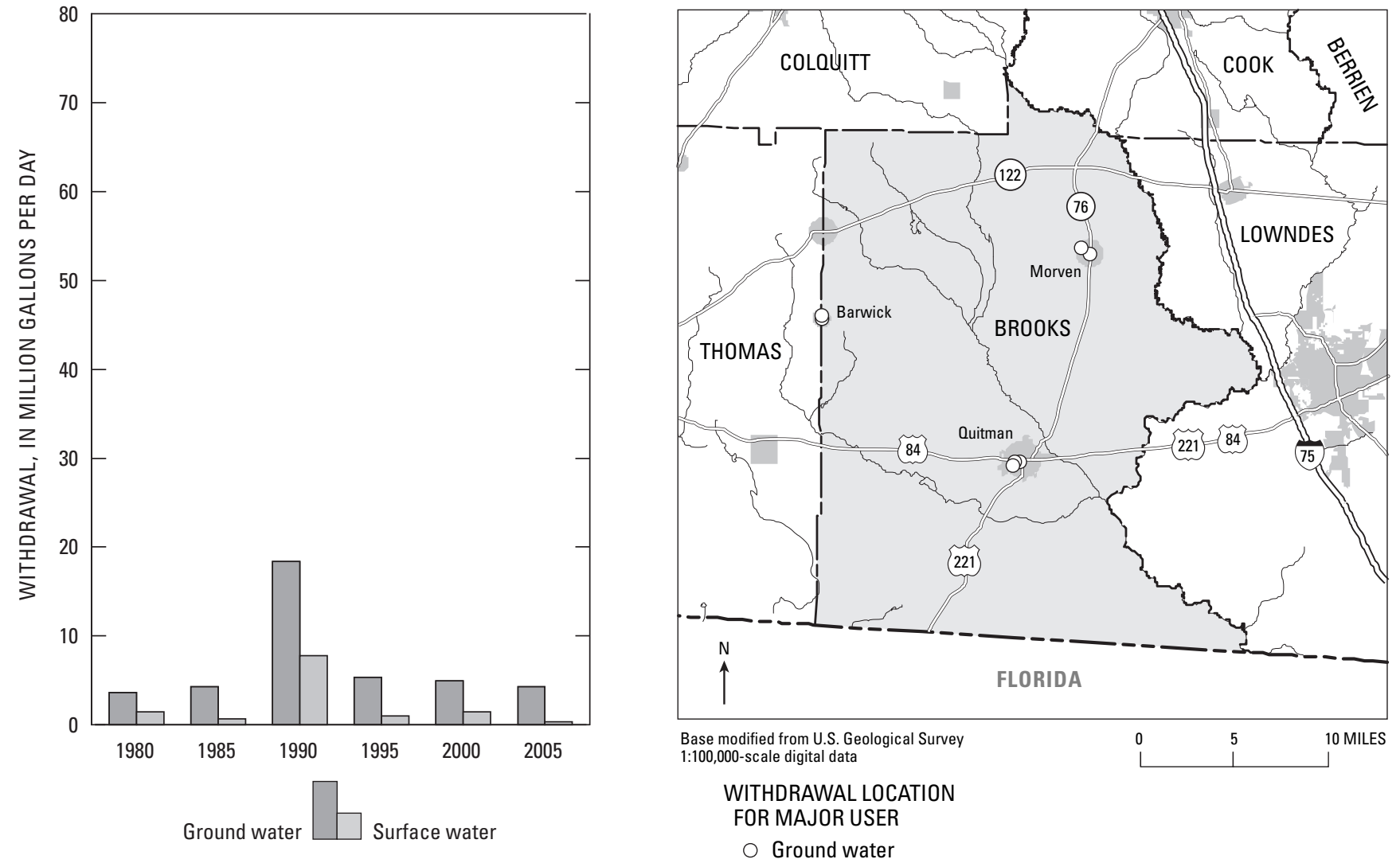


\section{BRYAN COUNTY}

Population: 28,549

Population served by public supply: $\quad 18,280$

Acres irrigated: 400

Hydroelectric use (Mgal/d): $\quad 0.00$

2005 WITHDRAWALS, IN MILLION GALLONS PER DAY

\begin{tabular}{|c|c|c|c|c|c|c|c|}
\hline & $\begin{array}{l}\text { Public } \\
\text { Supply }\end{array}$ & $\begin{array}{l}\text { Domestic \& } \\
\text { Commercial }\end{array}$ & $\begin{array}{c}\text { Industrial \& } \\
\text { Mining }\end{array}$ & Irrigation & Livestock & $\begin{array}{l}\text { Thermo- } \\
\text { electric }\end{array}$ & Totals \\
\hline Ground Water & 1.73 & 0.82 & 0.00 & 0.59 & 0.00 & 0.00 & 3.14 \\
\hline Surface Water & 0.00 & 0.00 & 0.00 & 0.00 & 0.01 & 0.00 & 0.01 \\
\hline TOTALS & 1.73 & 0.82 & 0.00 & 0.59 & 0.01 & 0.00 & 3.15 \\
\hline
\end{tabular}

Withdrawals by Major Public Suppliers (Mgal/d):

Name

City of Pembroke

City of Richmond Hill
GW

0.23

1.06
SW 0.00 0.00
Withdrawals by Major Industrial Groups (Mgal/d):

SIC

GW

SW
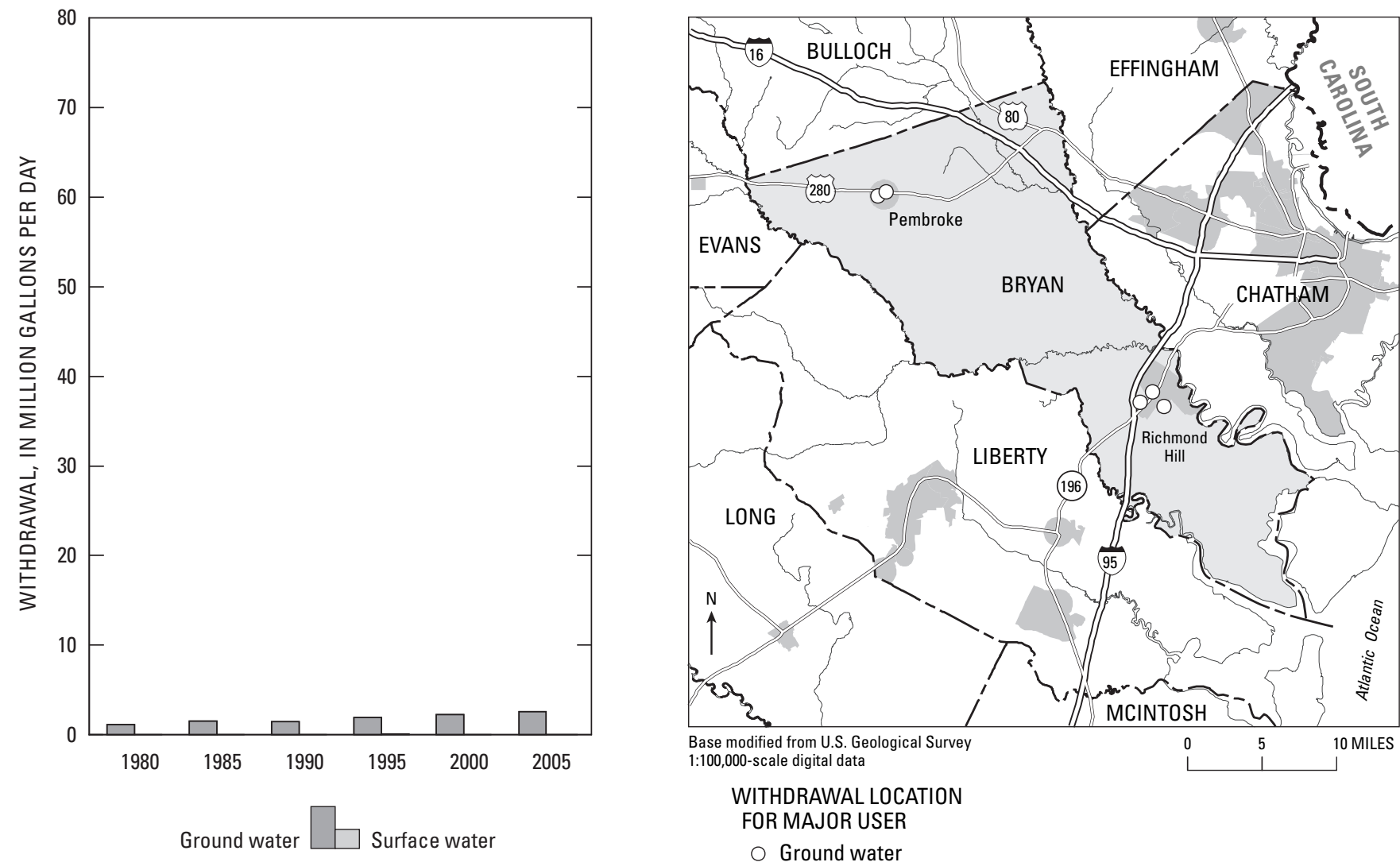


\section{BULLOCH COUNTY}

Population: 61,454

Population served by public supply: 48,470

Acres irrigated: 22,270

Hydroelectric use (Mgal/d): $\quad 0.00$

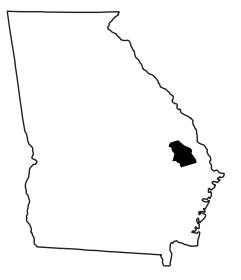

2005 WITHDRAWALS, IN MILLION GALLONS PER DAY

\begin{tabular}{|c|c|c|c|c|c|c|c|}
\hline & $\begin{array}{l}\text { Public } \\
\text { Supply }\end{array}$ & $\begin{array}{l}\text { Domestic \& } \\
\text { Commercial }\end{array}$ & $\begin{array}{c}\text { Industrial \& } \\
\text { Mining }\end{array}$ & Irrigation & Livestock & $\begin{array}{l}\text { Thermo- } \\
\text { electric }\end{array}$ & Totals \\
\hline Ground Water & 4.01 & 1.37 & 0.03 & 7.07 & 0.07 & 0.00 & 12.55 \\
\hline Surface Water & 0.00 & 0.00 & 0.00 & 4.58 & 0.16 & 0.00 & 4.74 \\
\hline TOTALS & 4.01 & 1.37 & 0.03 & 11.65 & 0.23 & 0.00 & 17.29 \\
\hline
\end{tabular}

Withdrawals by Major Public Suppliers (Mgal/d):

Name

Town of Brooklet

GW SW

Nevils Water Association

Town of Portal

$0.12 \quad 0.00$

$0.04 \quad 0.00$

$0.07 \quad 0.00$

Register Water System $\quad 0.02 \quad 0.00$

City of Statesboro
Withdrawals by Major Industrial Groups (Mgal/d):

SIC

33 - Primary metals
GW

0.03
SW

0.00
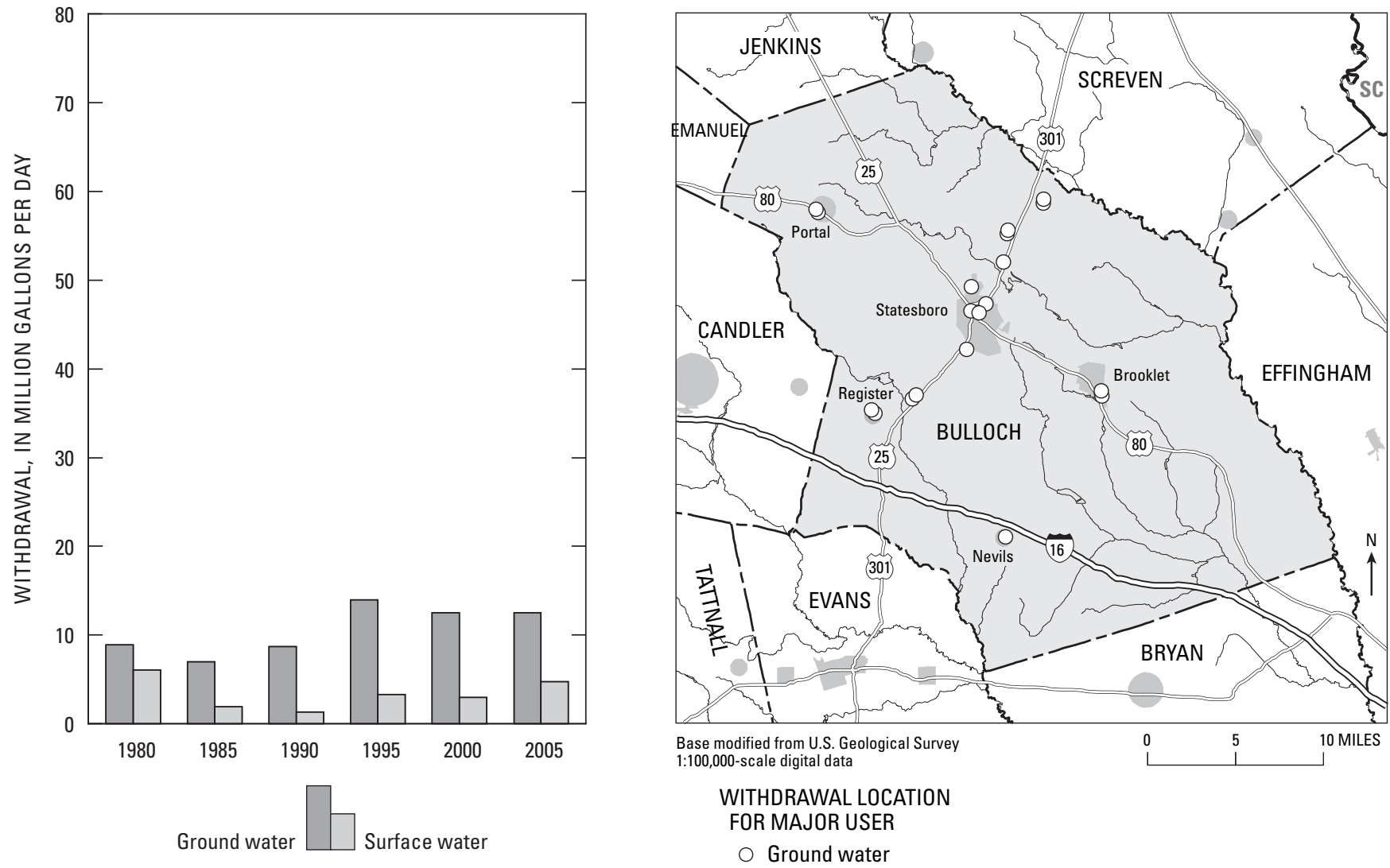


\section{BURKE COUNTY}

Population: 23,299

Population served by public supply: 8,390

Acres irrigated: 28,300

Hydroelectric use (Mgal/d): 0.00

2005 WITHDRAWALS, IN MILLION GALLONS PER DAY

\begin{tabular}{|c|c|c|c|c|c|c|c|}
\hline & $\begin{array}{l}\text { Public } \\
\text { Supply }\end{array}$ & $\begin{array}{l}\text { Domestic \& } \\
\text { Commercial }\end{array}$ & $\begin{array}{c}\text { Industrial \& } \\
\text { Mining }\end{array}$ & Irrigation & Livestock & $\begin{array}{l}\text { Thermo- } \\
\text { electric }\end{array}$ & Totals \\
\hline Ground Water & 0.97 & 1.12 & 0.05 & 11.66 & 0.05 & 0.85 & 14.70 \\
\hline Surface Water & 0.14 & 0.00 & 0.00 & 4.28 & 0.19 & 64.51 & 69.12 \\
\hline TOTALS & 1.11 & 1.12 & 0.05 & 15.94 & 0.24 & 65.36 & 83.82 \\
\hline
\end{tabular}

Withdrawals by Major Public Suppliers (Mgal/d):

\section{Name}

Town of Girard

City of Midville

Town of Sardis

City of Vidette

City of Waynesboro
SW

0.00

$0.06 \quad 0.00$

$0.07 \quad 0.00$

$0.01 \quad 0.00$

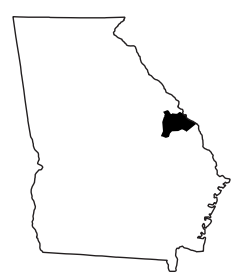

Withdrawals by Major Industrial Groups (Mgal/d):

SIC GW SW

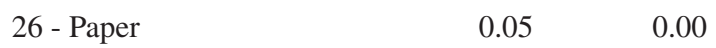
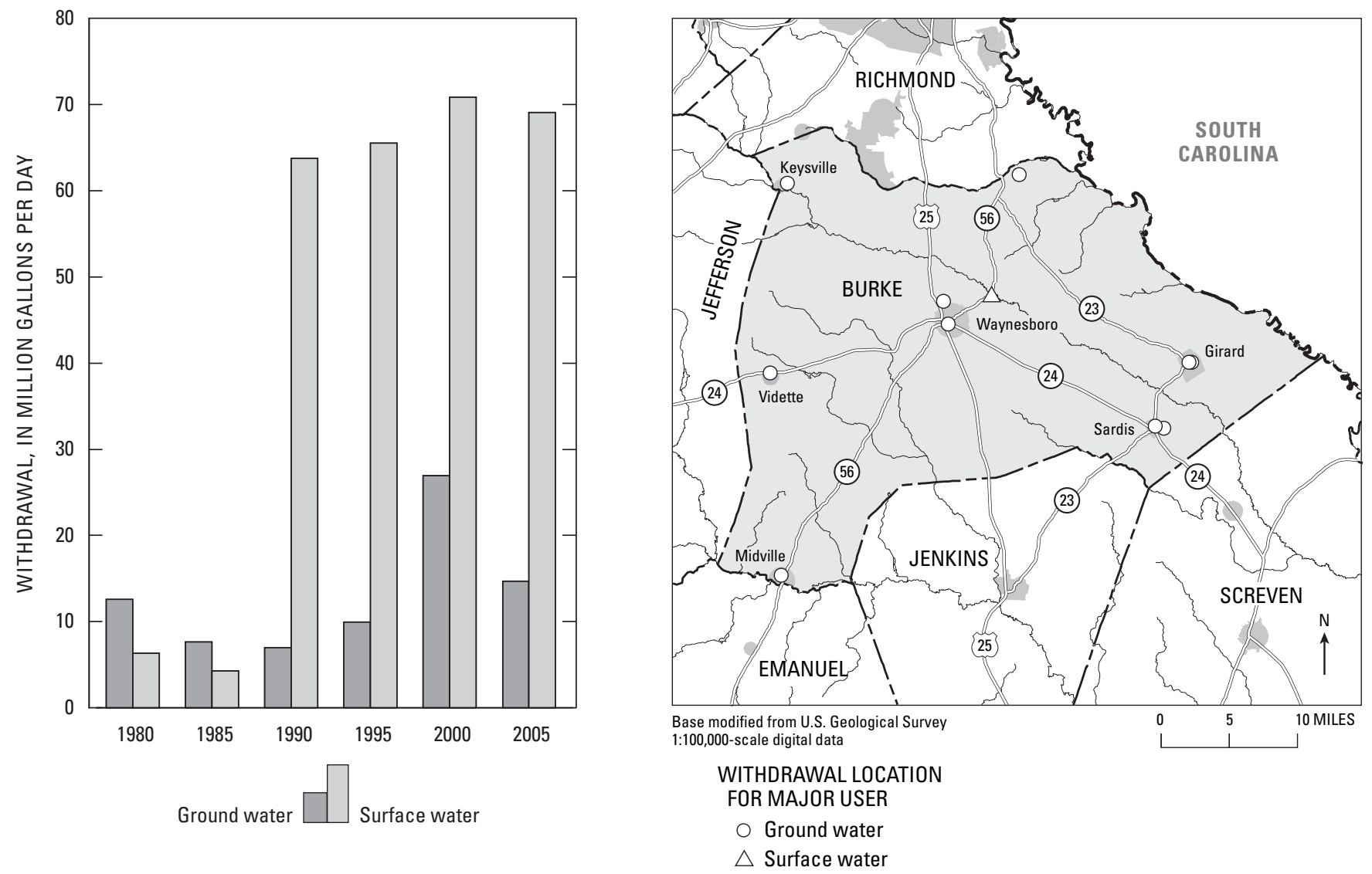


\section{BUTTS COUNTY}

Population: 21,045

Population served by public supply: $\quad 16,840$

Acres irrigated: 210

Hydroelectric use (Mgal/d): $\quad 1,070.95$

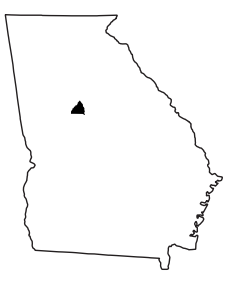

2005 WITHDRAWALS, IN MILLION GALLONS PER DAY

\begin{tabular}{|c|c|c|c|c|c|c|c|}
\hline & $\begin{array}{l}\text { Public } \\
\text { Supply }\end{array}$ & $\begin{array}{l}\text { Domestic \& } \\
\text { Commercial }\end{array}$ & $\begin{array}{c}\text { Industrial \& } \\
\text { Mining }\end{array}$ & Irrigation & Livestock & $\begin{array}{l}\text { Thermo- } \\
\text { electric }\end{array}$ & Totals \\
\hline Ground Water & 0.14 & 0.32 & 0.00 & 0.00 & 0.00 & 0.00 & 0.46 \\
\hline Surface Water & 2.79 & 0.00 & 0.00 & 0.21 & 0.04 & 0.00 & 3.04 \\
\hline TOTALS & 2.93 & 0.32 & 0.00 & 0.21 & 0.04 & 0.00 & 3.50 \\
\hline
\end{tabular}

Withdrawals by Major Public Suppliers (Mgal/d):

Name

Butts County Water \&

GW

SW

Sewer Authority

City of Jackson

City of Flovilla

Town of Jenkinsburg

0.00

2.50

0.09

0.00

0.02

0.00

0.29

0.00
Withdrawals by Major Industrial Groups (Mgal/d):

SIC

GW

SW
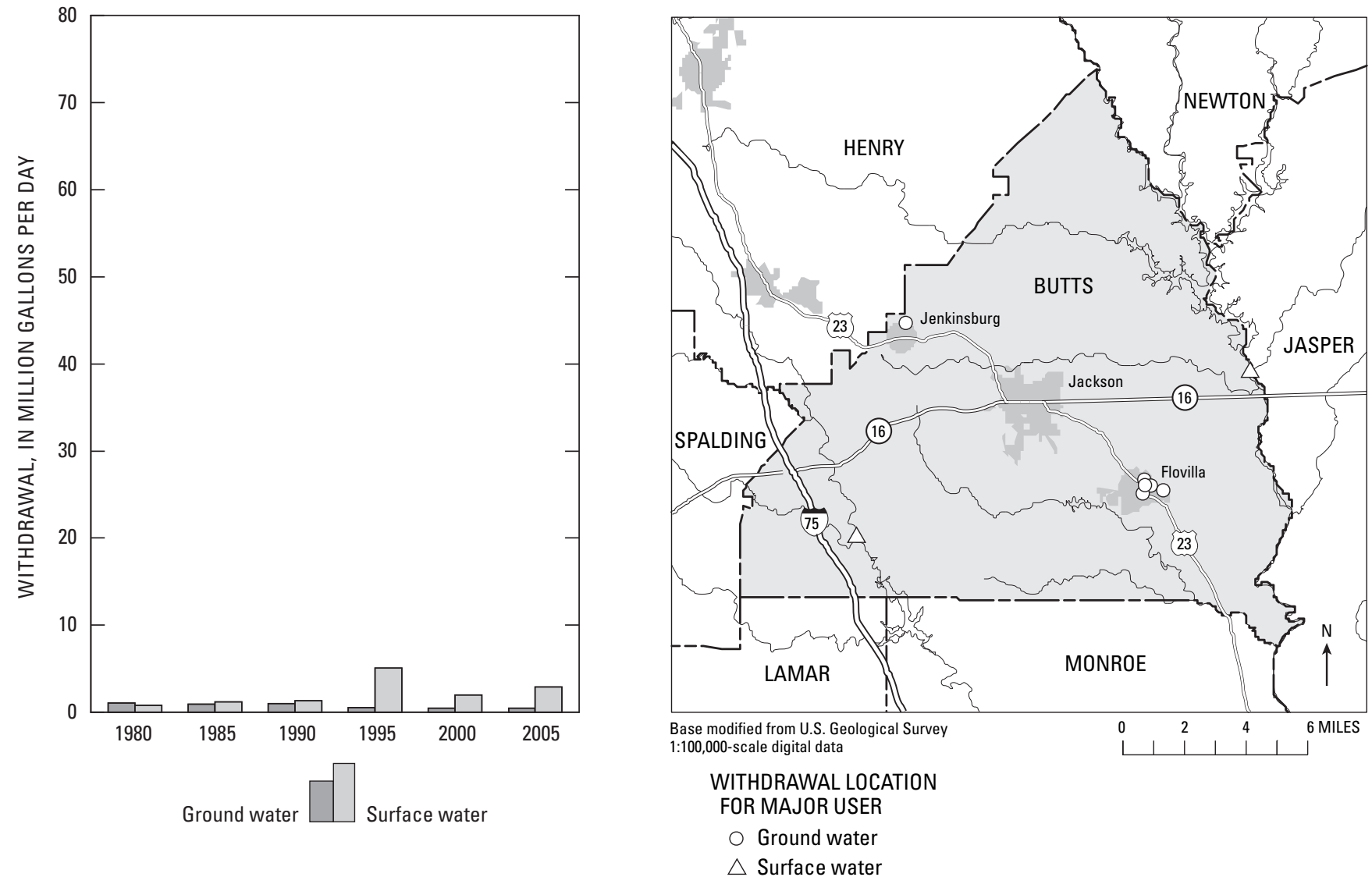


\section{CALHOUN COUNTY}

Population: 5,972

Population served by public supply: $\quad 5,170$

Acres irrigated: 48,880

Hydroelectric use (Mgal/d): $\quad 0.00$

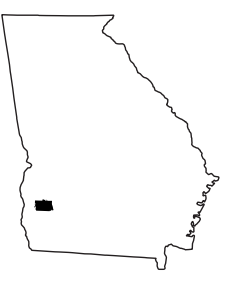

2005 WITHDRAWALS, IN MILLION GALLONS PER DAY

\begin{tabular}{|c|c|c|c|c|c|c|c|}
\hline & $\begin{array}{l}\text { Public } \\
\text { Supply }\end{array}$ & $\begin{array}{l}\text { Domestic \& } \\
\text { Commercial }\end{array}$ & $\begin{array}{c}\text { Industrial \& } \\
\text { Mining }\end{array}$ & Irrigation & Livestock & $\begin{array}{l}\text { Thermo- } \\
\text { electric }\end{array}$ & Totals \\
\hline Ground Water & 0.78 & 0.06 & 0.00 & 16.39 & 0.02 & 0.00 & 17.25 \\
\hline Surface Water & 0.00 & 0.00 & 0.00 & 9.72 & 0.06 & 0.00 & 9.78 \\
\hline TOTALS & 0.78 & 0.06 & 0.00 & 26.11 & 0.08 & 0.00 & 27.03 \\
\hline
\end{tabular}

Withdrawals by Major Public Suppliers (Mgal/d):

\section{Name}

City of Arlington

City of Edison

City of Leary

City of Morgan

\section{GW}

0.29

0.18

0.08

0.23
SW

0.00

0.00

0.00

0.00
Withdrawals by Major Industrial Groups (Mgal/d):

SIC

None
SW

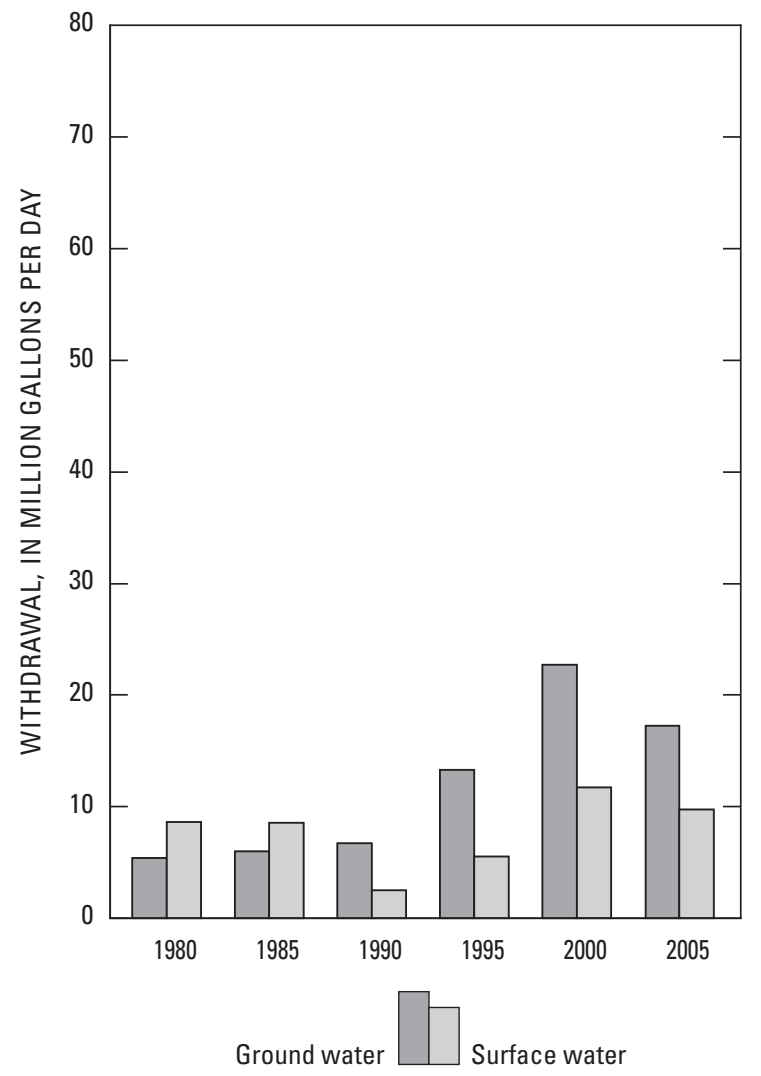

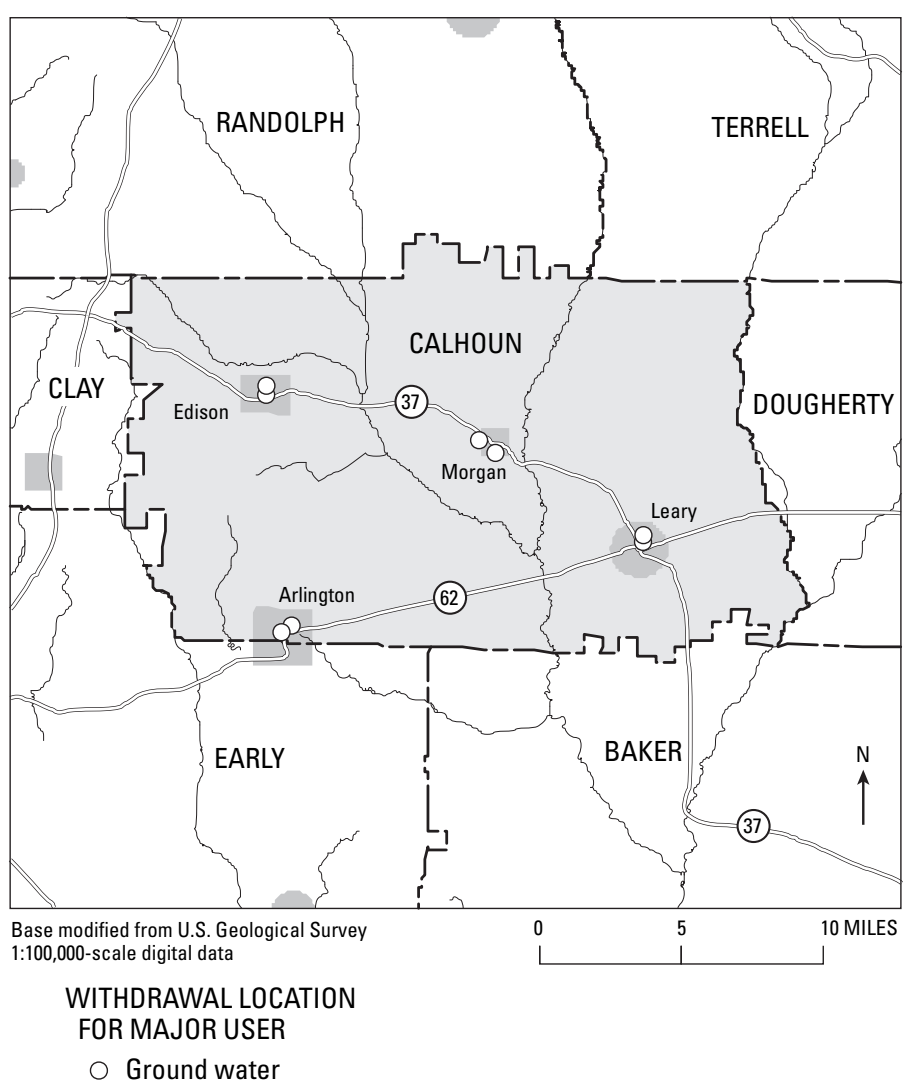




\section{CAMDEN COUNTY}

Population: 45,759

Population served by public supply: $\quad 31,170$

Acres irrigated: 690

Hydroelectric use (Mgal/d): $\quad 0.00$

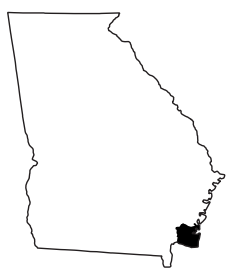

2005 WITHDRAWALS, IN MILLION GALLONS PER DAY

\begin{tabular}{|c|c|c|c|c|c|c|c|}
\hline & $\begin{array}{l}\text { Public } \\
\text { Supply }\end{array}$ & $\begin{array}{l}\text { Domestic \& } \\
\text { Commercial }\end{array}$ & $\begin{array}{c}\text { Industrial \& } \\
\text { Mining }\end{array}$ & Irrigation & Livestock & $\begin{array}{l}\text { Thermo- } \\
\text { electric }\end{array}$ & Totals \\
\hline Ground Water & 4.06 & 2.01 & 0.07 & 1.38 & 0.05 & 0.00 & 7.57 \\
\hline Surface Water & 0.00 & 0.00 & 0.00 & 0.00 & 0.01 & 0.00 & 0.01 \\
\hline TOTALS & 4.06 & 2.01 & 0.07 & 1.38 & 0.06 & 0.00 & 7.58 \\
\hline
\end{tabular}

Withdrawals by Major Public Suppliers (Mgal/d):

Name

GW

SW

City of Kingsland

City of St. Marys

1.45

0.00

City of Woodbine

1.48

0.00

0.14

0.00

USN Base Support
Withdrawals by Major Industrial Groups (Mgal/d):

SIC

26 - Paper

GW

0.01

SW

28 - Chemicals

0.06

0.00

0.00
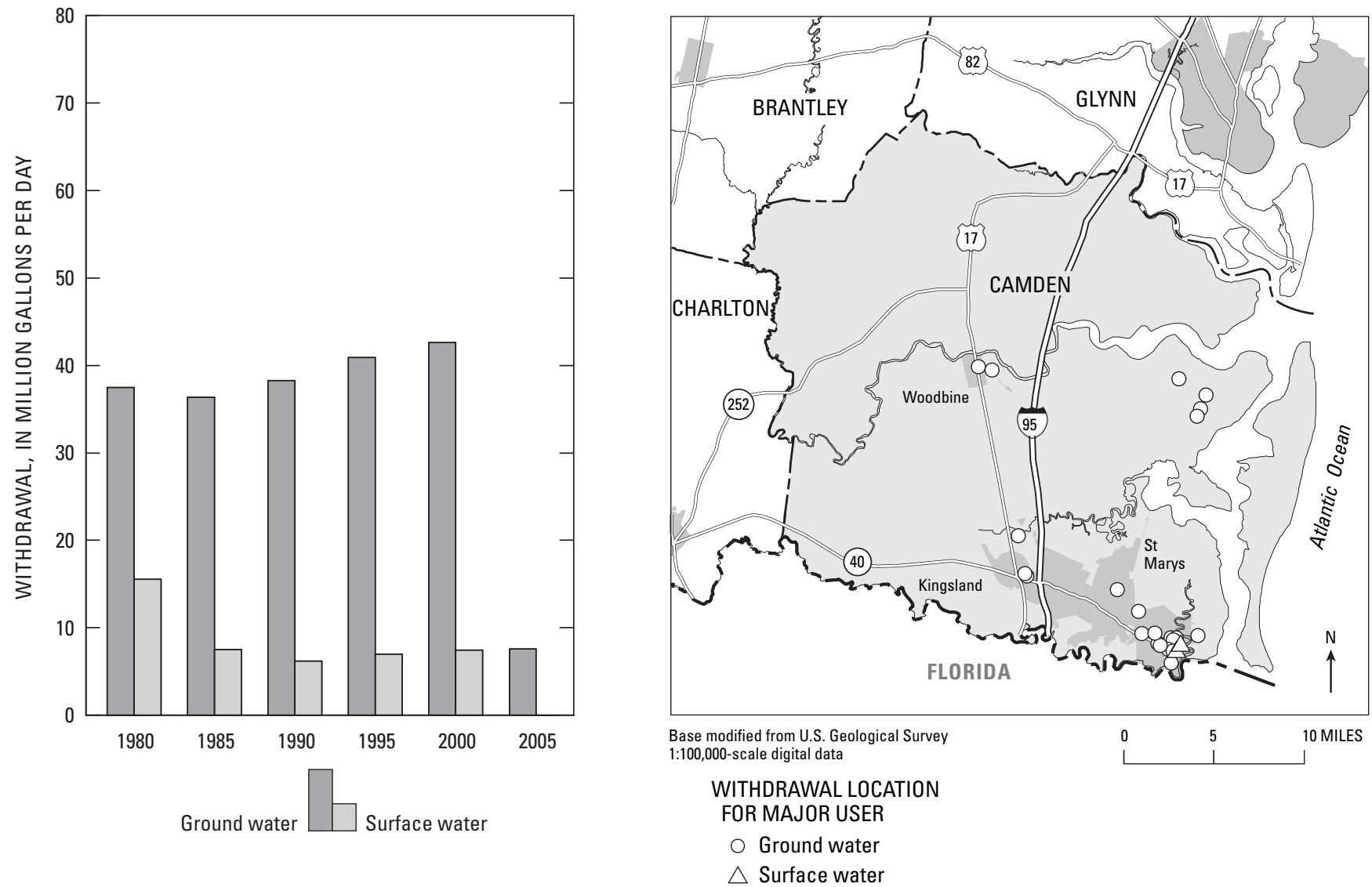


\section{CANDLER COUNTY}

Population: 10,321

Population served by public supply: $\quad 4,380$

Acres irrigated: 16,900

Hydroelectric use (Mgal/d): $\quad 0.00$

2005 WITHDRAWALS, IN MILLION GALLONS PER DAY

\begin{tabular}{|c|c|c|c|c|c|c|c|}
\hline & $\begin{array}{l}\text { Public } \\
\text { Supply }\end{array}$ & $\begin{array}{l}\text { Domestic \& } \\
\text { Commercial }\end{array}$ & $\begin{array}{c}\text { Industrial \& } \\
\text { Mining }\end{array}$ & Irrigation & Livestock & $\begin{array}{l}\text { Thermo- } \\
\text { electric }\end{array}$ & Totals \\
\hline Ground Water & 0.46 & 0.45 & 0.00 & 4.03 & 0.02 & 0.00 & 4.96 \\
\hline Surface Water & 0.00 & 0.00 & 0.00 & 5.98 & 0.19 & 0.00 & 6.17 \\
\hline TOTALS & 0.46 & 0.45 & 0.00 & 10.01 & 0.21 & 0.00 & 11.13 \\
\hline
\end{tabular}

Withdrawals by Major Public Suppliers (Mgal/d):

Name

City of Metter

Town of Pulaski
GW SW

$0.44 \quad 0.00$

$0.02 \quad 0.00$
Withdrawals by Major Industrial Groups (Mgal/d):

SIC

GW

SW
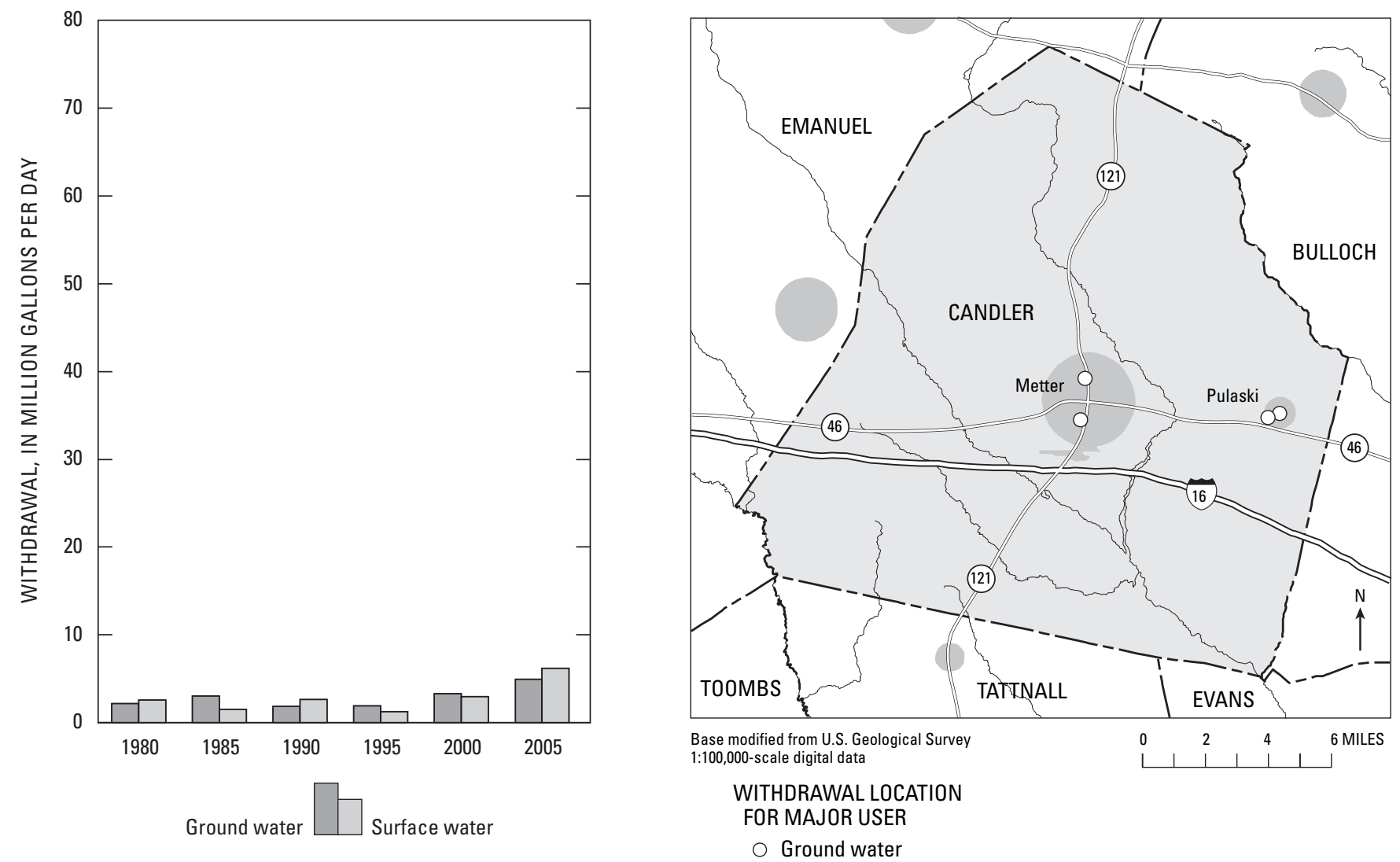


\section{CARROLL COUNTY}

Population: 105,453

Population served by public supply: $\quad 82,870$

Acres irrigated: 440

Hydroelectric use (Mgal/d): 0.00

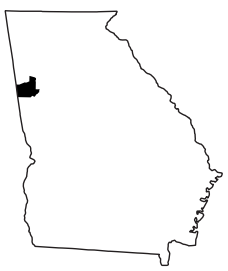

2005 WITHDRAWALS, IN MILLION GALLONS PER DAY

\begin{tabular}{|c|c|c|c|c|c|c|c|}
\hline & $\begin{array}{l}\text { Public } \\
\text { Supply }\end{array}$ & $\begin{array}{l}\text { Domestic \& } \\
\text { Commercial }\end{array}$ & $\begin{array}{c}\text { Industrial \& } \\
\text { Mining }\end{array}$ & Irrigation & Livestock & $\begin{array}{l}\text { Thermo- } \\
\text { electric }\end{array}$ & Totals \\
\hline Ground Water & 0.63 & 1.69 & 0.25 & 0.00 & 0.02 & 0.00 & 2.59 \\
\hline Surface Water & 11.05 & 0.00 & 0.09 & 0.00 & 0.69 & 0.00 & 11.83 \\
\hline TOTALS & 11.68 & 1.69 & 0.34 & 0.00 & 0.71 & 0.00 & 14.42 \\
\hline
\end{tabular}

Withdrawals by Major Public Suppliers (Mgal/d):

Name

GW SW

City of Bowdon

$0.00 \quad 0.75$

City of Carrollton

0.00

0.08

0.02

0.00

0.05

0.07

0.40

Town of Whitesburg

Carroll County Water System
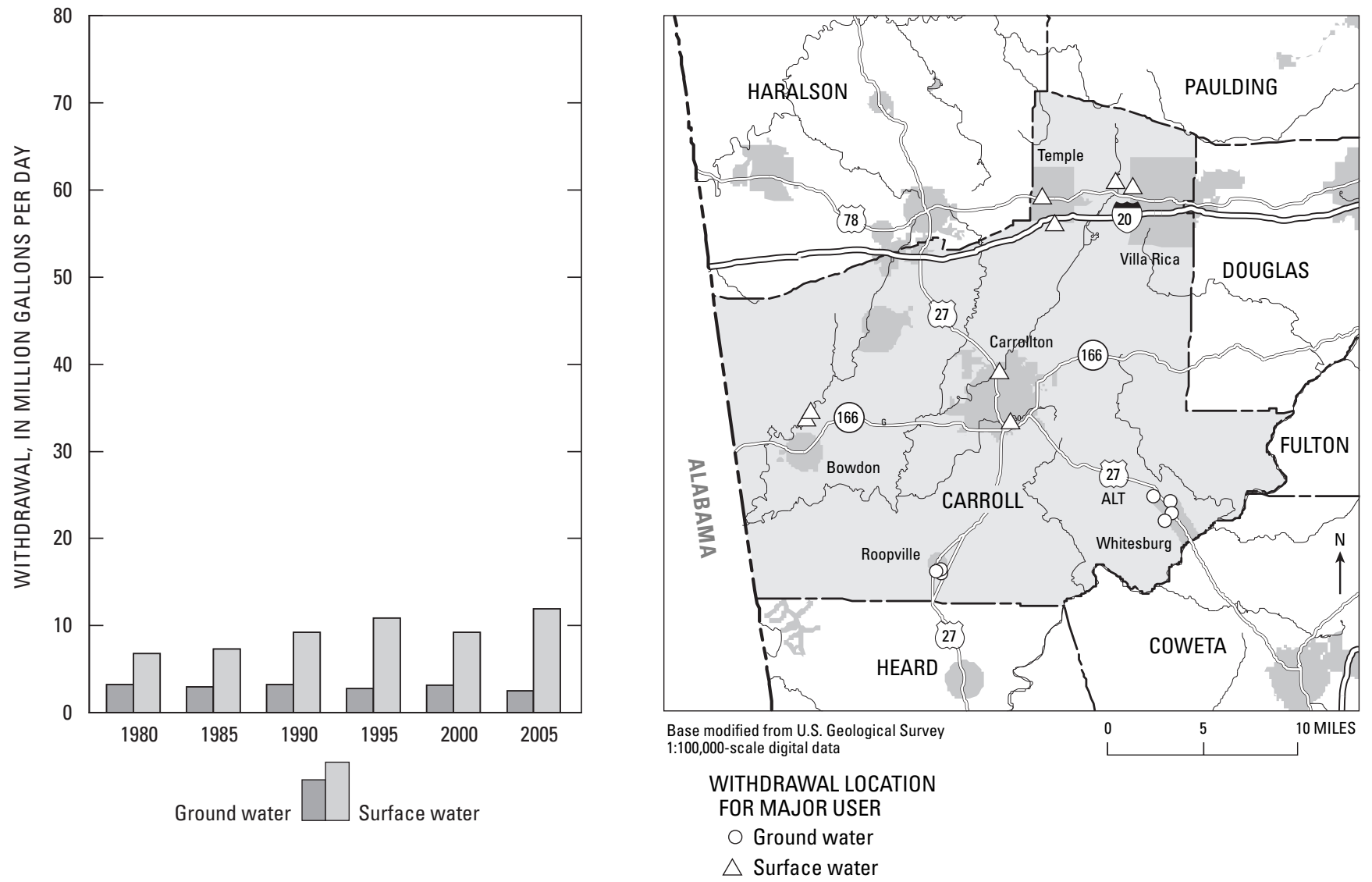

$\begin{array}{lcc}\text { SIC } & \text { GW } & \text { SW } \\ 33 \text { - Primary metals } & 0.00 & 0.09\end{array}$




\section{CATOOSA COUNTY}

Population: 60,813

Population served by public supply: $\quad 51,340$

Acres irrigated: 690

Hydroelectric use (Mgal/d): $\quad 0.00$

2005 WITHDRAWALS, IN MILLION GALLONS PER DAY

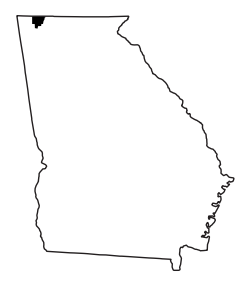

\begin{tabular}{lccccccc}
\hline & $\begin{array}{c}\text { Public } \\
\text { Supply }\end{array}$ & $\begin{array}{c}\text { Domestic \& } \\
\text { Commercial }\end{array}$ & $\begin{array}{c}\text { Industrial \& } \\
\text { Mining }\end{array}$ & Irrigation & Livestock & $\begin{array}{c}\text { Thermo- } \\
\text { electric }\end{array}$ & Totals \\
\hline Ground Water & 4.12 & 0.71 & 0.05 & 0.26 & 0.02 & 0.00 & 5.16 \\
Surface Water & 0.56 & 0.00 & 0.00 & 0.79 & 0.18 & 0.00 & 1.53 \\
TOTALS & 4.68 & 0.71 & 0.05 & 1.05 & 0.20 & 0.00 & 6.69 \\
\hline
\end{tabular}

Withdrawals by Major Public Suppliers (Mgal/d):

$\begin{array}{lcc}\text { Name } & \text { GW } & \text { SW } \\ \text { Catoosa County Water System } & 4.12 & 0.00 \\ \text { City of Ringgold } & 0.00 & 0.56\end{array}$

Withdrawals by Major Industrial Groups (Mgal/d):

SIC

GW

SW

City of Ringgold

None
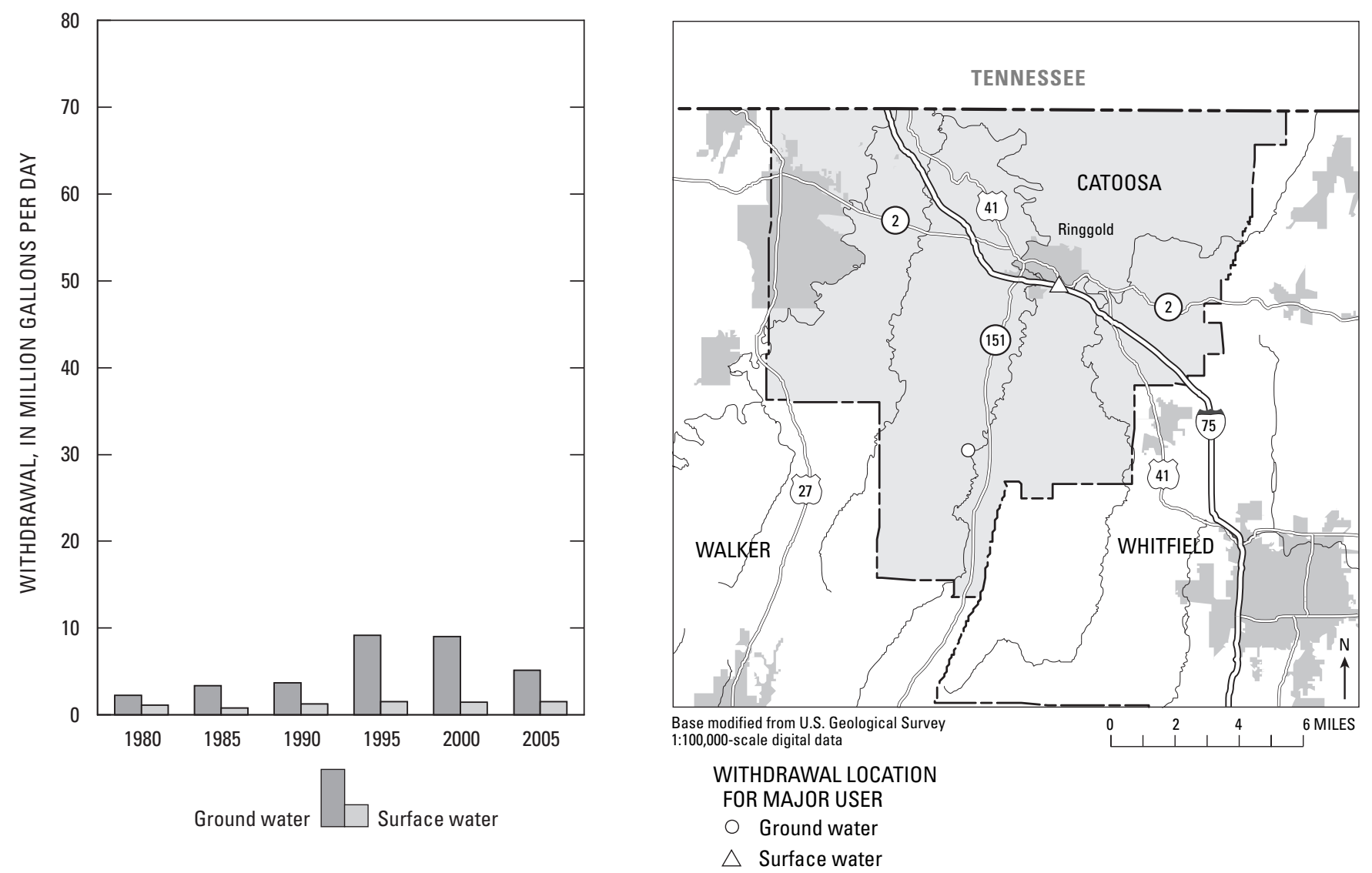


\section{CHARLTON COUNTY}

Population: 10,790

Population served by public supply: $\quad 4,460$

Acres irrigated: 120

Hydroelectric use (Mgal/d): $\quad 0.00$

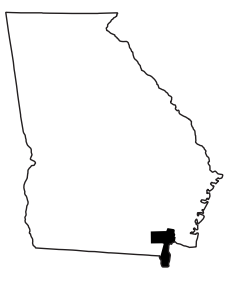

2005 WITHDRAWALS, IN MILLION GALLONS PER DAY

\begin{tabular}{|c|c|c|c|c|c|c|c|}
\hline & $\begin{array}{l}\text { Public } \\
\text { Supply }\end{array}$ & $\begin{array}{l}\text { Domestic \& } \\
\text { Commercial }\end{array}$ & $\begin{array}{c}\text { Industrial \& } \\
\text { Mining }\end{array}$ & Irrigation & Livestock & $\begin{array}{l}\text { Thermo- } \\
\text { electric }\end{array}$ & Totals \\
\hline Ground Water & 0.73 & 0.48 & 0.00 & 0.00 & 0.00 & 0.00 & 1.21 \\
\hline Surface Water & 0.00 & 0.00 & 0.00 & 0.01 & 0.02 & 0.00 & 0.03 \\
\hline TOTALS & 0.73 & 0.48 & 0.00 & 0.01 & 0.02 & 0.00 & 1.24 \\
\hline
\end{tabular}

Withdrawals by Major Public Suppliers (Mgal/d):

Name

GW SW

City of Folkston

0.72

0.00
Withdrawals by Major Industrial Groups (Mgal/d):

SIC

GW

SW
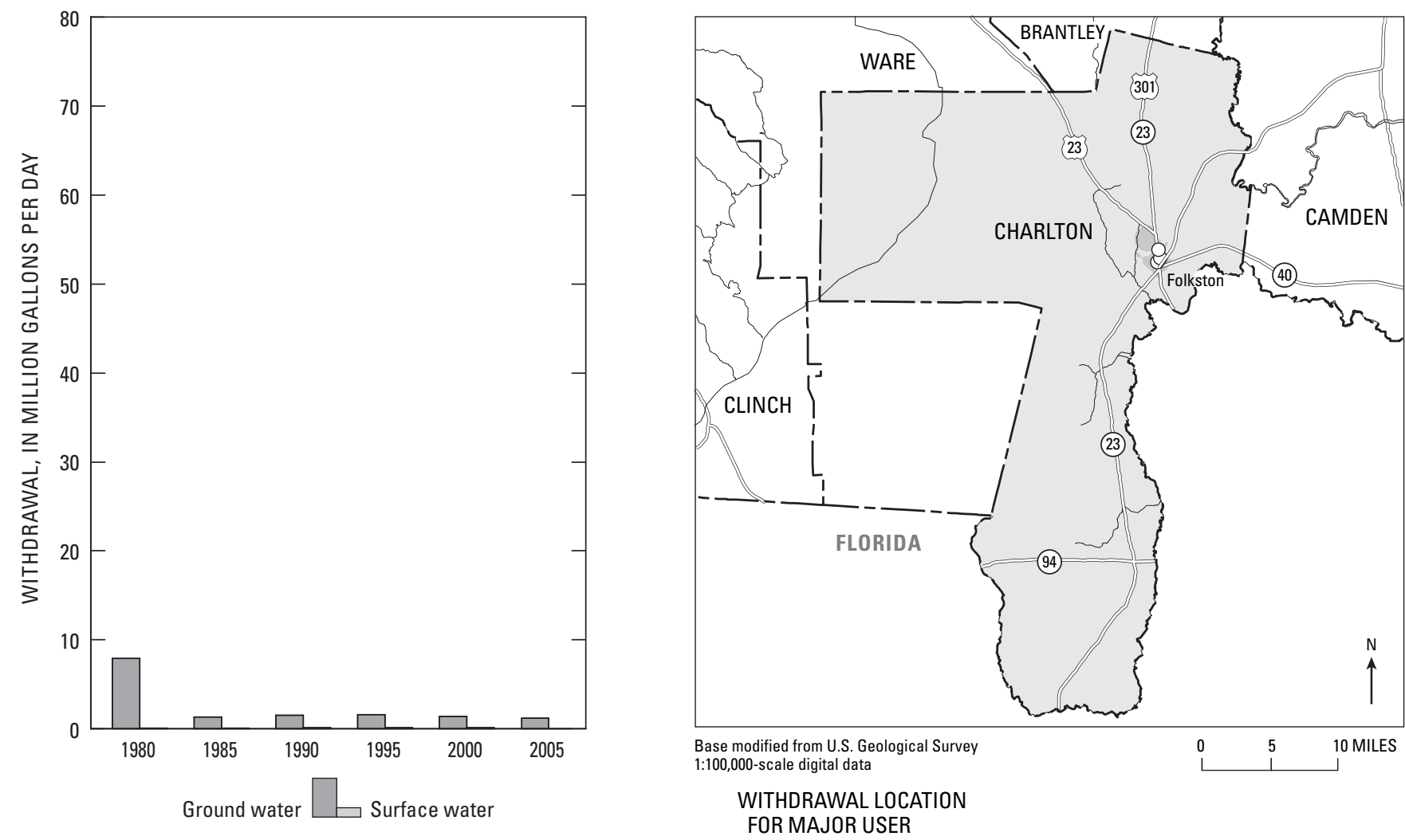

$O$ Ground water 


\section{CHATHAM COUNTY}

Population: 238,410

Population served by public supply: $\quad 208,610$

Acres irrigated: 1,580

Hydroelectric use (Mgal/d): $\quad 0.00$

2005 WITHDRAWALS, IN MILLION GALLONS PER DAY

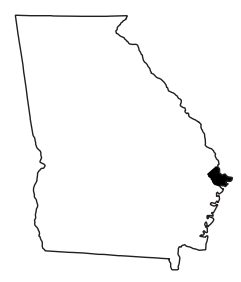

\begin{tabular}{|c|c|c|c|c|c|c|c|}
\hline & $\begin{array}{l}\text { Public } \\
\text { Supply }\end{array}$ & $\begin{array}{l}\text { Domestic \& } \\
\text { Commercial }\end{array}$ & $\begin{array}{c}\text { Industrial \& } \\
\text { Mining }\end{array}$ & Irrigation & Livestock & $\begin{array}{l}\text { Thermo- } \\
\text { electric }\end{array}$ & Totals \\
\hline Ground Water & 33.52 & 5.06 & 21.44 & 2.35 & 0.00 & 2.60 & 64.97 \\
\hline Surface Water & 0.00 & 0.00 & 20.54 & 0.00 & 0.01 & 158.58 & 179.13 \\
\hline TOTALS & 33.52 & 5.06 & 41.98 & $2.35^{*}$ & 0.01 & 161.18 & 244.10 \\
\hline
\end{tabular}

Withdrawals by Major Public Suppliers (Mgal/d):

\section{Name}

City of Garden City

Hunter Army Airfield

Town of Pooler

City of Port Wentworth

City of Savannah

Skidaway Island Utilities

City of Tybee Island

City of Savannah-Glen Robin

\section{GW}

1.16

0.78

0.63

0.29

23.98

1.60

0.86

0.64

Withdrawals by Major Industrial Groups (Mgal/d):

SW

0.00

0.00

0.00

0.00

0.00

0.00

0.00

0.00
SIC

20 - Food

26 - Paper

28 - Chemicals

29 - Petroleum

GW

0.44

16.53

3.95

0.37

*Some irrigation withdrawals included in commercial category

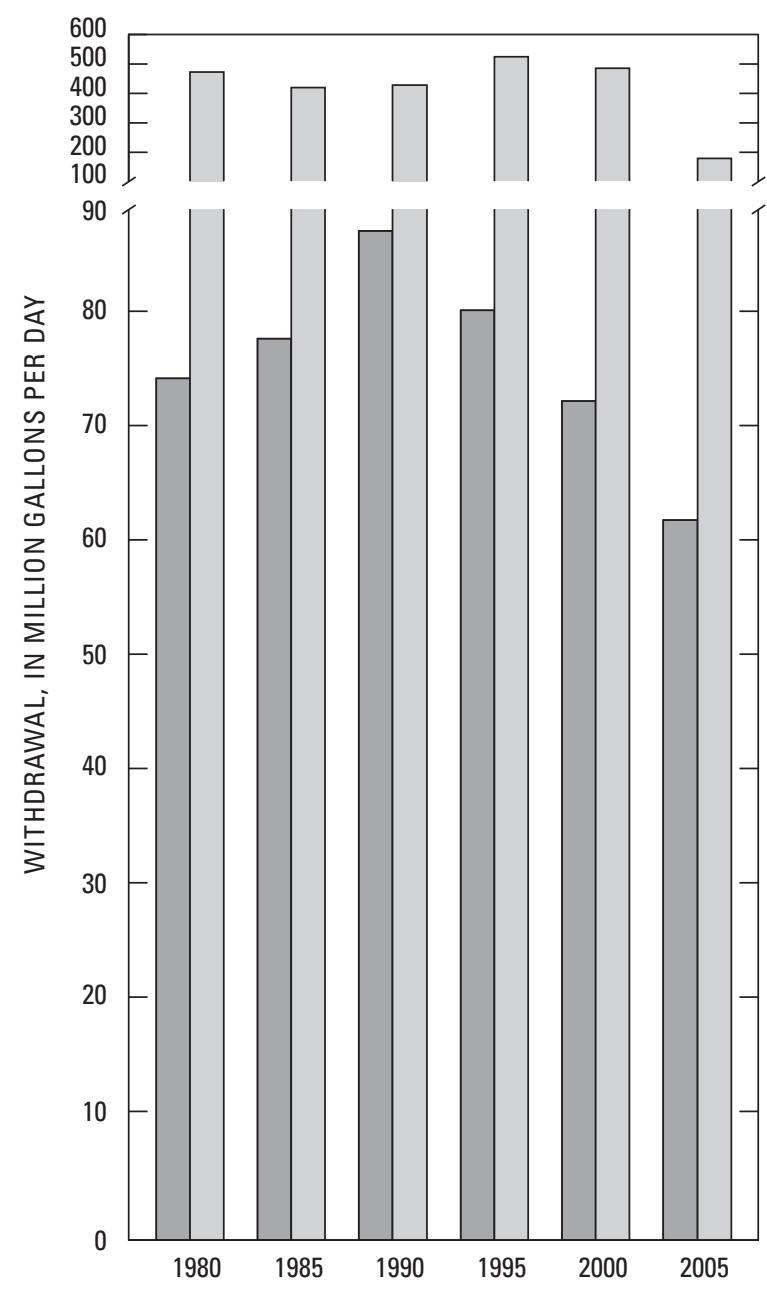

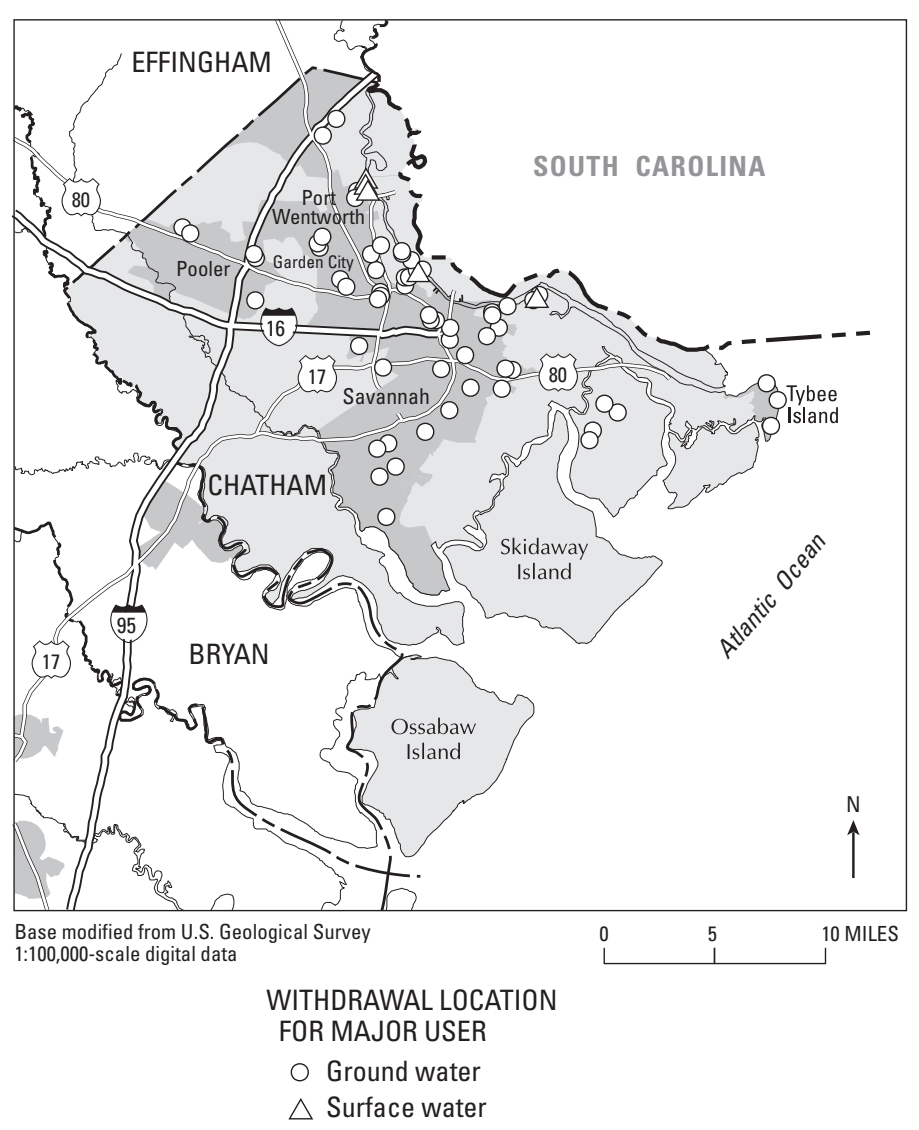




\section{CHATTAHOOCHEE COUNTY}

Population: 14,679

Population served by public supply: $\quad 14,390$

Acres irrigated: 100

Hydroelectric use (Mgal/d): $\quad 0.00$

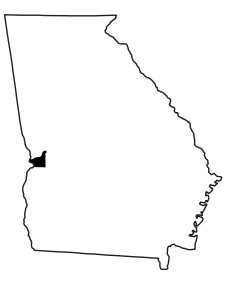

2005 WITHDRAWALS, IN MILLION GALLONS PER DAY

\begin{tabular}{lccccccc}
\hline & $\begin{array}{c}\text { Public } \\
\text { Supply }\end{array}$ & $\begin{array}{c}\text { Domestic \& } \\
\text { Commercial }\end{array}$ & $\begin{array}{c}\text { Industrial \& } \\
\text { Mining }\end{array}$ & Irrigation & Livestock & $\begin{array}{c}\text { Thermo- } \\
\text { electric }\end{array}$ & Totals \\
\hline Ground Water & 0.34 & 0.02 & 0.00 & 0.15 & 0.00 & 0.00 & 0.51 \\
Surface Water & 0.69 & 6.22 & 0.00 & 0.00 & 0.01 & 0.00 & 6.92 \\
TOTALS & 1.03 & 6.24 & 0.00 & 0.15 & 0.01 & 0.00 & 7.43 \\
\hline
\end{tabular}

Withdrawals by Major Public Suppliers (Mgal/d):

Name

Chattahoochee County

Water System

City of Cusseta

\section{GW}

0.34

0.00

SW

0.00

0.69
Withdrawals by Major Industrial Groups (Mgal/d):

SIC

GW

SW
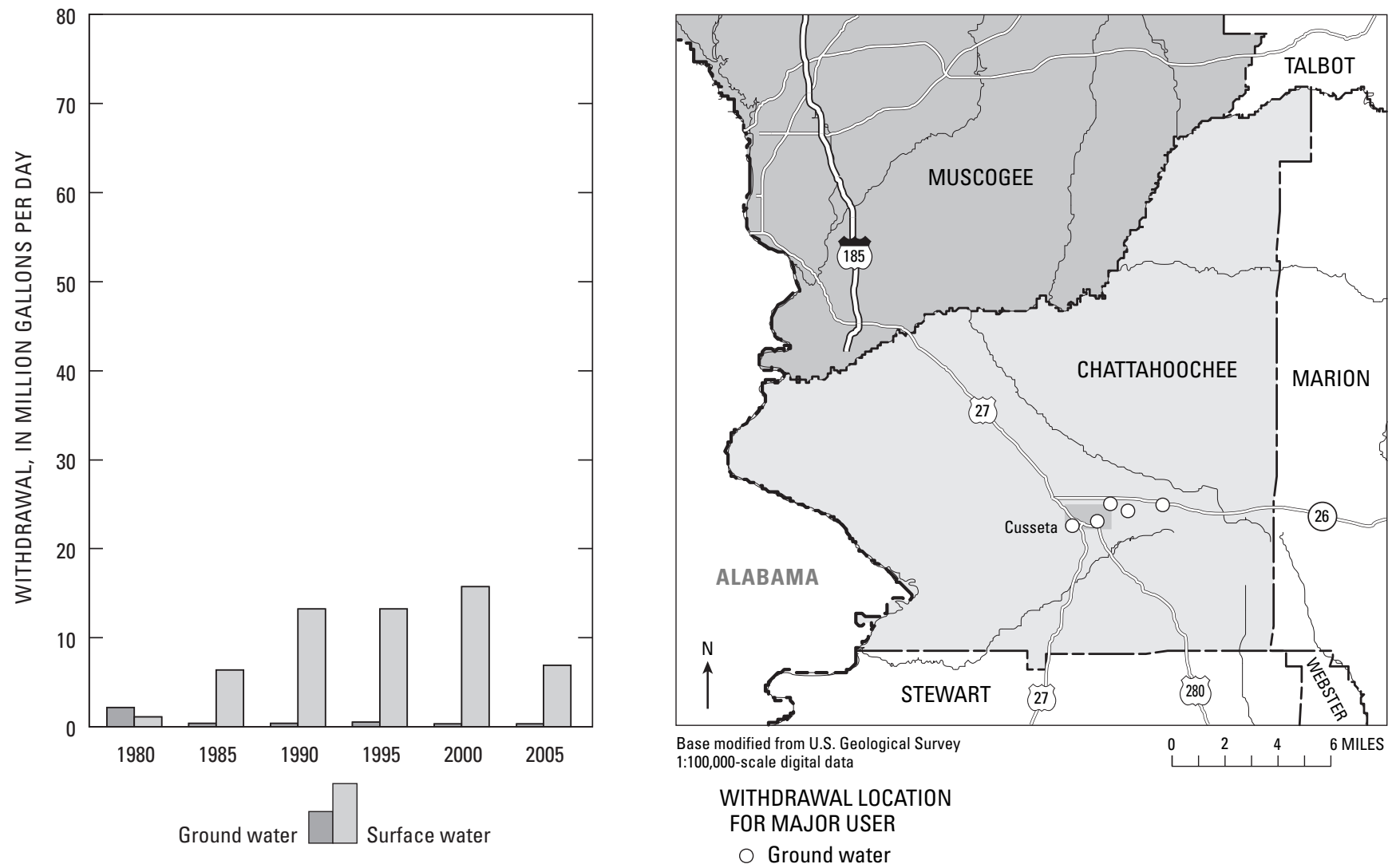


\section{CHATTOOGA COUNTY}

Population: 26,570

Population served by public supply: 22,720

Acres irrigated: 50

Hydroelectric use (Mgal/d): $\quad 0.00$

2005 WITHDRAWALS, IN MILLION GALLONS PER DAY

\begin{tabular}{|c|c|c|c|c|c|c|c|}
\hline & $\begin{array}{l}\text { Public } \\
\text { Supply }\end{array}$ & $\begin{array}{l}\text { Domestic \& } \\
\text { Commercial }\end{array}$ & $\begin{array}{c}\text { Industrial \& } \\
\text { Mining }\end{array}$ & Irrigation & Livestock & $\begin{array}{l}\text { Thermo- } \\
\text { electric }\end{array}$ & Totals \\
\hline Ground Water & 1.31 & 0.29 & 4.58 & 0.00 & 0.39 & 0.00 & 6.57 \\
\hline Surface Water & 2.05 & 0.00 & 2.74 & 0.07 & 0.85 & 0.00 & 5.71 \\
\hline TOTALS & 3.36 & 0.29 & 7.32 & 0.07 & 1.24 & 0.00 & 12.28 \\
\hline
\end{tabular}

Withdrawals by Major Public Suppliers (Mgal/d):

Name

GW SW

Chattooga County Water System $\quad 0.51 \quad 0.00$

Town of Lyerly

Town of Menlo

0.15

0.05

0.00

0.45

0.15
SW
0.00

0.00

0.00

2.05

0.00

0.00
Withdrawals by Major Industrial Groups (Mgal/d):

SIC GW SW
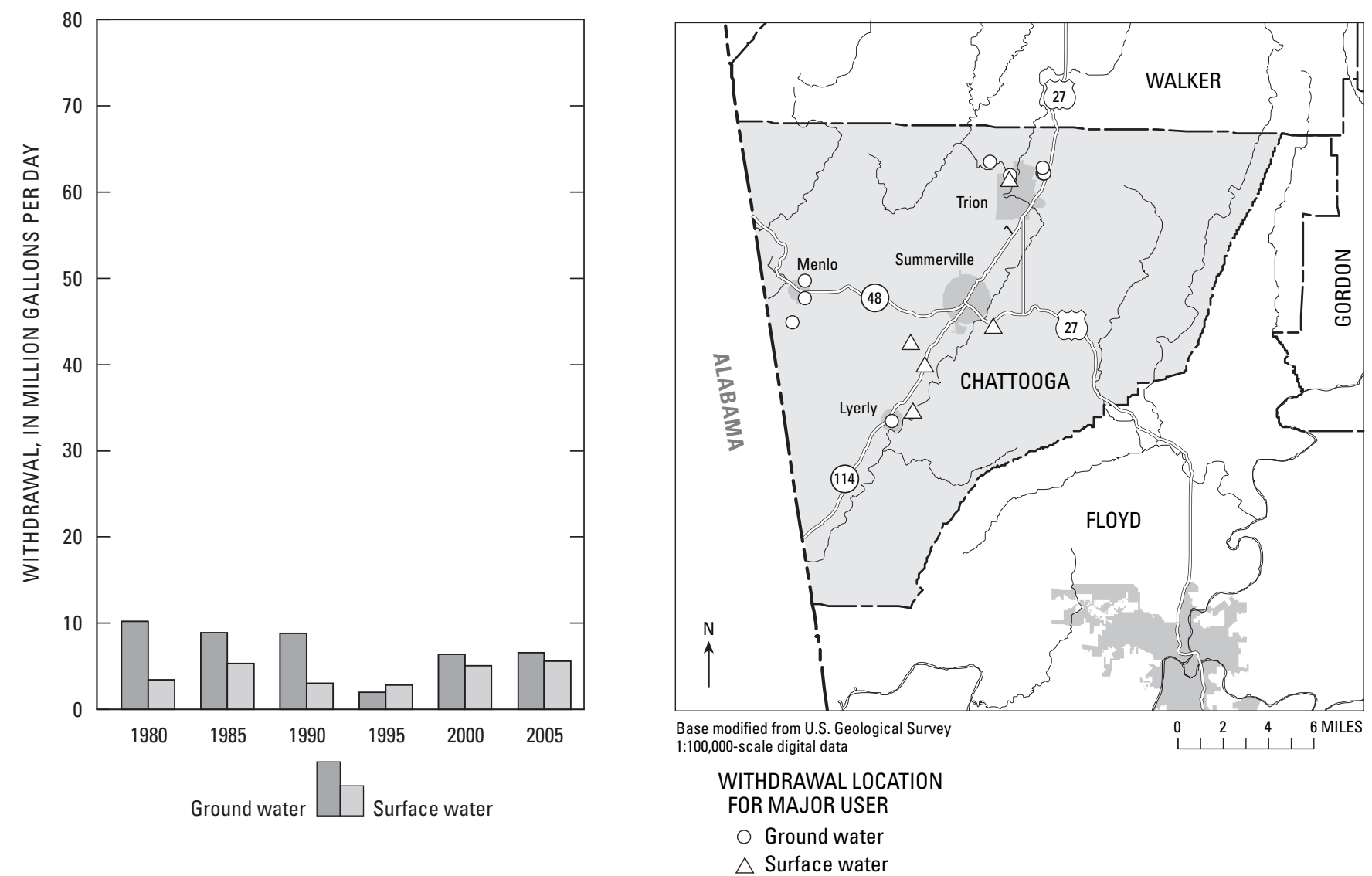


\section{CHEROKEE COUNTY}

Population: 184,211

Population served by public supply: $\quad 115,320$

Acres irrigated: 1,330

Hydroelectric use (Mgal/d): 0.00

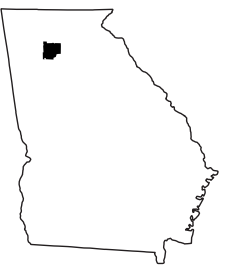

2005 WITHDRAWALS, IN MILLION GALLONS PER DAY

\begin{tabular}{|c|c|c|c|c|c|c|c|}
\hline & $\begin{array}{l}\text { Public } \\
\text { Supply }\end{array}$ & $\begin{array}{l}\text { Domestic \& } \\
\text { Commercial }\end{array}$ & $\begin{array}{c}\text { Industrial \& } \\
\text { Mining }\end{array}$ & Irrigation & Livestock & $\begin{array}{l}\text { Thermo- } \\
\text { electric }\end{array}$ & Totals \\
\hline Ground Water & 0.30 & 5.18 & 0.57 & 0.60 & 0.02 & 0.00 & 6.67 \\
\hline Surface Water & 18.64 & 0.00 & 1.95 & 1.78 & 0.31 & 0.00 & 22.68 \\
\hline TOTALS & 18.94 & 5.18 & 2.52 & 2.38 & 0.33 & 0.00 & 29.35 \\
\hline
\end{tabular}

Withdrawals by Major Public Suppliers (Mgal/d):

Name

GW SW

City of Ball Ground

$0.14 \quad 0.00$

City of Canton

Cherokee County

Water System

$0.00 \quad 2.83$

$0.00 \quad 15.81$
Withdrawals by Major Industrial Groups (Mgal/d):

SIC

20 - Food

GW

SW
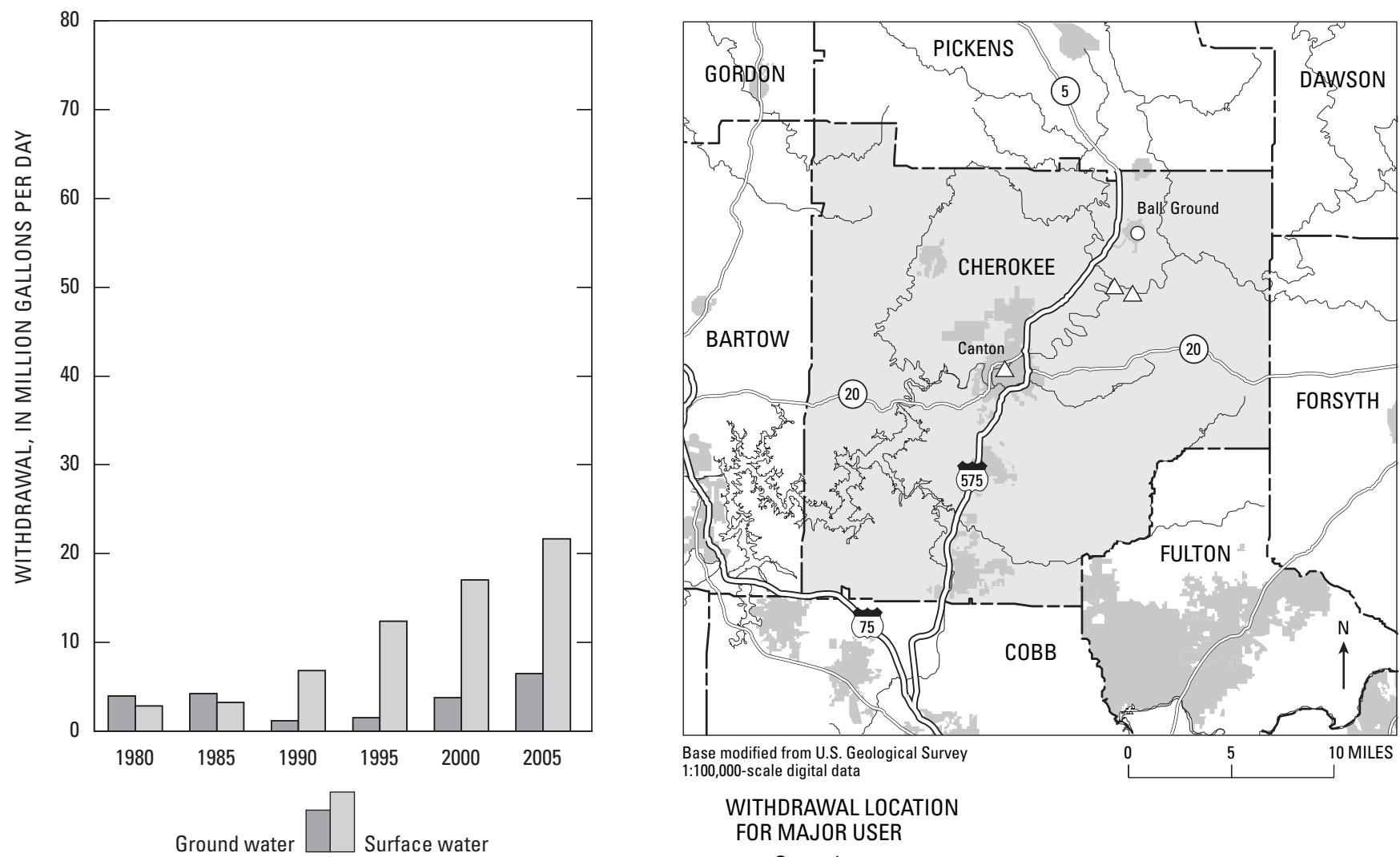

WITHDRAWAL LOCATION

FOR MAJOR USER

O Ground water

$\triangle$ Surface water 


\section{CLARKE COUNTY}

Population: 104,439

Population served by public supply: $\quad 104,220$

Acres irrigated: 760

Hydroelectric use (Mgal/d): 77.67

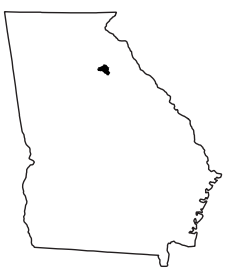

2005 WITHDRAWALS, IN MILLION GALLONS PER DAY

\begin{tabular}{|c|c|c|c|c|c|c|c|}
\hline & $\begin{array}{l}\text { Public } \\
\text { Supply }\end{array}$ & $\begin{array}{l}\text { Domestic \& } \\
\text { Commercial }\end{array}$ & $\begin{array}{c}\text { Industrial \& } \\
\text { Mining }\end{array}$ & Irrigation & Livestock & $\begin{array}{l}\text { Thermo- } \\
\text { electric }\end{array}$ & Totals \\
\hline Ground Water & 0.24 & 0.02 & 0.00 & 0.24 & 0.00 & 0.00 & 0.50 \\
\hline Surface Water & 6.29 & 0.00 & 0.00 & 0.69 & 0.09 & 0.00 & 7.07 \\
\hline TOTALS & 6.53 & 0.02 & 0.00 & 0.93 & 0.09 & 0.00 & 7.57 \\
\hline
\end{tabular}

Withdrawals by Major Public Suppliers (Mgal/d):

Name

GW

0.00

SW

Athens-Clarke County

Public Utility
Withdrawals by Major Industrial Groups (Mgal/d):

SIC

GW

SW

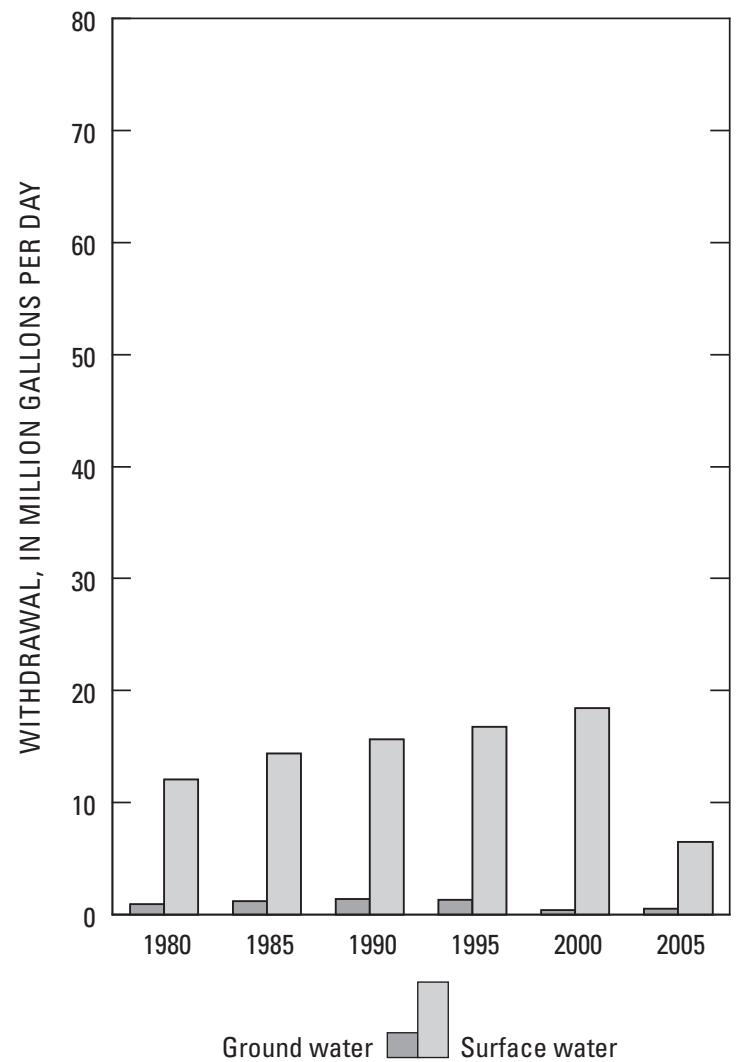

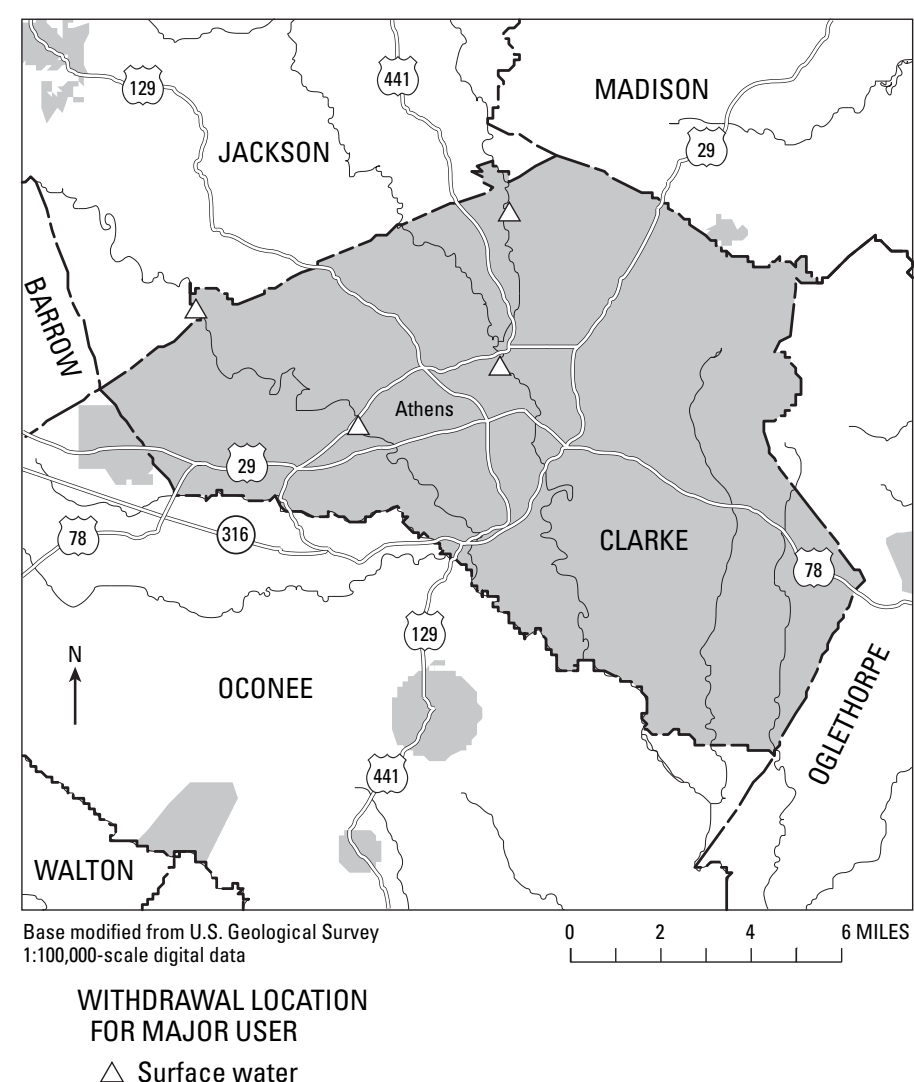




\section{CLAY COUNTY}

Population: 3,242

Population served by public supply: 1,840

Acres irrigated: 420

Hydroelectric use (Mgal/d): $\quad 5,439.48$

2005 WITHDRAWALS, IN MILLION GALLONS PER DAY

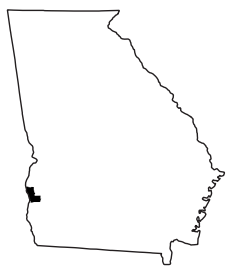

\begin{tabular}{|c|c|c|c|c|c|c|c|}
\hline & $\begin{array}{l}\text { Public } \\
\text { Supply }\end{array}$ & $\begin{array}{l}\text { Domestic \& } \\
\text { Commercial }\end{array}$ & $\begin{array}{c}\text { Industrial \& } \\
\text { Mining }\end{array}$ & Irrigation & Livestock & $\begin{array}{l}\text { Thermo- } \\
\text { electric }\end{array}$ & Totals \\
\hline Ground Water & 0.26 & 0.11 & 0.00 & 0.20 & 0.01 & 0.00 & 0.58 \\
\hline Surface Water & 0.00 & 0.00 & 0.00 & 0.06 & 0.02 & 0.00 & 0.08 \\
\hline TOTALS & 0.26 & 0.11 & 0.00 & 0.26 & 0.03 & 0.00 & 0.66 \\
\hline
\end{tabular}

Withdrawals by Major Public Suppliers (Mgal/d):

Name

Town of Bluffton

GW

City of Fort Gaines

$\begin{array}{ll}0.01 & 0.00 \\ 0.24 & 0.00\end{array}$

Withdrawals by Major Industrial Groups (Mgal/d):

SIC

None
SW

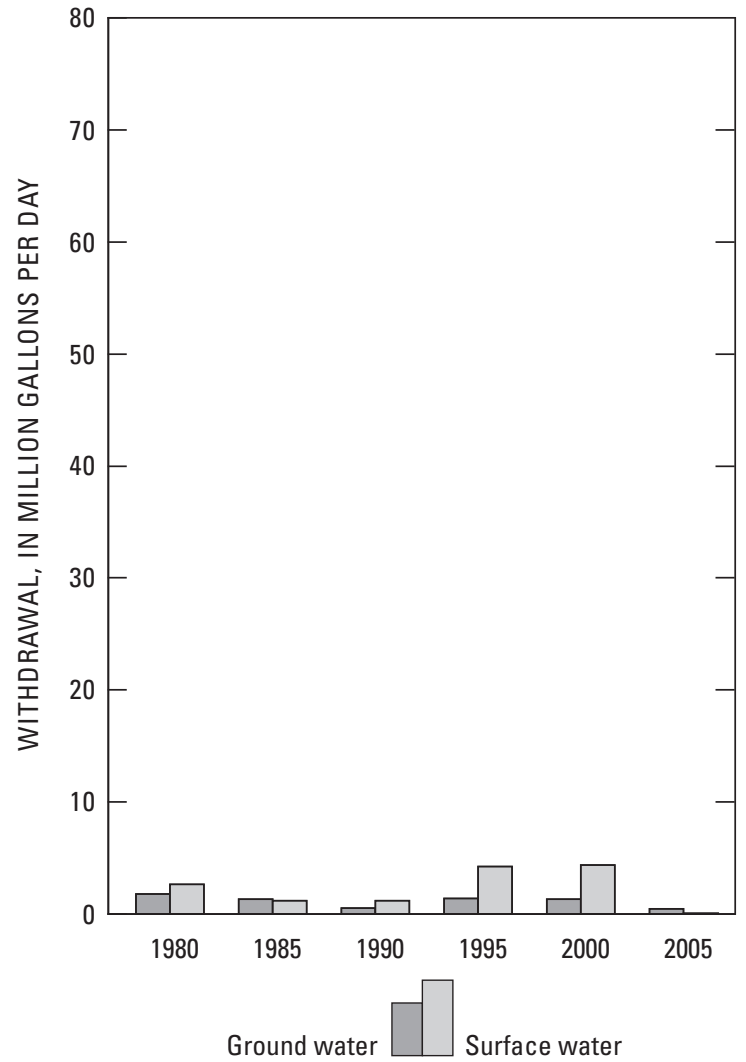

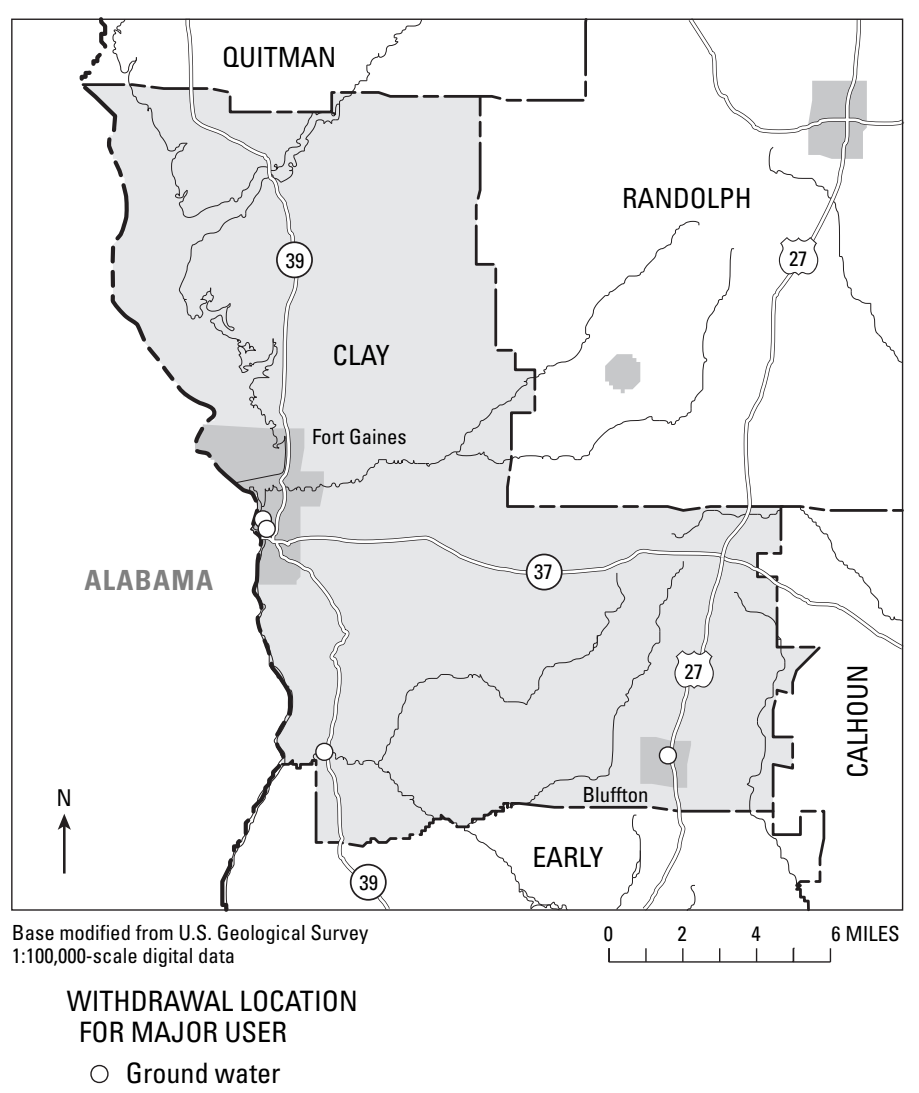




\section{CLAYTON COUNTY}

Population: 267,966

Population served by public supply: 258,980

Acres irrigated: 590

Hydroelectric use (Mgal/d): $\quad 0.00$

2005 WITHDRAWALS, IN MILLION GALLONS PER DAY

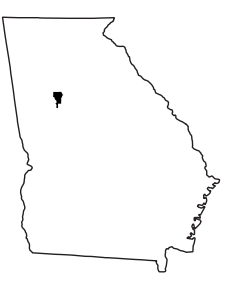

\begin{tabular}{|c|c|c|c|c|c|c|c|}
\hline & $\begin{array}{l}\text { Public } \\
\text { Supply }\end{array}$ & $\begin{array}{l}\text { Domestic \& } \\
\text { Commercial }\end{array}$ & $\begin{array}{c}\text { Industrial \& } \\
\text { Mining }\end{array}$ & Irrigation & Livestock & $\begin{array}{l}\text { Thermo- } \\
\text { electric }\end{array}$ & Totals \\
\hline Ground Water & 0.02 & 1.94 & 0.37 & 0.00 & 0.00 & 0.00 & 2.33 \\
\hline Surface Water & 29.94 & 0.00 & 0.01 & 0.88 & 0.01 & 0.00 & 30.84 \\
\hline TOTALS & 29.96 & 1.94 & 0.38 & 0.88 & 0.01 & 0.00 & 33.17 \\
\hline
\end{tabular}

Withdrawals by Major Public Suppliers (Mgal/d):

\section{Name}

Clayton County Water Authority-Shoal Creek

Clayton County Water Authority (water source located in Henry County)
Withdrawals by Major Industrial Groups (Mgal/d):

SIC

GW

SW

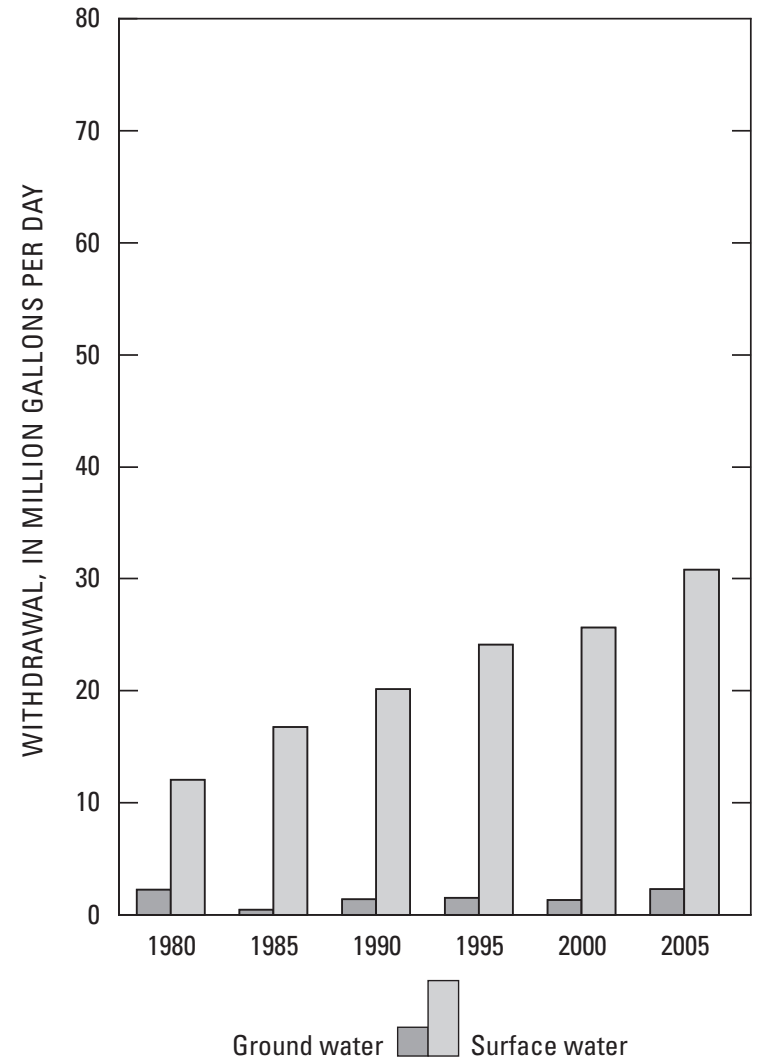

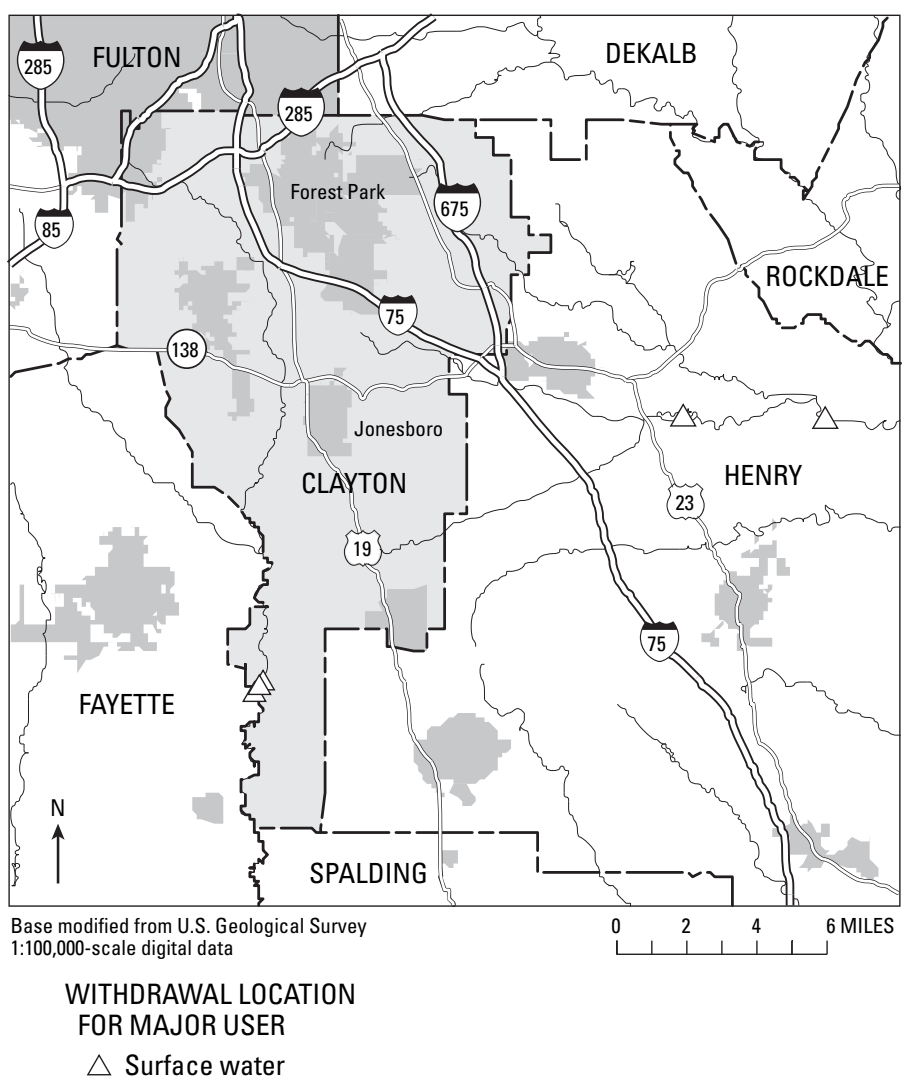




\section{CLINCH COUNTY}

Population: 6,996

Population served by public supply: $\quad 3,310$

Acres irrigated: 980

Hydroelectric use (Mgal/d): $\quad 0.00$

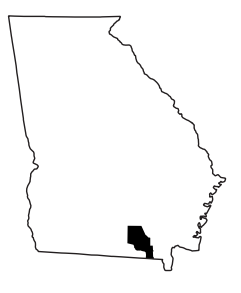

2005 WITHDRAWALS, IN MILLION GALLONS PER DAY

\begin{tabular}{|c|c|c|c|c|c|c|c|}
\hline & $\begin{array}{l}\text { Public } \\
\text { Supply }\end{array}$ & $\begin{array}{l}\text { Domestic \& } \\
\text { Commercial }\end{array}$ & $\begin{array}{c}\text { Industrial \& } \\
\text { Mining }\end{array}$ & Irrigation & Livestock & $\begin{array}{l}\text { Thermo- } \\
\text { electric }\end{array}$ & Totals \\
\hline Ground Water & 0.49 & 0.28 & 0.04 & 0.25 & 0.74 & 0.00 & 1.80 \\
\hline Surface Water & 0.00 & 0.00 & 0.00 & 0.06 & 0.02 & 0.00 & 0.08 \\
\hline TOTALS & 0.49 & 0.28 & 0.04 & 0.31 & 0.76 & 0.00 & 1.88 \\
\hline
\end{tabular}

Withdrawals by Major Public Suppliers (Mgal/d):

Name

Town of DuPont

GW

0.01

SW

City of Homerville

0.48

0.00

Withdrawals by Major Industrial Groups (Mgal/d):

SIC

GW

SW

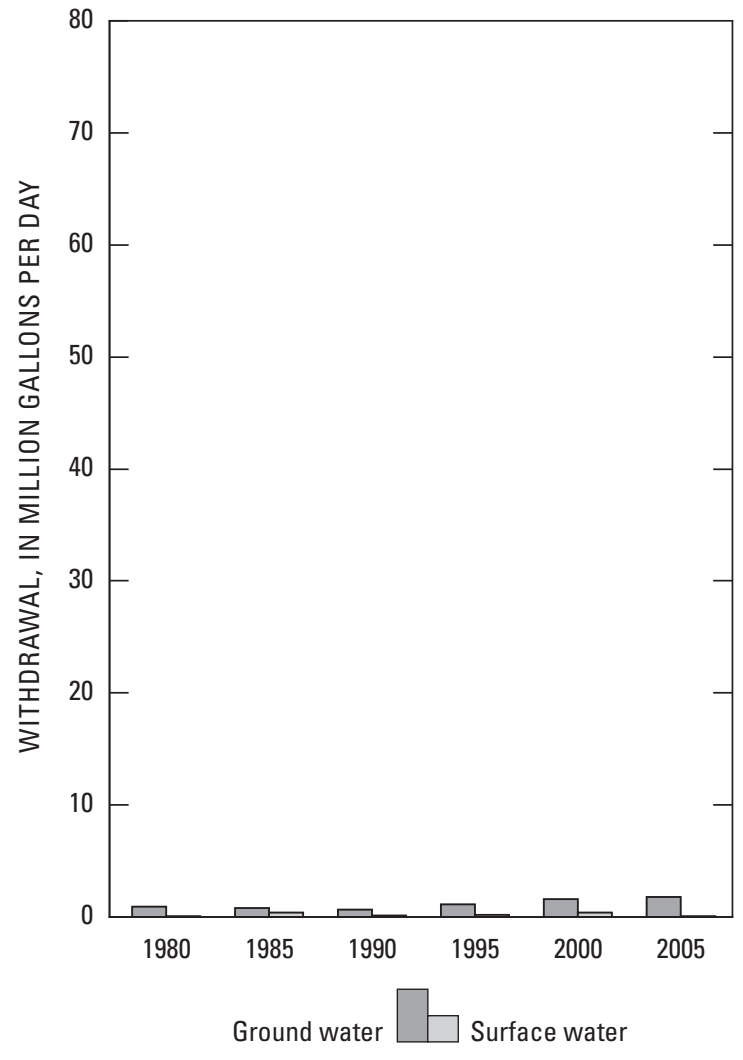

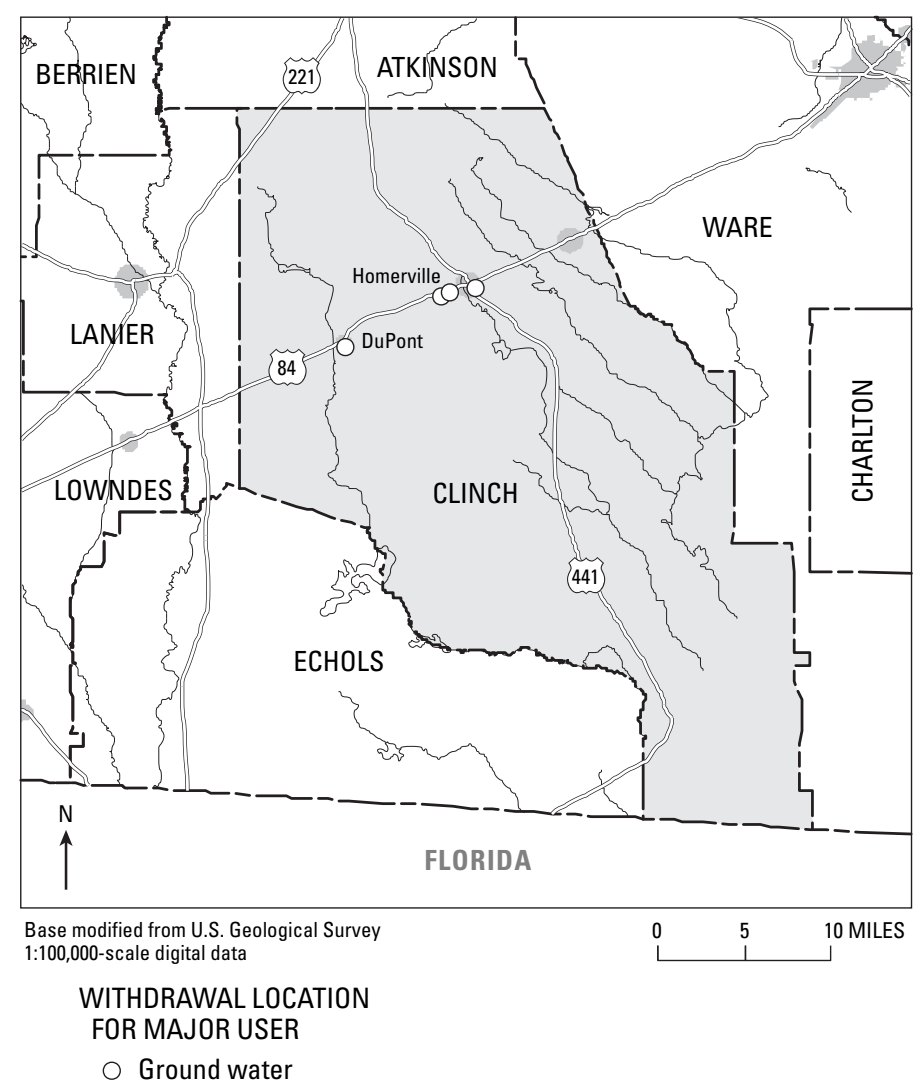




\section{COBB COUNTY}

Population: 663,818

Population served by public supply: 650,540

Acres irrigated: 2,100

Hydroelectric use (Mgal/d): $\quad 0.00$

2005 WITHDRAWALS, IN MILLION GALLONS PER DAY

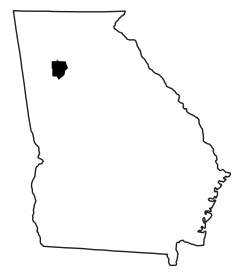

\begin{tabular}{|c|c|c|c|c|c|c|c|}
\hline & $\begin{array}{l}\text { Public } \\
\text { Supply }\end{array}$ & $\begin{array}{l}\text { Domestic \& } \\
\text { Commercial }\end{array}$ & $\begin{array}{c}\text { Industrial \& } \\
\text { Mining }\end{array}$ & Irrigation & Livestock & $\begin{array}{l}\text { Thermo- } \\
\text { electric }\end{array}$ & Totals \\
\hline Ground Water & 0.00 & 1.00 & 0.80 & 0.02 & 0.01 & 0.00 & 1.83 \\
\hline Surface Water & 91.36 & 0.00 & 0.37 & 2.91 & 0.02 & 362.58 & 457.24 \\
\hline TOTALS & 91.36 & 1.00 & 1.17 & 2.93 & 0.03 & 362.58 & 459.07 \\
\hline
\end{tabular}

Withdrawals by Major Public Suppliers (Mgal/d):

\section{Name}

GW SW

Cobb County-Marietta

$0.00 \quad 46.95$

Water Authority

Cobb County-Marietta Water

Authority (water source

located in Bartow County)

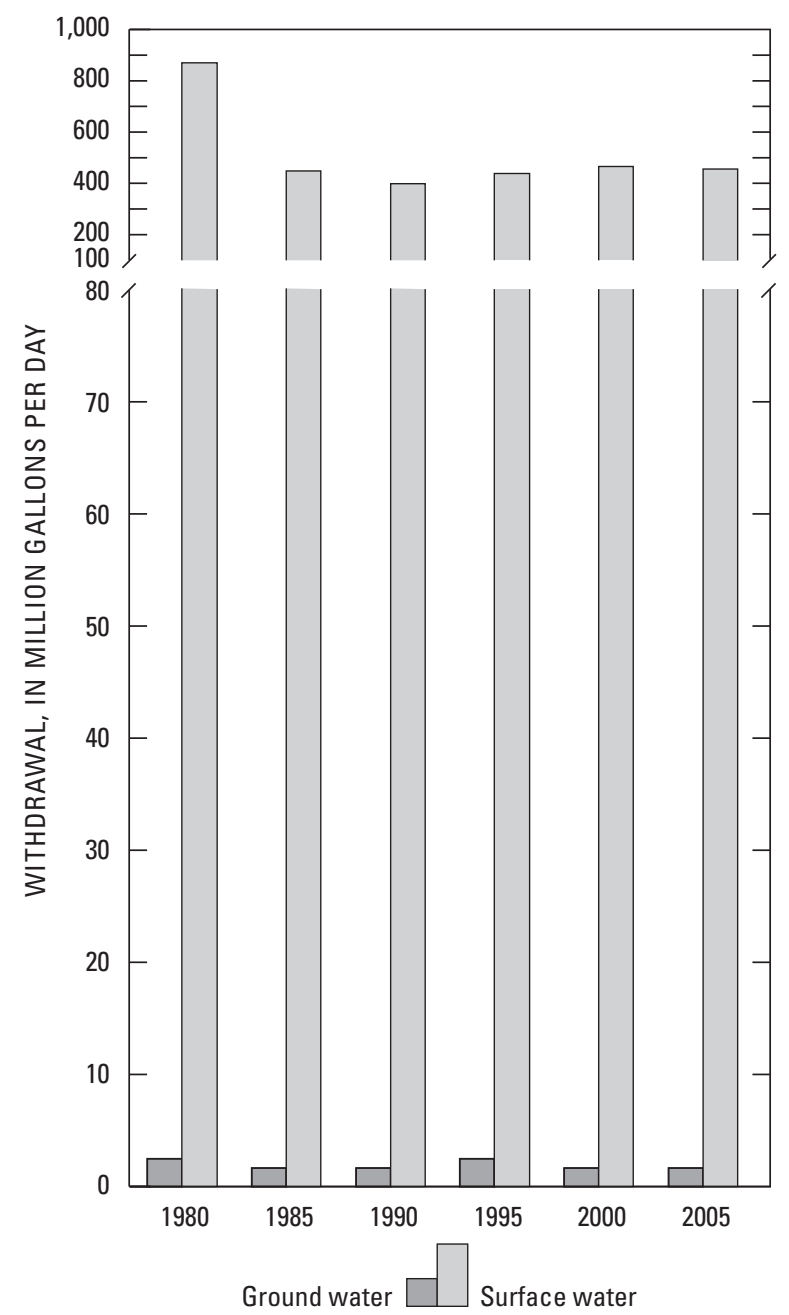

Withdrawals by Major Industrial Groups (Mgal/d):

$\begin{array}{lcc}\text { SIC } & \text { GW } & \text { SW } \\ 26 \text { - Paper } & 0.00 & 0.36\end{array}$

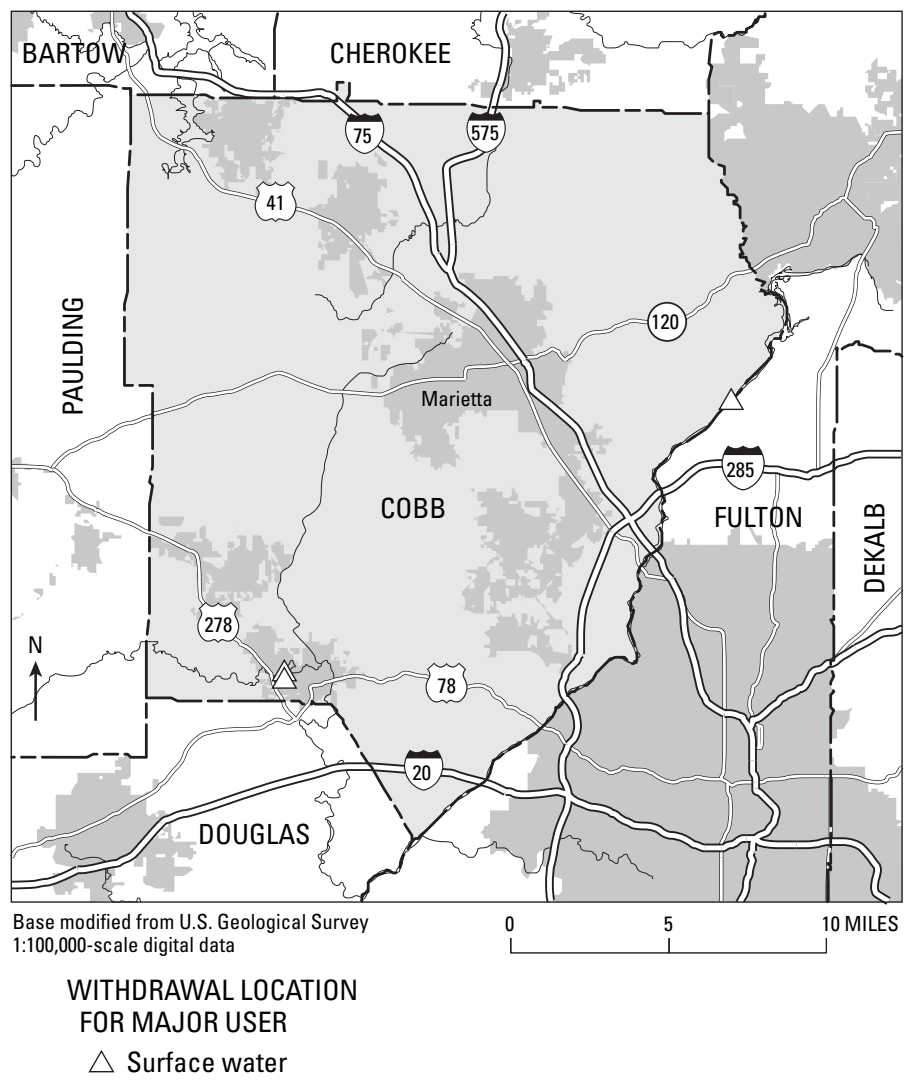




\section{COFFEE COUNTY}

Population: 39,674

Population served by public supply: $\quad 17,900$

Acres irrigated: 22,690

Hydroelectric use (Mgal/d): 0.00

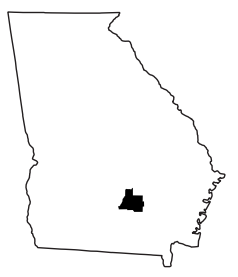

2005 WITHDRAWALS, IN MILLION GALLONS PER DAY

\begin{tabular}{lccccccrr}
\hline & $\begin{array}{c}\text { Public } \\
\text { Supply }\end{array}$ & $\begin{array}{c}\text { Domestic \& } \\
\text { Commercial }\end{array}$ & $\begin{array}{c}\text { Industrial \& } \\
\text { Mining }\end{array}$ & Irrigation & Livestock & \multicolumn{2}{c}{$\begin{array}{c}\text { Thermo- } \\
\text { electric }\end{array}$} & Totals \\
\cline { 1 - 5 } & 4.68 & 1.63 & 0.00 & 4.05 & 0.07 & 0.00 & 10.43 \\
Ground Water & 4.68 & 0.00 & 0.00 & 5.10 & 0.60 & 0.00 & 5.70 \\
Surface Water & 0.00 & 1.63 & 0.00 & 9.15 & 0.67 & 0.00 & 16.13 \\
\hline
\end{tabular}

Withdrawals by Major Public Suppliers (Mgal/d):

Name

City of Ambrose

City of Broxton

City of Douglas

City of Nicholls
GW SW

$0.03 \quad 0.00$

$0.00 \quad 0.00$

$4.04 \quad 0.00$

$0.29 \quad 0.00$
Withdrawals by Major Industrial Groups (Mgal/d):

SIC

None
SW
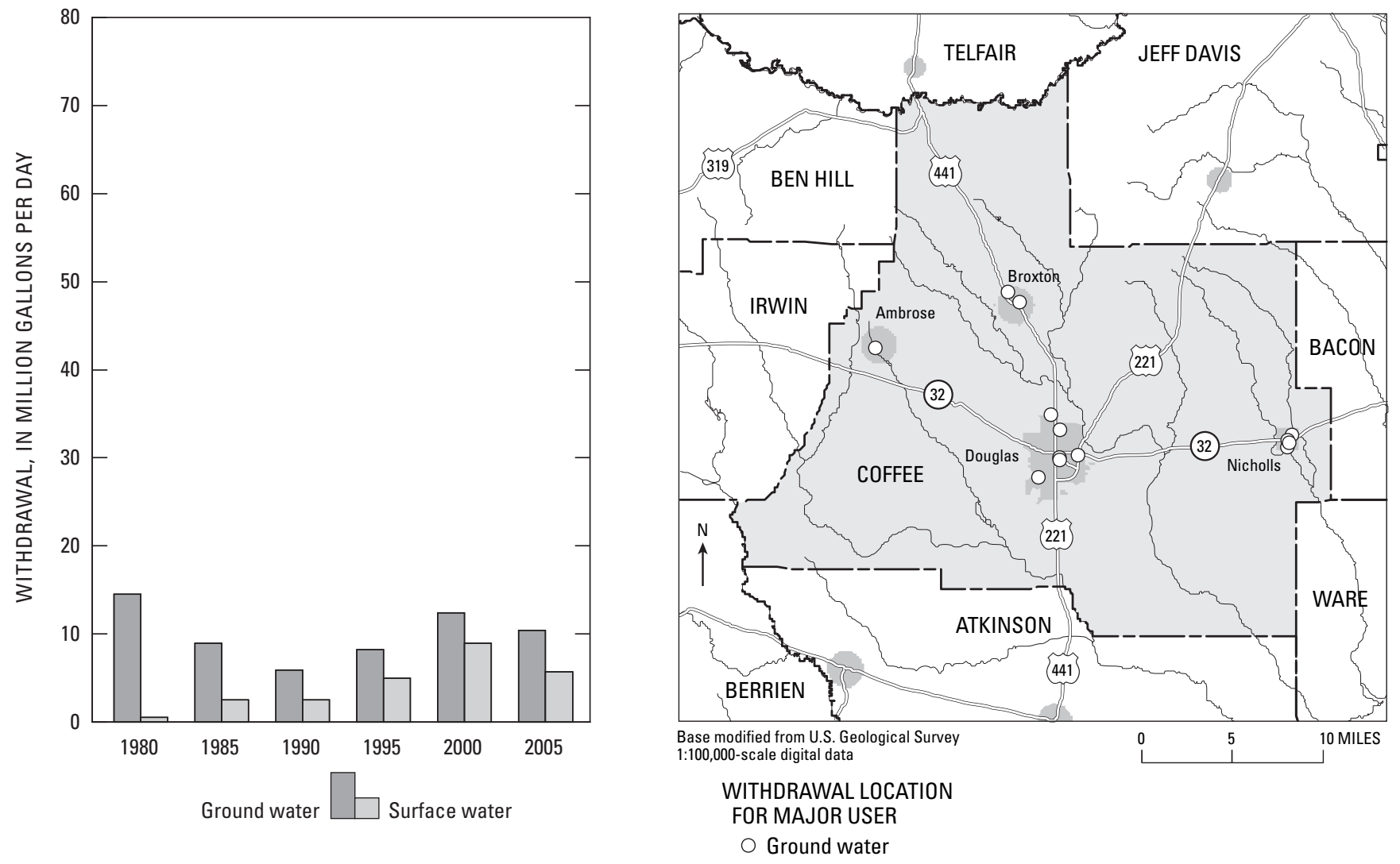


\section{COLOUITT COUNTY}

Population: 43,915

Population served by public supply: 24,260

Acres irrigated: 46,100

Hydroelectric use (Mgal/d): $\quad 0.00$

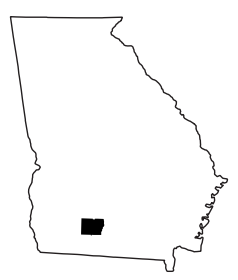

2005 WITHDRAWALS, IN MILLION GALLONS PER DAY

\begin{tabular}{|c|c|c|c|c|c|c|c|}
\hline & $\begin{array}{l}\text { Public } \\
\text { Supply }\end{array}$ & $\begin{array}{l}\text { Domestic \& } \\
\text { Commercial }\end{array}$ & $\begin{array}{c}\text { Industrial \& } \\
\text { Mining }\end{array}$ & Irrigation & Livestock & $\begin{array}{l}\text { Thermo- } \\
\text { electric }\end{array}$ & Totals \\
\hline Ground Water & 3.84 & 1.47 & 0.33 & 9.65 & 0.07 & 0.00 & 15.36 \\
\hline Surface Water & 0.00 & 0.00 & 0.00 & 21.11 & 0.28 & 0.00 & 21.39 \\
\hline TOTALS & 3.84 & 1.47 & 0.33 & 30.76 & 0.35 & 0.00 & 36.75 \\
\hline
\end{tabular}

Withdrawals by Major Public Suppliers (Mgal/d):

$\begin{array}{lcc}\text { Name } & \text { GW } & \text { SW } \\ \text { Town of Berlin } & 0.04 & 0.00 \\ \text { City of Doerun } & 0.08 & 0.00 \\ \text { City of Ellenton } & 0.03 & 0.00 \\ \text { Town of Funston } & 0.03 & 0.00 \\ \text { City of Moultrie } & 3.33 & 0.00 \\ \text { Town of Norman Park } & 0.00 & 0.00 \\ \text { Town of Riverside } & 0.07 & 0.00\end{array}$

Withdrawals by Major Industrial Groups (Mgal/d):

$\begin{array}{lcc}\text { SIC } & \text { GW } & \text { SW } \\ 20 \text { - Food } & 0.32 & 0.00\end{array}$
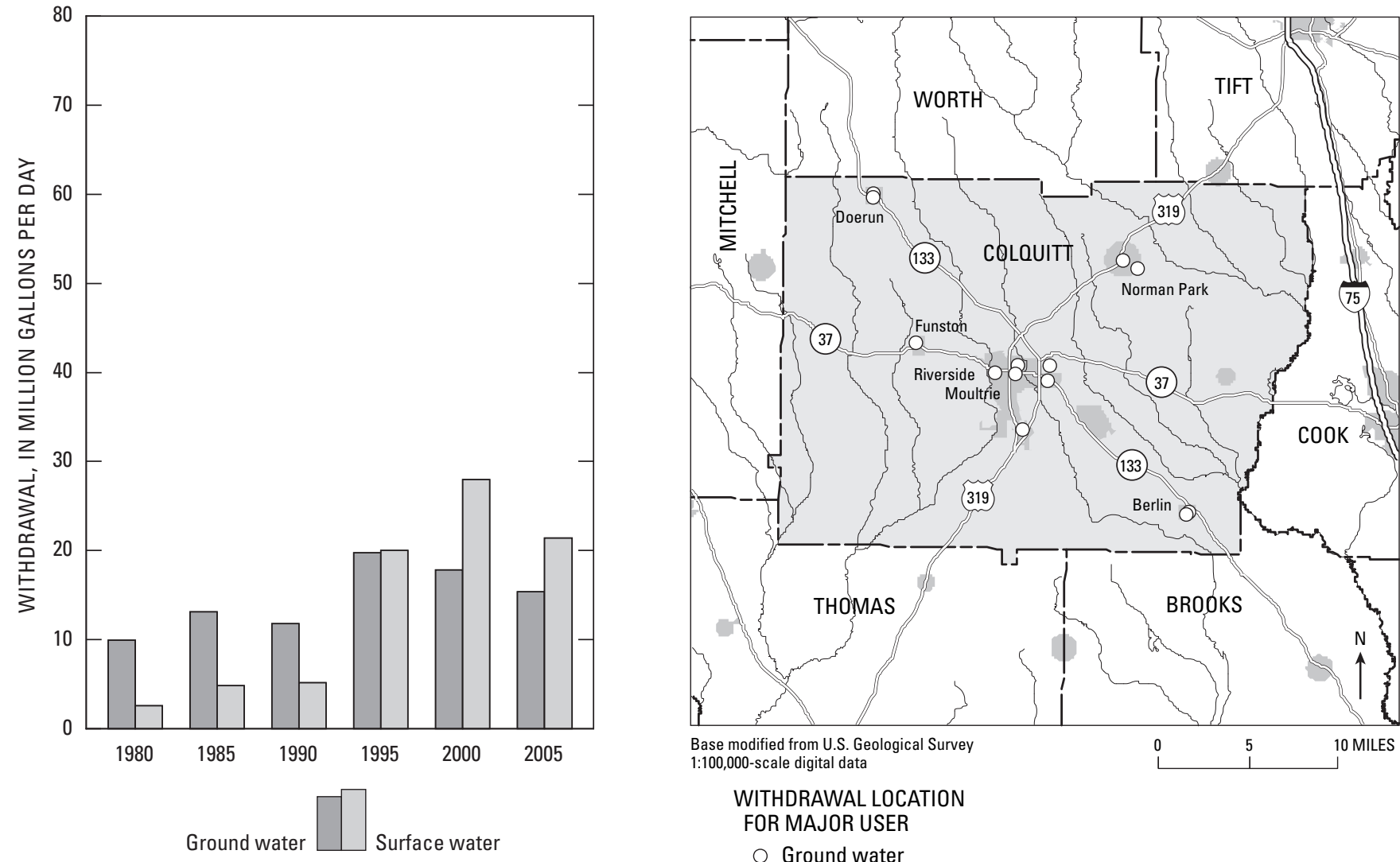


\section{COLUMBIA COUNTY}

Population: 103,812

Population served by public supply: $\quad 90,710$

Acres irrigated: 480

Hydroelectric use (Mgal/d): $\quad 3,855.20$

2005 WITHDRAWALS, IN MILLION GALLONS PER DAY

\begin{tabular}{|c|c|c|c|c|c|c|c|}
\hline & $\begin{array}{l}\text { Public } \\
\text { Supply }\end{array}$ & $\begin{array}{l}\text { Domestic \& } \\
\text { Commercial }\end{array}$ & $\begin{array}{c}\text { Industrial \& } \\
\text { Mining }\end{array}$ & Irrigation & Livestock & $\begin{array}{l}\text { Thermo- } \\
\text { electric }\end{array}$ & Totals \\
\hline Ground Water & 0.18 & 0.98 & 0.88 & 0.13 & 0.00 & 0.00 & 2.17 \\
\hline Surface Water & 12.37 & 0.00 & 0.01 & 1.00 & 0.03 & 0.00 & 13.41 \\
\hline TOTALS & 12.55 & 0.98 & 0.89 & 1.13 & 0.03 & 0.00 & 15.58 \\
\hline
\end{tabular}

Withdrawals by Major Public Suppliers (Mgal/d):

Name

GW SW

Columbia County

Water System

City of Grovetown

City of Harlem
$0.00 \quad 12.37$

$0.13 \quad 0.00$

$0.02 \quad 0.00$

Withdrawals by Major Industrial Groups (Mgal/d):

SIC

GW SW

20 - Food

0.07

0.00
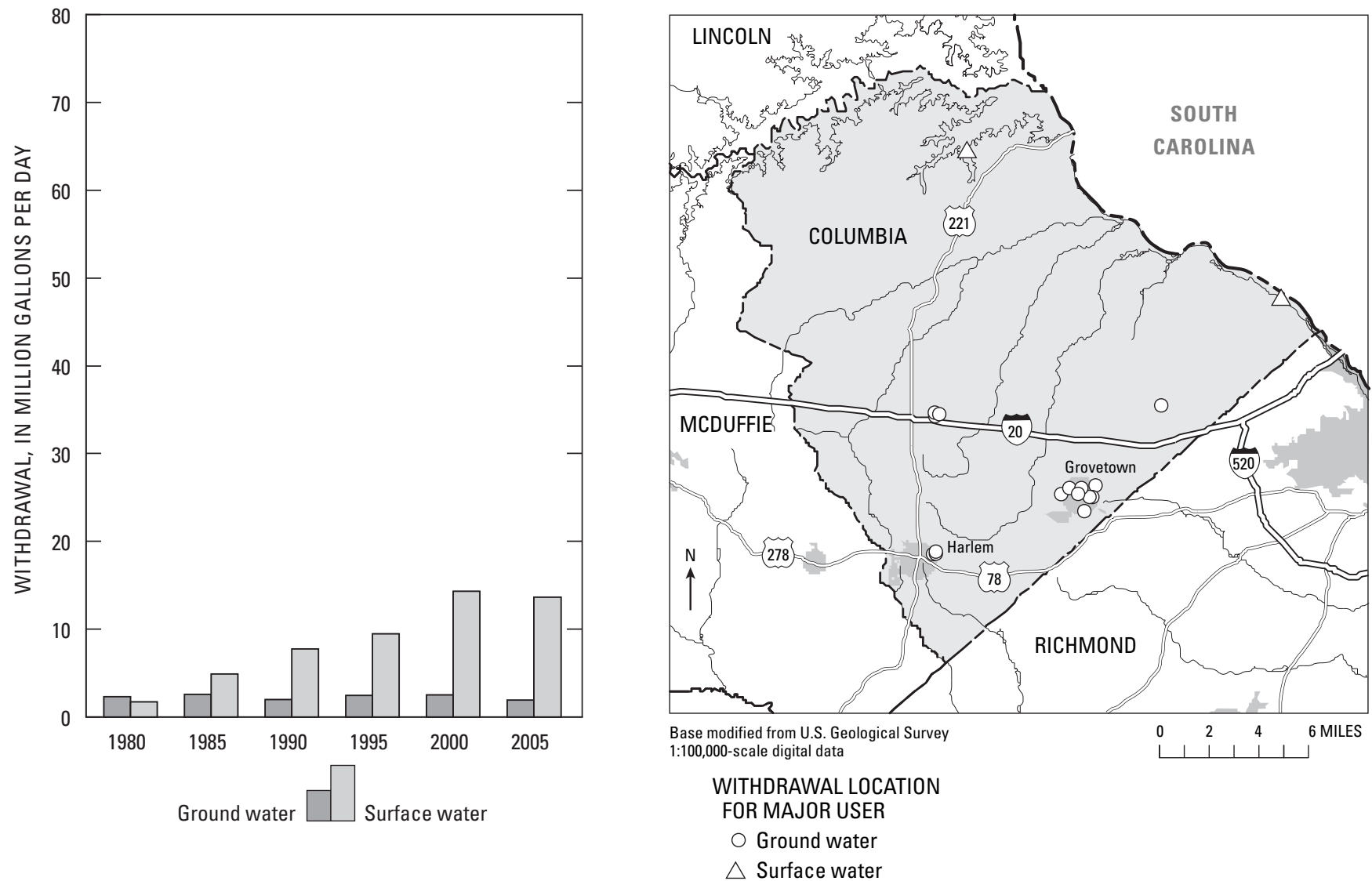


\section{COOK COUNTY}

Population: 16,366

Population served by public supply: 9,110

Acres irrigated: 18,150

Hydroelectric use (Mgal/d): $\quad 0.00$

2005 WITHDRAWALS, IN MILLION GALLONS PER DAY

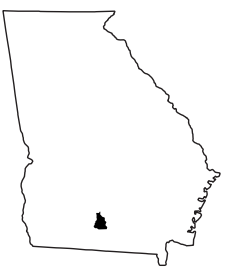

\begin{tabular}{|c|c|c|c|c|c|c|c|}
\hline & $\begin{array}{l}\text { Public } \\
\text { Supply }\end{array}$ & $\begin{array}{l}\text { Domestic \& } \\
\text { Commercial }\end{array}$ & $\begin{array}{c}\text { Industrial \& } \\
\text { Mining }\end{array}$ & Irrigation & Livestock & $\begin{array}{l}\text { Thermo- } \\
\text { electric }\end{array}$ & Totals \\
\hline Ground Water & 2.09 & 0.54 & 0.17 & 5.10 & 0.01 & 0.00 & 7.91 \\
\hline Surface Water & 0.00 & 0.00 & 0.00 & 3.18 & 0.04 & 0.00 & 3.22 \\
\hline TOTALS & 2.09 & 0.54 & 0.17 & 8.28 & 0.05 & 0.00 & 11.13 \\
\hline
\end{tabular}

Withdrawals by Major Public Suppliers (Mgal/d):

Name

City of Adel

Town of Cecil

Town of Lenox

Town of Sparks

GW

1.53

0.03

0.08

0.38
SW

0.00

0.00

0.00

0.00
Withdrawals by Major Industrial Groups (Mgal/d):

SIC

GW

SW
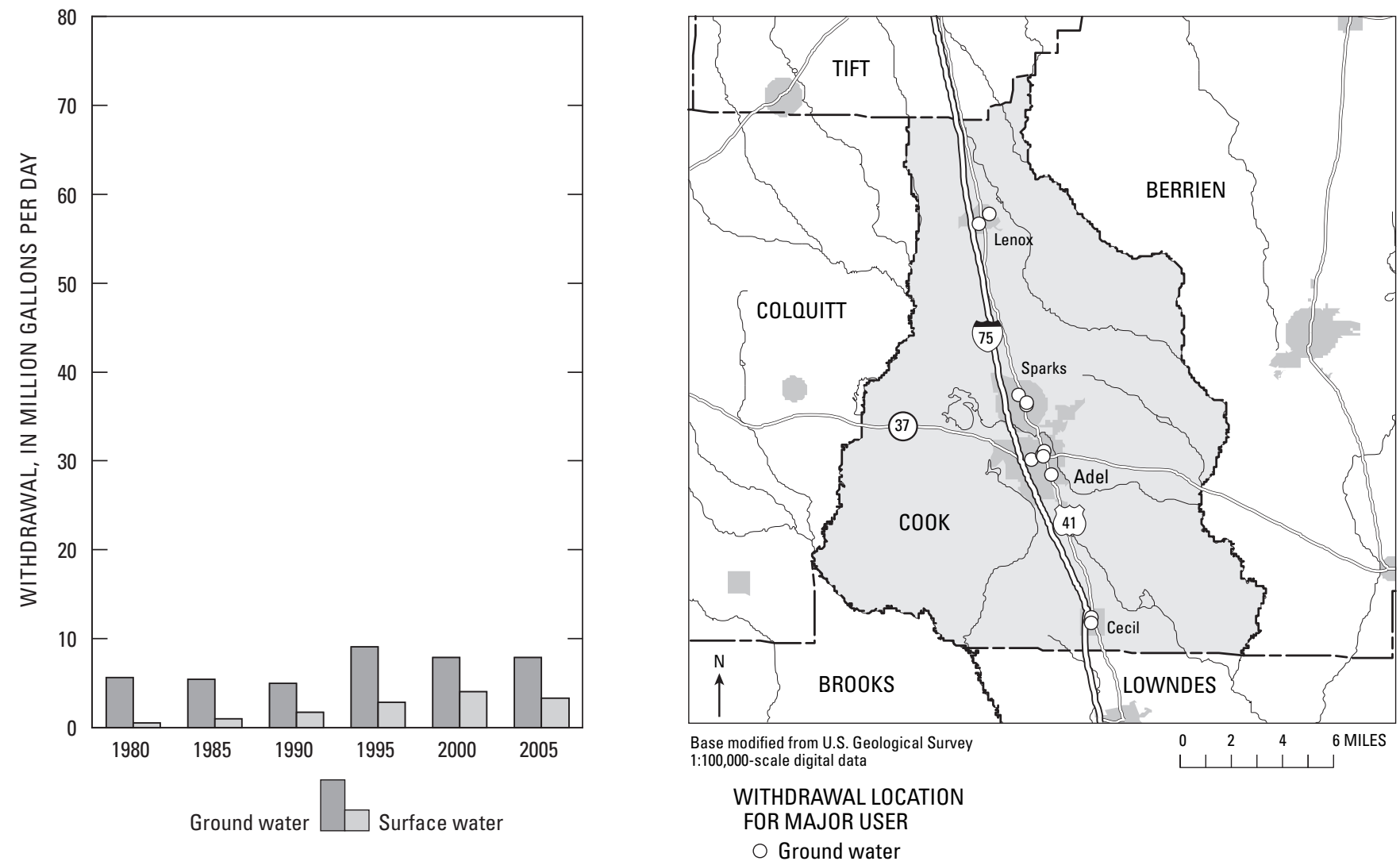


\section{COWETA COUNTY}

Population: 109,903

Population served by public supply: 104,330

Acres irrigated: 1,180

Hydroelectric use (Mgal/d): 0.00

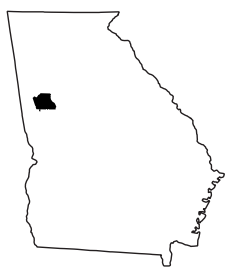

2005 WITHDRAWALS, IN MILLION GALLONS PER DAY

\begin{tabular}{|c|c|c|c|c|c|c|c|}
\hline & $\begin{array}{l}\text { Public } \\
\text { Supply }\end{array}$ & $\begin{array}{l}\text { Domestic \& } \\
\text { Commercial }\end{array}$ & $\begin{array}{c}\text { Industrial \& } \\
\text { Mining }\end{array}$ & Irrigation & Livestock & $\begin{array}{l}\text { Thermo- } \\
\text { electric }\end{array}$ & Totals \\
\hline Ground Water & 1.04 & 0.52 & 0.28 & 0.00 & 0.00 & 0.00 & 1.84 \\
\hline Surface Water & 7.15 & 0.00 & 0.00 & 2.33 & 0.11 & 31.08 & 40.67 \\
\hline TOTALS & 8.19 & 0.52 & 0.28 & 2.33 & 0.11 & 31.08 & 42.51 \\
\hline
\end{tabular}

Withdrawals by Major Public Suppliers (Mgal/d):

Name

GW SW

Town of Moreland

0.03

0.00

City of Newnan

Town of Turin

0.00

0.04

Coweta County Water System

City of Senoia

Withdrawals by Major Industrial Groups (Mgal/d):

SIC

GW

SW
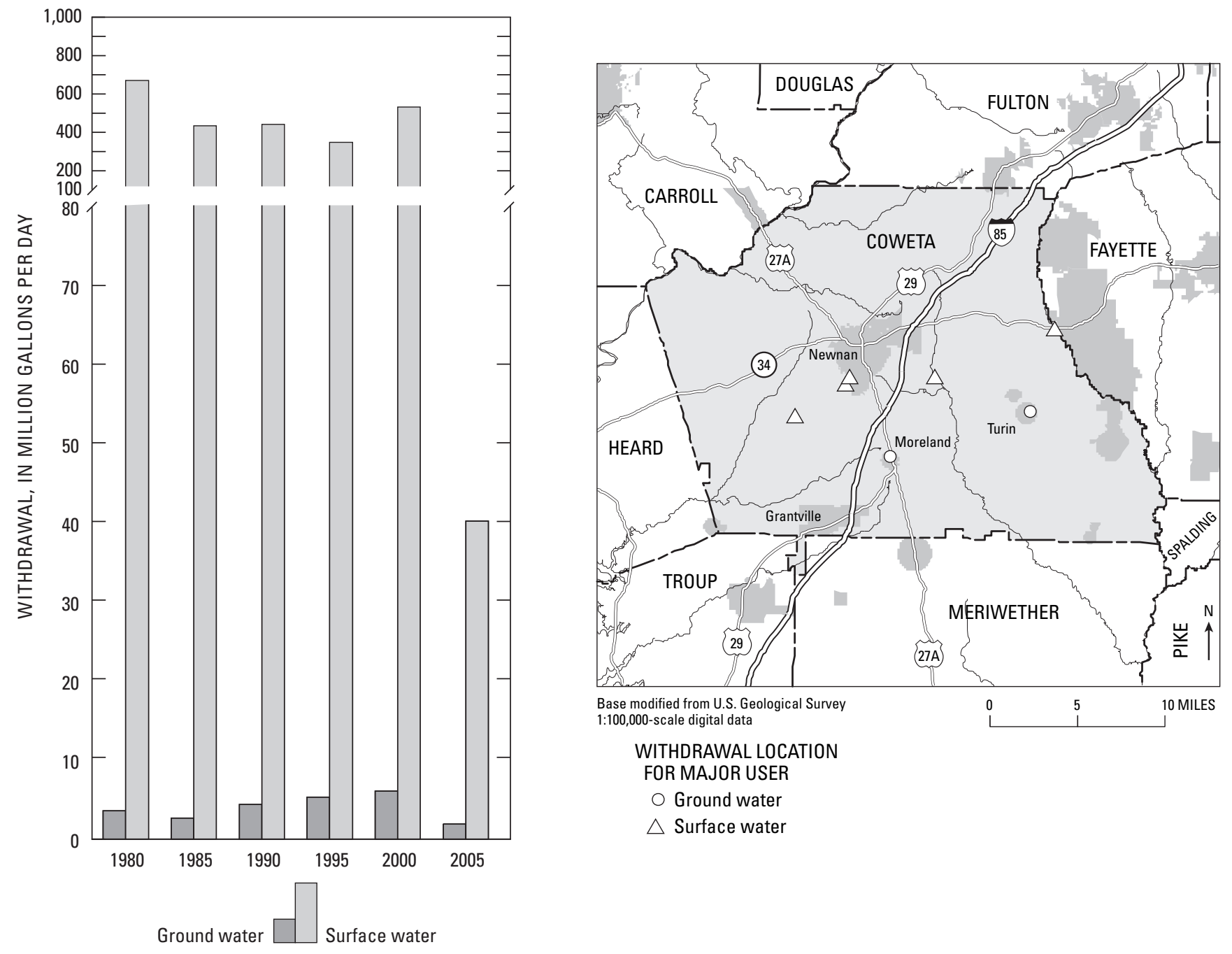


\section{CRAWFORD COUNTY}

Population: 12,874

Population served by public supply: 2,760

Acres irrigated: 3,170

Hydroelectric use (Mgal/d): 0.00

2005 WITHDRAWALS, IN MILLION GALLONS PER DAY

\begin{tabular}{|c|c|c|c|c|c|c|c|}
\hline & $\begin{array}{l}\text { Public } \\
\text { Supply }\end{array}$ & $\begin{array}{l}\text { Domestic \& } \\
\text { Commercial }\end{array}$ & $\begin{array}{c}\text { Industrial \& } \\
\text { Mining }\end{array}$ & Irrigation & Livestock & $\begin{array}{l}\text { Thermo- } \\
\text { electric }\end{array}$ & Totals \\
\hline Ground Water & 0.28 & 0.76 & 0.43 & 2.65 & 0.00 & 0.00 & 4.12 \\
\hline Surface Water & 0.00 & 0.00 & 0.01 & 0.57 & 0.17 & 0.00 & 0.75 \\
\hline TOTALS & 0.28 & 0.76 & 0.44 & 3.22 & 0.17 & 0.00 & 4.87 \\
\hline
\end{tabular}

Withdrawals by Major Public Suppliers (Mgal/d):

Name

City of Roberta

GW

0.16

SW

Crawford County Board of Commissioners
Withdrawals by Major Industrial Groups (Mgal/d):

SIC

GW

SW
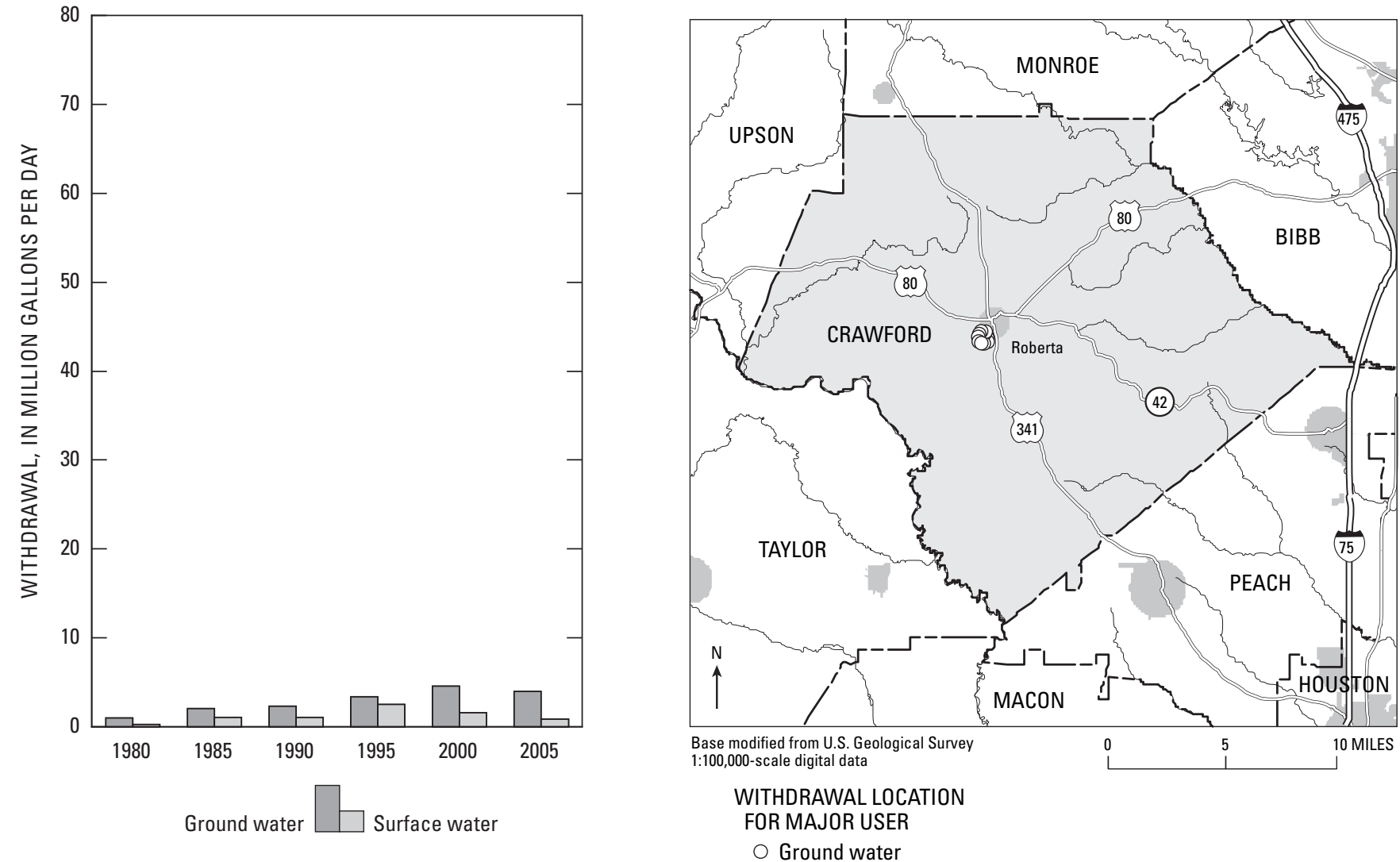


\section{CRISP COUNTY}

Population: 22,017

Population served by public supply: 17,170

Acres irrigated: 29,070

Hydroelectric use (Mgal/d): 0.00

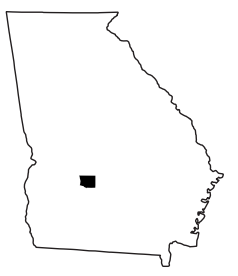

2005 WITHDRAWALS, IN MILLION GALLONS PER DAY

\begin{tabular}{|c|c|c|c|c|c|c|c|}
\hline & $\begin{array}{l}\text { Public } \\
\text { Supply }\end{array}$ & $\begin{array}{l}\text { Domestic \& } \\
\text { Commercial }\end{array}$ & $\begin{array}{c}\text { Industrial \& } \\
\text { Mining }\end{array}$ & Irrigation & Livestock & $\begin{array}{l}\text { Thermo- } \\
\text { electric }\end{array}$ & Totals \\
\hline Ground Water & 2.10 & 0.36 & 0.07 & 15.00 & 0.02 & 0.00 & 17.55 \\
\hline Surface Water & 0.00 & 0.00 & 0.00 & 1.20 & 0.05 & 0.00 & 1.25 \\
\hline TOTALS & 2.10 & 0.36 & 0.07 & 16.20 & 0.07 & 0.00 & 18.80 \\
\hline
\end{tabular}

Withdrawals by Major Public Suppliers (Mgal/d):

Name

GW

0.02

SW

Town of Arabi

1.79

City of Cordele

0.21

0.00

0.00

0.00
Withdrawals by Major Industrial Groups (Mgal/d):

SIC

26 - Paper

GW

SW

0.00
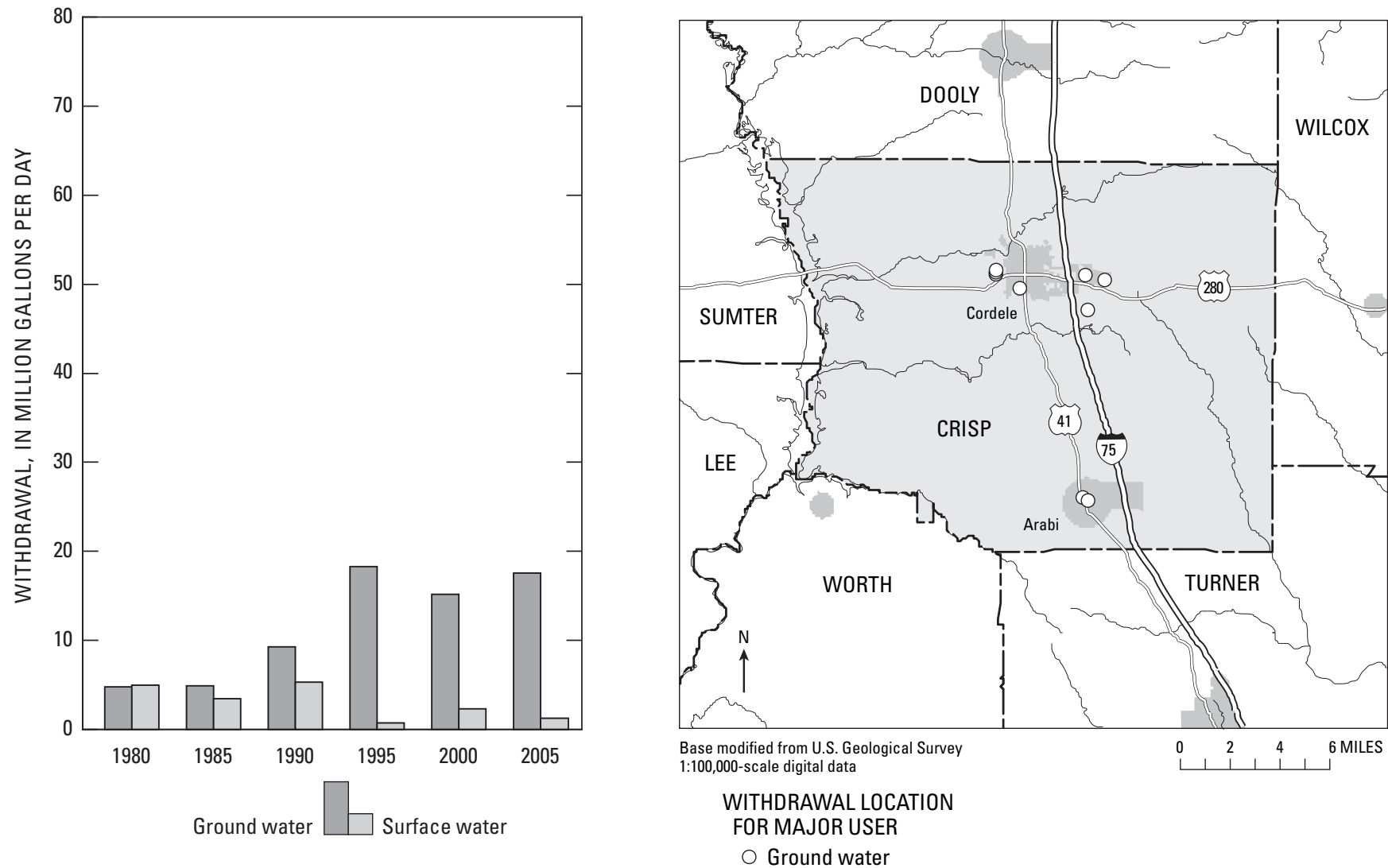


\section{DADE COUNTY}

Population: 16,040

Population served by public supply: $\quad 16,000$

Acres irrigated: 320

Hydroelectric use (Mgal/d): $\quad 0.00$

2005 WITHDRAWALS, IN MILLION GALLONS PER DAY

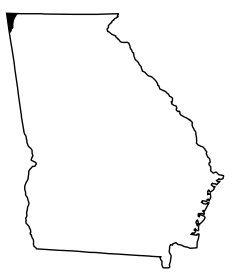

\begin{tabular}{lccccccc}
\hline & $\begin{array}{c}\text { Public } \\
\text { Supply }\end{array}$ & $\begin{array}{c}\text { Domestic \& } \\
\text { Commercial }\end{array}$ & $\begin{array}{c}\text { Industrial \& } \\
\text { Mining }\end{array}$ & Irrigation & Livestock & $\begin{array}{c}\text { Thermo- } \\
\text { electric }\end{array}$ & Totals \\
\hline Ground Water & 0.00 & 0.00 & 0.00 & 0.15 & 0.01 & 0.00 & 0.16 \\
Surface Water & 2.35 & 0.00 & 0.00 & 0.31 & 0.08 & 0.00 & 2.74 \\
TOTALS & 2.35 & 0.00 & 0.00 & 0.46 & 0.09 & 0.00 & 2.90 \\
\hline
\end{tabular}

Withdrawals by Major Public Suppliers (Mgal/d):

Name

Dade Water System

SW

2.35
Withdrawals by Major Industrial Groups (Mgal/d):

SIC

None
GW

SW
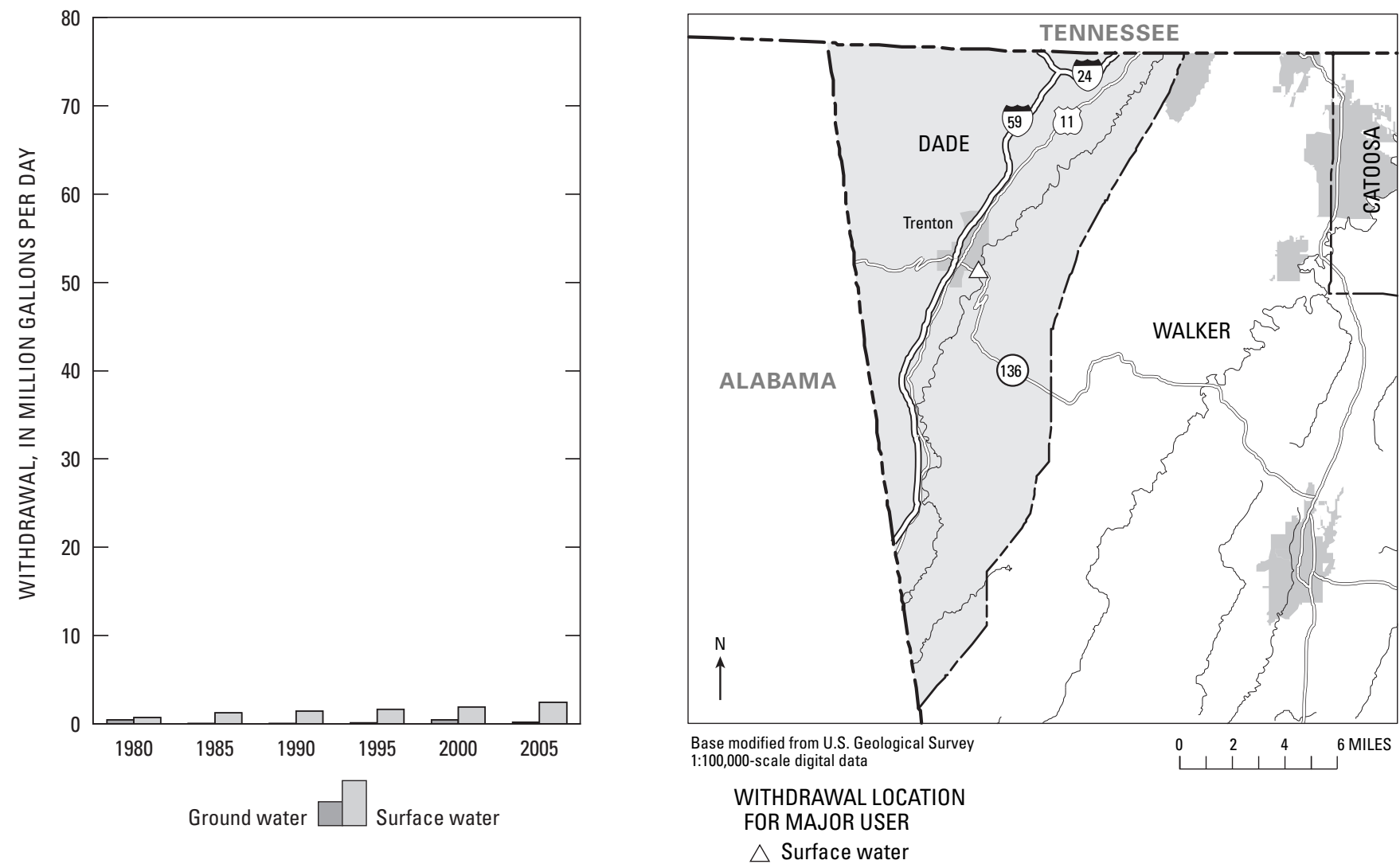


\section{DAWSON COUNTY}

Population: 19,731

Population served by public supply: 13,340

Acres irrigated: 290

Hydroelectric use (Mgal/d): $\quad 0.00$

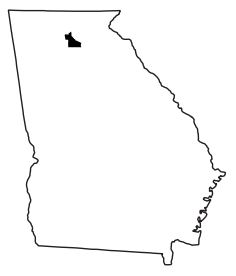

2005 WITHDRAWALS, IN MILLION GALLONS PER DAY

\begin{tabular}{|c|c|c|c|c|c|c|c|}
\hline & $\begin{array}{l}\text { Public } \\
\text { Supply }\end{array}$ & $\begin{array}{l}\text { Domestic \& } \\
\text { Commercial }\end{array}$ & $\begin{array}{c}\text { Industrial \& } \\
\text { Mining }\end{array}$ & Irrigation & Livestock & $\begin{array}{l}\text { Thermo- } \\
\text { electric }\end{array}$ & Totals \\
\hline Ground Water & 0.00 & 0.48 & 0.00 & 0.00 & 0.00 & 0.00 & 0.48 \\
\hline Surface Water & 1.60 & 0.00 & 0.00 & 0.39 & 0.24 & 0.00 & 2.23 \\
\hline TOTALS & 1.60 & 0.48 & 0.00 & 0.39 & 0.24 & 0.00 & 2.71 \\
\hline
\end{tabular}

Withdrawals by Major Public Suppliers (Mgal/d):

Name

GW SW

Etowah Water \&

0.00

1.50

0.00

0.10
Sewer Authority

Town of Dawsonville

$0.00 \quad 0.10$

Withdrawals by Major Industrial Groups (Mgal/d):

SIC

GW

SW
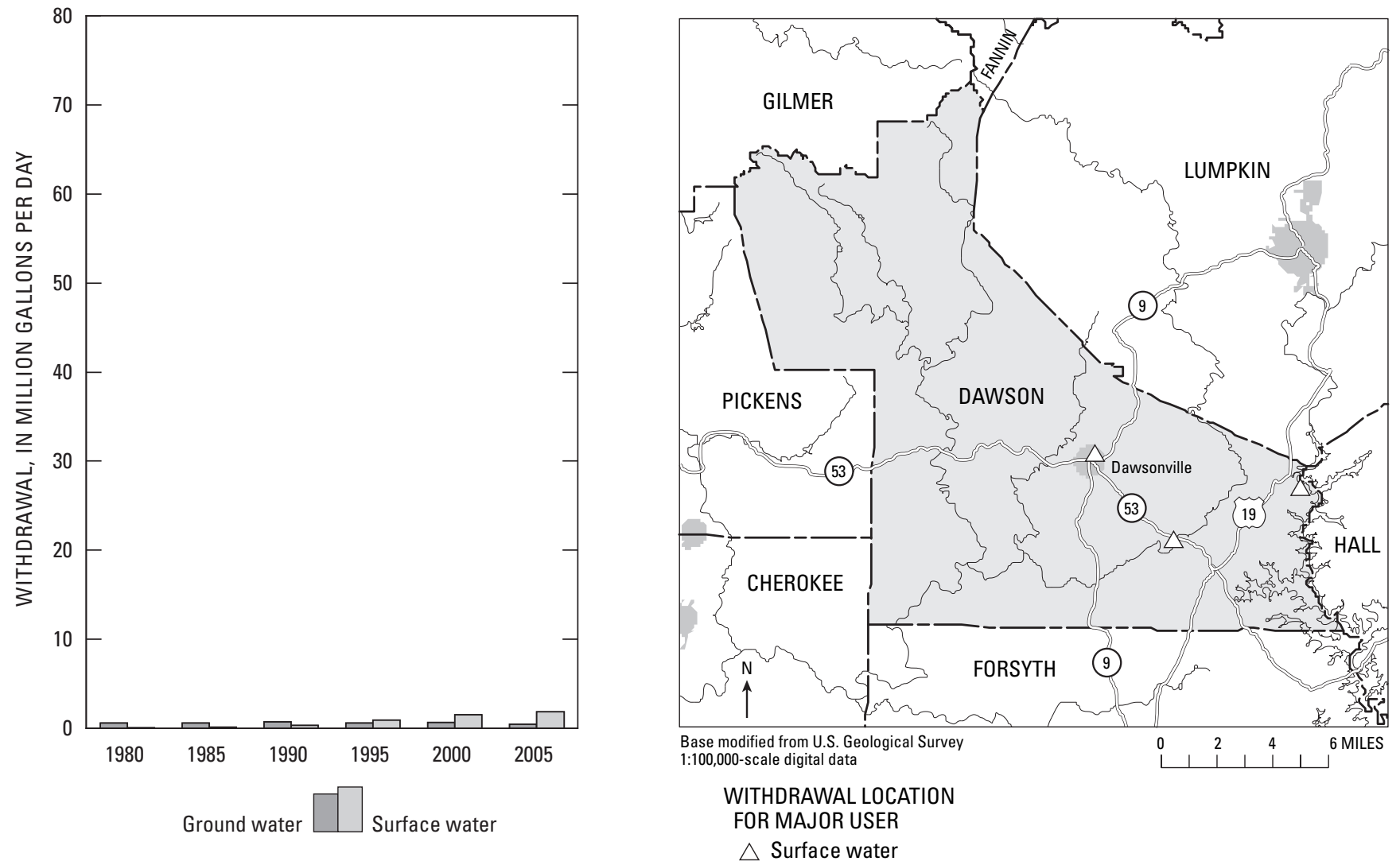


\section{DECATUR COUNTY}

Population: 28,618

Population served by public supply: 14,390

Acres irrigated: 65,000

Hydroelectric use (Mgal/d): $\quad 0.00$

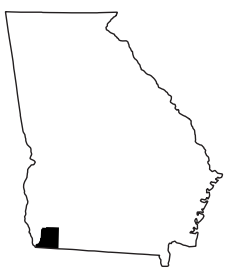

2005 WITHDRAWALS, IN MILLION GALLONS PER DAY

\begin{tabular}{|c|c|c|c|c|c|c|c|}
\hline & $\begin{array}{l}\text { Public } \\
\text { Supply }\end{array}$ & $\begin{array}{l}\text { Domestic \& } \\
\text { Commercial }\end{array}$ & $\begin{array}{c}\text { Industrial \& } \\
\text { Mining }\end{array}$ & Irrigation & Livestock & $\begin{array}{l}\text { Thermo- } \\
\text { electric }\end{array}$ & Totals \\
\hline Ground Water & 2.87 & 1.45 & 0.60 & 34.79 & 0.16 & 0.00 & 39.87 \\
\hline Surface Water & 0.00 & 0.00 & 0.54 & 1.85 & 0.07 & 0.00 & 2.46 \\
\hline TOTALS & 2.87 & 1.45 & 1.14 & 36.64 & 0.23 & 0.00 & 42.33 \\
\hline
\end{tabular}

Withdrawals by Major Public Suppliers (Mgal/d):

Name

Town of Attapulgus

City of Bainbridge

Town of Brinson

Town of Climax
GW

0.06

2.69

0.02

0.03
Withdrawals by Major Industrial Groups (Mgal/d):

SIC

GW

0.30

0.05

SW

0.00

32 - Stone, clay
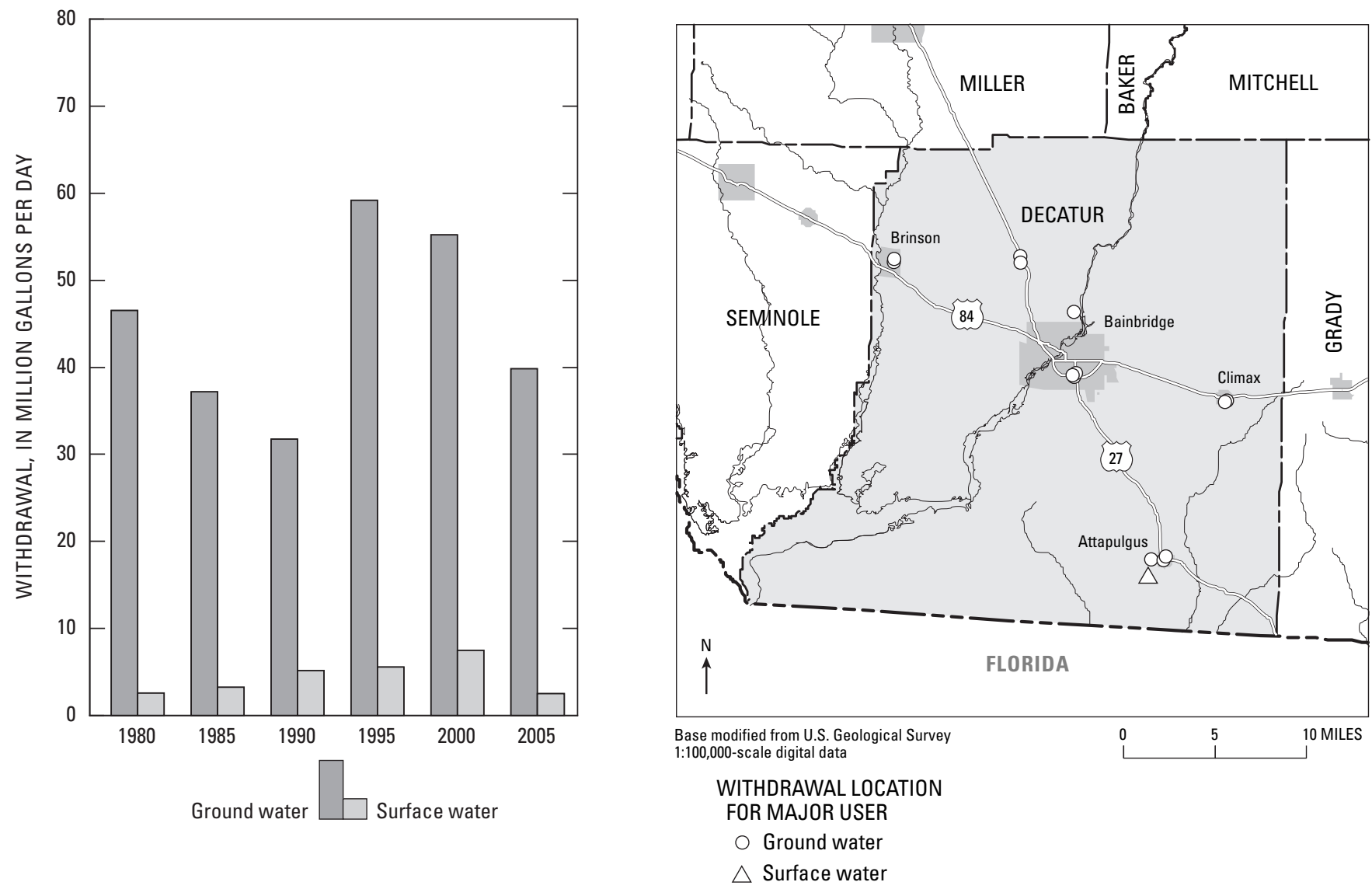


\section{DEKALB COUNTY}

Population: 677,959

Population served by public supply: $\quad 637,280$

Acres irrigated: 1,180

Hydroelectric use (Mgal/d): $\quad 0.00$

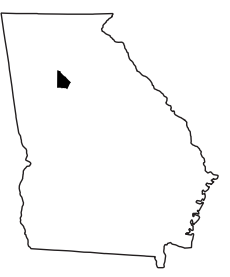

2005 WITHDRAWALS, IN MILLION GALLONS PER DAY

\begin{tabular}{|c|c|c|c|c|c|c|c|}
\hline & $\begin{array}{l}\text { Public } \\
\text { Supply }\end{array}$ & $\begin{array}{l}\text { Domestic \& } \\
\text { Commercial }\end{array}$ & $\begin{array}{c}\text { Industrial \& } \\
\text { Mining }\end{array}$ & Irrigation & Livestock & $\begin{array}{l}\text { Thermo- } \\
\text { electric }\end{array}$ & Totals \\
\hline Ground Water & 0.00 & 3.05 & 0.96 & 0.00 & 0.00 & 0.00 & 4.01 \\
\hline Surface Water & $83.05^{\mathrm{a}}$ & 0.00 & 0.01 & 1.77 & 0.00 & 0.00 & 84.83 \\
\hline TOTALS & 83.05 & 3.05 & 0.97 & 1.77 & 0.00 & 0.00 & 88.84 \\
\hline
\end{tabular}

Withdrawals by Major Public Suppliers (Mgal/d):

Name GW SW

DeKalb County Water System $\quad 0.00 \quad 83.05^{\mathrm{a}}$
Withdrawals by Major Industrial Groups (Mgal/d):

SIC

None
GW

SW

${ }^{a}$ Water used in DeKalb County but withdrawn from Fulton County.
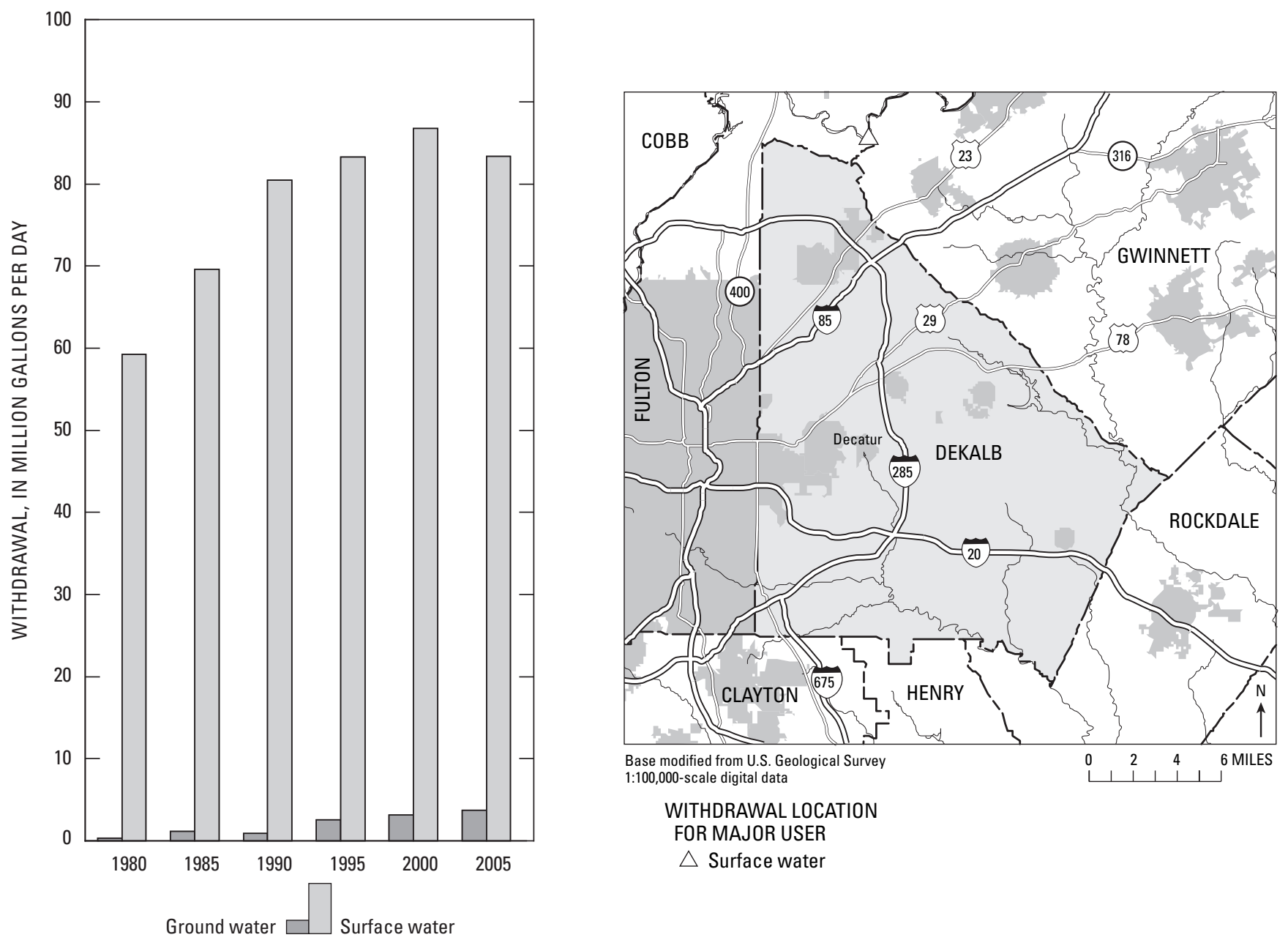


\section{DODGE COUNTY}

Population: $\quad 19,574$

Population served by public supply: 7,330

Acres irrigated: 22,530

Hydroelectric use (Mgal/d): $\quad 0.00$

2005 WITHDRAWALS, IN MILLION GALLONS PER DAY

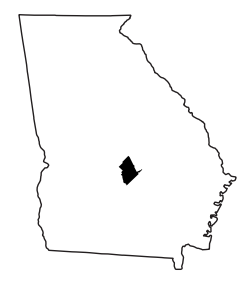

\begin{tabular}{|c|c|c|c|c|c|c|c|}
\hline & $\begin{array}{l}\text { Public } \\
\text { Supply }\end{array}$ & $\begin{array}{l}\text { Domestic \& } \\
\text { Commercial }\end{array}$ & $\begin{array}{c}\text { Industrial \& } \\
\text { Mining }\end{array}$ & Irrigation & Livestock & $\begin{array}{l}\text { Thermo- } \\
\text { electric }\end{array}$ & Totals \\
\hline Ground Water & 0.72 & 0.92 & 0.00 & 3.20 & 0.75 & 0.00 & 5.59 \\
\hline Surface Water & 0.00 & 0.00 & 0.00 & 14.24 & 0.10 & 0.00 & 14.34 \\
\hline TOTALS & 0.72 & 0.92 & 0.00 & 17.44 & 0.85 & 0.00 & 19.93 \\
\hline
\end{tabular}

Withdrawals by Major Public Suppliers (Mgal/d):

Name

GW

Town of Chauncey

Town of Chester

City of Eastman

Town of Rhine

0.03

SW

0.08

0.58

0.03

Withdrawals by Major Industrial Groups (Mgal/d):

SIC

GW

SW

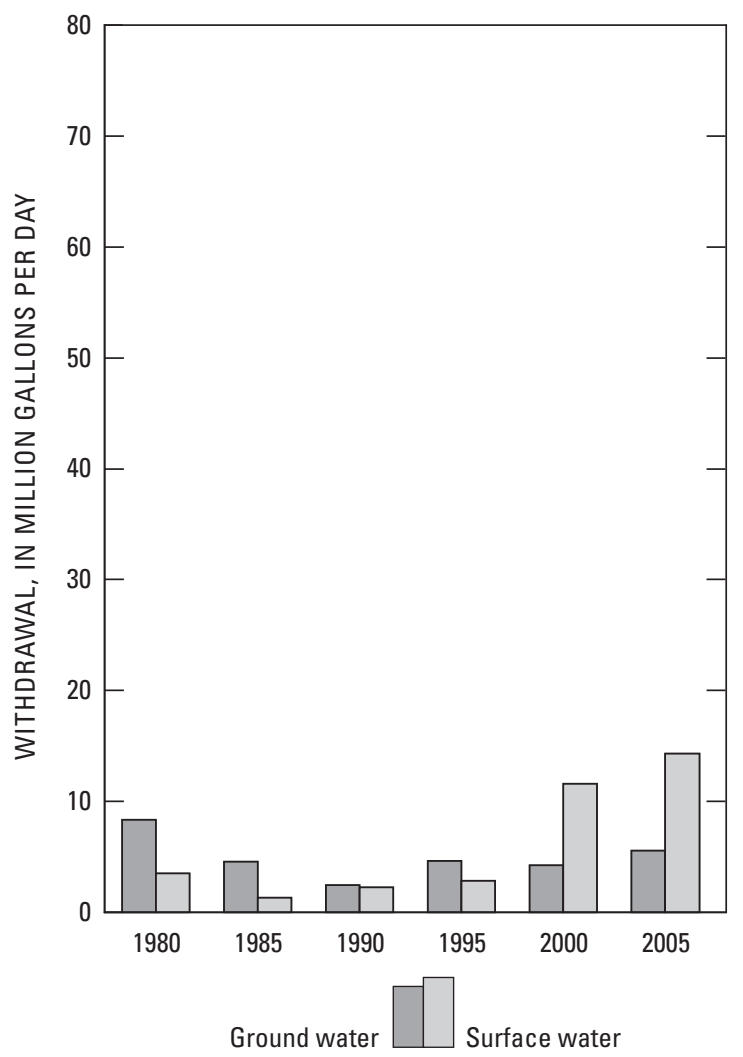

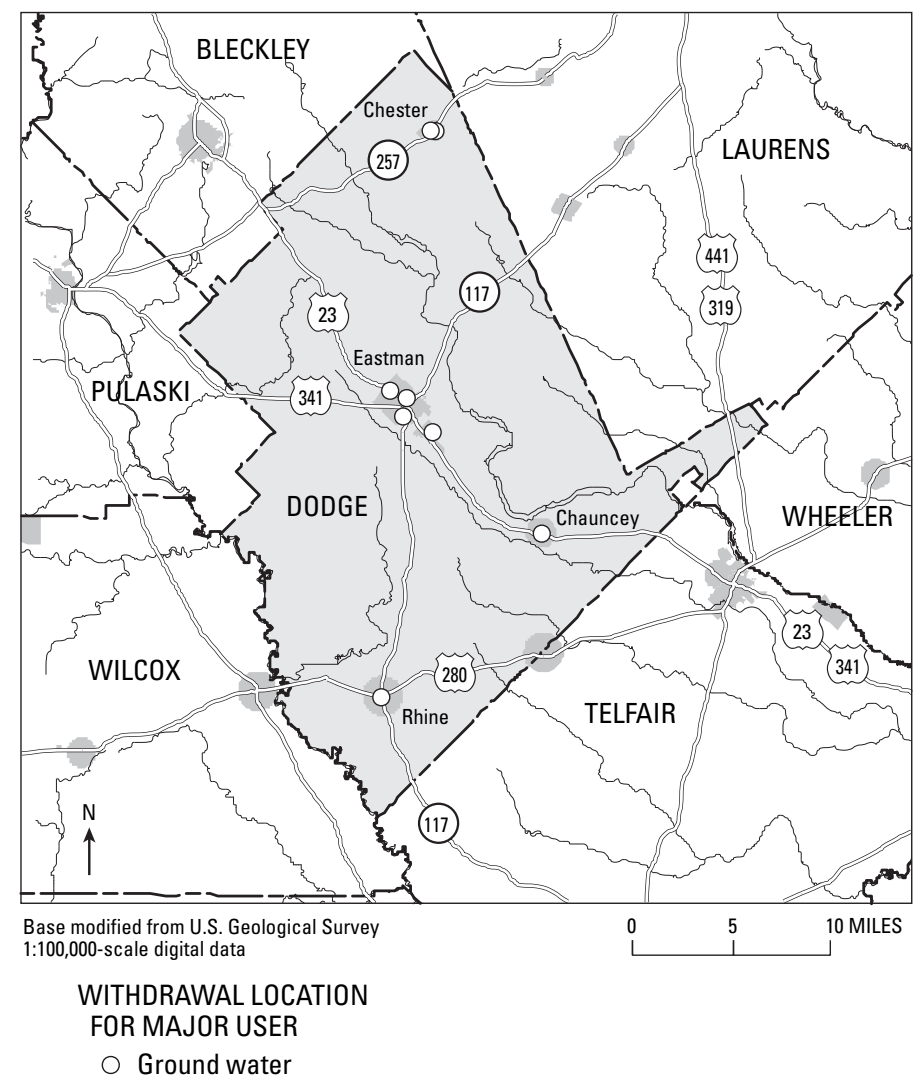




\section{DOOLY COUNTY}

Population: 11,749

Population served by public supply: $\quad 7,740$

Acres irrigated: 44,500

Hydroelectric use (Mgal/d): $\quad 0.00$

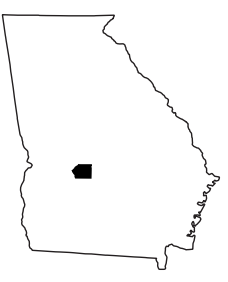

2005 WITHDRAWALS, IN MILLION GALLONS PER DAY

\begin{tabular}{|c|c|c|c|c|c|c|c|}
\hline & $\begin{array}{l}\text { Public } \\
\text { Supply }\end{array}$ & $\begin{array}{l}\text { Domestic \& } \\
\text { Commercial }\end{array}$ & $\begin{array}{c}\text { Industrial \& } \\
\text { Mining }\end{array}$ & Irrigation & Livestock & $\begin{array}{l}\text { Thermo- } \\
\text { electric }\end{array}$ & Totals \\
\hline Ground Water & 2.21 & 0.30 & 0.00 & 22.25 & 0.03 & 0.00 & 24.79 \\
\hline Surface Water & 0.00 & 0.00 & 0.00 & 1.34 & 0.16 & 0.00 & 1.50 \\
\hline TOTALS & 2.21 & 0.30 & 0.00 & 23.59 & 0.19 & 0.00 & 26.29 \\
\hline
\end{tabular}

Withdrawals by Major Public Suppliers (Mgal/d):

Name

Town of Byromville

GW

0.03

SW

Town of Lilly

City of Pinehurst

City of Unadilla

City of Vienna
0.02

0.03

0.52

1.59

Withdrawals by Major Industrial Groups (Mgal/d):

SIC

GW

SW
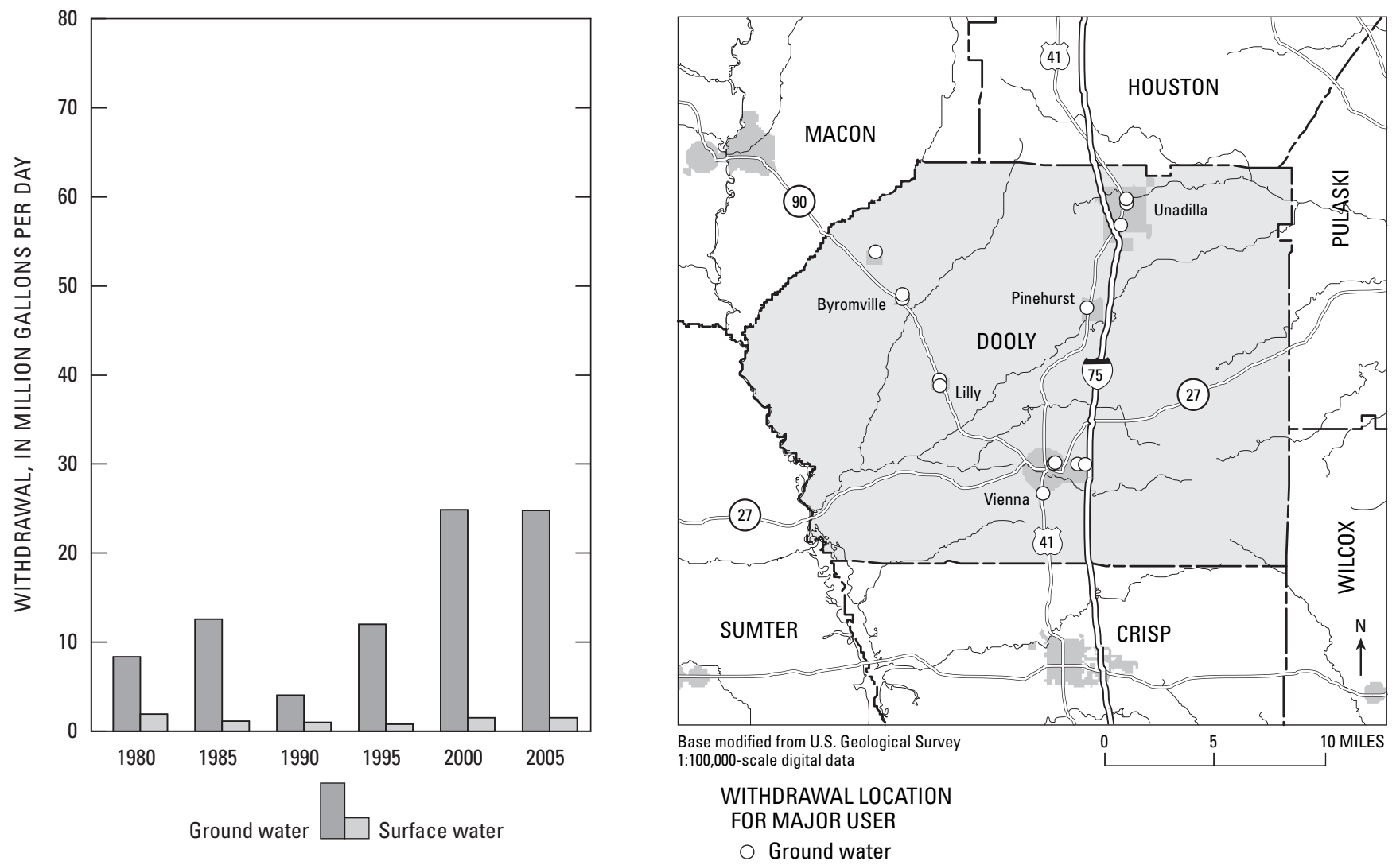


\section{DOUGHERTY COUNTY}

Population: 94,882

Population served by public supply: $\quad 91,170$

Acres irrigated: 23,350

Hydroelectric use (Mgal/d): $\quad 1,000.32$

2005 WITHDRAWALS, IN MILLION GALLONS PER DAY

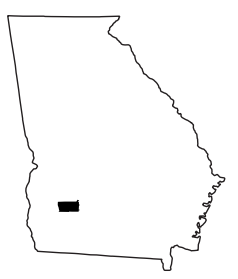

\begin{tabular}{|c|c|c|c|c|c|c|c|}
\hline & $\begin{array}{l}\text { Public } \\
\text { Supply }\end{array}$ & $\begin{array}{l}\text { Domestic \& } \\
\text { Commercial }\end{array}$ & $\begin{array}{c}\text { Industrial \& } \\
\text { Mining }\end{array}$ & Irrigation & Livestock & $\begin{array}{l}\text { Thermo- } \\
\text { electric }\end{array}$ & Totals \\
\hline Ground Water & 13.73 & 0.90 & 13.62 & 9.48 & 0.02 & 0.05 & 37.80 \\
\hline Surface Water & 0.00 & 0.00 & 0.00 & 1.49 & 0.01 & 120.09 & 121.59 \\
\hline TOTALS & 13.73 & 0.90 & 13.62 & 10.97 & 0.03 & 120.14 & 159.39 \\
\hline
\end{tabular}

Withdrawals by Major Public Suppliers (Mgal/d):

$\begin{array}{lcc}\text { Name } & \text { GW } & \text { SW } \\ \text { City of Albany } & 13.33 & 0.00 \\ \text { Putney Water System } & 0.02 & 0.00\end{array}$

Withdrawals by Major Industrial Groups (Mgal/d):

$\begin{array}{lcc}\text { SIC } & \text { GW } & \text { SW } \\ 20 \text { - Food } & 2.19 & 0.00 \\ 26 \text { - Paper } & 6.64 & 0.00 \\ 28 \text { - Chemicals } & 4.61 & 0.00 \\ 30 \text { - Rubber } & 0.10 & 0.00 \\ 32 \text { - Stone, clay } & 0.04 & 0.00\end{array}$
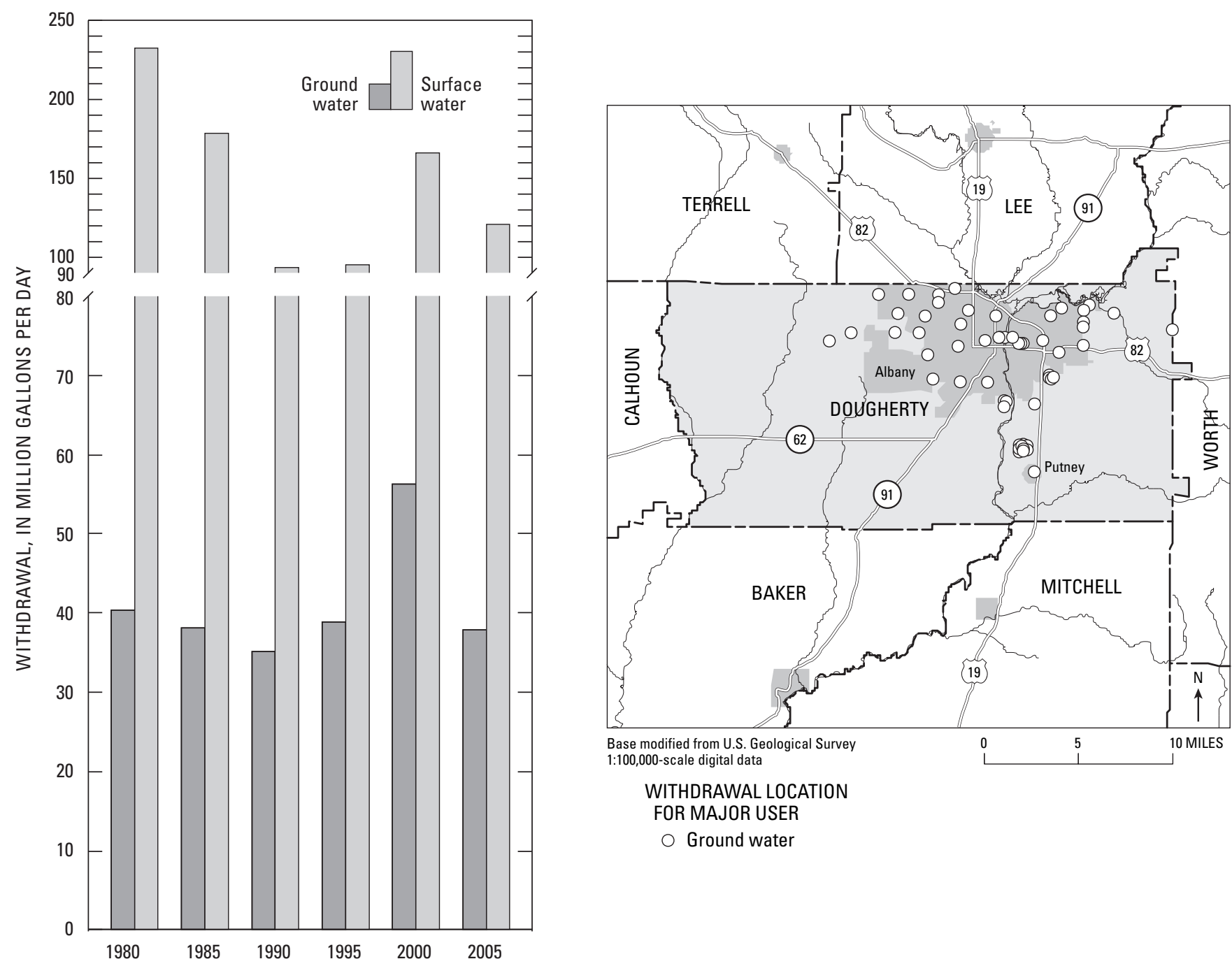


\section{DOUGLAS COUNTY}

Population: 112,760

Population served by public supply: 73,820

Acres irrigated: 1,500

Hydroelectric use (Mgal/d): $\quad 0.00$

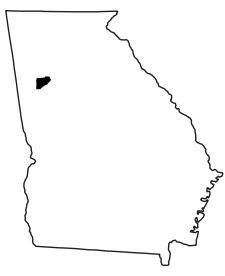

2005 WITHDRAWALS, IN MILLION GALLONS PER DAY

\begin{tabular}{|c|c|c|c|c|c|c|c|}
\hline & $\begin{array}{l}\text { Public } \\
\text { Supply }\end{array}$ & $\begin{array}{l}\text { Domestic \& } \\
\text { Commercial }\end{array}$ & $\begin{array}{c}\text { Industrial \& } \\
\text { Mining }\end{array}$ & Irrigation & Livestock & $\begin{array}{l}\text { Thermo- } \\
\text { electric }\end{array}$ & Totals \\
\hline Ground Water & 0.06 & 2.92 & 0.93 & 0.00 & 0.01 & 0.00 & 3.92 \\
\hline Surface Water & 12.33 & 0.00 & 0.01 & 1.77 & 0.02 & 0.00 & 14.13 \\
\hline TOTALS & 12.39 & 2.92 & 0.94 & 1.77 & 0.03 & 0.00 & 18.05 \\
\hline
\end{tabular}

Withdrawals by Major Public Suppliers (Mgal/d):

Name

GW

Douglasville-Douglas County

Water \& Sewer Authority

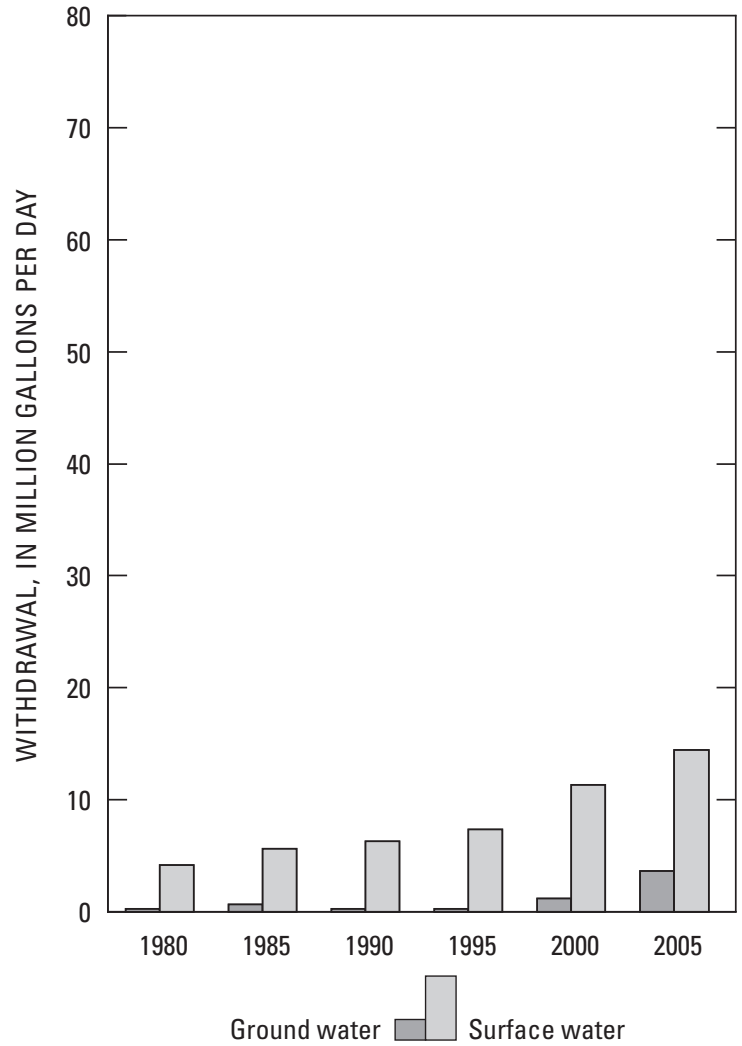

Withdrawals by Major Industrial Groups (Mgal/d):

SIC

GW

SW

None

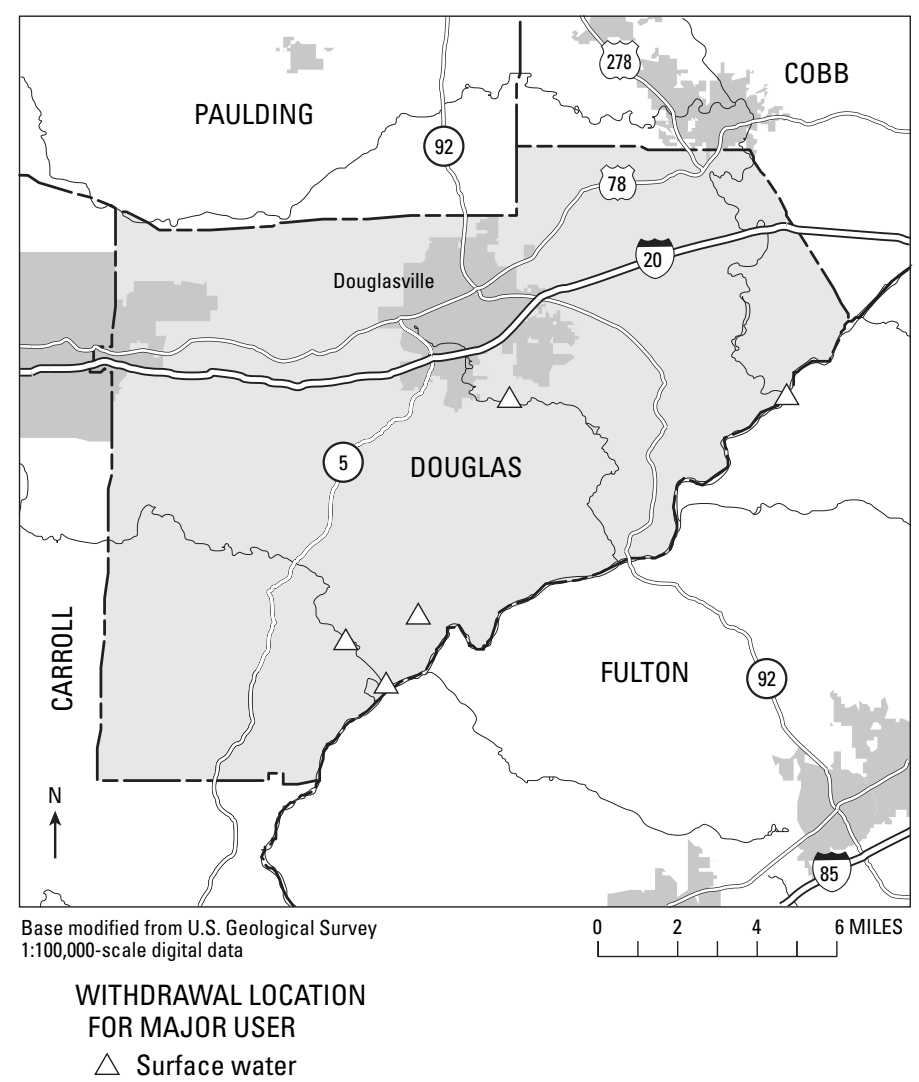




\section{EARLY COUNTY}

Population: 12,056

Population served by public supply: 6,100

Acres irrigated: 56,080

Hydroelectric use (Mgal/d): $\quad 0.00$

2005 WITHDRAWALS, IN MILLION GALLONS PER DAY

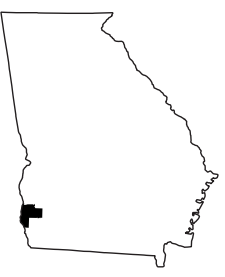

\begin{tabular}{lccccccc}
\hline & $\begin{array}{c}\text { Public } \\
\text { Supply }\end{array}$ & $\begin{array}{c}\text { Domestic \& } \\
\text { Commercial }\end{array}$ & $\begin{array}{c}\text { Industrial \& } \\
\text { Mining }\end{array}$ & Irrigation & Livestock & $\begin{array}{c}\text { Thermo- } \\
\text { electric }\end{array}$ & Totals \\
\hline Ground Water & 0.90 & 0.45 & 0.42 & 11.79 & 0.10 & 0.00 & 13.66 \\
Surface Water & 0.00 & 0.00 & 107.14 & 3.02 & 0.08 & 0.00 & 110.24 \\
TOTALS & 0.90 & 0.45 & 107.56 & 14.81 & 0.18 & 0.00 & 123.90 \\
\hline
\end{tabular}

Withdrawals by Major Public Suppliers (Mgal/d):

Name GW SW

City of Blakely

Town of Damascus

Town of Jakin

$\begin{array}{ll}0.86 & 0.00 \\ 0.02 & 0.00 \\ 0.02 & 0.00\end{array}$

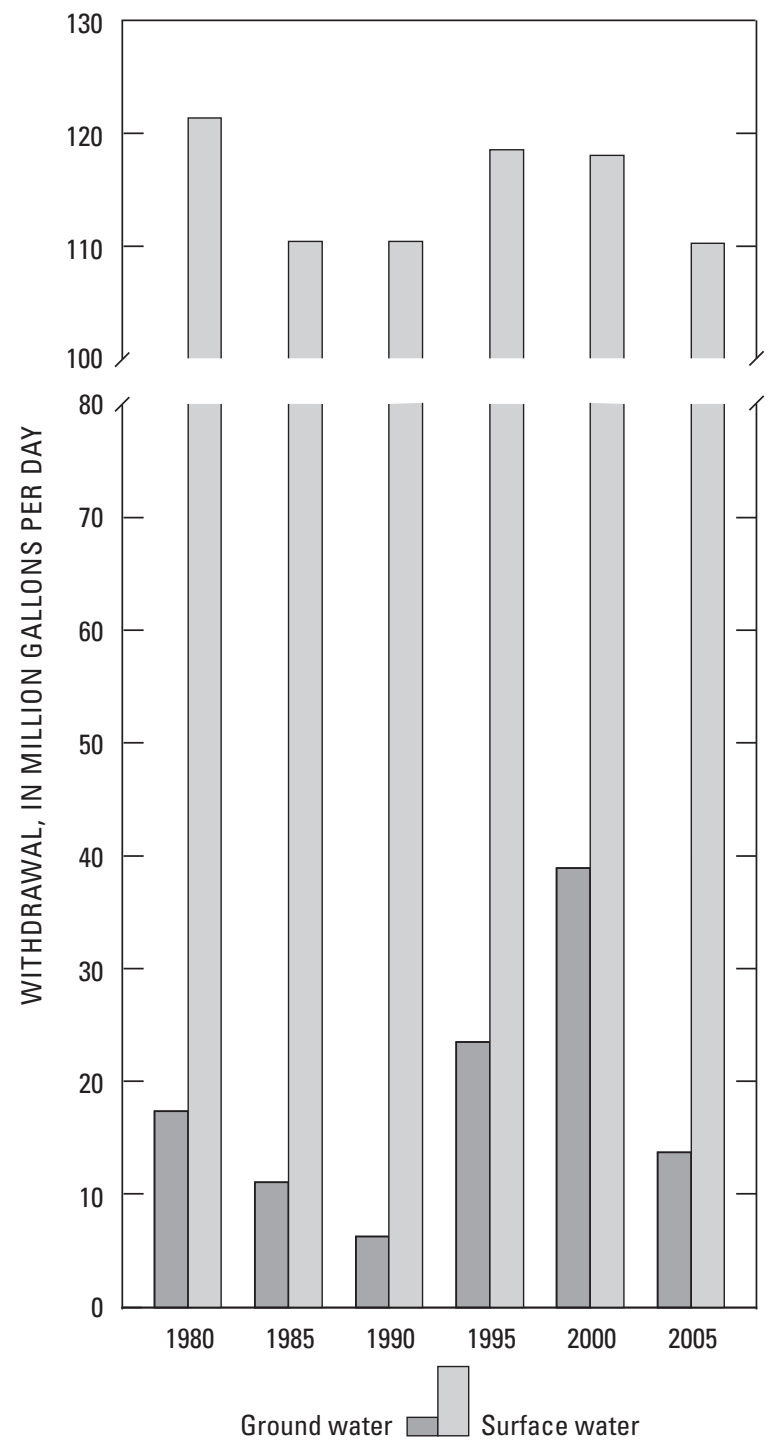

Withdrawals by Major Industrial Groups (Mgal/d):

$\begin{array}{lcr}\text { SIC } & \text { GW } & \text { SW } \\ \text { 26 - Paper } & 0.08 & 107.14 \\ \text { 36 - Electrical Machinery } & 0.34 & 0.00\end{array}$

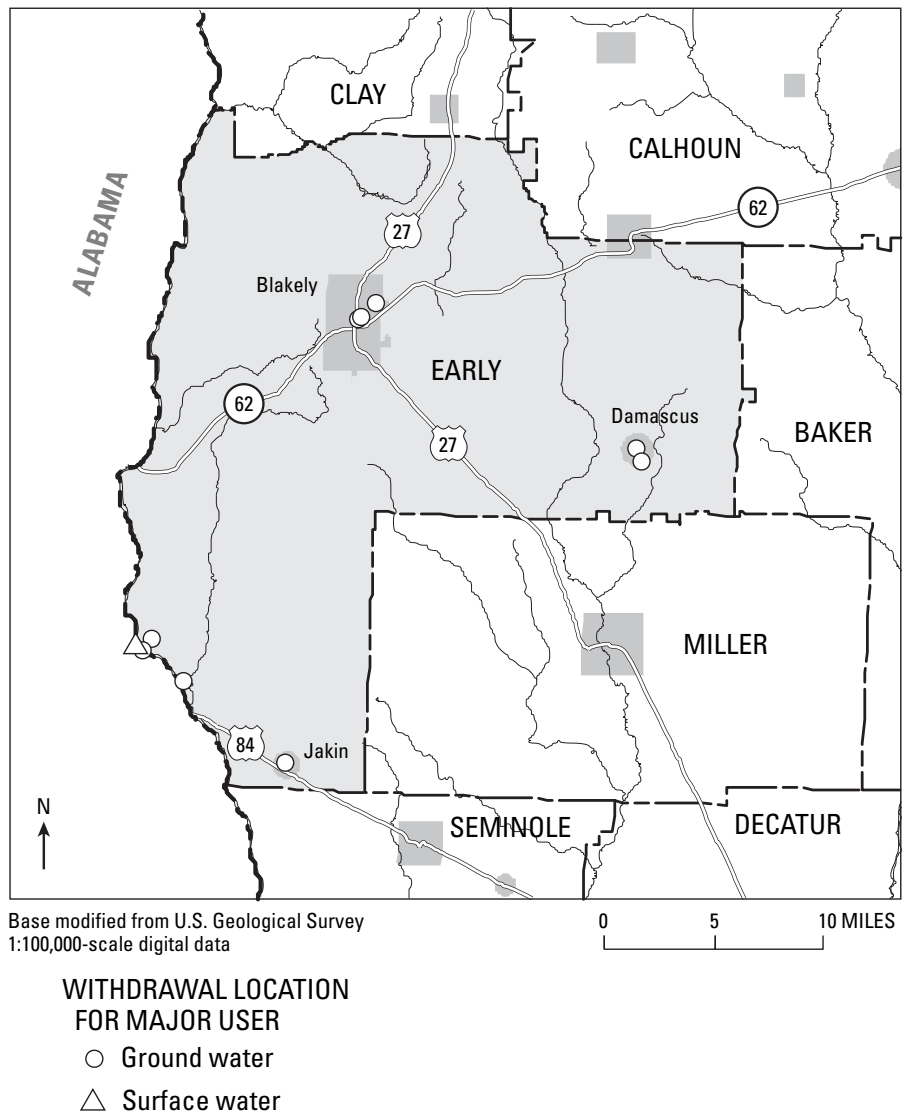




\section{ECHOLS COUNTY}

Population: 4,253

Population served by public supply: 710

Acres irrigated: 3,320

Hydroelectric use (Mgal/d): 0.00

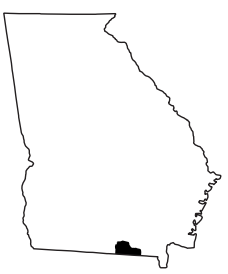

2005 WITHDRAWALS, IN MILLION GALLONS PER DAY

\begin{tabular}{|c|c|c|c|c|c|c|c|}
\hline & $\begin{array}{l}\text { Public } \\
\text { Supply }\end{array}$ & $\begin{array}{l}\text { Domestic \& } \\
\text { Commercial }\end{array}$ & $\begin{array}{c}\text { Industrial \& } \\
\text { Mining }\end{array}$ & Irrigation & Livestock & $\begin{array}{l}\text { Thermo- } \\
\text { electric }\end{array}$ & Totals \\
\hline Ground Water & 0.05 & 0.27 & 0.00 & 5.46 & 0.00 & 0.00 & 5.78 \\
\hline Surface Water & 0.00 & 0.00 & 0.00 & 0.49 & 0.01 & 0.00 & 0.50 \\
\hline TOTALS & 0.05 & 0.27 & 0.00 & 5.95 & 0.01 & 0.00 & 6.28 \\
\hline
\end{tabular}

Withdrawals by Major Public Suppliers (Mgal/d):

Name

GW

Echols County Water Authority
SW 0.00
Withdrawals by Major Industrial Groups (Mgal/d):

SIC

GW

SW

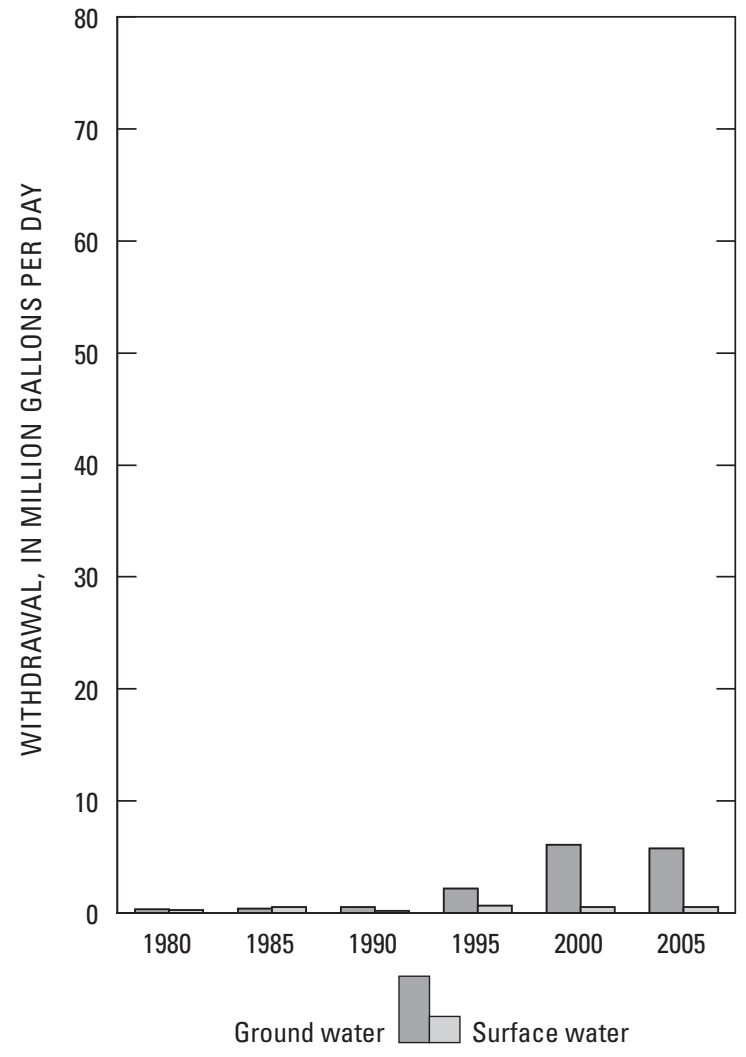

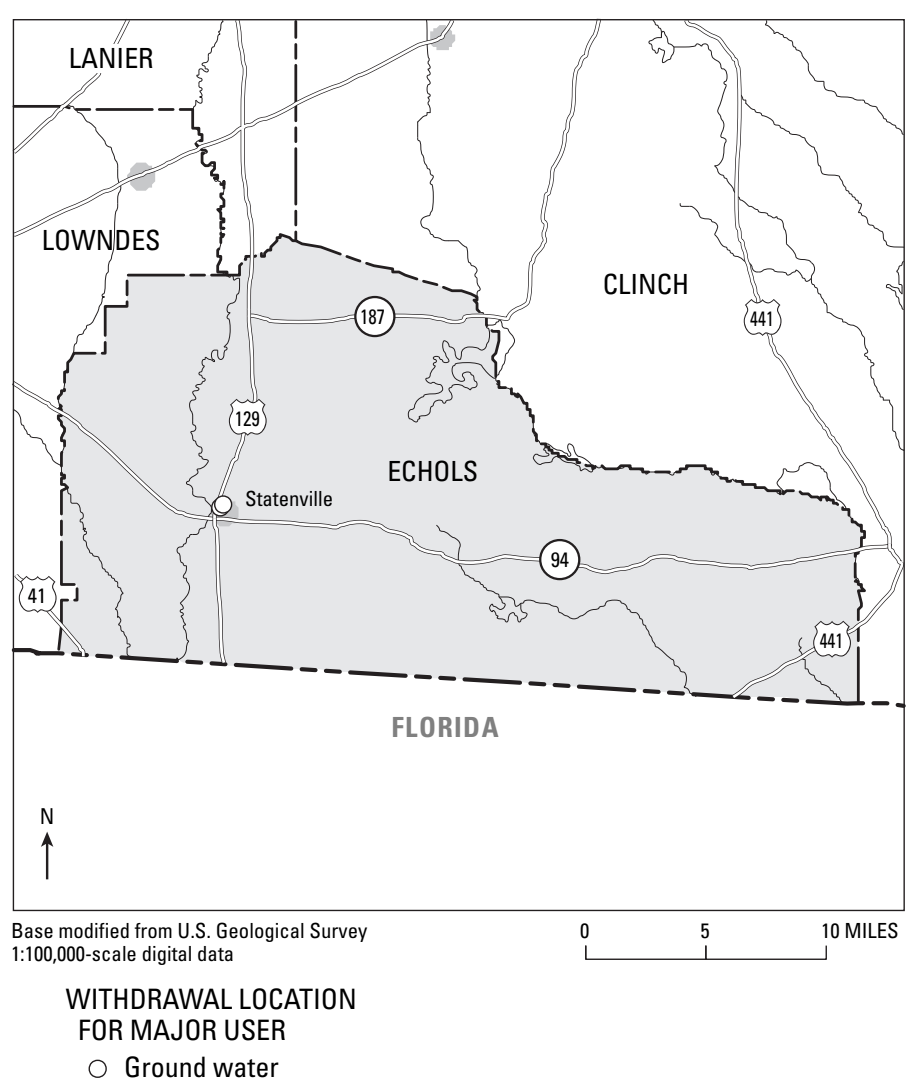




\section{EFFINGHAM COUNTY}

Population: 46,924

Population served by public supply: 25,840

Acres irrigated: 1,100

Hydroelectric use (Mgal/d): $\quad 0.00$

2005 WITHDRAWALS, IN MILLION GALLONS PER DAY

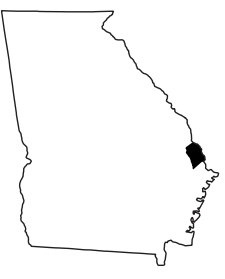

\begin{tabular}{lccccccrr}
\hline & $\begin{array}{c}\text { Public } \\
\text { Supply }\end{array}$ & $\begin{array}{c}\text { Domestic \& } \\
\text { Commercial }\end{array}$ & $\begin{array}{c}\text { Industrial \& } \\
\text { Mining }\end{array}$ & Irrigation & Livestock & \multicolumn{2}{c}{$\begin{array}{c}\text { Thermo- } \\
\text { electric }\end{array}$} & Totals \\
\hline Ground Water & 1.96 & 1.62 & 2.07 & 0.23 & 0.04 & 0.04 & 5.96 \\
Surface Water & 34.33 & 0.00 & 15.77 & 0.29 & 0.03 & 94.49 & 144.91 \\
TOTALS & 36.29 & 1.62 & 17.84 & 0.52 & 0.07 & 94.53 & 150.87 \\
\hline
\end{tabular}

Withdrawals by Major Public Suppliers (Mgal/d):

Name

Coastal Water \& Sewer Commission

City of Guyton

Town of Rincon

GW

0.13

SW

0.20

0.73

0.32

City of Springfield

Savannah Industrial and Domestic

0.00

0.00

0.00

0.00

34.33

Withdrawals by Major Industrial Groups (Mgal/d):

SIC GW SW

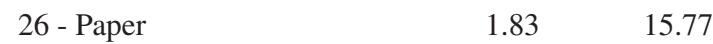

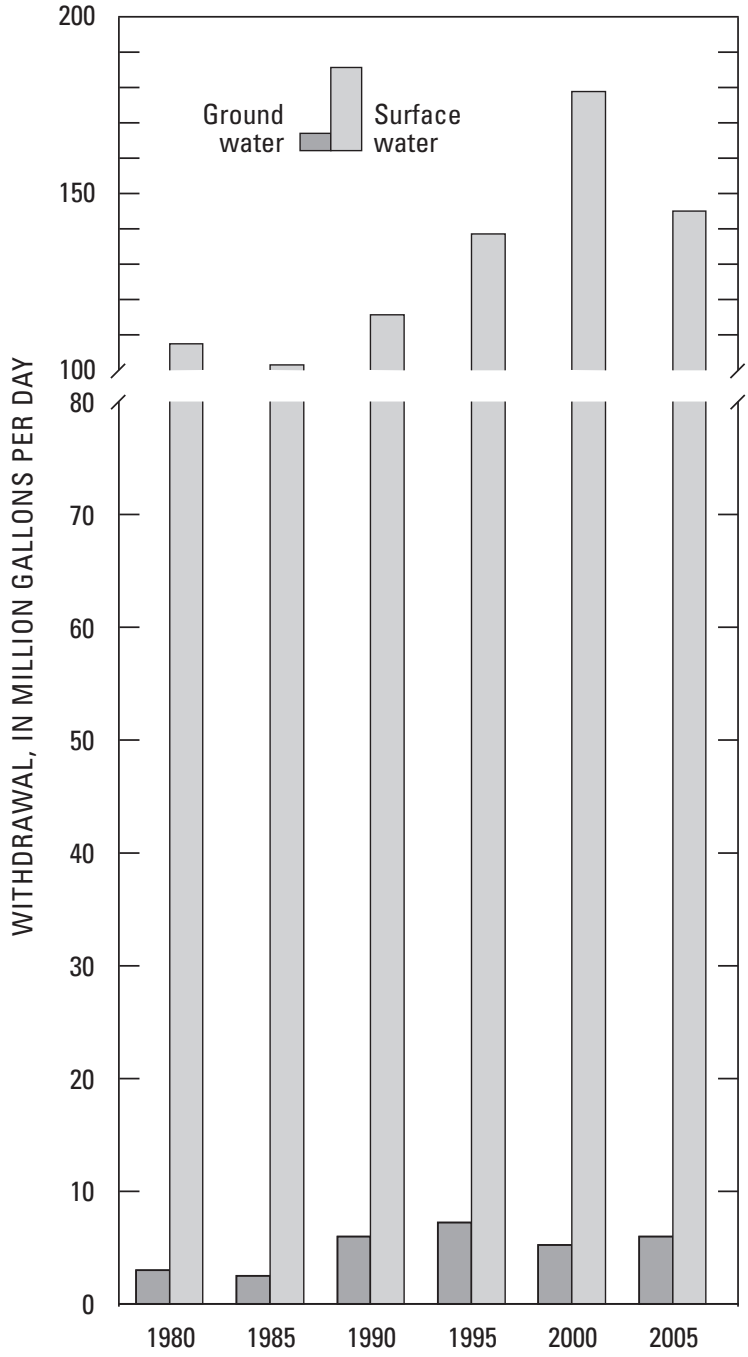

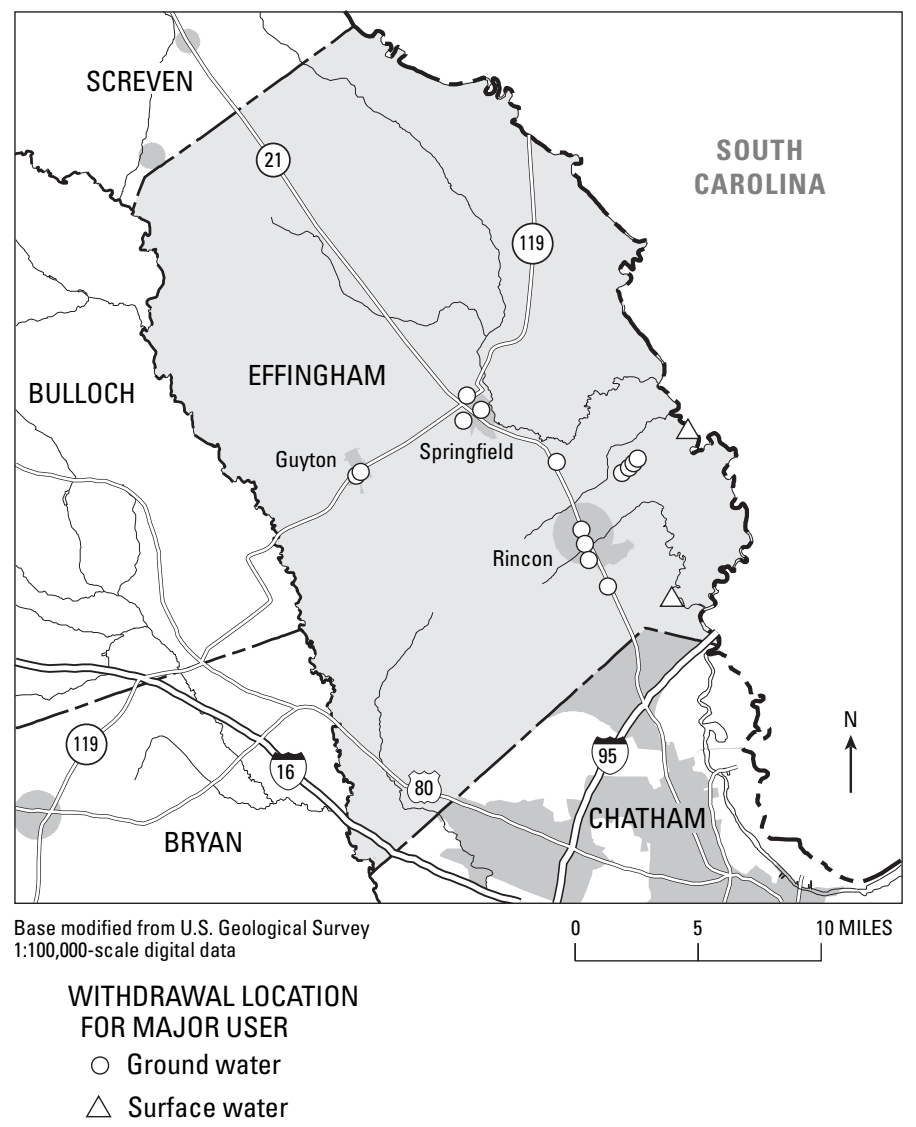




\section{ELBERT COUNTY}

Population: 20,799

Population served by public supply: 10,200

Acres irrigated: 550

Hydroelectric use (Mgal/d): $\quad 2,414.79$

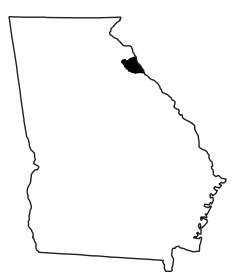

2005 WITHDRAWALS, IN MILLION GALLONS PER DAY

\begin{tabular}{|c|c|c|c|c|c|c|c|}
\hline & $\begin{array}{l}\text { Public } \\
\text { Supply }\end{array}$ & $\begin{array}{l}\text { Domestic \& } \\
\text { Commercial }\end{array}$ & $\begin{array}{c}\text { Industrial \& } \\
\text { Mining }\end{array}$ & Irrigation & Livestock & $\begin{array}{l}\text { Thermo- } \\
\text { electric }\end{array}$ & Totals \\
\hline Ground Water & 0.11 & 0.80 & 0.04 & 0.00 & 0.02 & 0.00 & 0.97 \\
\hline Surface Water & 1.73 & 0.00 & 0.00 & 0.45 & 0.25 & 0.00 & 2.43 \\
\hline TOTALS & 1.84 & 0.80 & 0.04 & 0.45 & 0.27 & 0.00 & 3.40 \\
\hline
\end{tabular}

Withdrawals by Major Public Suppliers (Mgal/d):

Name

City of Bowman

City of Elberton
GW

0.09

0.00
SW

0.00

1.73
Withdrawals by Major Industrial Groups (Mgal/d):

SIC

None
GW

SW
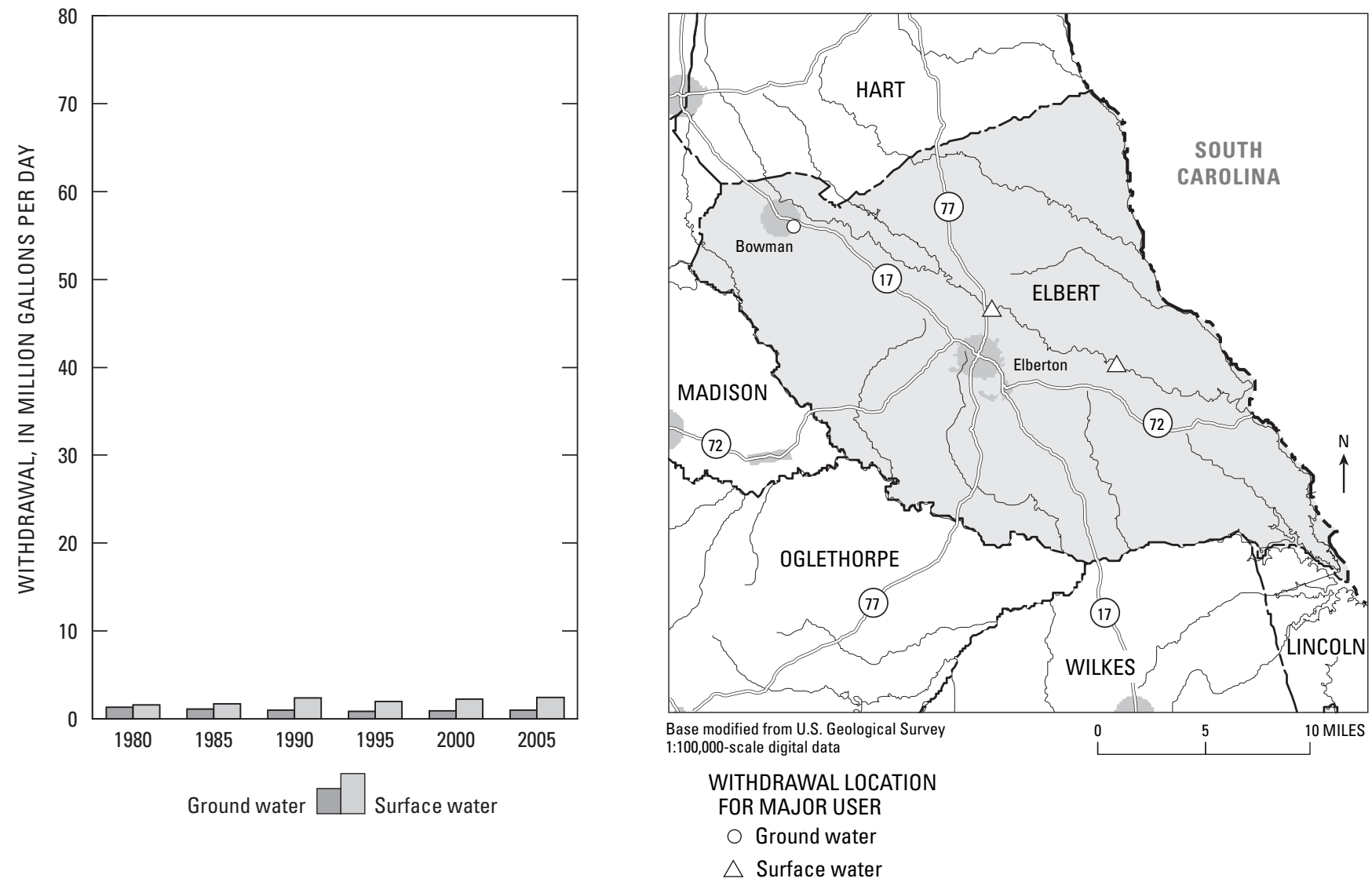


\section{EMANUEL COUNTY}

Population: 22,108

Population served by public supply: 11,990

Acres irrigated: $\quad 6,820$

Hydroelectric use (Mgal/d): $\quad 0.00$

2005 WITHDRAWALS, IN MILLION GALLONS PER DAY

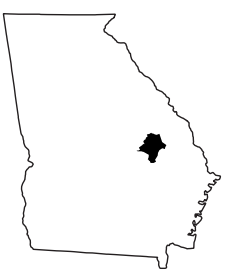

\begin{tabular}{lccccccc}
\hline & $\begin{array}{c}\text { Public } \\
\text { Supply }\end{array}$ & $\begin{array}{c}\text { Domestic \& } \\
\text { Commercial }\end{array}$ & $\begin{array}{c}\text { Industrial \& } \\
\text { Mining }\end{array}$ & Irrigation & Livestock & $\begin{array}{c}\text { Thermo- } \\
\text { electric }\end{array}$ & Totals \\
\hline Ground Water & 2.03 & 0.76 & 0.98 & 2.34 & 0.07 & 0.00 & 6.18 \\
Surface Water & 0.00 & 0.00 & 0.00 & 0.36 & 0.12 & 0.00 & 0.48 \\
TOTALS & 2.03 & 0.76 & 0.98 & 2.70 & 0.19 & 0.00 & 6.66 \\
\hline
\end{tabular}

Withdrawals by Major Public Suppliers (Mgal/d):

$\begin{array}{lcc}\text { Name } & \text { GW } & \text { SW } \\ \text { City of Adrian } & 0.05 & 0.00 \\ \text { Town of Garfield } & 0.03 & 0.00 \\ \text { Town of Nunez } & 0.01 & 0.00 \\ \text { Town of Oak Park } & 0.03 & 0.00 \\ \text { Town of Stillmore } & 0.05 & 0.00 \\ \text { Town of Summertown } & 0.02 & 0.00 \\ \text { City of Swainsboro } & 1.54 & 0.00 \\ \text { City of Twin City } & 0.27 & 0.00\end{array}$

Withdrawals by Major Industrial Groups (Mgal/d):

$\begin{array}{lcc}\text { SIC } & \text { GW } & \text { SW } \\ 20 \text { - Food } & 0.98 & 0.00\end{array}$
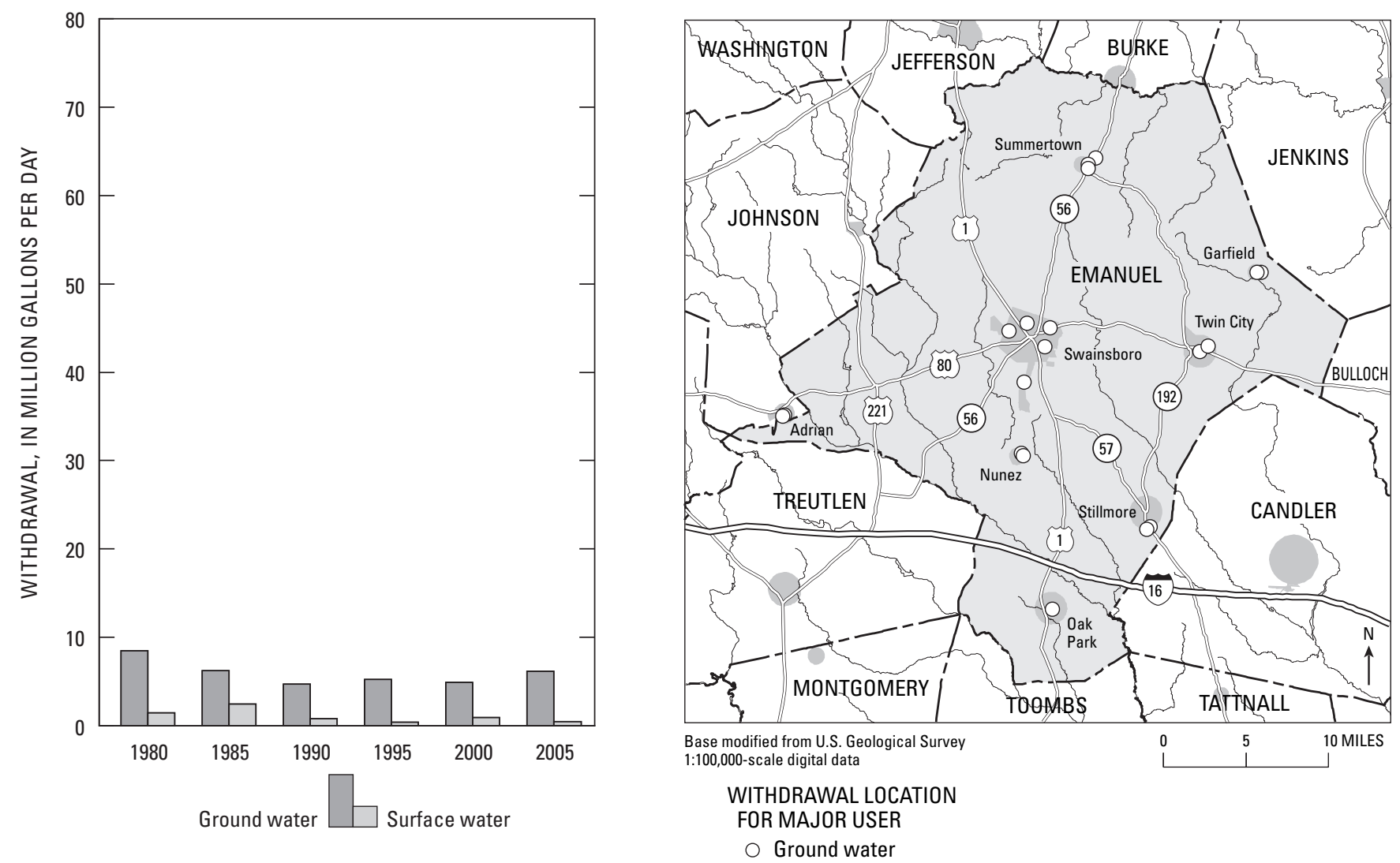


\section{EVANS COUNTY}

Population: 11,443

Population served by public supply: $\quad 5,880$

Acres irrigated: $\quad 4,890$

Hydroelectric use (Mgal/d): $\quad 0.00$

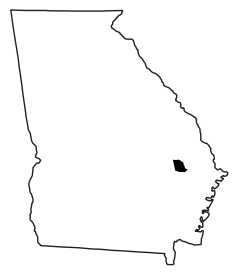

2005 WITHDRAWALS, IN MILLION GALLONS PER DAY

\begin{tabular}{|c|c|c|c|c|c|c|c|}
\hline & $\begin{array}{l}\text { Public } \\
\text { Supply }\end{array}$ & $\begin{array}{l}\text { Domestic \& } \\
\text { Commercial }\end{array}$ & $\begin{array}{c}\text { Industrial \& } \\
\text { Mining }\end{array}$ & Irrigation & Livestock & $\begin{array}{l}\text { Thermo- } \\
\text { electric }\end{array}$ & Totals \\
\hline Ground Water & 0.56 & 0.42 & 1.55 & 0.38 & 0.01 & 0.00 & 2.92 \\
\hline Surface Water & 0.00 & 0.00 & 0.00 & 1.20 & 0.12 & 0.00 & 1.32 \\
\hline TOTALS & 0.56 & 0.42 & 1.55 & 1.58 & 0.13 & 0.00 & 4.24 \\
\hline
\end{tabular}

Withdrawals by Major Public Suppliers (Mgal/d):

$\begin{array}{lcc}\text { Name } & \text { GW } & \text { SW } \\ \text { City of Bellville } & 0.03 & 0.00 \\ \text { City of Claxton } & 0.42 & 0.00 \\ \text { City of Daisy } & 0.02 & 0.00 \\ \text { City of Hagan } & 0.08 & 0.00\end{array}$

Withdrawals by Major Industrial Groups (Mgal/d):

$\begin{array}{lcc}\text { SIC } & \text { GW } & \text { SW } \\ 20 \text { - Food } & 1.55 & 0.00\end{array}$
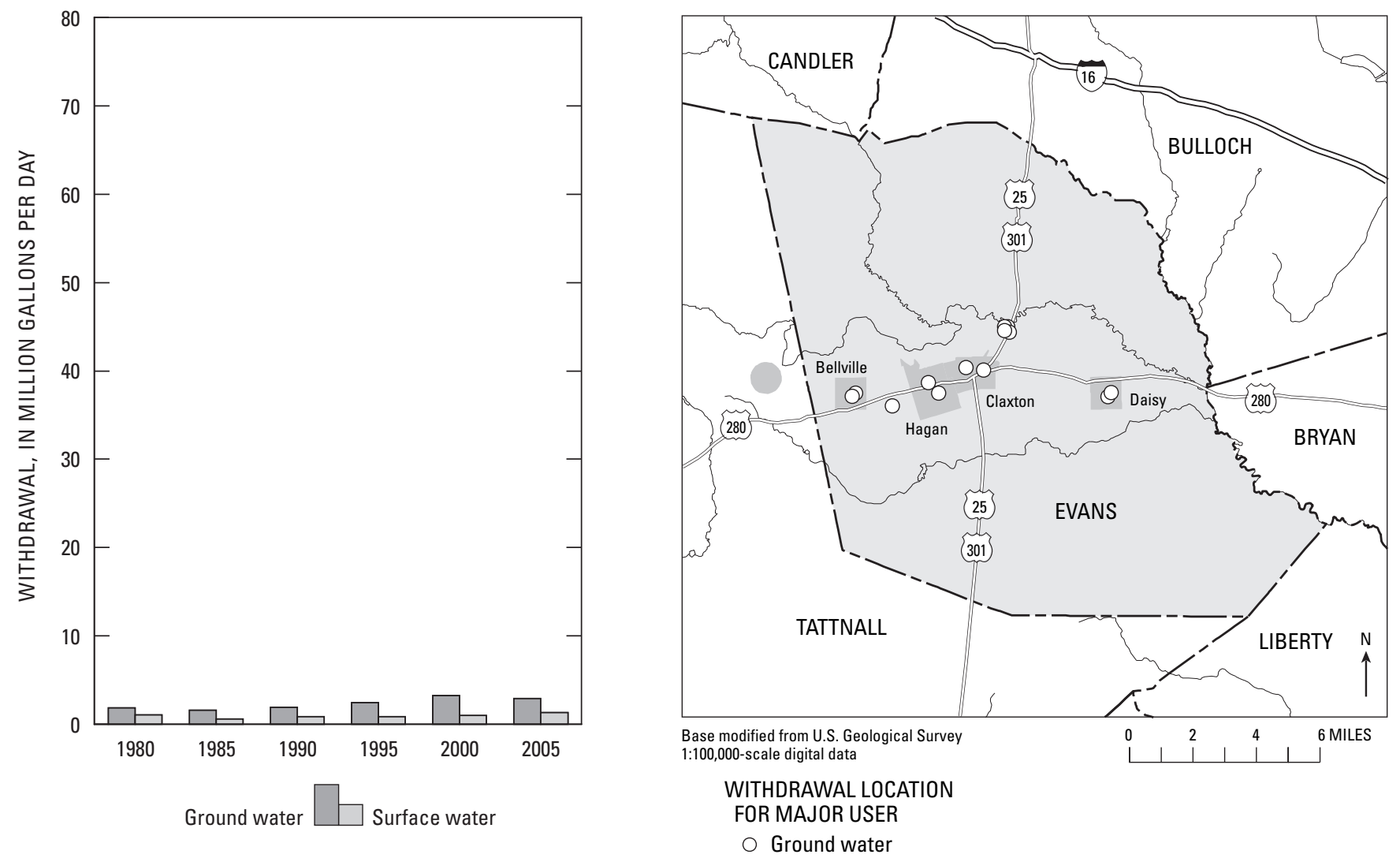


\section{FANNIN COUNTY}

Population: 21,887

Population served by public supply: 11,760

Acres irrigated: 210

Hydroelectric use (Mgal/d): 525.21

2005 WITHDRAWALS, IN MILLION GALLONS PER DAY

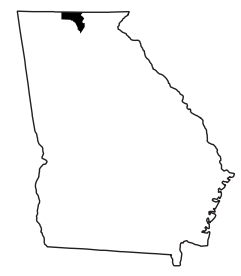

\begin{tabular}{lccccccc}
\hline & $\begin{array}{c}\text { Public } \\
\text { Supply }\end{array}$ & $\begin{array}{c}\text { Domestic \& } \\
\text { Commercial }\end{array}$ & $\begin{array}{c}\text { Industrial \& } \\
\text { Mining }\end{array}$ & Irrigation & Livestock & $\begin{array}{c}\text { Thermo- } \\
\text { electric }\end{array}$ & Totals \\
\hline Ground Water & 0.17 & 0.76 & 0.00 & 0.03 & 0.00 & 0.00 & 0.96 \\
Surface Water & 1.61 & 0.00 & 0.00 & 0.02 & 0.18 & 0.00 & 1.81 \\
TOTALS & 1.78 & 0.76 & 0.00 & 0.05 & 0.18 & 0.00 & 2.77 \\
\hline
\end{tabular}

Withdrawals by Major Public Suppliers (Mgal/d):

Name

City of Blue Ridge

City of McCaysville

Town of Mineral Bluff

Town of Morganton
GW SW

$0.00 \quad 0.84$

$0.00 \quad 0.76$

$0.05 \quad 0.00$

$0.11 \quad 0.00$
Withdrawals by Major Industrial Groups (Mgal/d):

SIC

None
GW

SW
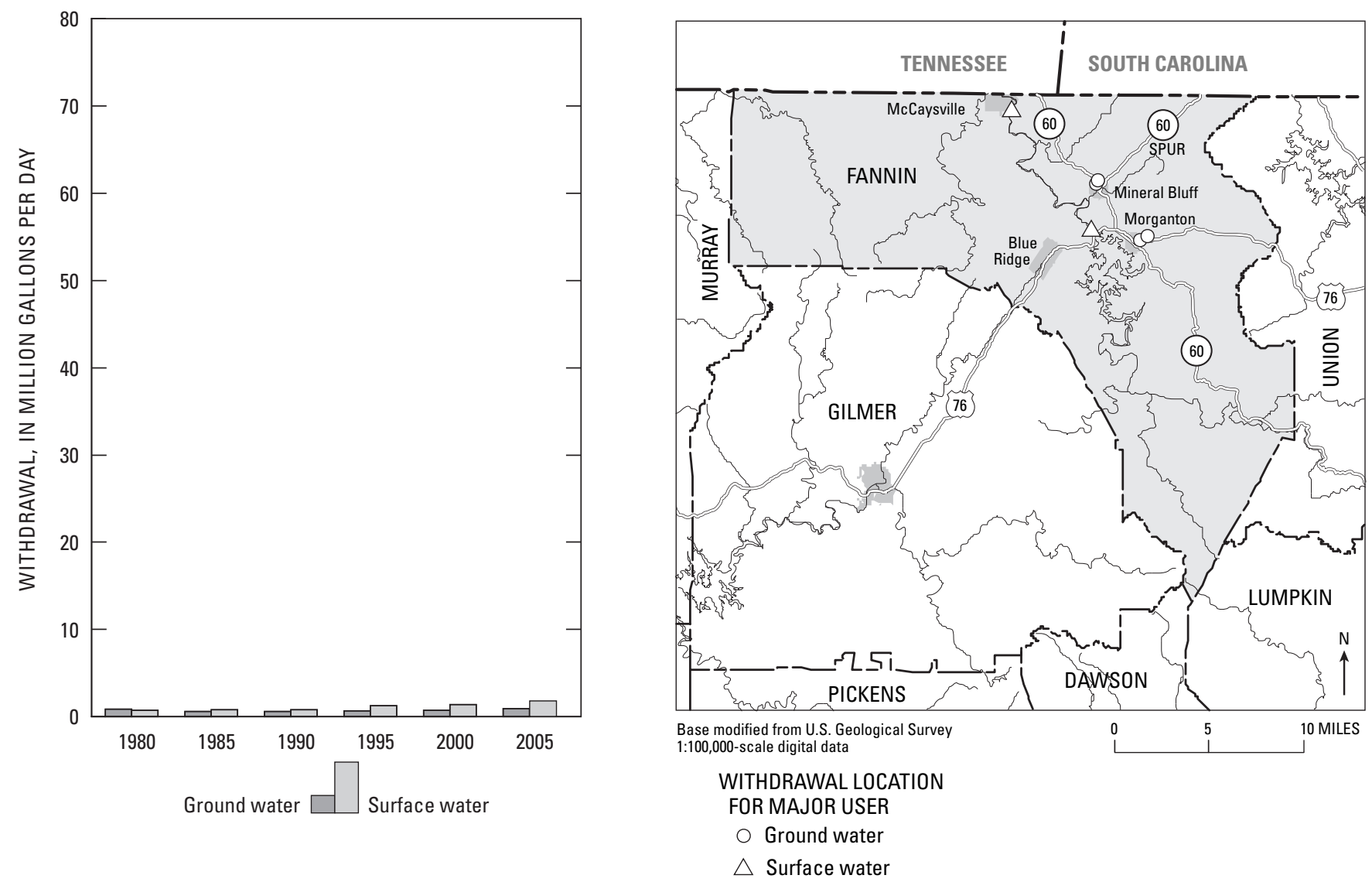


\section{FAYETTE COUNTY}

Population: 104,248

Population served by public supply: 81,100

Acres irrigated: 840

Hydroelectric use (Mgal/d): $\quad 0.00$

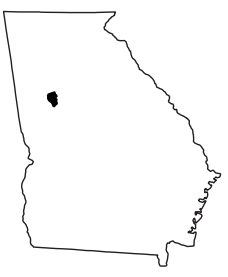

2005 WITHDRAWALS, IN MILLION GALLONS PER DAY

\begin{tabular}{|c|c|c|c|c|c|c|c|}
\hline & $\begin{array}{l}\text { Public } \\
\text { Supply }\end{array}$ & $\begin{array}{l}\text { Domestic \& } \\
\text { Commercial }\end{array}$ & $\begin{array}{c}\text { Industrial \& } \\
\text { Mining }\end{array}$ & Irrigation & Livestock & $\begin{array}{l}\text { Thermo- } \\
\text { electric }\end{array}$ & Totals \\
\hline Ground Water & 0.93 & 1.74 & 0.59 & 0.33 & 0.00 & 0.00 & 3.59 \\
\hline Surface Water & 10.19 & 0.00 & 0.01 & 0.96 & 0.04 & 0.00 & 11.20 \\
\hline TOTALS & 11.12 & 1.74 & 0.60 & 1.29 & 0.04 & 0.00 & 14.79 \\
\hline
\end{tabular}

Withdrawals by Major Public Suppliers (Mgal/d):

Name GW

Town of Brooks $\quad 0.02 \quad 0.00$

Fayette County Water System $\quad 0.12 \quad 8.41$

City of Fayetteville $\quad 0.61 \quad 1.27$

Board of Commission of $\quad 0.00 \quad 0.51$

Fayette County
Withdrawals by Major Industrial Groups (Mgal/d):

SIC

GW

SW
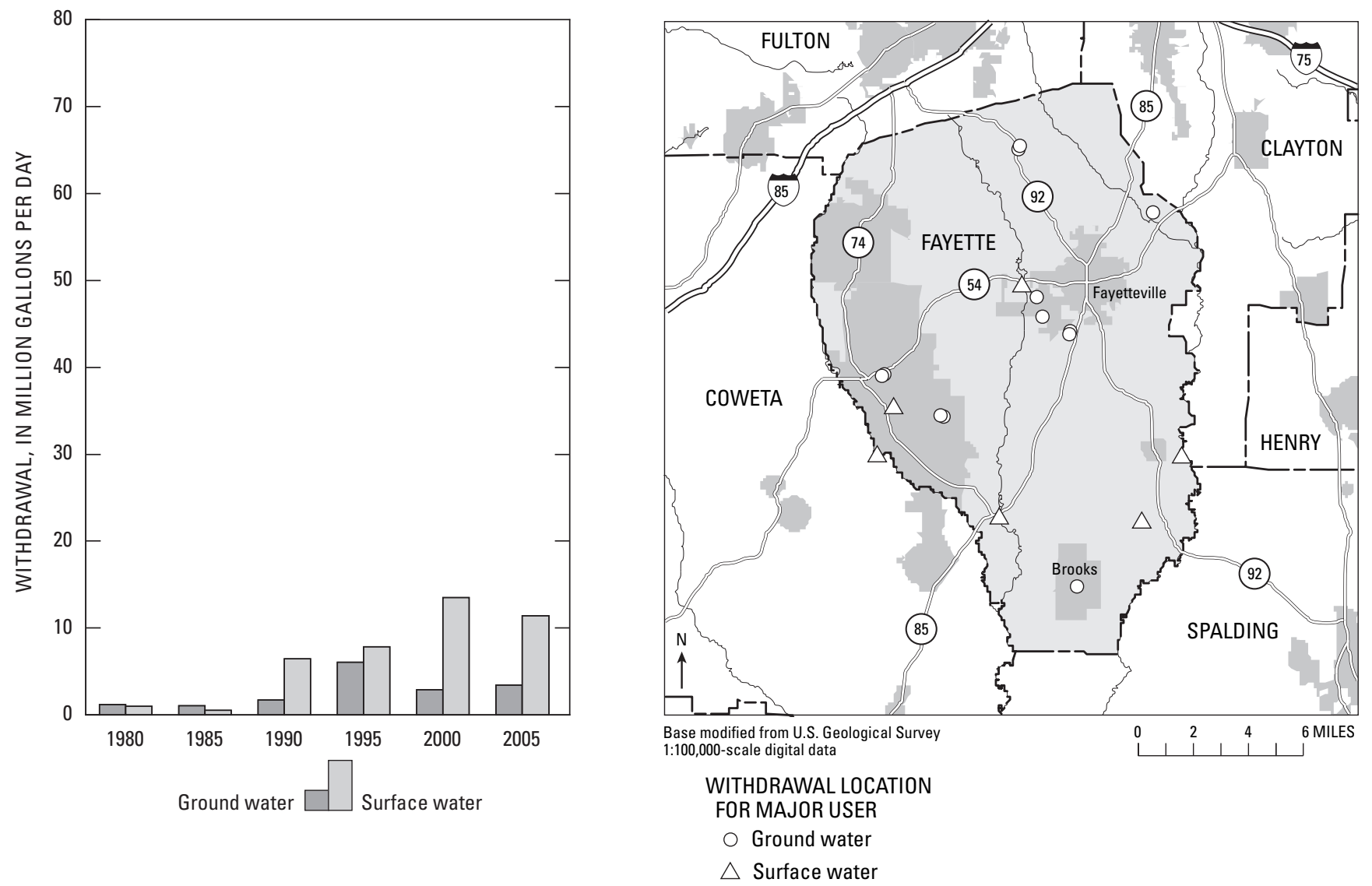


\section{FLOYD COUNTY}

Population: 94,198

Population served by public supply: 93,350

Acres irrigated: 2,230

Hydroelectric use (Mgal/d): $\quad 0.00$

2005 WITHDRAWALS, IN MILLION GALLONS PER DAY

\begin{tabular}{|c|c|c|c|c|c|c|c|}
\hline & $\begin{array}{l}\text { Public } \\
\text { Supply }\end{array}$ & $\begin{array}{l}\text { Domestic \& } \\
\text { Commercial }\end{array}$ & $\begin{array}{c}\text { Industrial \& } \\
\text { Mining }\end{array}$ & Irrigation & Livestock & $\begin{array}{l}\text { Thermo- } \\
\text { electric }\end{array}$ & Totals \\
\hline Ground Water & 0.84 & 0.17 & 1.12 & 1.51 & 0.02 & 0.00 & 3.66 \\
\hline Surface Water & 12.55 & 0.30 & 25.76 & 1.96 & 10.06 & 535.00 & 565.63 \\
\hline TOTALS & 13.39 & 0.47 & 26.88 & 3.47 & 10.08 & 535.00 & 589.29 \\
\hline
\end{tabular}

Withdrawals by Major Public Suppliers (Mgal/d):

\section{Name}

City of Cave Spring

Floyd County Water System

City of Rome
GW SW

$0.55 \quad 0.00$

$0.29 \quad 2.57$

$0.00 \quad 9.98$
Withdrawals by Major Industrial Groups (Mgal/d):

SIC GW SW

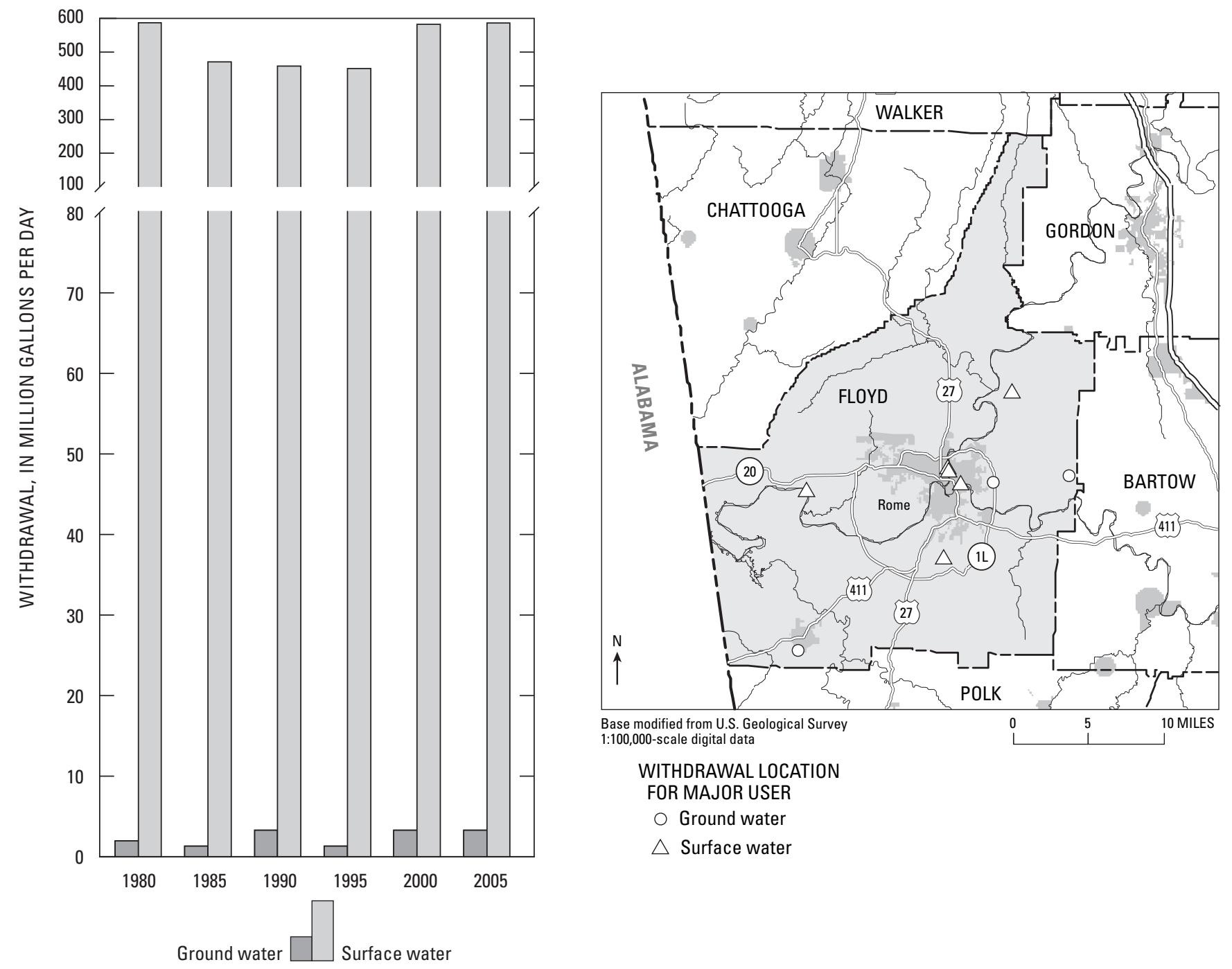




\section{FORSYTH COUNTY}

Population: 140,393

Population served by public supply: 98,400

Acres irrigated: 590

Hydroelectric use (Mgal/d): $\quad 1,343.36$

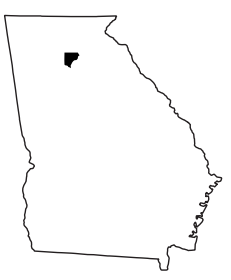

2005 WITHDRAWALS, IN MILLION GALLONS PER DAY

\begin{tabular}{|c|c|c|c|c|c|c|c|}
\hline & $\begin{array}{l}\text { Public } \\
\text { Supply }\end{array}$ & $\begin{array}{l}\text { Domestic \& } \\
\text { Commercial }\end{array}$ & $\begin{array}{c}\text { Industrial \& } \\
\text { Mining }\end{array}$ & Irrigation & Livestock & $\begin{array}{l}\text { Thermo- } \\
\text { electric }\end{array}$ & Totals \\
\hline Ground Water & 0.13 & 3.15 & 1.05 & 0.00 & 0.00 & 0.00 & 4.33 \\
\hline Surface Water & 17.00 & 0.00 & 0.10 & 0.24 & 6.85 & 0.00 & 24.19 \\
\hline TOTALS & 17.13 & 3.15 & 1.15 & 0.24 & 6.85 & 0.00 & 28.52 \\
\hline
\end{tabular}

Withdrawals by Major Public Suppliers (Mgal/d):

Name

GW SW

City of Cumming

$0.00 \quad 10.51$

Forsyth County Water Systen
Withdrawals by Major Industrial Groups (Mgal/d):

SIC

None
GW

SW

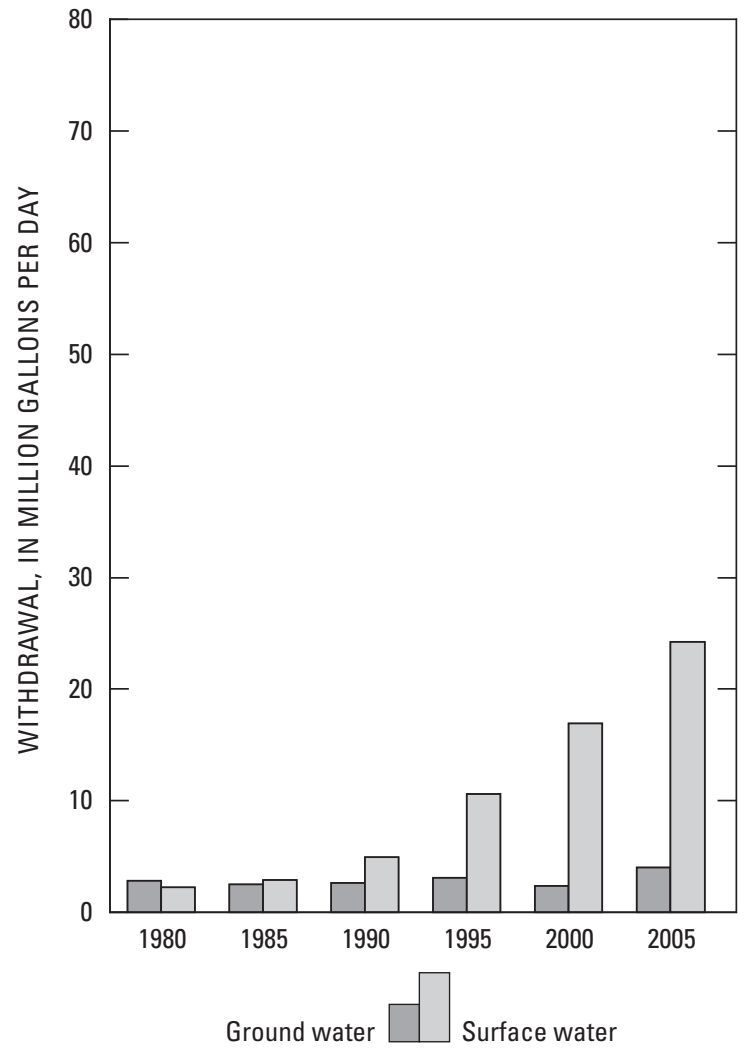

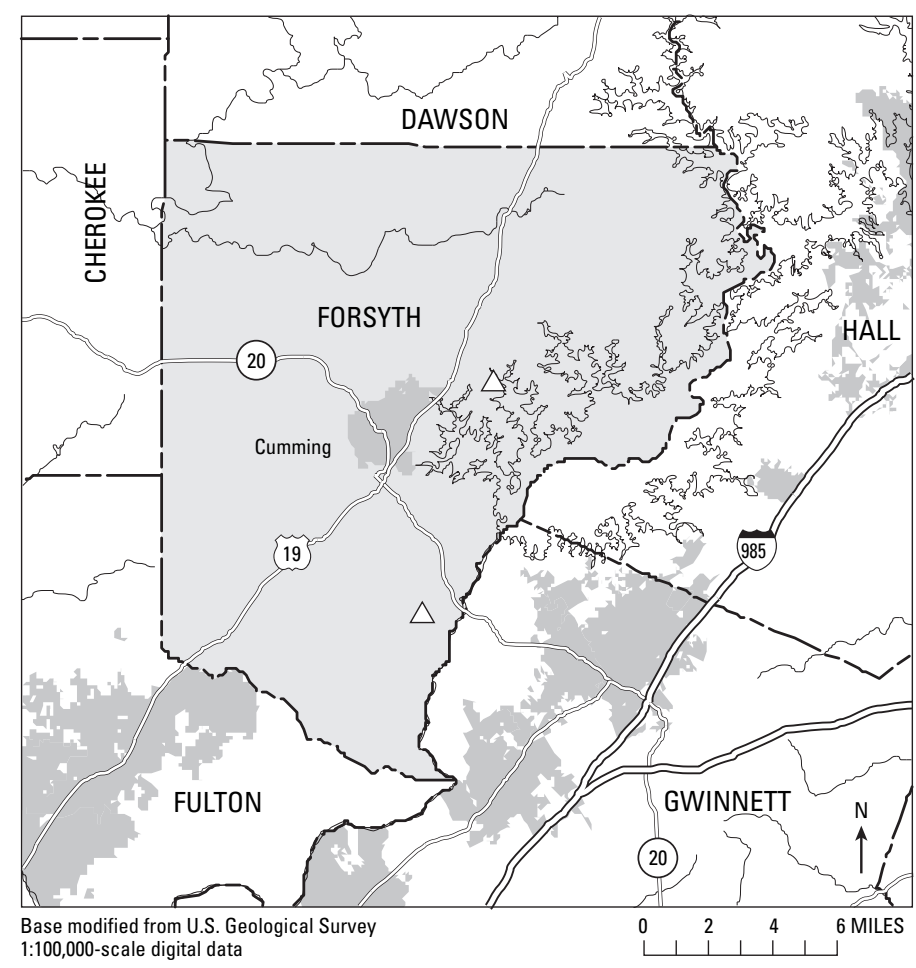

WITHDRAWAL LOCATION

FOR MAJOR USER

$\triangle$ Surface water 


\section{FRANKLIN COUNTY}

Population: 21,590

Population served by public supply: $\quad 10,490$

Acres irrigated: 400

Hydroelectric use (Mgal/d): $\quad 1,222.26$

2005 WITHDRAWALS, IN MILLION GALLONS PER DAY

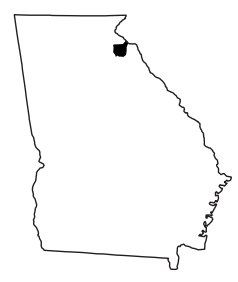

\begin{tabular}{|c|c|c|c|c|c|c|c|}
\hline & $\begin{array}{l}\text { Public } \\
\text { Supply }\end{array}$ & $\begin{array}{l}\text { Domestic \& } \\
\text { Commercial }\end{array}$ & $\begin{array}{c}\text { Industrial \& } \\
\text { Mining }\end{array}$ & Irrigation & Livestock & $\begin{array}{l}\text { Thermo- } \\
\text { electric }\end{array}$ & Totals \\
\hline Ground Water & 0.21 & 0.83 & 0.00 & 0.00 & 0.02 & 0.00 & 1.06 \\
\hline Surface Water & 1.72 & 0.00 & 0.00 & 0.26 & 1.12 & 0.00 & 3.10 \\
\hline TOTALS & 1.93 & 0.83 & 0.00 & 0.26 & 1.14 & 0.00 & 4.16 \\
\hline
\end{tabular}

Withdrawals by Major Public Suppliers (Mgal/d):

Name

City of Canon

City of Carnesville

City of Franklin Springs

City of Lavonia

City of Royston

GW

0.07

0.04

0.10

0.00

0.00
SW

0.00

0.00

0.00

1.41

0.31
Withdrawals by Major Industrial Groups (Mgal/d):

SIC

GW

SW
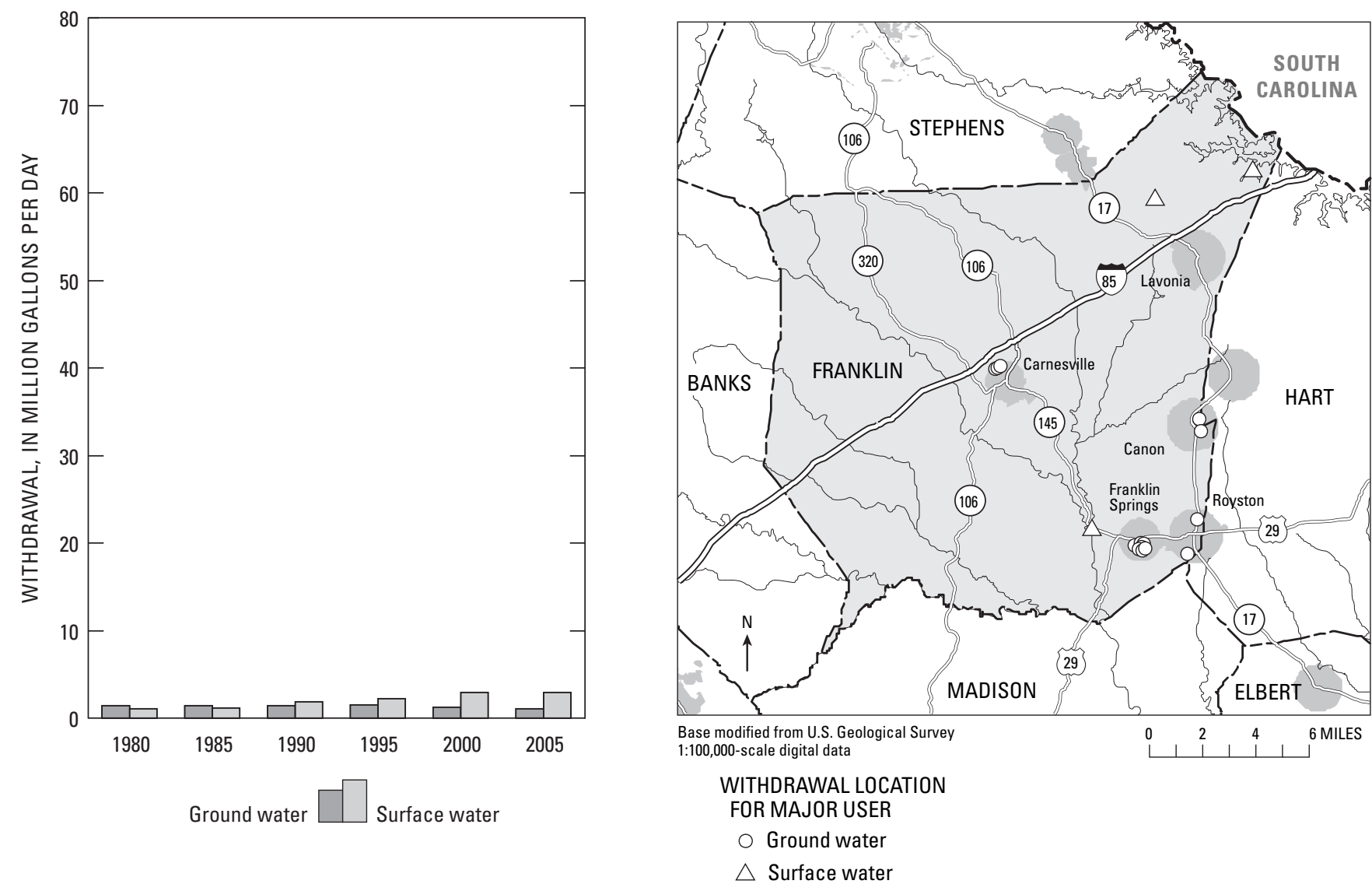


\section{FULTON COUNTY}

Population: 915,623

Population served by public supply: 899,140

Acres irrigated: 2,970

Hydroelectric use (Mgal/d): 0.00

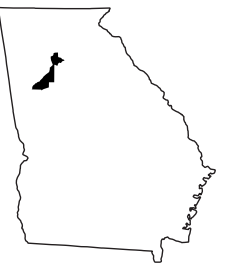

2005 WITHDRAWALS, IN MILLION GALLONS PER DAY

\begin{tabular}{|c|c|c|c|c|c|c|c|}
\hline & $\begin{array}{l}\text { Public } \\
\text { Supply }\end{array}$ & $\begin{array}{l}\text { Domestic \& } \\
\text { Commercial }\end{array}$ & $\begin{array}{c}\text { Industrial \& } \\
\text { Mining }\end{array}$ & Irrigation & Livestock & $\begin{array}{l}\text { Thermo- } \\
\text { electric }\end{array}$ & Totals \\
\hline Ground Water & 0.00 & 1.24 & 1.22 & 0.00 & 0.01 & 0.00 & 2.47 \\
\hline Surface Water & 154.29 & 0.40 & 0.12 & 4.41 & 0.05 & 0.00 & 159.27 \\
\hline TOTALS & 154.29 & 1.64 & 1.34 & 4.41 & 0.06 & 0.00 & 161.74 \\
\hline
\end{tabular}

Withdrawals by Major Public Suppliers (Mgal/d):

Name

Atlanta-Fulton County Water

GW SW

Resources Comm

City of Atlanta

$0.00 \quad 42.80$

$0.00 \quad 100.47$

City of East Point (water source

located in Douglas County)

City of Roswell

0.00

0.00

1.19
Withdrawals by Major Industrial Groups (Mgal/d):

SIC

GW

SW
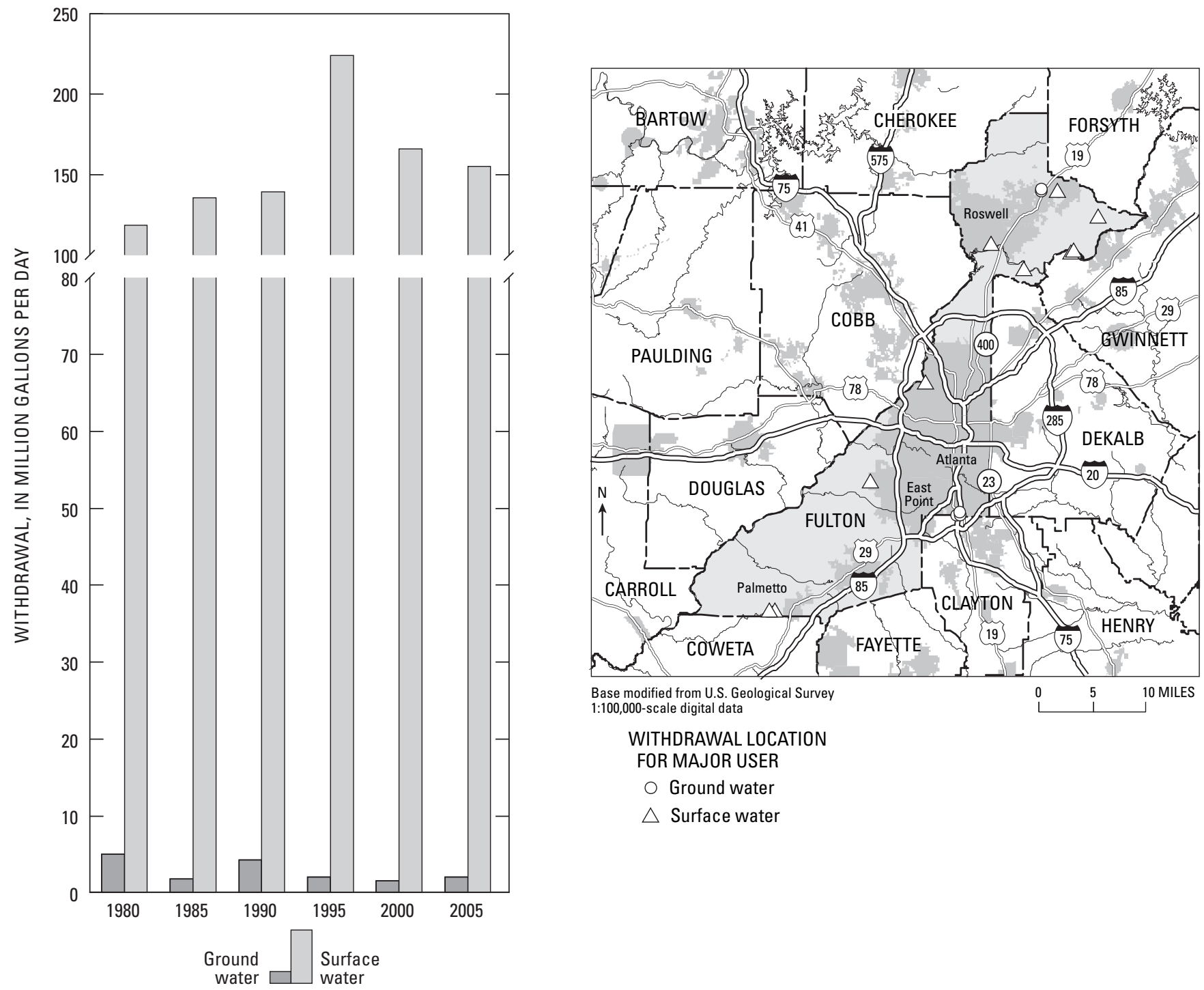


\section{GILMER COUNTY}

Population: 27,335

Population served by public supply: 12,210

Acres irrigated: 120

Hydroelectric use (Mgal/d): 0.00

2005 WITHDRAWALS, IN MILLION GALLONS PER DAY

\begin{tabular}{|c|c|c|c|c|c|c|c|}
\hline & $\begin{array}{l}\text { Public } \\
\text { Supply }\end{array}$ & $\begin{array}{l}\text { Domestic \& } \\
\text { Commercial }\end{array}$ & $\begin{array}{c}\text { Industrial \& } \\
\text { Mining }\end{array}$ & Irrigation & Livestock & $\begin{array}{l}\text { Thermo- } \\
\text { electric }\end{array}$ & Totals \\
\hline Ground Water & 0.10 & 1.13 & 5.78 & 0.00 & 0.00 & 0.00 & 7.01 \\
\hline Surface Water & 3.12 & 0.00 & 0.00 & 0.17 & 0.64 & 0.00 & 3.93 \\
\hline TOTALS & 3.22 & 1.13 & 5.78 & 0.17 & 0.64 & 0.00 & 10.94 \\
\hline
\end{tabular}

Withdrawals by Major Public Suppliers (Mgal/d):

\section{Name}

East Ellijay

Ellijay-Gilmer County

Water System

$\begin{array}{cc}\text { GW } & \text { SW } \\ 0.05 & 0.00 \\ 0.00 & 3.12\end{array}$

Withdrawals by Major Industrial Groups (Mgal/d):

SIC

32 - Stone, clay

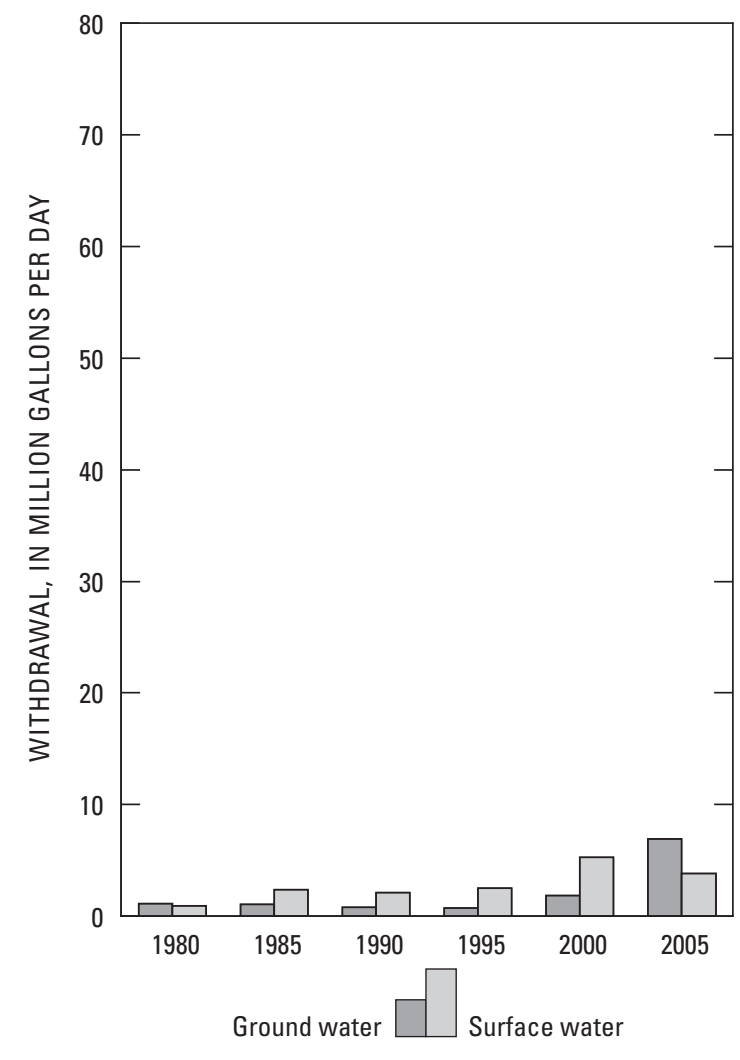

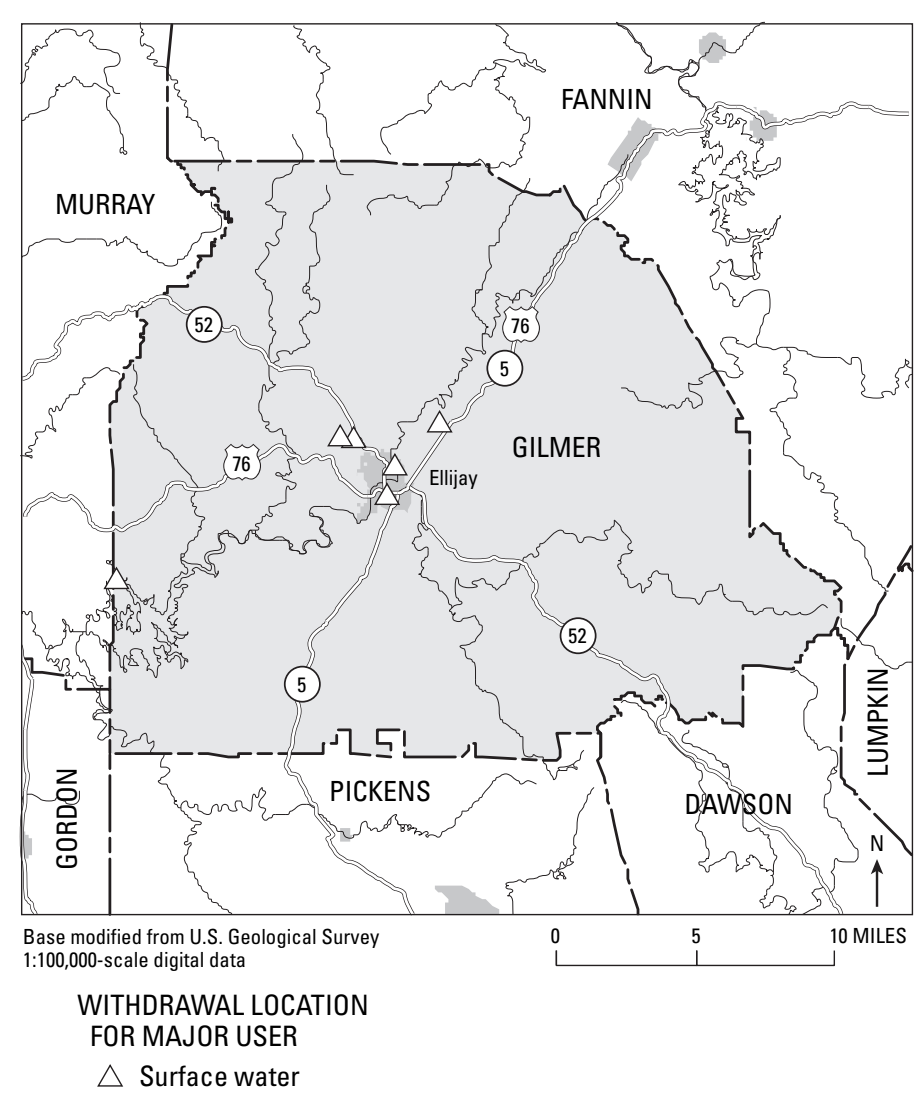




\section{GLASCOCK COUNTY}

Population: 2,705

Population served by public supply: $\quad 1,090$

Acres irrigated: 110

Hydroelectric use (Mgal/d): $\quad 0.00$

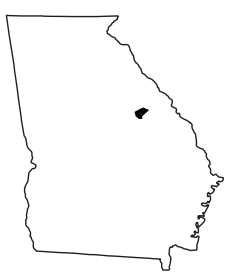

2005 WITHDRAWALS, IN MILLION GALLONS PER DAY

\begin{tabular}{|c|c|c|c|c|c|c|c|}
\hline & $\begin{array}{l}\text { Public } \\
\text { Supply }\end{array}$ & $\begin{array}{l}\text { Domestic \& } \\
\text { Commercial }\end{array}$ & $\begin{array}{c}\text { Industrial \& } \\
\text { Mining }\end{array}$ & Irrigation & Livestock & $\begin{array}{l}\text { Thermo- } \\
\text { electric }\end{array}$ & Totals \\
\hline Ground Water & 0.08 & 0.12 & 0.05 & 0.00 & 0.01 & 0.00 & 0.26 \\
\hline Surface Water & 0.00 & 0.00 & 0.00 & 0.04 & 0.03 & 0.00 & 0.07 \\
\hline TOTALS & 0.08 & 0.12 & 0.05 & 0.04 & 0.04 & 0.00 & 0.33 \\
\hline
\end{tabular}

Withdrawals by Major Public Suppliers (Mgal/d):

Name

GW SW

City of Gibson

Town of Mitchell

$0.06 \quad 0.00$

$0.02 \quad 0.00$

Withdrawals by Major Industrial Groups (Mgal/d):

32 - Stone, clay
GW

0.05
SW 0.00
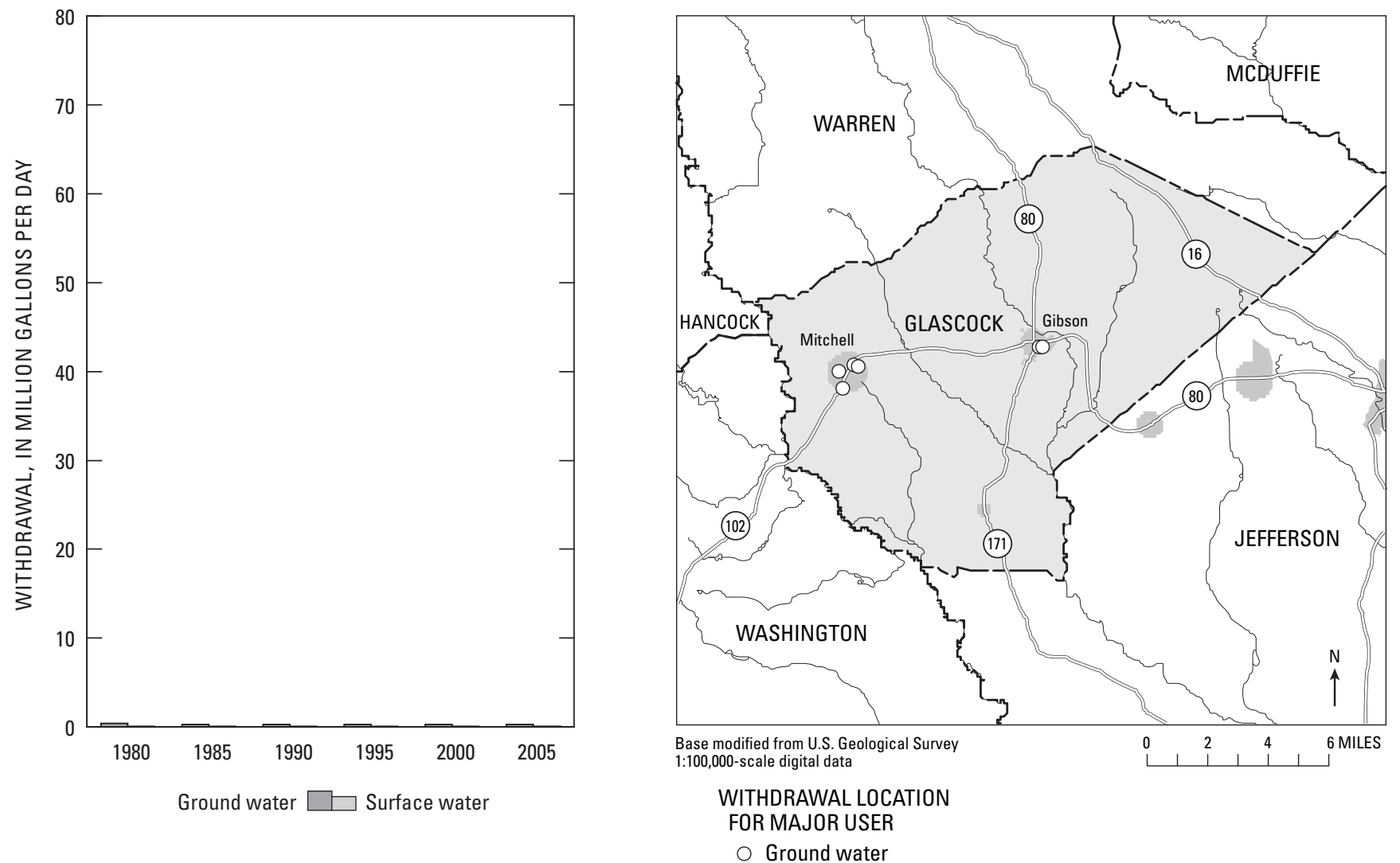


\section{GLYNN COUNTY}

Population: 71,874

Population served by public supply: 62,600

Acres irrigated: 1,290

Hydroelectric use (Mgal/d): $\quad 0.00$

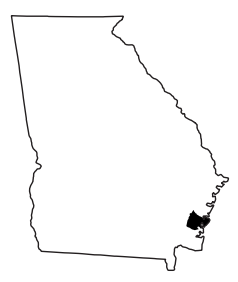

2005 WITHDRAWALS, IN MILLION GALLONS PER DAY

\begin{tabular}{|c|c|c|c|c|c|c|c|}
\hline & $\begin{array}{l}\text { Public } \\
\text { Supply }\end{array}$ & $\begin{array}{l}\text { Domestic \& } \\
\text { Commercial }\end{array}$ & $\begin{array}{c}\text { Industrial \& } \\
\text { Mining }\end{array}$ & Irrigation & Livestock & $\begin{array}{l}\text { Thermo- } \\
\text { electric }\end{array}$ & Totals \\
\hline Ground Water & 9.85 & 4.37 & 41.93 & 0.00 & 0.00 & 0.02 & 56.17 \\
\hline Surface Water & 0.00 & 0.00 & 22.58 & 0.00 & 0.04 & 36.94 & 59.56 \\
\hline TOTALS & 9.85 & 4.37 & 64.51 & 0.00 & 0.04 & 36.96 & 115.73 \\
\hline
\end{tabular}

Withdrawals by Major Public Suppliers (Mgal/d):

\section{Name}

City of Brunswick

Jekyll Island Water Authority

Glynn County Board of Commissioners-

St. Simons Island W\&S

Sea Island Services

\section{$\mathrm{GW}$}

4.57

1.06

2.76

0.88
SW

0.00

0.00

0.00

0.00
Withdrawals by Major Industrial Groups (Mgal/d):

SIC

20 - Food

GW SW

$0.34 \quad 0.00$

26 - Paper

$33.11 \quad 22.58$

28 - Chemicals

8.28

0.00

32 - Stone, clay

0.17

0.00
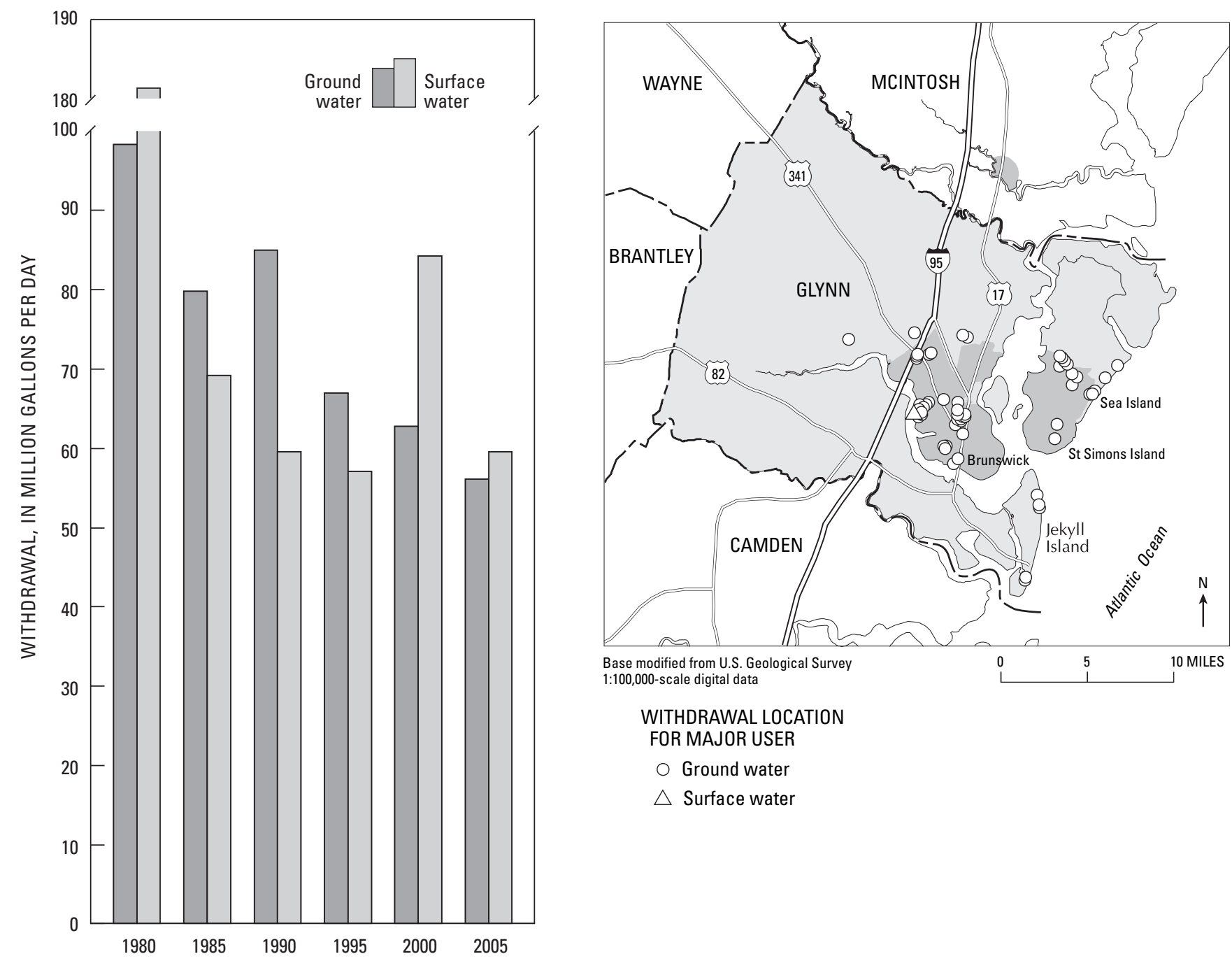


\section{GORDON COUNTY}

Population: 50,279

Population served by public supply: $\quad 37,940$

Acres irrigated: 2,020

Hydroelectric use (Mgal/d): $\quad 0.00$

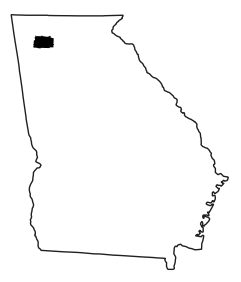

2005 WITHDRAWALS, IN MILLION GALLONS PER DAY

\begin{tabular}{|c|c|c|c|c|c|c|c|}
\hline & $\begin{array}{l}\text { Public } \\
\text { Supply }\end{array}$ & $\begin{array}{l}\text { Domestic \& } \\
\text { Commercial }\end{array}$ & $\begin{array}{c}\text { Industrial \& } \\
\text { Mining }\end{array}$ & Irrigation & Livestock & $\begin{array}{l}\text { Thermo- } \\
\text { electric }\end{array}$ & Totals \\
\hline Ground Water & 3.55 & 0.93 & 0.09 & 0.00 & 0.02 & 0.00 & 4.59 \\
\hline Surface Water & 9.16 & 0.00 & 0.00 & 0.73 & 0.63 & 0.00 & 10.52 \\
\hline TOTALS & 12.71 & 0.93 & 0.09 & 0.73 & 0.65 & 0.00 & 15.11 \\
\hline
\end{tabular}

Withdrawals by Major Public Suppliers (Mgal/d):

Name

City of Calhoun

City of Fairmount

$\mathrm{GW}$

3.55

0.00

SW

9.10

0.06
Withdrawals by Major Industrial Groups (Mgal/d):

SIC GW SW

22 - Textiles

0.09

0.00
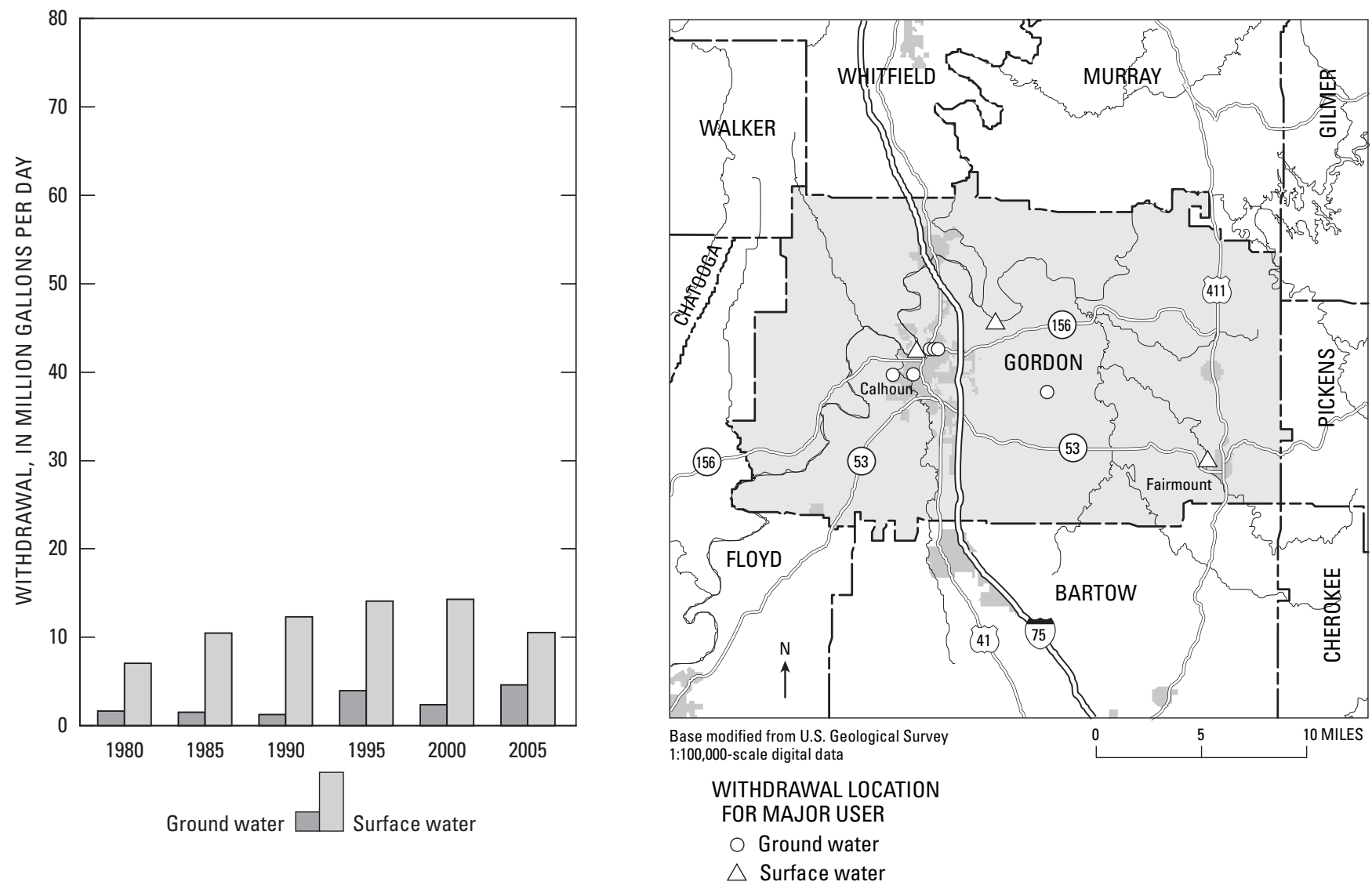


\section{GRADY COUNTY}

Population: 24,466

Population served by public supply: 12,970

Acres irrigated: 15,130

Hydroelectric use (Mgal/d): $\quad 0.00$

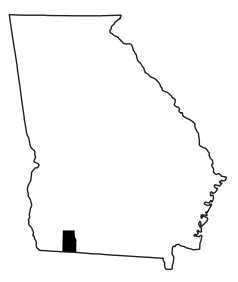

2005 WITHDRAWALS, IN MILLION GALLONS PER DAY

\begin{tabular}{|c|c|c|c|c|c|c|c|}
\hline & $\begin{array}{l}\text { Public } \\
\text { Supply }\end{array}$ & $\begin{array}{l}\text { Domestic \& } \\
\text { Commercial }\end{array}$ & $\begin{array}{c}\text { Industrial \& } \\
\text { Mining }\end{array}$ & Irrigation & Livestock & $\begin{array}{l}\text { Thermo- } \\
\text { electric }\end{array}$ & Totals \\
\hline Ground Water & 1.85 & 0.86 & 0.35 & 2.78 & 0.04 & 0.00 & 5.88 \\
\hline Surface Water & 0.00 & 0.00 & 0.00 & 4.30 & 0.18 & 0.00 & 4.48 \\
\hline TOTALS & 1.85 & 0.86 & 0.35 & 7.08 & 0.22 & 0.00 & 10.36 \\
\hline
\end{tabular}

Withdrawals by Major Public Suppliers (Mgal/d):

Name

GW

City of Cairo

1.70

SW

City of Whigham

0.08

Withdrawals by Major Industrial Groups (Mgal/d):

SIC

GW

SW

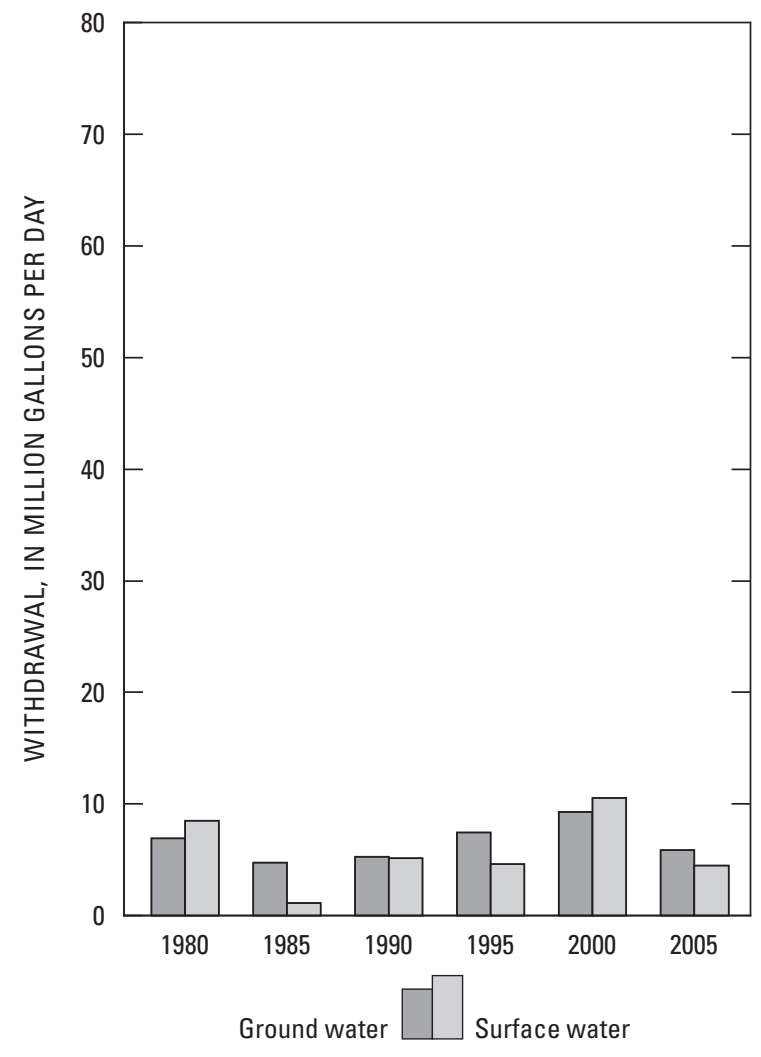

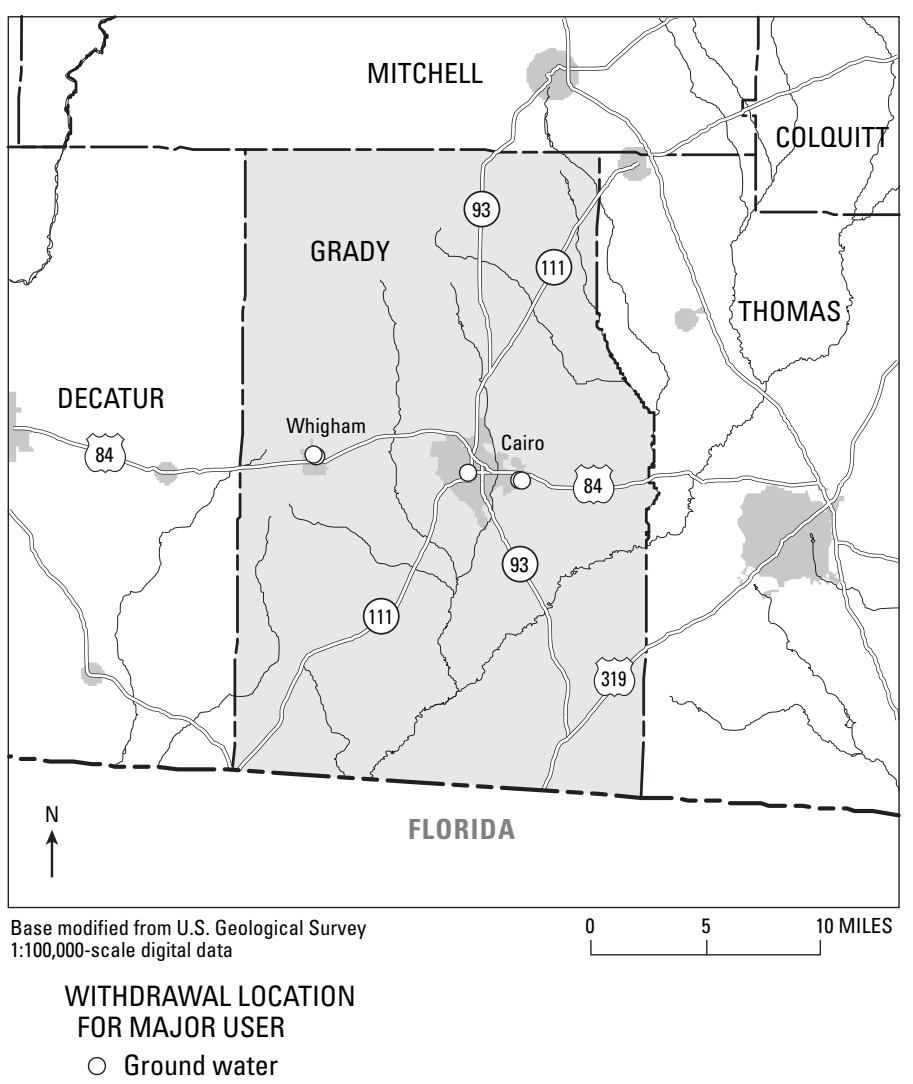




\section{GREENE COUNTY}

Population: 15,693

Population served by public supply: 11,870

Acres irrigated: 860

Hydroelectric use (Mgal/d): $\quad 0.00$

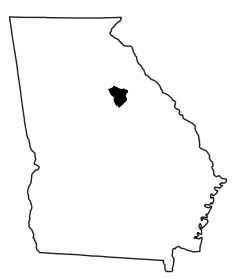

2005 WITHDRAWALS, IN MILLION GALLONS PER DAY

\begin{tabular}{|c|c|c|c|c|c|c|c|}
\hline & $\begin{array}{l}\text { Public } \\
\text { Supply }\end{array}$ & $\begin{array}{l}\text { Domestic \& } \\
\text { Commercial }\end{array}$ & $\begin{array}{c}\text { Industrial \& } \\
\text { Mining }\end{array}$ & Irrigation & Livestock & $\begin{array}{l}\text { Thermo- } \\
\text { electric }\end{array}$ & Totals \\
\hline Ground Water & 0.55 & 0.29 & 0.11 & 0.00 & 0.00 & 0.00 & 0.95 \\
\hline Surface Water & 0.90 & 0.00 & 0.05 & 1.74 & 0.27 & 0.00 & 2.96 \\
\hline TOTALS & 1.45 & 0.29 & 0.16 & 1.74 & 0.27 & 0.00 & 3.91 \\
\hline
\end{tabular}

Withdrawals by Major Public Suppliers (Mgal/d):

Name

GW SW

City of Greensboro

0.00

0.74

Town of Siloam

0.03

City of Union Point

Town of White Plains

City of Woodville

Piedmont Water Resources

Withdrawals by Major Industrial Groups (Mgal/d):

32 - Stone, clay
GW

0.00
SW 0.05
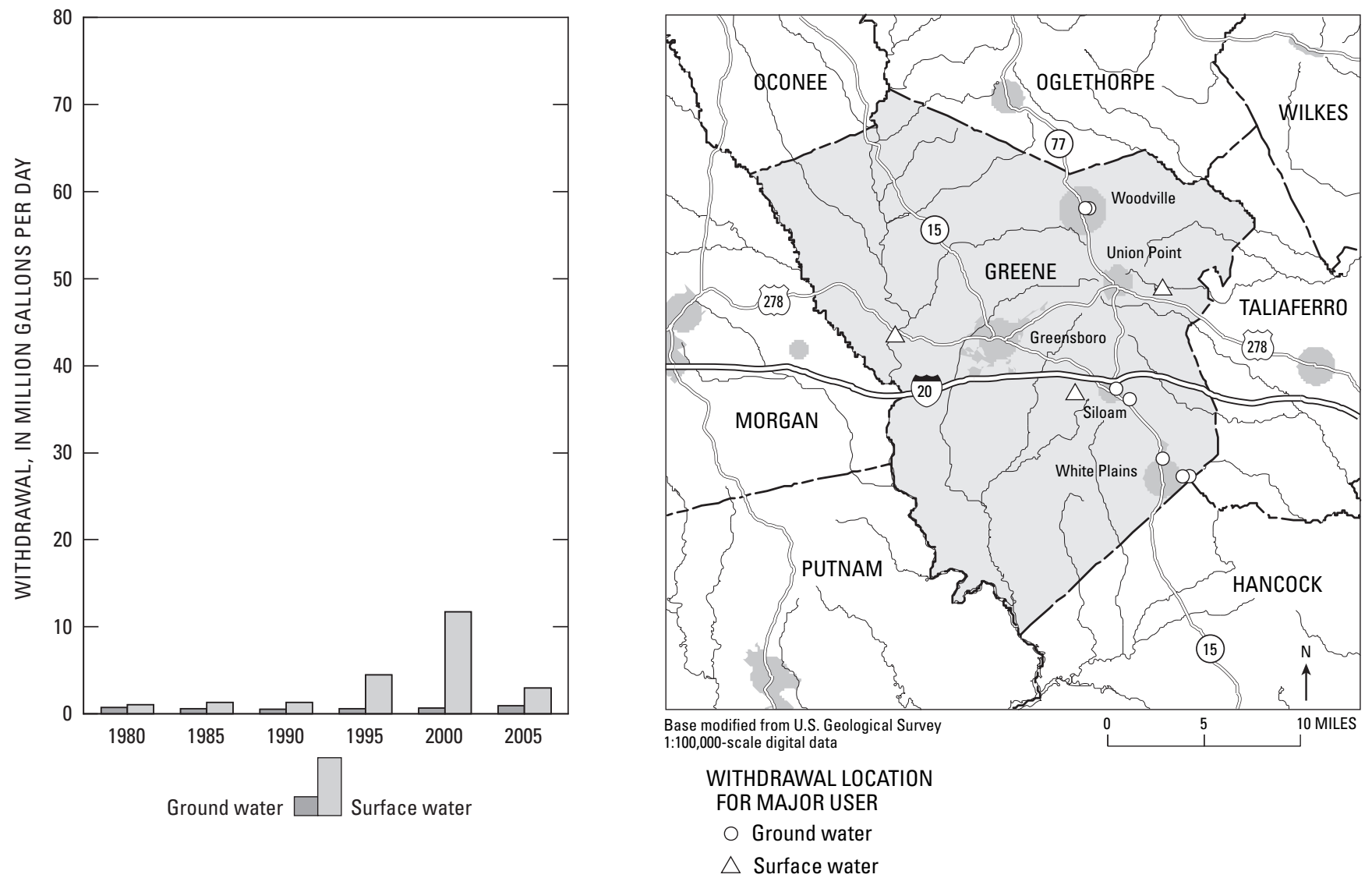


\section{GWINNETT COUNTY}

Population: 726,273

Population served by public supply: $\quad 726,270$

Acres irrigated: 3,020

Hydroelectric use (Mgal/d): $\quad 0.00$

2005 WITHDRAWALS, IN MILLION GALLONS PER DAY

\begin{tabular}{|c|c|c|c|c|c|c|c|}
\hline & $\begin{array}{l}\text { Public } \\
\text { Supply }\end{array}$ & $\begin{array}{l}\text { Domestic \& } \\
\text { Commercial }\end{array}$ & $\begin{array}{c}\text { Industrial \& } \\
\text { Mining }\end{array}$ & Irrigation & Livestock & $\begin{array}{l}\text { Thermo- } \\
\text { electric }\end{array}$ & Totals \\
\hline Ground Water & 0.38 & 0.00 & 1.19 & 1.95 & 0.01 & 0.00 & 3.53 \\
\hline Surface Water & 87.52 & 0.00 & 0.02 & 3.09 & 0.04 & 0.00 & 90.67 \\
\hline TOTALS & 87.90 & 0.00 & 1.21 & 5.04 & 0.05 & 0.00 & 94.20 \\
\hline
\end{tabular}

\section{Withdrawals by Major Public Suppliers (Mgal/d):}

Name

GW SW

Grayson Water System

$0.10 \quad 0.00$

City of Suwannee

City of Lawrenceville

City of Duluth

City of Norcross

City of Buford

Gwinnett W\&S Authority (water source located in Hall County

$0.04 \quad 0.00$

$0.14 \quad 0.00$

$0.00 \quad 1.84$

$0.00 \quad 0.36$

$0.00 \quad 1.44$

$0.00 \quad 83.56$
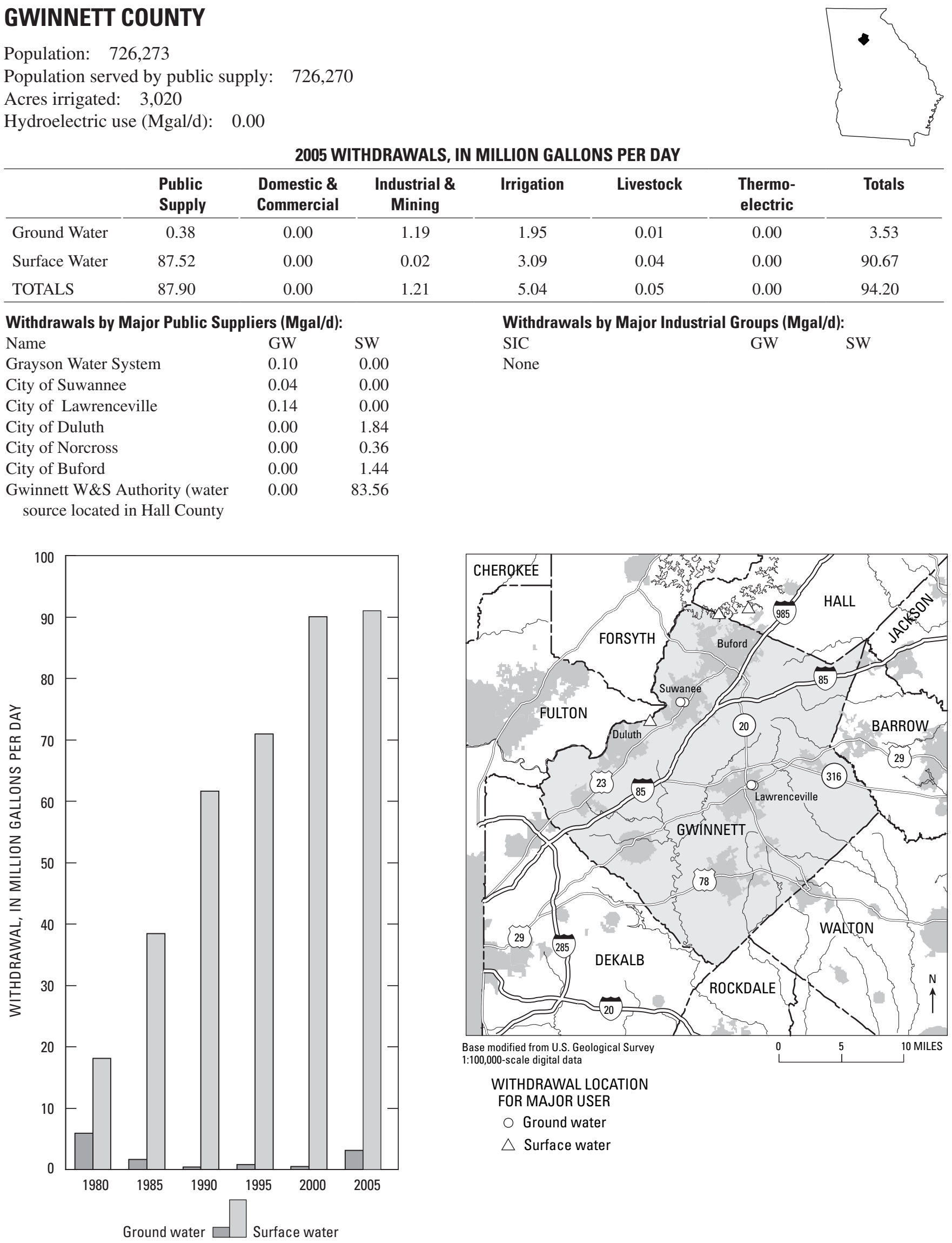

Withdrawals by Major Industrial Groups (Mgal/d):

SIC

GW

SW

None 


\section{HABERSHAM COUNTY}

Population: 39,603

Population served by public supply: 22,080

Acres irrigated: 310

Hydroelectric use (Mgal/d): $\quad 1,038.63$

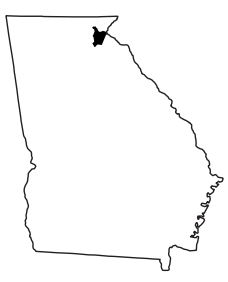

2005 WITHDRAWALS, IN MILLION GALLONS PER DAY

\begin{tabular}{|c|c|c|c|c|c|c|c|}
\hline & $\begin{array}{l}\text { Public } \\
\text { Supply }\end{array}$ & $\begin{array}{l}\text { Domestic \& } \\
\text { Commercial }\end{array}$ & $\begin{array}{c}\text { Industrial \& } \\
\text { Mining }\end{array}$ & Irrigation & Livestock & $\begin{array}{l}\text { Thermo- } \\
\text { electric }\end{array}$ & Totals \\
\hline Ground Water & 0.75 & 1.32 & 0.15 & 0.00 & 0.02 & 0.00 & 2.24 \\
\hline Surface Water & 5.15 & 0.00 & 0.00 & 0.50 & 5.31 & 0.00 & 10.96 \\
\hline TOTALS & 5.90 & 1.32 & 0.15 & 0.50 & 5.33 & 0.00 & 13.20 \\
\hline
\end{tabular}

Withdrawals by Major Public Suppliers (Mgal/d):

Name

Town of Alto

Town of Baldwin

City of Clarkesville

City of Cornelia

City of Demorest

Town of Mount Airy
GW SW

$0.37 \quad 0.00$

$0.00 \quad 2.05$

$0.00 \quad 0.63$

$0.00 \quad 2.47$

$0.25 \quad 0.00$

$0.05 \quad 0.00$
Withdrawals by Major Industrial Groups (Mgal/d):

SIC

GW

SW
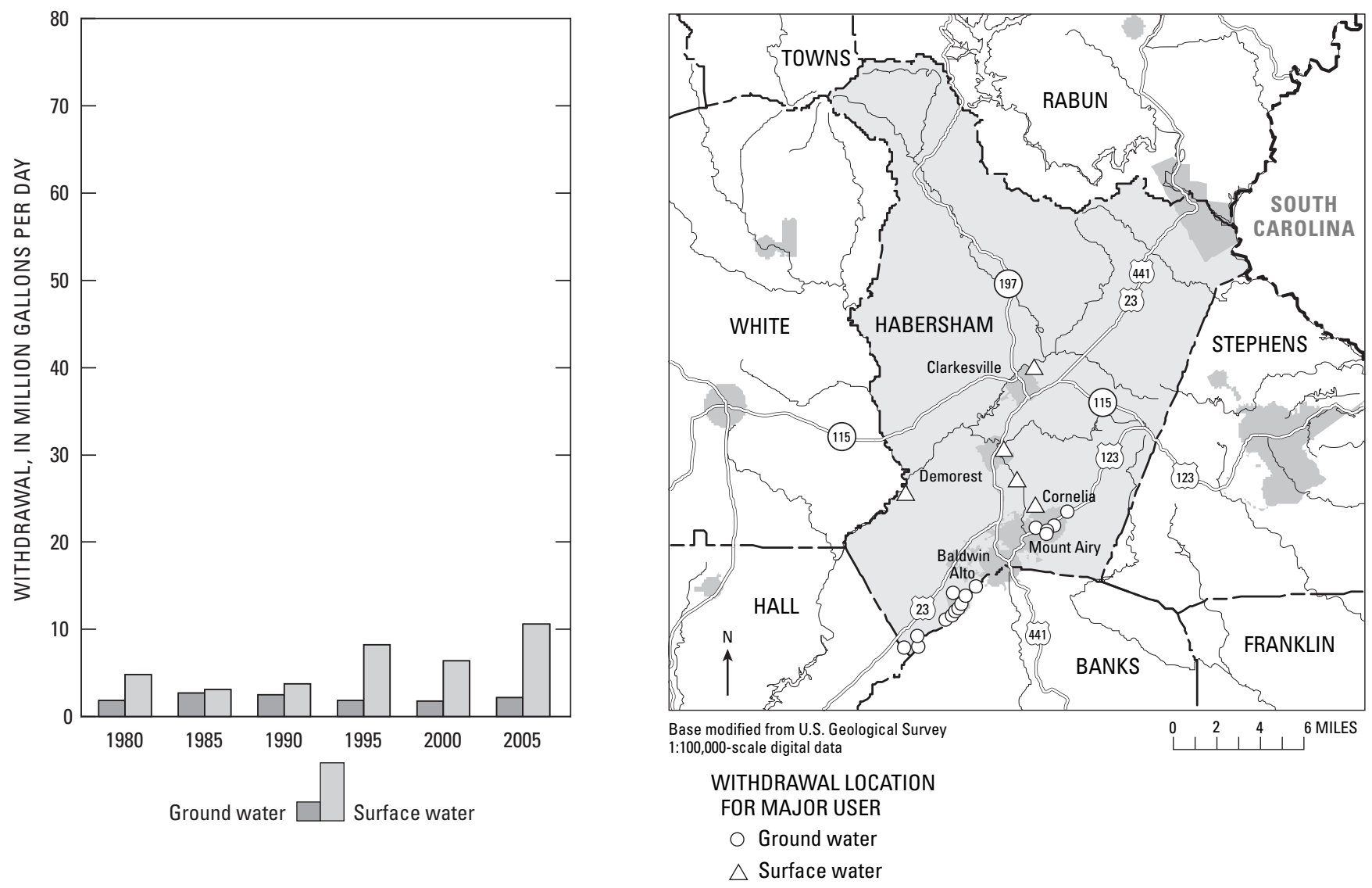


\section{HALL COUNTY}

Population: 165,771

Population served by public supply: $\quad 125,790$

Acres irrigated: 910

Hydroelectric use (Mgal/d): $\quad 0.00$

2005 WITHDRAWALS, IN MILLION GALLONS PER DAY

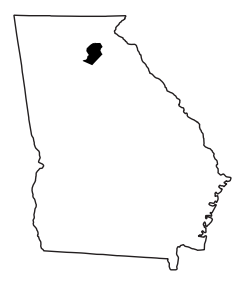

\begin{tabular}{|c|c|c|c|c|c|c|c|}
\hline & $\begin{array}{l}\text { Public } \\
\text { Supply }\end{array}$ & $\begin{array}{l}\text { Domestic \& } \\
\text { Commercial }\end{array}$ & $\begin{array}{c}\text { Industrial \& } \\
\text { Mining }\end{array}$ & Irrigation & Livestock & $\begin{array}{l}\text { Thermo- } \\
\text { electric }\end{array}$ & Totals \\
\hline Ground Water & 0.51 & 2.18 & 1.40 & 0.09 & 0.00 & 0.00 & 4.18 \\
\hline Surface Water & 17.86 & 0.11 & 0.08 & 0.68 & 0.80 & 0.00 & 19.53 \\
\hline TOTALS & 18.37 & 2.29 & 1.48 & 0.77 & 0.80 & 0.00 & 23.71 \\
\hline
\end{tabular}

Withdrawals by Major Public Suppliers (Mgal/d):

Name

Town of Flowery Branch

City of Gainesville

City of Lula
GW SW

$0.20 \quad 0.00$

$0.00 \quad 17.86$

$0.16 \quad 0.00$
Withdrawals by Major Industrial Groups (Mgal/d):

SIC

20 - Food

22 - Textiles
GW SW

$0.65 \quad 0.00$

$0.00 \quad 0.07$
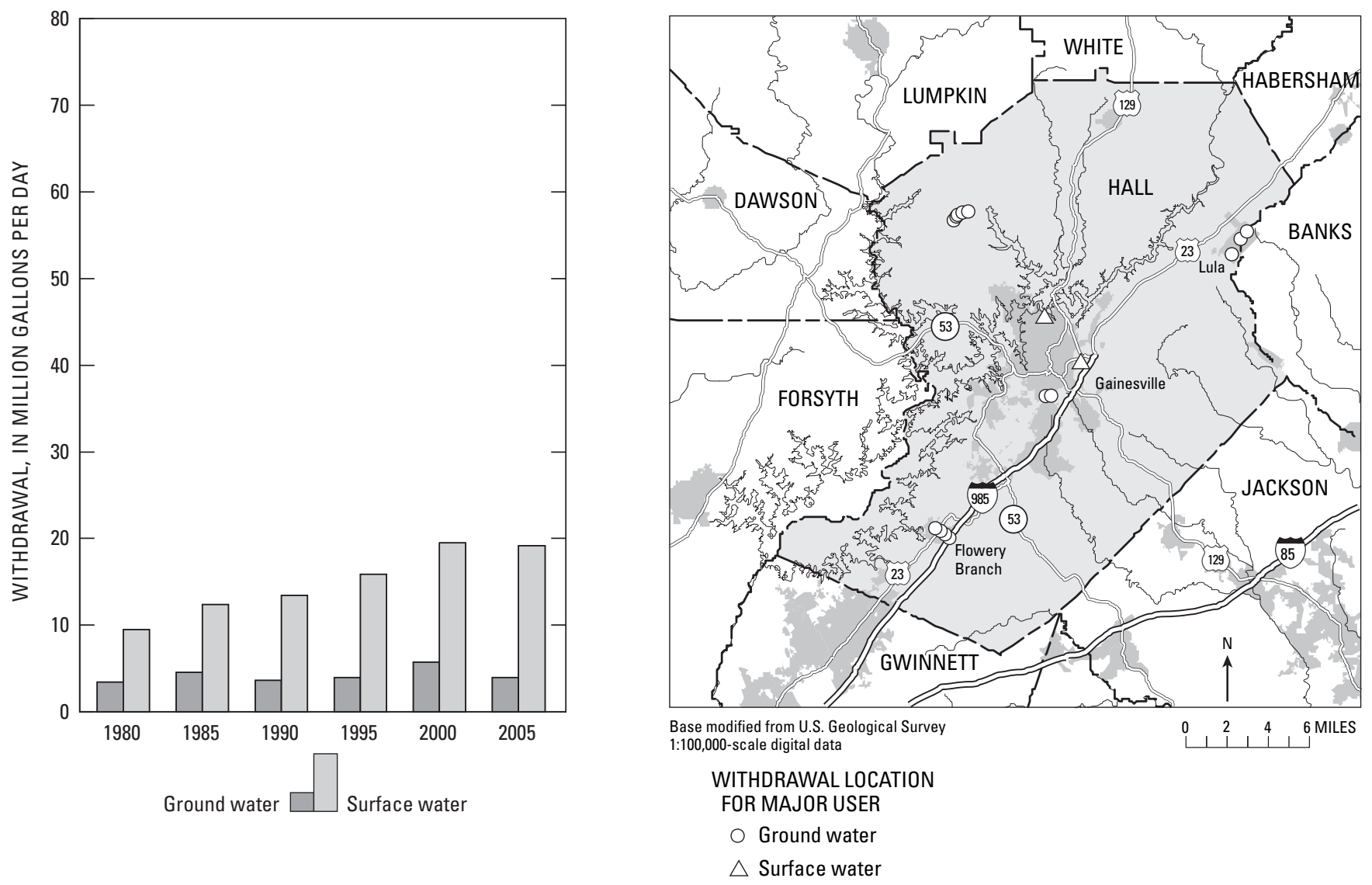


\section{HANCOCK COUNTY}

Population: 9,643

Population served by public supply: $\quad 6,110$

Acres irrigated: 40

Hydroelectric use (Mgal/d): 0.00

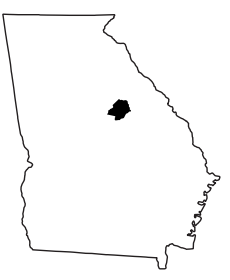

2005 WITHDRAWALS, IN MILLION GALLONS PER DAY

\begin{tabular}{|c|c|c|c|c|c|c|c|}
\hline & $\begin{array}{l}\text { Public } \\
\text { Supply }\end{array}$ & $\begin{array}{l}\text { Domestic \& } \\
\text { Commercial }\end{array}$ & $\begin{array}{c}\text { Industrial \& } \\
\text { Mining }\end{array}$ & Irrigation & Livestock & $\begin{array}{l}\text { Thermo- } \\
\text { electric }\end{array}$ & Totals \\
\hline Ground Water & 0.17 & 0.26 & 0.12 & 0.00 & 0.00 & 0.00 & 0.55 \\
\hline Surface Water & 0.85 & 0.00 & 0.00 & 0.04 & 0.04 & 0.00 & 0.93 \\
\hline TOTALS & 1.02 & 0.26 & 0.12 & 0.04 & 0.04 & 0.00 & 1.48 \\
\hline
\end{tabular}

Withdrawals by Major Public Suppliers (Mgal/d):

Name

Devereux Water System

GW

0.02

SW

City of Sparta

0.00

0.00

Withdrawals by Major Industrial Groups (Mgal/d):

SIC

GW

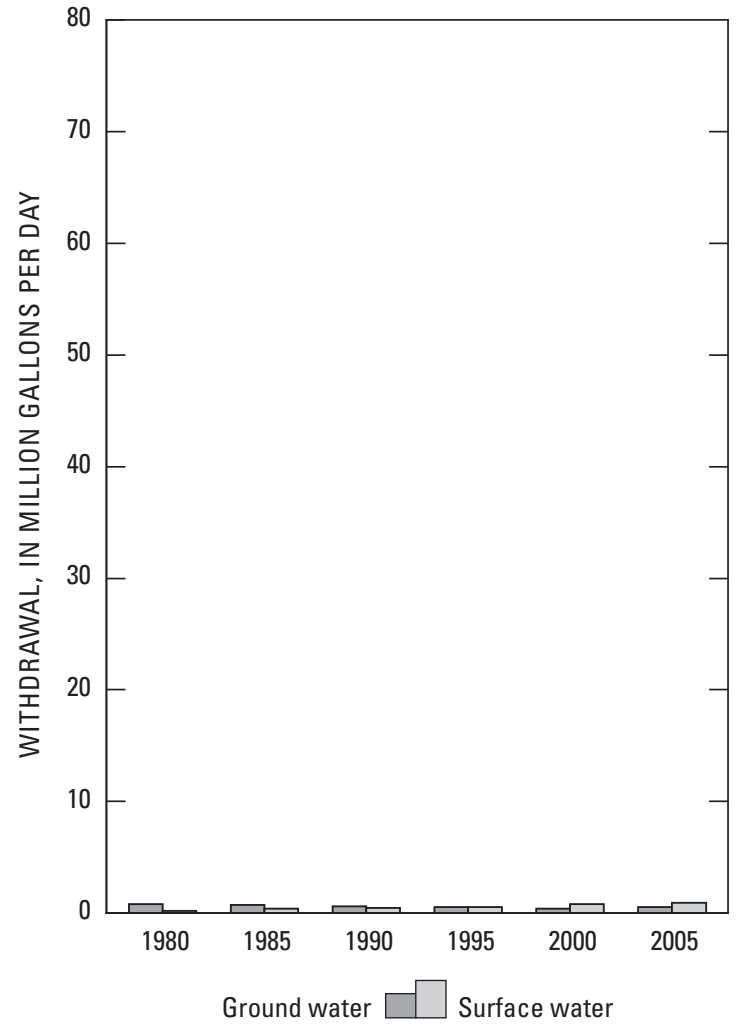

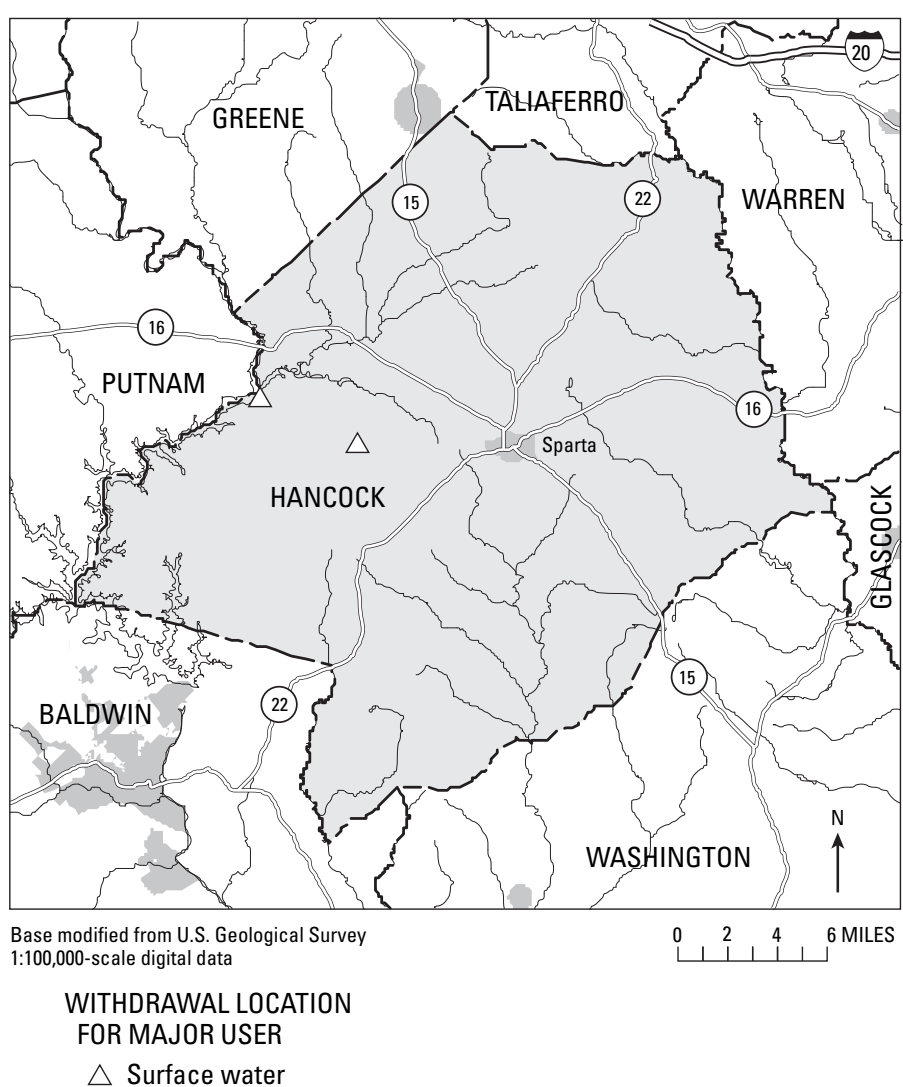




\section{HARALSON COUNTY}

Population: 28,338

Population served by public supply: 28,060

Acres irrigated: 150

Hydroelectric use (Mgal/d): $\quad 0.00$

2005 WITHDRAWALS, IN MILLION GALLONS PER DAY

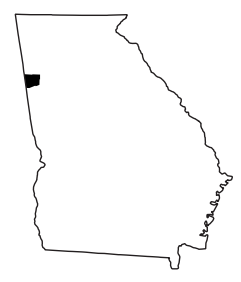

\begin{tabular}{|c|c|c|c|c|c|c|c|}
\hline & $\begin{array}{l}\text { Public } \\
\text { Supply }\end{array}$ & $\begin{array}{l}\text { Domestic \& } \\
\text { Commercial }\end{array}$ & $\begin{array}{c}\text { Industrial \& } \\
\text { Mining }\end{array}$ & Irrigation & Livestock & $\begin{array}{l}\text { Thermo- } \\
\text { electric }\end{array}$ & Totals \\
\hline Ground Water & 0.00 & 0.02 & 0.00 & 0.00 & 0.01 & 0.00 & 0.03 \\
\hline Surface Water & 2.36 & 0.00 & 0.00 & 0.85 & 0.12 & 0.00 & 3.33 \\
\hline TOTALS & 2.36 & 0.02 & 0.00 & 0.85 & 0.13 & 0.00 & 3.36 \\
\hline
\end{tabular}

Withdrawals by Major Public Suppliers (Mgal/d):

Name

City of Bremen

Haralson County

Water Authority
GW SW

$0.00 \quad 0.32$

$0.00 \quad 2.05$
Withdrawals by Major Industrial Groups (Mgal/d):

SIC

None
SW
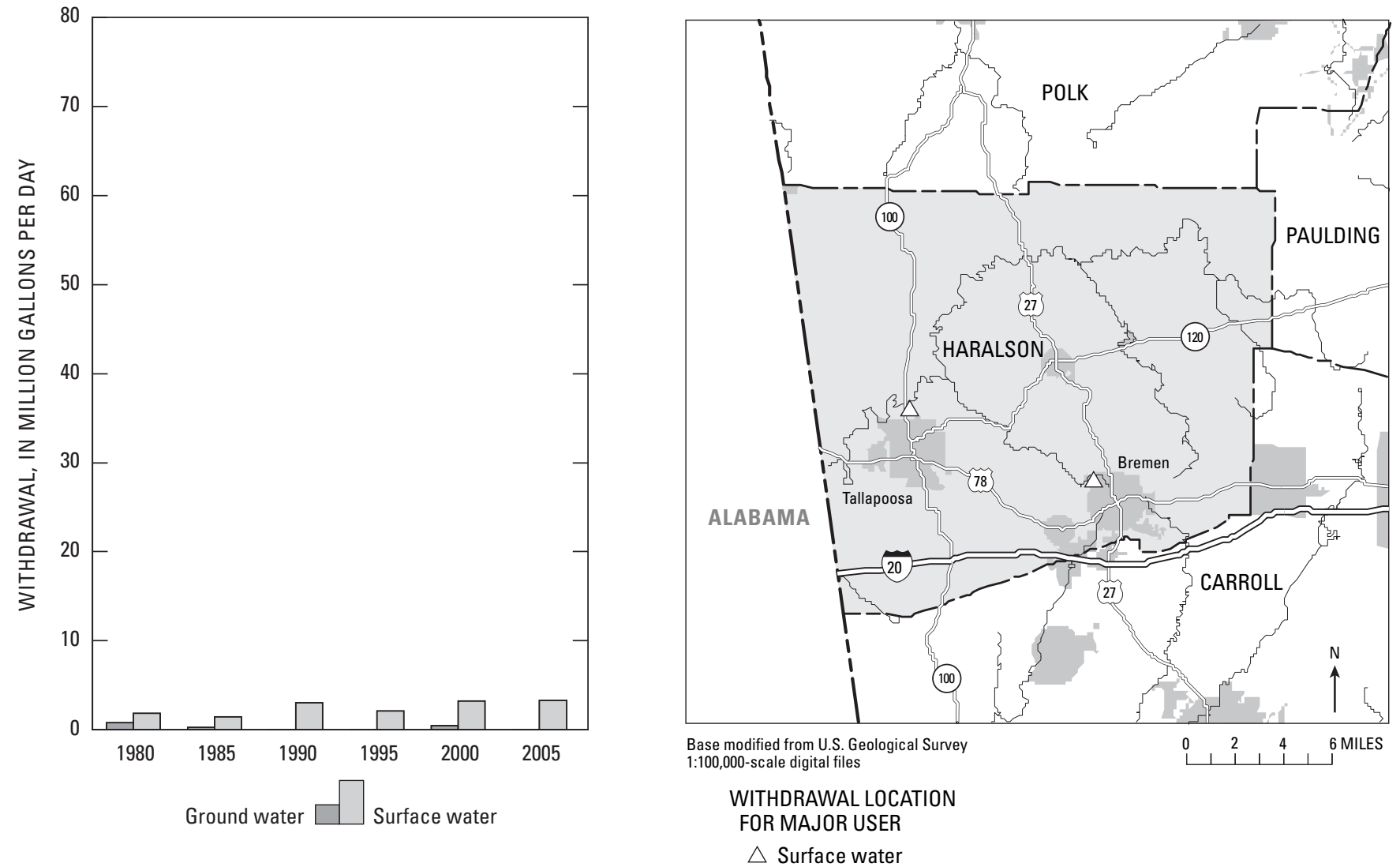


\section{HARRIS COUNTY}

Population: 27,779

Population served by public supply: $\quad 17,160$

Acres irrigated: 640

Hydroelectric use (Mgal/d): 7,375.31

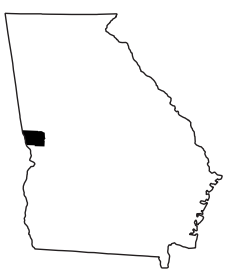

2005 WITHDRAWALS, IN MILLION GALLONS PER DAY

\begin{tabular}{lccccccc}
\hline & $\begin{array}{c}\text { Public } \\
\text { Supply }\end{array}$ & $\begin{array}{c}\text { Domestic \& } \\
\text { Commercial }\end{array}$ & $\begin{array}{c}\text { Industrial \& } \\
\text { Mining }\end{array}$ & Irrigation & Livestock & $\begin{array}{c}\text { Thermo- } \\
\text { electric }\end{array}$ & Totals \\
\hline Ground Water & 0.27 & 0.87 & 0.24 & 0.11 & 0.00 & 0.00 & 1.49 \\
Surface Water & 7.11 & 0.00 & 2.16 & 0.63 & 0.07 & 1.20 & 11.17 \\
TOTALS & 7.38 & 0.87 & 2.40 & 0.74 & 0.07 & 1.20 & 12.66 \\
\hline
\end{tabular}

Withdrawals by Major Public Suppliers (Mgal/d):

Name

GW SW

City of Hamilton

$0.08 \quad 0.00$

Harris County Waterworks

Town of Pine Mountain

City of Shiloh

$0.00 \quad 2.31$

$0.00 \quad 0.09$

Town of Waverly Hall

Chat Valley Water Supply

$0.06 \quad 0.00$

Withdrawals by Major Industrial Groups (Mgal/d):

SIC

None
GW

SW
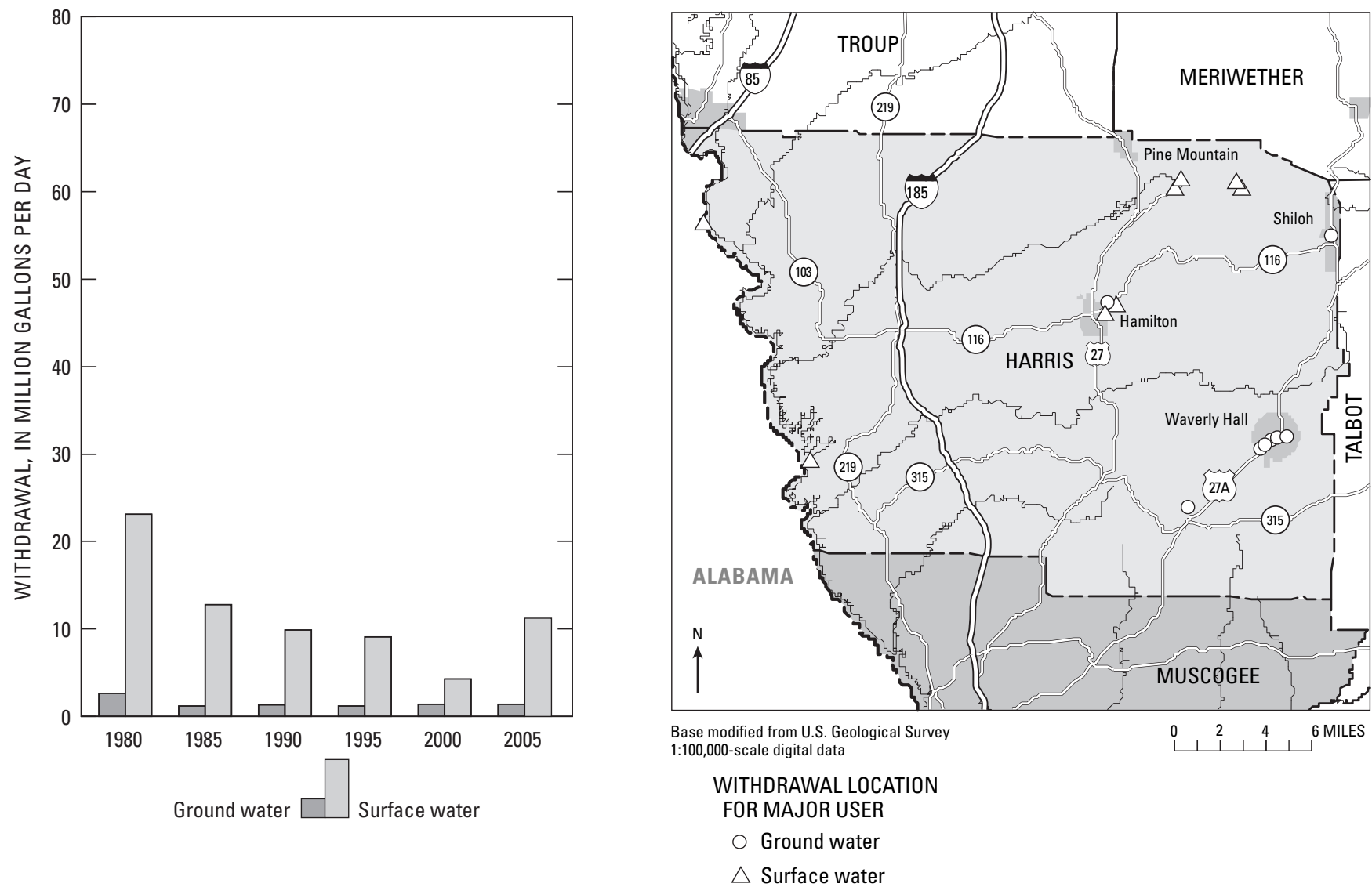


\section{HART COUNTY}

Population: 24,036

Population served by public supply: $\quad 8,250$

Acres irrigated: 800

Hydroelectric use (Mgal/d): $3,267.35$

2005 WITHDRAWALS, IN MILLION GALLONS PER DAY

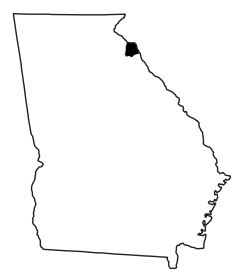

\begin{tabular}{|c|c|c|c|c|c|c|c|}
\hline & $\begin{array}{l}\text { Public } \\
\text { Supply }\end{array}$ & $\begin{array}{l}\text { Domestic \& } \\
\text { Commercial }\end{array}$ & $\begin{array}{c}\text { Industrial \& } \\
\text { Mining }\end{array}$ & Irrigation & Livestock & $\begin{array}{l}\text { Thermo- } \\
\text { electric }\end{array}$ & Totals \\
\hline Ground Water & 0.12 & 1.18 & 0.11 & 0.00 & 0.00 & 0.00 & 1.41 \\
\hline Surface Water & 1.19 & 0.00 & 0.00 & 0.53 & 0.65 & 0.00 & 2.37 \\
\hline TOTALS & 1.31 & 1.18 & 0.11 & 0.53 & 0.65 & 0.00 & 3.78 \\
\hline
\end{tabular}

Withdrawals by Major Public Suppliers (Mgal/d):

\section{Name}

Town of Bowersville

City of Hartwell

$\begin{array}{ll}\text { GW } & \text { SW } \\ 0.03 & 0.00 \\ 0.00 & 1.19\end{array}$

Withdrawals by Major Industrial Groups (Mgal/d):

SIC

32 - Stone, clay

GW

0.11

SW

0.00
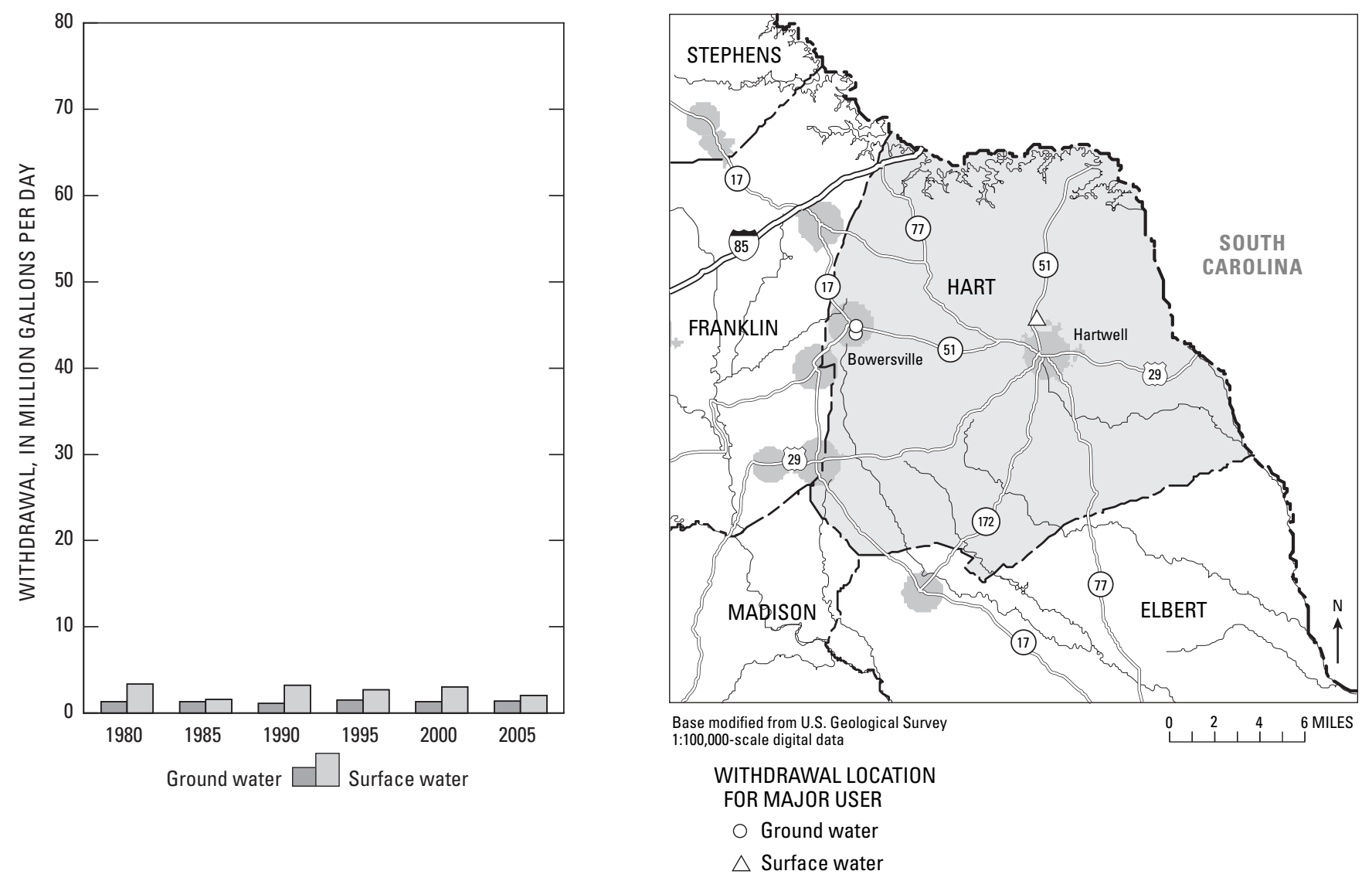


\section{HEARD COUNTY}

Population: 11,346

Population served by public supply: $\quad 8,220$

Acres irrigated: 0

Hydroelectric use (Mgal/d): $\quad 0.00$

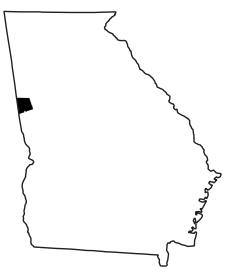

2005 WITHDRAWALS, IN MILLION GALLONS PER DAY

\begin{tabular}{|c|c|c|c|c|c|c|c|}
\hline & $\begin{array}{l}\text { Public } \\
\text { Supply }\end{array}$ & $\begin{array}{l}\text { Domestic \& } \\
\text { Commercial }\end{array}$ & $\begin{array}{c}\text { Industrial \& } \\
\text { Mining }\end{array}$ & Irrigation & Livestock & $\begin{array}{l}\text { Thermo- } \\
\text { electric }\end{array}$ & Totals \\
\hline Ground Water & 0.08 & 0.23 & 0.09 & 0.00 & 0.00 & 0.00 & 0.40 \\
\hline Surface Water & 0.98 & 0.00 & 0.00 & 0.00 & 0.18 & 64.33 & 65.49 \\
\hline TOTALS & 1.06 & 0.23 & 0.09 & 0.00 & 0.18 & 64.33 & 65.89 \\
\hline
\end{tabular}

Withdrawals by Major Public Suppliers (Mgal/d):

Name

GW SW

Ephesus Water System

0.08

0.00

Franklin/Heard County

Water Authority
Withdrawals by Major Industrial Groups (Mgal/d):

SIC

GW

SW
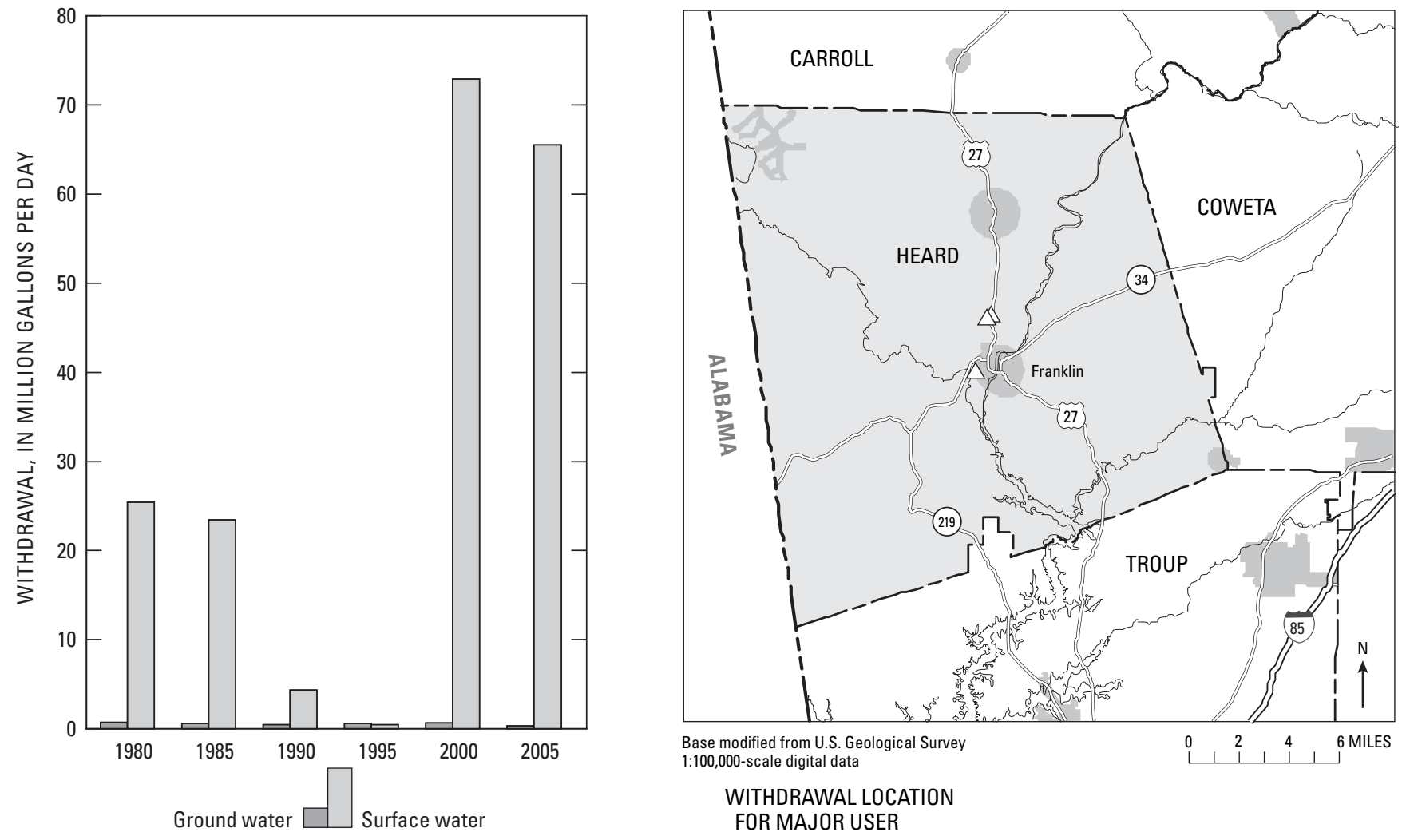

$\triangle$ Surface water 


\section{HENRY COUNTY}

Population: 167,848

Population served by public supply: $\quad 157,780$

Acres irrigated: 300

Hydroelectric use (Mgal/d): $\quad 0.00$

2005 WITHDRAWALS, IN MILLION GALLONS PER DAY

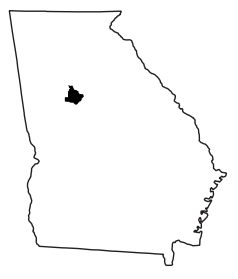

\begin{tabular}{|c|c|c|c|c|c|c|c|}
\hline & $\begin{array}{l}\text { Public } \\
\text { Supply }\end{array}$ & $\begin{array}{l}\text { Domestic \& } \\
\text { Commercial }\end{array}$ & $\begin{array}{c}\text { Industrial \& } \\
\text { Mining }\end{array}$ & Irrigation & Livestock & $\begin{array}{l}\text { Thermo- } \\
\text { electric }\end{array}$ & Totals \\
\hline Ground Water & 0.43 & 0.76 & 0.62 & 0.00 & 0.01 & 0.00 & 1.82 \\
\hline Surface Water & $16.48^{\mathrm{a}}$ & 0.00 & 0.01 & 0.27 & 0.09 & 0.00 & 16.85 \\
\hline TOTALS & 16.91 & 0.76 & 0.63 & 0.27 & 0.10 & 0.00 & 18.67 \\
\hline
\end{tabular}

Withdrawals by Major Public Suppliers (Mgal/d):

$\begin{array}{lcr}\text { Name } & \text { GW } & \text { SW } \\ \text { City of Hampton } & 0.11 & 0.00 \\ \text { Henry County W\&S } & 0.00 & 15.21^{\mathrm{a}} \\ \text { City of Locust Grove } & 0.00 & 0.08 \\ \text { City of McDonough } & 0.02 & 1.18 \\ \text { City of Stockbridge } & 0.22 & 0.00\end{array}$

Withdrawals by Major Industrial Groups (Mgal/d):

SIC GW SW

None

${ }^{a}$ Water used in Henry County but $15.21 \mathrm{Mgal} / \mathrm{d}$ withdrawn from Butts County.
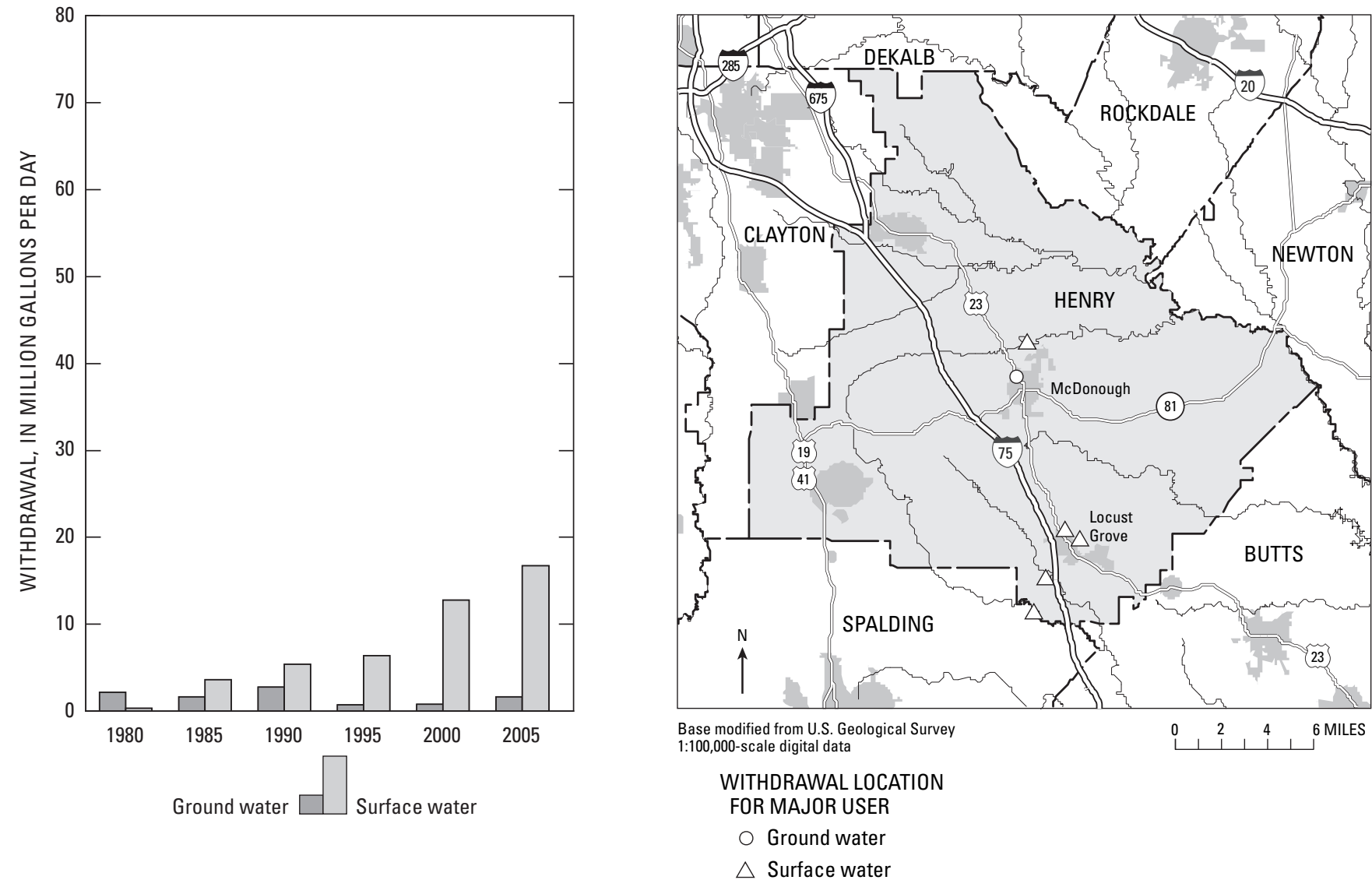


\section{HOUSTON COUNTY}

Population: 126,163

Population served by public supply: 122,820

Acres irrigated: 11,510

Hydroelectric use (Mgal/d): $\quad 0.00$

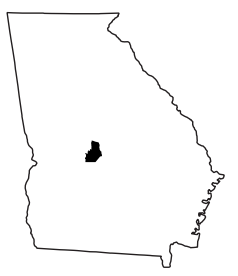

2005 WITHDRAWALS, IN MILLION GALLONS PER DAY

\begin{tabular}{|c|c|c|c|c|c|c|c|}
\hline & $\begin{array}{l}\text { Public } \\
\text { Supply }\end{array}$ & $\begin{array}{l}\text { Domestic \& } \\
\text { Commercial }\end{array}$ & $\begin{array}{c}\text { Industrial \& } \\
\text { Mining }\end{array}$ & Irrigation & Livestock & $\begin{array}{l}\text { Thermo- } \\
\text { electric }\end{array}$ & Totals \\
\hline Ground Water & 20.61 & 1.12 & 2.73 & 5.14 & 0.03 & 0.00 & 29.63 \\
\hline Surface Water & 0.00 & 0.00 & 0.01 & 0.49 & 0.11 & 0.00 & 0.61 \\
\hline TOTALS & 20.61 & 1.12 & 2.74 & 5.63 & 0.14 & 0.00 & 30.24 \\
\hline
\end{tabular}

Withdrawals by Major Public Suppliers (Mgal/d):

Name

City of Centerville

GW

City of Elberta

Houston County Board of Commissioners

Houston County Board of Commissioners

Houston County Board of Commissioners

City of Perry

City of Warner Robbins

\subsection{2}

0.35

0.10

7.92

0.10

1.94

6.96

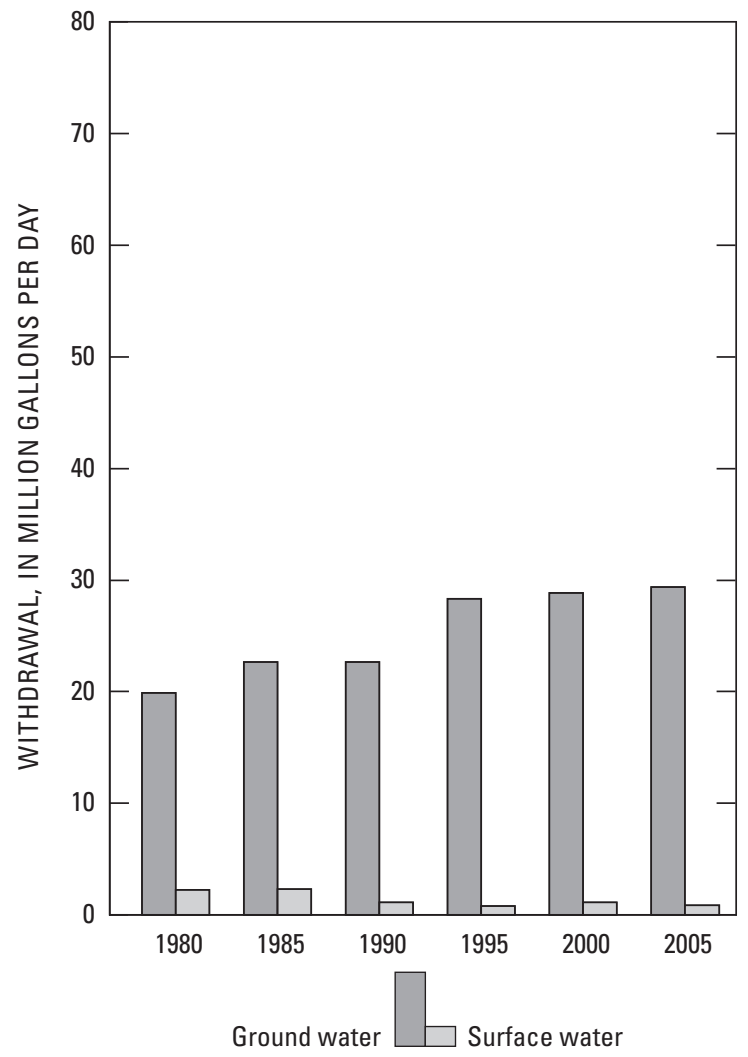

Withdrawals by Major Industrial Groups (Mgal/d):
SIC

20 - Food

32 - Stone, clay
GW

1.72

0.24
SW

0.00

0.00

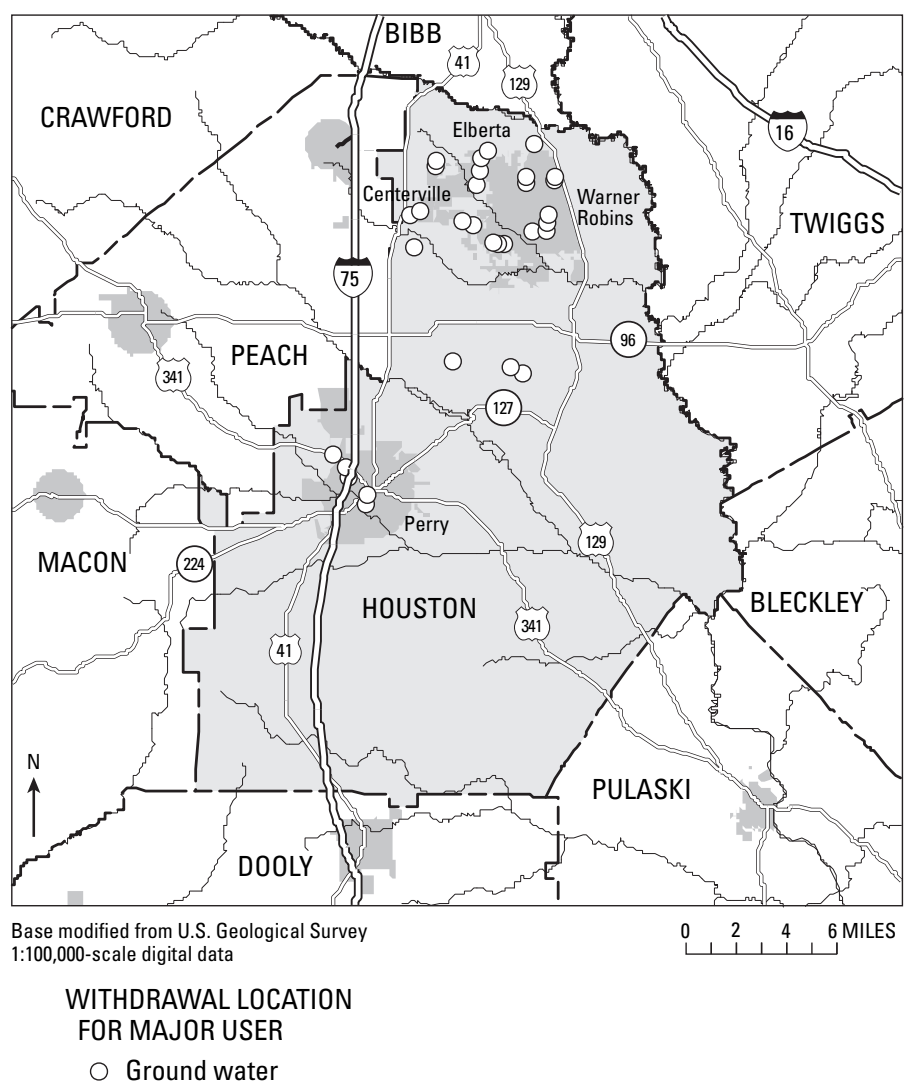




\section{IRWIN COUNTY}

Population: 10,093

Population served by public supply: 3,760

Acres irrigated: 38,460

Hydroelectric use (Mgal/d): $\quad 0.00$

2005 WITHDRAWALS, IN MILLION GALLONS PER DAY

\begin{tabular}{|c|c|c|c|c|c|c|c|}
\hline & $\begin{array}{l}\text { Public } \\
\text { Supply }\end{array}$ & $\begin{array}{l}\text { Domestic \& } \\
\text { Commercial }\end{array}$ & $\begin{array}{c}\text { Industrial \& } \\
\text { Mining }\end{array}$ & Irrigation & Livestock & $\begin{array}{l}\text { Thermo- } \\
\text { electric }\end{array}$ & Totals \\
\hline Ground Water & 0.57 & 0.47 & 0.00 & 9.14 & 0.02 & 0.00 & 10.20 \\
\hline Surface Water & 0.00 & 0.00 & 0.00 & 7.31 & 0.06 & 0.00 & 7.37 \\
\hline TOTALS & 0.57 & 0.47 & 0.00 & 16.45 & 0.08 & 0.00 & 17.57 \\
\hline
\end{tabular}

Withdrawals by Major Public Suppliers (Mgal/d):

Name GW SW

Irwinville Waterworks, Inc. $\quad 0.01 \quad 0.00$

Withdrawals by Major Industrial Groups (Mgal/d):

Irwinville Waterv
City of Mystic

W

SIC

GW

SW

City of Ocilla

0.02

None

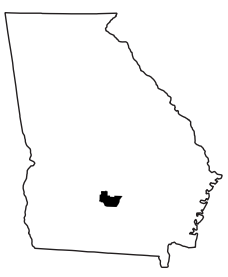

$0.53-0.00$



\section{JACKSON COUNTY}

Population: 52,292

Population served by public supply: 49,610

Acres irrigated: 330

Hydroelectric use (Mgal/d): $\quad 0.00$

2005 WITHDRAWALS, IN MILLION GALLONS PER DAY

\begin{tabular}{|c|c|c|c|c|c|c|c|}
\hline & $\begin{array}{l}\text { Public } \\
\text { Supply }\end{array}$ & $\begin{array}{l}\text { Domestic \& } \\
\text { Commercial }\end{array}$ & $\begin{array}{c}\text { Industrial \& } \\
\text { Mining }\end{array}$ & Irrigation & Livestock & $\begin{array}{l}\text { Thermo- } \\
\text { electric }\end{array}$ & Totals \\
\hline Ground Water & 0.52 & 0.20 & 0.69 & 0.00 & 0.07 & 0.00 & 1.48 \\
\hline Surface Water & 19.52 & 0.00 & 0.00 & 0.57 & 0.93 & 0.00 & 21.02 \\
\hline TOTALS & 20.04 & 0.20 & 0.69 & 0.57 & 1.00 & 0.00 & 22.50 \\
\hline
\end{tabular}

Withdrawals by Major Public Suppliers (Mgal/d):

Name

Town of Braselton

City of Hoschton

City of Jefferson

Nicholson Water Association

Upper Oconee Basin Water Authority

City of Commerce (water source

located in Banks County)

\section{GW}

0.14

0.03

0.00

0.20

0.00

0.00
Withdrawals by Major Industrial Groups (Mgal/d):

SIC

20 - Food
SW

0.00
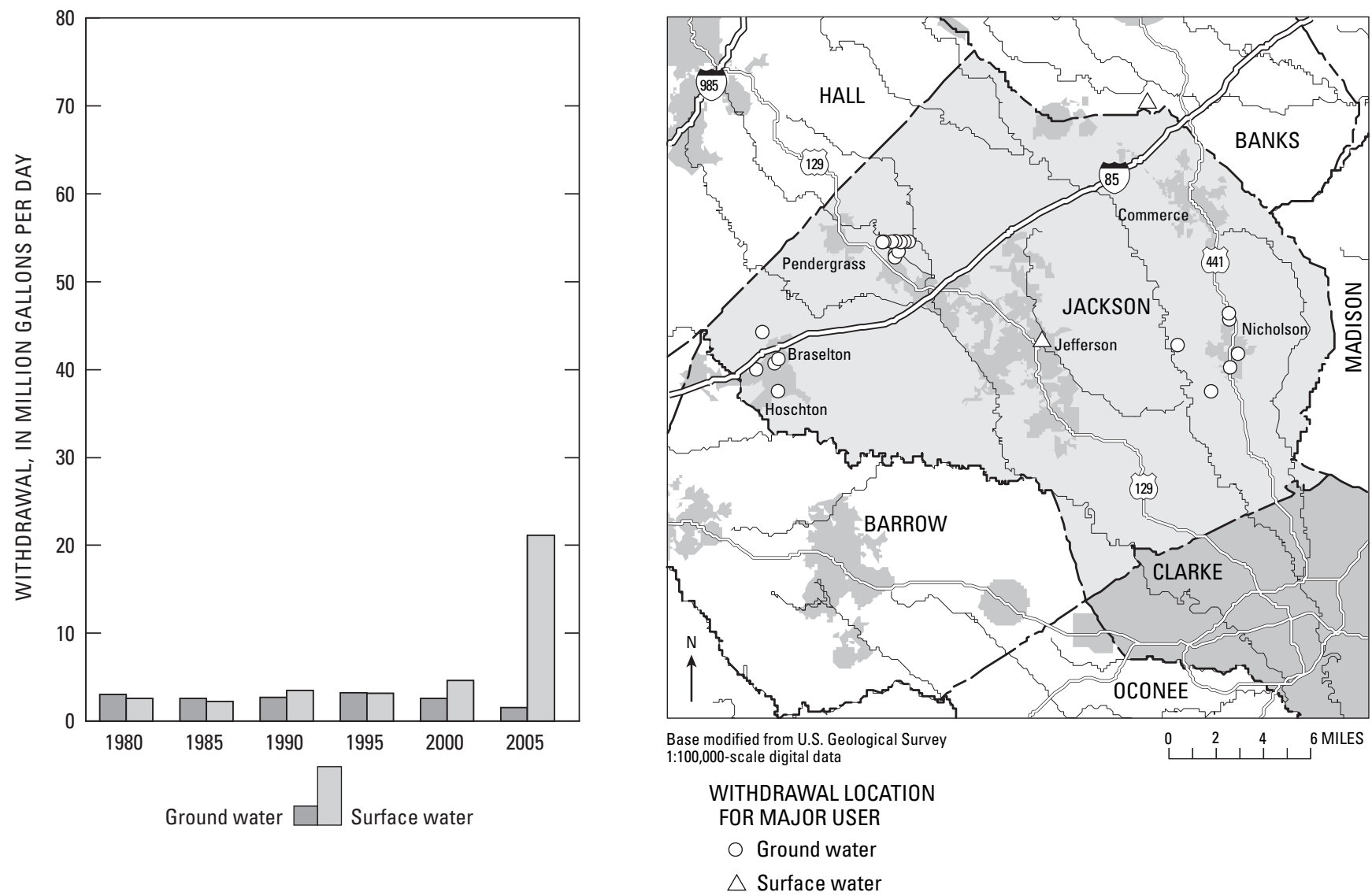


\section{JASPER COUNTY}

Population: 13,147

Population served by public supply: $\quad 4,420$

Acres irrigated: 150

Hydroelectric use (Mgal/d): $\quad 0.00$

2005 WITHDRAWALS, IN MILLION GALLONS PER DAY

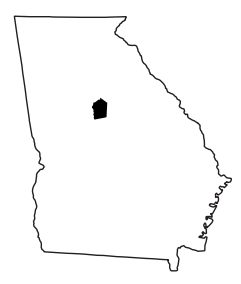

\begin{tabular}{|c|c|c|c|c|c|c|c|}
\hline & $\begin{array}{l}\text { Public } \\
\text { Supply }\end{array}$ & $\begin{array}{l}\text { Domestic \& } \\
\text { Commercial }\end{array}$ & $\begin{array}{c}\text { Industrial \& } \\
\text { Mining }\end{array}$ & Irrigation & Livestock & $\begin{array}{l}\text { Thermo- } \\
\text { electric }\end{array}$ & Totals \\
\hline Ground Water & 0.09 & 1.04 & 0.12 & 0.00 & 0.00 & 0.00 & 1.25 \\
\hline Surface Water & 0.46 & 0.00 & 0.66 & 0.22 & 0.20 & 0.00 & 1.54 \\
\hline TOTALS & 0.55 & 1.04 & 0.78 & 0.22 & 0.20 & 0.00 & 2.79 \\
\hline
\end{tabular}

Withdrawals by Major Public Suppliers (Mgal/d):

\section{Name}

City of Monticello

Town of Shady Dale

$\begin{array}{cc}\text { GW } & \text { SW } \\ 0.00 & 0.46 \\ 0.03 & 0.00\end{array}$

Withdrawals by Major Industrial Groups (Mgal/d):

$\begin{array}{lcc}\text { SIC } & \text { GW } & \text { SW } \\ 32 \text { - Stone, clay } & 0.00 & 0.66\end{array}$
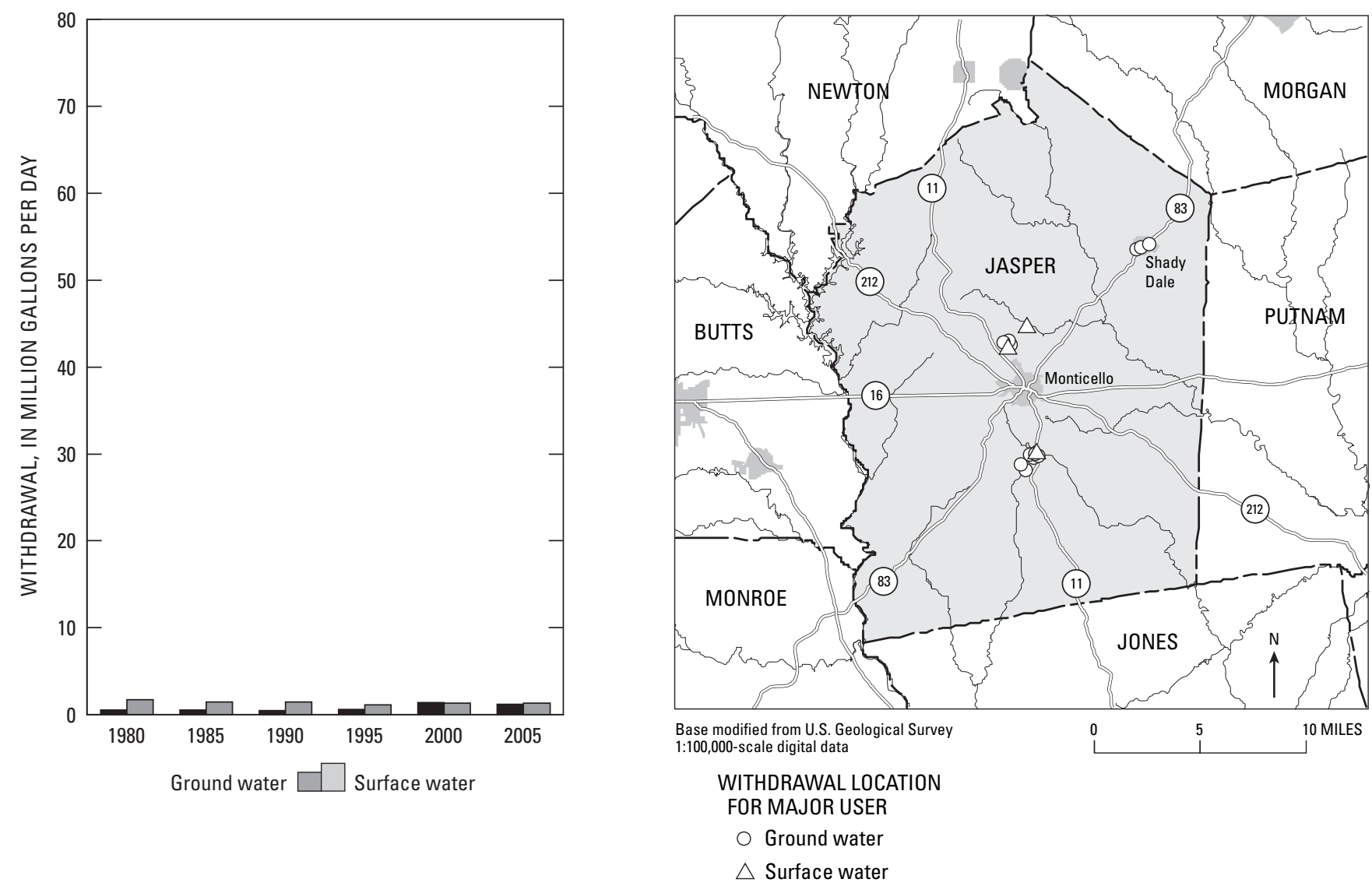


\section{JEFF DAVIS COUNTY}

Population: 13,083

Population served by public supply: $\quad 6,240$

Acres irrigated: 13,180

Hydroelectric use (Mgal/d): $\quad 0.00$

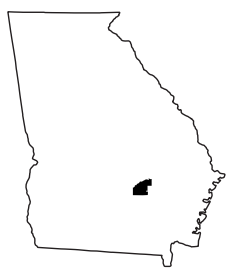

2005 WITHDRAWALS, IN MILLION GALLONS PER DAY

\begin{tabular}{|c|c|c|c|c|c|c|c|}
\hline & $\begin{array}{l}\text { Public } \\
\text { Supply }\end{array}$ & $\begin{array}{l}\text { Domestic \& } \\
\text { Commercial }\end{array}$ & $\begin{array}{c}\text { Industrial \& } \\
\text { Mining }\end{array}$ & Irrigation & Livestock & $\begin{array}{l}\text { Thermo- } \\
\text { electric }\end{array}$ & Totals \\
\hline Ground Water & 0.44 & 0.51 & 0.33 & 1.98 & 0.09 & 0.00 & 3.35 \\
\hline Surface Water & 0.00 & 0.00 & 0.00 & 2.10 & 0.13 & 0.00 & 2.23 \\
\hline TOTALS & 0.44 & 0.51 & 0.33 & 4.08 & 0.22 & 0.00 & 5.58 \\
\hline
\end{tabular}

Withdrawals by Major Public Suppliers (Mgal/d):

Name

GW SW

City of Denton

0.03

0.00

0.40

0.00
Withdrawals by Major Industrial Groups (Mgal/d):

SIC

GW

SW

22 - Textiles

0.33

0.00

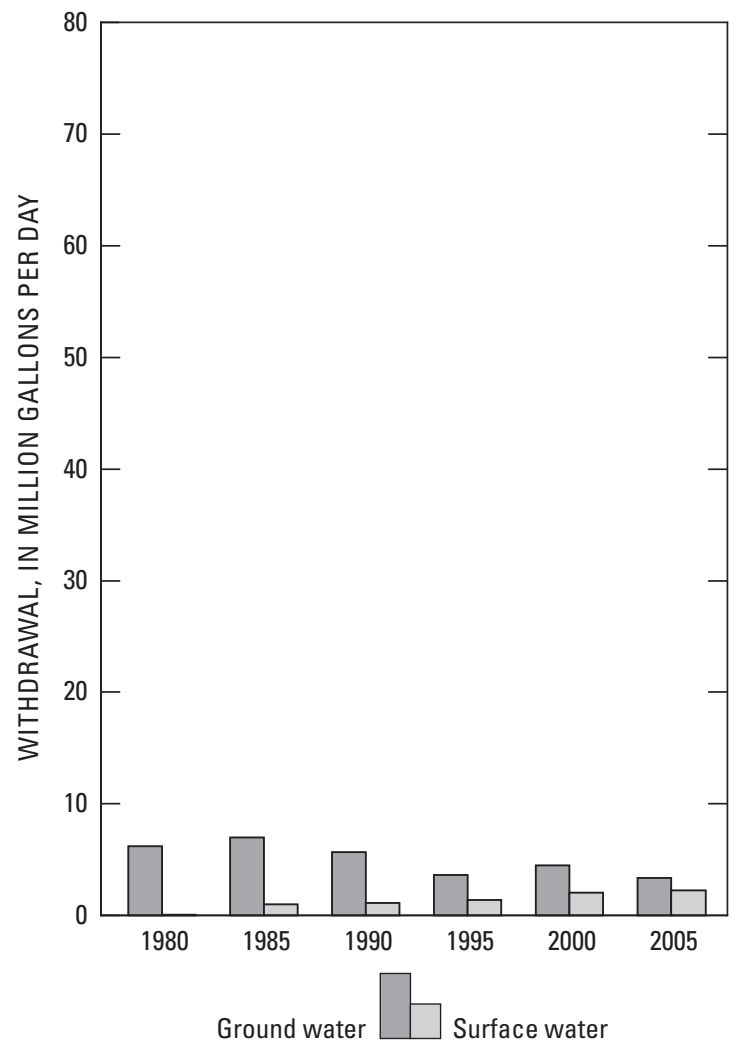

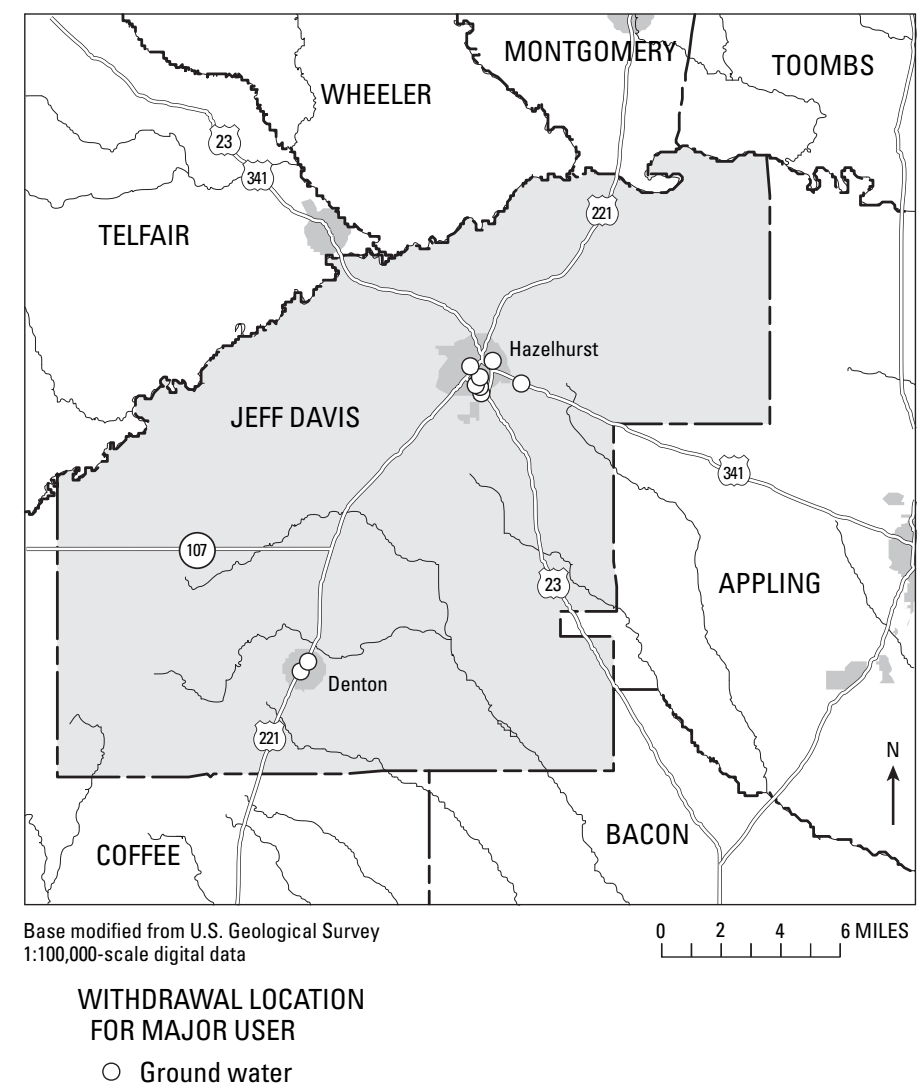




\section{JEFFERSON COUNTY}

Population: 16,926

Population served by public supply: $\quad 8,950$

Acres irrigated: 16,920

Hydroelectric use (Mgal/d): $\quad 0.00$

2005 WITHDRAWALS, IN MILLION GALLONS PER DAY

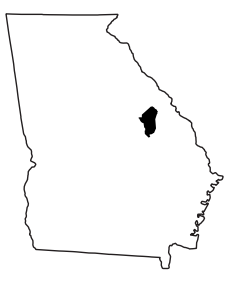

\begin{tabular}{lccccccr}
\hline & $\begin{array}{c}\text { Public } \\
\text { Supply }\end{array}$ & $\begin{array}{c}\text { Domestic \& } \\
\text { Commercial }\end{array}$ & $\begin{array}{c}\text { Industrial \& } \\
\text { Mining }\end{array}$ & Irrigation & Livestock & $\begin{array}{c}\text { Thermo- } \\
\text { electric }\end{array}$ & Totals \\
\hline Ground Water & 1.51 & 0.60 & 4.22 & 4.67 & 1.17 & 0.00 & 12.17 \\
Surface Water & 0.00 & 0.00 & 3.36 & 4.73 & 0.26 & 0.00 & 8.35 \\
TOTALS & 1.51 & 0.60 & 7.58 & 9.40 & 1.43 & 0.00 & 20.52 \\
\hline
\end{tabular}

Withdrawals by Major Public Suppliers (Mgal/d):

$\begin{array}{lcc}\text { Name } & \text { GW } & \text { SW } \\ \text { Town of Avera } & 0.02 & 0.00 \\ \text { Town of Bartow } & 0.04 & 0.00 \\ \text { City of Louisville } & 0.76 & 0.00 \\ \text { Town of Stapleton } & 0.04 & 0.00 \\ \text { City of Wadley } & 0.20 & 0.00 \\ \text { City of Wrens } & 0.45 & 0.00\end{array}$

Withdrawals by Major Industrial Groups (Mgal/d):

$\begin{array}{lcc}\text { SIC } & \text { GW } & \text { SW } \\ 32 \text { - Stone, clay } & 4.07 & 3.36\end{array}$
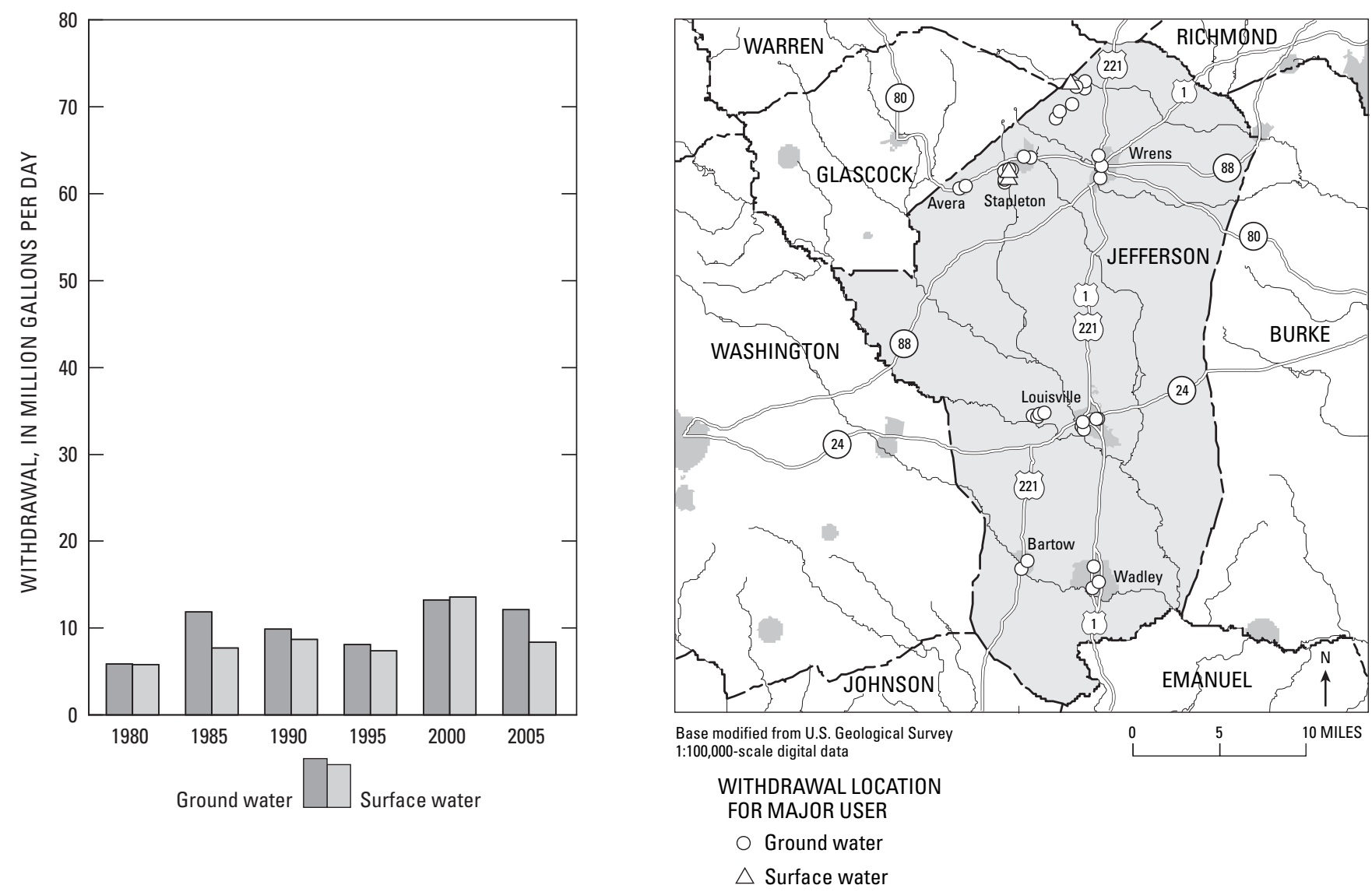


\section{JENKINS COUNTY}

Population: 8,729

Population served by public supply: $\quad 4,150$

Acres irrigated: 10,420

Hydroelectric use (Mgal/d): $\quad 0.00$

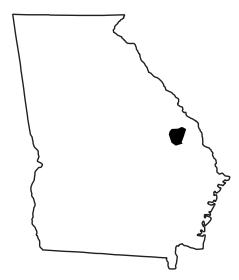

2005 WITHDRAWALS, IN MILLION GALLONS PER DAY

\begin{tabular}{|c|c|c|c|c|c|c|c|}
\hline & $\begin{array}{l}\text { Public } \\
\text { Supply }\end{array}$ & $\begin{array}{l}\text { Domestic \& } \\
\text { Commercial }\end{array}$ & $\begin{array}{c}\text { Industrial \& } \\
\text { Mining }\end{array}$ & Irrigation & Livestock & $\begin{array}{l}\text { Thermo- } \\
\text { electric }\end{array}$ & Totals \\
\hline Ground Water & 0.42 & 0.34 & 0.00 & 2.84 & 0.07 & 0.00 & 3.67 \\
\hline Surface Water & 0.00 & 0.00 & 0.00 & 1.11 & 0.11 & 0.00 & 1.22 \\
\hline TOTALS & 0.42 & 0.34 & 0.00 & 3.95 & 0.18 & 0.00 & 4.89 \\
\hline
\end{tabular}

Withdrawals by Major Public Suppliers (Mgal/d):

Name

City of Millen

GW SW

Perkins Water Authority

0.00

0.01

0.00

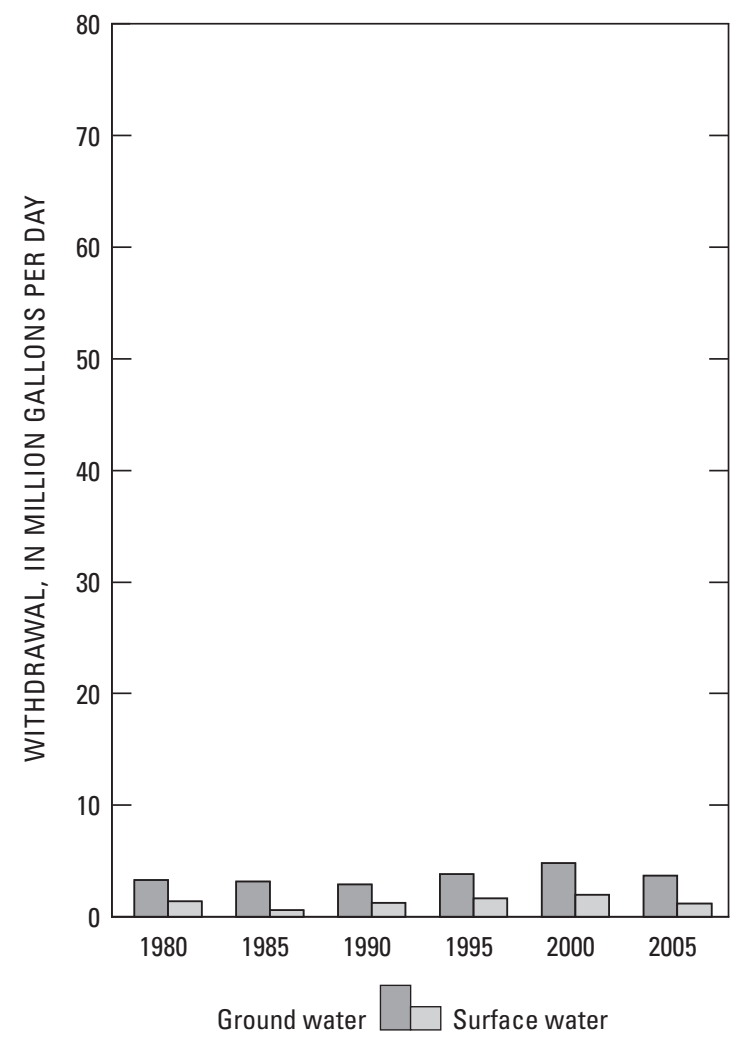

Withdrawals by Major Industrial Groups (Mgal/d):

SIC

GW

SW 


\section{JOHNSON COUNTY}

Population: $\quad 9,538$

Population served by public supply: $\quad 2,930$

Acres irrigated: 4,010

Hydroelectric use (Mgal/d): $\quad 0.00$

2005 WITHDRAWALS, IN MILLION GALLONS PER DAY

\begin{tabular}{|c|c|c|c|c|c|c|c|}
\hline & $\begin{array}{l}\text { Public } \\
\text { Supply }\end{array}$ & $\begin{array}{l}\text { Domestic \& } \\
\text { Commercial }\end{array}$ & $\begin{array}{c}\text { Industrial \& } \\
\text { Mining }\end{array}$ & Irrigation & Livestock & $\begin{array}{l}\text { Thermo- } \\
\text { electric }\end{array}$ & Totals \\
\hline Ground Water & 0.52 & 0.50 & 0.00 & 1.37 & 0.22 & 0.00 & 2.61 \\
\hline Surface Water & 0.00 & 0.00 & 0.00 & 0.37 & 0.06 & 0.00 & 0.43 \\
\hline TOTALS & 0.52 & 0.50 & 0.00 & 1.74 & 0.28 & 0.00 & 3.04 \\
\hline
\end{tabular}

Withdrawals by Major Public Suppliers (Mgal/d):

Name

Town of Kite

GW SW

Scott Water \& Sewer Authority

City of Wrightsville

$\begin{array}{ll}0.02 & 0.00 \\ 0.02 & 0.00 \\ 0.49 & 0.00\end{array}$

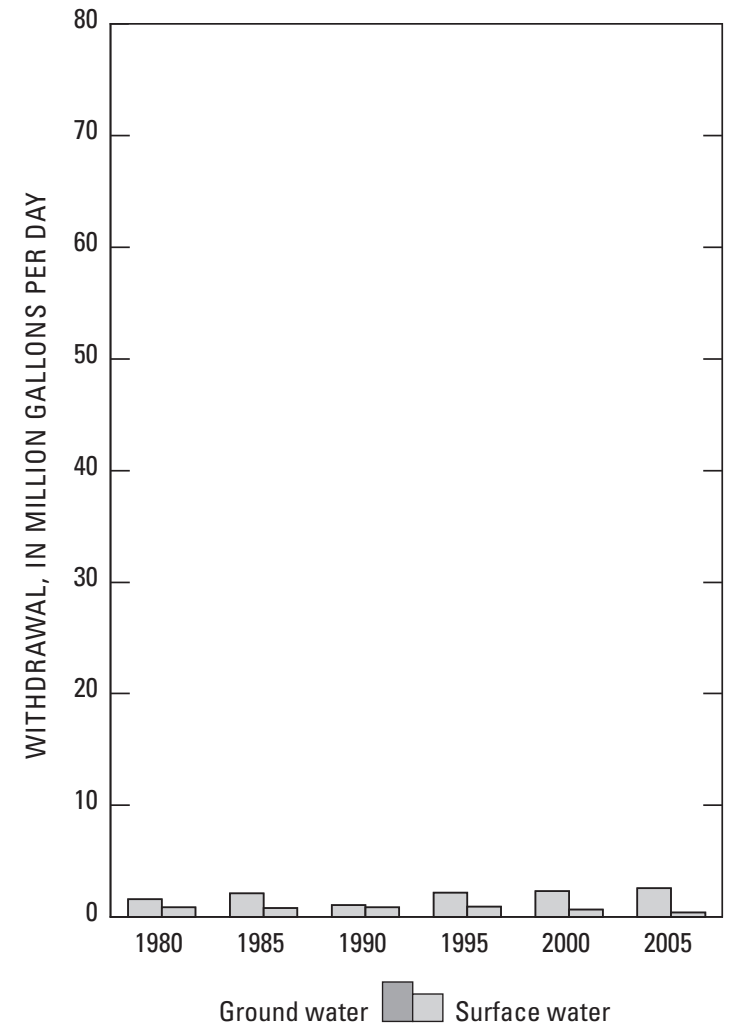

Withdrawals by Major Industrial Groups (Mgal/d):

SIC

GW

SW

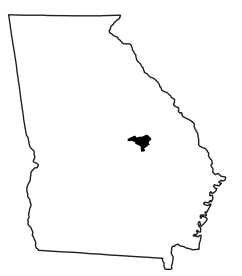

None

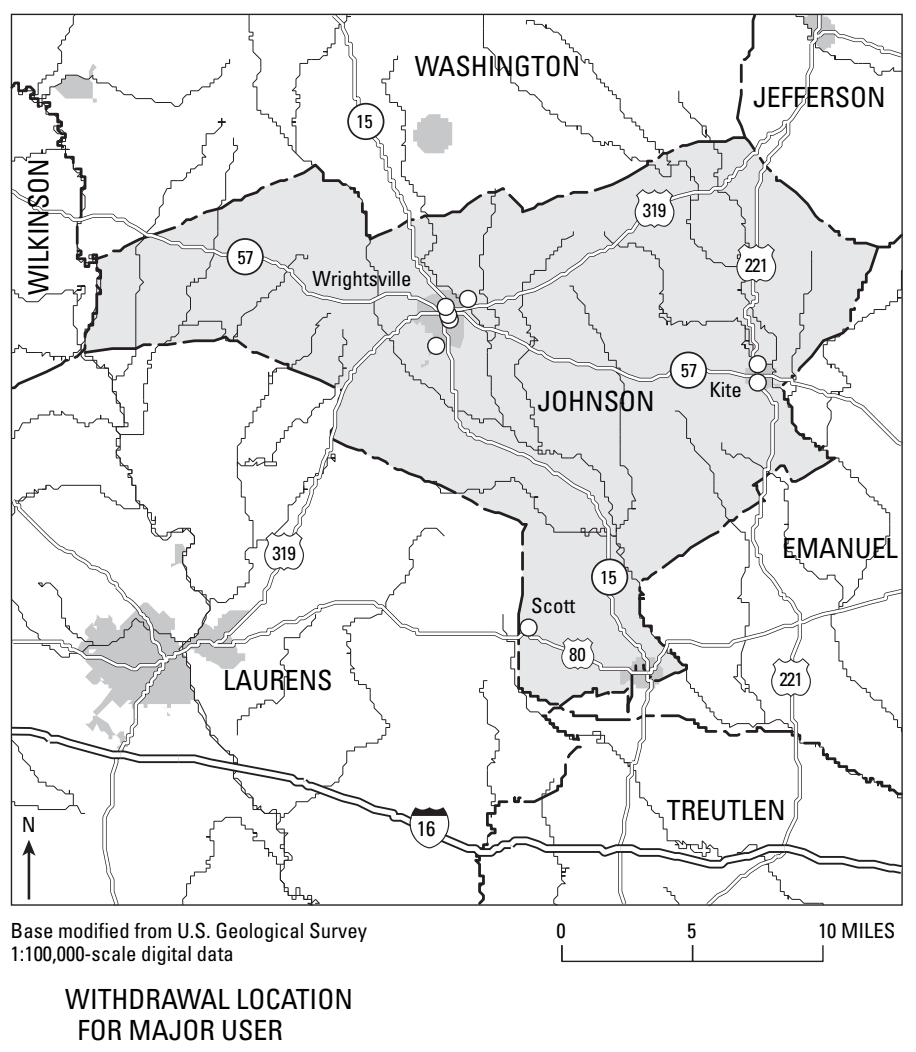




\section{JONES COUNTY}

Population: 26,836

Population served by public supply: 12,440

Acres irrigated: 270

Hydroelectric use (Mgal/d): $\quad 0.00$

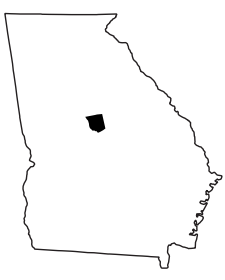

2005 WITHDRAWALS, IN MILLION GALLONS PER DAY

\begin{tabular}{|c|c|c|c|c|c|c|c|}
\hline & $\begin{array}{l}\text { Public } \\
\text { Supply }\end{array}$ & $\begin{array}{l}\text { Domestic \& } \\
\text { Commercial }\end{array}$ & $\begin{array}{c}\text { Industrial \& } \\
\text { Mining }\end{array}$ & Irrigation & Livestock & $\begin{array}{l}\text { Thermo- } \\
\text { electric }\end{array}$ & Totals \\
\hline Ground Water & 1.12 & 1.08 & 1.68 & 0.02 & 0.00 & 0.00 & 3.90 \\
\hline Surface Water & 0.00 & 0.00 & 0.02 & 0.23 & 0.10 & 0.00 & 0.35 \\
\hline TOTALS & 1.12 & 1.08 & 1.70 & 0.25 & 0.10 & 0.00 & 4.25 \\
\hline
\end{tabular}

Withdrawals by Major Public Suppliers (Mgal/d):

Name

City of Gray

GW $\quad$ SW

Haddock Water Commission

Jones County Water Commission
0.51

0.00

0.06

0.52
Withdrawals by Major Industrial Groups (Mgal/d):

SIC

GW

SW
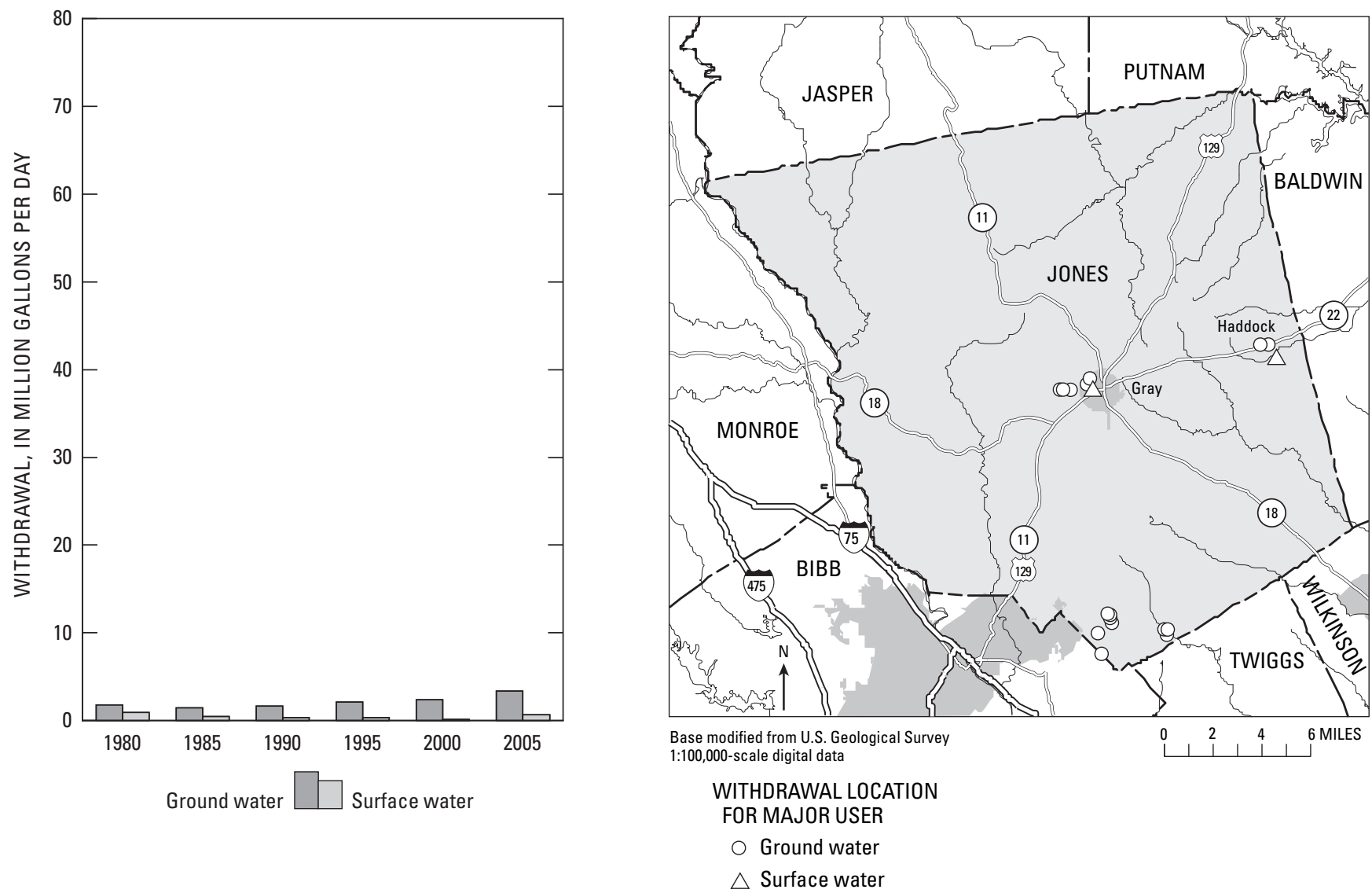


\section{LAMAR COUNTY}

Population: 16,378

Population served by public supply: 7,250

Acres irrigated: 1,470

Hydroelectric use (Mgal/d): $\quad 0.00$

2005 WITHDRAWALS, IN MILLION GALLONS PER DAY

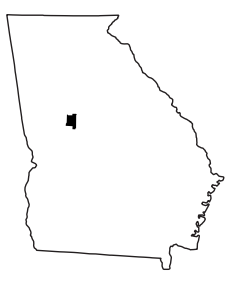

\begin{tabular}{lccccccc}
\hline & $\begin{array}{c}\text { Public } \\
\text { Supply }\end{array}$ & $\begin{array}{c}\text { Domestic \& } \\
\text { Commercial }\end{array}$ & $\begin{array}{c}\text { Industrial \& } \\
\text { Mining }\end{array}$ & Irrigation & Livestock & $\begin{array}{c}\text { Thermo- } \\
\text { electric }\end{array}$ & Totals \\
\hline Ground Water & 0.00 & 0.68 & 0.00 & 0.04 & 0.00 & 0.00 & 0.72 \\
Surface Water & 1.31 & 0.00 & 0.00 & 0.69 & 0.18 & 0.00 & 2.18 \\
TOTALS & 1.31 & 0.68 & 0.00 & 0.73 & 0.18 & 0.00 & 2.90 \\
\hline
\end{tabular}

Withdrawals by Major Public Suppliers (Mgal/d):

Name

City of Barnesville
SW 1.31
Withdrawals by Major Industrial Groups (Mgal/d):

SIC

None
SW

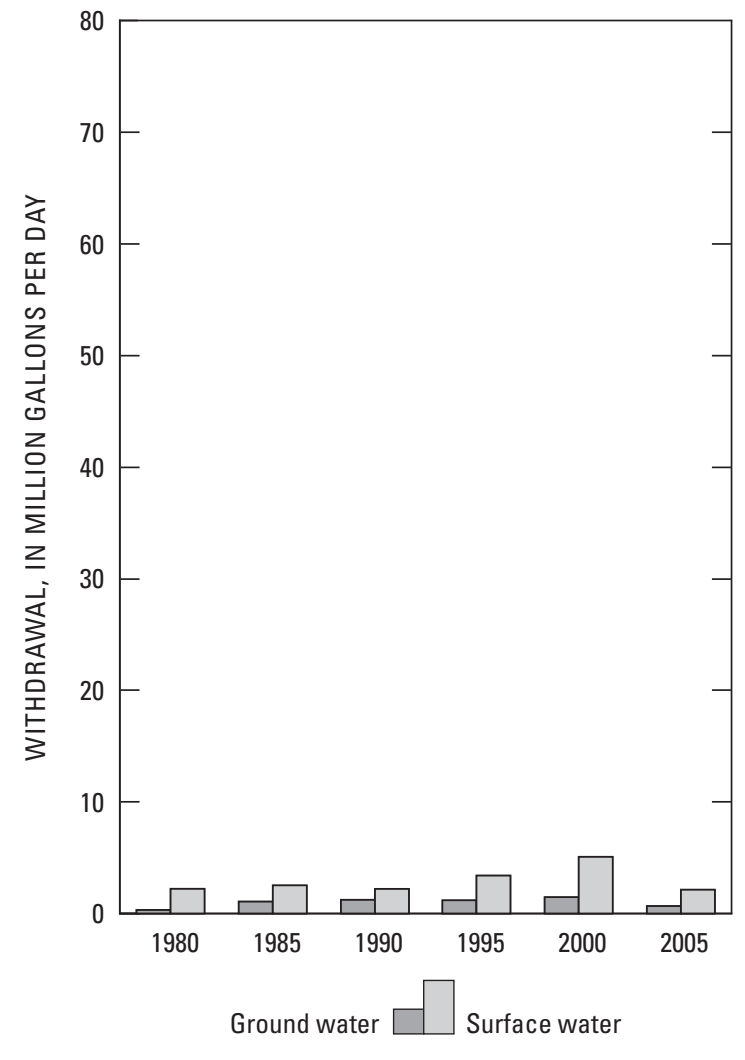

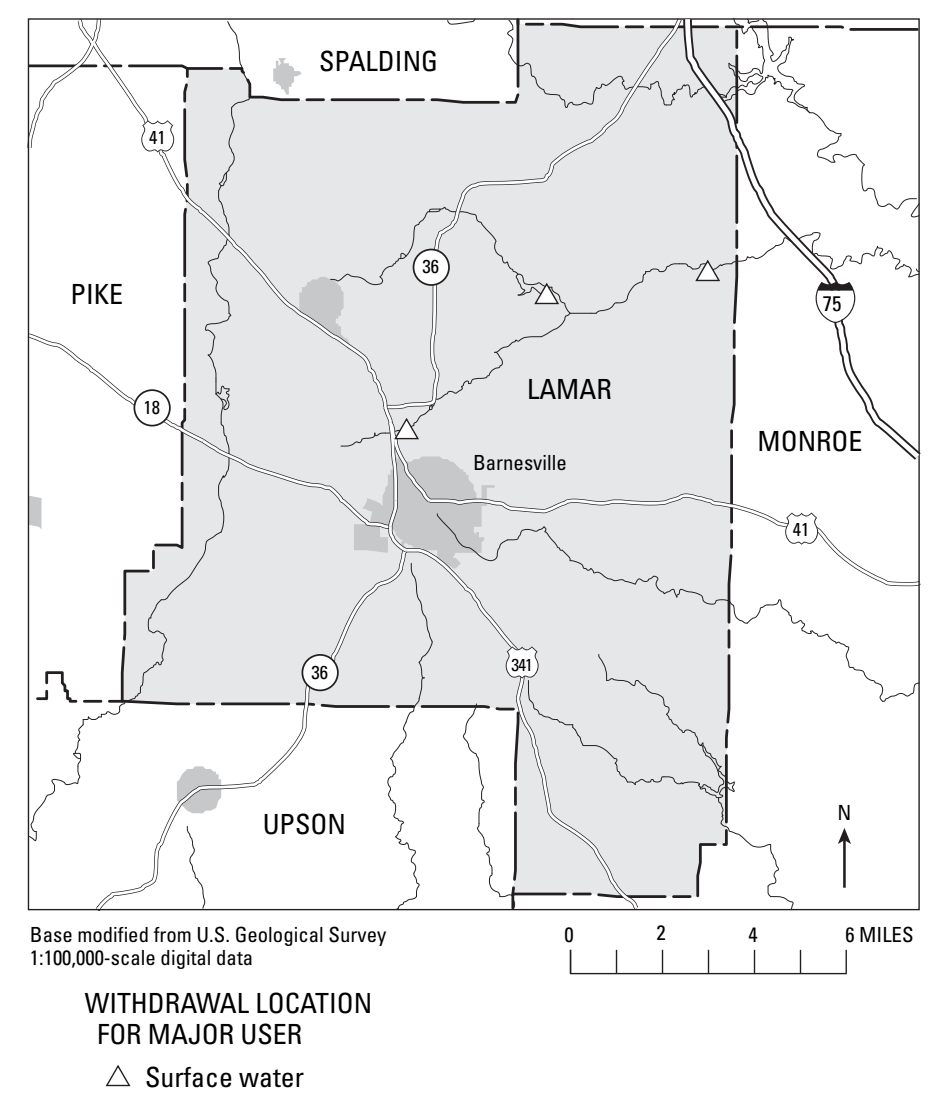




\section{LANIER COUNTY}

Population: 7,553

Population served by public supply: $\quad 2,940$

Acres irrigated: 3,100

Hydroelectric use (Mgal/d): $\quad 0.00$

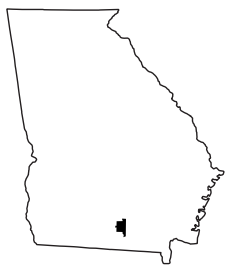

2005 WITHDRAWALS, IN MILLION GALLONS PER DAY

\begin{tabular}{|c|c|c|c|c|c|c|c|}
\hline & $\begin{array}{l}\text { Public } \\
\text { Supply }\end{array}$ & $\begin{array}{l}\text { Domestic \& } \\
\text { Commercial }\end{array}$ & $\begin{array}{c}\text { Industrial \& } \\
\text { Mining }\end{array}$ & Irrigation & Livestock & $\begin{array}{l}\text { Thermo- } \\
\text { electric }\end{array}$ & Totals \\
\hline Ground Water & 0.45 & 0.35 & 0.12 & 1.05 & 0.01 & 0.00 & 1.98 \\
\hline Surface Water & 0.00 & 0.00 & 0.00 & 0.18 & 0.01 & 0.00 & 0.19 \\
\hline TOTALS & 0.45 & 0.35 & 0.12 & 1.23 & 0.02 & 0.00 & 2.17 \\
\hline
\end{tabular}

Withdrawals by Major Public Suppliers (Mgal/d):

Name

City of Lakeland

GW

0.43

SW

0.00
Withdrawals by Major Industrial Groups (Mgal/d):

SIC

GW

SW
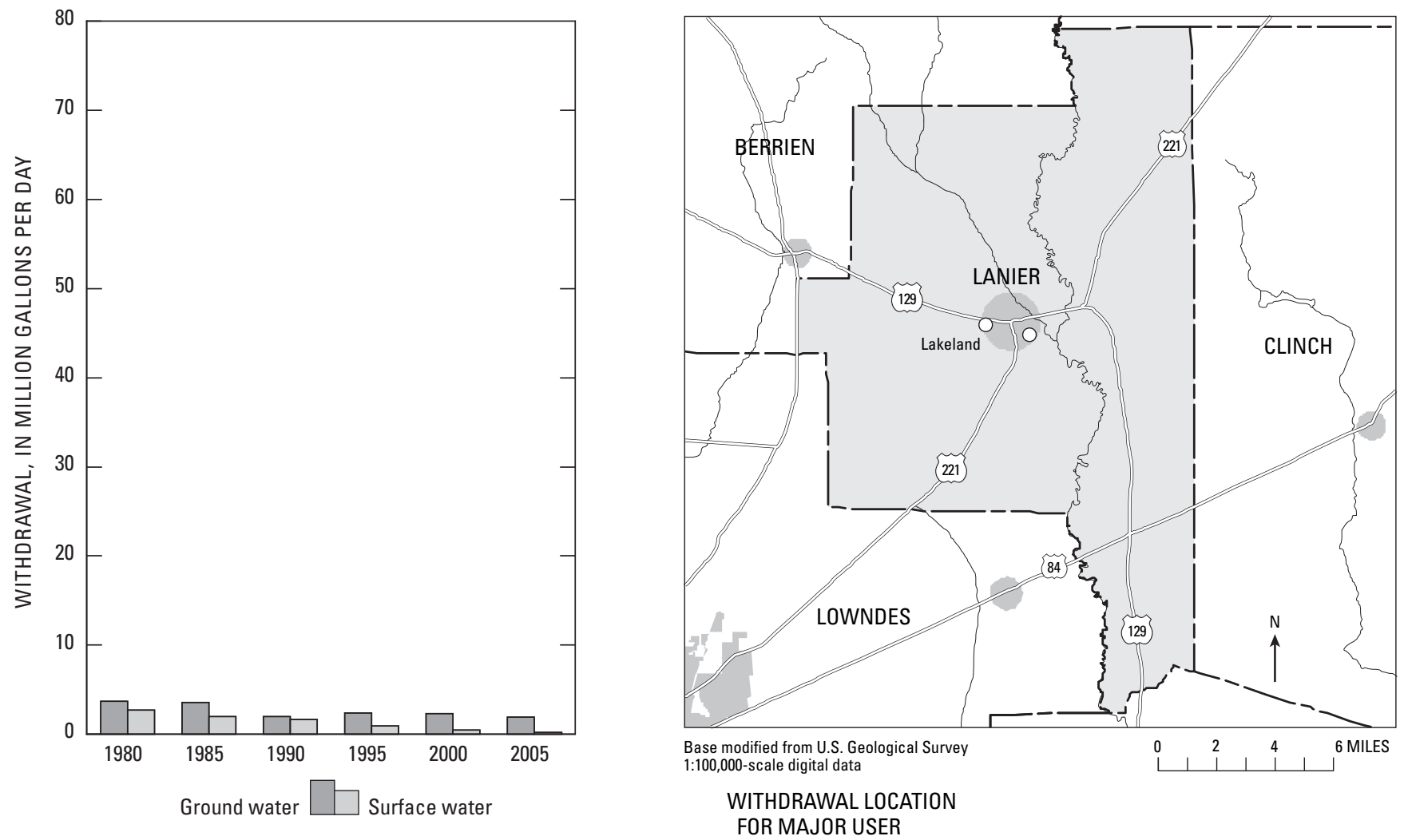

O Ground water 


\section{LAURENS COUNTY}

Population: 46,896

Population served by public supply: 25,030

Acres irrigated: 12,550

Hydroelectric use (Mgal/d): $\quad 0.00$

2005 WITHDRAWALS, IN MILLION GALLONS PER DAY

\begin{tabular}{|c|c|c|c|c|c|c|c|}
\hline & $\begin{array}{l}\text { Public } \\
\text { Supply }\end{array}$ & $\begin{array}{l}\text { Domestic \& } \\
\text { Commercial }\end{array}$ & $\begin{array}{c}\text { Industrial \& } \\
\text { Mining }\end{array}$ & Irrigation & Livestock & $\begin{array}{l}\text { Thermo- } \\
\text { electric }\end{array}$ & Totals \\
\hline Ground Water & 0.90 & 1.64 & 1.08 & 5.94 & 0.03 & 0.00 & 9.59 \\
\hline Surface Water & 3.04 & 0.00 & 15.82 & 1.56 & 0.11 & 0.00 & 20.53 \\
\hline TOTALS & 3.94 & 1.64 & 16.90 & 7.50 & 0.14 & 0.00 & 30.12 \\
\hline
\end{tabular}

Withdrawals by Major Public Suppliers (Mgal/d):

$\begin{array}{lcc}\text { Name } & \text { GW } & \text { SW } \\ \text { Town of Cadwell } & 0.03 & 0.00 \\ \text { Town of Dexter } & 0.06 & 0.00 \\ \text { City of Dublin } & 0.11 & 3.04 \\ \text { City of Dudley } & 0.04 & 0.00 \\ \text { Town of East Dublin } & 0.47 & 0.00 \\ \text { Town of Montrose } & 0.00 & 0.00 \\ \text { Town of Rentz } & 0.09 & 0.00 \\ \text { City of Allentown } & 0.02 & 0.00\end{array}$

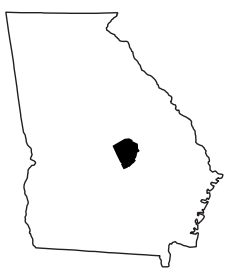

Withdrawals by Major Industrial Groups (Mgal/d):

$\begin{array}{lcc}\text { SIC } & \text { GW } & \text { SW } \\ 22 \text { - Textiles } & 1.08 & 0.65 \\ 26 \text { - Chemicals } & 0.00 & 15.18\end{array}$
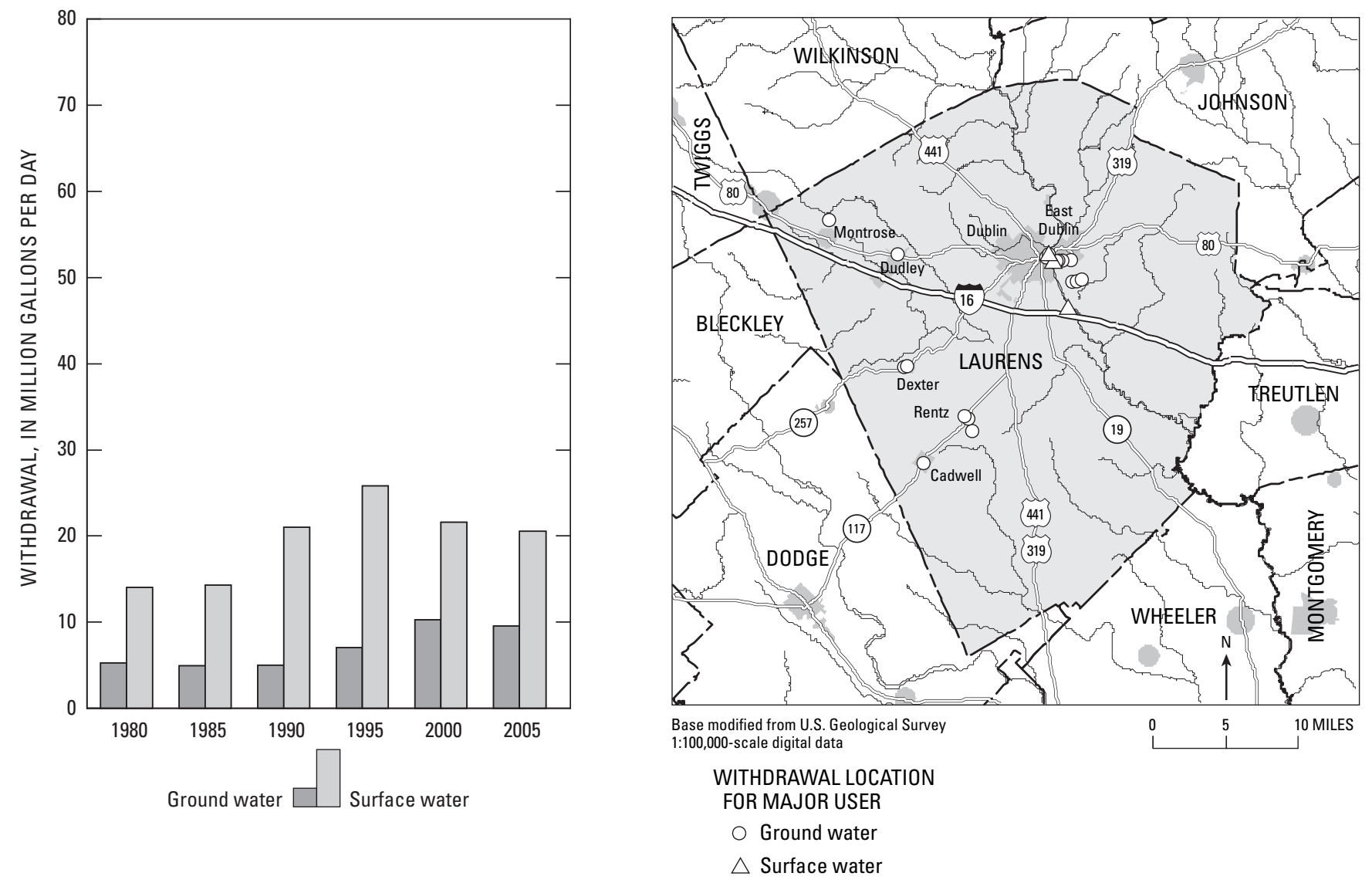


\section{LEE COUNTY}

Population: 31,099

Population served by public supply: $\quad 15,250$

Acres irrigated: 44,600

Hydroelectric use (Mgal/d): $\quad 0.00$

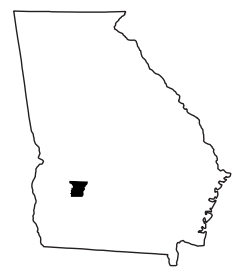

2005 WITHDRAWALS, IN MILLION GALLONS PER DAY

\begin{tabular}{|c|c|c|c|c|c|c|c|}
\hline & $\begin{array}{l}\text { Public } \\
\text { Supply }\end{array}$ & $\begin{array}{l}\text { Domestic \& } \\
\text { Commercial }\end{array}$ & $\begin{array}{c}\text { Industrial \& } \\
\text { Mining }\end{array}$ & Irrigation & Livestock & $\begin{array}{l}\text { Thermo- } \\
\text { electric }\end{array}$ & Totals \\
\hline Ground Water & 1.82 & 1.19 & 0.13 & 3.06 & 0.00 & 0.00 & 6.20 \\
\hline Surface Water & 0.00 & 0.00 & 0.00 & 1.22 & 0.14 & 0.00 & 1.36 \\
\hline TOTALS & 1.82 & 1.19 & 0.13 & 4.28 & 0.14 & 0.00 & 7.56 \\
\hline
\end{tabular}

Withdrawals by Major Public Suppliers (Mgal/d):

Name

GW SW

Lee County Utilities Authority

City of Smithville

$\begin{array}{ll}1.40 & 0.00 \\ 0.05 & 0.00\end{array}$

$0.05 \quad 0.00$
Withdrawals by Major Industrial Groups (Mgal/d):

SIC

None
GW

SW
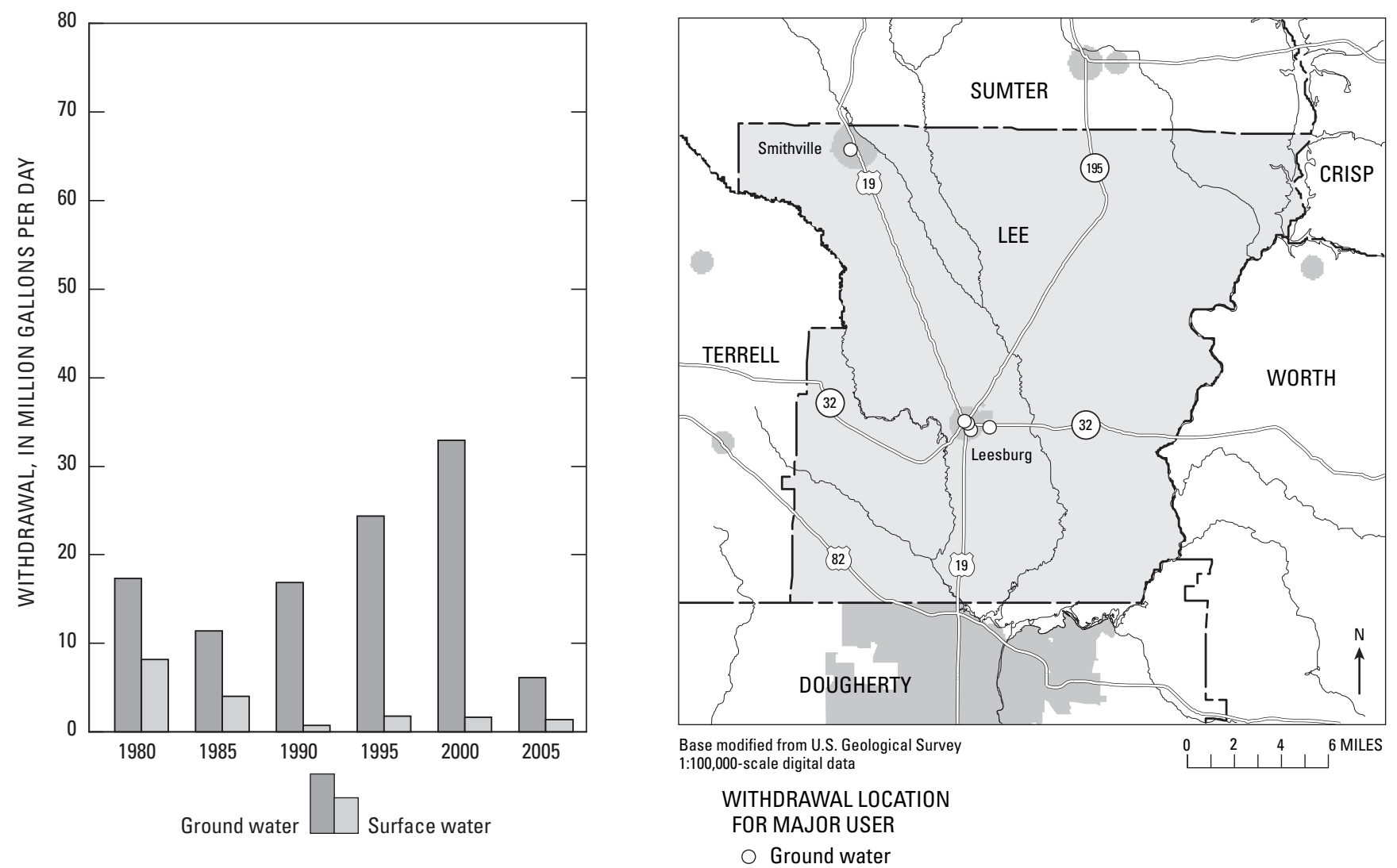


\section{LIBERTY COUNTY}

Population: 57,544

Population served by public supply: 48,340

Acres irrigated: 90

Hydroelectric use (Mgal/d): $\quad 0.00$

2005 WITHDRAWALS, IN MILLION GALLONS PER DAY

\begin{tabular}{|c|c|c|c|c|c|c|c|}
\hline & $\begin{array}{l}\text { Public } \\
\text { Supply }\end{array}$ & $\begin{array}{l}\text { Domestic \& } \\
\text { Commercial }\end{array}$ & $\begin{array}{c}\text { Industrial \& } \\
\text { Mining }\end{array}$ & Irrigation & Livestock & $\begin{array}{l}\text { Thermo- } \\
\text { electric }\end{array}$ & Totals \\
\hline Ground Water & 4.84 & 2.48 & 8.26 & 0.00 & 0.00 & 0.00 & 15.58 \\
\hline Surface Water & 0.00 & 0.00 & 0.00 & 0.20 & 0.01 & 0.00 & 0.21 \\
\hline TOTALS & 4.84 & 2.48 & 8.26 & 0.20 & 0.01 & 0.00 & 15.79 \\
\hline
\end{tabular}

Withdrawals by Major Public Suppliers (Mgal/d):

\section{Name}

City of Hinesville

City of Midway

City of Walthourville

City of Riceboro
SW

0.00

$\begin{array}{ll}0.23 & 0.00 \\ 0.43 & 0.00\end{array}$

$0.62 \quad 0.00$

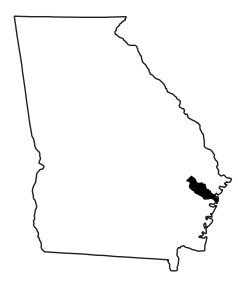

Withdrawals by Major Industrial Groups (Mgal/d):

$\begin{array}{lcc}\text { SIC } & \text { GW } & \text { SW } \\ 26 \text { - Paper } & 8.20 & 0.00\end{array}$
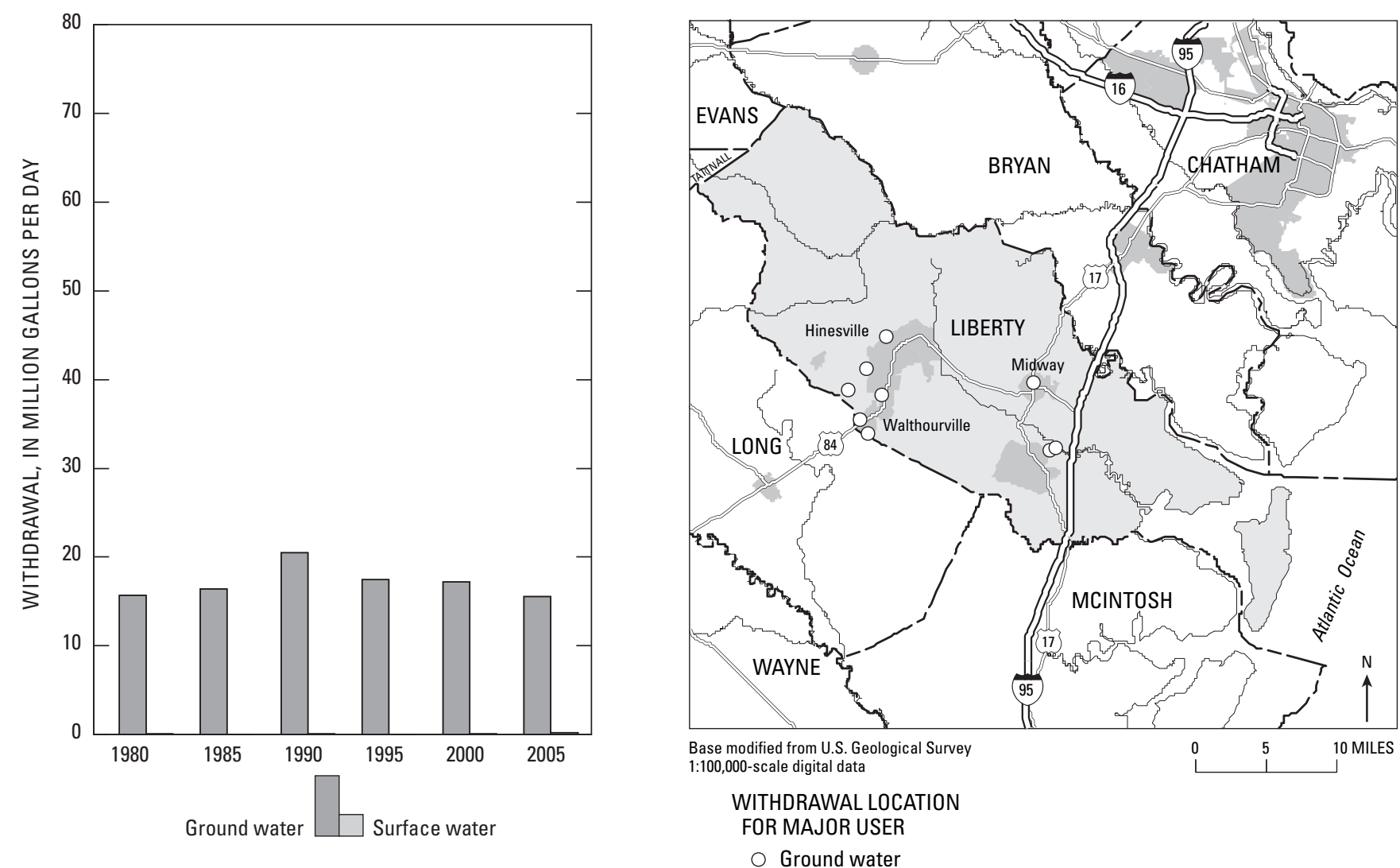


\section{LINCOLN COUNTY}

Population: $\quad 8,207$

Population served by public supply: $\quad 5,840$

Acres irrigated: 110

Hydroelectric use (Mgal/d): $\quad 0.00$

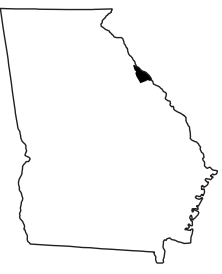

2005 WITHDRAWALS, IN MILLION GALLONS PER DAY

\begin{tabular}{|c|c|c|c|c|c|c|c|}
\hline & $\begin{array}{l}\text { Public } \\
\text { Supply }\end{array}$ & $\begin{array}{l}\text { Domestic \& } \\
\text { Commercial }\end{array}$ & $\begin{array}{c}\text { Industrial \& } \\
\text { Mining }\end{array}$ & Irrigation & Livestock & $\begin{array}{l}\text { Thermo- } \\
\text { electric }\end{array}$ & Totals \\
\hline Ground Water & 0.01 & 0.18 & 0.00 & 0.09 & 0.00 & 0.00 & 0.28 \\
\hline Surface Water & 0.38 & 0.00 & 0.00 & 0.18 & 0.07 & 0.00 & 0.63 \\
\hline TOTALS & 0.39 & 0.18 & 0.00 & 0.27 & 0.07 & 0.00 & 0.91 \\
\hline
\end{tabular}

Withdrawals by Major Public Suppliers (Mgal/d):

Name

City of Lincolnton

GW

0.00

SW

0.38
Withdrawals by Major Industrial Groups (Mgal/d):

SIC

GW

SW
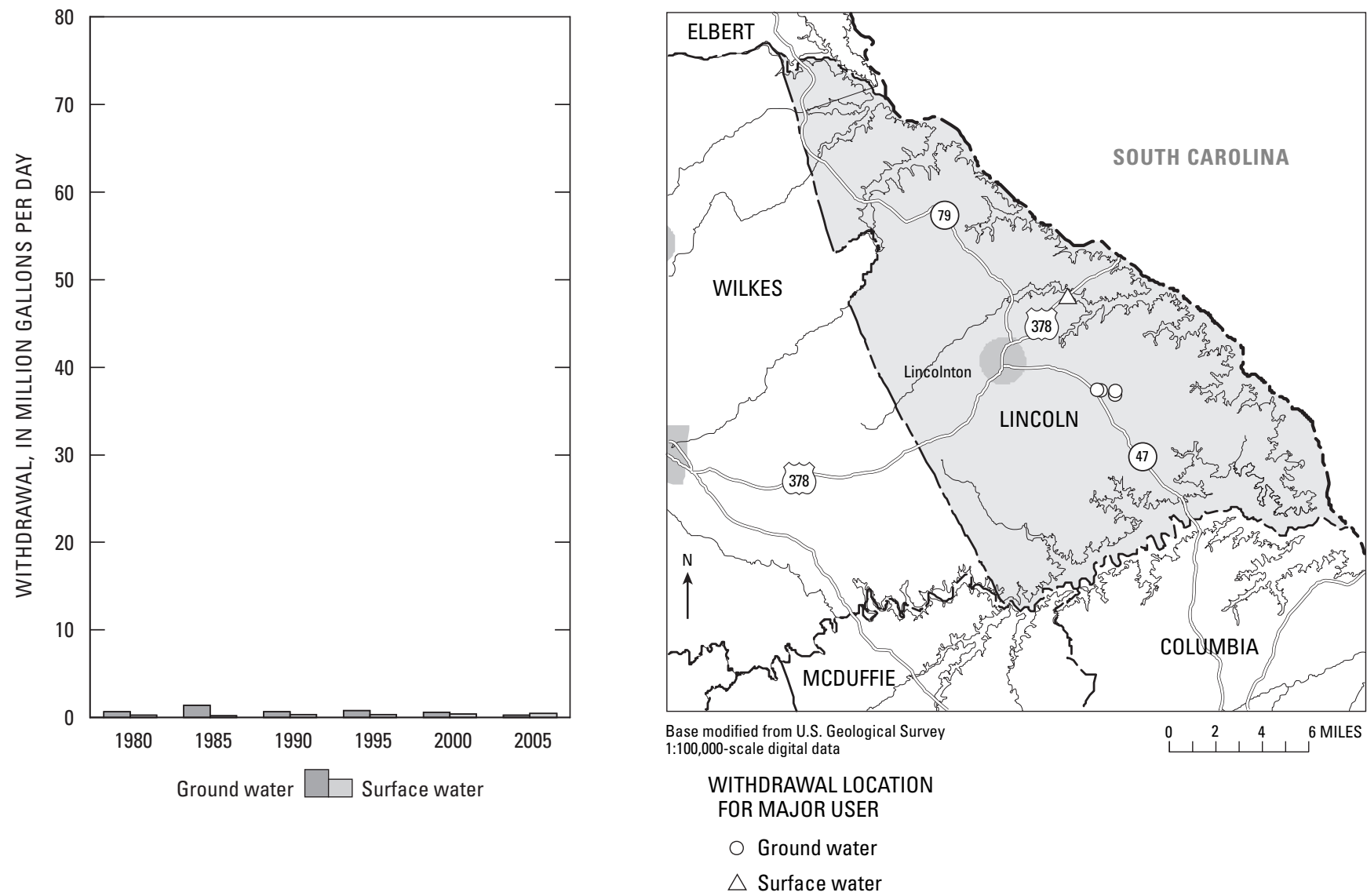


\section{LONG COUNTY}

Population: 11,083

Population served by public supply: 3,350

Acres irrigated: 740

Hydroelectric use (Mgal/d): $\quad 0.00$

2005 WITHDRAWALS, IN MILLION GALLONS PER DAY

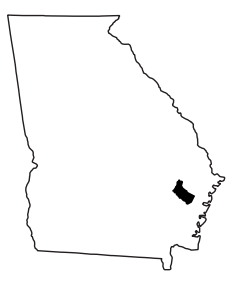

\begin{tabular}{|c|c|c|c|c|c|c|c|}
\hline & $\begin{array}{l}\text { Public } \\
\text { Supply }\end{array}$ & $\begin{array}{l}\text { Domestic \& } \\
\text { Commercial }\end{array}$ & $\begin{array}{l}\text { Industrial \& } \\
\text { Mining }\end{array}$ & Irrigation & Livestock & $\begin{array}{l}\text { Thermo- } \\
\text { electric }\end{array}$ & Totals \\
\hline Ground Water & 1.49 & 0.58 & 0.45 & 0.00 & 0.00 & 0.00 & 2.52 \\
\hline Surface Water & 0.00 & 0.00 & 0.01 & 0.40 & 0.05 & 0.00 & 0.46 \\
\hline TOTALS & 1.49 & 0.58 & 0.46 & 0.40 & 0.05 & 0.00 & 2.98 \\
\hline
\end{tabular}

Withdrawals by Major Public Suppliers (Mgal/d):

Name

City of Ludowici
SW 0.00
Withdrawals by Major Industrial Groups (Mgal/d):

SIC

None
SW
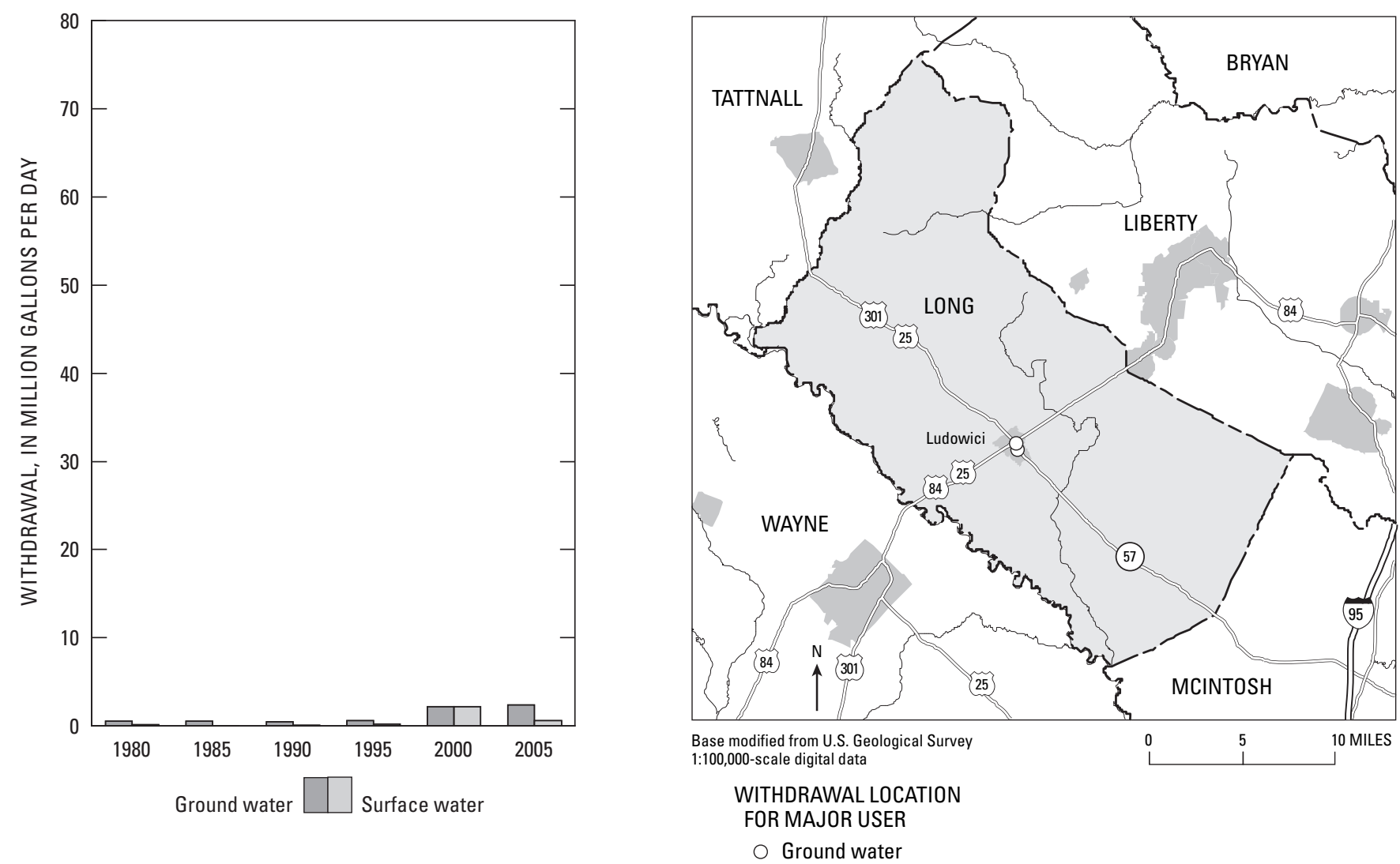


\section{LOWNDES COUNTY}

Population: 96,705

Population served by public supply: $\quad 79,830$

Acres irrigated: 13,680

Hydroelectric use (Mgal/d): $\quad 0.00$

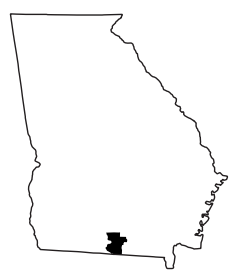

2005 WITHDRAWALS, IN MILLION GALLONS PER DAY

\begin{tabular}{|c|c|c|c|c|c|c|c|}
\hline & $\begin{array}{l}\text { Public } \\
\text { Supply }\end{array}$ & $\begin{array}{l}\text { Domestic \& } \\
\text { Commercial }\end{array}$ & $\begin{array}{c}\text { Industrial \& } \\
\text { Mining }\end{array}$ & Irrigation & Livestock & $\begin{array}{l}\text { Thermo- } \\
\text { electric }\end{array}$ & Totals \\
\hline Ground Water & 10.59 & 1.65 & 9.04 & 7.99 & 0.03 & 0.00 & 29.30 \\
\hline Surface Water & 0.00 & 0.00 & 0.00 & 1.37 & 0.05 & 0.00 & 1.42 \\
\hline TOTALS & 10.59 & 1.65 & 9.04 & 9.36 & 0.08 & 0.00 & 30.72 \\
\hline
\end{tabular}

Withdrawals by Major Public Suppliers (Mgal/d):

Name

GW SW

Town of Clyattville

City of Hahira

Town of Lake Park

Lowndes County Water System

0.02

0.26

0.04

1.79

0.05

City of Valdosta
SW
0.00

0.00

0.00

0.00

0.00

0.00

Withdrawals by Major Industrial Groups (Mgal/d):

SIC

26 - Paper

28 - Chemicals

36 - Electrical machinery
GW

8.11

0.85

0.08
SW

0.00

0.00

0.00
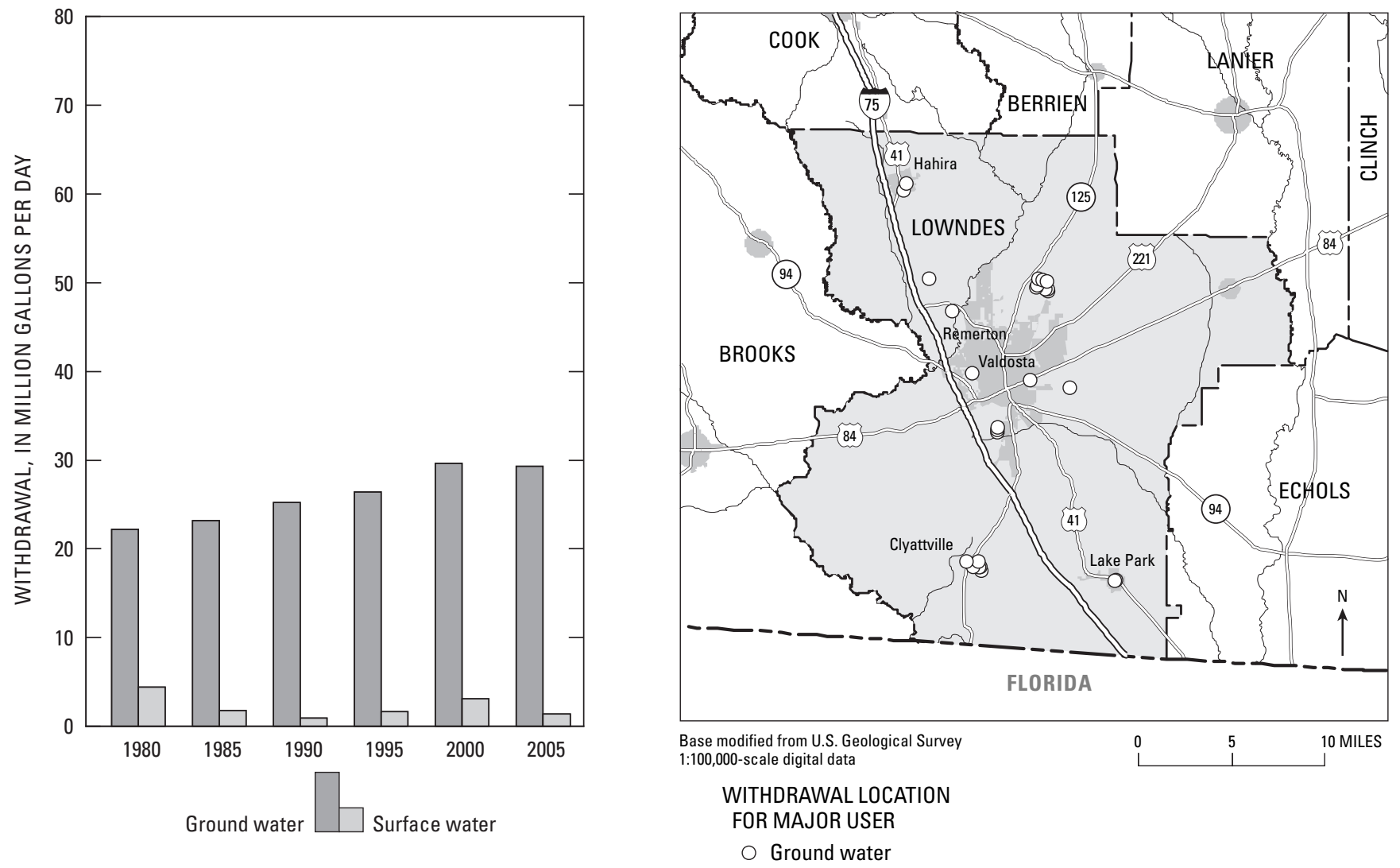


\section{LUMPKIN COUNTY}

Population: 24,324

Population served by public supply: 4,450

Acres irrigated: 190

Hydroelectric use (Mgal/d): $\quad 0.00$

2005 WITHDRAWALS, IN MILLION GALLONS PER DAY

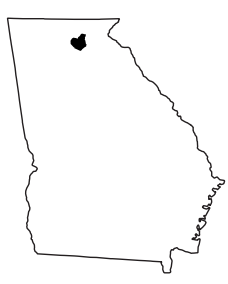

\begin{tabular}{|c|c|c|c|c|c|c|c|}
\hline & $\begin{array}{l}\text { Public } \\
\text { Supply }\end{array}$ & $\begin{array}{l}\text { Domestic \& } \\
\text { Commercial }\end{array}$ & $\begin{array}{c}\text { Industrial \& } \\
\text { Mining }\end{array}$ & Irrigation & Livestock & $\begin{array}{l}\text { Thermo- } \\
\text { electric }\end{array}$ & Totals \\
\hline Ground Water & 0.41 & 1.49 & 0.00 & 0.03 & 0.00 & 0.00 & 1.93 \\
\hline Surface Water & 0.80 & 0.00 & 0.14 & 0.15 & 0.22 & 0.00 & 1.31 \\
\hline TOTALS & 1.21 & 1.49 & 0.14 & 0.18 & 0.22 & 0.00 & 3.24 \\
\hline
\end{tabular}

Withdrawals by Major Public Suppliers (Mgal/d):

Name

City of Dahlonega

GW

0.30

SW

0.80
Withdrawals by Major Industrial Groups (Mgal/d):

SIC

14 - Mining

GW

0.00

SW

0.14
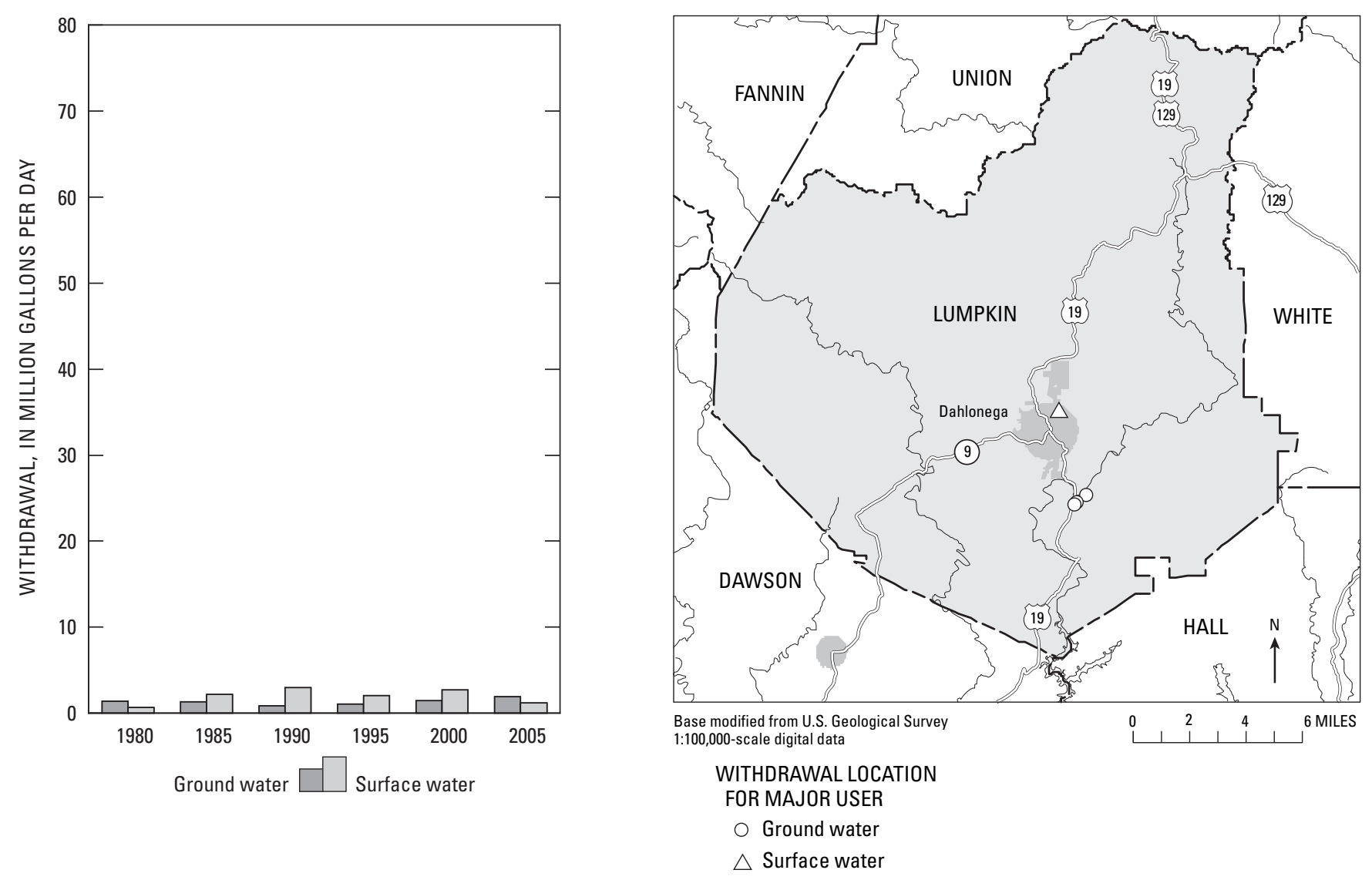


\section{MACON COUNTY}

Population: 13,745

Population served by public supply: 2,720

Acres irrigated: 28,210

Hydroelectric use (Mgal/d): 0.00

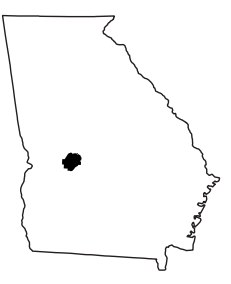

2005 WITHDRAWALS, IN MILLION GALLONS PER DAY

\begin{tabular}{|c|c|c|c|c|c|c|c|}
\hline & $\begin{array}{l}\text { Public } \\
\text { Supply }\end{array}$ & $\begin{array}{l}\text { Domestic \& } \\
\text { Commercial }\end{array}$ & $\begin{array}{c}\text { Industrial \& } \\
\text { Mining }\end{array}$ & Irrigation & Livestock & $\begin{array}{l}\text { Thermo- } \\
\text { electric }\end{array}$ & Totals \\
\hline Ground Water & 1.59 & 0.44 & 0.96 & 13.77 & 0.12 & 0.00 & 16.88 \\
\hline Surface Water & 0.00 & 0.00 & 9.61 & 4.49 & 0.49 & 0.00 & 14.59 \\
\hline TOTALS & 1.59 & 0.44 & 10.57 & 18.26 & 0.61 & 0.00 & 31.47 \\
\hline
\end{tabular}

Withdrawals by Major Public Suppliers (Mgal/d):

Name

Town of Ideal

Town of Marshallville

City of Montezuma

City of Oglethorpe
GW SW

$0.04 \quad 0.00$

$0.14 \quad 0.00$

$0.94 \quad 0.00$

$0.47 \quad 0.00$
Withdrawals by Major Industrial Groups (Mgal/d):

$\begin{array}{lcc}\text { SIC } & \text { GW } & \text { SW } \\ 20 \text { - Food } & 0.46 & 0.00 \\ 26 \text { - Paper } & 0.47 & 9.61 \\ 32 \text { - Stone, clay } & 0.03 & 0.00\end{array}$
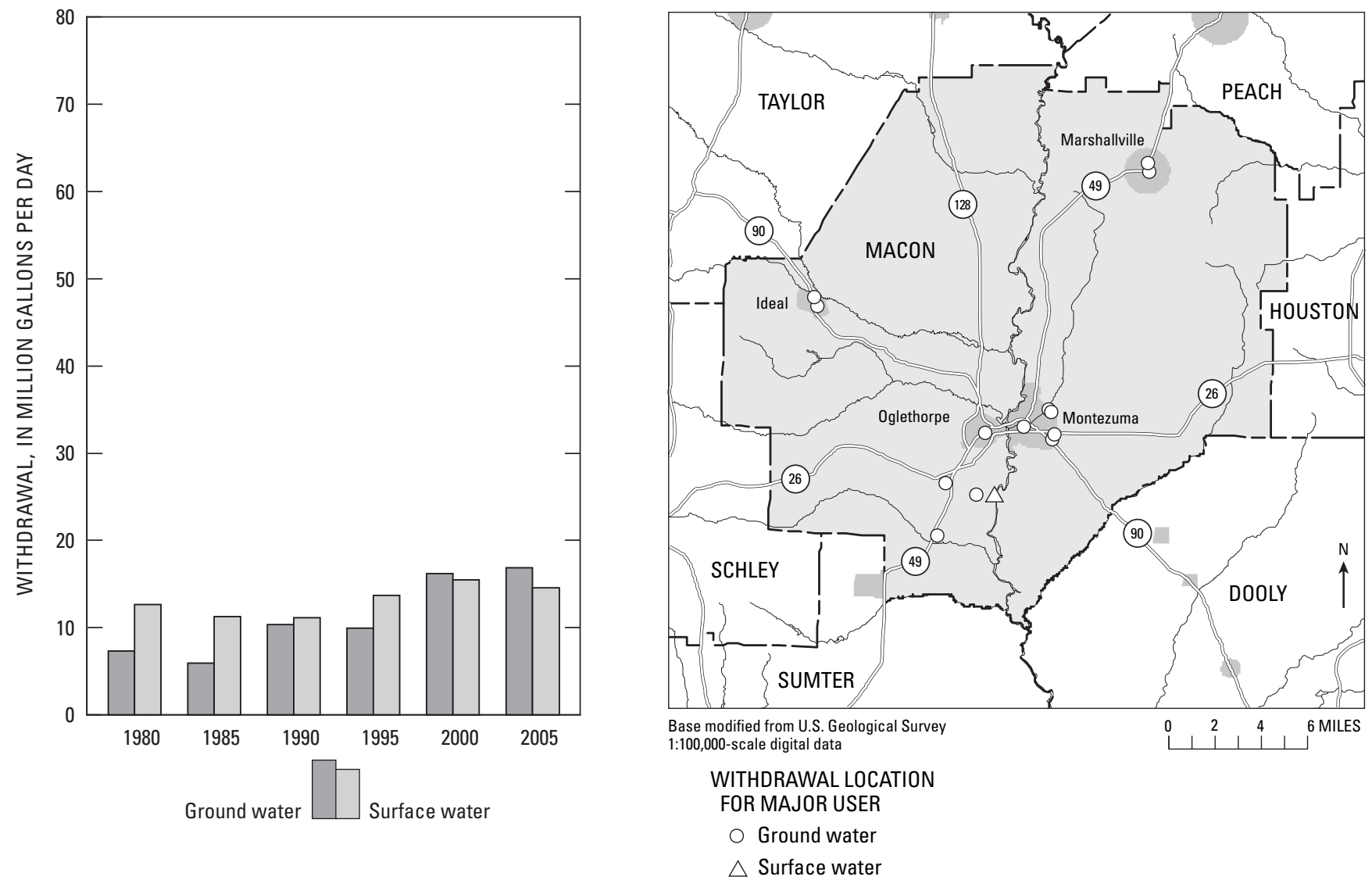


\section{MADISON COUNTY}

Population: 27,289

Population served by public supply: $\quad 12,830$

Acres irrigated: 340

Hydroelectric use (Mgal/d): $\quad 0.00$

2005 WITHDRAWALS, IN MILLION GALLONS PER DAY

\begin{tabular}{|c|c|c|c|c|c|c|c|}
\hline & $\begin{array}{l}\text { Public } \\
\text { Supply }\end{array}$ & $\begin{array}{l}\text { Domestic \& } \\
\text { Commercial }\end{array}$ & $\begin{array}{c}\text { Industrial \& } \\
\text { Mining }\end{array}$ & Irrigation & Livestock & $\begin{array}{l}\text { Thermo- } \\
\text { electric }\end{array}$ & Totals \\
\hline Ground Water & 0.42 & 1.64 & 0.09 & 0.09 & 0.00 & 0.00 & 2.24 \\
\hline Surface Water & 0.00 & 0.00 & 0.21 & 0.25 & 0.86 & 0.00 & 1.32 \\
\hline TOTALS & 0.42 & 1.64 & 0.30 & 0.34 & 0.86 & 0.00 & 3.56 \\
\hline
\end{tabular}

Withdrawals by Major Public Suppliers (Mgal/d):

Name

Town of Carlton

City of Colbert

Town of Comer

City of Danielsville

City of Ila
GW SW

$0.02 \quad 0.00$

$0.09 \quad 0.00$

$0.09 \quad 0.00$

$0.11 \quad 0.00$

$0.02 \quad 0.00$

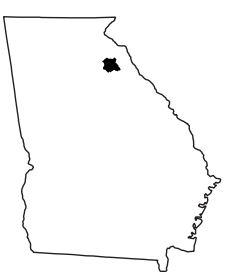

Withdrawals by Major Industrial Groups (Mgal/d):

SIC

32 - Stone, clay
GW

0.00
SW

0.21
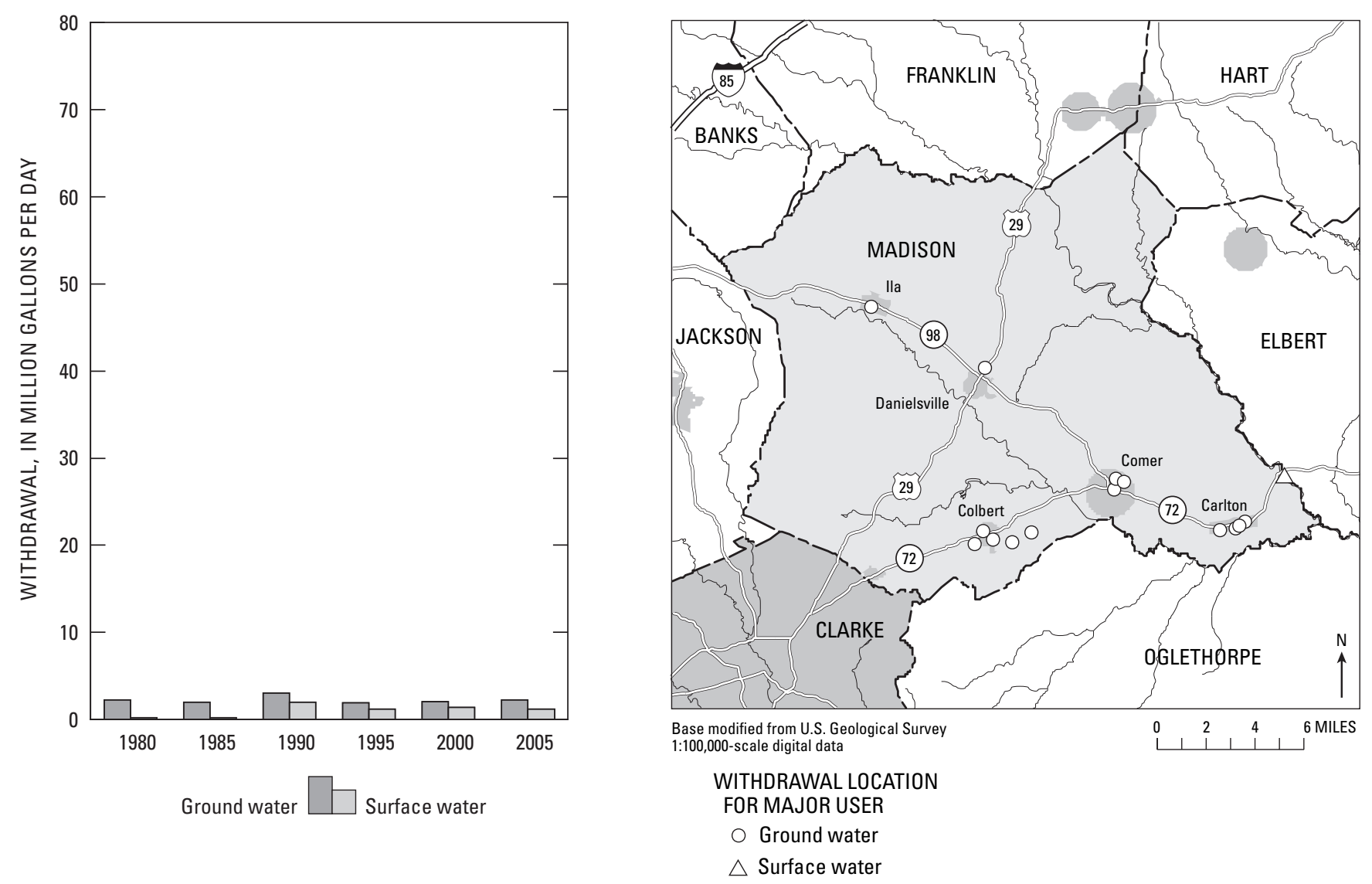


\section{MARION COUNTY}

Population: 7,244

Population served by public supply: $\quad 8,300$

Acres irrigated: 3,690

Hydroelectric use (Mgal/d): 0.00

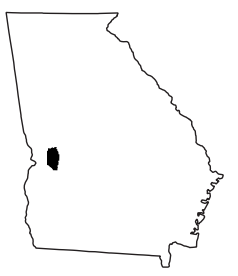

2005 WITHDRAWALS, IN MILLION GALLONS PER DAY

\begin{tabular}{|c|c|c|c|c|c|c|c|}
\hline & $\begin{array}{l}\text { Public } \\
\text { Supply }\end{array}$ & $\begin{array}{l}\text { Domestic \& } \\
\text { Commercial }\end{array}$ & $\begin{array}{c}\text { Industrial \& } \\
\text { Mining }\end{array}$ & Irrigation & Livestock & $\begin{array}{l}\text { Thermo- } \\
\text { electric }\end{array}$ & Totals \\
\hline Ground Water & 1.91 & 0.49 & 0.29 & 0.19 & 0.01 & 0.00 & 2.89 \\
\hline Surface Water & 0.00 & 0.00 & 0.00 & 0.30 & 0.08 & 0.00 & 0.38 \\
\hline TOTALS & 1.91 & 0.49 & 0.29 & 0.49 & 0.09 & 0.00 & 3.27 \\
\hline
\end{tabular}

Withdrawals by Major Public Suppliers (Mgal/d):

Name

GW SW

City of Buena Vista

$1.44 \quad 0.00$

Marion County Water System
Withdrawals by Major Industrial Groups (Mgal/d):

SIC

None
GW

SW
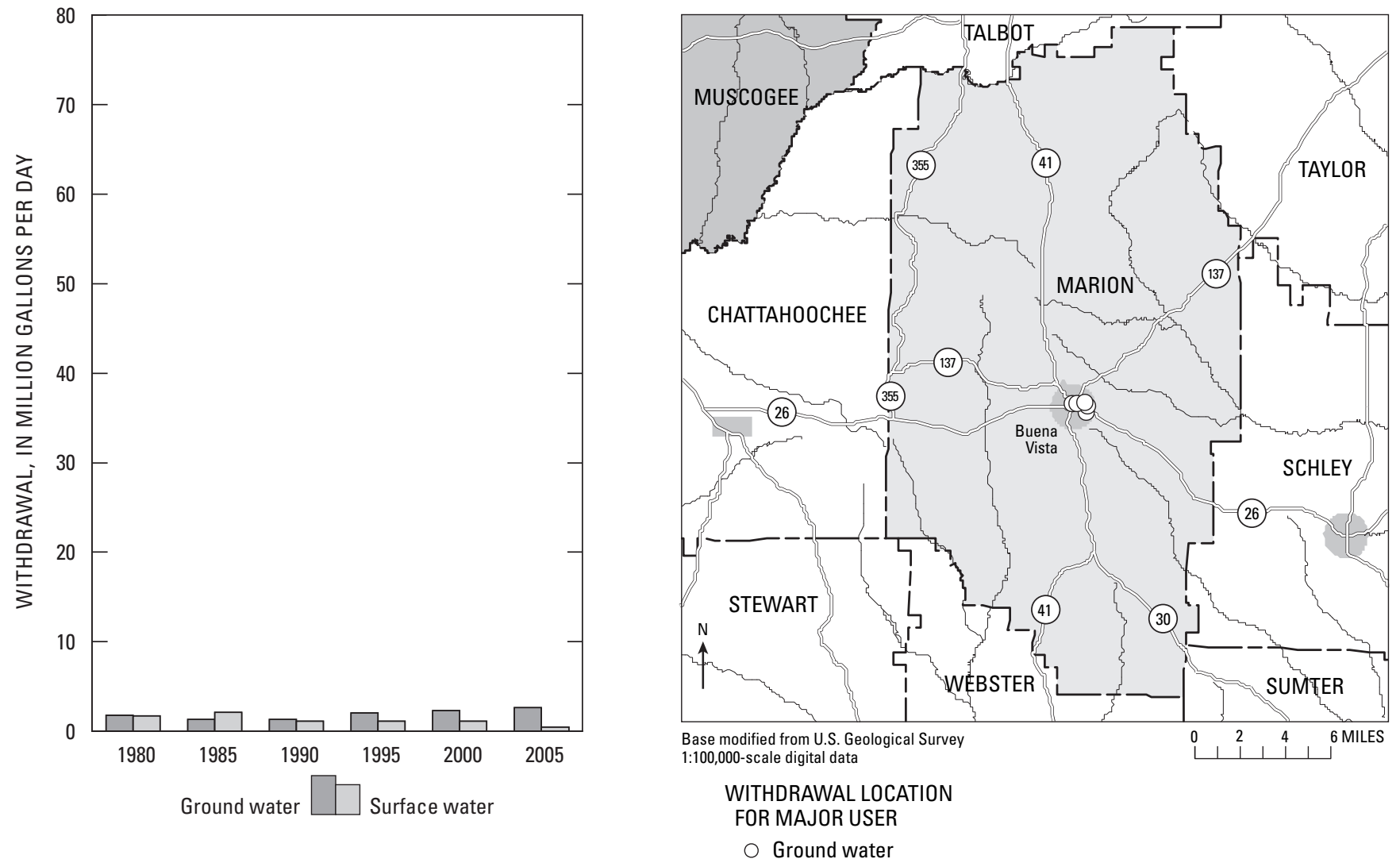


\section{MCDUFFIE COUNTY}

Population: 21,743

Population served by public supply: $\quad 7,910$

Acres irrigated: 1,560

Hydroelectric use (Mgal/d): $\quad 0.00$

2005 WITHDRAWALS, IN MILLION GALLONS PER DAY

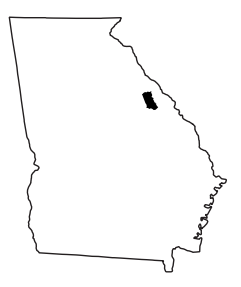

\begin{tabular}{|c|c|c|c|c|c|c|c|}
\hline & $\begin{array}{l}\text { Public } \\
\text { Supply }\end{array}$ & $\begin{array}{l}\text { Domestic \& } \\
\text { Commercial }\end{array}$ & $\begin{array}{c}\text { Industrial \& } \\
\text { Mining }\end{array}$ & Irrigation & Livestock & $\begin{array}{l}\text { Thermo- } \\
\text { electric }\end{array}$ & Totals \\
\hline Ground Water & 0.03 & 0.67 & 0.00 & 0.66 & 0.00 & 0.00 & 1.36 \\
\hline Surface Water & 2.58 & 0.00 & 0.00 & 1.77 & 0.14 & 0.00 & 4.49 \\
\hline TOTALS & 2.61 & 0.67 & 0.00 & 2.43 & 0.14 & 0.00 & 5.85 \\
\hline
\end{tabular}

Withdrawals by Major Public Suppliers (Mgal/d):

\section{Name}

Town of Dearing

GW

0.03

SW

Thomson-McDuffie County

Water Commission
Withdrawals by Major Industrial Groups (Mgal/d):

SIC

None
SW
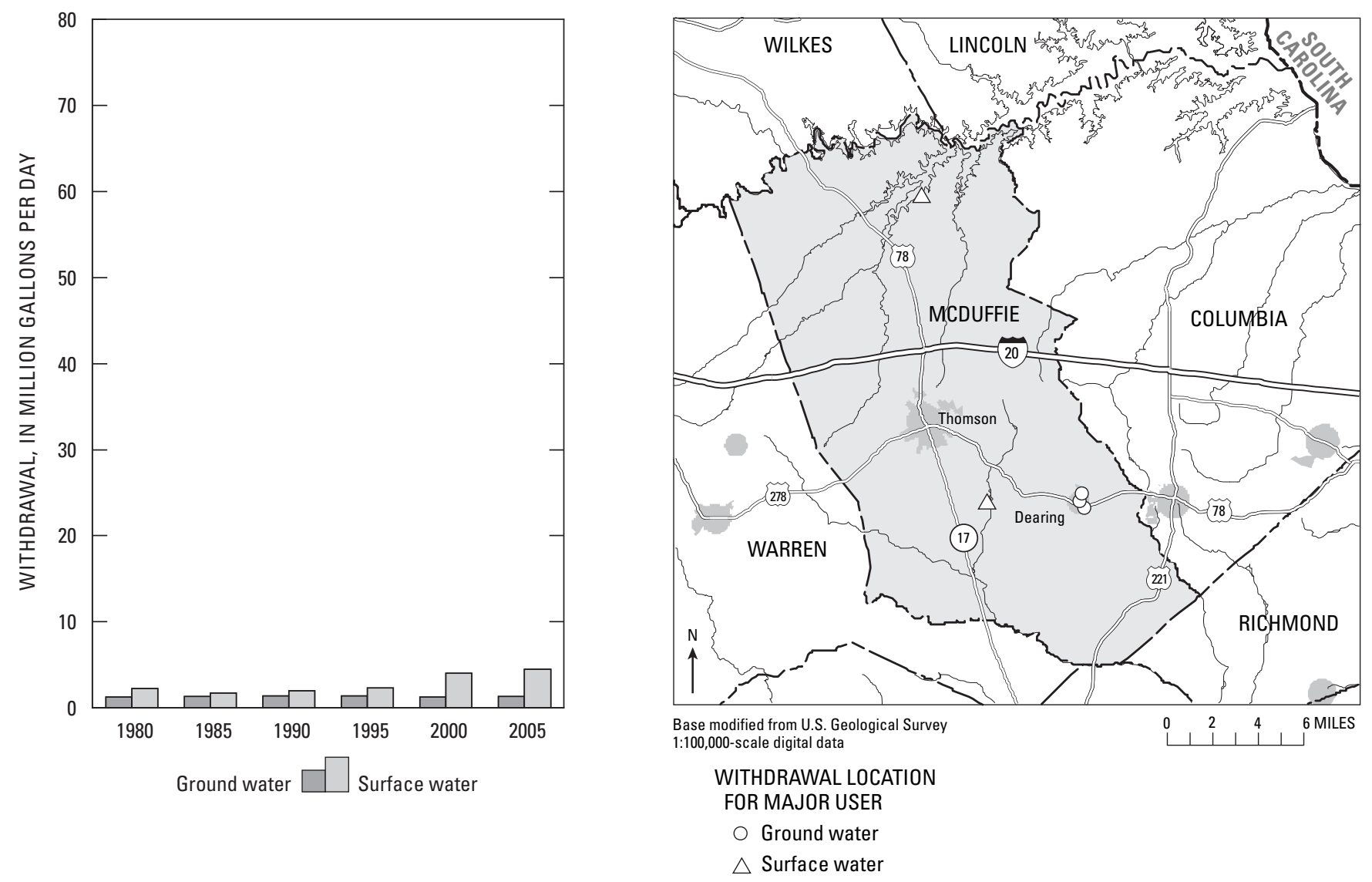


\section{MCINTOSH COUNTY}

Population: 11,068

Population served by public supply: $\quad 5,380$

Acres irrigated: 100

Hydroelectric use (Mgal/d): $\quad 0.00$

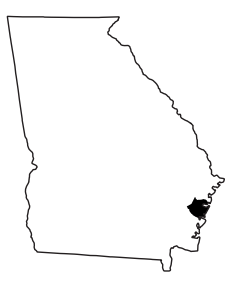

2005 WITHDRAWALS, IN MILLION GALLONS PER DAY

\begin{tabular}{|c|c|c|c|c|c|c|c|}
\hline & $\begin{array}{l}\text { Public } \\
\text { Supply }\end{array}$ & $\begin{array}{l}\text { Domestic \& } \\
\text { Commercial }\end{array}$ & $\begin{array}{c}\text { Industrial \& } \\
\text { Mining }\end{array}$ & Irrigation & Livestock & $\begin{array}{l}\text { Thermo- } \\
\text { electric }\end{array}$ & Totals \\
\hline Ground Water & 0.73 & 0.21 & 0.00 & 0.00 & 0.00 & 0.00 & 0.94 \\
\hline Surface Water & 0.00 & 0.00 & 0.00 & 0.15 & 0.00 & 0.00 & 0.15 \\
\hline TOTALS & 0.73 & 0.21 & 0.00 & 0.15 & 0.00 & 0.00 & 1.09 \\
\hline
\end{tabular}

Withdrawals by Major Public Suppliers (Mgal/d):

\section{Name}

City of Darien

GW

0.32

SW

Hog Hammock Commission
Withdrawals by Major Industrial Groups (Mgal/d):

SIC

GW

SW
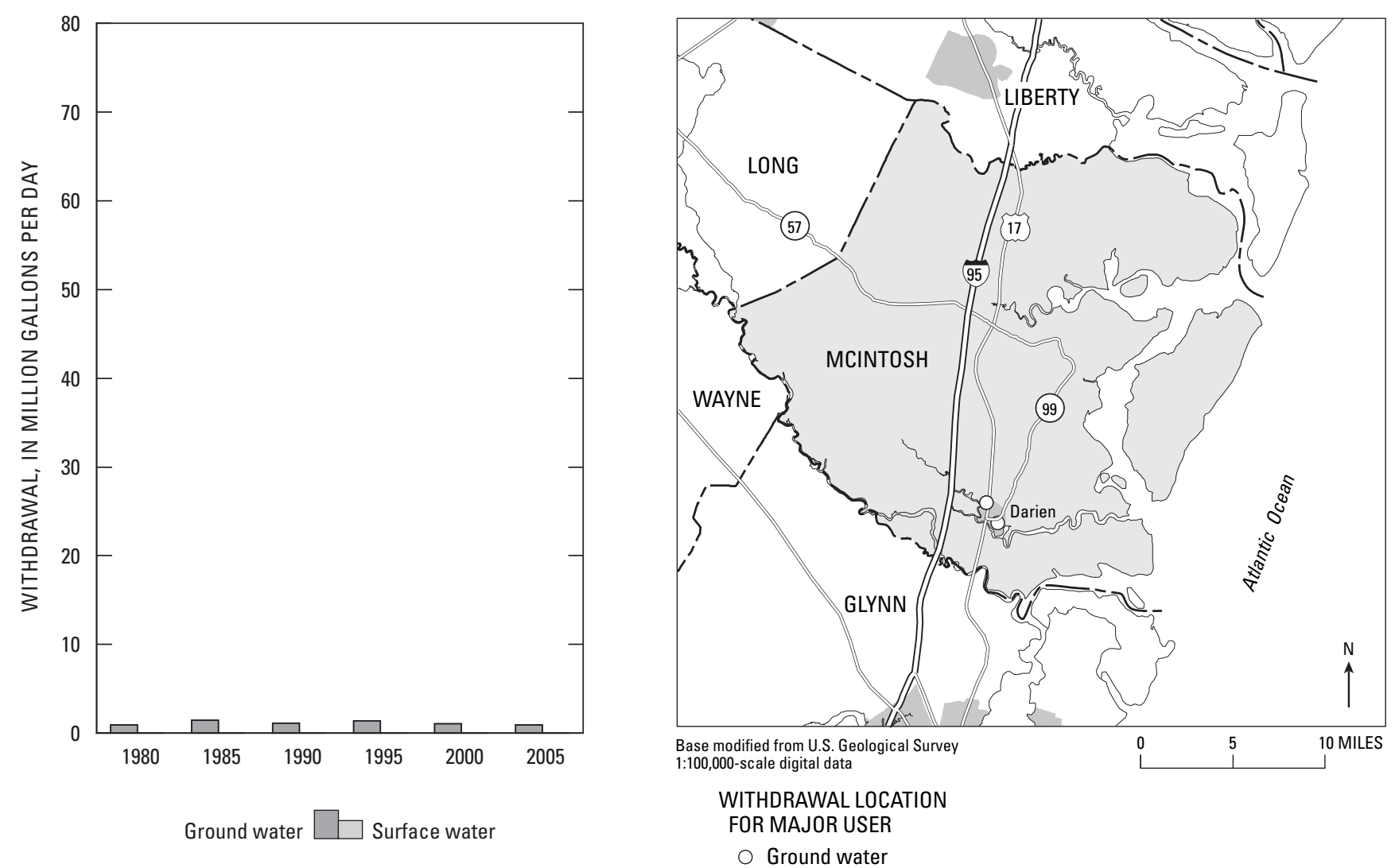


\section{MERIWETHER COUNTY}

Population: 22,919

Population served by public supply: $\quad 9,870$

Acres irrigated: 350

Hydroelectric use (Mgal/d): $\quad 0.00$

2005 WITHDRAWALS, IN MILLION GALLONS PER DAY

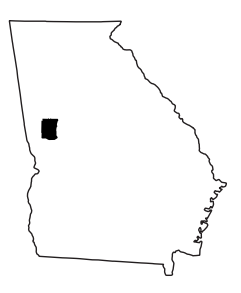

\begin{tabular}{lccccccc}
\hline & $\begin{array}{c}\text { Public } \\
\text { Supply }\end{array}$ & $\begin{array}{c}\text { Domestic \& } \\
\text { Commercial }\end{array}$ & $\begin{array}{c}\text { Industrial \& } \\
\text { Mining }\end{array}$ & Irrigation & Livestock & $\begin{array}{c}\text { Thermo- } \\
\text { electric }\end{array}$ & Totals \\
\hline Ground Water & 0.18 & 1.08 & 0.09 & 0.49 & 0.00 & 0.00 & 1.84 \\
Surface Water & 1.23 & 0.00 & 0.00 & 0.29 & 0.22 & 0.00 & 1.74 \\
TOTALS & 1.41 & 1.08 & 0.09 & 0.78 & 0.22 & 0.00 & 3.58 \\
\hline
\end{tabular}

Withdrawals by Major Public Suppliers (Mgal/d):

\section{Name}

Lone Oak Water System

Town of Luthersville

City of Warm Springs

Town of Woodbury

City of Manchester (water source located in Talbot County)

\section{$\mathrm{GW}$ \\ SW}

0.03

0.05

0.08

0.00

0.00
0.00

0.00

0.00

0.15

1.09
Withdrawals by Major Industrial Groups (Mgal/d):

SIC

None
GW

SW
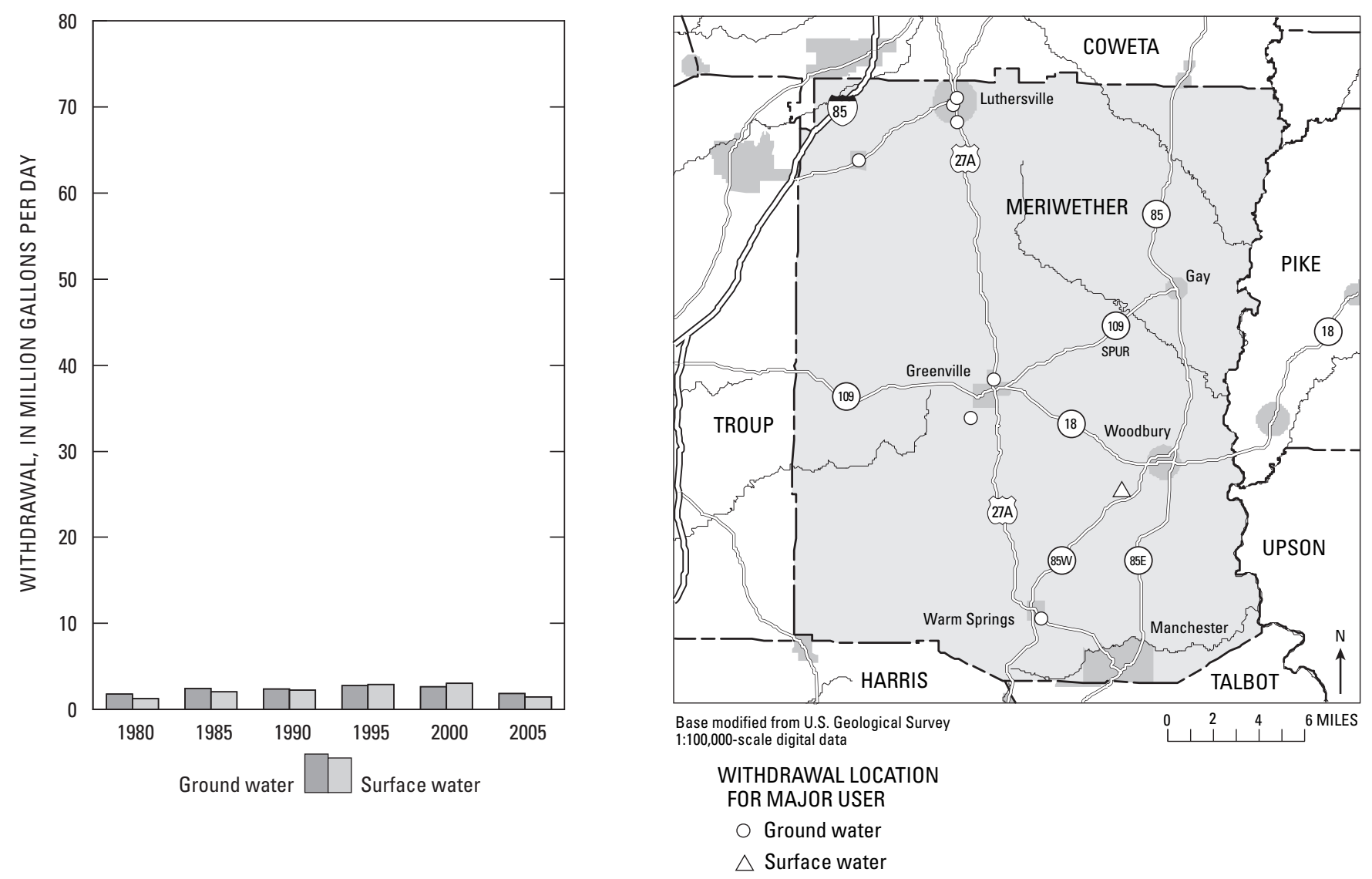


\section{MILLER COUNTY}

Population: 6,228

Population served by public supply: 2,450

Acres irrigated: 43,030

Hydroelectric use (Mgal/d): 0.00

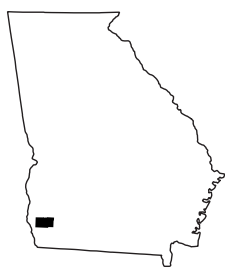

2005 WITHDRAWALS, IN MILLION GALLONS PER DAY

\begin{tabular}{|c|c|c|c|c|c|c|c|}
\hline & $\begin{array}{l}\text { Public } \\
\text { Supply }\end{array}$ & $\begin{array}{l}\text { Domestic \& } \\
\text { Commercial }\end{array}$ & $\begin{array}{c}\text { Industrial \& } \\
\text { Mining }\end{array}$ & Irrigation & Livestock & $\begin{array}{l}\text { Thermo- } \\
\text { electric }\end{array}$ & Totals \\
\hline Ground Water & 0.69 & 0.28 & 0.00 & 19.13 & 0.00 & 0.00 & 20.10 \\
\hline Surface Water & 0.00 & 0.00 & 0.00 & 1.88 & 0.11 & 0.00 & 1.99 \\
\hline TOTALS & 0.69 & 0.28 & 0.00 & 21.01 & 0.11 & 0.00 & 22.09 \\
\hline
\end{tabular}

Withdrawals by Major Public Suppliers (Mgal/d):

Name

City of Colquitt

GW

0.69

SW

0.00
Withdrawals by Major Industrial Groups (Mgal/d):

SIC

GW

SW
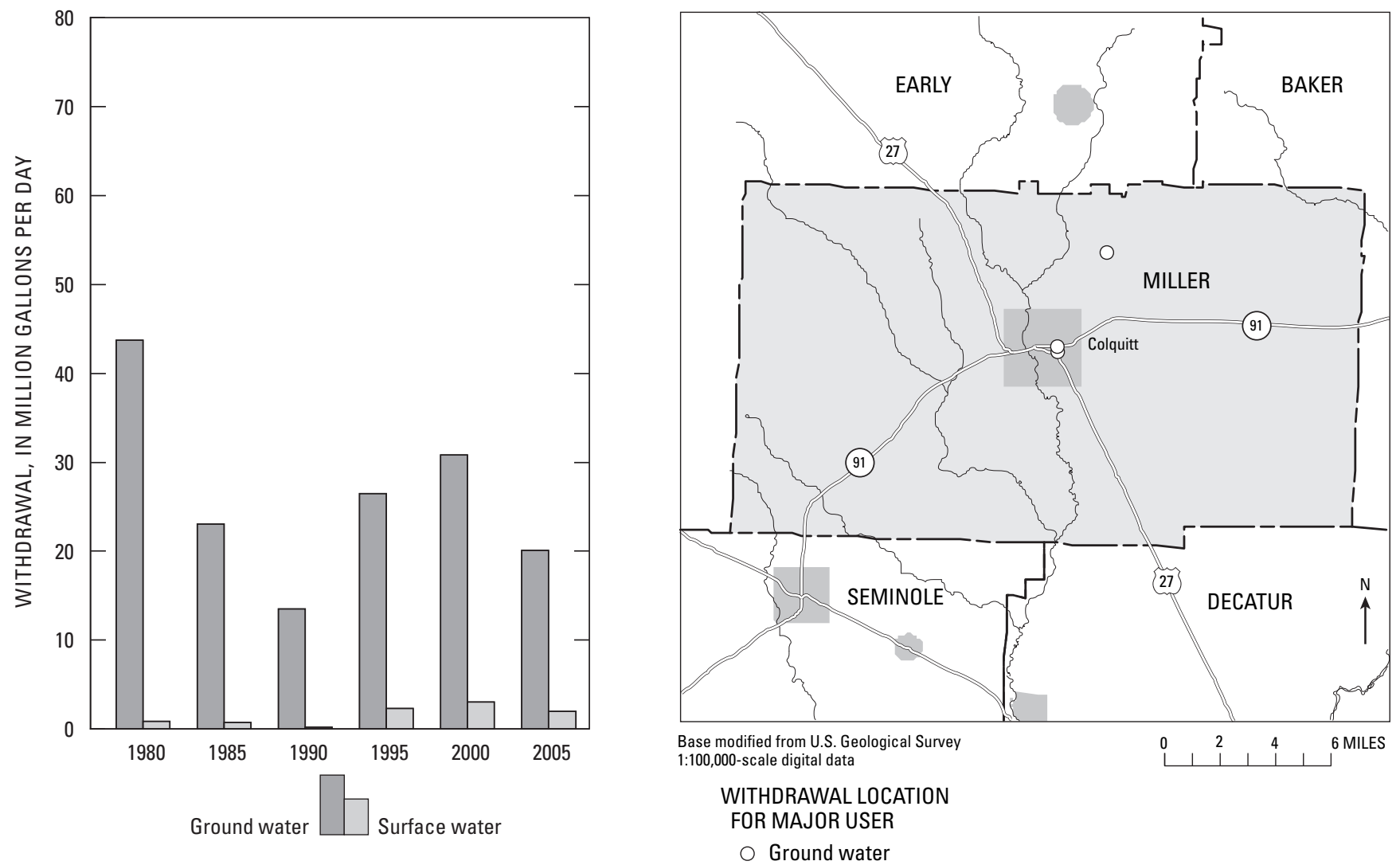


\section{MITCHELL COUNTY}

Population: 23,791

Population served by public supply: $\quad 12,880$

Acres irrigated: 78,180

Hydroelectric use (Mgal/d): $\quad 0.00$

2005 WITHDRAWALS, IN MILLION GALLONS PER DAY

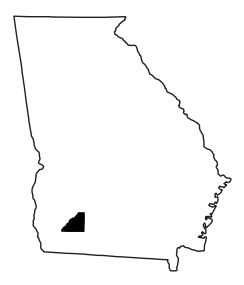

\begin{tabular}{|c|c|c|c|c|c|c|c|}
\hline & $\begin{array}{l}\text { Public } \\
\text { Supply }\end{array}$ & $\begin{array}{l}\text { Domestic \& } \\
\text { Commercial }\end{array}$ & $\begin{array}{c}\text { Industrial \& } \\
\text { Mining }\end{array}$ & Irrigation & Livestock & $\begin{array}{l}\text { Thermo- } \\
\text { electric }\end{array}$ & Totals \\
\hline Ground Water & 3.87 & 1.09 & 0.03 & 28.87 & 0.01 & 0.00 & 33.87 \\
\hline Surface Water & 0.00 & 0.00 & 0.00 & 5.12 & 0.68 & 0.00 & 5.80 \\
\hline TOTALS & 3.87 & 1.09 & 0.03 & 33.99 & 0.69 & 0.00 & 39.67 \\
\hline
\end{tabular}

Withdrawals by Major Public Suppliers (Mgal/d):

\section{Name}

City of Baconton

City of Camilla

Hinsonton Water Association

City of Pelham

Town of Sale City

GW

0.07

3.23

0.01

0.51

0.04
SW

0.00

0.00

0.00

0.00

0.00
Withdrawals by Major Industrial Groups (Mgal/d):

SIC

None
SW
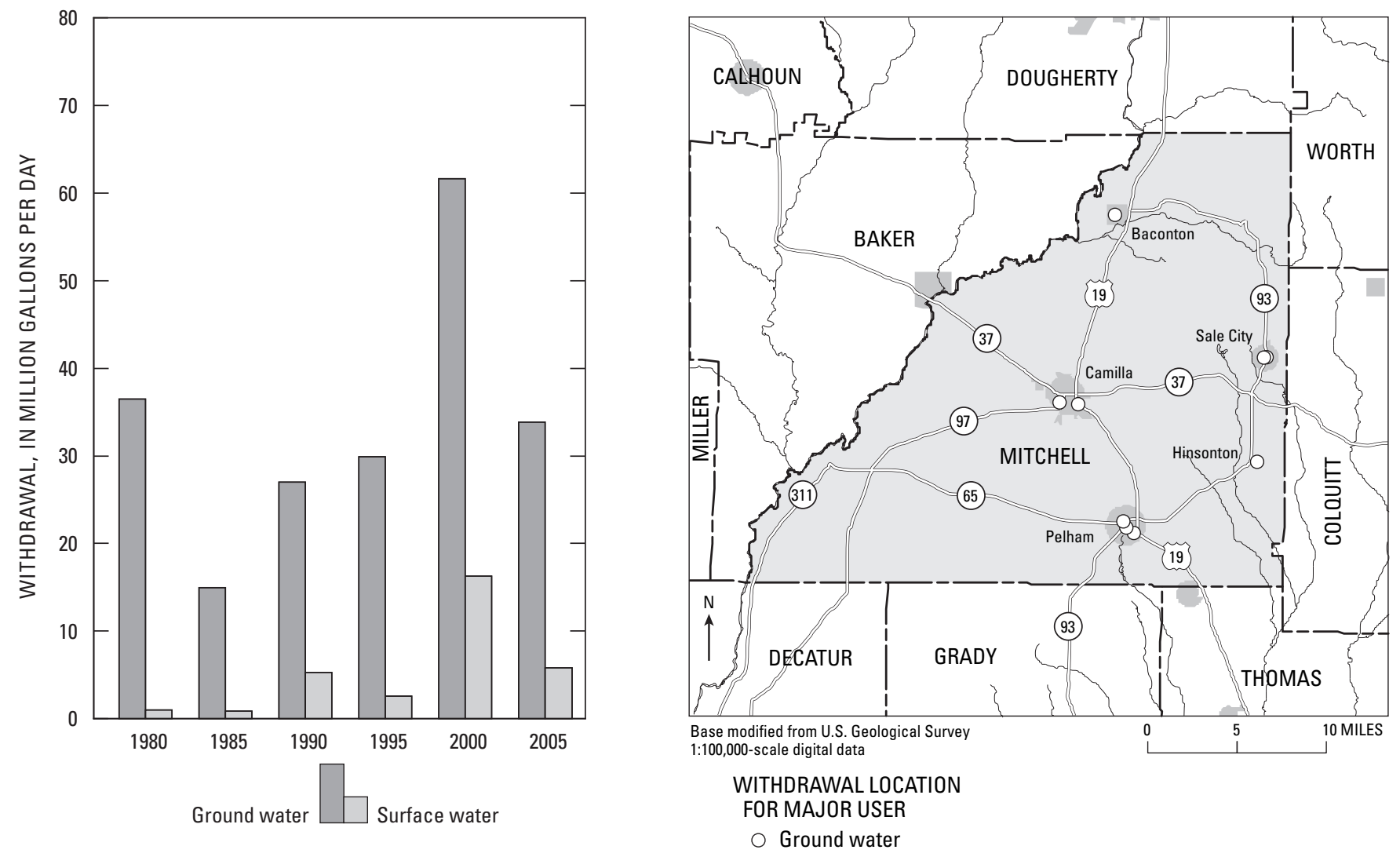


\section{MONROE COUNTY}

Population: 23,785

Population served by public supply: $\quad 8,240$

Acres irrigated: 100

Hydroelectric use (Mgal/d): $\quad 0.00$

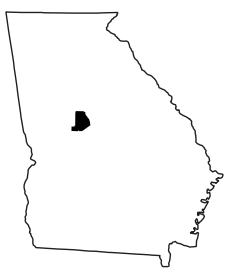

2005 WITHDRAWALS, IN MILLION GALLONS PER DAY

\begin{tabular}{lccccccc}
\hline & $\begin{array}{c}\text { Public } \\
\text { Supply }\end{array}$ & $\begin{array}{c}\text { Domestic \& } \\
\text { Commercial }\end{array}$ & $\begin{array}{c}\text { Industrial \& } \\
\text { Mining }\end{array}$ & Irrigation & Livestock & $\begin{array}{c}\text { Thermo- } \\
\text { electric }\end{array}$ & Totals \\
\cline { 1 - 5 } Ground Water & 0.15 & 1.17 & 0.69 & 0.00 & 0.00 & 0.02 & 2.03 \\
Surface Water & 1.28 & 0.00 & 0.01 & 0.15 & 0.33 & 59.00 & 60.77 \\
TOTALS & 1.43 & 1.17 & 0.70 & 0.15 & 0.33 & 59.02 & 62.80 \\
\hline
\end{tabular}

Withdrawals by Major Public Suppliers (Mgal/d):

Name

City of Culloden

City of Forsyth

$\mathrm{GW}$

0.02

0.00

SW

0.00

1.28
Withdrawals by Major Industrial Groups (Mgal/d):

SIC

None
GW SW
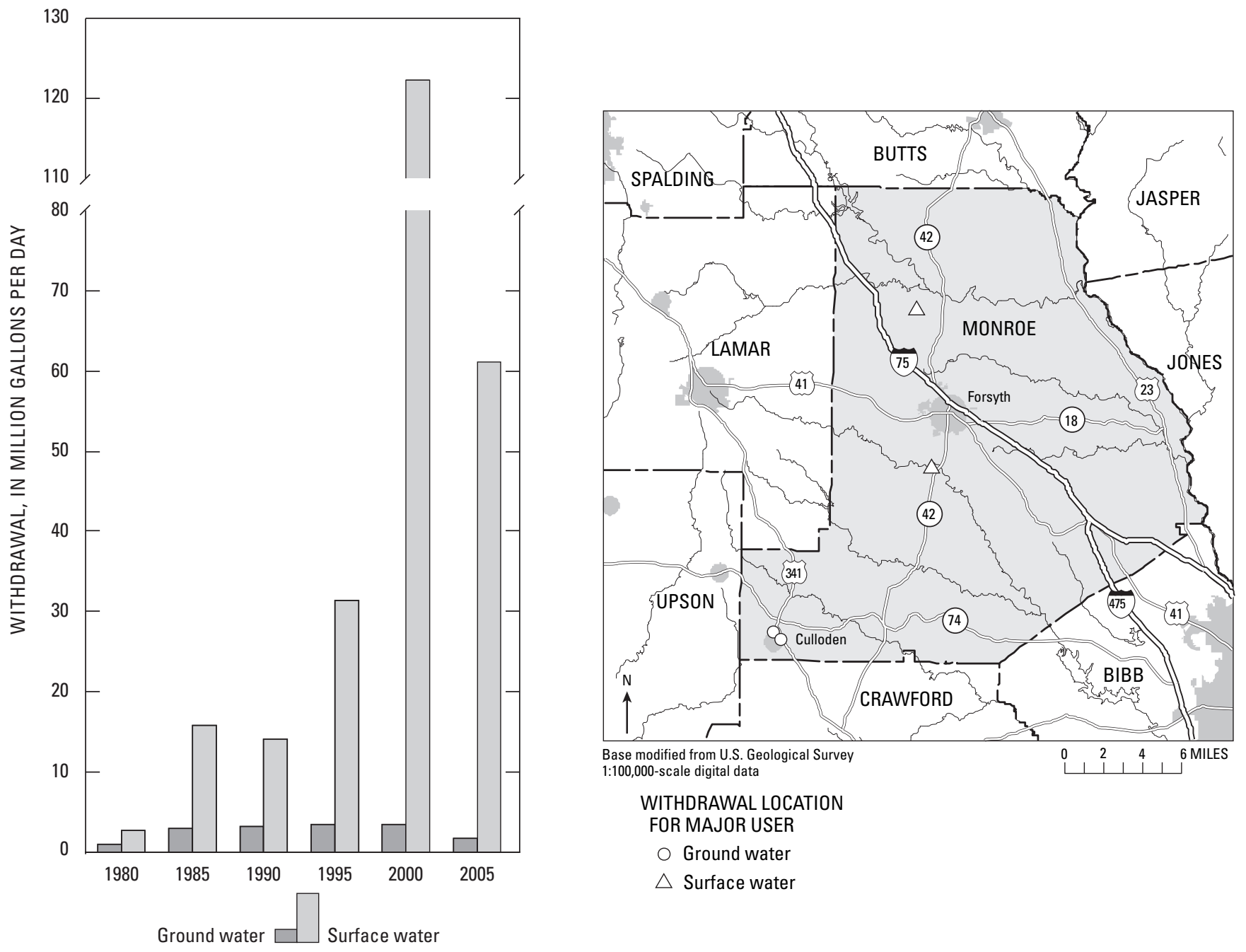


\section{MONTGOMERY COUNTY}

Population: 8,909

Population served by public supply: 5,430

Acres irrigated: 2,930

Hydroelectric use (Mgal/d): 0.00

2005 WITHDRAWALS, IN MILLION GALLONS PER DAY

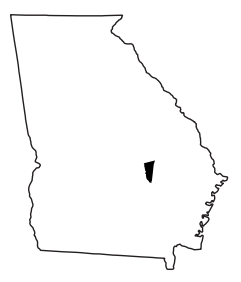

\begin{tabular}{|c|c|c|c|c|c|c|c|}
\hline & $\begin{array}{l}\text { Public } \\
\text { Supply }\end{array}$ & $\begin{array}{l}\text { Domestic \& } \\
\text { Commercial }\end{array}$ & $\begin{array}{c}\text { Industrial \& } \\
\text { Mining }\end{array}$ & Irrigation & Livestock & $\begin{array}{l}\text { Thermo- } \\
\text { electric }\end{array}$ & Totals \\
\hline Ground Water & 0.48 & 0.26 & 0.01 & 1.13 & 0.01 & 0.00 & 1.89 \\
\hline Surface Water & 0.00 & 0.00 & 0.00 & 0.52 & 0.05 & 0.00 & 0.57 \\
\hline TOTALS & 0.48 & 0.26 & 0.01 & 1.65 & 0.06 & 0.00 & 2.46 \\
\hline
\end{tabular}

Withdrawals by Major Public Suppliers (Mgal/d):

\section{Name}

Town of Ailey

Town of Alston

Charlotte Water Association

City of Mt. Vernon

Village of Tarrytown

Town of Uvalda
SW

0.00

0.00

0.00

0.00

0.00

0.00
Withdrawals by Major Industrial Groups (Mgal/d):

SIC

None
SW

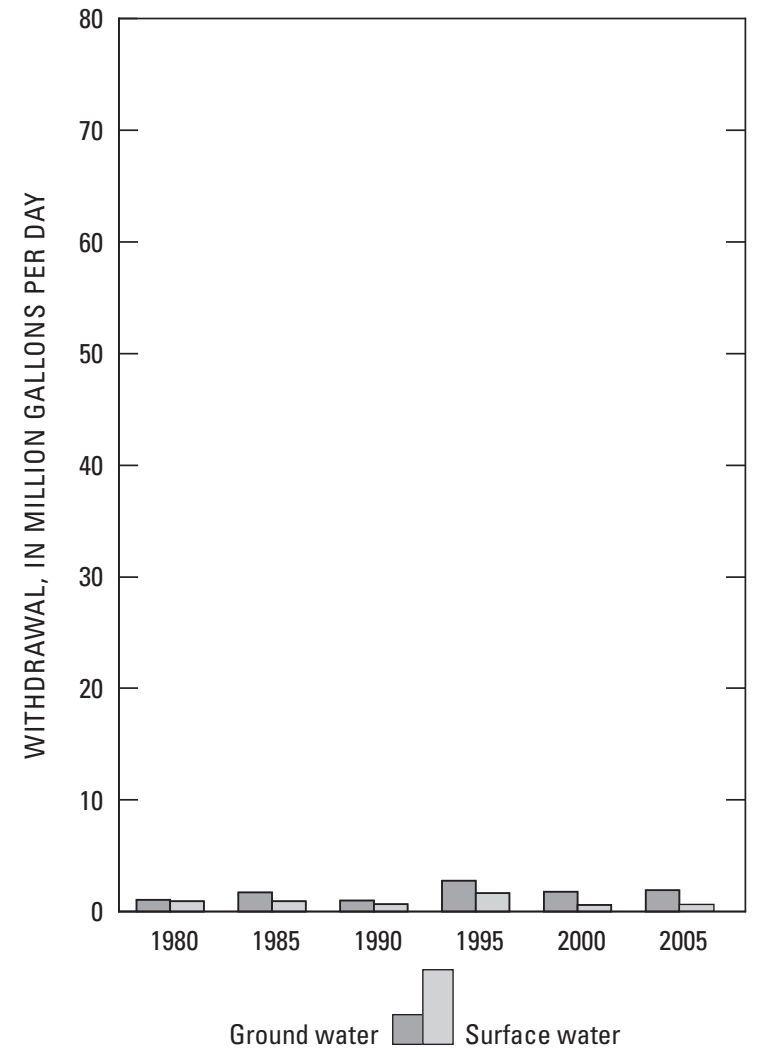

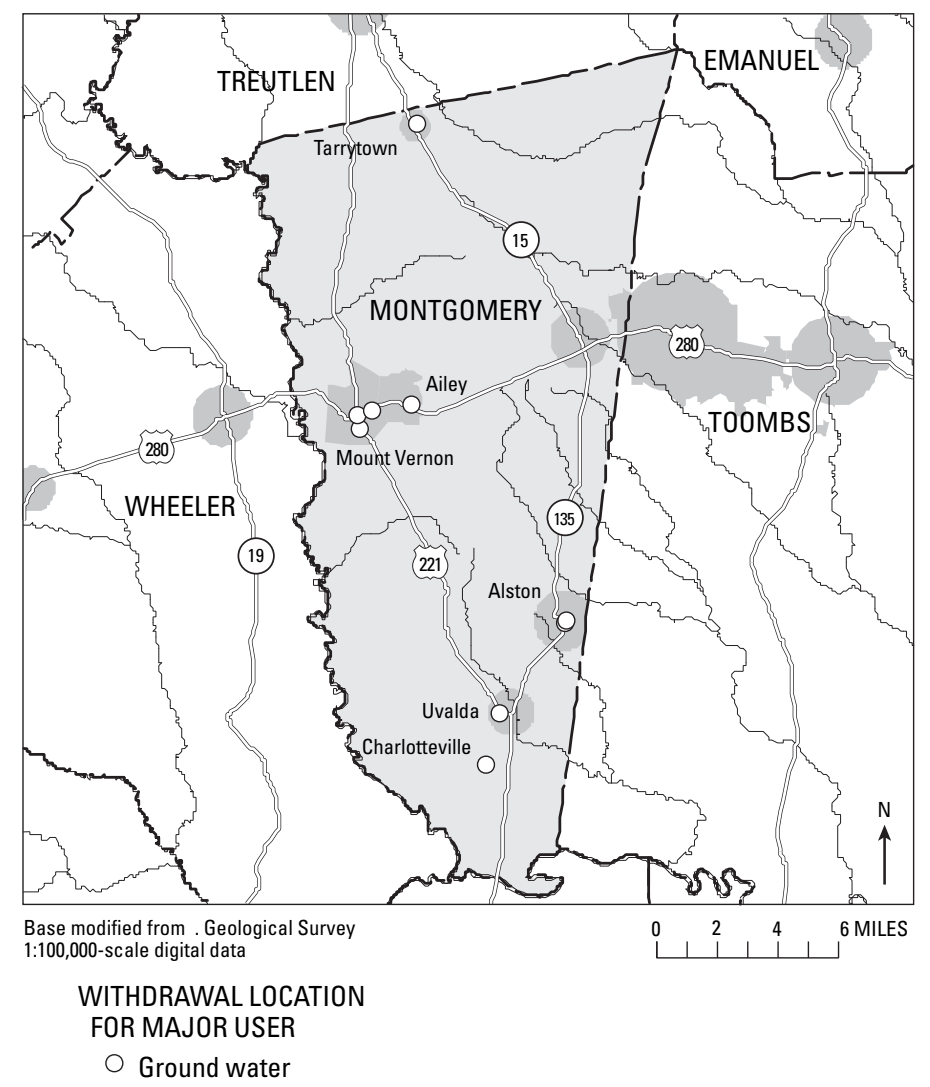




\section{MORGAN COUNTY}

Population: 17,492

Population served by public supply: $\quad 8,050$

Acres irrigated: 290

Hydroelectric use (Mgal/d): $\quad 0.00$

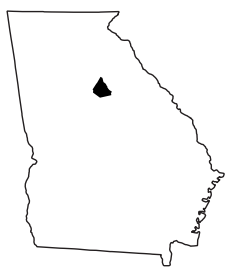

2005 WITHDRAWALS, IN MILLION GALLONS PER DAY

\begin{tabular}{|c|c|c|c|c|c|c|c|}
\hline & $\begin{array}{l}\text { Public } \\
\text { Supply }\end{array}$ & $\begin{array}{l}\text { Domestic \& } \\
\text { Commercial }\end{array}$ & $\begin{array}{c}\text { Industrial \& } \\
\text { Mining }\end{array}$ & Irrigation & Livestock & $\begin{array}{l}\text { Thermo- } \\
\text { electric }\end{array}$ & Totals \\
\hline Ground Water & 0.15 & 0.71 & 0.00 & 0.01 & 0.00 & 0.00 & 0.87 \\
\hline Surface Water & 1.30 & 0.00 & 0.14 & 0.10 & 0.51 & 0.00 & 2.05 \\
\hline TOTALS & 1.45 & 0.71 & 0.14 & 0.11 & 0.51 & 0.00 & 2.92 \\
\hline
\end{tabular}

Withdrawals by Major Public Suppliers (Mgal/d):

Name

Town of Buckhead

$\mathrm{GW}$

0.04

SW

City of Madison

Withdrawals by Major Industrial Groups (Mgal/d):

\section{SIC}

32 - Stone, clay
GW

0.00
SW

0.14
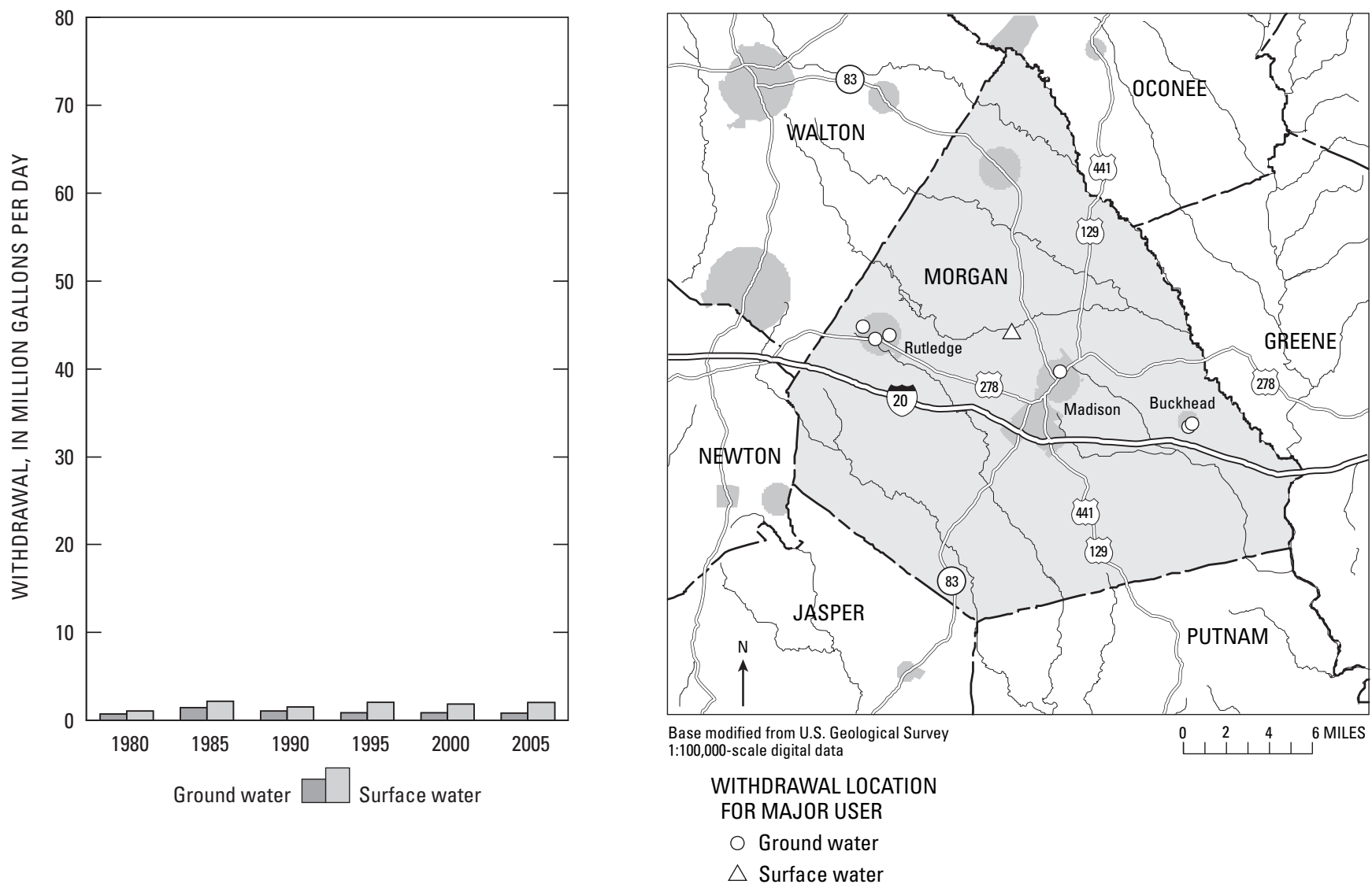


\section{MURRAY COUNTY}

Population: $\quad 40,812$

Population served by public supply: 16,220

Acres irrigated: 1,110

Hydroelectric use (Mgal/d): $\quad 1,126.98$

2005 WITHDRAWALS, IN MILLION GALLONS PER DAY

\begin{tabular}{|c|c|c|c|c|c|c|c|}
\hline & $\begin{array}{l}\text { Public } \\
\text { Supply }\end{array}$ & $\begin{array}{l}\text { Domestic \& } \\
\text { Commercial }\end{array}$ & $\begin{array}{c}\text { Industrial \& } \\
\text { Mining }\end{array}$ & Irrigation & Livestock & $\begin{array}{l}\text { Thermo- } \\
\text { electric }\end{array}$ & Totals \\
\hline Ground Water & 2.16 & 1.84 & 0.04 & 0.00 & 0.02 & 0.00 & 4.06 \\
\hline Surface Water & 1.26 & 0.00 & 0.00 & 0.78 & 0.19 & 0.00 & 2.23 \\
\hline TOTALS & 3.42 & 1.84 & 0.04 & 0.78 & 0.21 & 0.00 & 6.29 \\
\hline
\end{tabular}

Withdrawals by Major Public Suppliers (Mgal/d):

Name

GW

SW

Withdrawals by Major Industrial Groups (Mgal/d):

Chatsworth Waterworks Commission

1.44

0.00

SIC

GW

SW

City of Chatsworth

$0.71 \quad 1.26$
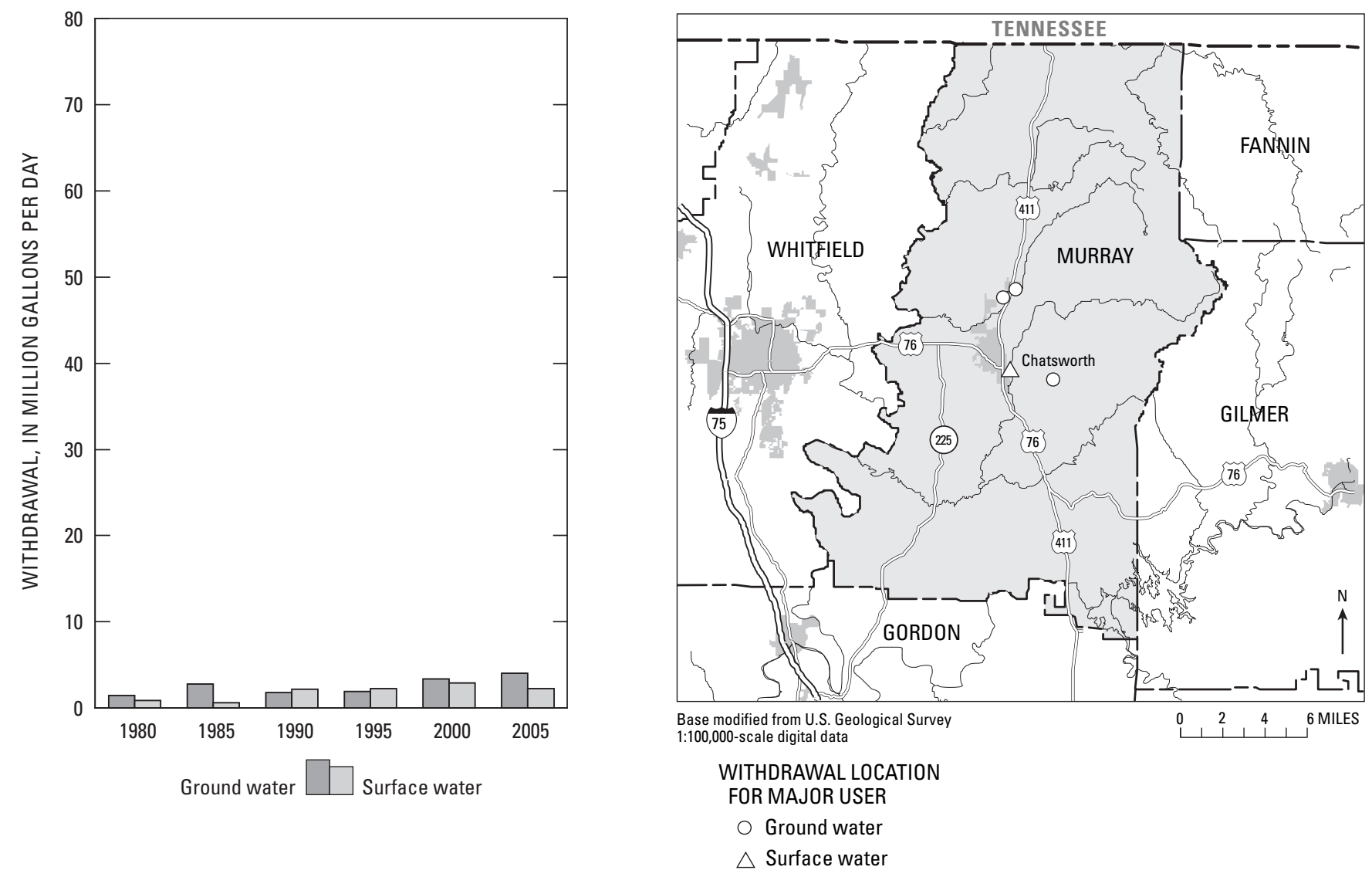


\section{MUSCOGEE COUNTY}

Population: 185,271

Population served by public supply: $\quad 185,270$

Acres irrigated: 790

Hydroelectric use (Mgal/d): $\quad 8,333.33$

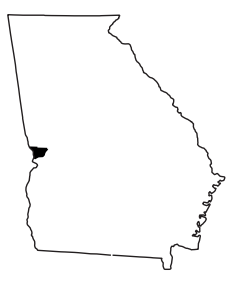

2005 WITHDRAWALS, IN MILLION GALLONS PER DAY

\begin{tabular}{|c|c|c|c|c|c|c|c|}
\hline & $\begin{array}{l}\text { Public } \\
\text { Supply }\end{array}$ & $\begin{array}{l}\text { Domestic \& } \\
\text { Commercial }\end{array}$ & $\begin{array}{c}\text { Industrial \& } \\
\text { Mining }\end{array}$ & Irrigation & Livestock & $\begin{array}{l}\text { Thermo- } \\
\text { electric }\end{array}$ & Totals \\
\hline Ground Water & 0.00 & 0.00 & 0.46 & 0.00 & 0.00 & 0.00 & 0.46 \\
\hline Surface Water & 32.22 & 0.00 & 0.01 & 1.18 & 0.02 & 0.00 & 33.43 \\
\hline TOTALS & 32.22 & 0.00 & 0.47 & 1.18 & 0.02 & 0.00 & 33.89 \\
\hline
\end{tabular}

Withdrawals by Major Public Suppliers (Mgal/d):

Name

Smith Water Authority

City of Columbus

$\begin{array}{cr}\text { GW } & \text { SW } \\ 0.00 & 2.38 \\ 0.00 & 29.85\end{array}$

Withdrawals by Major Industrial Groups (Mgal/d):

SIC

28 - Chemicals
GW

0.03
SW

0.00
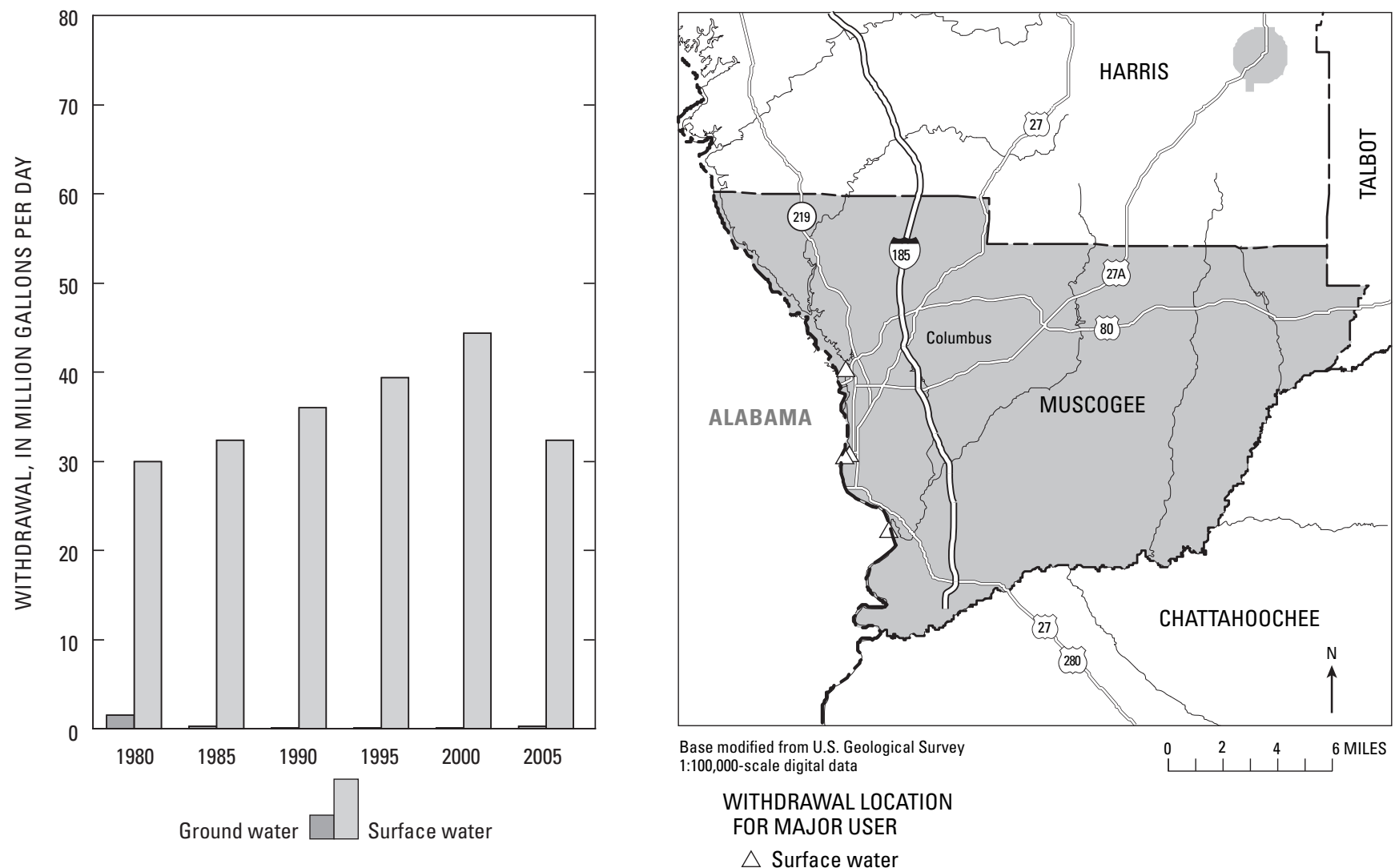


\section{NEWTON COUNTY}

Population: 86,713

Population served by public supply: 59,650

Acres irrigated: 250

Hydroelectric use (Mgal/d): 39.35

2005 WITHDRAWALS, IN MILLION GALLONS PER DAY

\begin{tabular}{|c|c|c|c|c|c|c|c|}
\hline & $\begin{array}{l}\text { Public } \\
\text { Supply }\end{array}$ & $\begin{array}{l}\text { Domestic \& } \\
\text { Commercial }\end{array}$ & $\begin{array}{c}\text { Industrial \& } \\
\text { Mining }\end{array}$ & Irrigation & Livestock & $\begin{array}{l}\text { Thermo- } \\
\text { electric }\end{array}$ & Totals \\
\hline Ground Water & 1.18 & 2.03 & 0.32 & 0.47 & 0.01 & 0.00 & 4.01 \\
\hline Surface Water & 10.07 & 0.00 & 0.00 & 0.47 & 0.09 & 0.00 & 10.63 \\
\hline TOTALS & 11.25 & 2.03 & 0.32 & 0.94 & 0.10 & 0.00 & 14.64 \\
\hline
\end{tabular}

Withdrawals by Major Public Suppliers (Mgal/d):

Name

Newton County-Almond Community

City of Covington

Town of Mansfield

Town of Newborn

City of Oxford

Newton County Board of Commissioners

Town of Porterdale

City of Oxford
GW

0.03

0.00

0.05

0.05

0.15

0.00

0.00

0.15

Withdrawals by Major Industrial Groups (Mgal/d):

SW

0.00

1.32

0.00

0.00

0.00

8.63

0.12

0.00
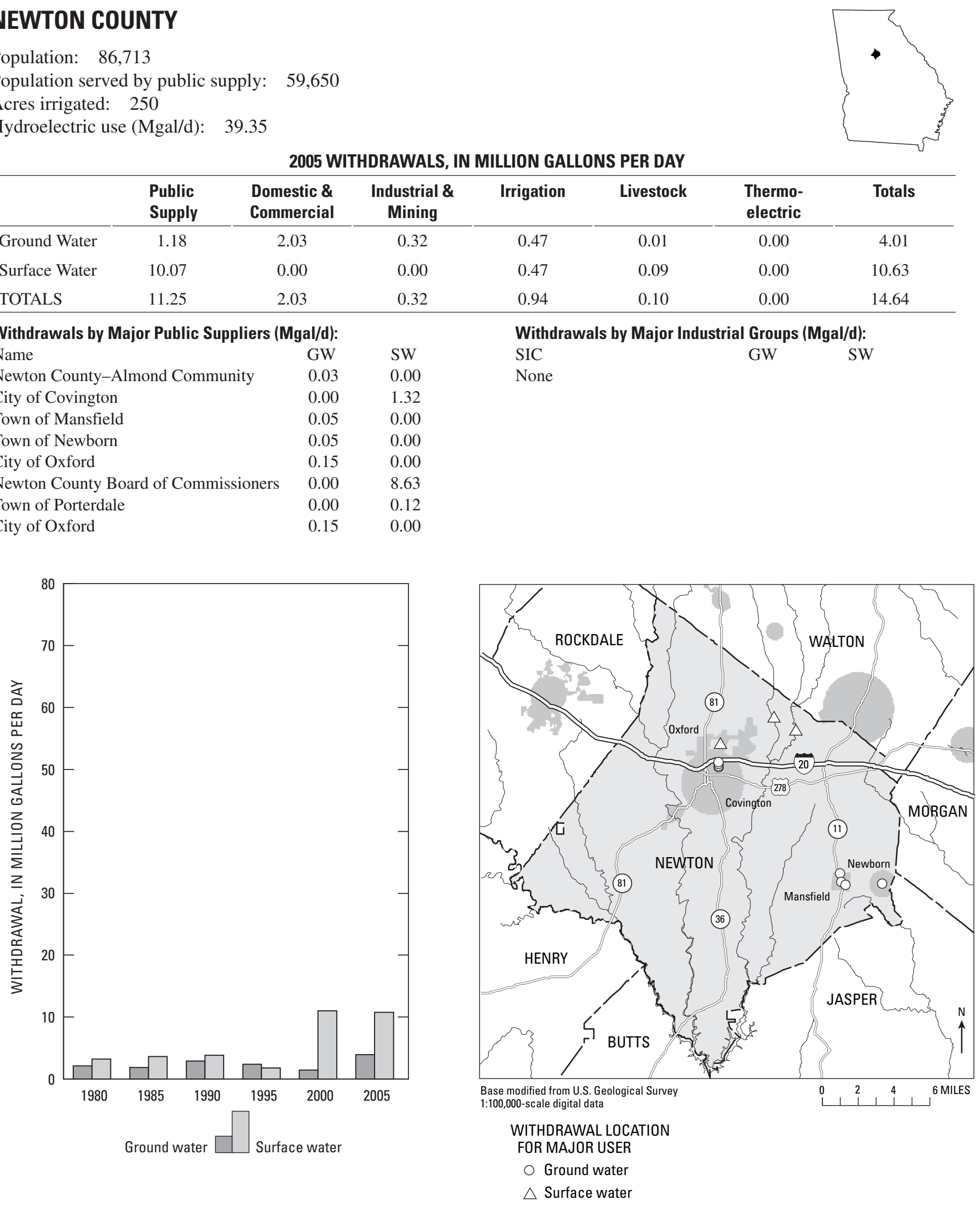


\section{OCONEE COUNTY}

Population: 29,748

Population served by public supply: $\quad 17,850$

Acres irrigated: 630

Hydroelectric use (Mgal/d): 160.33

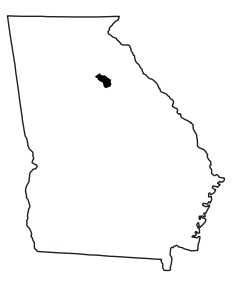

2005 WITHDRAWALS, IN MILLION GALLONS PER DAY

\begin{tabular}{|c|c|c|c|c|c|c|c|}
\hline & $\begin{array}{l}\text { Public } \\
\text { Supply }\end{array}$ & $\begin{array}{l}\text { Domestic \& } \\
\text { Commercial }\end{array}$ & $\begin{array}{c}\text { Industrial \& } \\
\text { Mining }\end{array}$ & Irrigation & Livestock & $\begin{array}{l}\text { Thermo- } \\
\text { electric }\end{array}$ & Totals \\
\hline Ground Water & 0.31 & 0.89 & 0.00 & 0.12 & 0.00 & 0.00 & 1.32 \\
\hline Surface Water & 0.00 & 0.00 & 0.00 & 0.37 & 0.32 & 0.00 & 0.69 \\
\hline TOTALS & 0.31 & 0.89 & 0.00 & 0.49 & 0.32 & 0.00 & 2.01 \\
\hline
\end{tabular}

Withdrawals by Major Public Suppliers (Mgal/d):

\section{Name}

Oconee County Public

Utilities Authority

$\begin{array}{cc}\text { GW } & \text { SW } \\ 0.04 & 0.00\end{array}$

Withdrawals by Major Industrial Groups (Mgal/d):

SIC

None
GW

SW
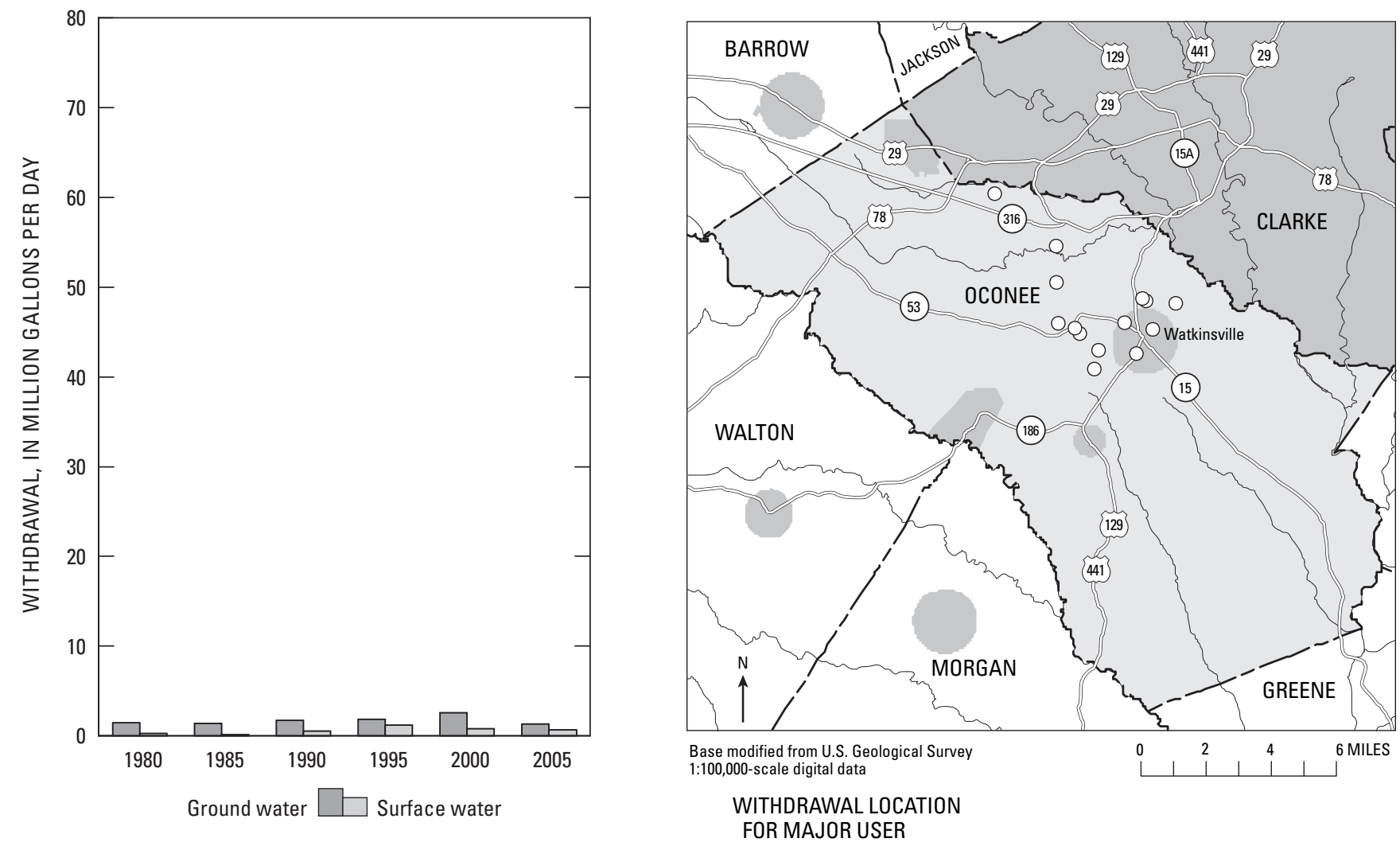

$\bigcirc$ Ground water 


\section{OGLETHORPE COUNTY}

Population: 13,609

Population served by public supply: 2,340

Acres irrigated: 450

Hydroelectric use (Mgal/d): $\quad 0.00$

2005 WITHDRAWALS, IN MILLION GALLONS PER DAY

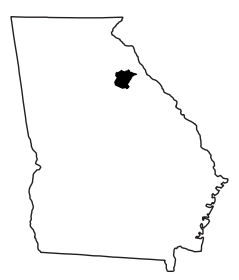

\begin{tabular}{lccccccc}
\hline & $\begin{array}{c}\text { Public } \\
\text { Supply }\end{array}$ & $\begin{array}{c}\text { Domestic \& } \\
\text { Commercial }\end{array}$ & $\begin{array}{c}\text { Industrial \& } \\
\text { Mining }\end{array}$ & Irrigation & Livestock & $\begin{array}{c}\text { Thermo- } \\
\text { electric }\end{array}$ & Totals \\
\hline Ground Water & 0.09 & 0.84 & 0.00 & 0.05 & 0.00 & 0.00 & 0.98 \\
Surface Water & 0.13 & 0.00 & 0.00 & 0.29 & 0.59 & 0.00 & 1.01 \\
TOTALS & 0.22 & 0.84 & 0.00 & 0.34 & 0.59 & 0.00 & 1.99 \\
\hline
\end{tabular}

Withdrawals by Major Public Suppliers (Mgal/d):

\section{Name}

City of Arnoldsville

City of Crawford

City of Lexington

Town of Maxeys
GW

0.02

0.00

0.04

0.02
Withdrawals by Major Industrial Groups (Mgal/d):

SIC

None
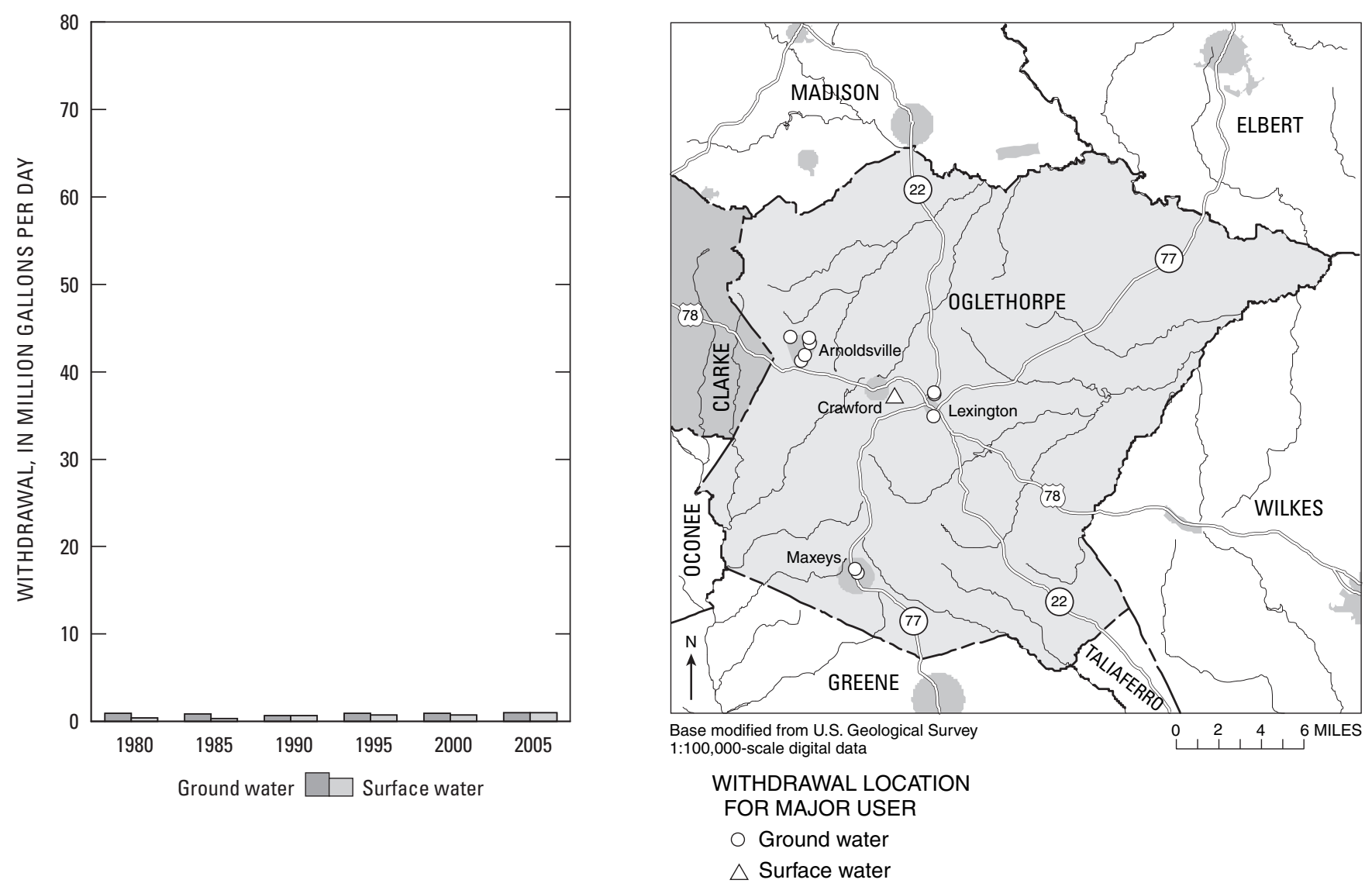


\section{PAULDING COUNTY}

Population: 112,411

Population served by public supply: 96,510

Acres irrigated: 200

Hydroelectric use (Mgal/d): 0.00

2005 WITHDRAWALS, IN MILLION GALLONS PER DAY

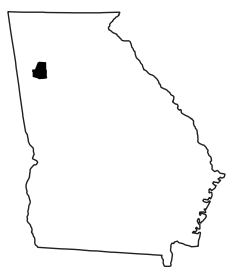

\begin{tabular}{|c|c|c|c|c|c|c|c|}
\hline & $\begin{array}{l}\text { Public } \\
\text { Supply }\end{array}$ & $\begin{array}{l}\text { Domestic \& } \\
\text { Commercial }\end{array}$ & $\begin{array}{c}\text { Industrial \& } \\
\text { Mining }\end{array}$ & Irrigation & Livestock & $\begin{array}{l}\text { Thermo- } \\
\text { electric }\end{array}$ & Totals \\
\hline Ground Water & 0.06 & 1.19 & 0.24 & 0.00 & 0.00 & 0.00 & 1.49 \\
\hline Surface Water & 3.05 & 0.00 & 0.00 & 0.11 & 0.10 & 0.00 & 3.26 \\
\hline TOTALS & 3.11 & 1.19 & 0.24 & 0.11 & 0.10 & 0.00 & 4.75 \\
\hline
\end{tabular}

Withdrawals by Major Public Suppliers (Mgal/d):

Name

City of Dallas

GW

City of Hiram

0.00

SW

Paulding County Water System
0.06

0.00
Withdrawals by Major Industrial Groups (Mgal/d):

SIC

GW

SW
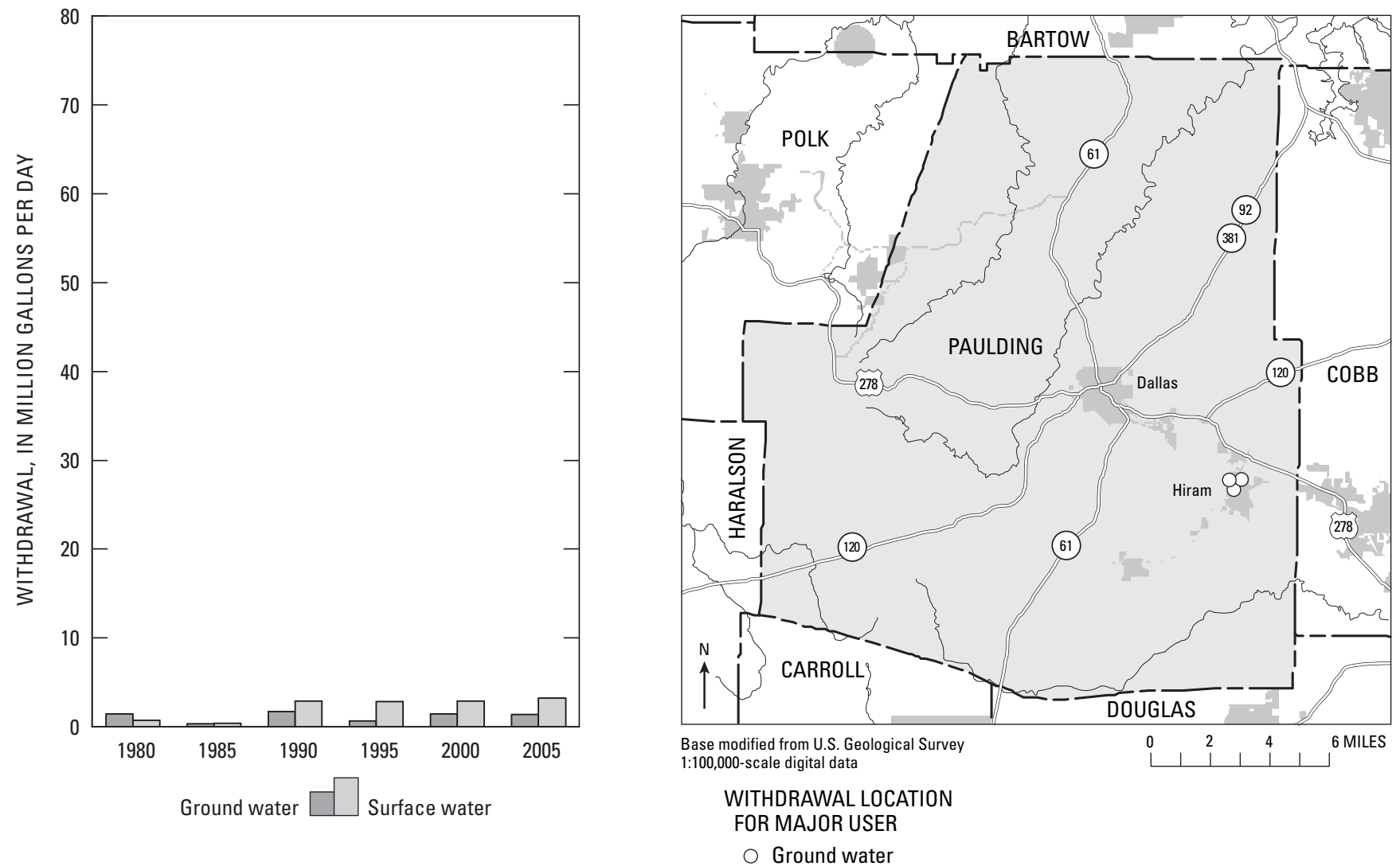


\section{PEACH COUNTY}

Population: 24,794

Population served by public supply: 17,100

Acres irrigated: 9,000

Hydroelectric use (Mgal/d): $\quad 0.00$

2005 WITHDRAWALS, IN MILLION GALLONS PER DAY

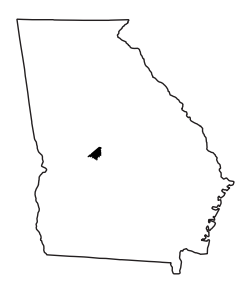

\begin{tabular}{lccccccr}
\hline & $\begin{array}{c}\text { Public } \\
\text { Supply }\end{array}$ & $\begin{array}{c}\text { Domestic \& } \\
\text { Commercial }\end{array}$ & $\begin{array}{c}\text { Industrial \& } \\
\text { Mining }\end{array}$ & Irrigation & Livestock & $\begin{array}{c}\text { Thermo- } \\
\text { electric }\end{array}$ & Totals \\
\hline Ground Water & 2.16 & 0.58 & 0.00 & 7.22 & 0.02 & 0.00 & 9.98 \\
Surface Water & 0.00 & 0.00 & 0.00 & 0.60 & 0.05 & 0.00 & 0.65 \\
TOTALS & 2.16 & 0.58 & 0.00 & 7.82 & 0.07 & 0.00 & 10.63 \\
\hline
\end{tabular}

Withdrawals by Major Public Suppliers (Mgal/d):

\section{Name}

City of Byron

City of Fort Valley
SW

0.00

0.00
Withdrawals by Major Industrial Groups (Mgal/d):

SIC

GW

SW
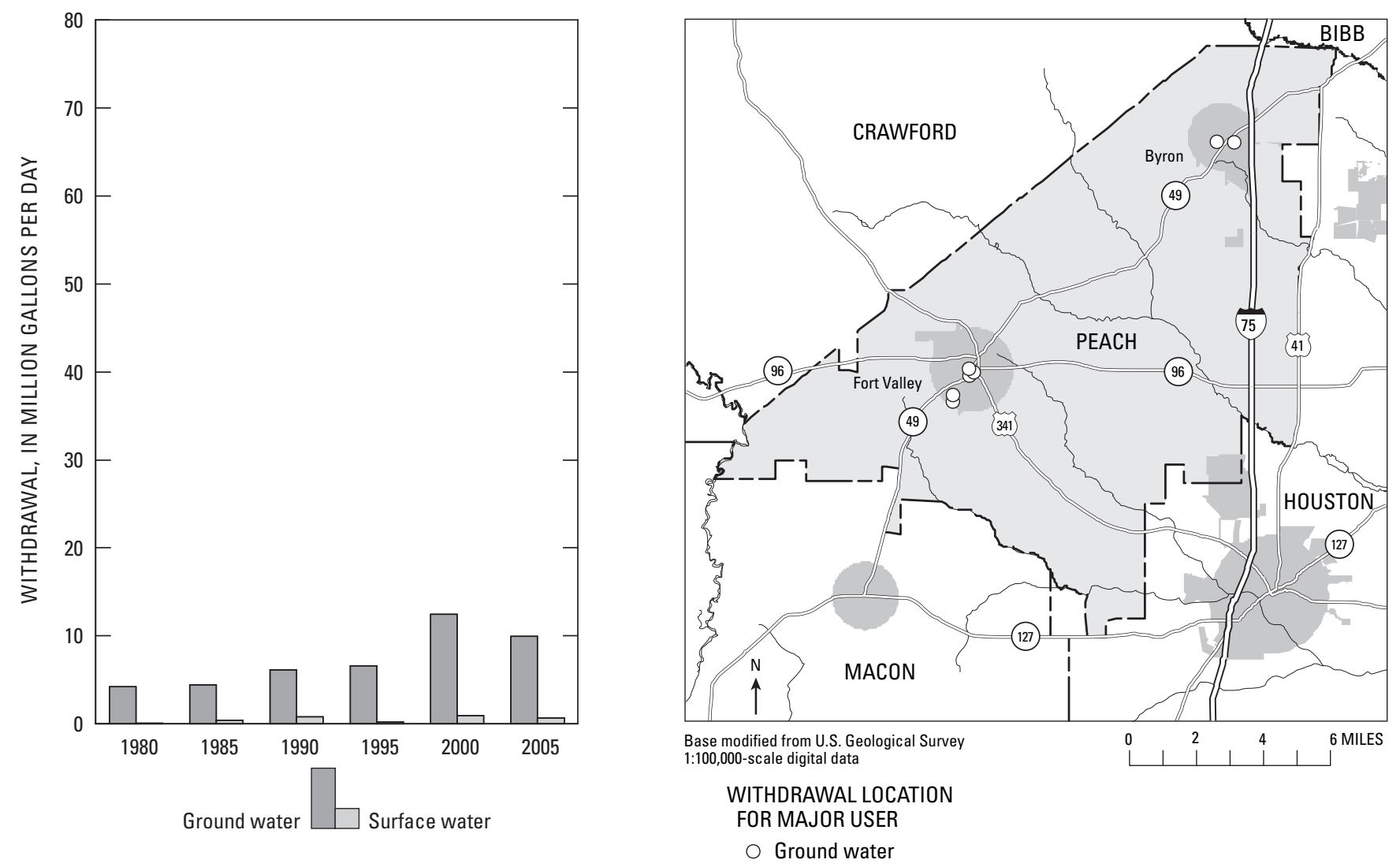


\section{PICKENS COUNTY}

Population: 28,442

Population served by public supply: 21,050

Acres irrigated: 770

Hydroelectric use (Mgal/d): $\quad 0.00$

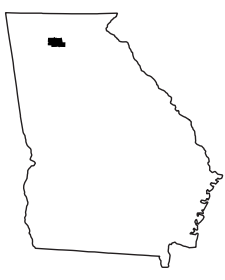

2005 WITHDRAWALS, IN MILLION GALLONS PER DAY

\begin{tabular}{|c|c|c|c|c|c|c|c|}
\hline & $\begin{array}{l}\text { Public } \\
\text { Supply }\end{array}$ & $\begin{array}{l}\text { Domestic \& } \\
\text { Commercial }\end{array}$ & $\begin{array}{l}\text { Industrial \& } \\
\text { Mining }\end{array}$ & Irrigation & Livestock & $\begin{array}{l}\text { Thermo- } \\
\text { electric }\end{array}$ & Totals \\
\hline Ground Water & 0.62 & 0.55 & 1.45 & 0.02 & 0.00 & 0.00 & 2.64 \\
\hline Surface Water & 1.55 & 0.00 & 0.01 & 0.34 & 0.32 & 0.00 & 2.22 \\
\hline TOTALS & 2.17 & 0.55 & 1.46 & 0.36 & 0.32 & 0.00 & 4.86 \\
\hline
\end{tabular}

Withdrawals by Major Public Suppliers (Mgal/d):

Name

Big Canoe Corporation

GW

0.00

SW

City of Jasper

0.48

0.06

City of Nelson

Pickens County Water Authority

Bent Tree Community
0.08

0.00
Withdrawals by Major Industrial Groups (Mgal/d):

$\begin{array}{lcc}\text { SIC } & \text { GW } & \text { SW } \\ 30 \text { - Rubber } & 0.00 & 0.01 \\ 32 \text { - Stone, clay } & 1.12 & 0.00\end{array}$
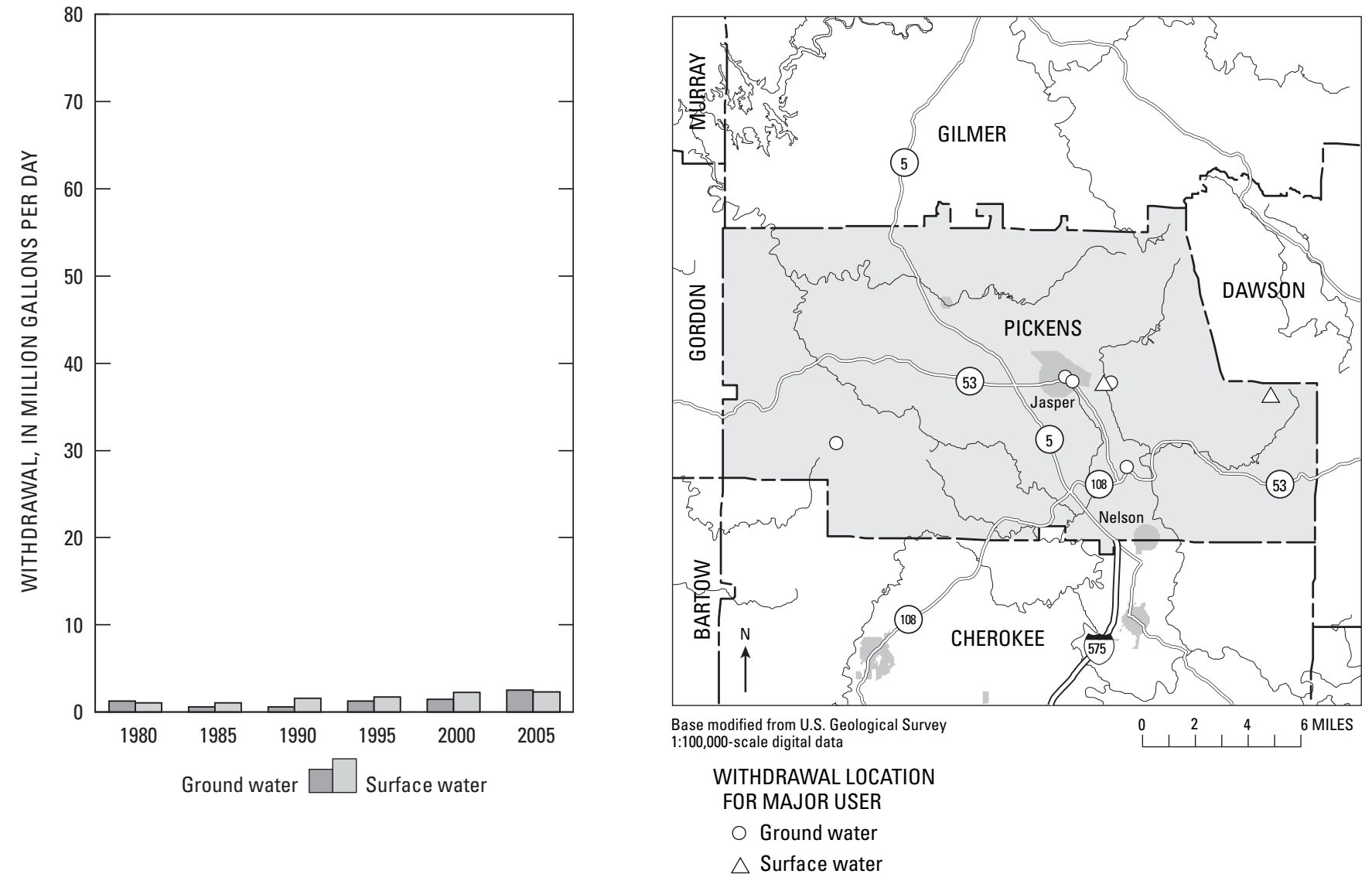


\section{PIERCE COUNTY}

Population: 17,119

Population served by public supply: $\quad 4,790$

Acres irrigated: 12,370

Hydroelectric use (Mgal/d): $\quad 0.00$

2005 WITHDRAWALS, IN MILLION GALLONS PER DAY

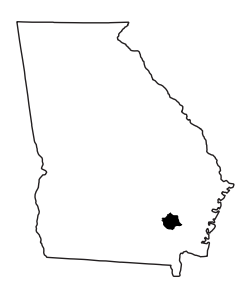

\begin{tabular}{lccccccc}
\hline & $\begin{array}{c}\text { Public } \\
\text { Supply }\end{array}$ & $\begin{array}{c}\text { Domestic \& } \\
\text { Commercial }\end{array}$ & $\begin{array}{c}\text { Industrial \& } \\
\text { Mining }\end{array}$ & Irrigation & Livestock & $\begin{array}{c}\text { Thermo- } \\
\text { electric }\end{array}$ & Totals \\
\hline Ground Water & 0.59 & 0.92 & 0.02 & 1.74 & 0.02 & 0.00 & 3.29 \\
Surface Water & 0.00 & 0.00 & 0.00 & 0.12 & 0.08 & 0.00 & 0.20 \\
TOTALS & 0.59 & 0.92 & 0.02 & 1.86 & 0.10 & 0.00 & 3.49 \\
\hline
\end{tabular}

Withdrawals by Major Public Suppliers (Mgal/d):

\section{Name}

City of Blackshear

City of Patterson
SW

0.00

0.00
Withdrawals by Major Industrial Groups (Mgal/d):

SIC

None
SW
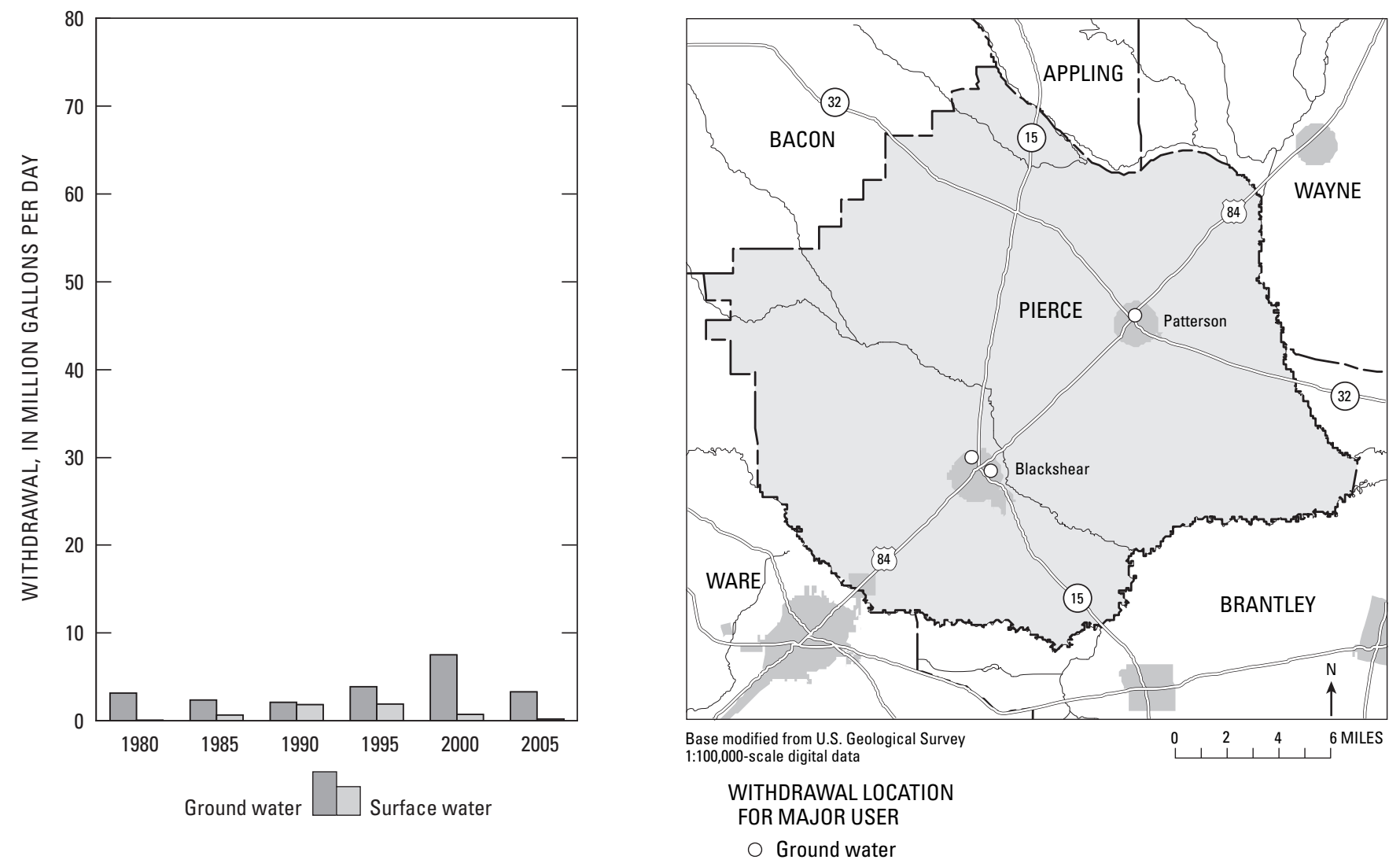


\section{PIKE COUNTY}

Population: 16,128

Population served by public supply: 3,610

Acres irrigated: 200

Hydroelectric use (Mgal/d): $\quad 0.00$

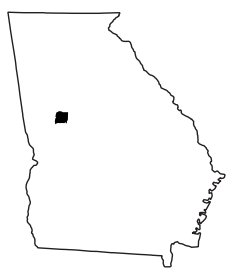

2005 WITHDRAWALS, IN MILLION GALLONS PER DAY

\begin{tabular}{|c|c|c|c|c|c|c|c|}
\hline & $\begin{array}{l}\text { Public } \\
\text { Supply }\end{array}$ & $\begin{array}{l}\text { Domestic \& } \\
\text { Commercial }\end{array}$ & $\begin{array}{c}\text { Industrial \& } \\
\text { Mining }\end{array}$ & Irrigation & Livestock & $\begin{array}{l}\text { Thermo- } \\
\text { electric }\end{array}$ & Totals \\
\hline Ground Water & 0.15 & 0.94 & 0.00 & 0.00 & 0.02 & 0.00 & 1.11 \\
\hline Surface Water & 4.34 & 0.00 & 0.00 & 0.29 & 0.18 & 0.00 & 4.81 \\
\hline TOTALS & 4.49 & 0.94 & 0.00 & 0.29 & 0.20 & 0.00 & 5.92 \\
\hline
\end{tabular}

Withdrawals by Major Public Suppliers (Mgal/d):

Name

GW SW

Town of Concord

0.07

0.00

City of Meansville

City of Molena

0.02

0.03

0.03

City of Griffin (water source located in Spalding County)
Withdrawals by Major Industrial Groups (Mgal/d):

SIC

GW

SW
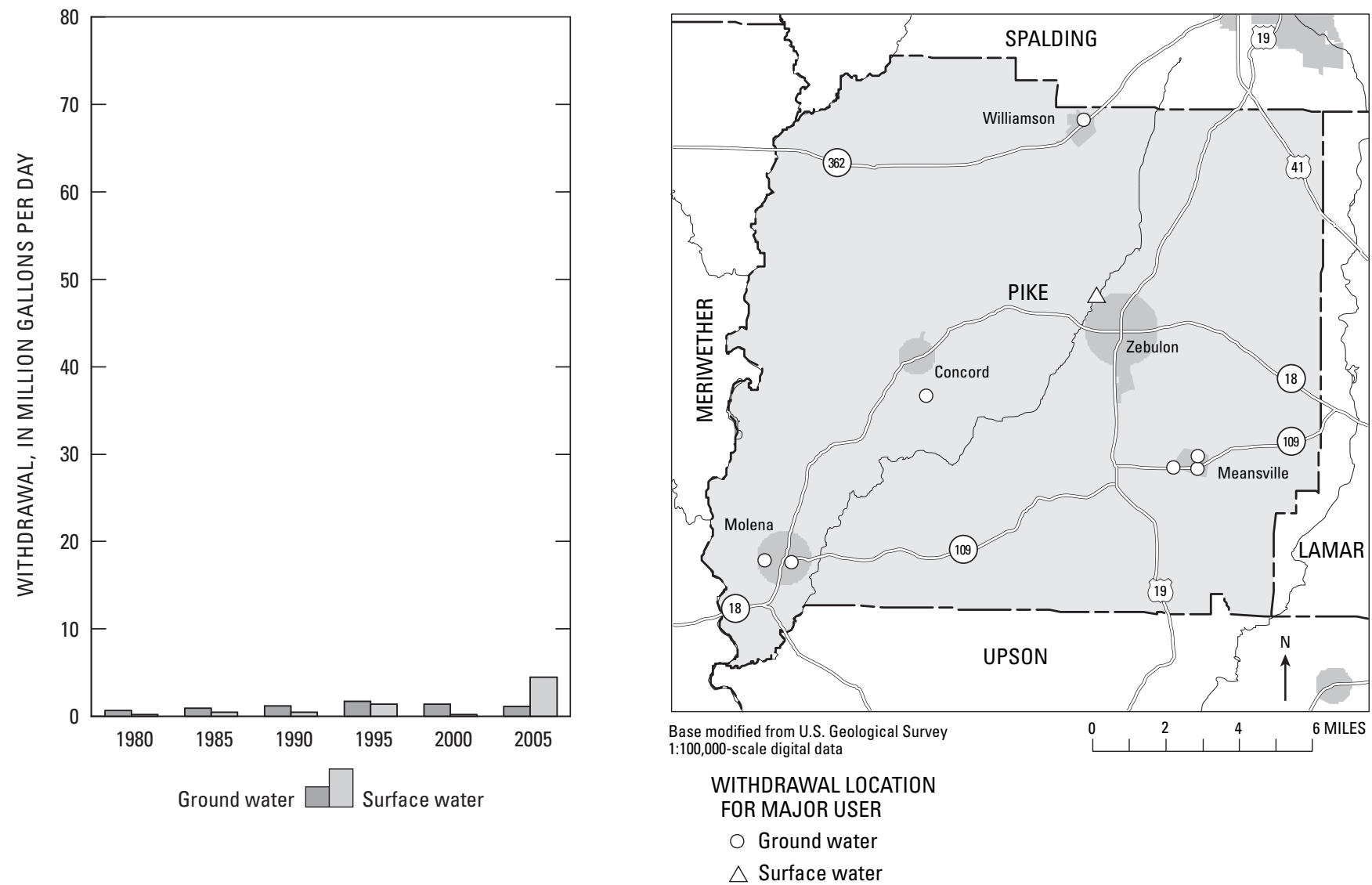


\section{POLK COUNTY}

Population: 40,479

Population served by public supply: 35,620

Acres irrigated: 520

Hydroelectric use (Mgal/d): $\quad 0.00$

2005 WITHDRAWALS, IN MILLION GALLONS PER DAY

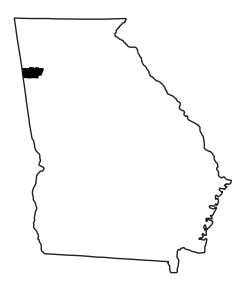

\begin{tabular}{lccccccc}
\hline & $\begin{array}{c}\text { Public } \\
\text { Supply }\end{array}$ & $\begin{array}{c}\text { Domestic \& } \\
\text { Commercial }\end{array}$ & $\begin{array}{c}\text { Industrial \& } \\
\text { Mining }\end{array}$ & Irrigation & Livestock & $\begin{array}{c}\text { Thermo- } \\
\text { electric }\end{array}$ & Totals \\
\hline Ground Water & 3.75 & 0.36 & 1.55 & 0.11 & 0.01 & 0.00 & 5.78 \\
Surface Water & 2.21 & 0.00 & 0.09 & 0.62 & 0.16 & 0.00 & 3.08 \\
TOTALS & 5.96 & 0.36 & 1.64 & 0.73 & 0.17 & 0.00 & 8.86 \\
\hline
\end{tabular}

Withdrawals by Major Public Suppliers (Mgal/d):

\section{Name}

City of Cedartown

Polk County Water Authority

City of Rockmart

$\begin{array}{ll}\text { GW } & \text { SW } \\ 2.27 & 0.00 \\ 0.00 & 2.22 \\ 1.48 & 0.00\end{array}$

Withdrawals by Major Industrial Groups (Mgal/d):

$\begin{array}{lcc}\text { SIC } & \text { GW } & \text { SW } \\ 28 \text { - Chemicals } & 1.35 & 0.00 \\ 29 \text { - Petroleum } & 0.00 & 0.09\end{array}$
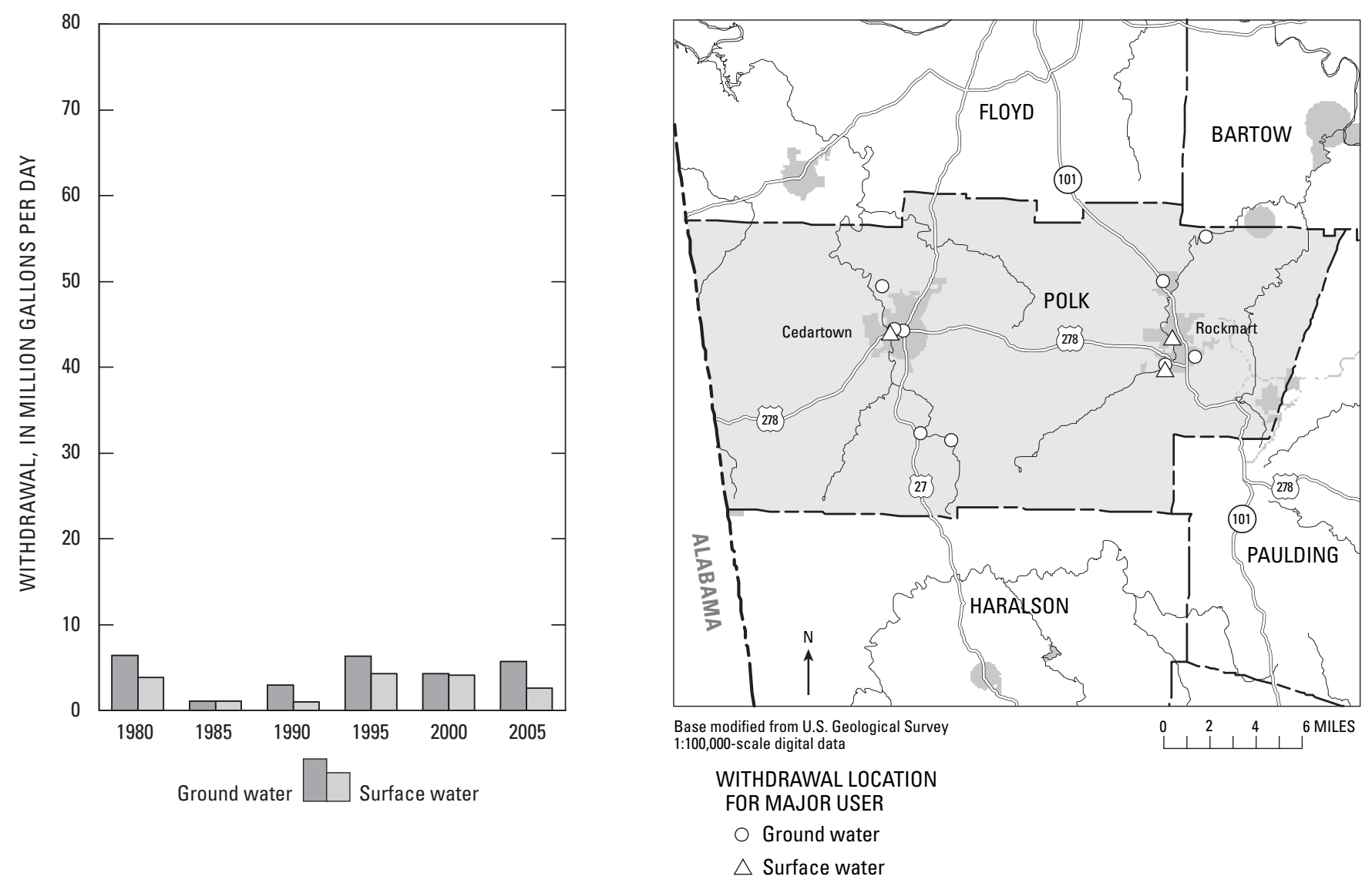


\section{PULASKI COUNTY}

Population: 9,737

Population served by public supply: $\quad 5,870$

Acres irrigated: 28,000

Hydroelectric use (Mgal/d): $\quad 0.00$

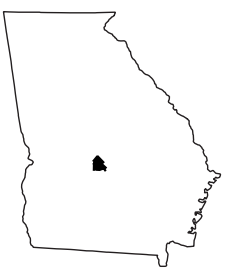

2005 WITHDRAWALS, IN MILLION GALLONS PER DAY

\begin{tabular}{|c|c|c|c|c|c|c|c|}
\hline & $\begin{array}{l}\text { Public } \\
\text { Supply }\end{array}$ & $\begin{array}{l}\text { Domestic \& } \\
\text { Commercial }\end{array}$ & $\begin{array}{c}\text { Industrial \& } \\
\text { Mining }\end{array}$ & Irrigation & Livestock & $\begin{array}{l}\text { Thermo- } \\
\text { electric }\end{array}$ & Totals \\
\hline Ground Water & 0.85 & 0.29 & 0.65 & 10.17 & 0.01 & 0.00 & 11.97 \\
\hline Surface Water & 0.00 & 0.00 & 0.00 & 4.74 & 0.03 & 0.00 & 4.77 \\
\hline TOTALS & 0.85 & 0.29 & 0.65 & 14.91 & 0.04 & 0.00 & 16.74 \\
\hline
\end{tabular}

Withdrawals by Major Public Suppliers (Mgal/d):

Name

Hartford Water Authority

City of Hawkinsville

$\begin{array}{cc}\text { GW } & \text { SW } \\ 0.04 & 0.00 \\ 0.81 & 0.00\end{array}$

Withdrawals by Major Industrial Groups (Mgal/d):

SIC

26 - Paper
SW

0.00

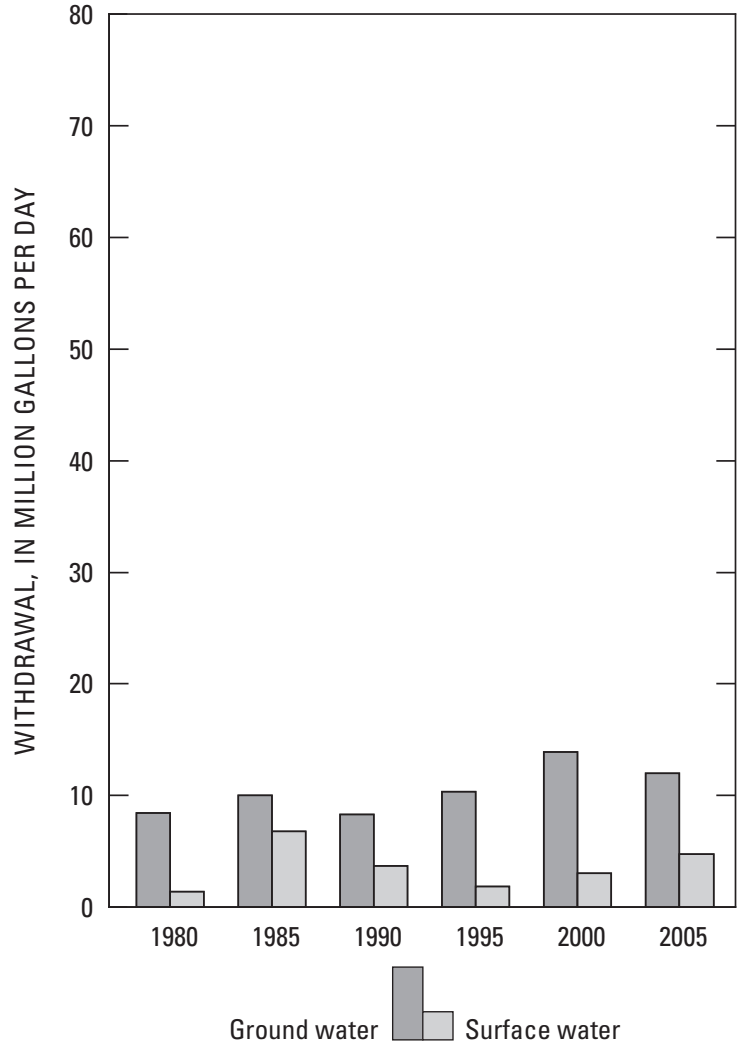

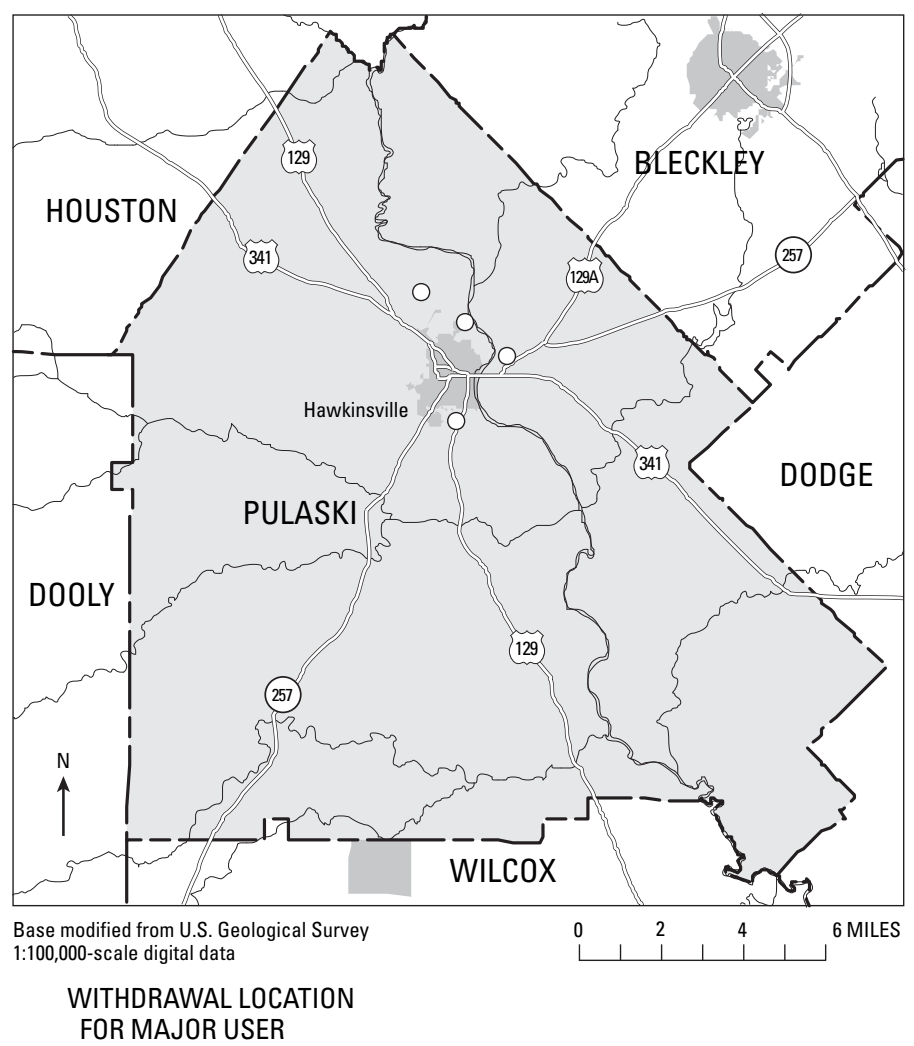




\section{PUTNAM COUNTY}

Population: 19,829

Population served by public supply: $\quad 11,940$

Acres irrigated: 1,380

Hydroelectric use (Mgal/d): 3,956.96

2005 WITHDRAWALS, IN MILLION GALLONS PER DAY

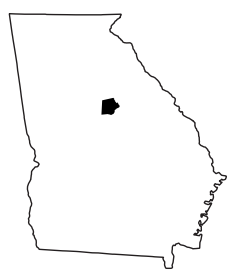

\begin{tabular}{lccccccrr}
\hline & $\begin{array}{c}\text { Public } \\
\text { Supply }\end{array}$ & $\begin{array}{c}\text { Domestic \& } \\
\text { Commercial }\end{array}$ & $\begin{array}{c}\text { Industrial \& } \\
\text { Mining }\end{array}$ & Irrigation & Livestock & \multicolumn{2}{c}{$\begin{array}{c}\text { Thermo- } \\
\text { electric }\end{array}$} & Totals \\
\hline Ground Water & 0.41 & 0.59 & 0.07 & 1.01 & 0.00 & 0.00 & 2.08 \\
Surface Water & 0.80 & 0.00 & 0.00 & 1.88 & 0.39 & $1,092.00$ & $1,095.07$ \\
TOTALS & 1.21 & 0.59 & 0.07 & 2.89 & 0.39 & $1,092.00$ & $1,097.15$ \\
\hline
\end{tabular}

Withdrawals by Major Public Suppliers (Mgal/d):

Name

City of Eatonton

GW

0.00

SW

0.80
Withdrawals by Major Industrial Groups (Mgal/d):

SIC

None
SW

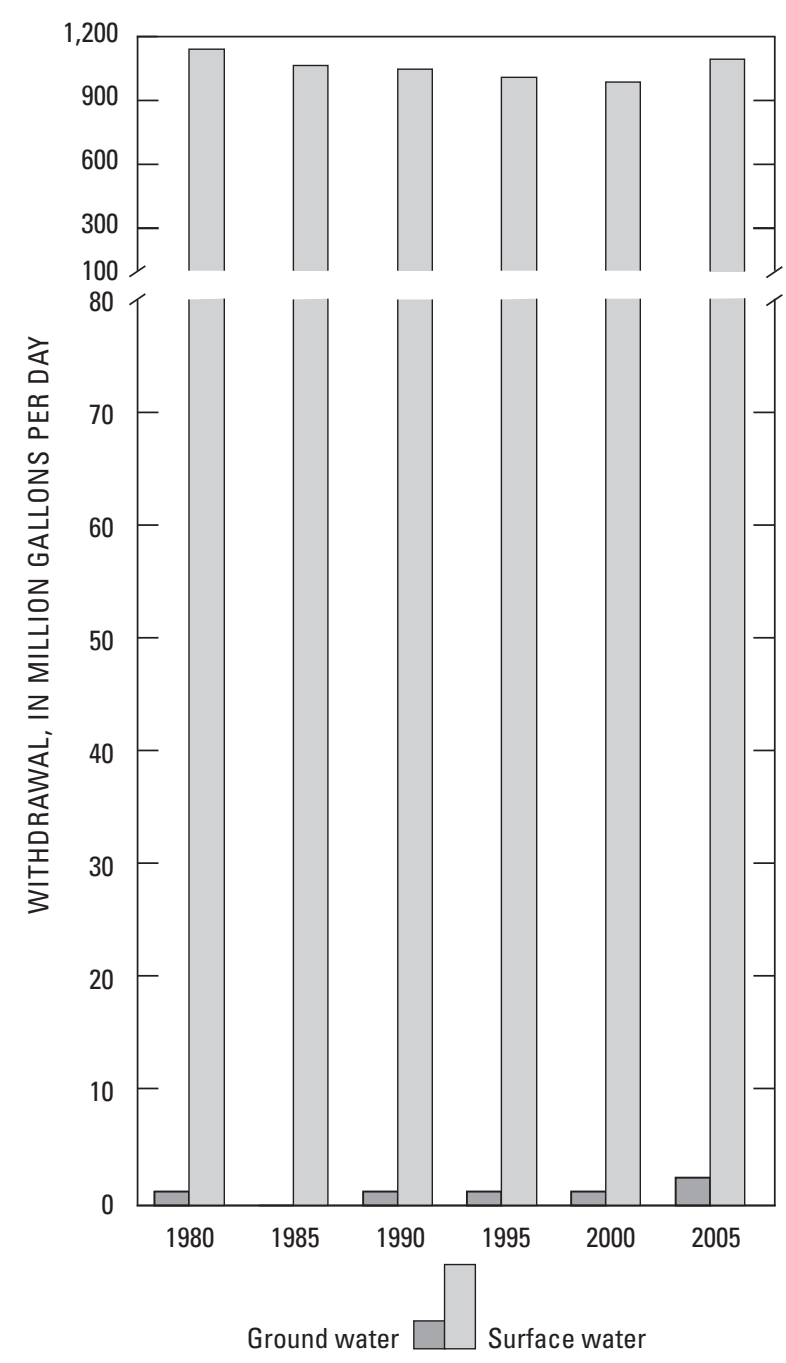

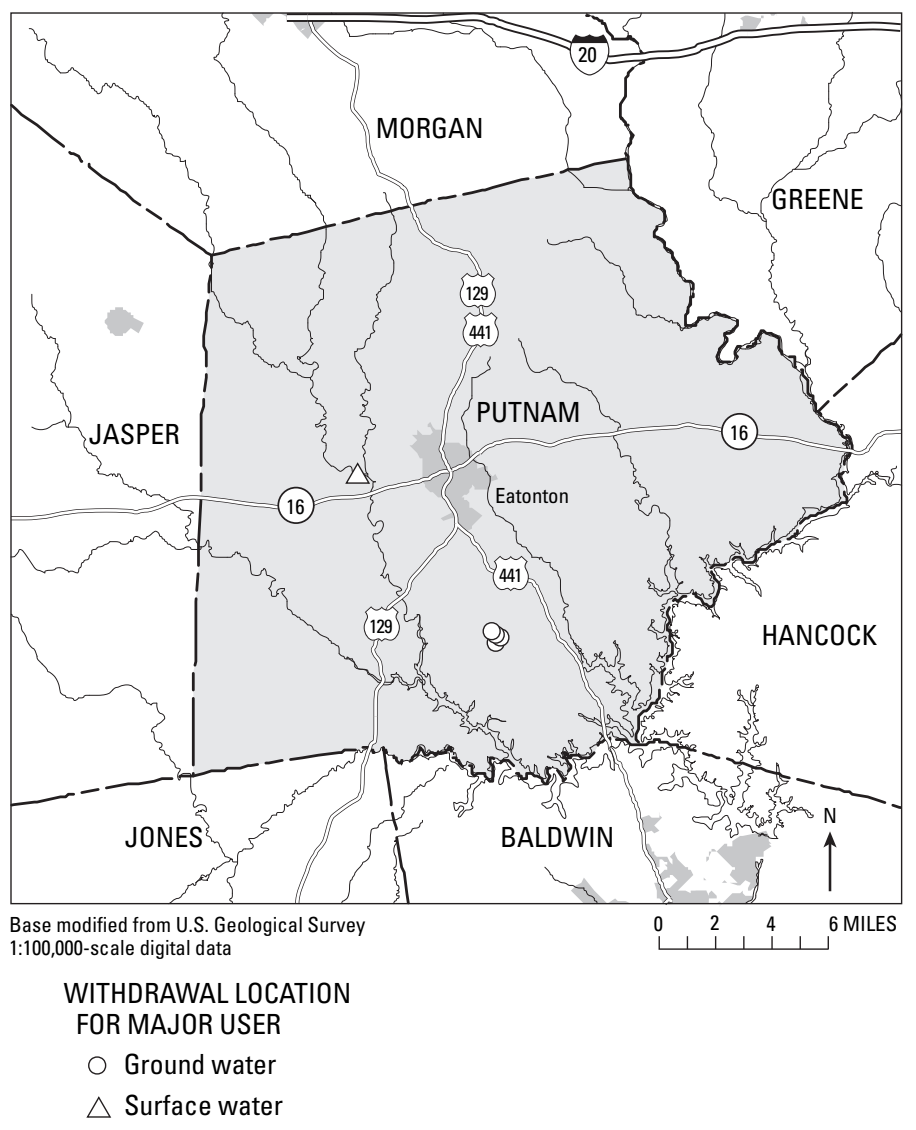




\section{QUITMAN COUNTY}

Population: 2,467

Population served by public supply: $\quad 1,900$

Acres irrigated: 260

Hydroelectric use (Mgal/d): 0.00

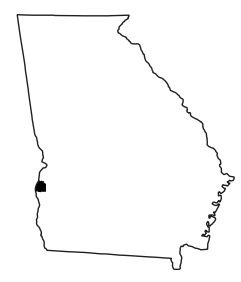

2005 WITHDRAWALS, IN MILLION GALLONS PER DAY

\begin{tabular}{|c|c|c|c|c|c|c|c|}
\hline & $\begin{array}{l}\text { Public } \\
\text { Supply }\end{array}$ & $\begin{array}{l}\text { Domestic \& } \\
\text { Commercial }\end{array}$ & $\begin{array}{c}\text { Industrial \& } \\
\text { Mining }\end{array}$ & Irrigation & Livestock & $\begin{array}{l}\text { Thermo- } \\
\text { electric }\end{array}$ & Totals \\
\hline Ground Water & 0.14 & 0.04 & 0.00 & 0.00 & 0.00 & 0.00 & 0.18 \\
\hline Surface Water & 0.00 & 0.00 & 0.00 & 0.02 & 0.01 & 0.00 & 0.03 \\
\hline TOTALS & 0.14 & 0.04 & 0.00 & 0.02 & 0.01 & 0.00 & 0.21 \\
\hline
\end{tabular}

Withdrawals by Major Public Suppliers (Mgal/d):

Name

Town of Georgetown

GW

0.07

SW

0.00
Withdrawals by Major Industrial Groups (Mgal/d):

SIC

None

\section{SW}
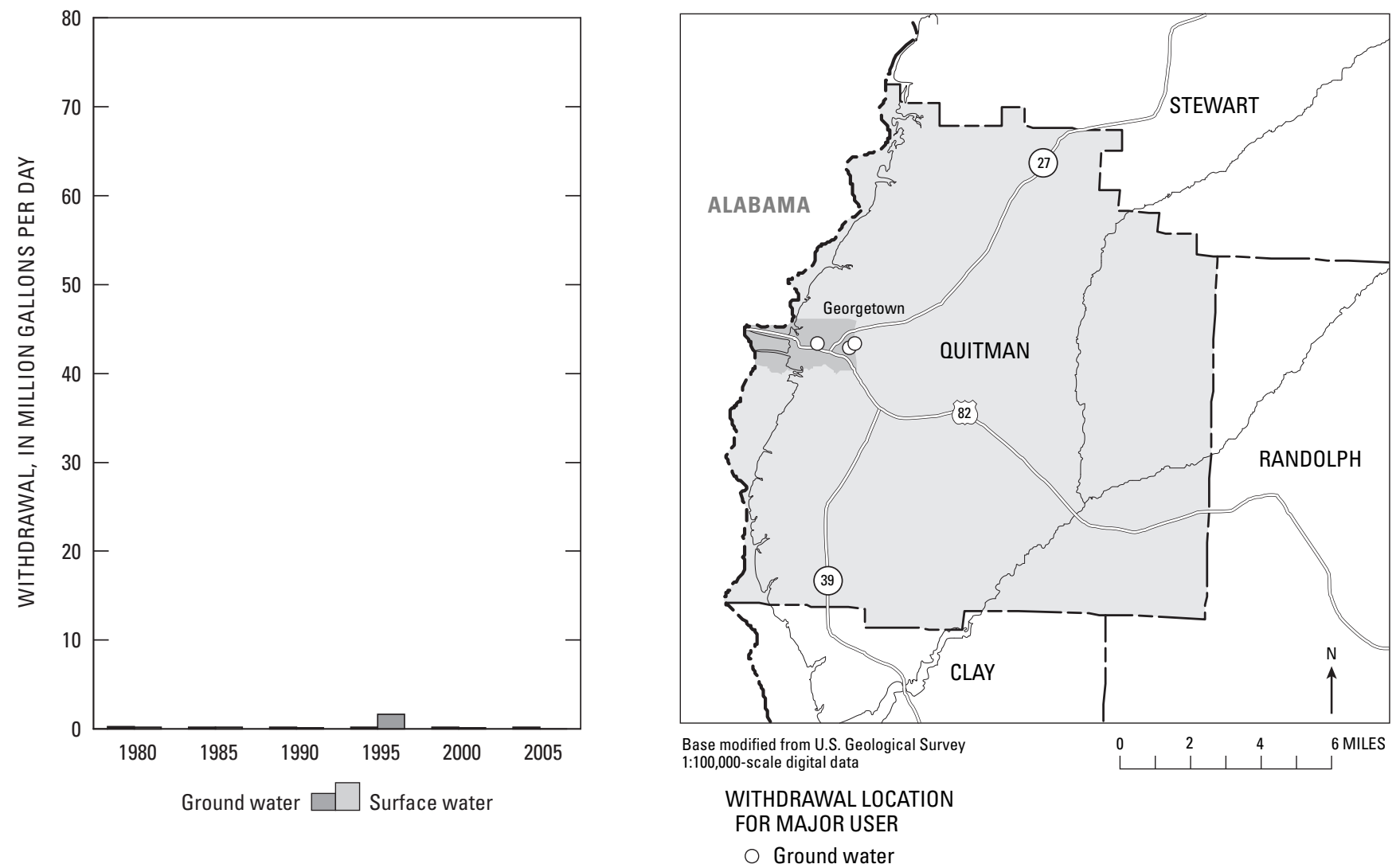


\section{RABUN COUNTY}

Population: 16,087

Population served by public supply: 11,740

Acres irrigated: 480

Hydroelectric use (Mgal/d): $\quad 1,181.69$

2005 WITHDRAWALS, IN MILLION GALLONS PER DAY

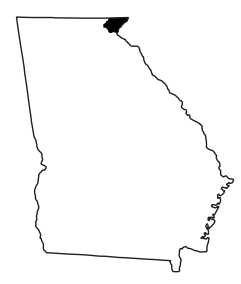

\begin{tabular}{lccccccc}
\hline & $\begin{array}{c}\text { Public } \\
\text { Supply }\end{array}$ & $\begin{array}{c}\text { Domestic \& } \\
\text { Commercial }\end{array}$ & $\begin{array}{c}\text { Industrial \& } \\
\text { Mining }\end{array}$ & Irrigation & Livestock & $\begin{array}{c}\text { Thermo- } \\
\text { electric }\end{array}$ & Totals \\
\hline Ground Water & 0.45 & 0.33 & 0.26 & 0.00 & 0.00 & 0.00 & 1.04 \\
Surface Water & 1.52 & 0.00 & 1.49 & 0.58 & 0.07 & 0.00 & 3.66 \\
TOTALS & 1.97 & 0.33 & 1.75 & 0.58 & 0.07 & 0.00 & 4.70 \\
\hline
\end{tabular}

Withdrawals by Major Public Suppliers (Mgal/d):

\section{Name}

City of Clayton

Town of Tallulah Falls

City of Sky Valley Resort

Clayton-Rabun County Water \& Sewer Authority
GW SW

$0.00 \quad 0.00$

$0.01 \quad 0.00$

$0.30 \quad 0.03$

$0.00 \quad 1.49$
Withdrawals by Major Industrial Groups (Mgal/d):

SIC

22 - Textiles

GW

0.11

SW

1.49
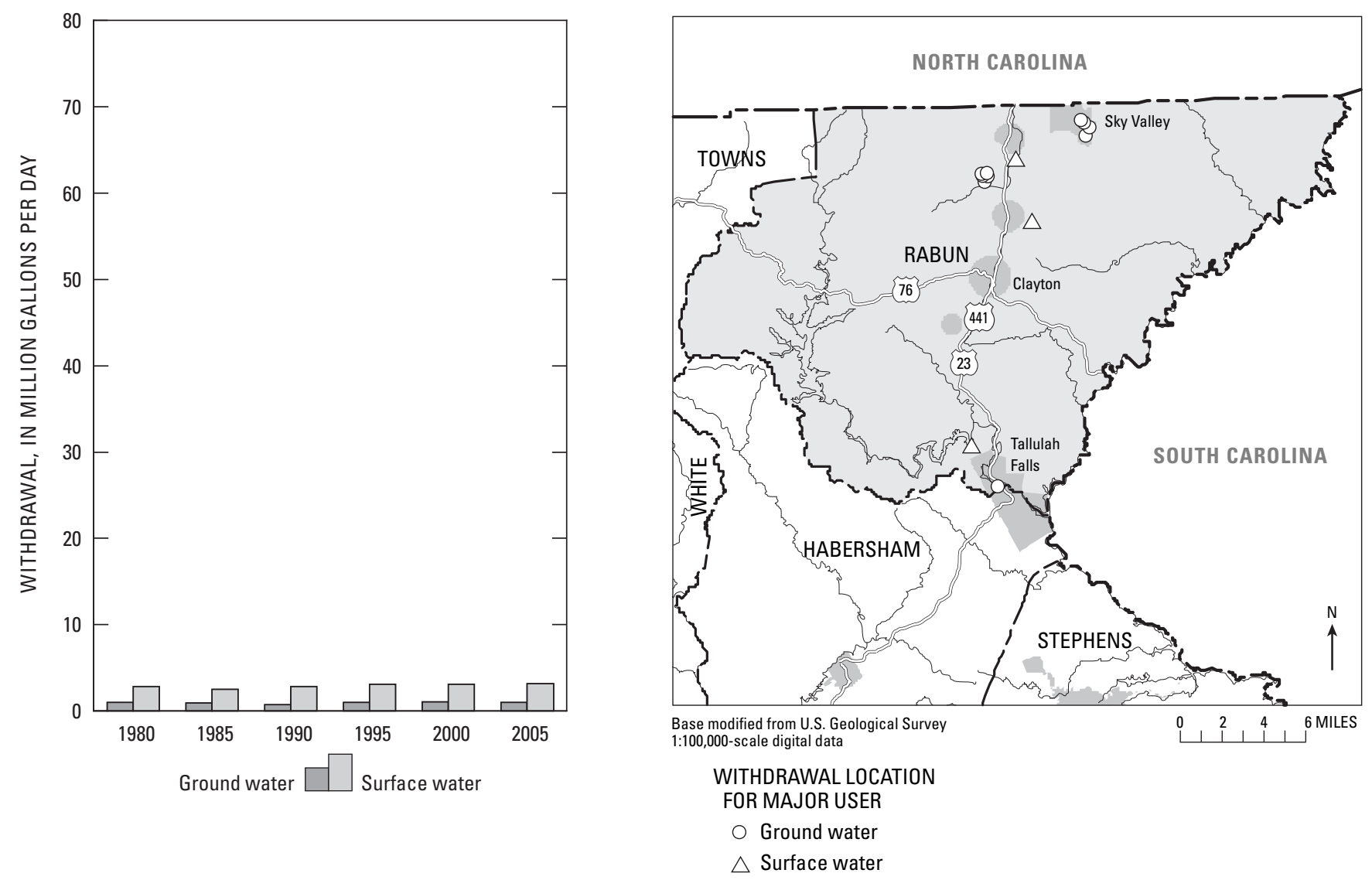


\section{RANDOLPH COUNTY}

Population: 7,310

Population served by public supply: 5,350

Acres irrigated: 26,210

Hydroelectric use (Mgal/d): $\quad 0.00$

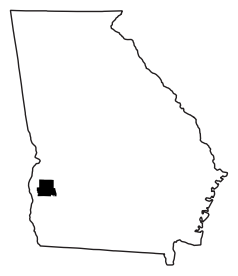

2005 WITHDRAWALS, IN MILLION GALLONS PER DAY

\begin{tabular}{|c|c|c|c|c|c|c|c|}
\hline & $\begin{array}{l}\text { Public } \\
\text { Supply }\end{array}$ & $\begin{array}{l}\text { Domestic \& } \\
\text { Commercial }\end{array}$ & $\begin{array}{c}\text { Industrial \& } \\
\text { Mining }\end{array}$ & Irrigation & Livestock & $\begin{array}{l}\text { Thermo- } \\
\text { electric }\end{array}$ & Totals \\
\hline Ground Water & 0.96 & 0.15 & 0.06 & 4.28 & 0.01 & 0.00 & 5.46 \\
\hline Surface Water & 0.00 & 0.00 & 0.00 & 4.92 & 0.04 & 0.00 & 4.96 \\
\hline TOTALS & 0.96 & 0.15 & 0.06 & 9.20 & 0.05 & 0.00 & 10.42 \\
\hline
\end{tabular}

Withdrawals by Major Public Suppliers (Mgal/d):

Name

City of Coleman

City of Cuthbert

City of Shellman
GW

0.02

0.69

0.10
SW

0.00

0.00

0.00
Withdrawals by Major Industrial Groups (Mgal/d):

SIC

20 - Food
SW

0.00
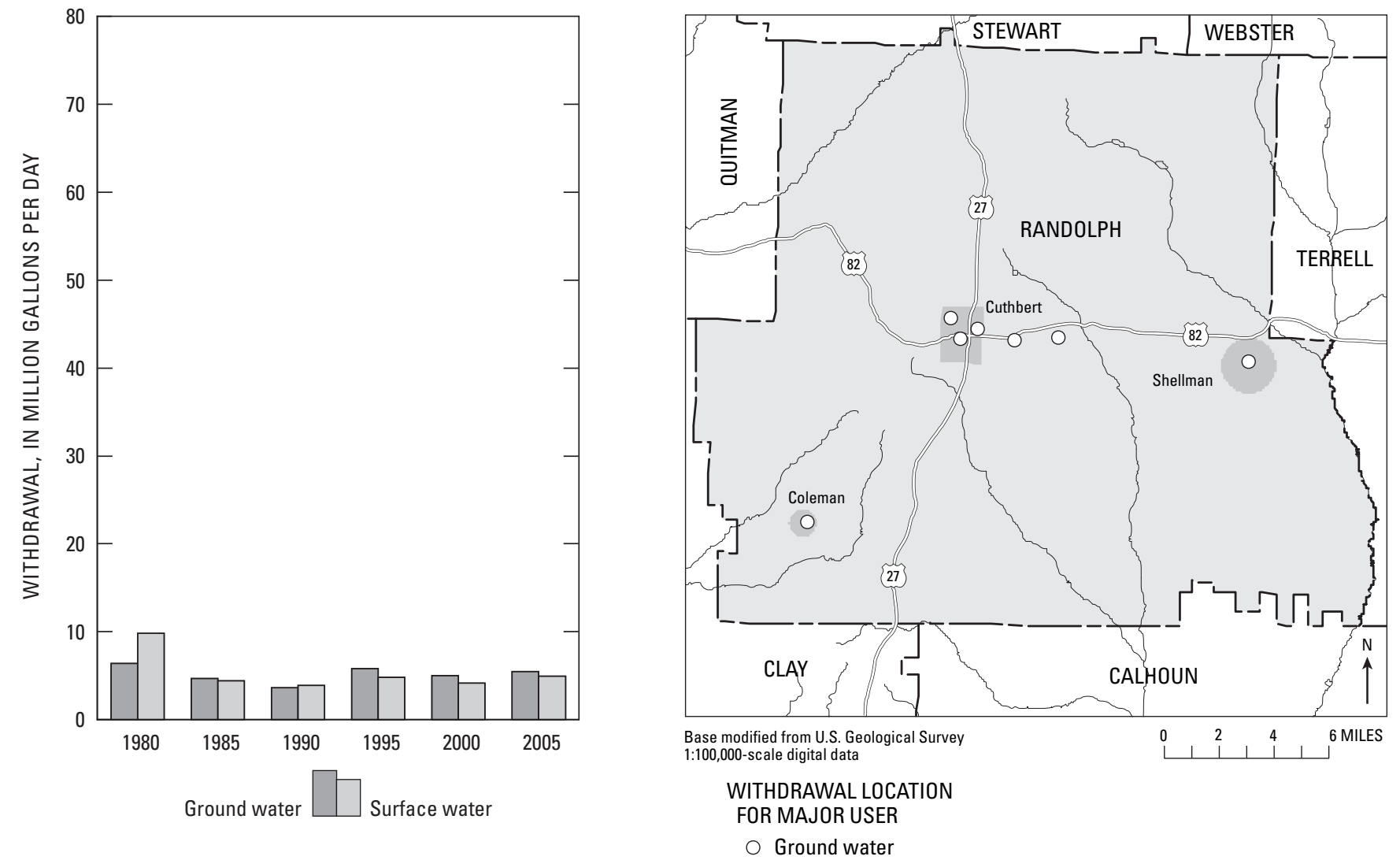


\section{RICHMOND COUNTY}

Population: 195,769

Population served by public supply: 191,860

Acres irrigated: 1,250

Hydroelectric use (Mgal/d): 1,573.96

\section{WITHDRAWALS, IN MILLION GALLONS PER DAY}

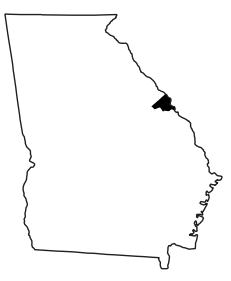

\begin{tabular}{|c|c|c|c|c|c|c|c|}
\hline & $\begin{array}{l}\text { Public } \\
\text { Supply }\end{array}$ & $\begin{array}{l}\text { Domestic \& } \\
\text { Commercial }\end{array}$ & $\begin{array}{c}\text { Industrial \& } \\
\text { Mining }\end{array}$ & Irrigation & Livestock & $\begin{array}{l}\text { Thermo- } \\
\text { electric }\end{array}$ & Tota \\
\hline Ground Water & 8.97 & 0.51 & 5.07 & 2.45 & 0.01 & 0.00 & 1 \\
\hline Surface Water & 34.16 & 1.93 & 64.21 & 0.54 & 0.02 & 0.00 & 10 \\
\hline TOTALS & 43.13 & 2.44 & 69.28 & 2.99 & 0.03 & 0.00 & $11^{\circ}$ \\
\hline \multicolumn{4}{|c|}{ Withdrawals by Major Public Suppliers (Mgal/d): } & \multicolumn{4}{|c|}{ Withdrawals by Major Industrial Groups (Mgal/d): } \\
\hline Name & & GW & & \multicolumn{2}{|l|}{ SIC } & $\mathrm{GW}$ & SW \\
\hline \multirow{2}{*}{\multicolumn{2}{|c|}{$\begin{array}{l}\text { Augusta-Richmond County } \\
\text { Water System }\end{array}$}} & 0.00 & 4.26 & \multirow{2}{*}{\multicolumn{2}{|c|}{$\begin{array}{l}14 \text { - Mining } \\
22 \text { - Textiles }\end{array}$}} & 0.02 & 0.00 \\
\hline & & & & & & 0.09 & 0.50 \\
\hline \multicolumn{2}{|l|}{ Town of Blythe } & 0.07 & 0.00 & \multicolumn{2}{|c|}{26 - Paper } & 0.00 & 53.76 \\
\hline & 0.34 & 0.00 & \multicolumn{2}{|c|}{28 - Chemicals } & 0.60 & 9.96 \\
\hline \multicolumn{2}{|c|}{$\begin{array}{l}\text { lown of Hepzlbah } \\
\text { Augusta-Richmond Utilities }\end{array}$} & 8.42 & 29.69 & \multicolumn{2}{|c|}{32 - Stone, clay } & 0.61 & 0.00 \\
\hline
\end{tabular}
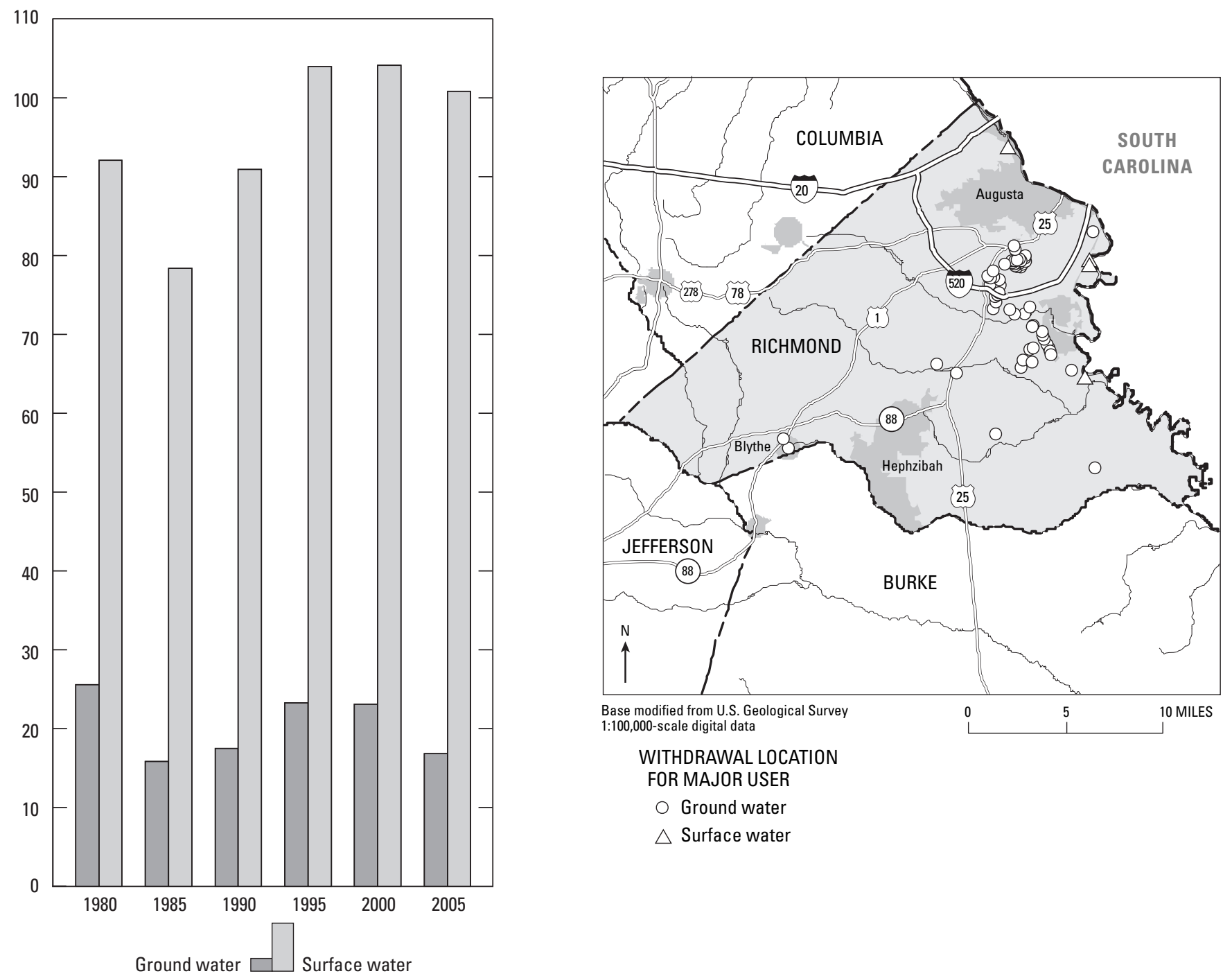


\section{ROCKDALE COUNTY}

Population: 78,545

Population served by public supply: $\quad 58,990$

Acres irrigated: 500

Hydroelectric use (Mgal/d): 41.96

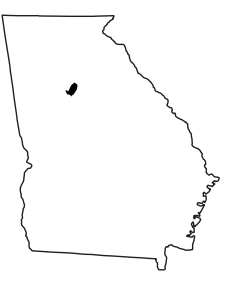

2005 WITHDRAWALS, IN MILLION GALLONS PER DAY

\begin{tabular}{|c|c|c|c|c|c|c|c|}
\hline & $\begin{array}{l}\text { Public } \\
\text { Supply }\end{array}$ & $\begin{array}{l}\text { Domestic \& } \\
\text { Commercial }\end{array}$ & $\begin{array}{c}\text { Industrial \& } \\
\text { Mining }\end{array}$ & Irrigation & Livestock & $\begin{array}{l}\text { Thermo- } \\
\text { electric }\end{array}$ & Totals \\
\hline Ground Water & 0.18 & 4.97 & 0.15 & 0.00 & 0.00 & 0.00 & 5.30 \\
\hline Surface Water & 12.36 & 0.00 & 0.00 & 0.31 & 0.02 & 0.00 & 12.69 \\
\hline TOTALS & 12.54 & 4.97 & 0.15 & 0.31 & 0.02 & 0.00 & 17.99 \\
\hline
\end{tabular}

Withdrawals by Major Public Suppliers (Mgal/d):

Name

City of Conyers

Milstead Water System

Rockdale County Board

of Commissioners
Withdrawals by Major Industrial Groups (Mgal/d):

SIC

GW

SW

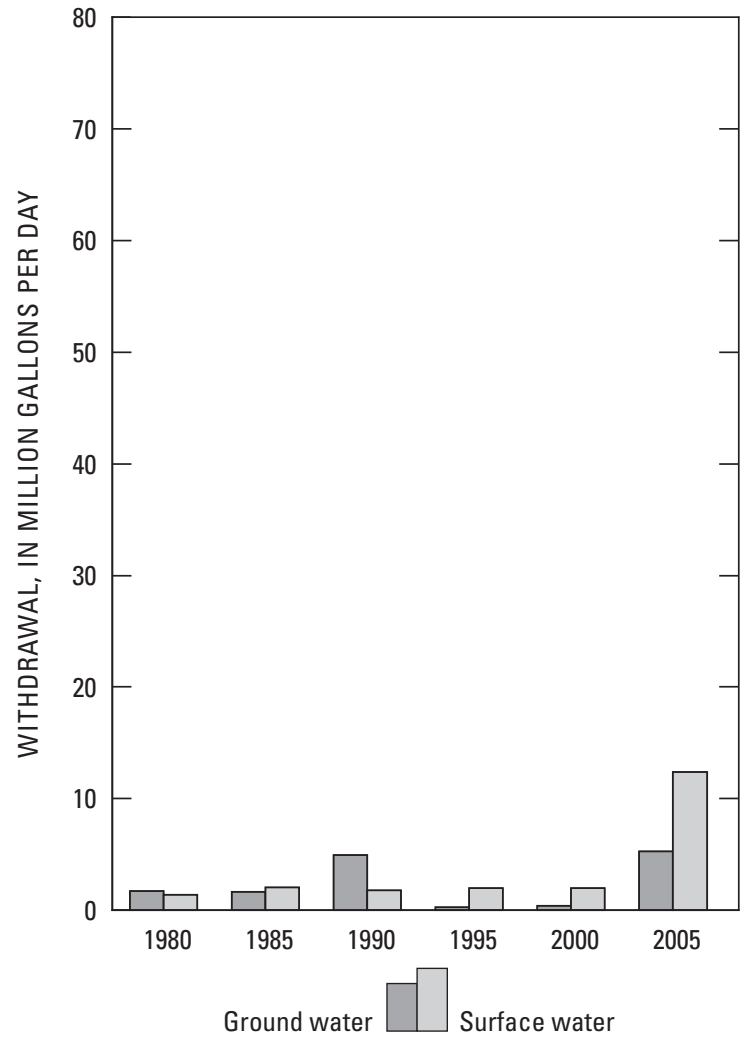

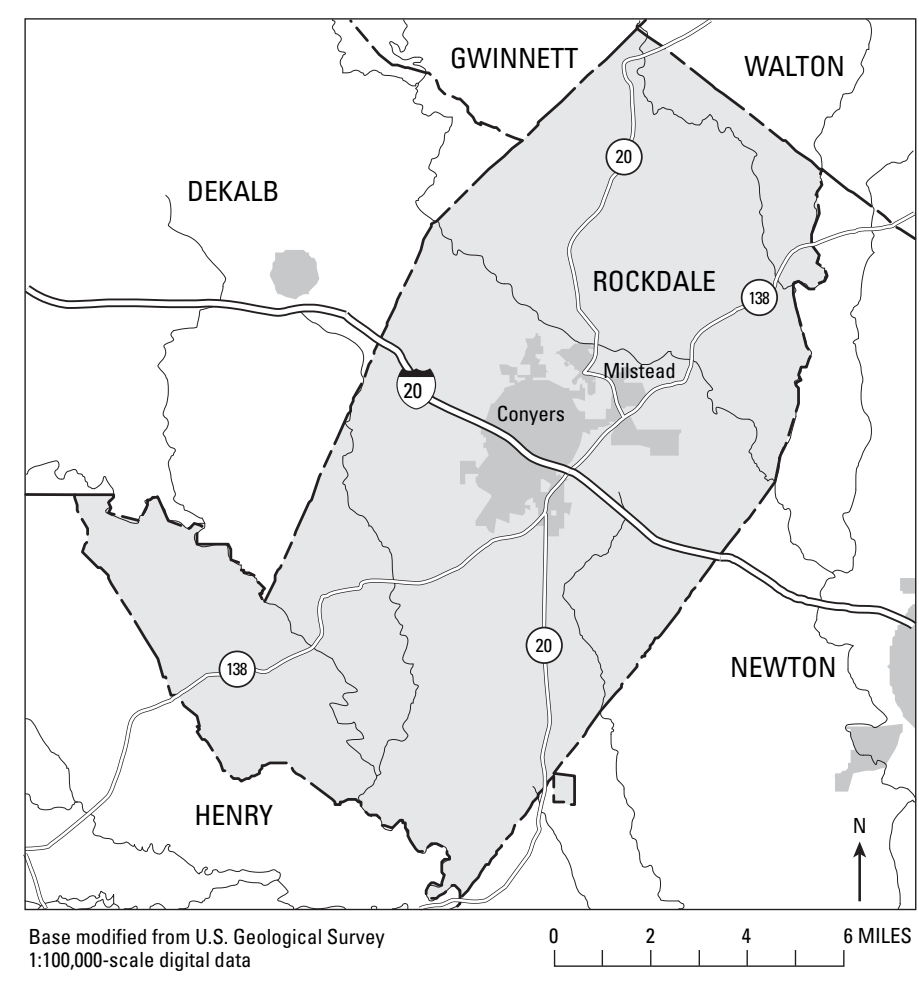




\section{SCHLEY COUNTY}

Population: 4,122

Population served by public supply: 3,450

Acres irrigated: 990

Hydroelectric use (Mgal/d): $\quad 0.00$

2005 WITHDRAWALS, IN MILLION GALLONS PER DAY

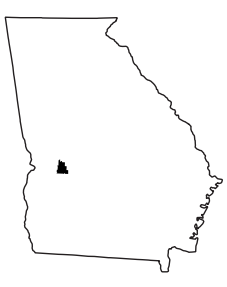

\begin{tabular}{lccccccc}
\hline & $\begin{array}{c}\text { Public } \\
\text { Supply }\end{array}$ & $\begin{array}{c}\text { Domestic \& } \\
\text { Commercial }\end{array}$ & $\begin{array}{c}\text { Industrial \& } \\
\text { Mining }\end{array}$ & Irrigation & Livestock & $\begin{array}{c}\text { Thermo- } \\
\text { electric }\end{array}$ & Totals \\
\cline { 1 - 5 } Ground Water & 0.63 & 0.05 & 0.00 & 0.03 & 0.01 & 0.00 & 0.72 \\
Surface Water & 0.00 & 0.00 & 0.00 & 0.25 & 0.07 & 0.00 & 0.32 \\
TOTALS & 0.63 & 0.05 & 0.00 & 0.28 & 0.08 & 0.00 & 1.04 \\
\hline
\end{tabular}

Withdrawals by Major Public Suppliers (Mgal/d):

$\begin{array}{lcc}\text { Name } & \text { GW } & \text { SW } \\ \text { City of Ellaville } & 0.26 & 0.00 \\ \text { Schley County Board } & 0.37 & 0.00\end{array}$

Withdrawals by Major Industrial Groups (Mgal/d):

SIC

GW

SW

Schley County Board

0.00

None

of Commissioners
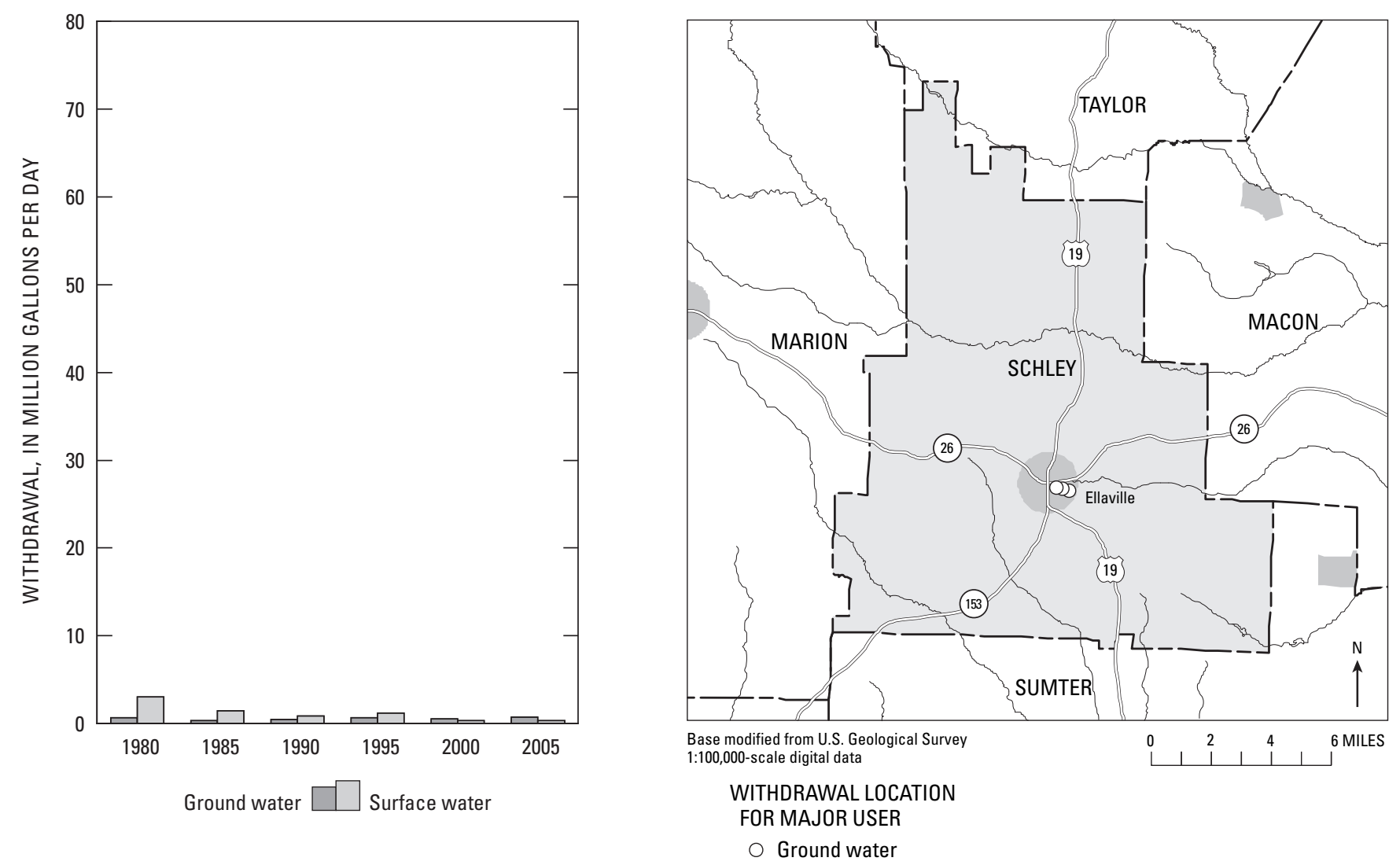


\section{SCREVEN COUNTY}

Population: 15,430

Population served by public supply: $\quad 6,340$

Acres irrigated: 25,000

Hydroelectric use (Mgal/d): $\quad 0.00$

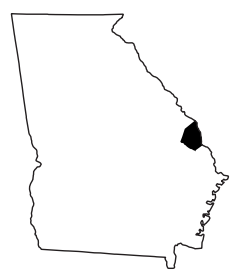

2005 WITHDRAWALS, IN MILLION GALLONS PER DAY

\begin{tabular}{|c|c|c|c|c|c|c|c|}
\hline & $\begin{array}{l}\text { Public } \\
\text { Supply }\end{array}$ & $\begin{array}{l}\text { Domestic \& } \\
\text { Commercial }\end{array}$ & $\begin{array}{c}\text { Industrial \& } \\
\text { Mining }\end{array}$ & Irrigation & Livestock & $\begin{array}{l}\text { Thermo- } \\
\text { electric }\end{array}$ & Totals \\
\hline Ground Water & 1.02 & 0.68 & 1.73 & 5.56 & 0.05 & 0.00 & 9.04 \\
\hline Surface Water & 0.00 & 0.00 & 0.00 & 5.23 & 0.06 & 0.00 & 5.29 \\
\hline TOTALS & 1.02 & 0.68 & 1.73 & 10.79 & 0.11 & 0.00 & 14.33 \\
\hline
\end{tabular}

Withdrawals by Major Public Suppliers (Mgal/d):

Name

City of Hiltonia

GW SW

Town of Newington

City of Oliver

City of Sylvania

0.00

$0.03 \quad 0.00$

$0.03 \quad 0.00$

$0.81 \quad 0.00$

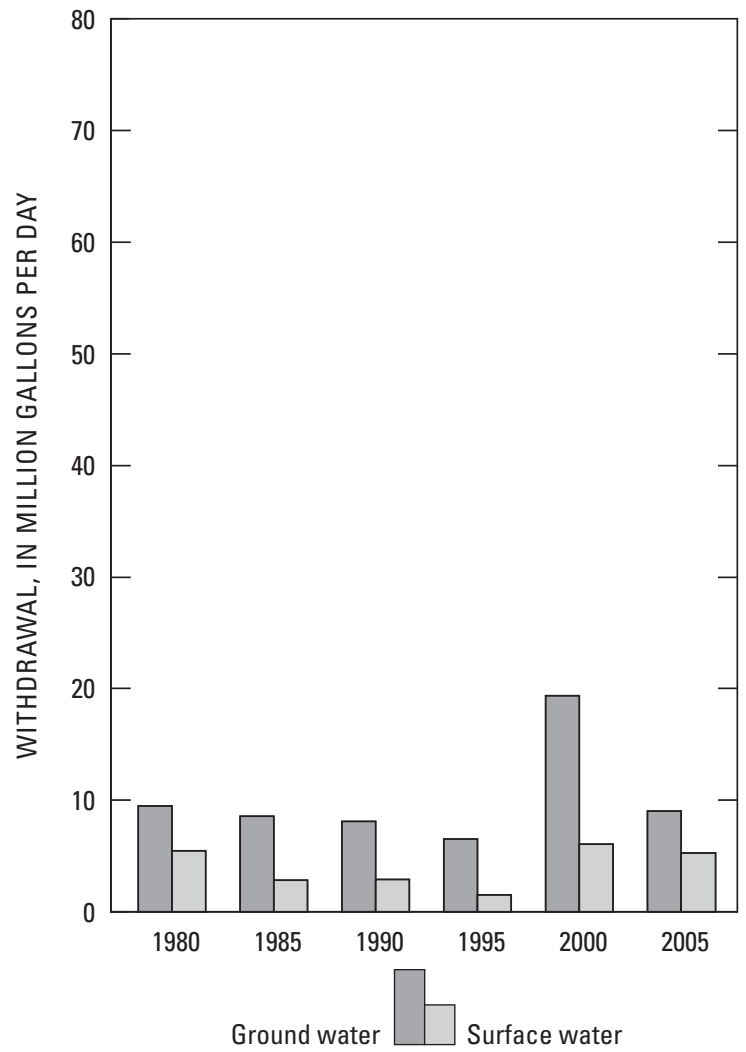

Withdrawals by Major Industrial Groups (Mgal/d):

SIC GW SW

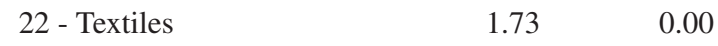

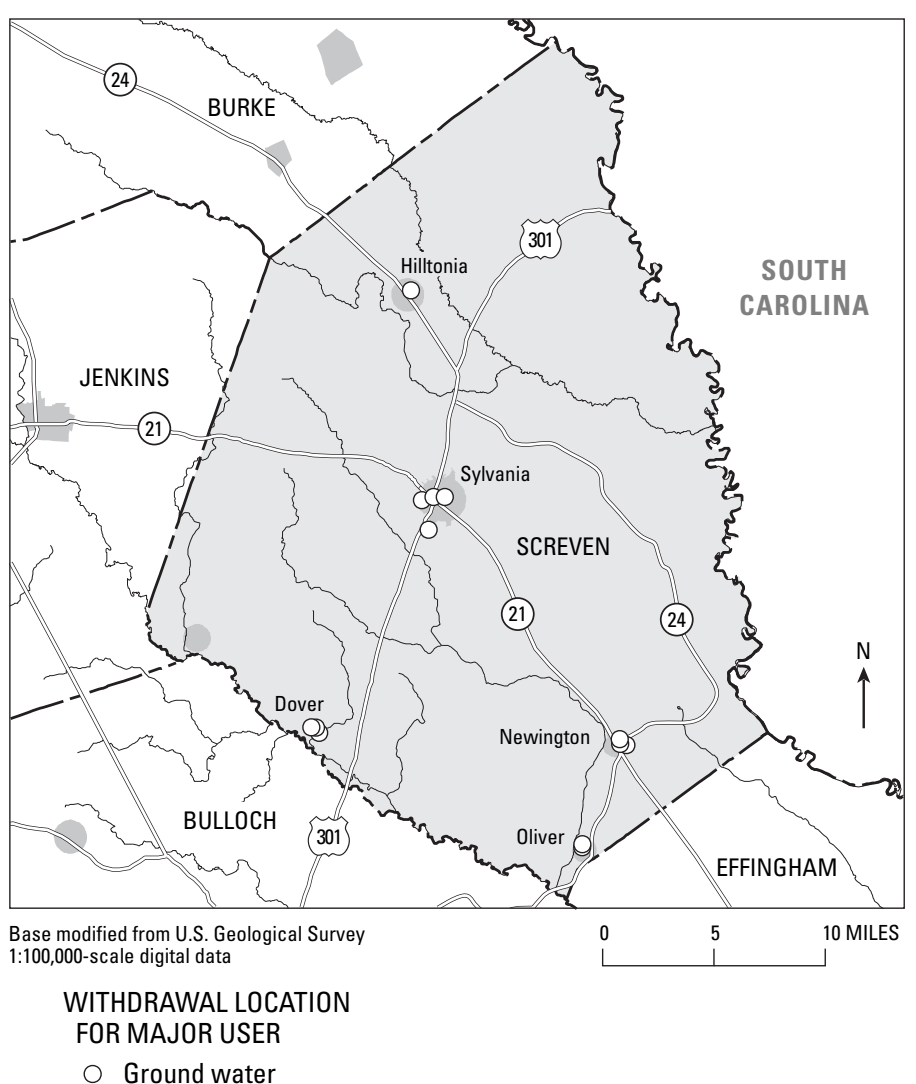




\section{SEMINOLE COUNTY}

Population: 9,226

Population served by public supply: 3,970

Acres irrigated: 57,550

Hydroelectric use (Mgal/d): $\quad 0.00$

2005 WITHDRAWALS, IN MILLION GALLONS PER DAY

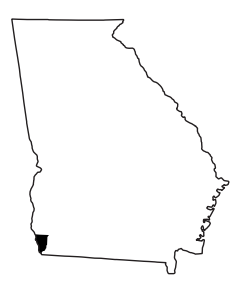

\begin{tabular}{|c|c|c|c|c|c|c|c|}
\hline & $\begin{array}{l}\text { Public } \\
\text { Supply }\end{array}$ & $\begin{array}{l}\text { Domestic \& } \\
\text { Commercial }\end{array}$ & $\begin{array}{c}\text { Industrial \& } \\
\text { Mining }\end{array}$ & Irrigation & Livestock & $\begin{array}{l}\text { Thermo- } \\
\text { electric }\end{array}$ & Totals \\
\hline Ground Water & 0.63 & 0.39 & 0.00 & 35.13 & 0.10 & 0.00 & 36.25 \\
\hline Surface Water & 0.00 & 0.00 & 0.00 & 3.17 & 0.02 & 0.00 & 3.19 \\
\hline TOTALS & 0.63 & 0.39 & 0.00 & 38.30 & 0.12 & 0.00 & 39.44 \\
\hline
\end{tabular}

Withdrawals by Major Public Suppliers (Mgal/d):

$\begin{array}{lcc}\text { Name } & \text { GW } & \text { SW } \\ \text { City of Donalsonville } & 0.56 & 0.00 \\ \text { Town of Iron City } & 0.03 & 0.00\end{array}$

Withdrawals by Major Industrial Groups (Mgal/d):

SIC

GW

SW

None
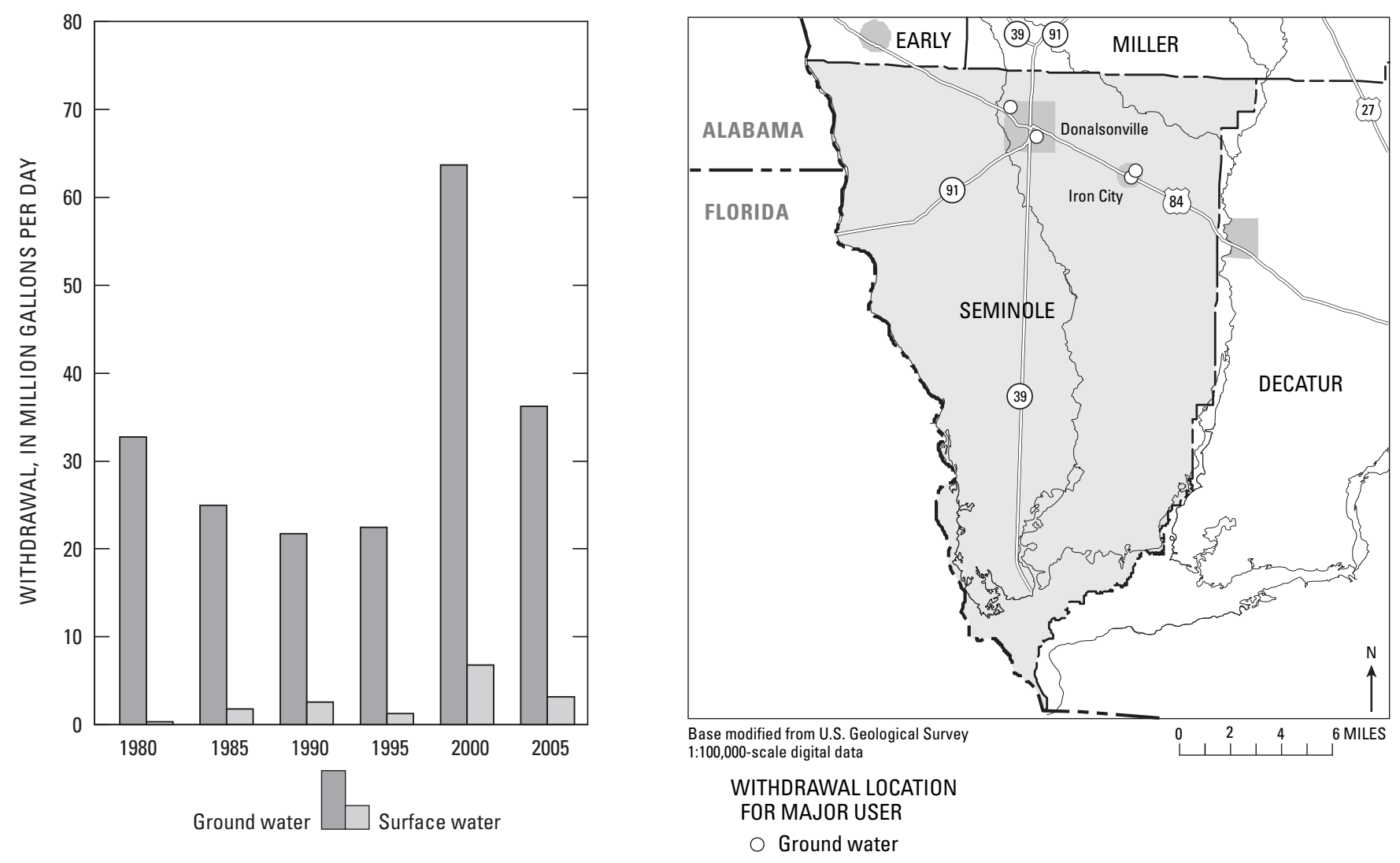


\section{SPALDING COUNTY}

Population: 61,289

Population served by public supply: $\quad 49,640$

Acres irrigated: 530

Hydroelectric use (Mgal/d): $\quad 0.00$

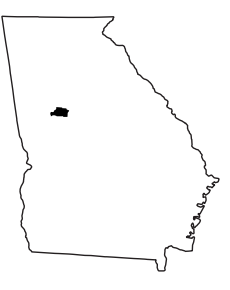

2005 WITHDRAWALS, IN MILLION GALLONS PER DAY

\begin{tabular}{|c|c|c|c|c|c|c|c|}
\hline & $\begin{array}{l}\text { Public } \\
\text { Supply }\end{array}$ & $\begin{array}{l}\text { Domestic \& } \\
\text { Commercial }\end{array}$ & $\begin{array}{c}\text { Industrial \& } \\
\text { Mining }\end{array}$ & Irrigation & Livestock & $\begin{array}{l}\text { Thermo- } \\
\text { electric }\end{array}$ & Totals \\
\hline Ground Water & 0.10 & 0.87 & 0.21 & 0.00 & 0.00 & 0.00 & 1.18 \\
\hline Surface Water & 7.97 & 0.00 & 0.00 & 0.81 & 0.07 & 0.00 & 8.85 \\
\hline TOTALS & 8.07 & 0.87 & 0.21 & 0.81 & 0.07 & 0.00 & 10.03 \\
\hline
\end{tabular}

Withdrawals by Major Public Suppliers (Mgal/d):

Name

GW SW

Withdrawals by Major Industrial Groups (Mgal/d):

City of Griffin

$0.00 \quad 7.97$

SIC

GW

SW

None
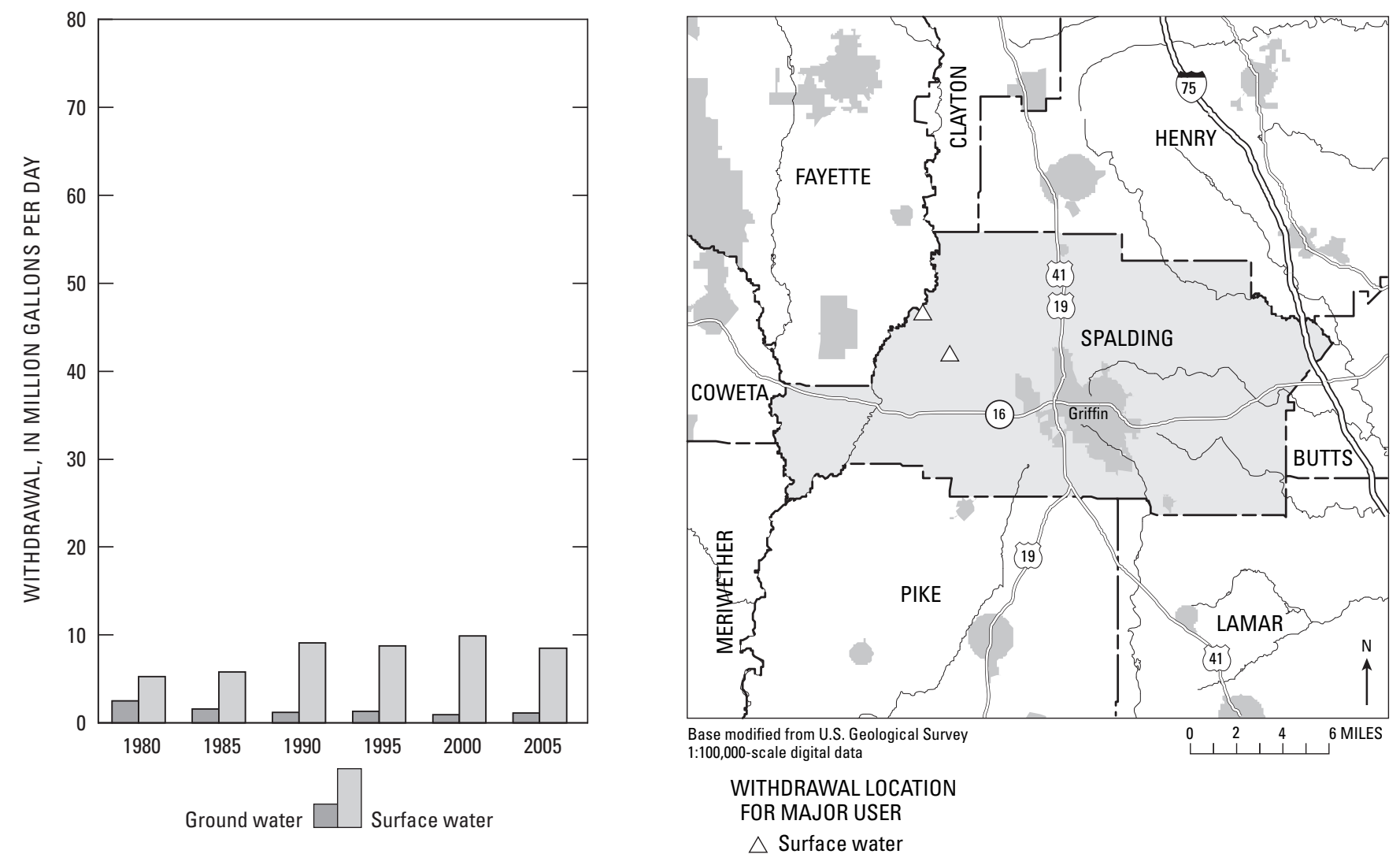


\section{STEPHENS COUNTY}

Population: 25,060

Population served by public supply: 22,550

Acres irrigated: 120

Hydroelectric use (Mgal/d): $\quad 1,077.19$

2005 WITHDRAWALS, IN MILLION GALLONS PER DAY

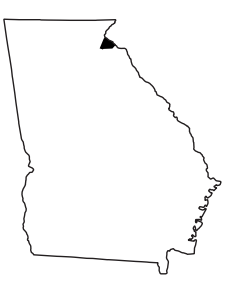

\begin{tabular}{|c|c|c|c|c|c|c|c|}
\hline & $\begin{array}{l}\text { Public } \\
\text { Supply }\end{array}$ & $\begin{array}{l}\text { Domestic \& } \\
\text { Commercial }\end{array}$ & $\begin{array}{c}\text { Industrial \& } \\
\text { Mining }\end{array}$ & Irrigation & Livestock & $\begin{array}{l}\text { Thermo- } \\
\text { electric }\end{array}$ & Totals \\
\hline Ground Water & 0.09 & 0.19 & 0.11 & 0.00 & 0.00 & 0.00 & 0.39 \\
\hline Surface Water & 3.88 & 0.00 & 0.00 & 0.07 & 0.23 & 0.00 & 4.18 \\
\hline TOTALS & 3.97 & 0.19 & 0.11 & 0.07 & 0.23 & 0.00 & 4.57 \\
\hline
\end{tabular}

Withdrawals by Major Public Suppliers (Mgal/d):

Name

Town of Martin

City of Toccoa
GW SW

$0.08 \quad 0.00$

$0.00 \quad 3.88$
Withdrawals by Major Industrial Groups (Mgal/d):

SIC

None
GW

SW
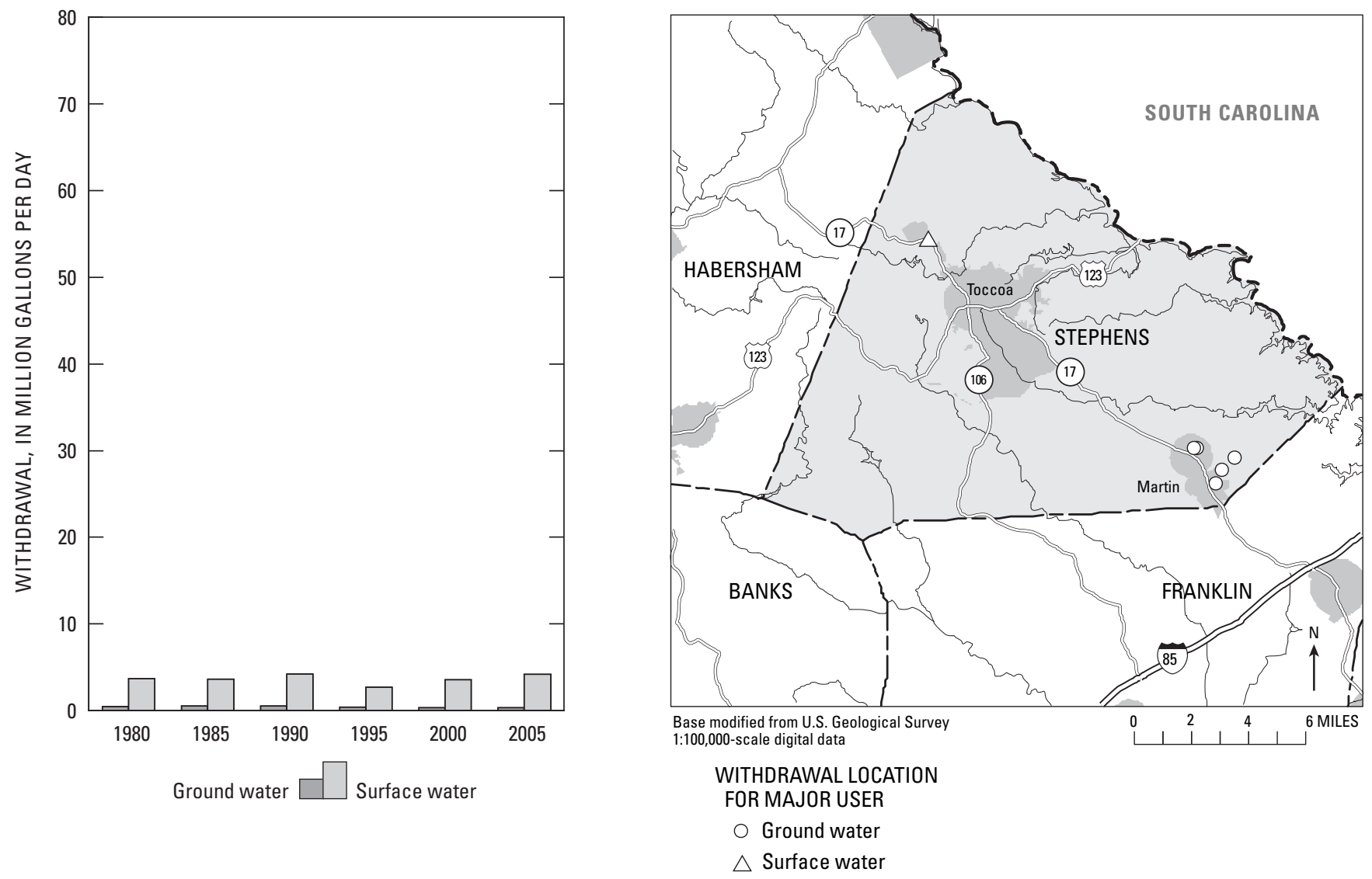


\section{STEWART COUNTY}

Population: 4,882

Population served by public supply: $\quad 4,350$

Acres irrigated: 2,210

Hydroelectric use (Mgal/d): $\quad 0.00$

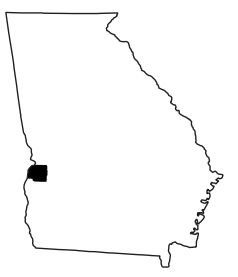

2005 WITHDRAWALS, IN MILLION GALLONS PER DAY

\begin{tabular}{|c|c|c|c|c|c|c|c|}
\hline & $\begin{array}{l}\text { Public } \\
\text { Supply }\end{array}$ & $\begin{array}{l}\text { Domestic \& } \\
\text { Commercial }\end{array}$ & $\begin{array}{c}\text { Industrial \& } \\
\text { Mining }\end{array}$ & Irrigation & Livestock & $\begin{array}{l}\text { Thermo- } \\
\text { electric }\end{array}$ & Totals \\
\hline Ground Water & 0.22 & 0.04 & 0.00 & 0.00 & 0.03 & 0.00 & 0.29 \\
\hline Surface Water & 0.00 & 0.00 & 0.00 & 0.78 & 0.03 & 0.00 & 0.81 \\
\hline TOTALS & 0.22 & 0.04 & 0.00 & 0.78 & 0.06 & 0.00 & 1.10 \\
\hline
\end{tabular}

Withdrawals by Major Public Suppliers (Mgal/d):

Name

City of Omaha

City of Lumpkin
GW

0.02

0.13
SW

0.00

0.00
Withdrawals by Major Industrial Groups (Mgal/d):

SIC

None
GW

SW
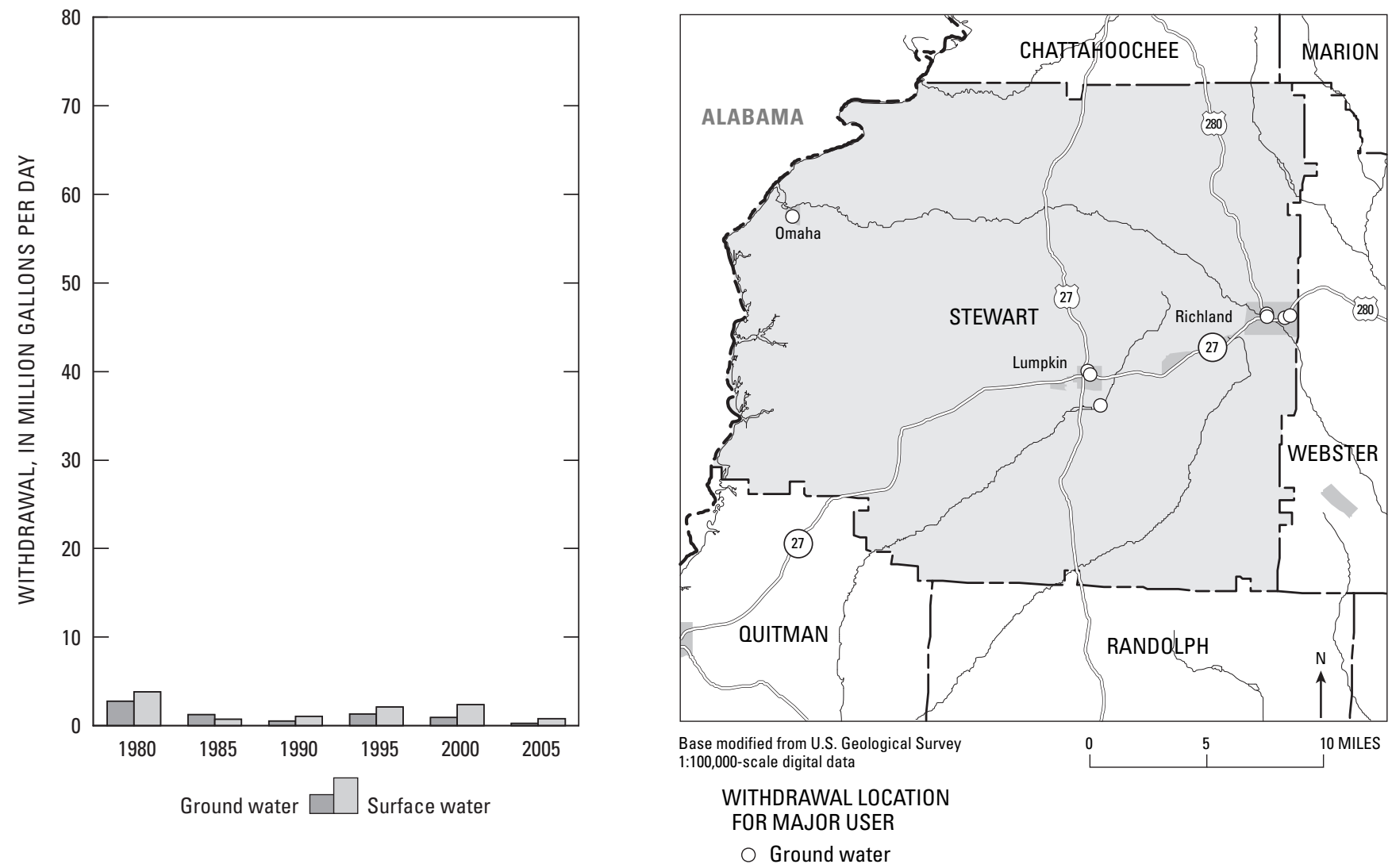


\section{SUMTER COUNTY}

Population: 32,912

Population served by public supply: 21,320

Acres irrigated: 52,550

Hydroelectric use (Mgal/d): $\quad 0.00$

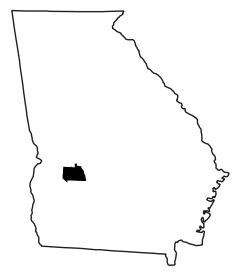

2005 WITHDRAWALS, IN MILLION GALLONS PER DAY

\begin{tabular}{|c|c|c|c|c|c|c|c|}
\hline & $\begin{array}{l}\text { Public } \\
\text { Supply }\end{array}$ & $\begin{array}{l}\text { Domestic \& } \\
\text { Commercial }\end{array}$ & $\begin{array}{c}\text { Industrial \& } \\
\text { Mining }\end{array}$ & Irrigation & Livestock & $\begin{array}{l}\text { Thermo- } \\
\text { electric }\end{array}$ & Totals \\
\hline Ground Water & 3.05 & 0.87 & 0.71 & 5.78 & 0.05 & 0.00 & 10.46 \\
\hline Surface Water & 0.00 & 0.00 & 0.00 & 3.32 & 0.18 & 0.00 & 3.50 \\
\hline TOTALS & 3.05 & 0.87 & 0.71 & 9.10 & 0.23 & 0.00 & 13.96 \\
\hline
\end{tabular}

Withdrawals by Major Public Suppliers (Mgal/d):

Name GW SW

Village of Andersonville $\quad 0.03 \quad 0.00$

$\begin{array}{lll}\text { City of Americus } & 2.87 & 0.00\end{array}$

Village of Desoto $\quad 0.03 \quad 0.00$

Village of Leslie $\quad 0.03 \quad 0.00$
Withdrawals by Major Industrial Groups (Mgal/d):

$\begin{array}{lcc}\text { SIC } & \text { GW } & \text { SW } \\ 32 \text { - Stone, clay } & 0.50 & 0.00\end{array}$

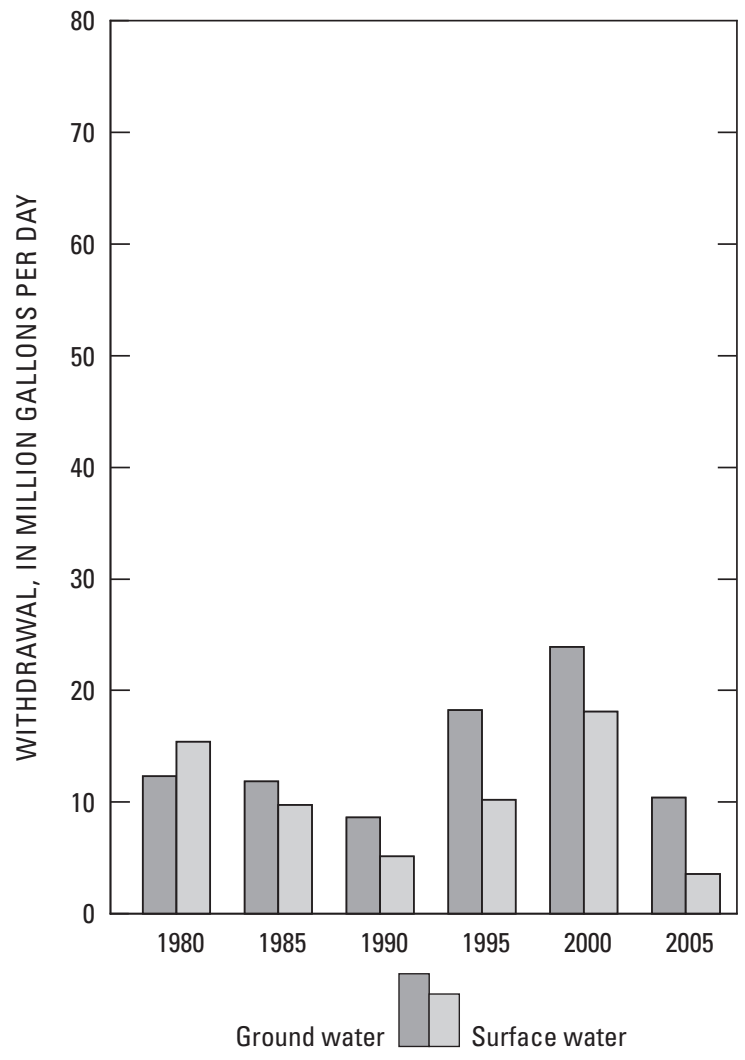

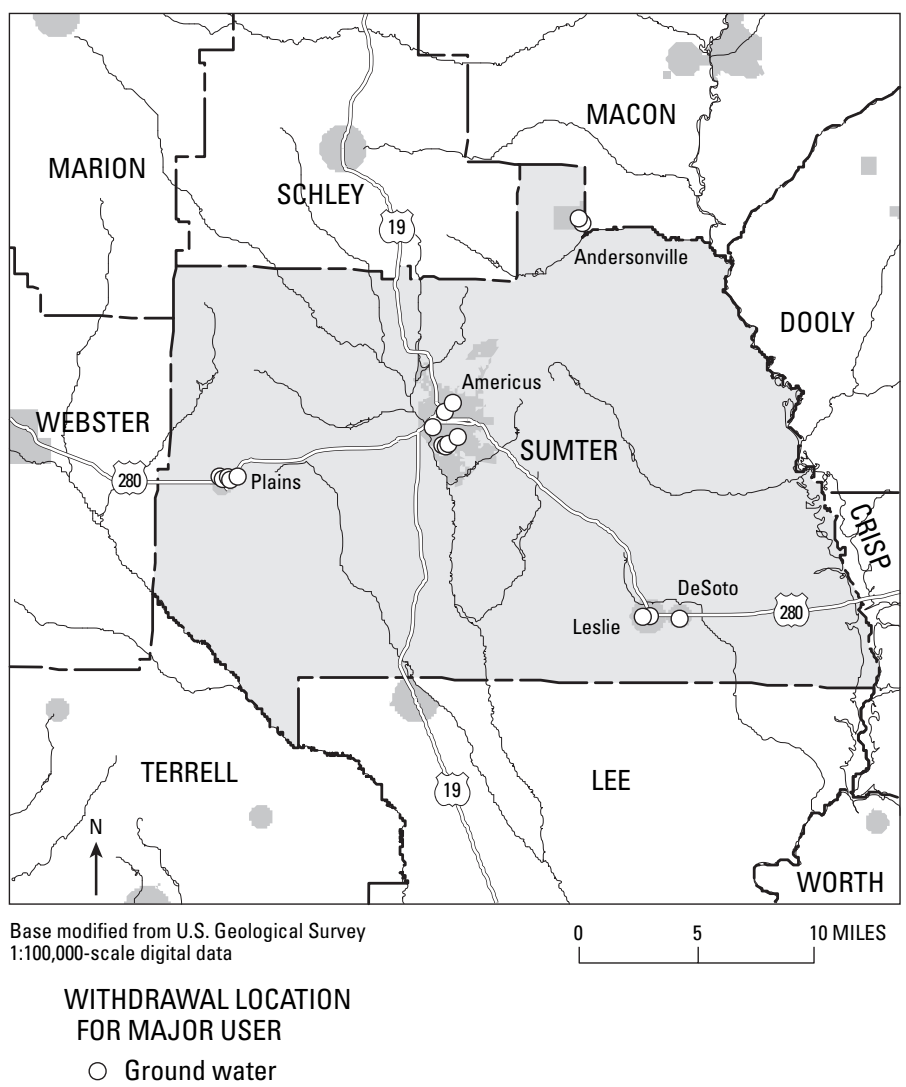




\section{TALBOT COUNTY}

Population: 6,709

Population served by public supply: 4,840

Acres irrigated: 0

Hydroelectric use (Mgal/d): $\quad 0.00$

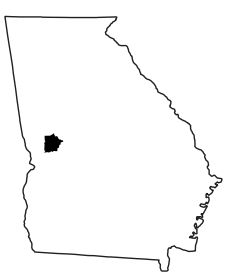

2005 WITHDRAWALS, IN MILLION GALLONS PER DAY

\begin{tabular}{lccccccc}
\hline & $\begin{array}{c}\text { Public } \\
\text { Supply }\end{array}$ & $\begin{array}{c}\text { Domestic \& } \\
\text { Commercial }\end{array}$ & $\begin{array}{c}\text { Industrial \& } \\
\text { Mining }\end{array}$ & Irrigation & Livestock & $\begin{array}{c}\text { Thermo- } \\
\text { electric }\end{array}$ & Totals \\
\hline Ground Water & 0.18 & 0.14 & 0.90 & 0.00 & 0.00 & 0.00 & 1.22 \\
Surface Water & 0.00 & 0.00 & 0.01 & 0.00 & 0.14 & 0.00 & 0.15 \\
TOTALS & 0.18 & 0.14 & 0.91 & 0.00 & 0.14 & 0.00 & 1.37 \\
\hline
\end{tabular}

Withdrawals by Major Public Suppliers (Mgal/d):

Name

Town of Geneva

GW SW

Town of Junction City

City of Talbotton

City of Woodland
0.02

0.01

0.09

0.05
Withdrawals by Major Industrial Groups (Mgal/d):

SIC

None
GW

SW
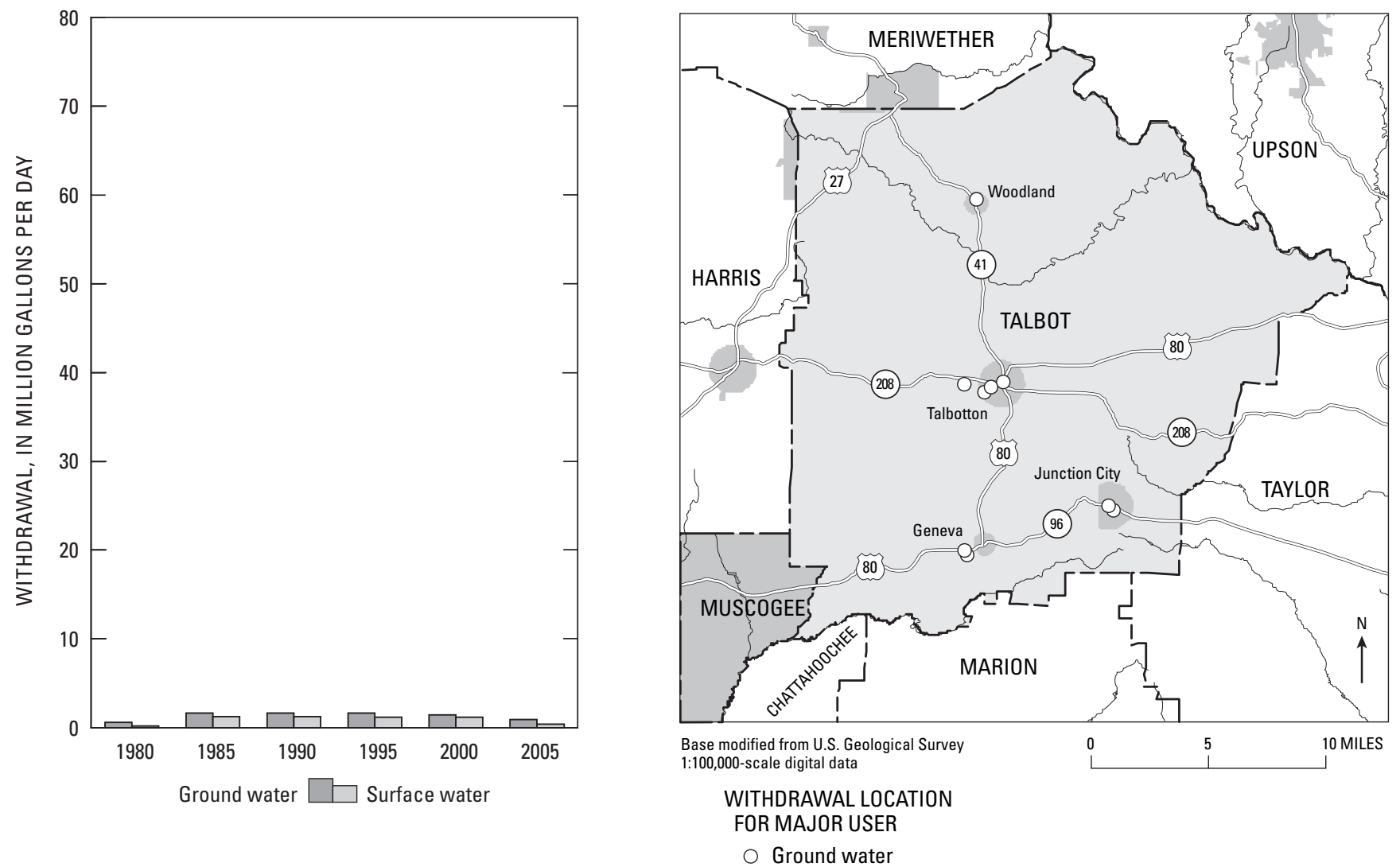


\section{TALIAFERRO COUNTY}

Population: 1,826

Population served by public supply: 700

Acres irrigated: 0

Hydroelectric use (Mgal/d): $\quad 0.00$

2005 WITHDRAWALS, IN MILLION GALLONS PER DAY

\begin{tabular}{|c|c|c|c|c|c|c|c|}
\hline & $\begin{array}{l}\text { Public } \\
\text { Supply }\end{array}$ & $\begin{array}{l}\text { Domestic \& } \\
\text { Commercial }\end{array}$ & $\begin{array}{c}\text { Industrial \& } \\
\text { Mining }\end{array}$ & Irrigation & Livestock & $\begin{array}{l}\text { Thermo- } \\
\text { electric }\end{array}$ & Totals \\
\hline Ground Water & 0.05 & 0.08 & 0.00 & 0.00 & 0.00 & 0.00 & 0.13 \\
\hline Surface Water & 0.00 & 0.00 & 0.00 & 0.00 & 0.06 & 0.00 & 0.06 \\
\hline TOTALS & 0.05 & 0.08 & 0.00 & 0.00 & 0.06 & 0.00 & 0.19 \\
\hline
\end{tabular}

Withdrawals by Major Public Suppliers (Mgal/d):

Name

City of Crawfordville

GW

0.05

SW

0.00
Withdrawals by Major Industrial Groups (Mgal/d):

SIC

GW

SW

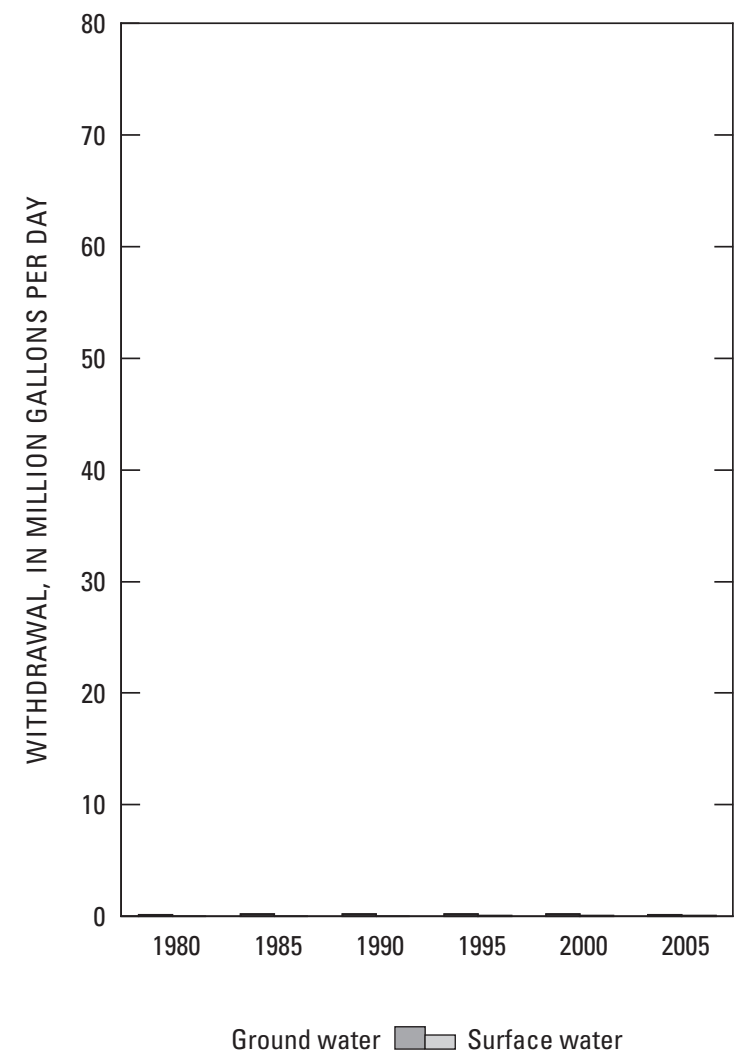

Ground water $\square \square$ Surface water

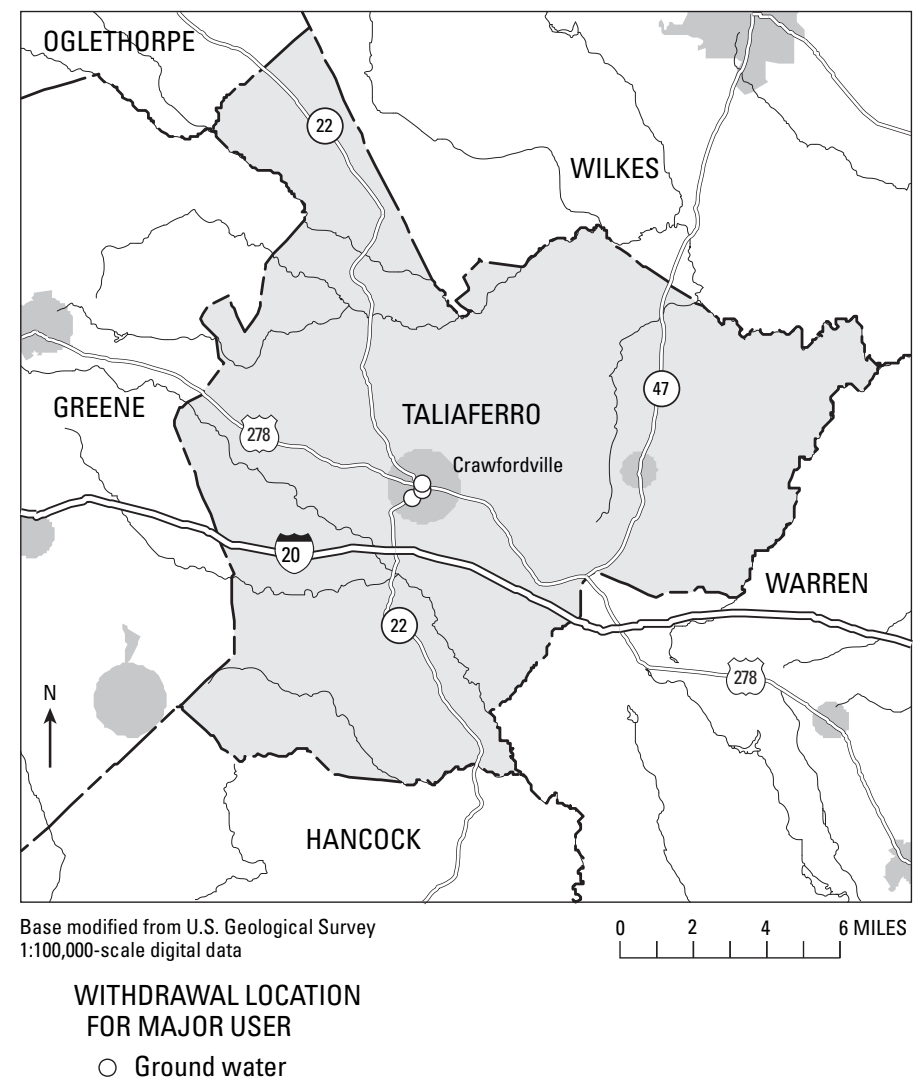




\section{TATTNALL COUNTY}

Population: 133,211

Population served by public supply: $\quad 9,270$

Acres irrigated: 26,580

Hydroelectric use (Mgal/d): $\quad 0.00$

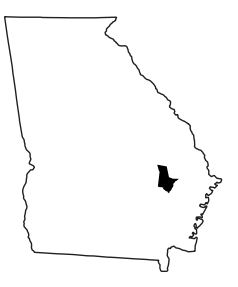

2005 WITHDRAWALS, IN MILLION GALLONS PER DAY

\begin{tabular}{lccccccc}
\hline & $\begin{array}{c}\text { Public } \\
\text { Supply }\end{array}$ & $\begin{array}{c}\text { Domestic \& } \\
\text { Commercial }\end{array}$ & $\begin{array}{c}\text { Industrial \& } \\
\text { Mining }\end{array}$ & Irrigation & Livestock & $\begin{array}{c}\text { Thermo- } \\
\text { electric }\end{array}$ & Totals \\
\hline Ground Water & 1.12 & 1.80 & 0.09 & 1.69 & 0.06 & 0.00 & 4.76 \\
Surface Water & 0.00 & 0.00 & 0.00 & 3.71 & 0.51 & 0.00 & 4.22 \\
TOTALS & 1.12 & 1.80 & 0.09 & 5.40 & 0.57 & 0.00 & 8.98 \\
\hline
\end{tabular}

Withdrawals by Major Public Suppliers (Mgal/d):

Name

City of Cobbtown

City of Collins

City of Glennville

City of Manassas

City of Reidsville

$\mathrm{GW}$

0.05

0.04

0.73

0.01

0.28
SW

0.00

0.00

0.00

0.00

0.00
Withdrawals by Major Industrial Groups (Mgal/d):

SIC

None
GW

SW
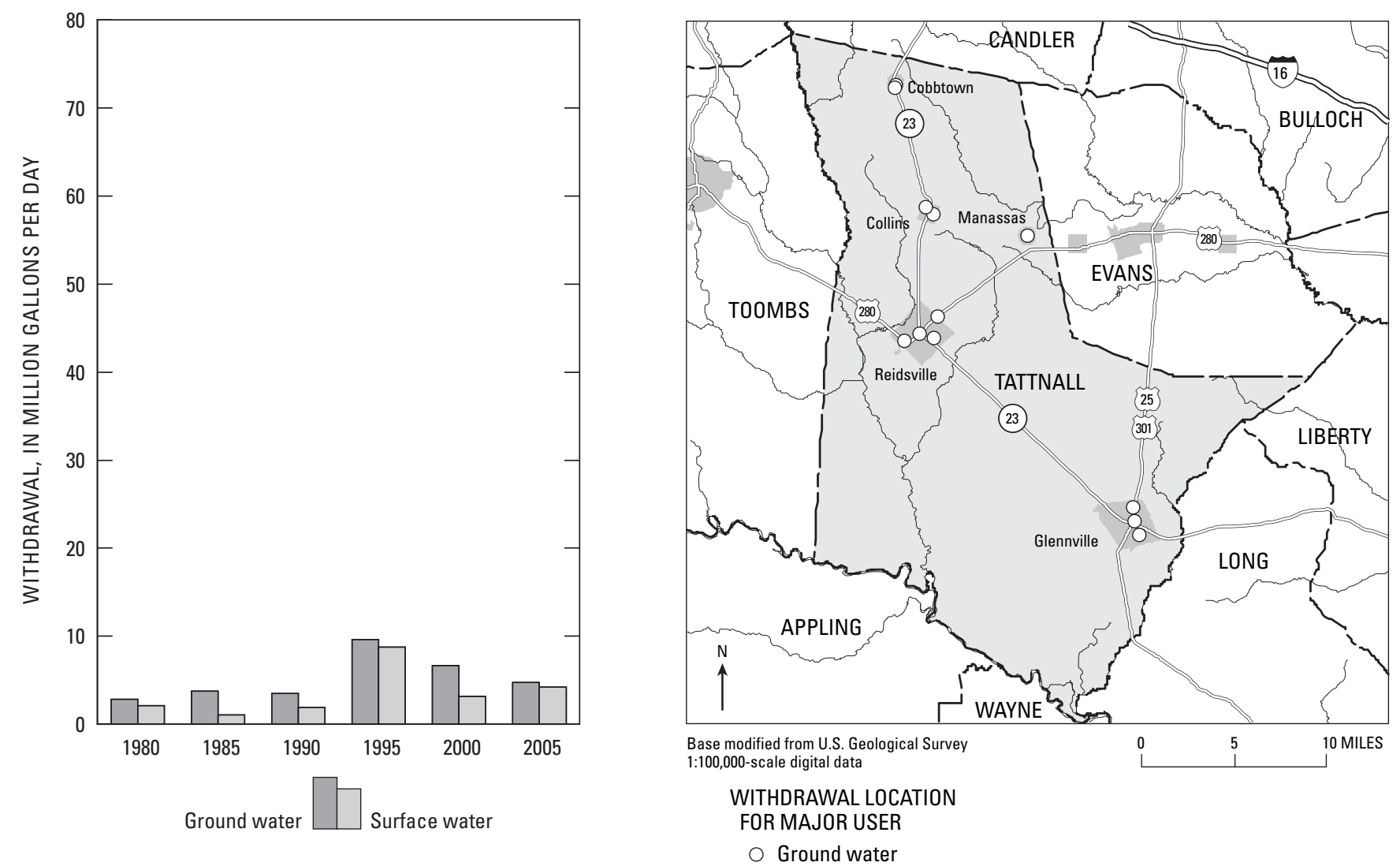


\section{TAYLOR COUNTY}

Population: 8,887

Population served by public supply: 4,700

Acres irrigated: 2,650

Hydroelectric use (Mgal/d): $\quad 0.00$

2005 WITHDRAWALS, IN MILLION GALLONS PER DAY

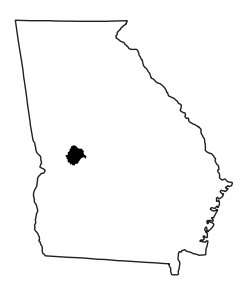

\begin{tabular}{lccccccc}
\hline & $\begin{array}{c}\text { Public } \\
\text { Supply }\end{array}$ & $\begin{array}{c}\text { Domestic \& } \\
\text { Commercial }\end{array}$ & $\begin{array}{c}\text { Industrial \& } \\
\text { Mining }\end{array}$ & Irrigation & Livestock & $\begin{array}{c}\text { Thermo- } \\
\text { electric }\end{array}$ & Totals \\
\hline Ground Water & 0.78 & 0.31 & 0.76 & 0.85 & 0.02 & 0.00 & 2.72 \\
Surface Water & 0.00 & 0.00 & 1.80 & 0.61 & 0.13 & 0.00 & 2.54 \\
TOTALS & 0.78 & 0.31 & 2.56 & 1.46 & 0.15 & 0.00 & 5.26 \\
\hline
\end{tabular}

Withdrawals by Major Public Suppliers (Mgal/d):

$\begin{array}{lcc}\text { Name } & \text { GW } & \text { SW } \\ \text { Town of Butler } & 0.61 & 0.00 \\ \text { Town of Reynolds } & 0.16 & 0.00\end{array}$

Withdrawals by Major Industrial Groups (Mgal/d):

SIC GW SW

32 - Stone, clay $\quad 0.00 \quad 1.79$
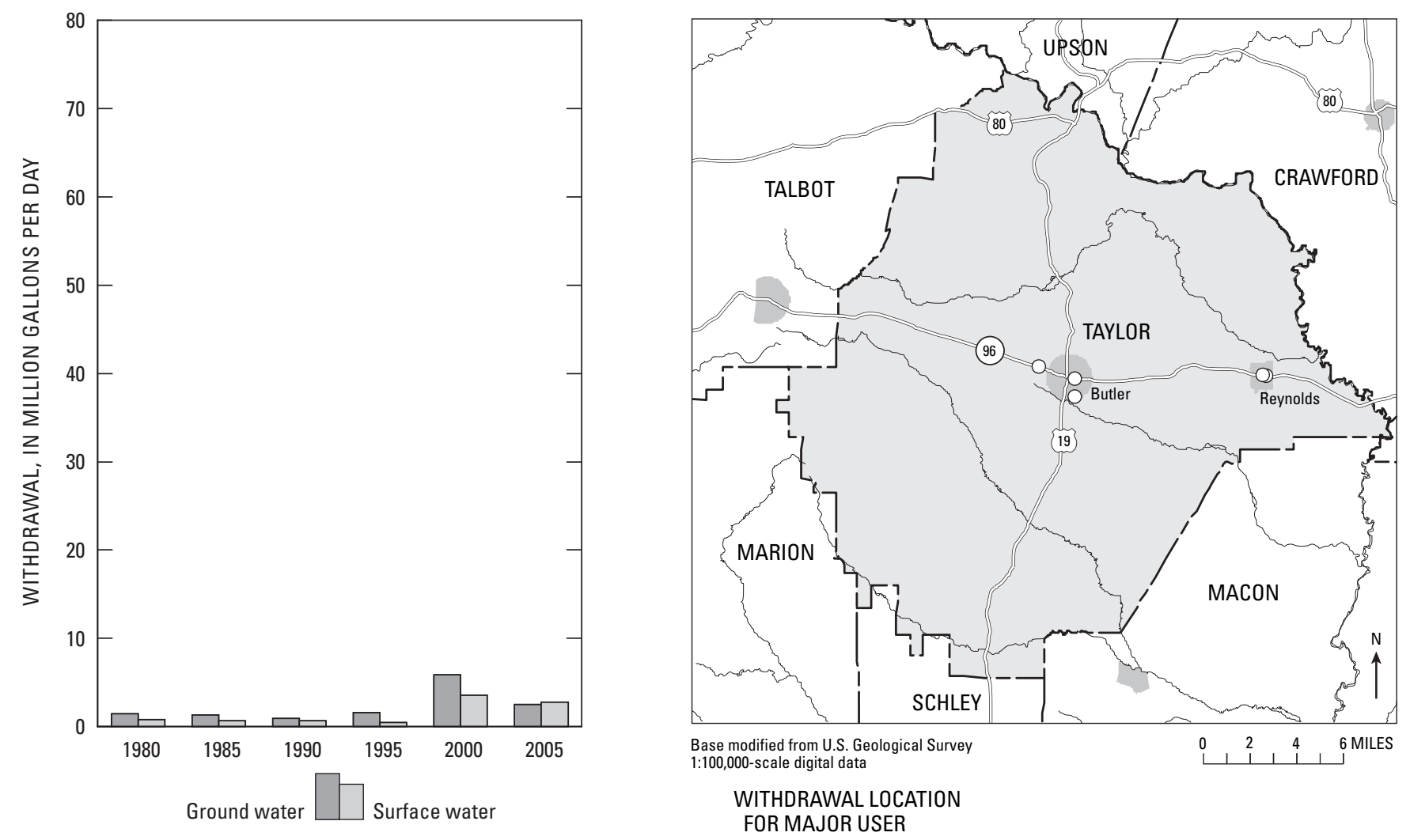

O Ground water 


\section{TELFAIR COUNTY}

Population: 13,205

Population served by public supply: $\quad 9,070$

Acres irrigated: $\quad 15,000$

Hydroelectric use (Mgal/d): $\quad 0.00$

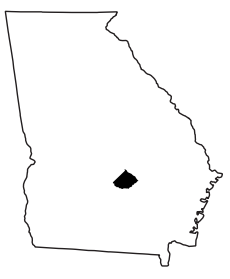

2005 WITHDRAWALS, IN MILLION GALLONS PER DAY

\begin{tabular}{|c|c|c|c|c|c|c|c|}
\hline & $\begin{array}{l}\text { Public } \\
\text { Supply }\end{array}$ & $\begin{array}{l}\text { Domestic \& } \\
\text { Commercial }\end{array}$ & $\begin{array}{c}\text { Industrial \& } \\
\text { Mining }\end{array}$ & Irrigation & Livestock & $\begin{array}{l}\text { Thermo- } \\
\text { electric }\end{array}$ & Totals \\
\hline Ground Water & 1.27 & 0.31 & 0.09 & 2.07 & 0.01 & 0.00 & 3.75 \\
\hline Surface Water & 0.00 & 0.00 & 0.00 & 0.95 & 0.05 & 0.00 & 1.00 \\
\hline TOTALS & 1.27 & 0.31 & 0.09 & 3.02 & 0.06 & 0.00 & 4.75 \\
\hline
\end{tabular}

Withdrawals by Major Public Suppliers (Mgal/d):

Name

Town of Helena

GW SW

Town of Jacksonville

City of Lumber City

City of McRae

Town of Milan

City of Scotland

0.19

0.00

0.65

0.08

0.00

0.02

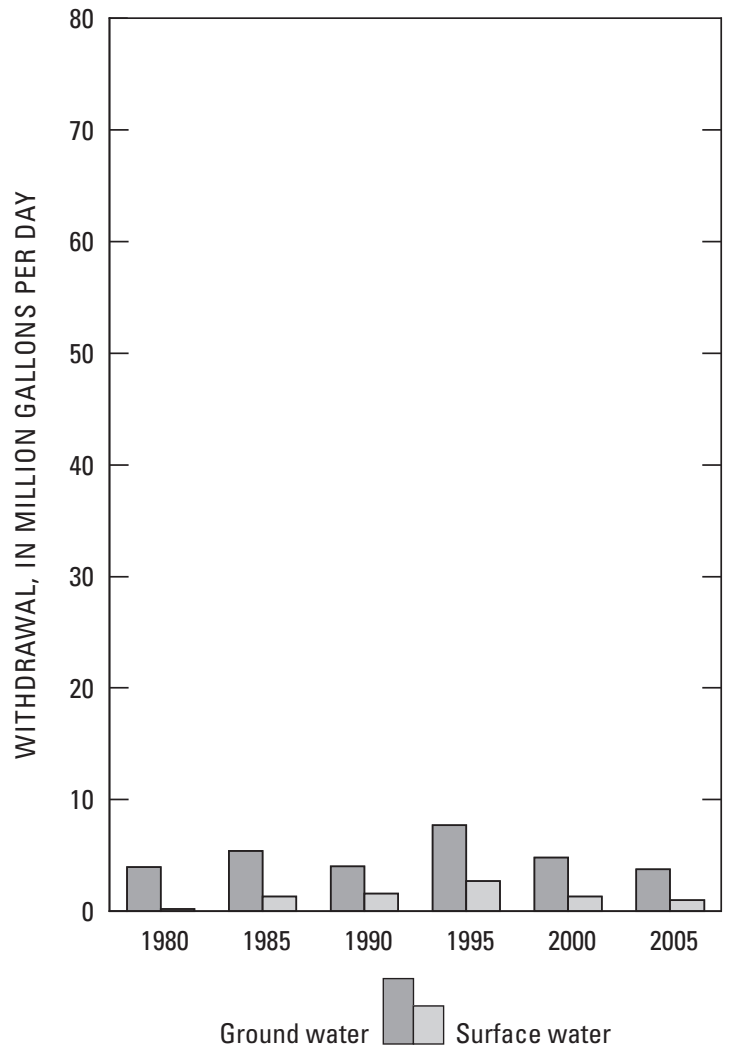

Withdrawals by Major Industrial Groups (Mgal/d):

SIC

GW

SW

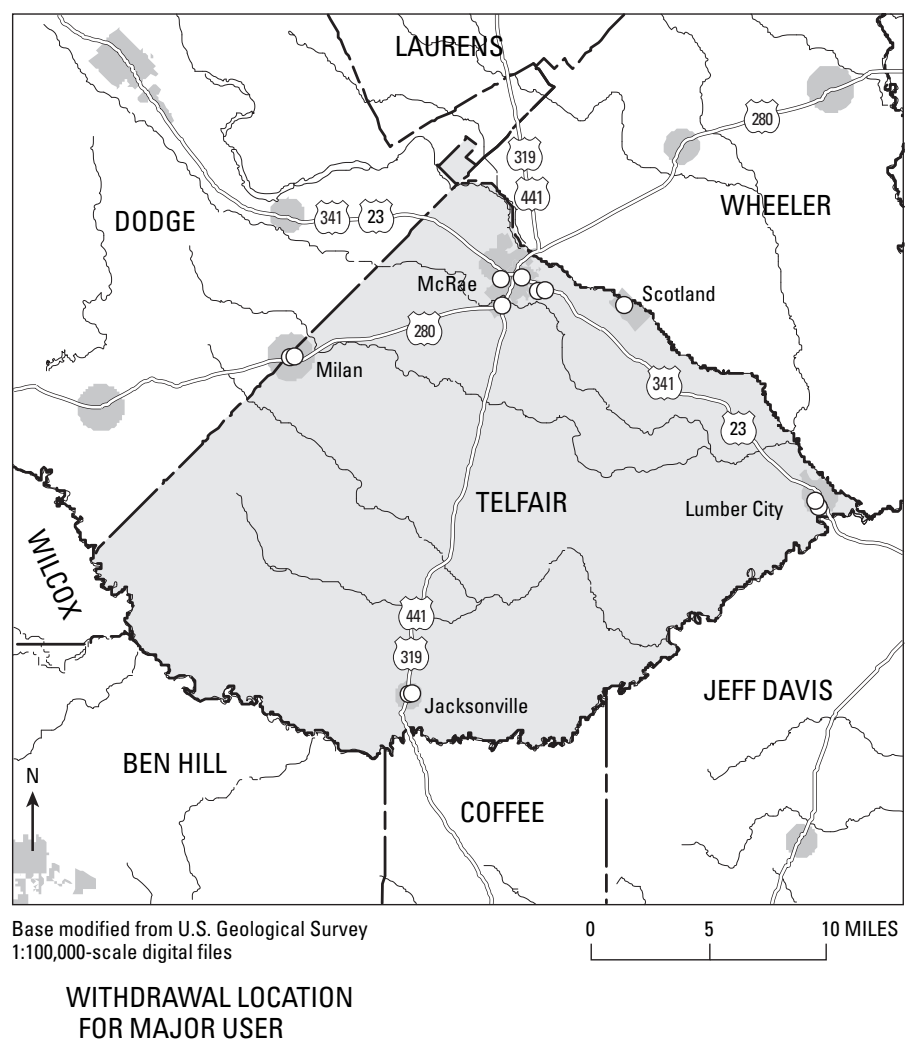




\section{TERRELL COUNTY}

Population: 10,711

Population served by public supply: 7,550

Acres irrigated: 28,630

Hydroelectric use (Mgal/d): $\quad 0.00$

2005 WITHDRAWALS, IN MILLION GALLONS PER DAY

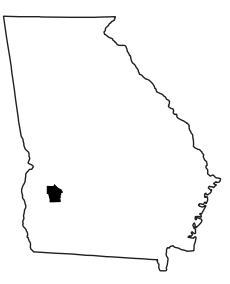

\begin{tabular}{lccccccc}
\hline & $\begin{array}{c}\text { Public } \\
\text { Supply }\end{array}$ & $\begin{array}{c}\text { Domestic \& } \\
\text { Commercial }\end{array}$ & $\begin{array}{c}\text { Industrial \& } \\
\text { Mining }\end{array}$ & Irrigation & Livestock & $\begin{array}{c}\text { Thermo- } \\
\text { electric }\end{array}$ & Totals \\
\hline Ground Water & 1.48 & 0.24 & 0.00 & 7.07 & 0.07 & 0.00 & 8.86 \\
Surface Water & 0.00 & 0.00 & 0.00 & 7.33 & 0.01 & 0.00 & 7.34 \\
TOTALS & 1.48 & 0.24 & 0.00 & 14.40 & 0.08 & 0.00 & 16.20 \\
\hline
\end{tabular}

Withdrawals by Major Public Suppliers (Mgal/d):

\section{Name}

Town of Bronwood

City of Dawson

Town of Parrott

Town of Sasser

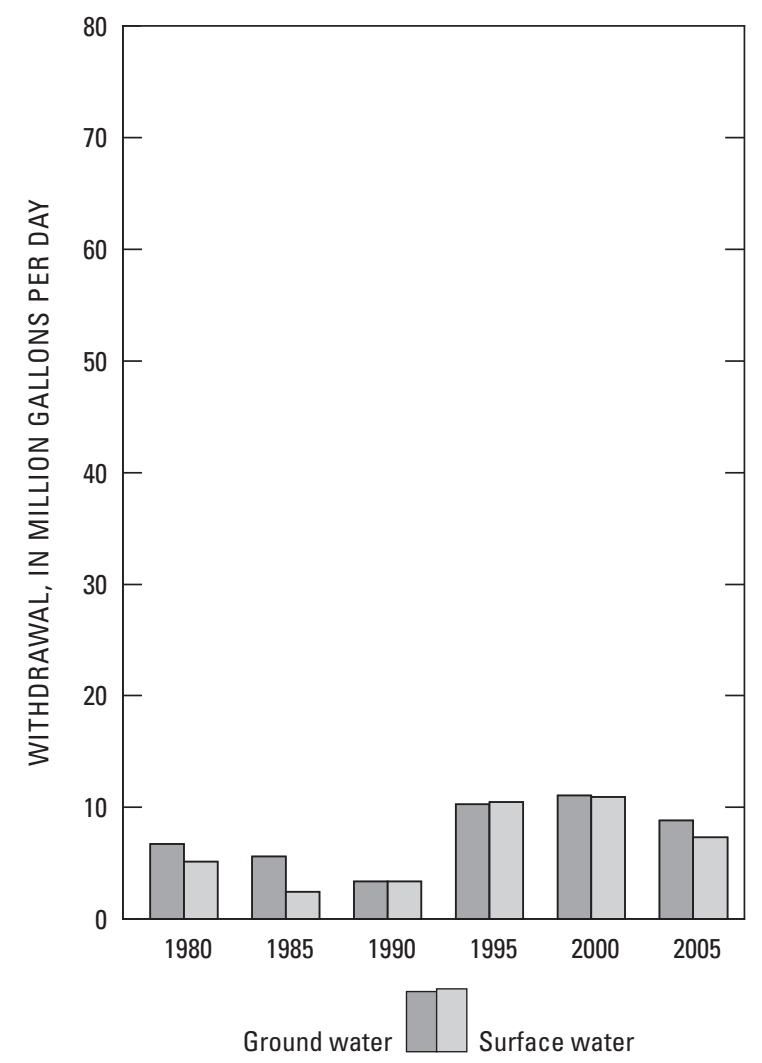

$0.04 \quad 0.00$

$\begin{array}{ll}1.37 & 0.00\end{array}$

$0.03 \quad 0.00$

$0.04 \quad 0.00$
Withdrawals by Major Industrial Groups (Mgal/d):

SIC

GW

SW

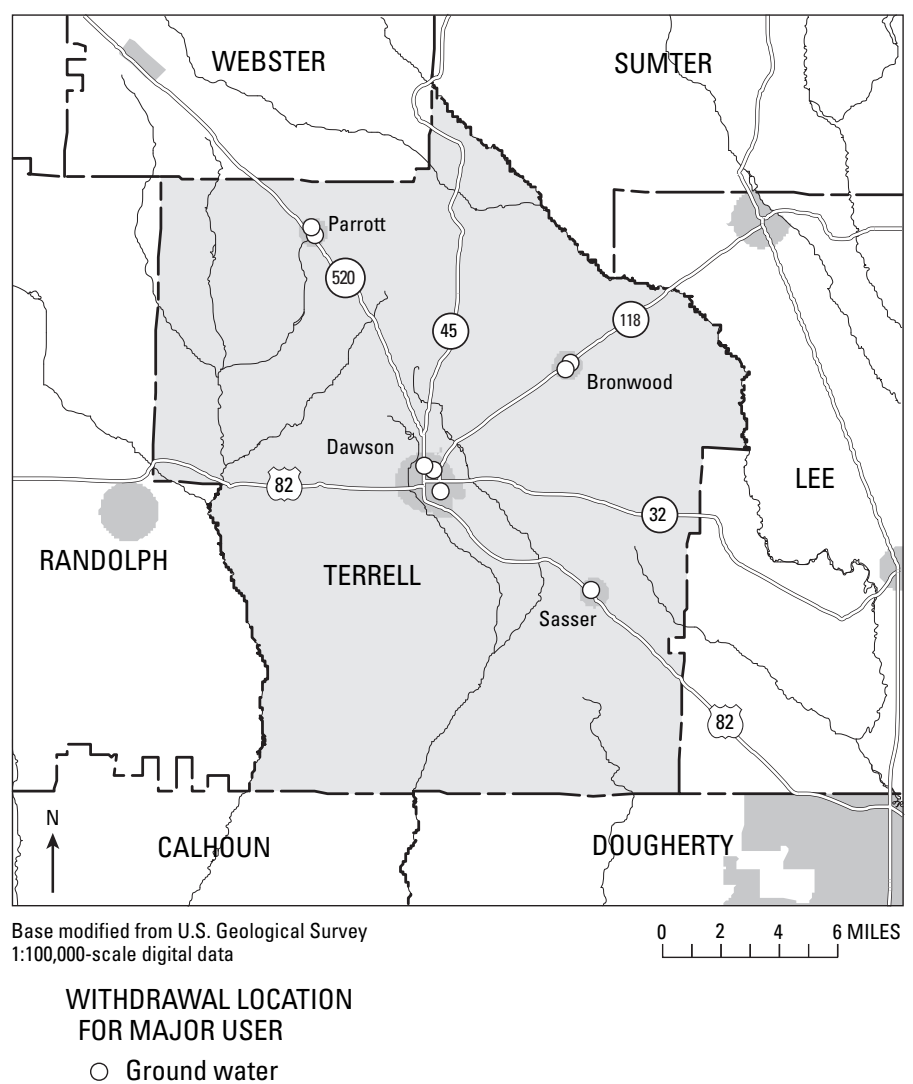




\section{THOMAS COUNTY}

Population: 44,692

Population served by public supply: 32,420

Acres irrigated: 13,950

Hydroelectric use (Mgal/d): $\quad 0.00$

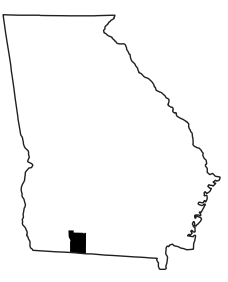

2005 WITHDRAWALS, IN MILLION GALLONS PER DAY

\begin{tabular}{|c|c|c|c|c|c|c|c|}
\hline & $\begin{array}{l}\text { Public } \\
\text { Supply }\end{array}$ & $\begin{array}{l}\text { Domestic \& } \\
\text { Commercial }\end{array}$ & $\begin{array}{c}\text { Industrial \& } \\
\text { Mining }\end{array}$ & Irrigation & Livestock & $\begin{array}{l}\text { Thermo- } \\
\text { electric }\end{array}$ & Totals \\
\hline Ground Water & 5.55 & 0.92 & 3.00 & 7.72 & 0.11 & 0.00 & 17.30 \\
\hline Surface Water & 0.00 & 0.00 & 0.04 & 2.68 & 0.12 & 0.00 & 2.84 \\
\hline TOTALS & 5.55 & 0.92 & 3.04 & 10.40 & 0.23 & 0.00 & 20.14 \\
\hline
\end{tabular}

Withdrawals by Major Public Suppliers (Mgal/d):

Name

GW SW

City of Thomasville-Waverly

0.10

0.00

City of Boston

0.20

0.10

0.09

0.14

0.11

0.12

4.50

0.00

0.00

0.00

City of Coolidge

Town of Ochlocknee

City of Pavo

City of Thomasville Water

\& Light Department

Withdrawals by Major Industrial Groups (Mgal/d):

SIC

20 - Food

28 - Chemicals

32 - Stone, clay
GW

0.02

0.12

0.10
SW

0.00

0.00

0.00
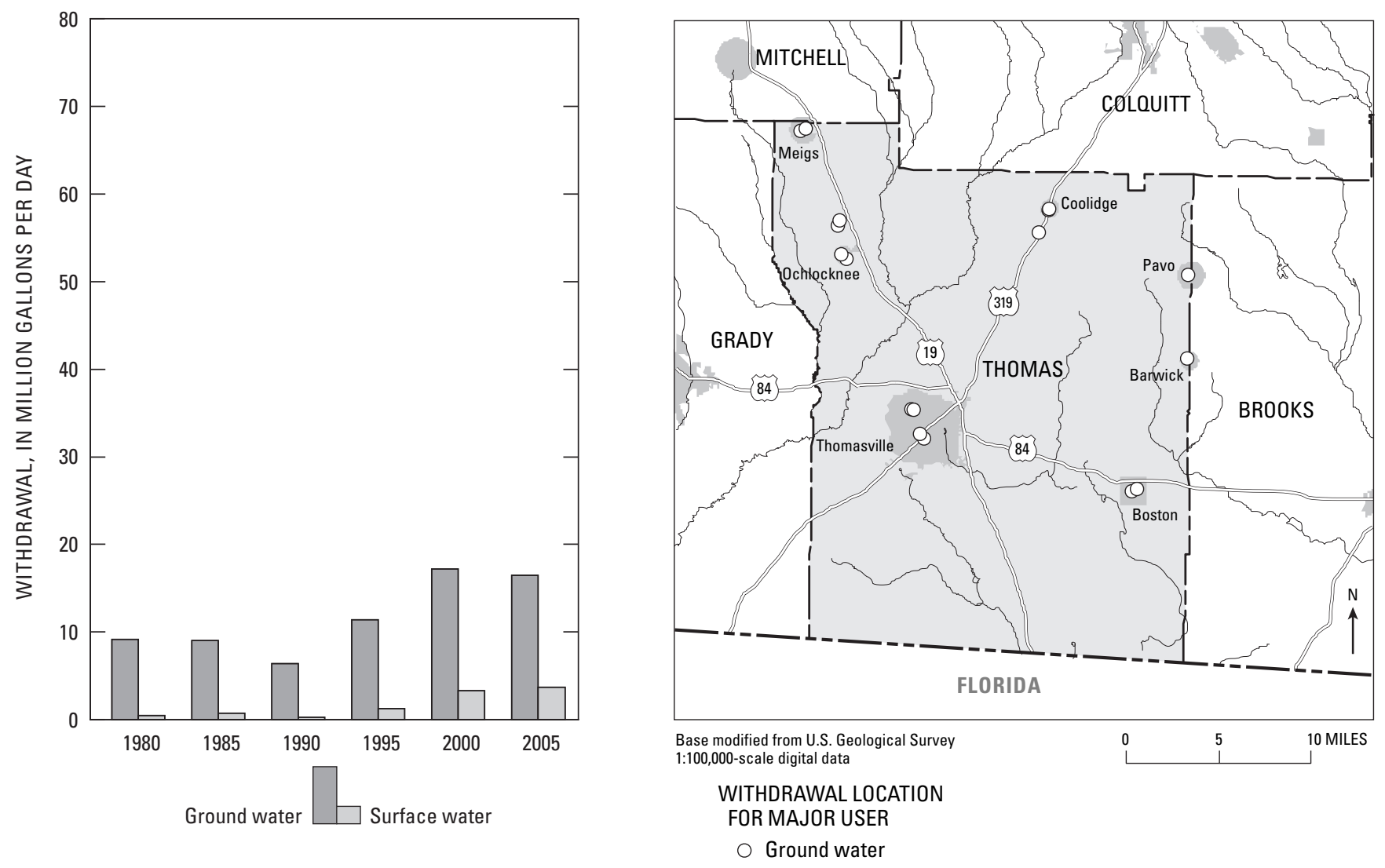


\section{TIFT COUNTY}

Population: 40,793

Population served by public supply: 29,180

Acres irrigated: 44,010

Hydroelectric use (Mgal/d): $\quad 0.00$

2005 WITHDRAWALS, IN MILLION GALLONS PER DAY

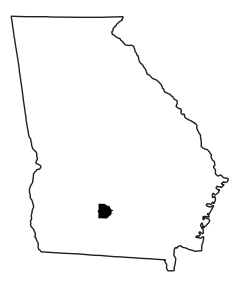

\begin{tabular}{lccccccr}
\hline & $\begin{array}{c}\text { Public } \\
\text { Supply }\end{array}$ & $\begin{array}{c}\text { Domestic \& } \\
\text { Commercial }\end{array}$ & $\begin{array}{c}\text { Industrial \& } \\
\text { Mining }\end{array}$ & Irrigation & Livestock & $\begin{array}{c}\text { Thermo- } \\
\text { electric }\end{array}$ & Totals \\
\hline Ground Water & 4.99 & 1.00 & 0.00 & 11.30 & 0.03 & 0.00 & 17.32 \\
Surface Water & 0.00 & 0.00 & 0.00 & 8.59 & 0.07 & 0.00 & 8.66 \\
TOTALS & 4.99 & 1.00 & 0.00 & 19.89 & 0.10 & 0.00 & 25.98 \\
\hline
\end{tabular}

Withdrawals by Major Public Suppliers (Mgal/d):

\section{Name}

City of Omega

City of Tifton

Town of Ty Ty
GW

0.13

4.68

0.10
SW 0.00 0.00 0.00
Withdrawals by Major Industrial Groups (Mgal/d):

SIC

None
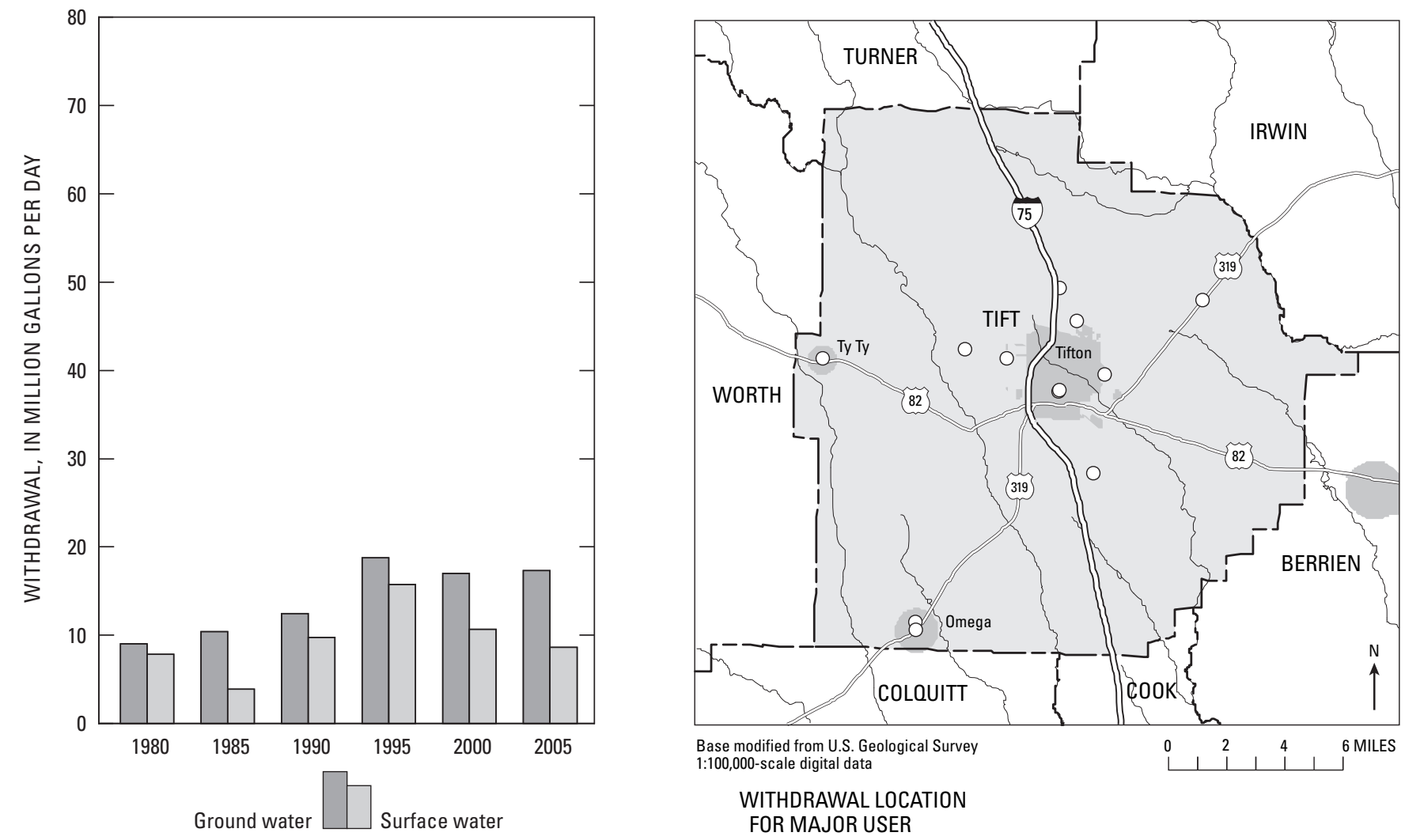

WITHDRAWAL LOCATION

FOR MAJOR USER

$\bigcirc$ Ground water 


\section{TOOMBS COUNTY}

Population: 27,274

Population served by public supply: 18,190

Acres irrigated: 14,800

Hydroelectric use (Mgal/d): $\quad 0.00$

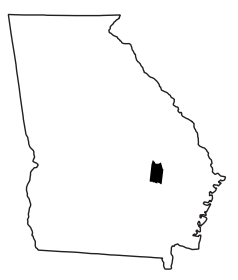

2005 WITHDRAWALS, IN MILLION GALLONS PER DAY

\begin{tabular}{|c|c|c|c|c|c|c|c|}
\hline & $\begin{array}{l}\text { Public } \\
\text { Supply }\end{array}$ & $\begin{array}{l}\text { Domestic \& } \\
\text { Commercial }\end{array}$ & $\begin{array}{c}\text { Industrial \& } \\
\text { Mining }\end{array}$ & Irrigation & Livestock & $\begin{array}{l}\text { Thermo- } \\
\text { electric }\end{array}$ & Totals \\
\hline Ground Water & 2.67 & 0.68 & 0.04 & 2.62 & 0.03 & 0.00 & 6.04 \\
\hline Surface Water & 0.00 & 0.00 & 0.00 & 4.91 & 0.13 & 0.00 & 5.04 \\
\hline TOTALS & 2.67 & 0.68 & 0.04 & 7.53 & 0.16 & 0.00 & 11.08 \\
\hline
\end{tabular}

Withdrawals by Major Public Suppliers (Mgal/d):

Name

City of Lyons

City of Santa Claus

City of Vidalia
GW SW

$\begin{array}{ll}0.88 & 0.00\end{array}$

$0.03 \quad 0.00$

$1.70 \quad 0.00$
Withdrawals by Major Industrial Groups (Mgal/d):

SIC

GW

SW
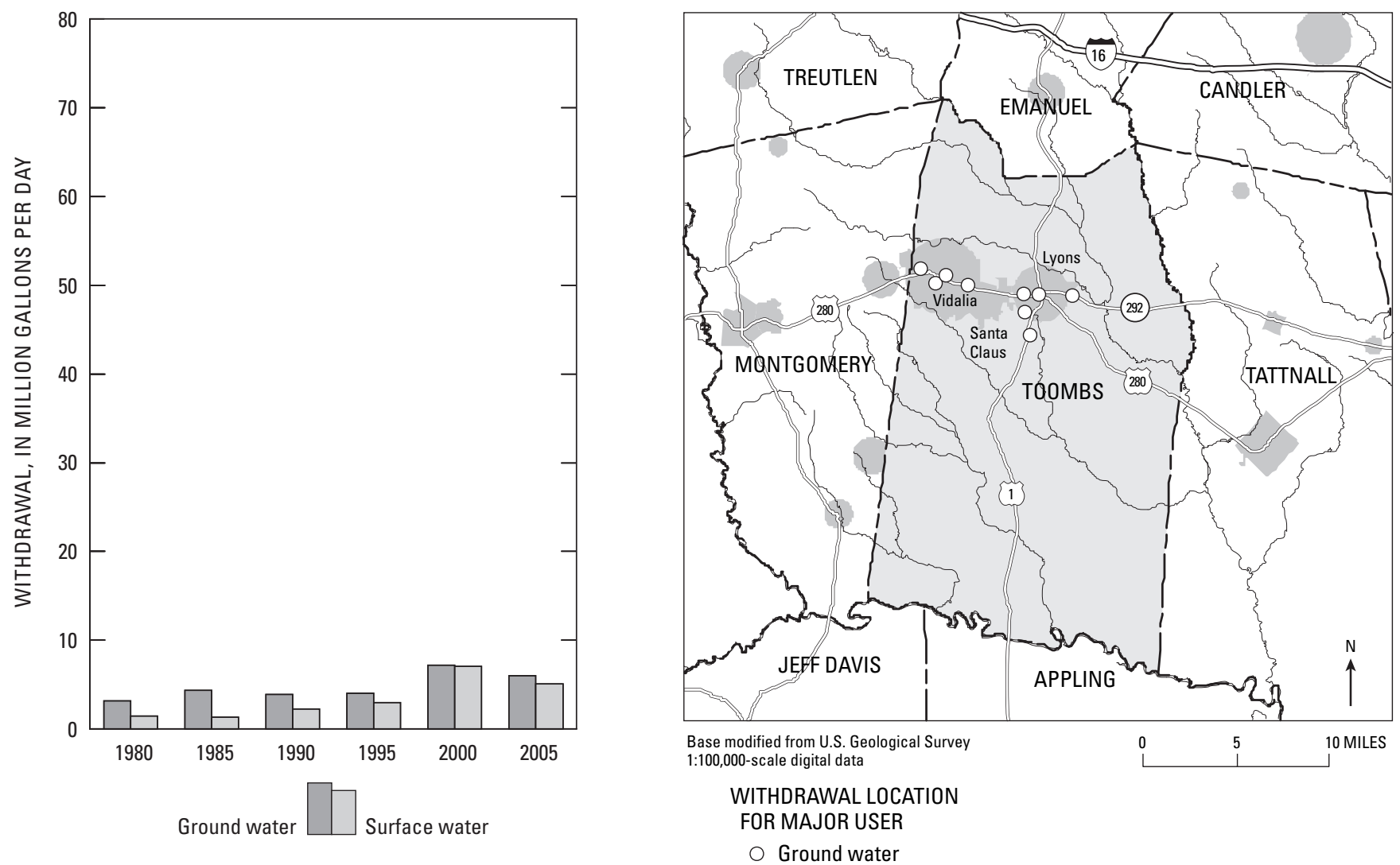


\section{TOWNS COUNTY}

Population: 10,315

Population served by public supply: $\quad 9,590$

Acres irrigated: 170

Hydroelectric use (Mgal/d): $\quad 0.00$

2005 WITHDRAWALS, IN MILLION GALLONS PER DAY

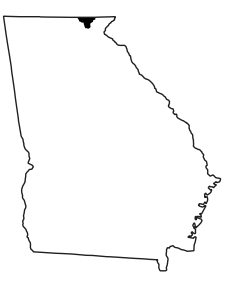

\begin{tabular}{lccccccc}
\hline & $\begin{array}{c}\text { Public } \\
\text { Supply }\end{array}$ & $\begin{array}{c}\text { Domestic \& } \\
\text { Commercial }\end{array}$ & $\begin{array}{c}\text { Industrial \& } \\
\text { Mining }\end{array}$ & Irrigation & Livestock & $\begin{array}{c}\text { Thermo- } \\
\text { electric }\end{array}$ & Totals \\
\hline Ground Water & 1.15 & 0.05 & 0.05 & 0.05 & 0.00 & 0.00 & 1.30 \\
Surface Water & 0.96 & 0.00 & 0.00 & 0.28 & 4.04 & 0.00 & 5.28 \\
TOTALS & 2.11 & 0.05 & 0.05 & 0.33 & 4.04 & 0.00 & 6.58 \\
\hline
\end{tabular}

Withdrawals by Major Public Suppliers (Mgal/d):

\section{Name}

Town of Hiawassee

City of Young Harris

Towns County Water System
GW SW

$0.00 \quad 0.96$

$0.13 \quad 0.00$

$1.02 \quad 0.00$
Withdrawals by Major Industrial Groups (Mgal/d):

SIC

None
SW
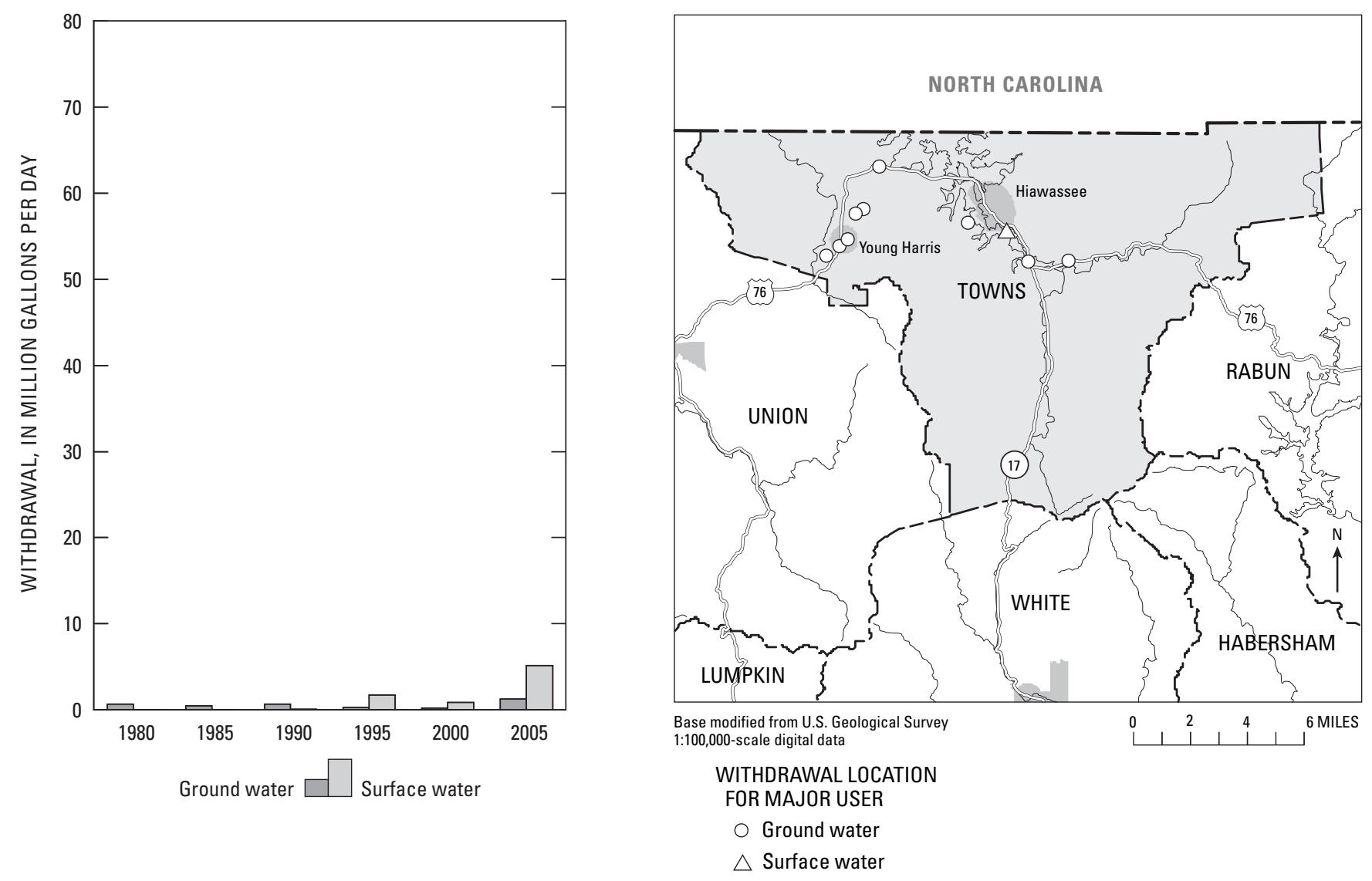


\section{TREUTLEN COUNTY}

Population: 6,753

Population served by public supply: 3,130

Acres irrigated: 1,350

Hydroelectric use (Mgal/d): 0.00

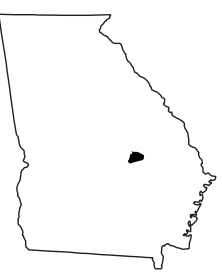

2005 WITHDRAWALS, IN MILLION GALLONS PER DAY

\begin{tabular}{|c|c|c|c|c|c|c|c|}
\hline & $\begin{array}{l}\text { Public } \\
\text { Supply }\end{array}$ & $\begin{array}{l}\text { Domestic \& } \\
\text { Commercial }\end{array}$ & $\begin{array}{c}\text { Industrial \& } \\
\text { Mining }\end{array}$ & Irrigation & Livestock & $\begin{array}{l}\text { Thermo- } \\
\text { electric }\end{array}$ & Totals \\
\hline Ground Water & 0.40 & 0.27 & 0.00 & 0.69 & 0.01 & 0.00 & 1.37 \\
\hline Surface Water & 0.00 & 0.00 & 0.00 & 0.16 & 0.01 & 0.00 & 0.17 \\
\hline TOTALS & 0.40 & 0.27 & 0.00 & 0.85 & 0.02 & 0.00 & 1.54 \\
\hline
\end{tabular}

Withdrawals by Major Public Suppliers (Mgal/d):

Name

City of Soperton

GW

0.39

SW

0.00
Withdrawals by Major Industrial Groups (Mgal/d):

SIC

GW

SW

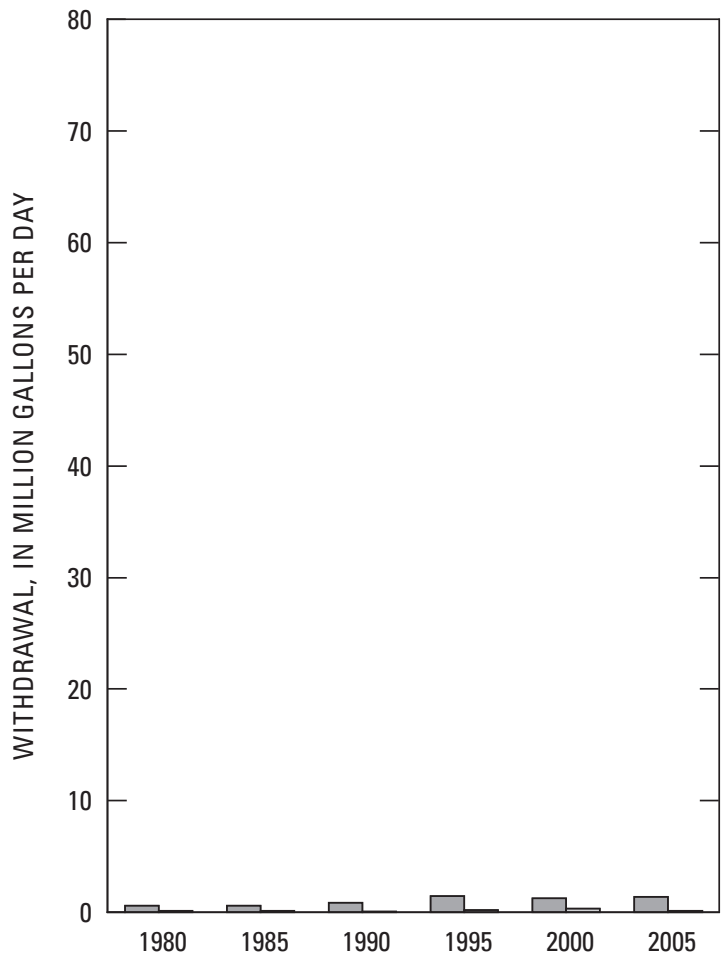

Ground water $\square$ Surface water

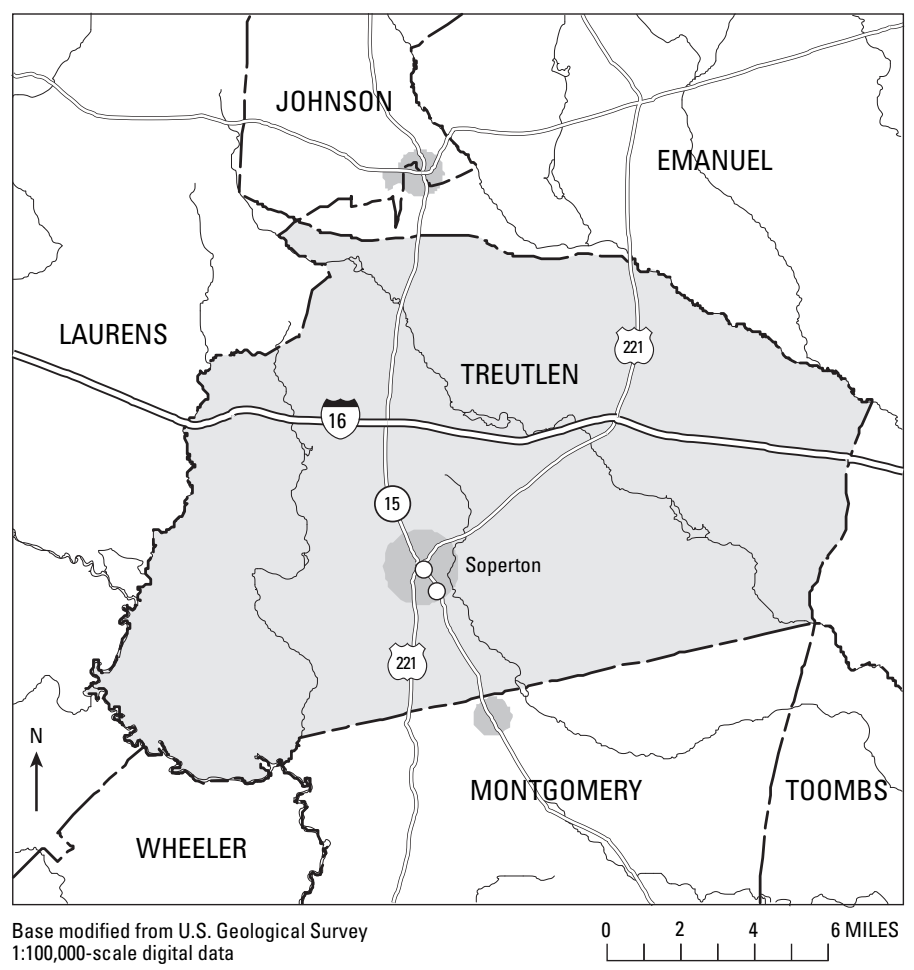

WITHDRAWAL LOCATION FOR MAJOR USER

O Ground water 


\section{TROUP COUNTY}

Population: 62,015

Population served by public supply: $\quad 50,170$

Acres irrigated: 390

Hydroelectric use (Mgal/d): $\quad 2,793.93$

\section{WITHDRAWALS, IN MILLION GALLONS PER DAY}

\begin{tabular}{lccccccc}
\hline & $\begin{array}{c}\text { Public } \\
\text { Supply }\end{array}$ & $\begin{array}{c}\text { Domestic \& } \\
\text { Commercial }\end{array}$ & $\begin{array}{c}\text { Industrial \& } \\
\text { Mining }\end{array}$ & Irrigation & Livestock & $\begin{array}{c}\text { Thermo- } \\
\text { electric }\end{array}$ & Totals \\
\hline Ground Water & 0.05 & 0.89 & 0.27 & 0.61 & 0.00 & 0.00 & 1.82 \\
Surface Water & 10.28 & 0.00 & 0.00 & 0.44 & 0.09 & 0.00 & 10.81 \\
TOTALS & 10.33 & 0.89 & 0.27 & 1.05 & 0.09 & 0.00 & 12.63 \\
\hline
\end{tabular}

Withdrawals by Major Public Suppliers (Mgal/d):

\section{Name}

City of Hogansville

City of Lagrange

Mountville Water Association

City of West Point
GW

0.00

0.00

0.03

0.00
SW

0.42

9.20

0.00

0.66

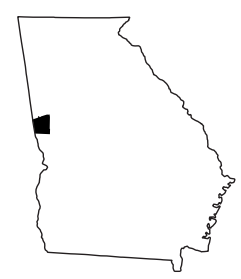

Withdrawals by Major Industrial Groups (Mgal/d):

$\begin{array}{lcc}\text { SIC } & \text { GW } & \text { SW } \\ 22 \text { - Textiles } & 0.12 & 0.00\end{array}$
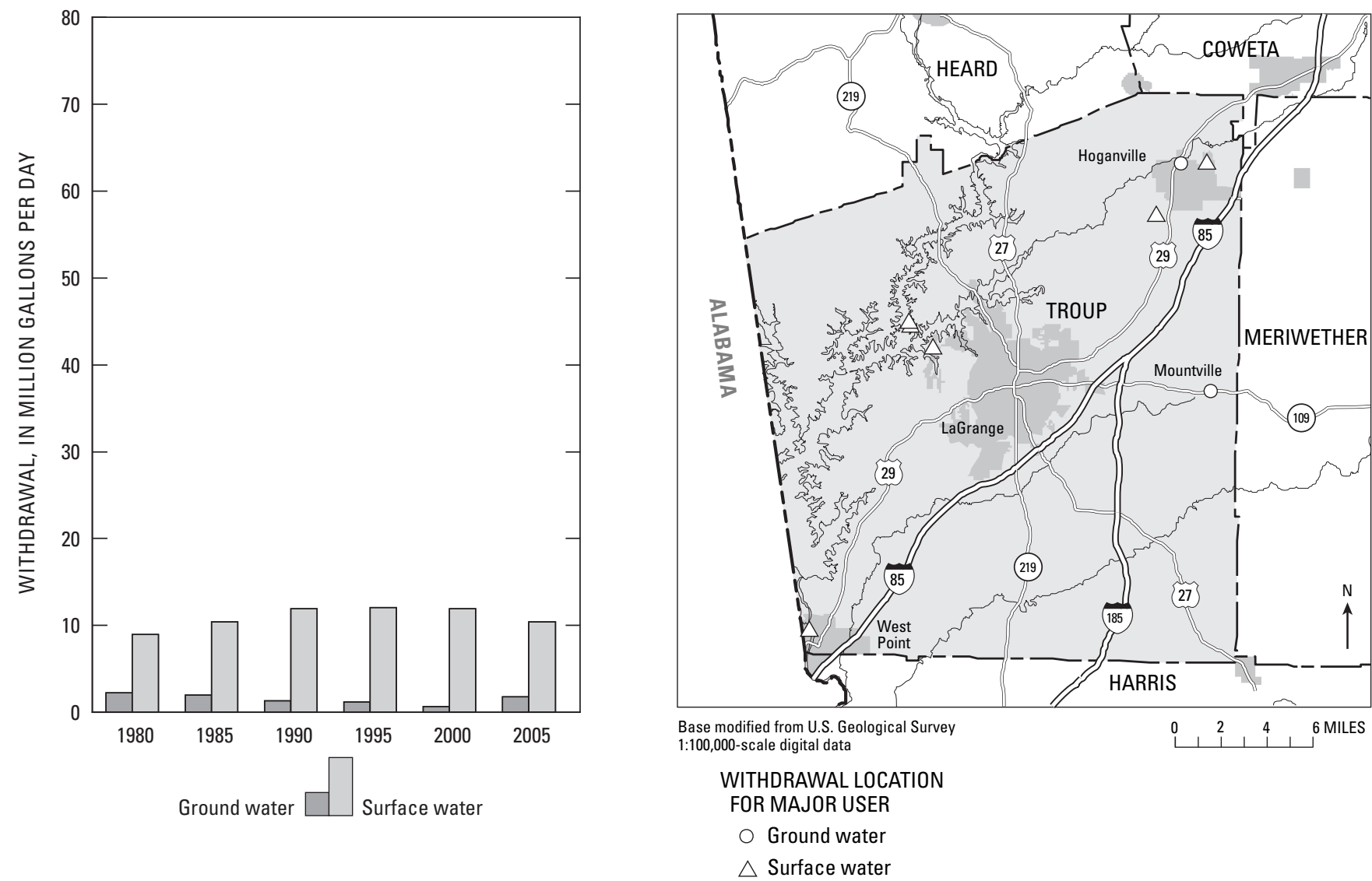


\section{TURNER COUNTY}

Population: $\quad 9,474$

Population served by public supply: $\quad 6,280$

Acres irrigated: 28,090

Hydroelectric use (Mgal/d): $\quad 0.00$

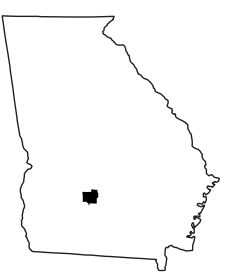

2005 WITHDRAWALS, IN MILLION GALLONS PER DAY

\begin{tabular}{|c|c|c|c|c|c|c|c|}
\hline & $\begin{array}{l}\text { Public } \\
\text { Supply }\end{array}$ & $\begin{array}{l}\text { Domestic \& } \\
\text { Commercial }\end{array}$ & $\begin{array}{c}\text { Industrial \& } \\
\text { Mining }\end{array}$ & Irrigation & Livestock & $\begin{array}{l}\text { Thermo- } \\
\text { electric }\end{array}$ & Totals \\
\hline Ground Water & 0.88 & 0.24 & 0.00 & 6.06 & 0.03 & 0.00 & 7.21 \\
\hline Surface Water & 0.00 & 0.00 & 0.00 & 8.55 & 0.11 & 0.00 & 8.66 \\
\hline TOTALS & 0.88 & 0.24 & 0.00 & 14.61 & 0.14 & 0.00 & 15.87 \\
\hline
\end{tabular}

Withdrawals by Major Public Suppliers (Mgal/d):

Name

City of Ashburn

Town of Rebecca

City of Sycamore
GW SW

$0.74 \quad 0.00$

$0.04 \quad 0.00$

$0.08 \quad 0.00$
Withdrawals by Major Industrial Groups (Mgal/d):

SIC

GW

SW
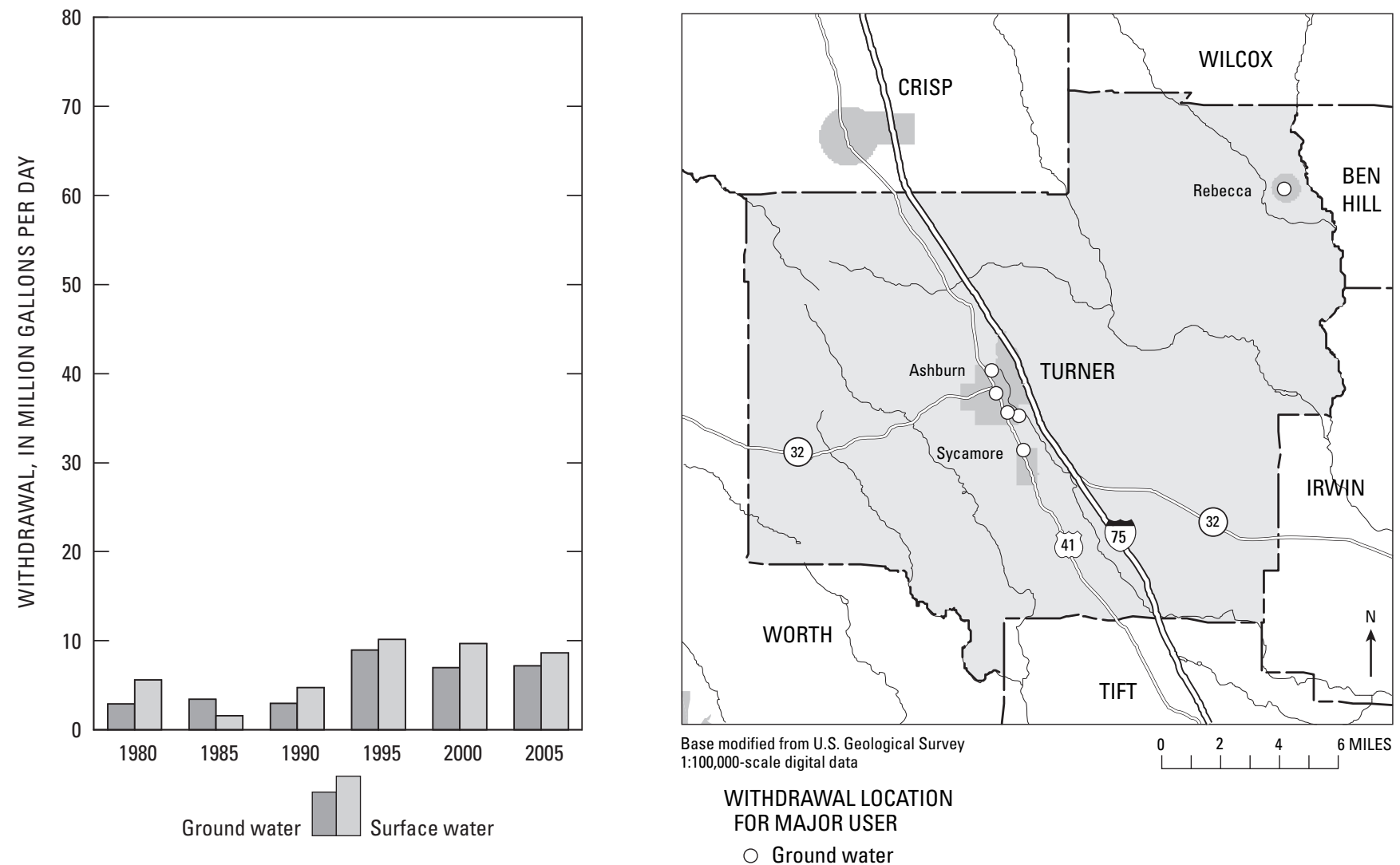


\section{TWIGGS COUNTY}

Population: 10,299

Population served by public supply: 2,400

Acres irrigated: 3,590

Hydroelectric use (Mgal/d): $\quad 0.00$

2005 WITHDRAWALS, IN MILLION GALLONS PER DAY

\begin{tabular}{|c|c|c|c|c|c|c|c|}
\hline & $\begin{array}{l}\text { Public } \\
\text { Supply }\end{array}$ & $\begin{array}{l}\text { Domestic \& } \\
\text { Commercial }\end{array}$ & $\begin{array}{c}\text { Industrial \& } \\
\text { Mining }\end{array}$ & Irrigation & Livestock & $\begin{array}{l}\text { Thermo- } \\
\text { electric }\end{array}$ & Totals \\
\hline Ground Water & 0.30 & 0.59 & 19.89 & 0.93 & 0.01 & 0.00 & 21.72 \\
\hline Surface Water & 0.00 & 0.00 & 0.00 & 0.47 & 0.01 & 0.00 & 0.48 \\
\hline TOTALS & 0.30 & 0.59 & 19.89 & 1.40 & 0.02 & 0.00 & 22.20 \\
\hline
\end{tabular}

Withdrawals by Major Public Suppliers (Mgal/d):

Name

Town of Danville

City of Jeffersonville
GW SW

$0.06 \quad 0.00$

$0.22 \quad 0.00$
Withdrawals by Major Industrial Groups (Mgal/d):

SIC

14 - Mining

32 - Stone, clay

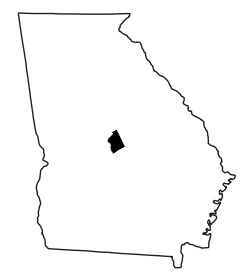

SW

$\begin{array}{rr}0.04 & 0.00 \\ 14.76 & 0.00\end{array}$
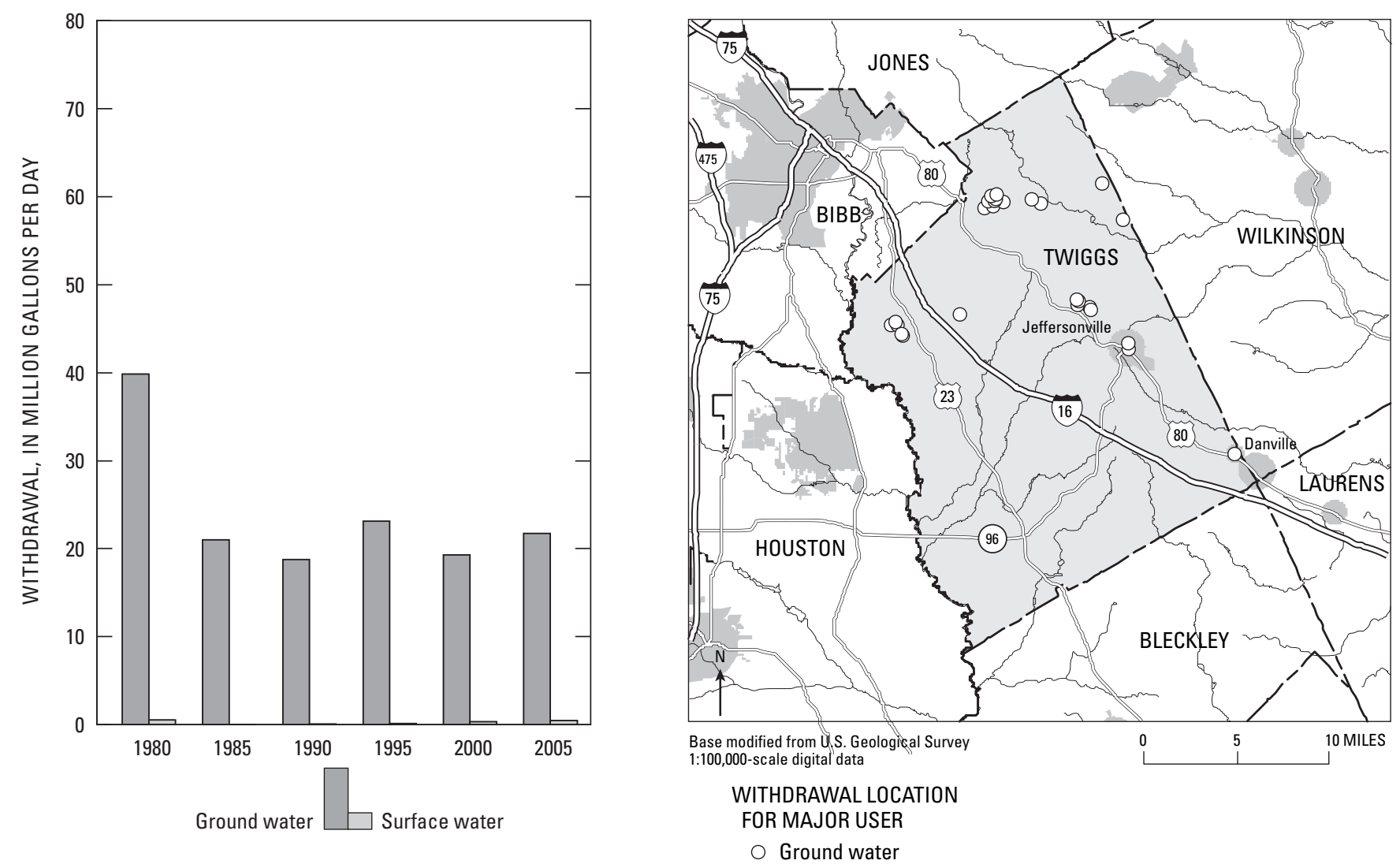


\section{UNION COUNTY}

Population: $\quad 19,782$

Population served by public supply: 12,860

Acres irrigated: 100

Hydroelectric use (Mgal/d): 378.36

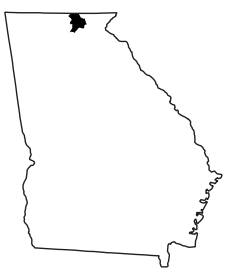

2005 WITHDRAWALS, IN MILLION GALLONS PER DAY

\begin{tabular}{|c|c|c|c|c|c|c|c|}
\hline & $\begin{array}{l}\text { Public } \\
\text { Supply }\end{array}$ & $\begin{array}{l}\text { Domestic \& } \\
\text { Commercial }\end{array}$ & $\begin{array}{c}\text { Industrial \& } \\
\text { Mining }\end{array}$ & Irrigation & Livestock & $\begin{array}{l}\text { Thermo- } \\
\text { electric }\end{array}$ & Totals \\
\hline Ground Water & 0.99 & 0.52 & 0.10 & 0.00 & 0.00 & 0.00 & 1.61 \\
\hline Surface Water & 0.92 & 0.00 & 0.00 & 0.15 & 5.40 & 0.00 & 6.47 \\
\hline TOTALS & 1.91 & 0.52 & 0.10 & 0.15 & 5.40 & 0.00 & 8.08 \\
\hline
\end{tabular}

Withdrawals by Major Public Suppliers (Mgal/d):

Name

GW

City of Blairsville

0.24

SW

Coosa Water Authority

Notla Water Authority
0.37

0.37
Withdrawals by Major Industrial Groups (Mgal/d):

SIC

GW

SW
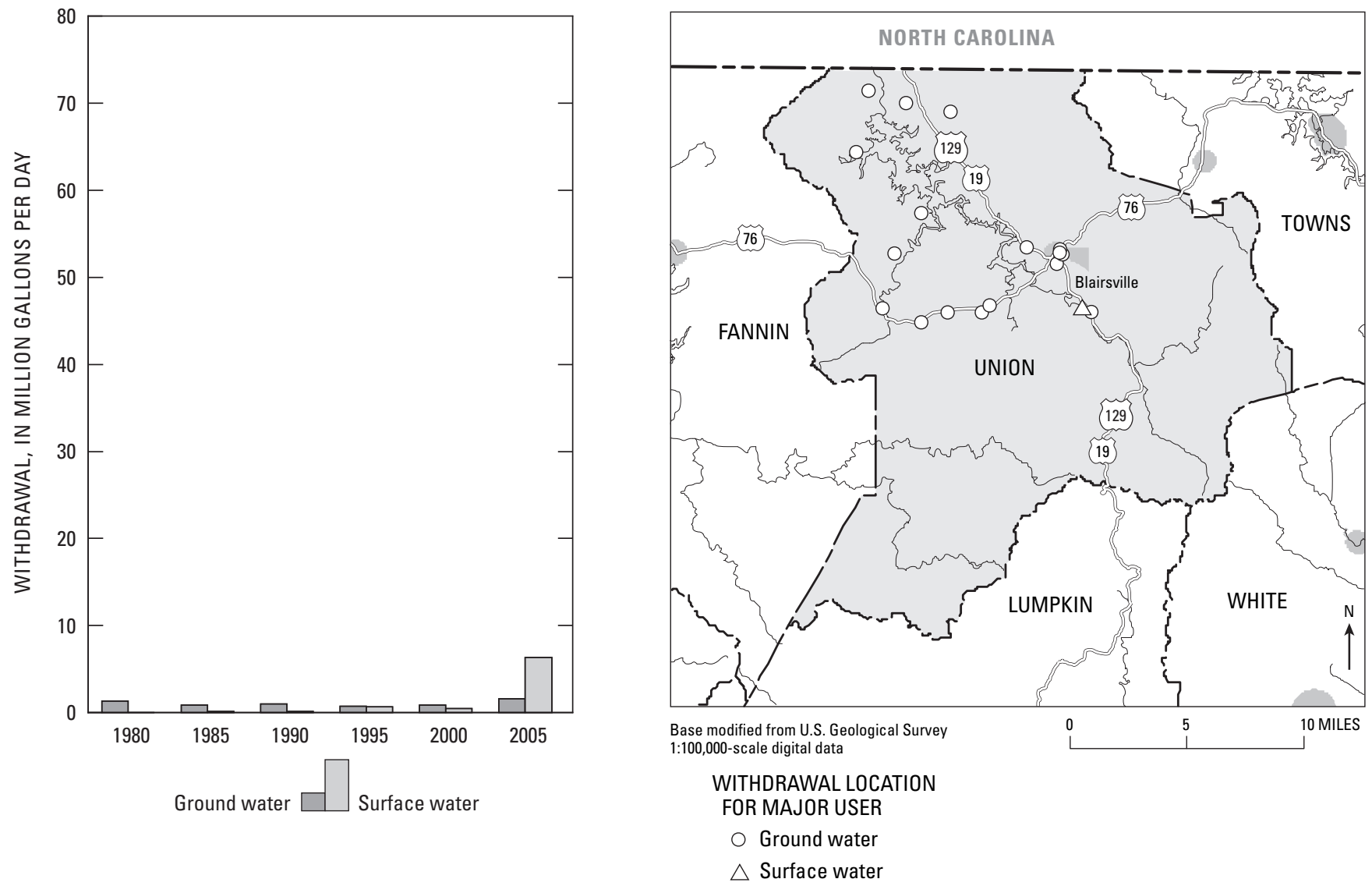


\section{UPSON COUNTY}

Population: 27,679

Population served by public supply: 12,270

Acres irrigated: 750

Hydroelectric use (Mgal/d): 0.00

\section{WITHDRAWALS, IN MILLION GALLONS PER DAY}

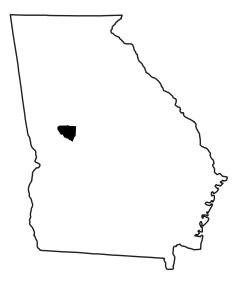

\begin{tabular}{|c|c|c|c|c|c|c|c|}
\hline & $\begin{array}{l}\text { Public } \\
\text { Supply }\end{array}$ & $\begin{array}{l}\text { Domestic \& } \\
\text { Commercial }\end{array}$ & $\begin{array}{c}\text { Industrial \& } \\
\text { Mining }\end{array}$ & Irrigation & Livestock & $\begin{array}{l}\text { Thermo- } \\
\text { electric }\end{array}$ & Totals \\
\hline Ground Water & 0.25 & 1.16 & 0.00 & 0.00 & 0.02 & 0.00 & 1.43 \\
\hline Surface Water & 2.61 & 0.00 & 0.23 & 0.50 & 0.26 & 0.00 & 3.60 \\
\hline TOTALS & 2.86 & 1.16 & 0.23 & 0.50 & 0.28 & 0.00 & 5.03 \\
\hline
\end{tabular}

Withdrawals by Major Public Suppliers (Mgal/d):

\section{Name}

City of Thomaston

Town of Yatesville
Withdrawals by Major Industrial Groups (Mgal/d):

SIC

22 - Textiles

GW

0.00

SW

0.15
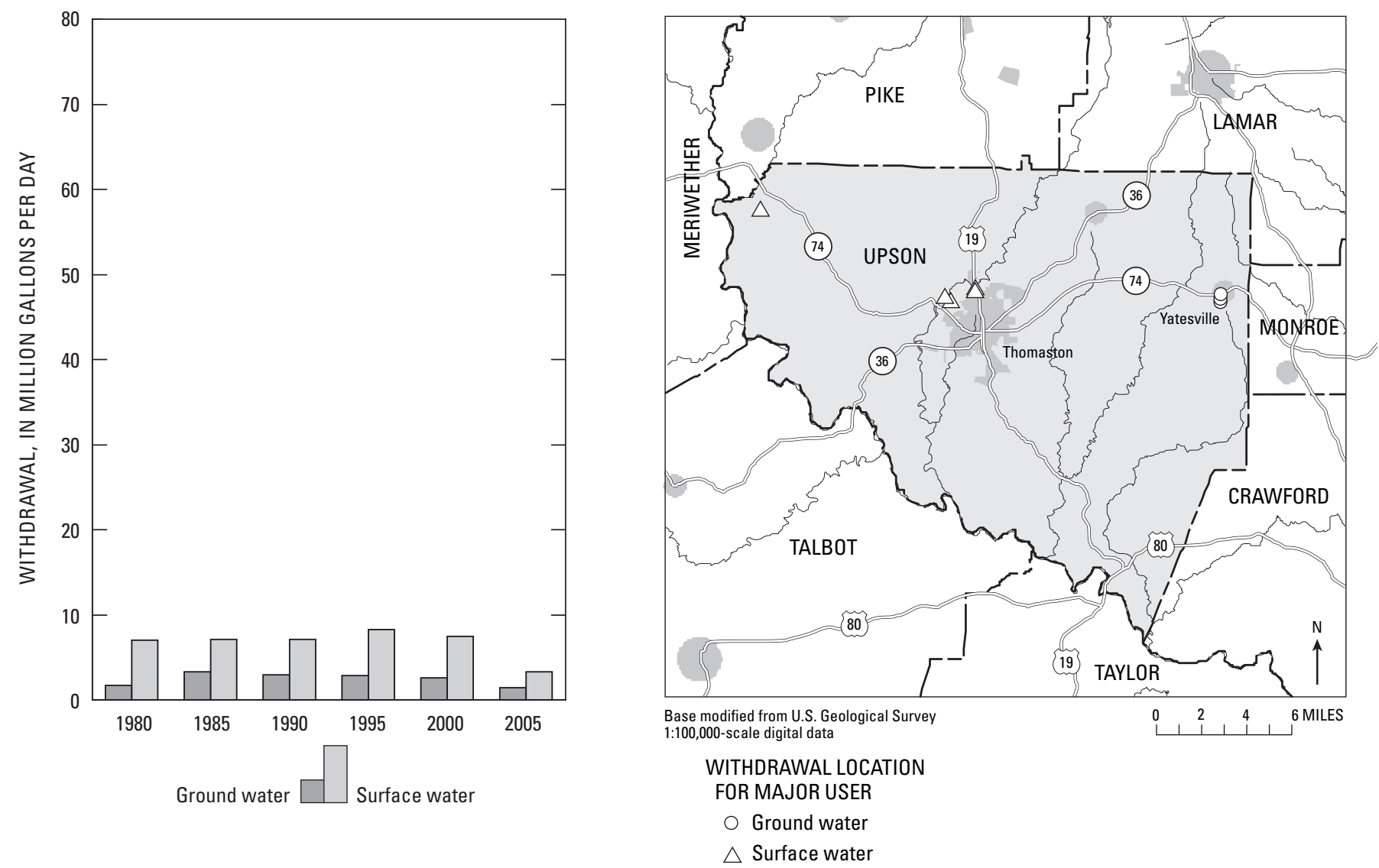


\section{WALKER COUNTY}

Population: $\quad 63,890$

Population served by public supply: $\quad 63,690$

Acres irrigated: 440

Hydroelectric use (Mgal/d): $\quad 0.00$

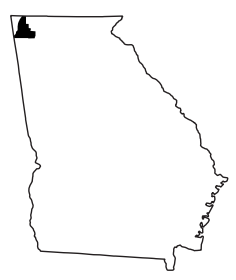

2005 WITHDRAWALS, IN MILLION GALLONS PER DAY

\begin{tabular}{|c|c|c|c|c|c|c|c|}
\hline & $\begin{array}{l}\text { Public } \\
\text { Supply }\end{array}$ & $\begin{array}{l}\text { Domestic \& } \\
\text { Commercial }\end{array}$ & $\begin{array}{c}\text { Industrial \& } \\
\text { Mining }\end{array}$ & Irrigation & Livestock & $\begin{array}{l}\text { Thermo- } \\
\text { electric }\end{array}$ & Totals \\
\hline Ground Water & 6.41 & 0.02 & 0.72 & 0.00 & 0.02 & 0.00 & 7.17 \\
\hline Surface Water & 1.20 & 0.00 & 0.51 & 0.71 & 0.33 & 0.00 & 2.75 \\
\hline TOTALS & 7.61 & 0.02 & 1.23 & 0.71 & 0.35 & 0.00 & 9.92 \\
\hline
\end{tabular}

Withdrawals by Major Public Suppliers (Mgal/d):

Name

Walker County Water

\& Sewer Authority

City of Lafayette

City of Chickamauga

Kensington Water \& Sewer

Walker County Rural Water

\& Sewer Authority
SW

$3.81 \quad 0.00$

0.97

0.91

0.47

0.25
Withdrawals by Major Industrial Groups (Mgal/d):

SIC

22 - Textiles

30 - Rubber
GW

0.15

0.47
SW

0.51

0.00
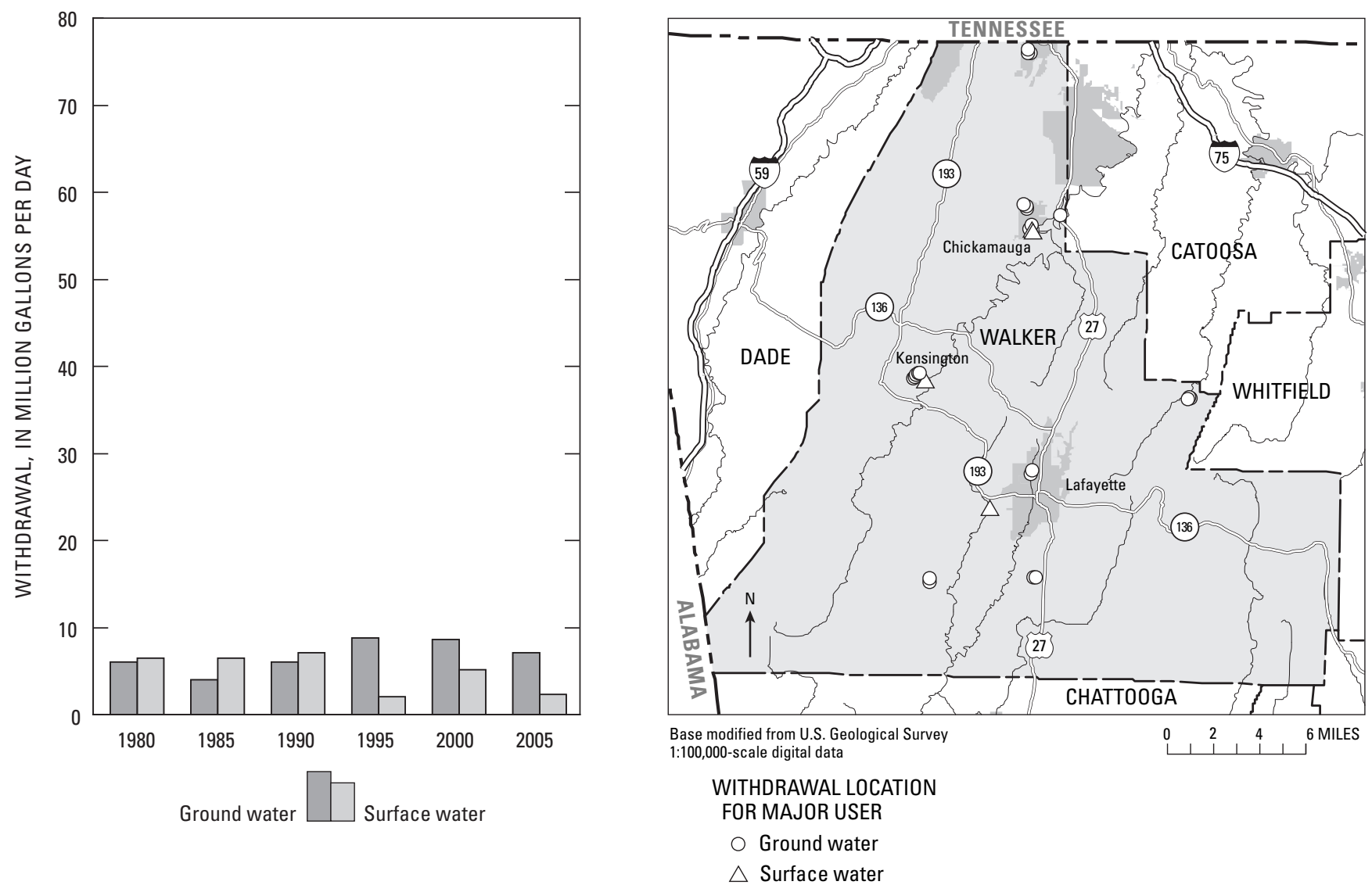


\section{WALTON COUNTY}

Population: 75,647

Population served by public supply: 40,850

Acres irrigated: 1,300

Hydroelectric use (Mgal/d): 0.00

2005 WITHDRAWALS, IN MILLION GALLONS PER DAY

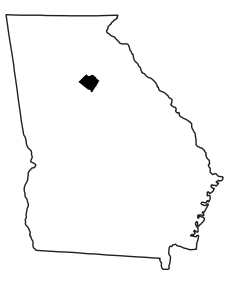

\begin{tabular}{|c|c|c|c|c|c|c|c|}
\hline & $\begin{array}{l}\text { Public } \\
\text { Supply }\end{array}$ & $\begin{array}{l}\text { Domestic \& } \\
\text { Commercial }\end{array}$ & $\begin{array}{c}\text { Industrial \& } \\
\text { Mining }\end{array}$ & Irrigation & Livestock & $\begin{array}{l}\text { Thermo- } \\
\text { electric }\end{array}$ & Totals \\
\hline Ground Water & 0.34 & 2.61 & 0.00 & 0.59 & 0.00 & 0.00 & 3.54 \\
\hline Surface Water & 4.86 & 0.00 & 0.00 & 2.09 & 0.26 & 0.00 & 7.21 \\
\hline TOTALS & 5.20 & 2.61 & 0.00 & 2.68 & 0.26 & 0.00 & 10.75 \\
\hline
\end{tabular}

Withdrawals by Major Public Suppliers (Mgal/d):

\section{Name}

Town of Jersey

Monroe Water, Light

\& Gas Company

City of Social Circle

City of Loganville
GW SW

$0.03 \quad 0.00$

$0.00 \quad 4.30$

$0.00 \quad 0.57$

$0.29 \quad 0.00$
Withdrawals by Major Industrial Groups (Mgal/d):

SIC

None
GW SW
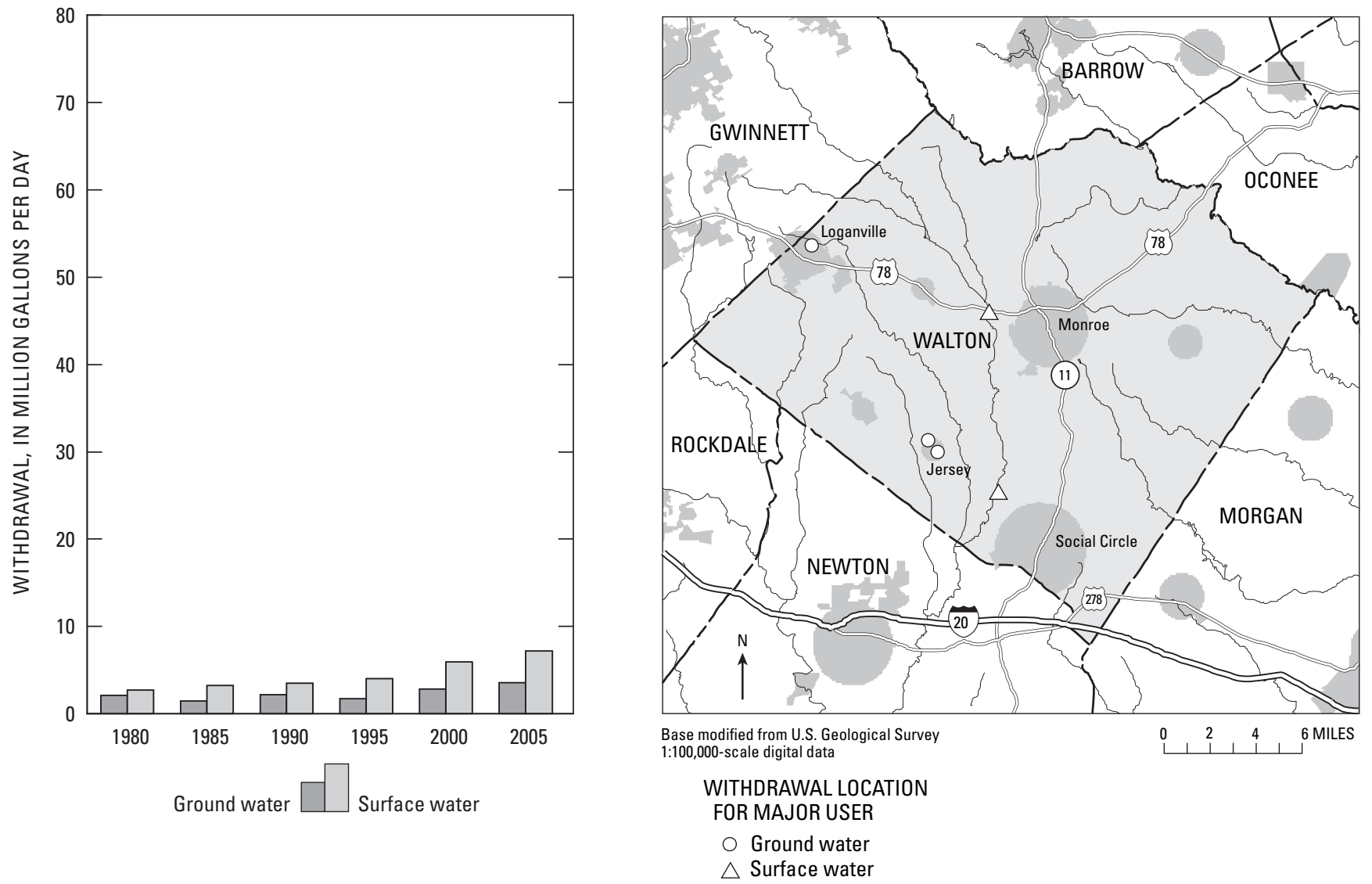


\section{WARE COUNTY}

Population: 34,492

Population served by public supply: $\quad 29,320$

Acres irrigated: 5,800

Hydroelectric use (Mgal/d): $\quad 0.00$



2005 WITHDRAWALS, IN MILLION GALLONS PER DAY

\begin{tabular}{|c|c|c|c|c|c|c|c|}
\hline & $\begin{array}{l}\text { Public } \\
\text { Supply }\end{array}$ & $\begin{array}{l}\text { Domestic \& } \\
\text { Commercial }\end{array}$ & $\begin{array}{c}\text { Industrial \& } \\
\text { Mining }\end{array}$ & Irrigation & Livestock & $\begin{array}{l}\text { Thermo- } \\
\text { electric }\end{array}$ & Totals \\
\hline Ground Water & 3.44 & 1.27 & 0.00 & 1.36 & 0.01 & 0.00 & 6.08 \\
\hline Surface Water & 0.00 & 0.00 & 0.00 & 0.65 & 0.12 & 0.00 & 0.77 \\
\hline TOTALS & 3.44 & 1.27 & 0.00 & 2.01 & 0.13 & 0.00 & 6.85 \\
\hline
\end{tabular}

Withdrawals by Major Public Suppliers (Mgal/d):

Name

City of Manor

GW

0.08

SW

City of Waycross

Satilla Regional W\&S Authority

$2.12 \quad 0.00$

Withdrawals by Major Industrial Groups (Mgal/d):

SIC

None
GW

SW

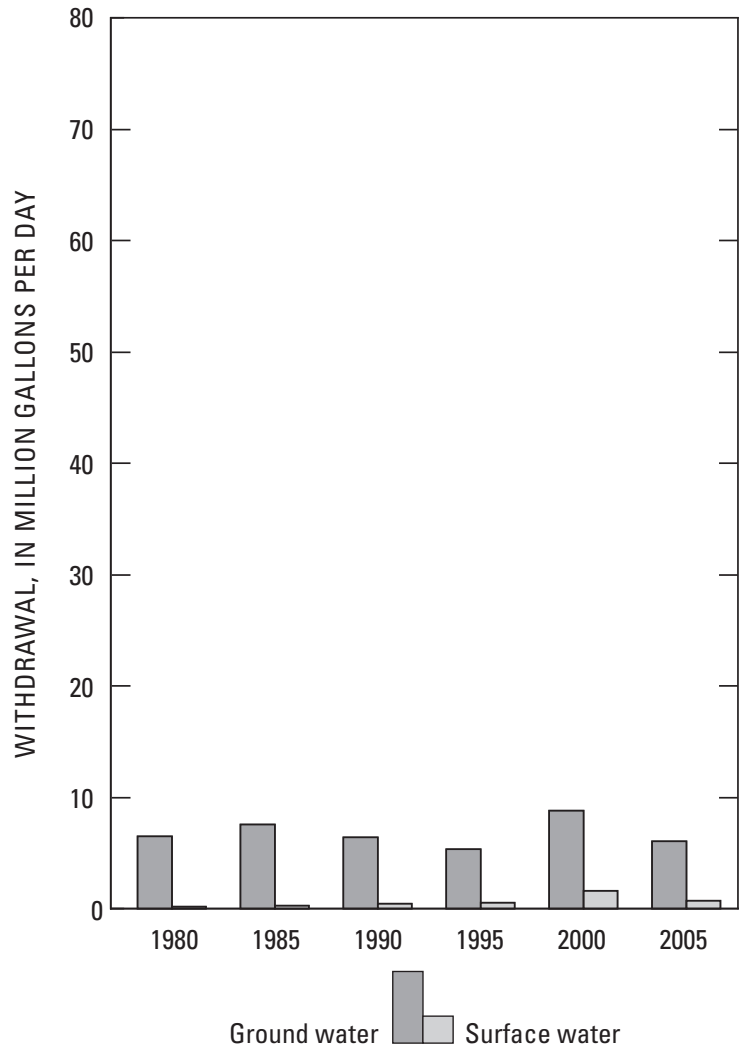

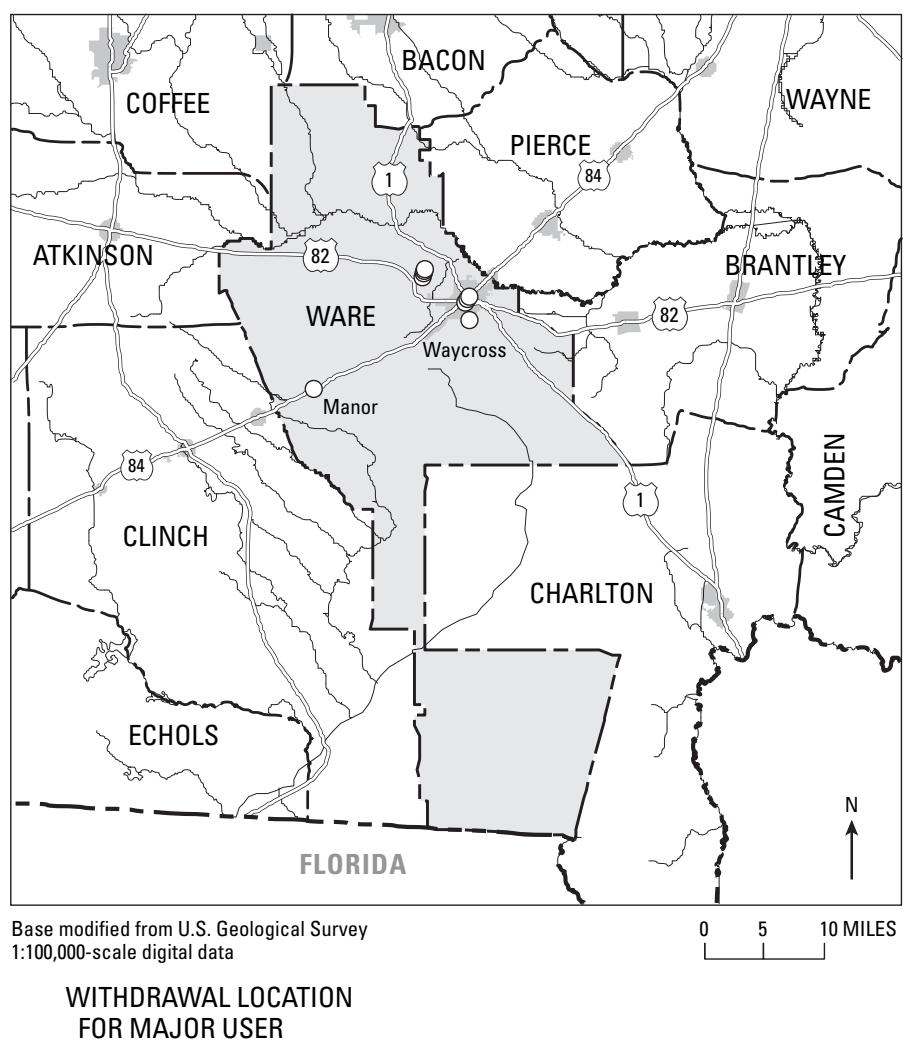




\section{WARREN COUNTY}

Population: 6,101

Population served by public supply: 3,420

Acres irrigated: 4,880

Hydroelectric use (Mgal/d): 0.00

2005 WITHDRAWALS, IN MILLION GALLONS PER DAY

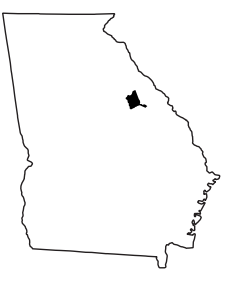

\begin{tabular}{|c|c|c|c|c|c|c|c|}
\hline & $\begin{array}{l}\text { Public } \\
\text { Supply }\end{array}$ & $\begin{array}{l}\text { Domestic \& } \\
\text { Commercial }\end{array}$ & $\begin{array}{c}\text { Industrial \& } \\
\text { Mining }\end{array}$ & Irrigation & Livestock & $\begin{array}{l}\text { Thermo- } \\
\text { electric }\end{array}$ & Totals \\
\hline Ground Water & 0.03 & 0.20 & 1.56 & 0.15 & 0.00 & 0.00 & 1.94 \\
\hline Surface Water & 0.36 & 0.00 & 0.61 & 1.59 & 0.10 & 0.00 & 2.66 \\
\hline TOTALS & 0.39 & 0.20 & 2.17 & 1.74 & 0.10 & 0.00 & 4.60 \\
\hline
\end{tabular}

Withdrawals by Major Public Suppliers (Mgal/d):

\section{Name}

Town of Camak

Town of Norwood

City of Warrenton
GW SW

$0.01 \quad 0.00$

$0.02 \quad 0.00$

$0.00 \quad 0.36$

Withdrawals by Major Industrial Groups (Mgal/d):

$\begin{array}{lcc}\text { SIC } & \text { GW } & \text { SW } \\ 32 \text { - Stone, clay } & 0.46 & 0.61\end{array}$
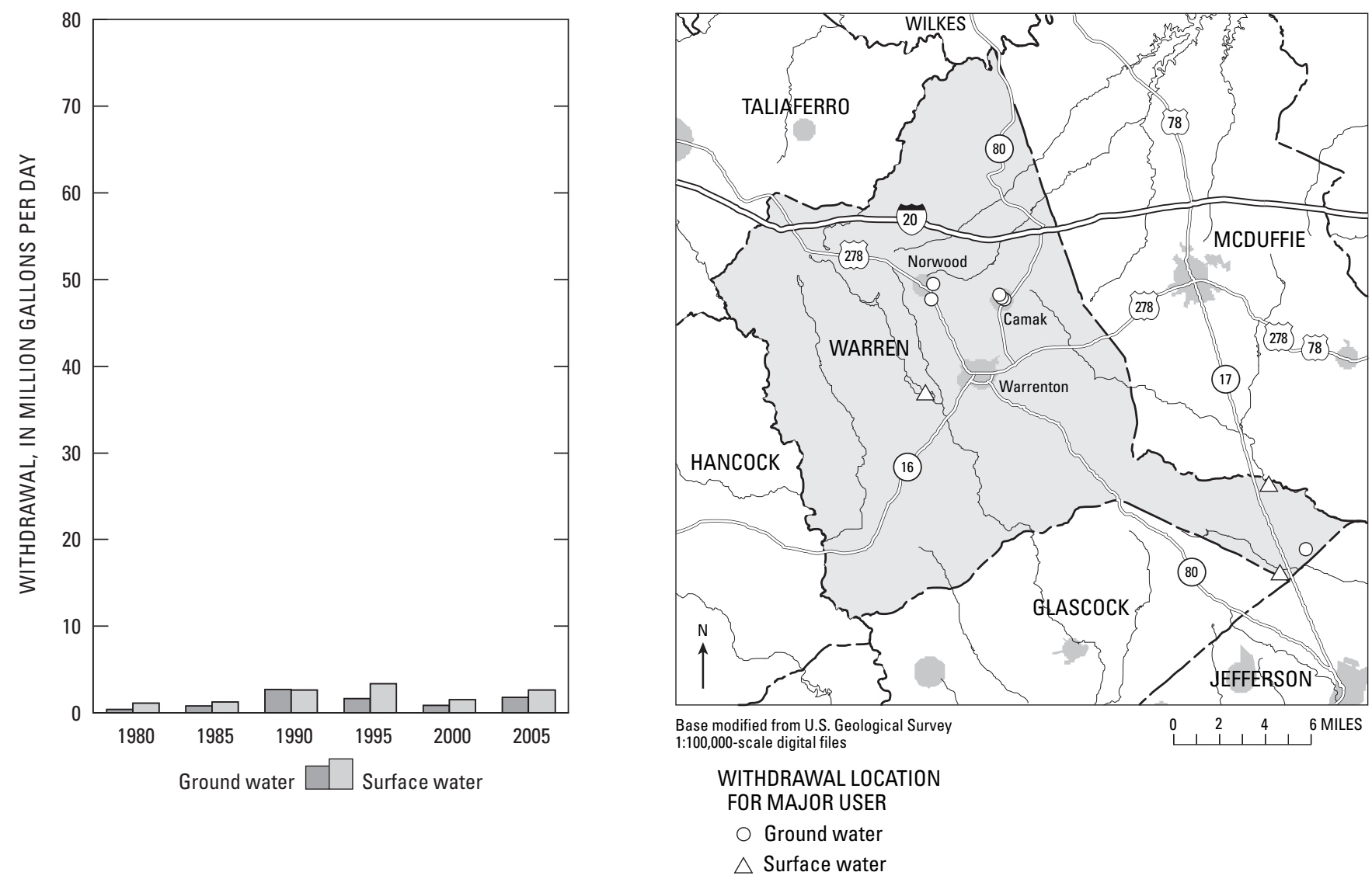


\section{WASHINGTON COUNTY}

Population: 20,118

Population served by public supply: 10,830

Acres irrigated: 7,720

Hydroelectric use (Mgal/d): $\quad 0.00$

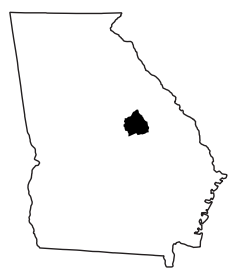

2005 WITHDRAWALS, IN MILLION GALLONS PER DAY

\begin{tabular}{|c|c|c|c|c|c|c|c|}
\hline & $\begin{array}{l}\text { Public } \\
\text { Supply }\end{array}$ & $\begin{array}{l}\text { Domestic \& } \\
\text { Commercial }\end{array}$ & $\begin{array}{c}\text { Industrial \& } \\
\text { Mining }\end{array}$ & Irrigation & Livestock & $\begin{array}{l}\text { Thermo- } \\
\text { electric }\end{array}$ & Totals \\
\hline Ground Water & 3.24 & 0.70 & 26.06 & 4.67 & 0.03 & 0.00 & 34.70 \\
\hline Surface Water & 0.00 & 0.00 & 0.00 & 0.92 & 0.11 & 0.00 & 1.03 \\
\hline TOTALS & 3.24 & 0.70 & 26.06 & 5.59 & 0.14 & 0.00 & 35.73 \\
\hline
\end{tabular}

Withdrawals by Major Public Suppliers (Mgal/d):

Name

GW SW

City of Davisboro

$0.18 \quad 0.00$

Town of Deepstep

0.50

0.00

Town of Harrison

0.07

0.04

0.03

2.03

0.36

0.03

Warthen Water Association

Town of Riddleville

City of Sandersville

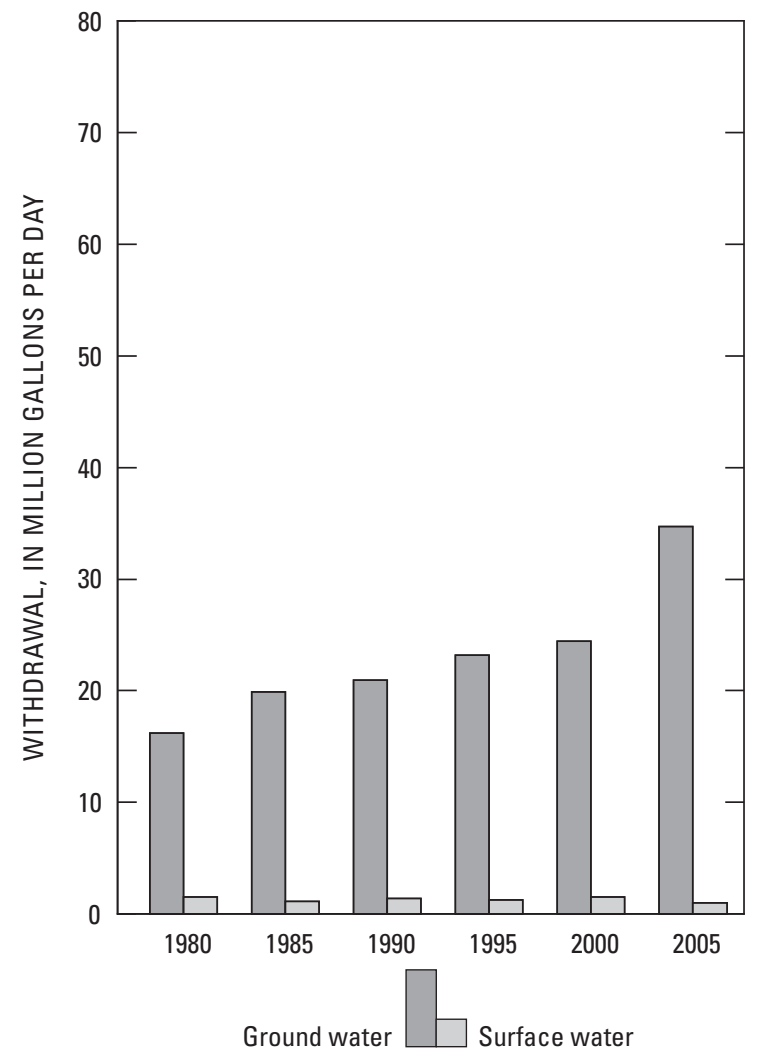

Withdrawals by Major Industrial Groups (Mgal/d):

$\begin{array}{lcc}\text { SIC } & \text { GW } & \text { SW } \\ 32 \text { - Stone, clay } & 18.45 & 0.00\end{array}$

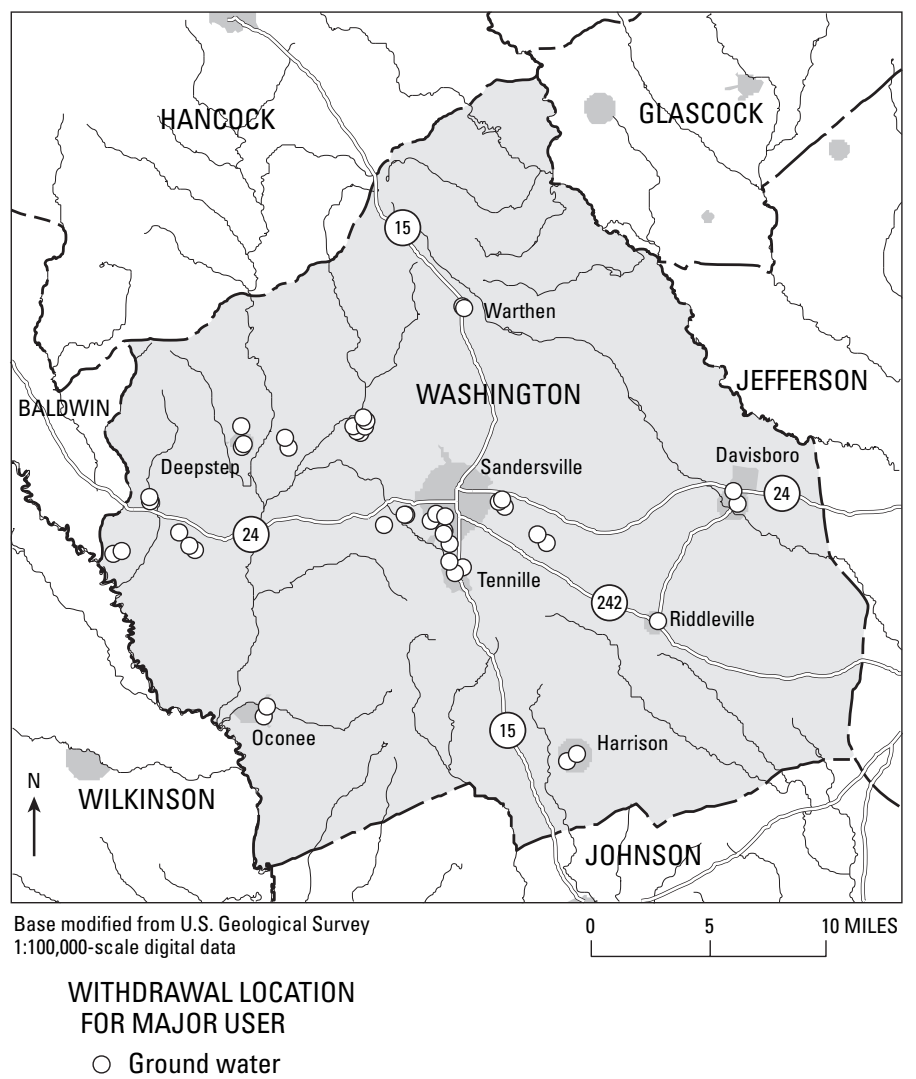




\section{WAYNE COUNTY}

Population: 28,390

Population served by public supply: $\quad 12,200$

Acres irrigated: $\quad 5,400$

Hydroelectric use (Mgal/d): $\quad 0.00$

2005 WITHDRAWALS, IN MILLION GALLONS PER DAY

\begin{tabular}{|c|c|c|c|c|c|c|c|}
\hline & $\begin{array}{l}\text { Public } \\
\text { Supply }\end{array}$ & $\begin{array}{l}\text { Domestic \& } \\
\text { Commercial }\end{array}$ & $\begin{array}{c}\text { Industrial \& } \\
\text { Mining }\end{array}$ & Irrigation & Livestock & $\begin{array}{l}\text { Thermo- } \\
\text { electric }\end{array}$ & Totals \\
\hline Ground Water & 2.09 & 1.21 & 59.23 & 2.04 & 0.01 & 0.00 & 64.58 \\
\hline Surface Water & 0.00 & 0.00 & 0.00 & 0.56 & 0.08 & 0.00 & 0.64 \\
\hline TOTALS & 2.09 & 1.21 & 59.23 & 2.60 & 0.09 & 0.00 & 65.22 \\
\hline
\end{tabular}

Withdrawals by Major Public Suppliers (Mgal/d):

Name

City of Jesup

Town of Odum

City of Screven
GW SW

$1.79 \quad 0.00$

$0.06 \quad 0.00$

$0.09 \quad 0.00$
Withdrawals by Major Industrial Groups (Mgal/d):

SIC

28 - Chemicals

GW

59.15

SW

0.00
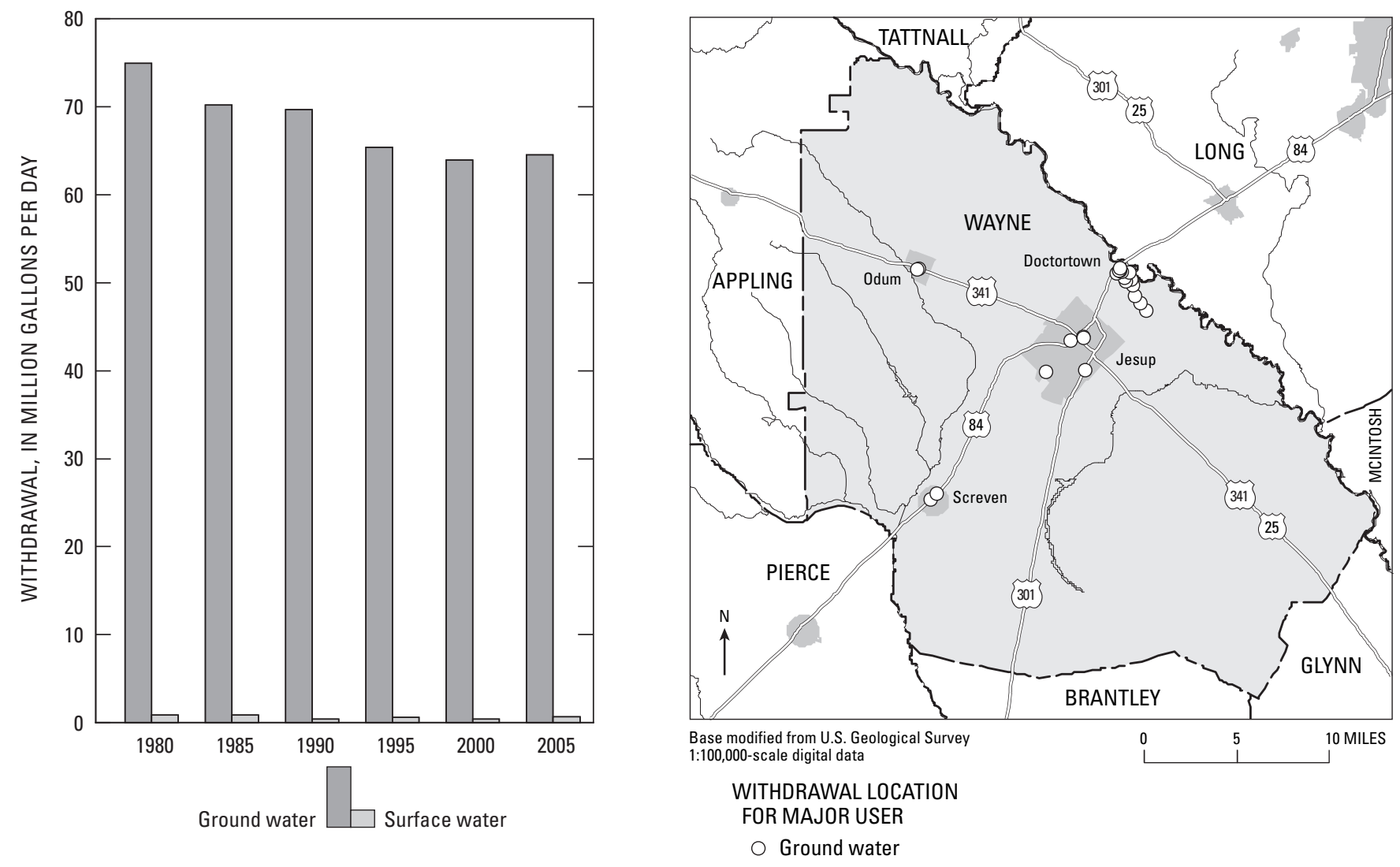


\section{WEBSTER COUNTY}

Population: 2,289

Population served by public supply: 1,240

Acres irrigated: 10,200

Hydroelectric use (Mgal/d): $\quad 0.00$

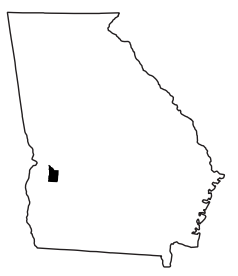

2005 WITHDRAWALS, IN MILLION GALLONS PER DAY

\begin{tabular}{|c|c|c|c|c|c|c|c|}
\hline & $\begin{array}{l}\text { Public } \\
\text { Supply }\end{array}$ & $\begin{array}{l}\text { Domestic \& } \\
\text { Commercial }\end{array}$ & $\begin{array}{c}\text { Industrial \& } \\
\text { Mining }\end{array}$ & Irrigation & Livestock & $\begin{array}{l}\text { Thermo- } \\
\text { electric }\end{array}$ & Totals \\
\hline Ground Water & 0.17 & 0.08 & 0.00 & 0.29 & 0.00 & 0.00 & 0.54 \\
\hline Surface Water & 0.00 & 0.00 & 0.00 & 2.35 & 0.05 & 0.00 & 2.40 \\
\hline TOTALS & 0.17 & 0.08 & 0.00 & 2.64 & 0.05 & 0.00 & 2.94 \\
\hline
\end{tabular}

Withdrawals by Major Public Suppliers (Mgal/d):

Name

City of Preston

Town of Weston
GW

0.15

0.02
SW

0.00

0.00
Withdrawals by Major Industrial Groups (Mgal/d):

SIC

None
GW

SW
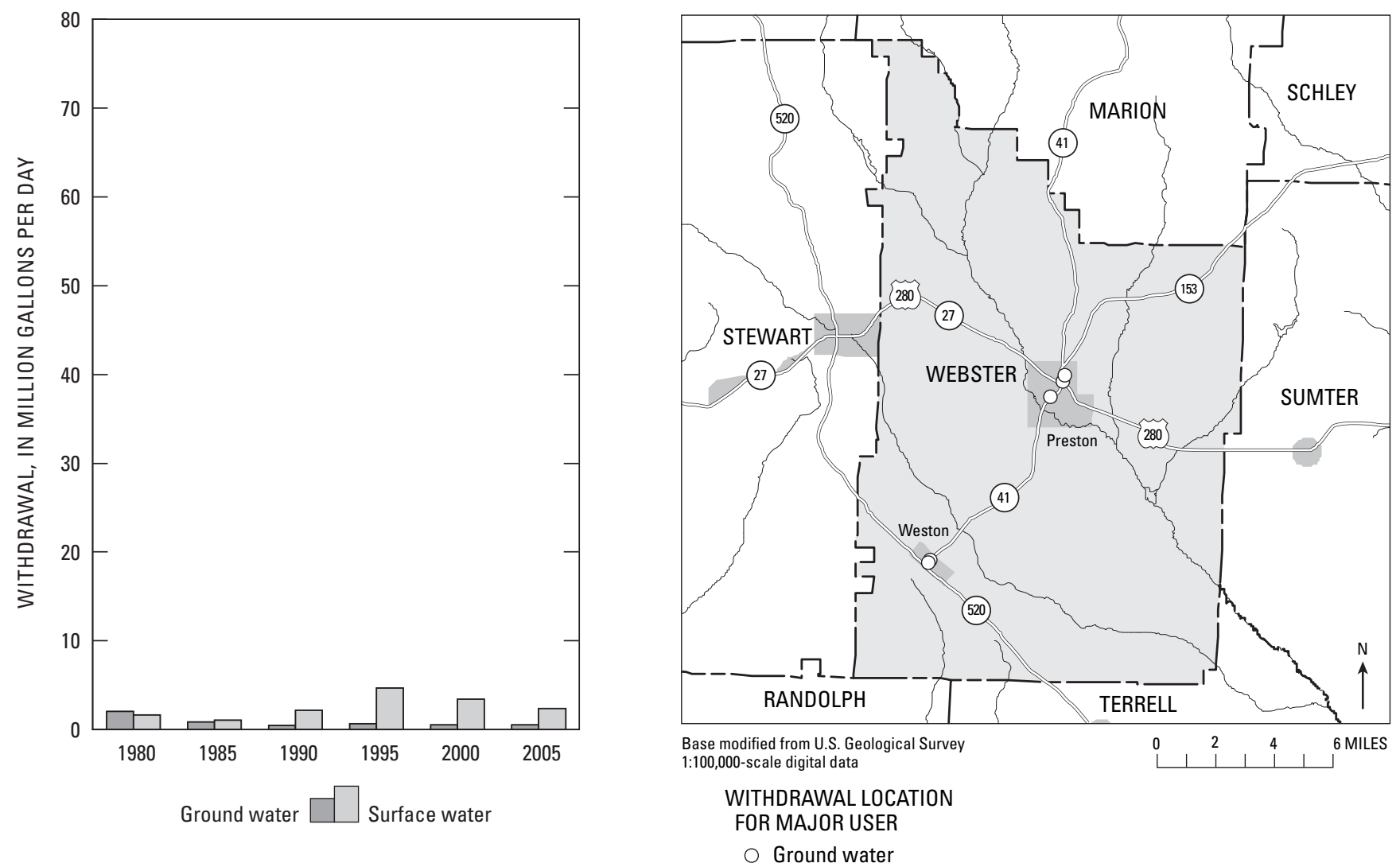


\section{WHEELER COUNTY}

Population: 6,706

Population served by public supply: 2,200

Acres irrigated: 7,540

Hydroelectric use (Mgal/d): $\quad 0.00$

2005 WITHDRAWALS, IN MILLION GALLONS PER DAY

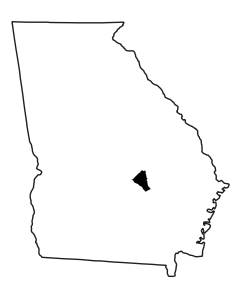

\begin{tabular}{|c|c|c|c|c|c|c|c|}
\hline & $\begin{array}{l}\text { Public } \\
\text { Supply }\end{array}$ & $\begin{array}{l}\text { Domestic \& } \\
\text { Commercial }\end{array}$ & $\begin{array}{c}\text { Industrial \& } \\
\text { Mining }\end{array}$ & Irrigation & Livestock & $\begin{array}{l}\text { Thermo- } \\
\text { electric }\end{array}$ & Totals \\
\hline Ground Water & 0.31 & 0.34 & 0.00 & 0.98 & 0.01 & 0.00 & 1.64 \\
\hline Surface Water & 0.00 & 0.00 & 0.00 & 3.19 & 0.02 & 0.00 & 3.21 \\
\hline TOTALS & 0.31 & 0.34 & 0.00 & 4.17 & 0.03 & 0.00 & 4.85 \\
\hline
\end{tabular}

Withdrawals by Major Public Suppliers (Mgal/d):

Name

City of Alamo

City of Glenwood
SW

0.00

0.00
Withdrawals by Major Industrial Groups (Mgal/d):

SIC

None
SW
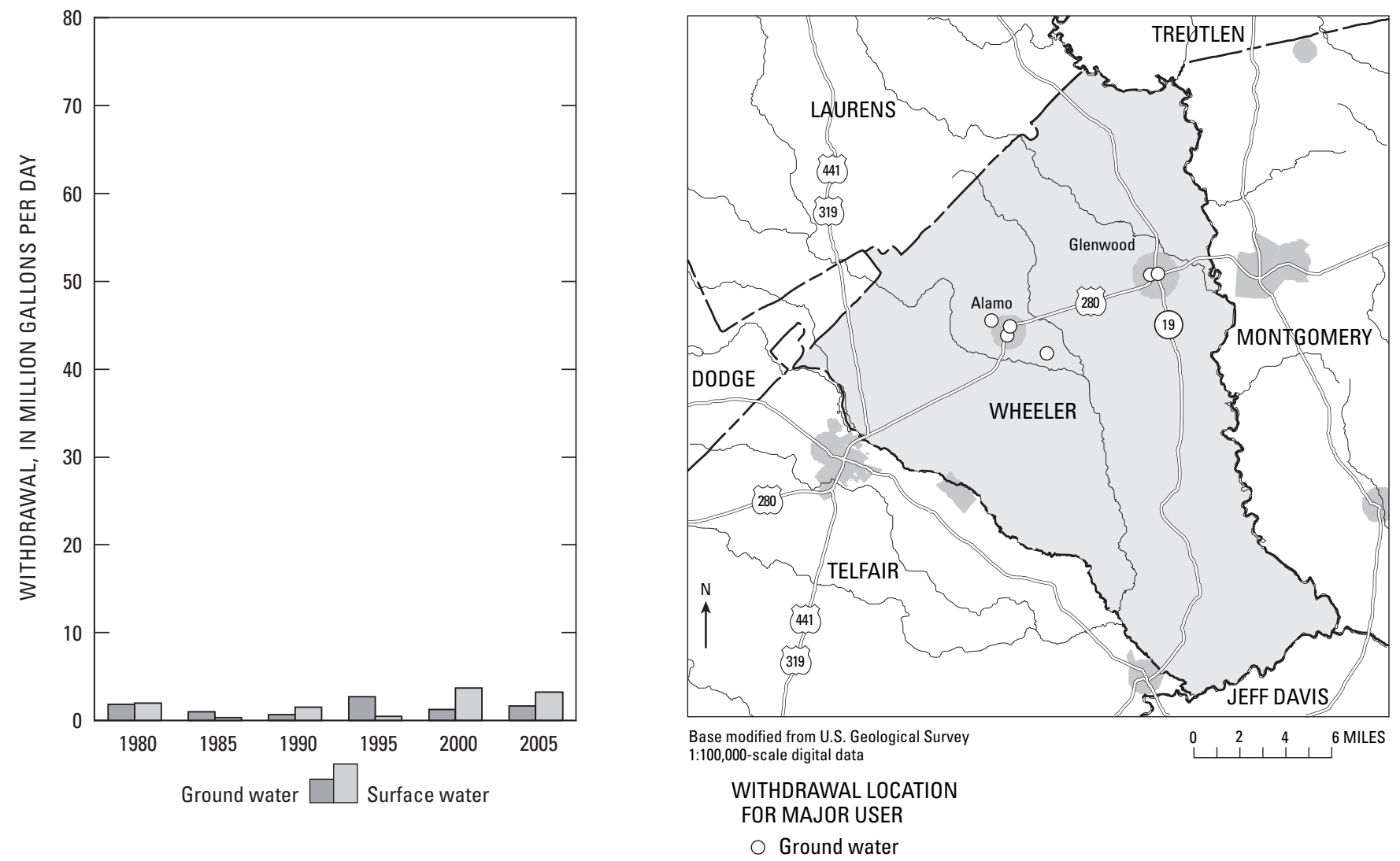


\section{WHITE COUNTY}

Population: 24,055

Population served by public supply: $\quad 12,630$

Acres irrigated: 220

Hydroelectric use (Mgal/d): $\quad 0.00$

2005 WITHDRAWALS, IN MILLION GALLONS PER DAY

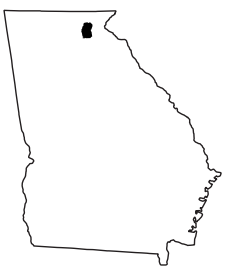

\begin{tabular}{lccccccc}
\hline & $\begin{array}{c}\text { Public } \\
\text { Supply }\end{array}$ & $\begin{array}{c}\text { Domestic \& } \\
\text { Commercial }\end{array}$ & $\begin{array}{c}\text { Industrial \& } \\
\text { Mining }\end{array}$ & Irrigation & Livestock & \multicolumn{1}{c}{$\begin{array}{c}\text { Thermo- } \\
\text { electric }\end{array}$} & Totals \\
\cline { 1 - 4 } Ground Water & 0.45 & 1.10 & 0.00 & 0.00 & 0.00 & 0.00 & 1.55 \\
Surface Water & 1.44 & 0.00 & 0.01 & 0.29 & 0.37 & 0.00 & 2.11 \\
TOTALS & 1.89 & 1.10 & 0.01 & 0.29 & 0.37 & 0.00 & 3.66 \\
\hline
\end{tabular}

Withdrawals by Major Public Suppliers (Mgal/d):

Name

GW SW

White County Water

$\&$ Sewer Authority

City of Cleveland

City of Helen

City of Robertstown

$0.00 \quad 1.44$

$0.25 \quad 0.00$

$0.14 \quad 0.00$

$0.01 \quad 0.00$
Withdrawals by Major Industrial Groups (Mgal/d):

SIC GW SW
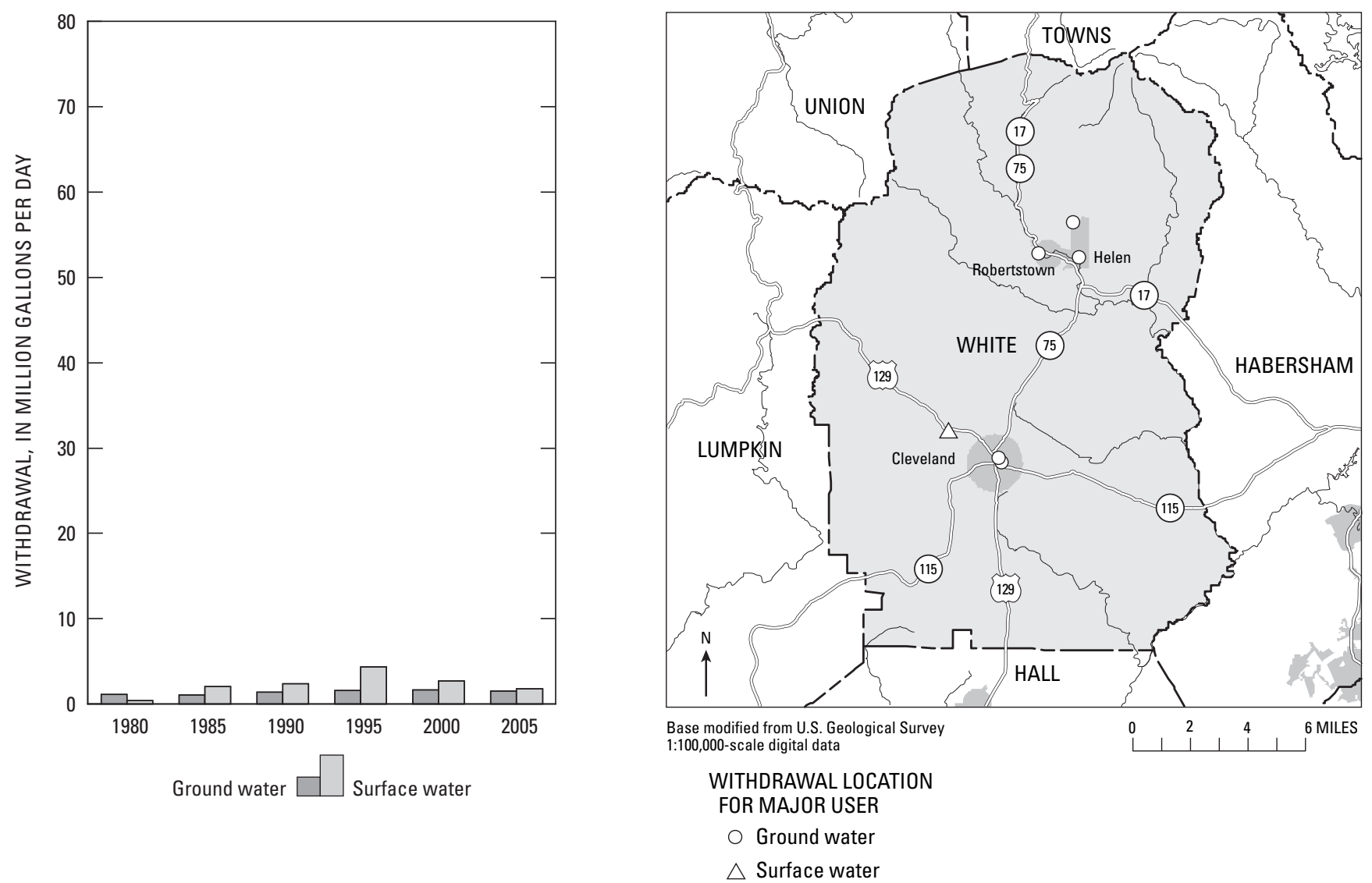


\section{WHITFIELD COUNTY}

Population: $\quad 90,889$

Population served by public supply: $\quad 89,980$

Acres irrigated: 350

Hydroelectric use (Mgal/d): $\quad 0.00$

2005 WITHDRAWALS, IN MILLION GALLONS PER DAY

\begin{tabular}{|c|c|c|c|c|c|c|c|}
\hline & $\begin{array}{l}\text { Public } \\
\text { Supply }\end{array}$ & $\begin{array}{l}\text { Domestic \& } \\
\text { Commercial }\end{array}$ & $\begin{array}{c}\text { Industrial \& } \\
\text { Mining }\end{array}$ & Irrigation & Livestock & $\begin{array}{l}\text { Thermo- } \\
\text { electric }\end{array}$ & Totals \\
\hline Ground Water & 0.00 & 0.07 & 0.15 & 0.00 & 0.11 & 0.00 & 0.33 \\
\hline Surface Water & 35.38 & 0.00 & 0.07 & 0.51 & 0.60 & 0.00 & 36.56 \\
\hline TOTALS & 35.38 & 0.07 & 0.22 & 0.51 & 0.71 & 0.00 & 36.89 \\
\hline
\end{tabular}

Withdrawals by Major Public Suppliers (Mgal/d):

Name GW SW

Dalton Utilities
Withdrawals by Major Industrial Groups (Mgal/d):

SIC

None

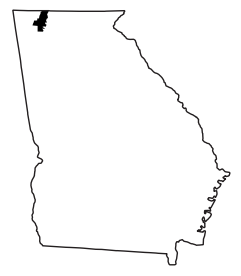

SW
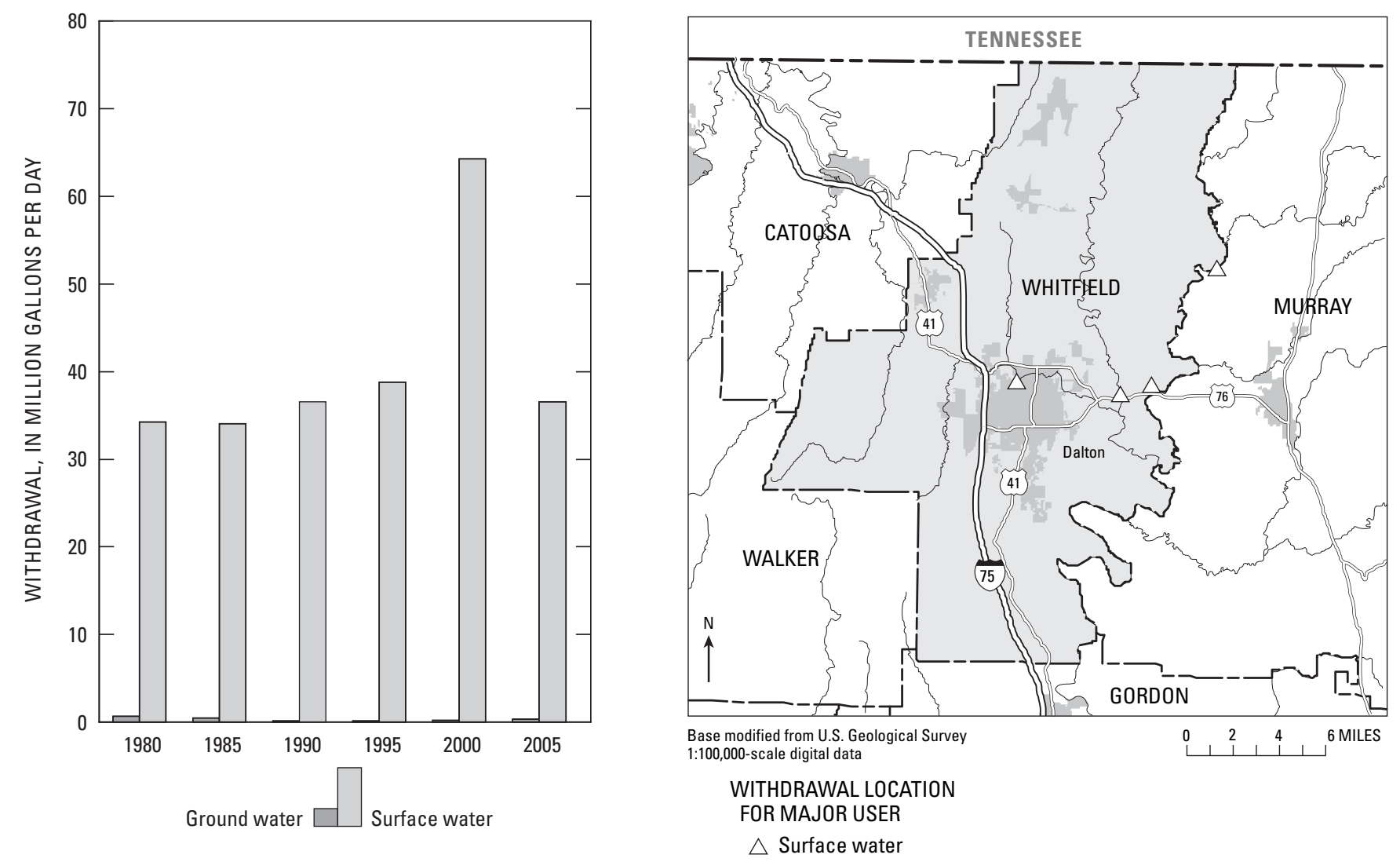


\section{WILCOX COUNTY}

Population: 8,721

Population served by public supply: $\quad 4,950$

Acres irrigated: 26,320

Hydroelectric use (Mgal/d): $\quad 0.00$

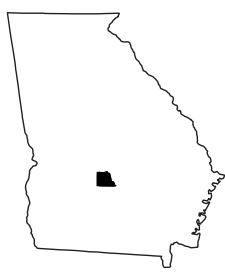

2005 WITHDRAWALS, IN MILLION GALLONS PER DAY

\begin{tabular}{|c|c|c|c|c|c|c|c|}
\hline & $\begin{array}{l}\text { Public } \\
\text { Supply }\end{array}$ & $\begin{array}{l}\text { Domestic \& } \\
\text { Commercial }\end{array}$ & $\begin{array}{c}\text { Industrial \& } \\
\text { Mining }\end{array}$ & Irrigation & Livestock & $\begin{array}{l}\text { Thermo- } \\
\text { electric }\end{array}$ & Totals \\
\hline Ground Water & 0.69 & 0.28 & 0.00 & 8.93 & 0.03 & 0.00 & 9.93 \\
\hline Surface Water & 0.00 & 0.00 & 0.00 & 2.49 & 0.16 & 0.00 & 2.65 \\
\hline TOTALS & 0.69 & 0.28 & 0.00 & 11.42 & 0.19 & 0.00 & 12.58 \\
\hline
\end{tabular}

Withdrawals by Major Public Suppliers (Mgal/d):

Name

GW SW

City of Abbeville

0.28

0.00

$0.08 \quad 0.00$

Town of Pineview

City of Pitts

City of Rochelle

Town of Seville
0.06

0.25

0.02
Withdrawals by Major Industrial Groups (Mgal/d):

SIC

None
GW

SW
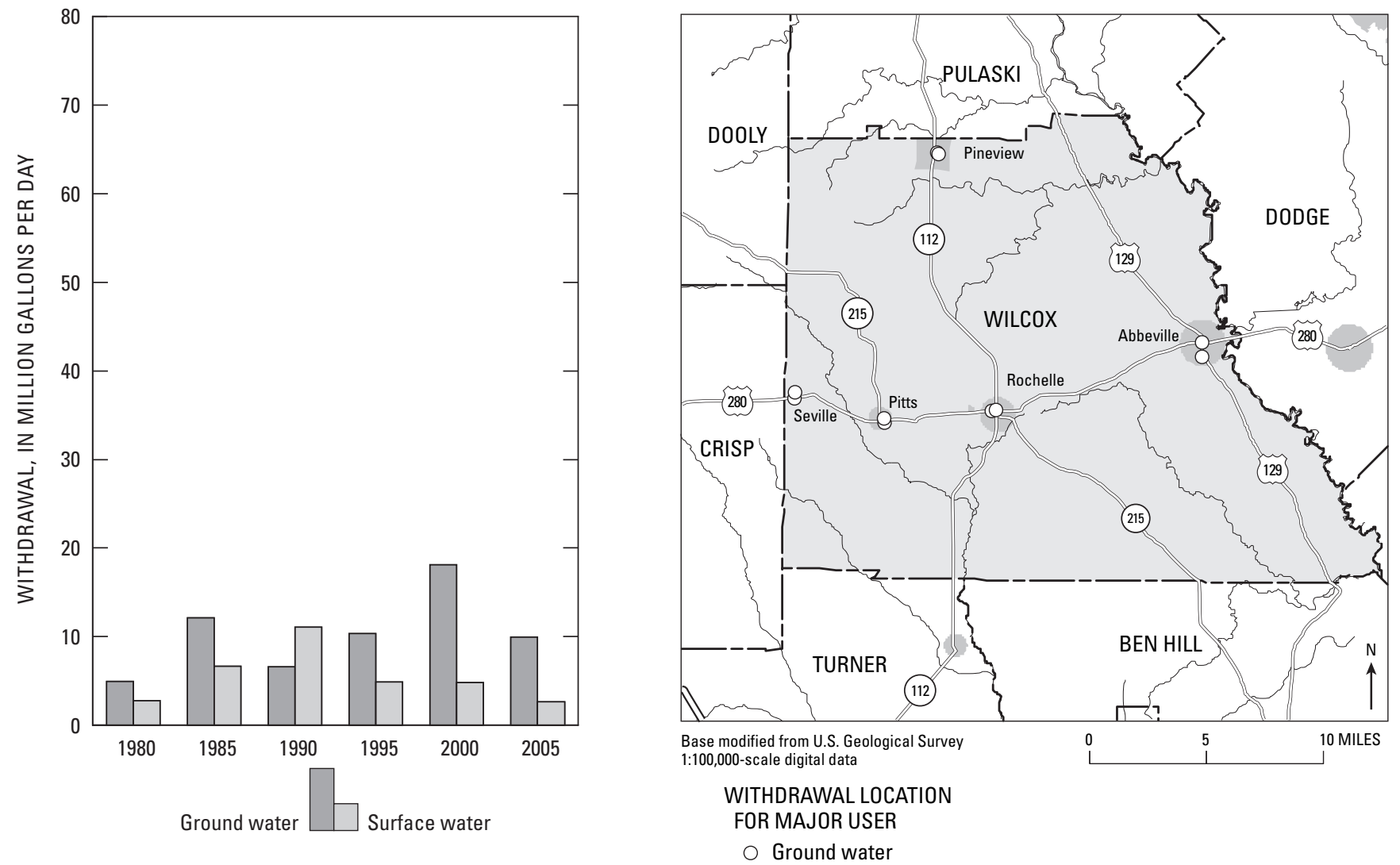


\section{WILKES COUNTY}

Population: 10,457

Population served by public supply: $\quad 5,130$

Acres irrigated: 50

Hydroelectric use (Mgal/d): $\quad 0.00$

2005 WITHDRAWALS, IN MILLION GALLONS PER DAY

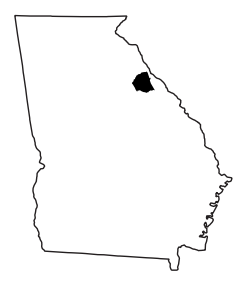

\begin{tabular}{|c|c|c|c|c|c|c|c|}
\hline & $\begin{array}{l}\text { Public } \\
\text { Supply }\end{array}$ & $\begin{array}{l}\text { Domestic \& } \\
\text { Commercial }\end{array}$ & $\begin{array}{c}\text { Industrial \& } \\
\text { Mining }\end{array}$ & Irrigation & Livestock & $\begin{array}{l}\text { Thermo- } \\
\text { electric }\end{array}$ & Totals \\
\hline Ground Water & 0.12 & 0.40 & 0.00 & 0.00 & 0.00 & 0.00 & 0.52 \\
\hline Surface Water & 1.08 & 0.00 & 0.00 & 0.02 & 0.32 & 0.00 & 1.42 \\
\hline TOTALS & 1.20 & 0.40 & 0.00 & 0.02 & 0.32 & 0.00 & 1.94 \\
\hline
\end{tabular}

Withdrawals by Major Public Suppliers (Mgal/d):

Name

Rayle Water Association

City of Tignall

City of Washington
GW

0.02

0.10

0.00
SW 0.00 0.00

1.09
Withdrawals by Major Industrial Groups (Mgal/d):

SIC

None
SW
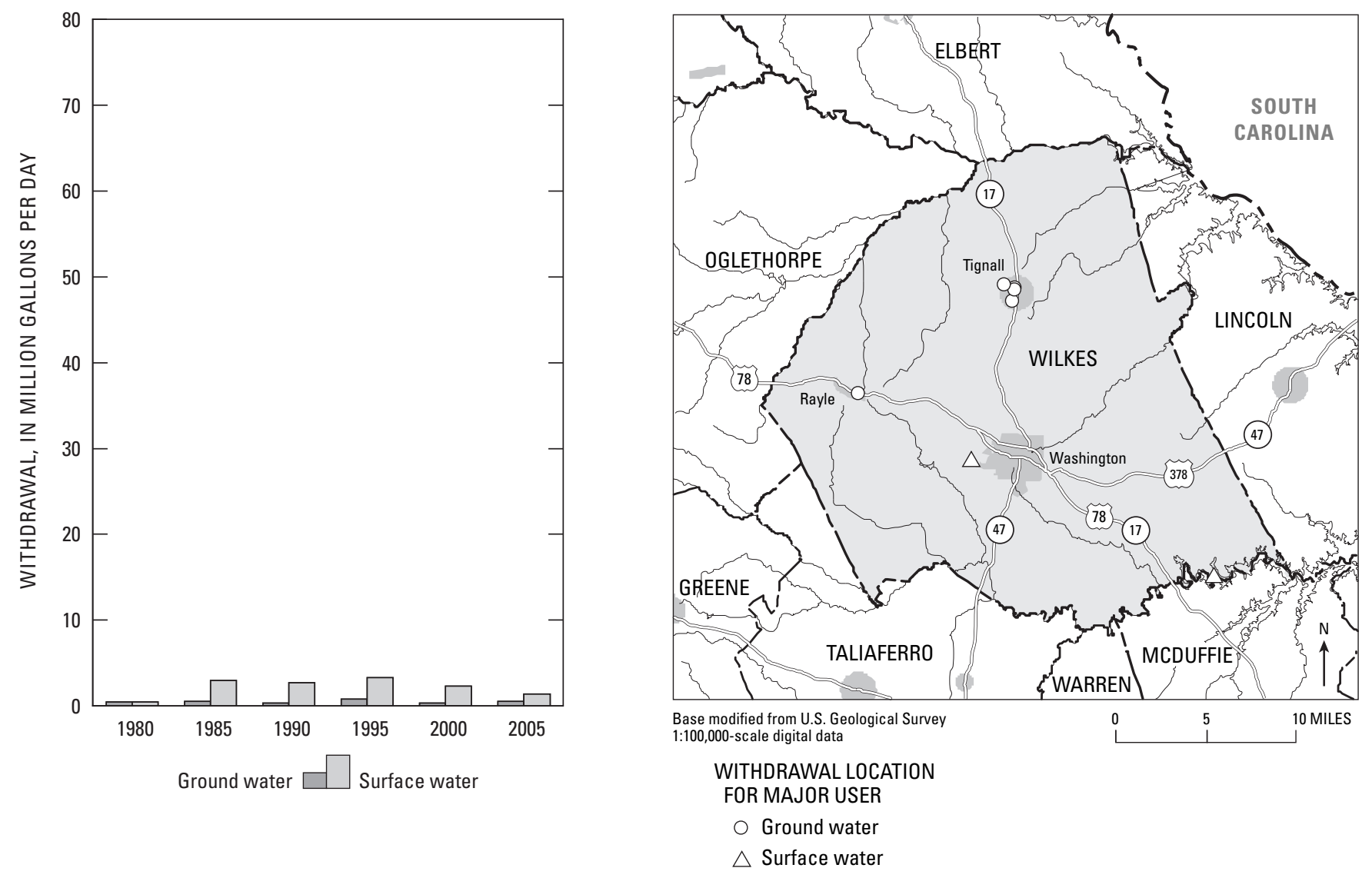


\section{WILKINSON COUNTY}

Population: 10,143

Population served by public supply: 7,050

Acres irrigated: 150

Hydroelectric use (Mgal/d): $\quad 0.00$

2005 WITHDRAWALS, IN MILLION GALLONS PER DAY

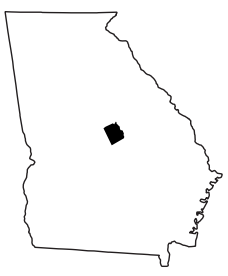

\begin{tabular}{lccccccrr}
\hline & $\begin{array}{c}\text { Public } \\
\text { Supply }\end{array}$ & $\begin{array}{c}\text { Domestic \& } \\
\text { Commercial }\end{array}$ & $\begin{array}{c}\text { Industrial \& } \\
\text { Mining }\end{array}$ & Irrigation & Livestock & \multicolumn{2}{c}{$\begin{array}{c}\text { Thermo- } \\
\text { electric }\end{array}$} & Totals \\
\cline { 2 - 5 } & 0.93 & 0.23 & 17.19 & 0.06 & 0.01 & 0.00 & 18.42 \\
Ground Water & 0.00 & 0.00 & 0.08 & 0.02 & 0.01 & 0.00 & 0.11 \\
Surface Water & 0.93 & 0.23 & 17.27 & 0.08 & 0.02 & 0.00 & 18.53 \\
\hline
\end{tabular}

Withdrawals by Major Public Suppliers (Mgal/d):

Name

Town of Allentown

Town of Gordon

Town of Irwinton

Town of Ivey

Town of McIntyre

Town of Toomsboro
GW

0.05

0.27

0.21

0.18

0.12

0.10
SW

0.00

0.00

0.00

0.00

0.00

0.00

Withdrawals by Major Industrial Groups (Mgal/d):

\section{SIC}

14 - Mining

32 - Stone, clay
GW

2.24

13.35
SW

0.00

0.08
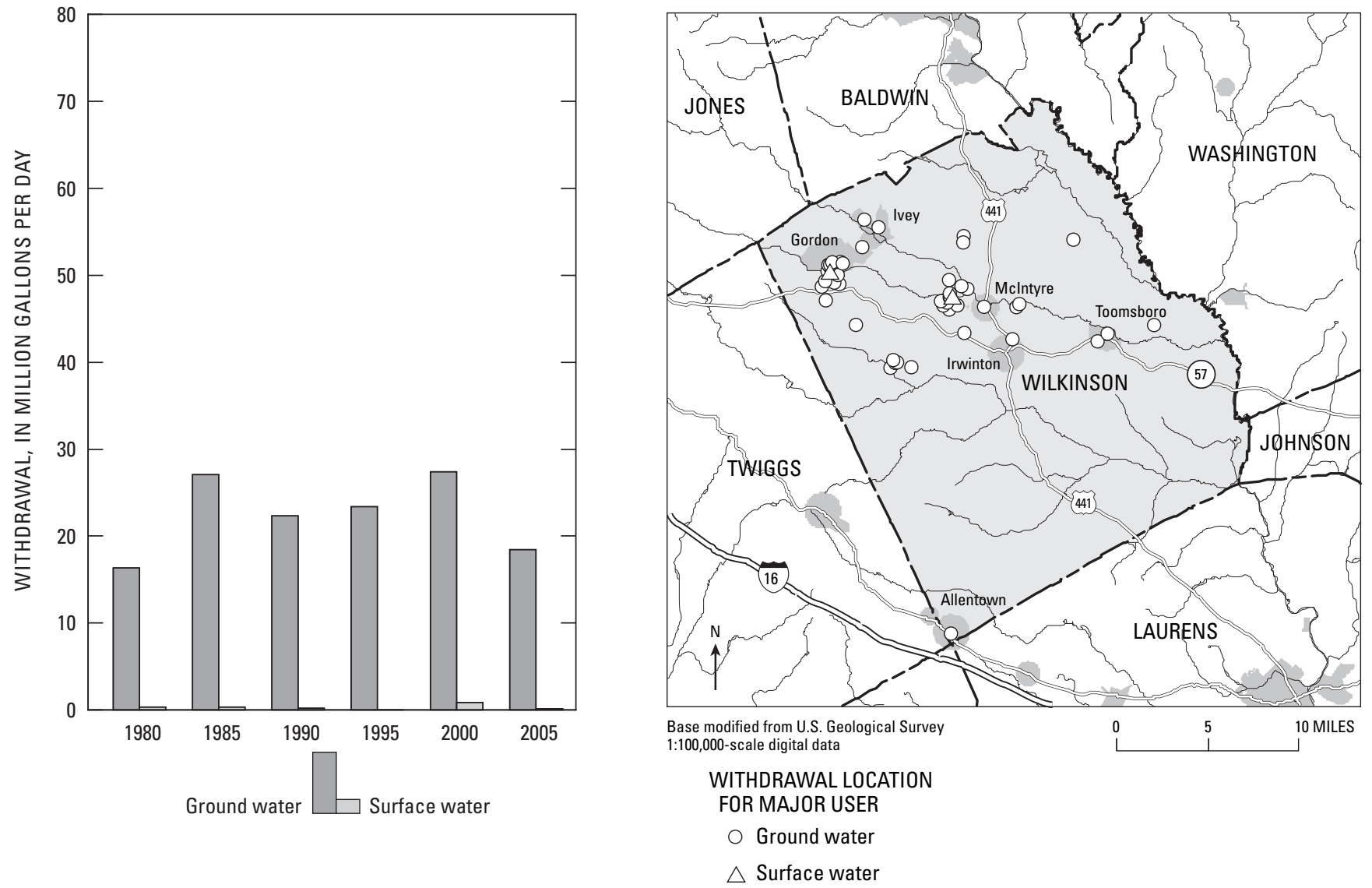


\section{WORTH COUNTY}

Population: 21,996

Population served by public supply: $\quad 10,090$

Acres irrigated: 46,670

Hydroelectric use (Mgal/d): $\quad 1,907.60$

2005 WITHDRAWALS, IN MILLION GALLONS PER DAY

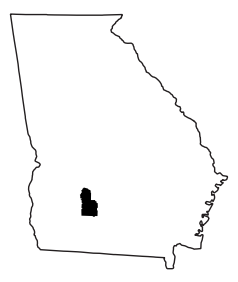

\begin{tabular}{|c|c|c|c|c|c|c|c|}
\hline & $\begin{array}{l}\text { Public } \\
\text { Supply }\end{array}$ & $\begin{array}{l}\text { Domestic \& } \\
\text { Commercial }\end{array}$ & $\begin{array}{c}\text { Industrial \& } \\
\text { Mining }\end{array}$ & Irrigation & Livestock & $\begin{array}{l}\text { Thermo- } \\
\text { electric }\end{array}$ & Totals \\
\hline Ground Water & 1.34 & 0.89 & 0.00 & 7.42 & 0.04 & 0.00 & 9.69 \\
\hline Surface Water & 0.00 & 0.00 & 0.00 & 3.24 & 0.10 & 0.76 & 4.10 \\
\hline TOTALS & 1.34 & 0.89 & 0.00 & 10.66 & 0.14 & 0.76 & 13.79 \\
\hline
\end{tabular}

Withdrawals by Major Public Suppliers (Mgal/d):

Name

Isabella Water System

GW

0.05

0.17

0.03

0.96

0.06

SW

0.00

0.00

Town of Sumner

City of Sylvester Water \& Light

City of Warwick
Withdrawals by Major Industrial Groups (Mgal/d):

SIC

GW

SW
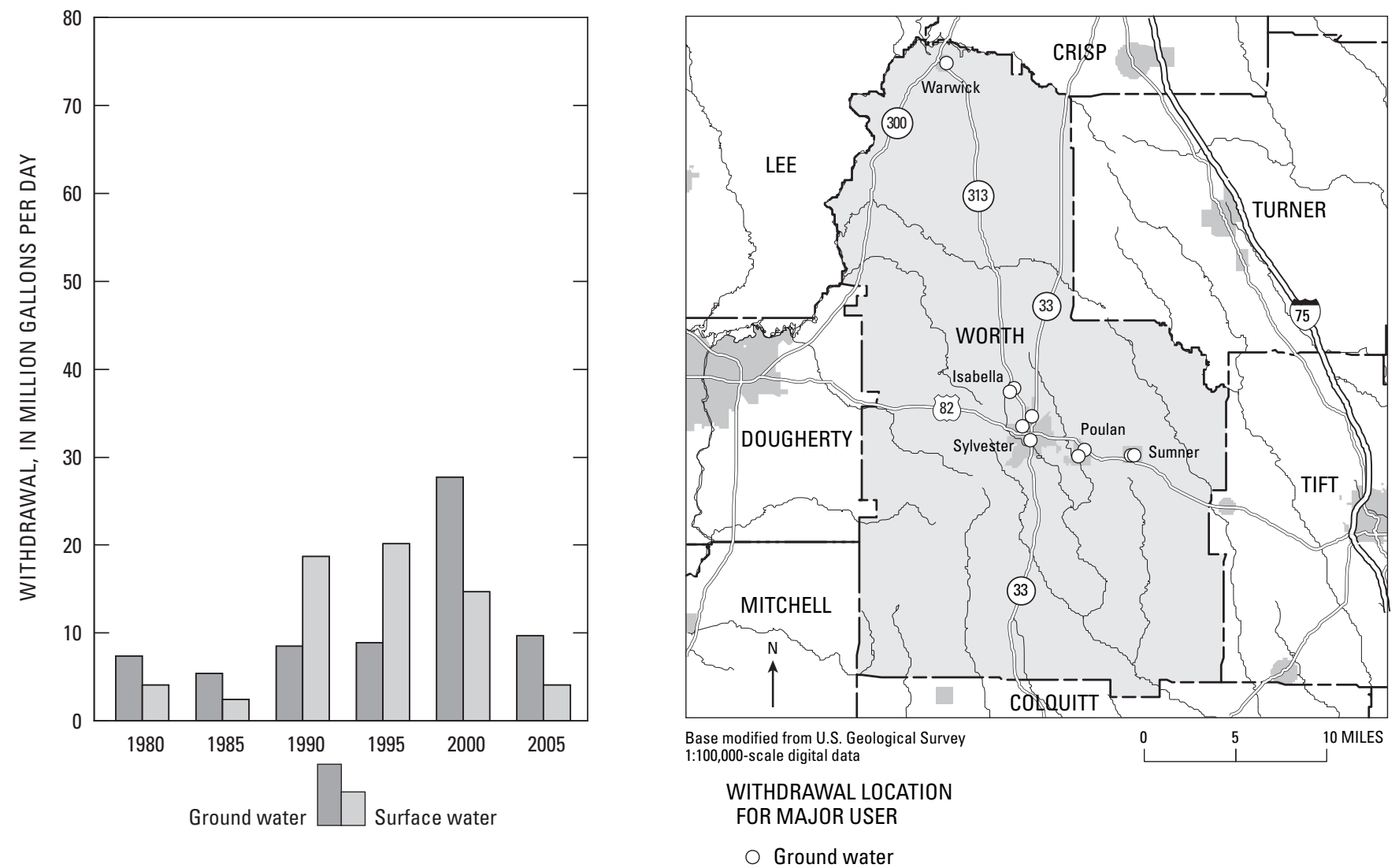
Appendix C. Water Withdrawal and Water Use by County in Georgia, 2005 (County of Source Withdrawal Location) 
Appendix C. Water withdrawal and water use by county in Georgia, 2005 (county of source withdrawal location).

[Values in shaded column are taken from table 6 and are repeated here for reference]

\begin{tabular}{|c|c|c|c|c|c|c|c|c|}
\hline County & \multicolumn{8}{|c|}{ Withdrawals, in million gallons per day } \\
\hline Appling & 0.93 & 0.93 & 0.85 & 0.00 & 3.09 & 0.38 & 57.77 & 63.02 \\
\hline Atkinson & 0.42 & 0.42 & 0.32 & 0.15 & 6.70 & 0.28 & 0.00 & 7.87 \\
\hline Baker & 0.11 & 0.11 & 0.25 & 0.00 & 33.31 & 0.72 & 0.00 & 34.39 \\
\hline Baldwin & 6.75 & 6.75 & 0.04 & 0.23 & 0.53 & 0.08 & 0.00 & 7.63 \\
\hline Banks & 2.01 & 0.69 & 0.93 & 0.00 & 0.15 & 0.69 & 0.00 & 3.78 \\
\hline Barrow & 5.01 & 5.01 & 2.10 & 0.54 & 0.63 & 0.33 & 0.00 & 8.61 \\
\hline Bibb & 0.14 & 25.53 & 1.85 & 15.57 & 1.06 & 0.06 & 0.00 & 18.68 \\
\hline Bleckley & 0.21 & 0.21 & 0.58 & 0.00 & 12.78 & 0.05 & 0.00 & 13.62 \\
\hline Brantley & 0.20 & 0.20 & 1.00 & 1.44 & 0.11 & 0.08 & 0.00 & 2.83 \\
\hline Brooks & 0.96 & 0.96 & 0.83 & 0.13 & 2.40 & 0.27 & 0.00 & 4.59 \\
\hline Bryan & 1.73 & 1.73 & 0.82 & 0.00 & 0.59 & 0.01 & 0.00 & 3.15 \\
\hline Bulloch & 4.01 & 4.01 & 1.37 & 0.03 & 11.65 & 0.23 & 0.00 & 17.29 \\
\hline Burke & 1.11 & 1.11 & 1.12 & 0.05 & 15.94 & 0.24 & 65.36 & 83.82 \\
\hline Butts & 2.93 & 2.93 & 0.32 & 0.00 & 0.21 & 0.04 & 0.00 & 3.50 \\
\hline Calhoun & 0.78 & 0.78 & 0.06 & 0.00 & 26.11 & 0.08 & 0.00 & 27.03 \\
\hline Chattahoochee & 1.03 & 1.03 & 6.24 & 0.00 & 0.15 & 0.01 & 0.00 & 7.43 \\
\hline Chattooga & 3.36 & 3.36 & 0.29 & 7.32 & 0.07 & 1.24 & 0.00 & 12.28 \\
\hline Cherokee & 18.94 & 18.94 & 5.18 & 2.52 & 2.38 & 0.33 & 0.00 & 29.35 \\
\hline Clarke & 6.53 & 6.53 & 0.02 & 0.00 & 0.93 & 0.09 & 0.00 & 7.57 \\
\hline Clay & 0.26 & 0.26 & 0.11 & 0.00 & 0.26 & 0.03 & 0.00 & 0.66 \\
\hline Clayton & 13.68 & 29.96 & 1.94 & 0.38 & 0.88 & 0.01 & 0.00 & 16.89 \\
\hline Clinch & 0.49 & 0.49 & 0.28 & 0.04 & 0.31 & 0.76 & 0.00 & 1.88 \\
\hline Cobb & 46.95 & 91.36 & 1.00 & 1.17 & 2.93 & 0.03 & 362.58 & 414.66 \\
\hline Coffee & 4.68 & 4.68 & 1.63 & 0.00 & 9.15 & 0.67 & 0.00 & 16.13 \\
\hline Colquitt & 3.84 & 3.84 & 1.47 & 0.33 & 30.76 & 0.35 & 0.00 & 36.75 \\
\hline Columbia & 12.55 & 12.55 & 0.98 & 0.89 & 1.13 & 0.03 & 0.00 & 15.58 \\
\hline Cook & 2.09 & 2.09 & 0.54 & 0.17 & 8.28 & 0.05 & 0.00 & 11.13 \\
\hline Coweta & 8.19 & 8.19 & 0.52 & 0.28 & 2.33 & 0.11 & 31.08 & 42.51 \\
\hline Crawford & 0.28 & 0.28 & 0.76 & 0.44 & 3.22 & 0.17 & 0.00 & 4.87 \\
\hline Crisp & 2.10 & 2.10 & 0.36 & 0.07 & 16.20 & 0.07 & 0.00 & 18.80 \\
\hline
\end{tabular}


Appendix C. Water withdrawal and water use by county in Georgia, 2005 (county of source withdrawal location).-Continued [Values in shaded column are taken from table 6 and are repeated here for reference]

\begin{tabular}{|c|c|c|c|c|c|c|c|c|}
\hline County & \multicolumn{8}{|c|}{ Withdrawals, in million gallons per day } \\
\hline Dade & 2.35 & 2.35 & 0.00 & 0.00 & 0.46 & 0.09 & 0.00 & 2.90 \\
\hline Dawson & 1.60 & 1.60 & 0.48 & 0.00 & 0.39 & 0.24 & 0.00 & 2.71 \\
\hline DeKalb & 0.00 & 83.05 & 3.05 & 0.97 & 1.77 & 0.00 & 0.00 & 5.79 \\
\hline Dodge & 0.72 & 0.72 & 0.92 & 0.00 & 17.44 & 0.85 & 0.00 & 19.93 \\
\hline Dooly & 2.21 & 2.21 & 0.30 & 0.00 & 23.59 & 0.19 & 0.00 & 26.29 \\
\hline Dougherty & 13.73 & 13.73 & 0.90 & 13.62 & 10.97 & 0.03 & 120.14 & 159.39 \\
\hline Effingham & 36.29 & 36.29 & 1.62 & 17.84 & 0.52 & 0.07 & 94.53 & 150.87 \\
\hline Elbert & 1.84 & 1.84 & 0.80 & 0.04 & 0.45 & 0.27 & 0.00 & 3.40 \\
\hline Emanuel & 2.03 & 2.03 & 0.76 & 0.98 & 2.70 & 0.19 & 0.00 & 6.66 \\
\hline Evans & 0.56 & 0.56 & 0.42 & 1.55 & 1.58 & 0.13 & 0.00 & 4.24 \\
\hline Fannin & 1.78 & 1.78 & 0.76 & 0.00 & 0.05 & 0.18 & 0.00 & 2.77 \\
\hline Fayette & 11.12 & 11.12 & 1.74 & 0.60 & 1.29 & 0.04 & 0.00 & 14.79 \\
\hline Floyd & 13.39 & 13.39 & 0.47 & 26.88 & 3.47 & 10.08 & 535.00 & 589.29 \\
\hline Forsyth & 17.13 & 17.13 & 3.15 & 1.15 & 0.24 & 6.85 & 0.00 & 28.52 \\
\hline Franklin & 1.93 & 1.93 & 0.83 & 0.00 & 0.26 & 1.14 & 0.00 & 4.16 \\
\hline Greene & 1.45 & 1.45 & 0.29 & 0.16 & 1.74 & 0.27 & 0.00 & 3.91 \\
\hline Gwinnett & 2.88 & 87.90 & 0.00 & 1.21 & 5.04 & 0.05 & 0.00 & 9.19 \\
\hline Habersham & 5.90 & 5.90 & 1.32 & 0.15 & 0.50 & 5.33 & 0.00 & 13.20 \\
\hline Hall & 103.37 & 18.37 & 2.29 & 1.48 & 0.77 & 0.80 & 0.00 & 108.71 \\
\hline Hancock & 1.02 & 1.02 & 0.26 & 0.12 & 0.04 & 0.04 & 0.00 & 1.48 \\
\hline Haralson & 2.36 & 2.36 & 0.02 & 0.00 & 0.85 & 0.13 & 0.00 & 3.36 \\
\hline Harris & 7.38 & 7.38 & 0.87 & 2.40 & 0.74 & 0.07 & 1.20 & 12.66 \\
\hline Hart & 1.31 & 1.31 & 1.18 & 0.11 & 0.53 & 0.65 & 0.00 & 3.78 \\
\hline Heard & 1.06 & 1.06 & 0.23 & 0.09 & 0.00 & 0.18 & 64.33 & 65.89 \\
\hline Henry & 33.19 & 16.91 & 0.76 & 0.63 & 0.27 & 0.10 & 0.00 & 34.95 \\
\hline Houston & 20.61 & 20.61 & 1.12 & 2.74 & 5.63 & 0.14 & 0.00 & 30.24 \\
\hline Irwin & 0.57 & 0.57 & 0.47 & 0.00 & 16.45 & 0.08 & 0.00 & 17.57 \\
\hline Jackson & 18.72 & 20.04 & 0.20 & 0.69 & 0.57 & 1.00 & 0.00 & 21.18 \\
\hline Jasper & 0.55 & 0.55 & 1.04 & 0.78 & 0.22 & 0.20 & 0.00 & 2.79 \\
\hline Jeff Davis & 0.44 & 0.44 & 0.51 & 0.33 & 4.08 & 0.22 & 0.00 & 5.58 \\
\hline
\end{tabular}


Appendix C. Water withdrawal and water use by county in Georgia, 2005 (county of source withdrawal location).-Continued [Values in shaded column are taken from table 6 and are repeated here for reference]

\begin{tabular}{|c|c|c|c|c|c|c|c|c|}
\hline County & \multicolumn{8}{|c|}{ Withdrawals, in million gallons per day } \\
\hline Jefferson & 1.51 & 1.51 & 0.60 & 7.58 & 9.40 & 1.43 & 0.00 & 20.52 \\
\hline Jenkins & 0.42 & 0.42 & 0.34 & 0.00 & 3.95 & 0.18 & 0.00 & 4.89 \\
\hline Jones & 26.51 & 1.12 & 1.08 & 1.70 & 0.25 & 0.10 & 0.00 & 29.64 \\
\hline Lamar & 1.31 & 1.31 & 0.68 & 0.00 & 0.73 & 0.18 & 0.00 & 2.90 \\
\hline Lanier & 0.45 & 0.45 & 0.35 & 0.12 & 1.23 & 0.02 & 0.00 & 2.17 \\
\hline Laurens & 3.94 & 3.94 & 1.64 & 16.90 & 7.50 & 0.14 & 0.00 & 30.12 \\
\hline Long & 1.49 & 1.49 & 0.58 & 0.46 & 0.40 & 0.05 & 0.00 & 2.98 \\
\hline Lowndes & 10.59 & 10.59 & 1.65 & 9.04 & 9.36 & 0.08 & 0.00 & 30.72 \\
\hline Lumpkin & 1.21 & 1.21 & 1.49 & 0.14 & 0.18 & 0.22 & 0.00 & 3.24 \\
\hline McDuffie & 2.61 & 2.61 & 0.67 & 0.00 & 2.43 & 0.14 & 0.00 & 5.85 \\
\hline McIntosh & 0.73 & 0.73 & 0.21 & 0.00 & 0.15 & 0.00 & 0.00 & 1.09 \\
\hline Macon & 1.59 & 1.59 & 0.44 & 10.57 & 18.26 & 0.61 & 0.00 & 31.47 \\
\hline Madison & 0.42 & 0.42 & 1.64 & 0.30 & 0.34 & 0.86 & 0.00 & 3.56 \\
\hline Marion & 1.91 & 1.91 & 0.49 & 0.29 & 0.49 & 0.09 & 0.00 & 3.27 \\
\hline Meriwether & 0.33 & 1.41 & 1.08 & 0.09 & 0.78 & 0.22 & 0.00 & 2.50 \\
\hline Muscogee & 32.22 & 32.22 & 0.00 & 0.47 & 1.18 & 0.02 & 0.00 & 33.89 \\
\hline Newton & 11.25 & 11.25 & 2.03 & 0.32 & 0.94 & 0.10 & 0.00 & 14.64 \\
\hline Oconee & 0.31 & 0.31 & 0.89 & 0.00 & 0.49 & 0.32 & 0.00 & 2.01 \\
\hline Oglethorpe & 0.22 & 0.22 & 0.84 & 0.00 & 0.34 & 0.59 & 0.00 & 1.99 \\
\hline Paulding & 3.11 & 3.11 & 1.19 & 0.24 & 0.11 & 0.10 & 0.00 & 4.75 \\
\hline Peach & 2.16 & 2.16 & 0.58 & 0.00 & 7.82 & 0.07 & 0.00 & 10.63 \\
\hline Pickens & 2.17 & 2.17 & 0.55 & 1.46 & 0.36 & 0.32 & 0.00 & 4.86 \\
\hline Pierce & 0.59 & 0.59 & 0.92 & 0.02 & 1.86 & 0.10 & 0.00 & 3.49 \\
\hline Pike & 0.15 & 4.49 & 0.94 & 0.00 & 0.29 & 0.20 & 0.00 & 1.58 \\
\hline Polk & 5.96 & 5.96 & 0.36 & 1.64 & 0.73 & 0.17 & 0.00 & 8.86 \\
\hline Pulaski & 0.85 & 0.85 & 0.29 & 0.65 & 14.91 & 0.04 & 0.00 & 16.74 \\
\hline Putnam & 1.21 & 1.21 & 0.59 & 0.07 & 2.89 & 0.39 & $1,092.00$ & $1,097.15$ \\
\hline Quitman & 0.14 & 0.14 & 0.04 & 0.00 & 0.02 & 0.01 & 0.00 & 0.21 \\
\hline Rabun & 1.97 & 1.97 & 0.33 & 1.75 & 0.58 & 0.07 & 0.00 & 4.70 \\
\hline Randolph & 0.96 & 0.96 & 0.15 & 0.06 & 9.20 & 0.05 & 0.00 & 10.42 \\
\hline
\end{tabular}


Appendix C. Water withdrawal and water use by county in Georgia, 2005 (county of source withdrawal location).-Continued [Values in shaded column are taken from table 6 and are repeated here for reference]

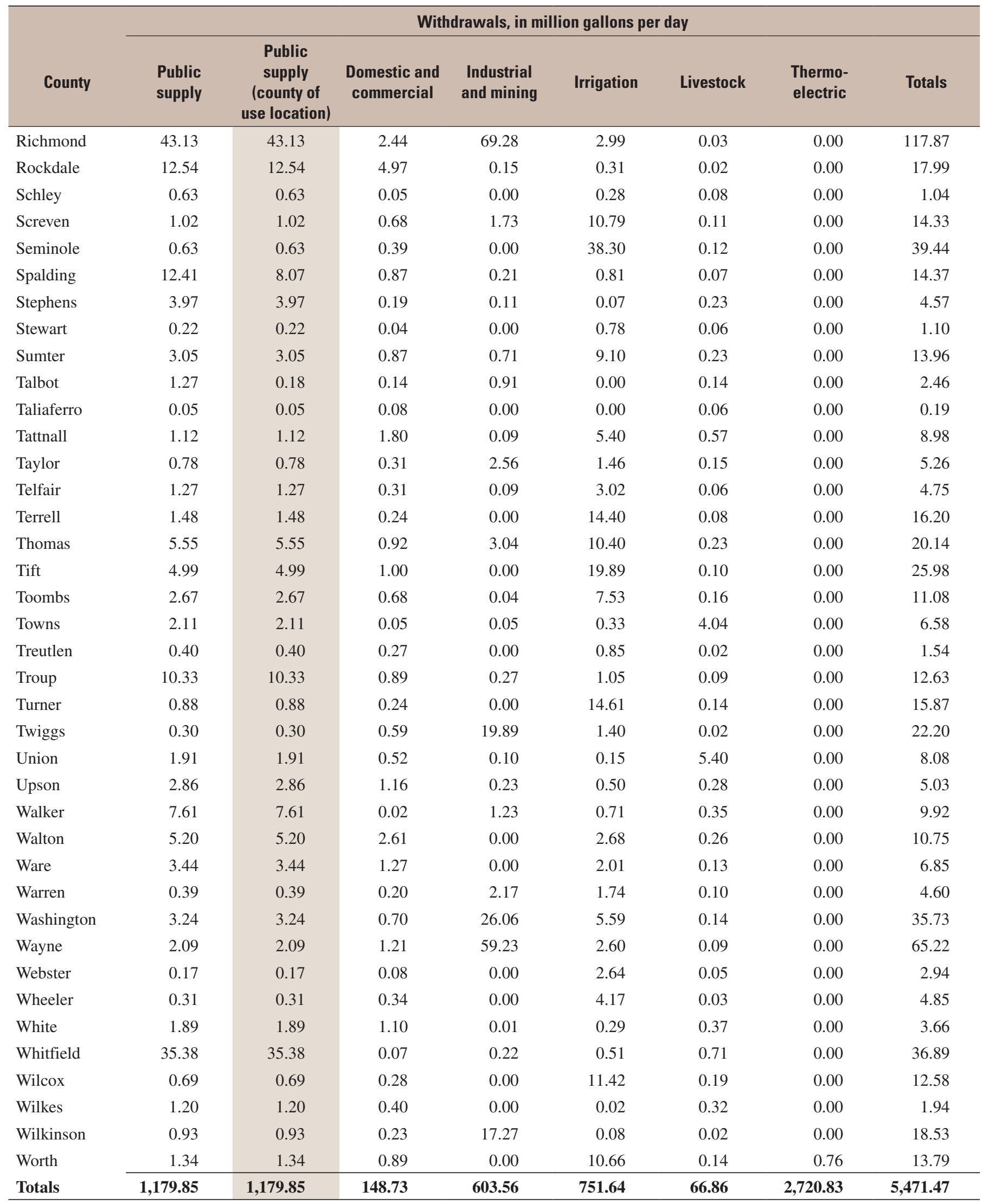


Manuscript approved for publication, December 17, 2008

Edited by Kay E. Hedrick

Graphics and layout by Caryl J. Wipperfurth and Bonnie J. Turcott

For more information concerning the research in this report, contact

USGS Georgia Water Science Center

3039 Amwiler Road

Atlanta, Georgia 30360

telephone: 770-903-9100

http://ga.water.usgs.gov 